\title{
The Arts-Integrated Curriculum (AIC) and its Possible Impact on the Self-Concepts of Adolescent Girls and Their Perceptions of Beauty: A Phenomenological Study
}

Miriam Roth Douglas
West Virginia University

Follow this and additional works at: https://researchrepository.wvu.edu/etd

\section{Recommended Citation}

Douglas, Miriam Roth, "The Arts-Integrated Curriculum (AIC) and its Possible Impact on the Self-Concepts of Adolescent Girls and Their Perceptions of Beauty: A Phenomenological Study" (2011). Graduate Theses, Dissertations, and Problem Reports. 3474.

https://researchrepository.wvu.edu/etd/3474

This Dissertation is protected by copyright and/or related rights. It has been brought to you by the The Research Repository @ WVU with permission from the rights-holder(s). You are free to use this Dissertation in any way that is permitted by the copyright and related rights legislation that applies to your use. For other uses you must obtain permission from the rights-holder(s) directly, unless additional rights are indicated by a Creative Commons license in the record and/ or on the work itself. This Dissertation has been accepted for inclusion in WVU Graduate Theses, Dissertations, and Problem Reports collection by an authorized administrator of The Research Repository @ WVU.

For more information, please contact researchrepository@mail.wvu.edu. 
The Arts-Integrated Curriculum (AIC) and its Possible Impact on the Self-Concepts of Adolescent Girls and Their Perceptions of Beauty:

A Phenomenological Study

Miriam Roth Douglas

Dissertation submitted to the

College of Human Resources \& Education

at West Virginia University

in partial fulfillment of the requirements

for the degree of

Doctor of Philosophy

in

Education

Joy Faini Saab, Ed.D., Chair

Cynthia Chalupa, Ph.D.

Chauncey, Goff, Ph.D.

Deborah Janson, Ph.D.

Sam Stack, Ph.D.

Pamela Whitehouse, Ed.D.

Department of Human Resources \& Education

Morgantown, West Virginia

2011

Keywords: Arts Integration, Arts-Integrated Curriculum (AIC), Self-Concept,

Adolescence, Girls, Perception of Beauty, Body Image, Phenomenology

Copyright 2011 Miriam Roth Douglas 


\section{Abstract \\ The Arts-Integrated Curriculum (AIC) and its Possible Impact on the Self-Concepts of Adolescent Girls and Their Perceptions of Beauty: A Phenomenological Study \\ Miriam Roth Douglas}

This study examines what phenomena may be associated with the self-concept and perceptions of beauty of adolescent girls who completed an Arts-Integrated Curriculum (AIC) in Elementary School. It is designed to add to the descriptive data on the perceptions of beauty that students, teachers, and parents have, especially with regard to girls' concept of self in relation to the AIC learning experiences. This work is driven by a qualitative research method known as phenomenology, which describes the lived experiences of a phenomenon for a group of people (Patton, 2005). The main research data were collected from interviews with ten participants: four students, three parents, two teachers, and a university liaison. Participants offered rich narratives that reflected their unique experiences. The interviews were driven by three minor research questions that helped to explore the main phenomena of the AIC experience: 1) How do young ladies (referring to adolescent girls) who completed an AIC describe their experience? 2) How do their parents describe it? 3) How do their teachers describe it? In addition to the interviews, the process of writing memos (memoing) and conducting follow-ups on the interviews and peer reviews, and referencing a related study from Devono (2009), support the triangulation of the data. The phenomena that were discovered are encouraging and indicate that participants report their perceptions of the importance of the arts and in AIC in the schools. Participants shared an interest in music and art, positive descriptions and characterizations of art, positive self-concept through arts, interdisciplinary learning, an appreciation for beautiful things, and a secure sense of self. The results of this study thus reveal personal implications reflecting the student perspective, societal-practical implications representing the parental and community perspective, and professional-intellectual implications that are relevant to the field of education, including schools' or teachers' perspectives. 


\section{Dedication}

Love many things,

for therein lies the true strength,

and whosoever loves much

performs much, and can accomplish much,

and what is done in LOVE

is done well.

Vincent van Gogh
Man soll lieben, so viel man kann, und darin liegt die wahre Stärke, und wer viel liebt, der tut auch viel und vermag viel, und was in LIEBE getan wird, das wird gut getan.

Vincent van Gogh

I thankfully dedicate this with LOVE to my family:

My parents Ernst and Gertrud,

my brother Alexander with Maris, Yannes and Luca,

and my husband Jarod.

Thanks for loving me! 


\section{Acknowledgements}

During the pursuit of my degree, it sometimes was not easy to have homes, families, and friends on two different continents. Nevertheless, it makes it a lot easier to be blessed with the most wonderful people on either side:

I would like to thank my doctoral committee, Dr. Cynthia Chalupa, Dr. Chauncey Goff, Dr. Deborah Janson, Dr. Samuel Stack, and Dr. Pamela Whitehouse. Special thanks goes to Dr. Joy Faini Saab my committee chair. Each of you contributed in your very special way to the success of this project. Dr. Chalupa, you always pointed me in the right direction when cultural and philosophical concepts got in my way. Dr. Goff, you truly knew how to make me think about the question of the "why" of this study. Dr. Janson, you understood to constantly provide support and constructive criticism. Dr. Stack, you sparked my interest in philosophical concepts and helped me to discover phenomenology as my research method. Dr. Whitehouse, you provided me with literature suggestions on qualitative research and women studies that make up the foundation of this work. Dr. Saab, you were all that a doctoral student could ask for in a committee chair and even more. You knew when to challenge, to encourage, or just to listen.

I would like to thank all my interviewees for their willingness to take the time to share their arts integration experiences with me. Special thanks goes to BUSY and BAND; MOTIVATED, INVOLVED, and Dr. S.; CARING, PIANO, and DRAWING; LOVING and FLUTE. Without your input, this study would not have been possible.

I would like to thank all my friends for being understanding and supportive throughout this whole process. Furthermore, I would like to thank those that helpfully contributed some of their time, knowledge, and effort toward this study. Jennifer V-K., you generously provided impeccable transcription services at an unparalleled speed. Jeffrey P., you lent your special talent of paying attention to detail by offering to edit this work. Angela R., you showed your gift of 
always being willing to help on a personal and professional level. Sandra S., you never stopped believing that I could achieve this goal of earning a doctorate.

I also would like to thank my family on this and the other side of the ocean for just being there for me, whenever I needed them.

Jim and Rita D., you were always so supportive and did not hesitate to help in any way. You and all the other family members are such a wonderful family to me. Jacqueline O., you always showed your interest and care for what I do. Emma H. and Irmgard S. (godmother), you and the roles you play in my life contributed immensely to what I have now achieved.

Special thanks goes to my parents Ernst und Gertrud R., for never ceasing to support, encourage, and believe in me. You are there for me at all times, you find time to listen no matter how busy you are, you would move mountains if you had to. It is so good to know that I am blessed with such wonderful and very special parents. There are just no words to thank you for what you have done and still do for me until this day. You have made me who I am today-this is your degree!

I also would like to thank my brother Alexander R. You have always been the perfect "big brother" to me that everyone could hope for. You always take care of me and make sure I am not getting hurt, no matter in which part of the world you are. I learned so much from you and would not be where I am today without you and your words of encouragement.

Finally, I would like to thank my most wonderful husband Jarod. Without his unending love, support, and understanding, it would not have been possible to put this dream into reality. You were there for me from the very beginning when the idea of this project was born. You accompanied me on a long and sometimes difficult path. Finally, you are on my side to share this big achievement. It takes a very special person, one who is willing to share success and disappointment - I am so thankful you are the one. 


\section{Table of Contents}

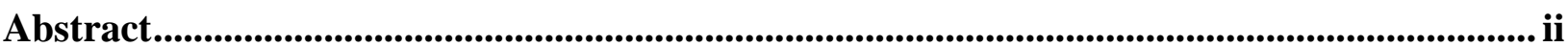

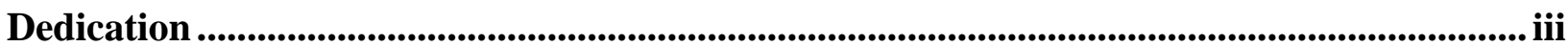

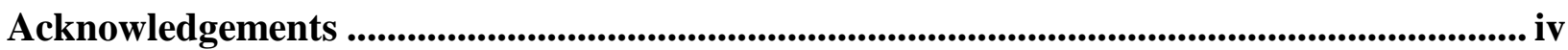

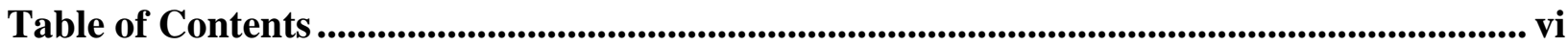

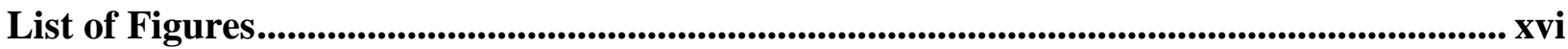

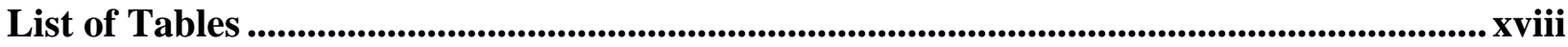

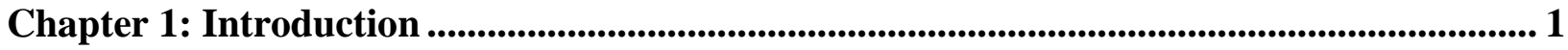

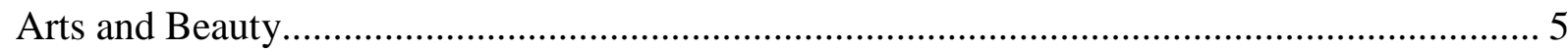

Ongoing change in arts and culture affects beauty standards (Dewey, 1934/1980/2005)..... 5

Changes and "multiple realities" of culture demand "wide-awakeness" (Greene, 1978)..... 6

Culturally complex signifying chains and (im)perfect body images (Butler, 1993). ............ 6

Standardization, instead of individualization, of culture (Eisner, 2002b).......................... 7

The comparison factor - an impossible-to-achieve image struggle (Bordo, 2003) ............... 9

The importance of the arts in public spaces and their support by political figures and

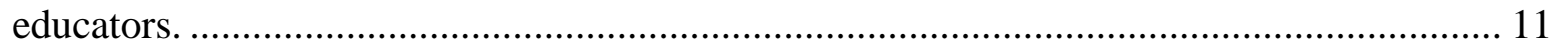

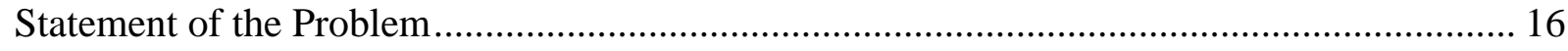

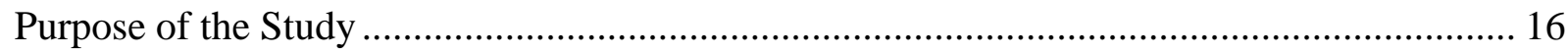

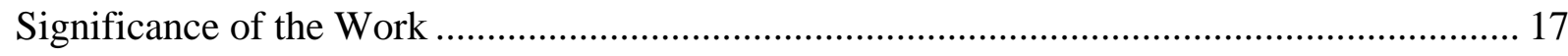

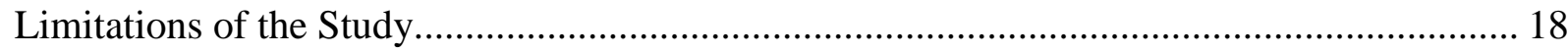

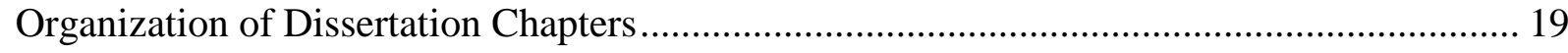

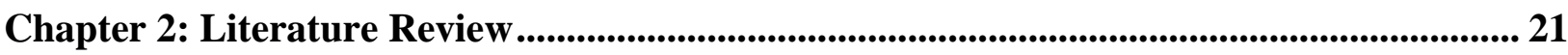




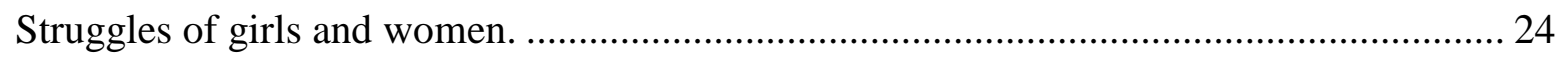

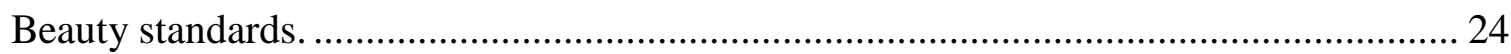

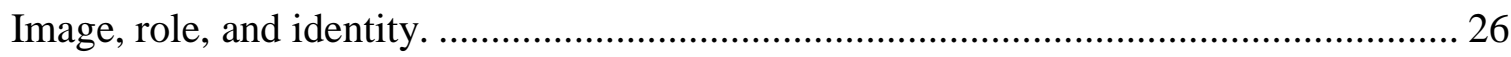

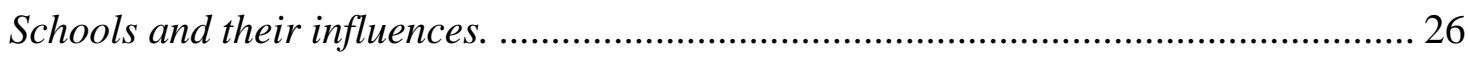

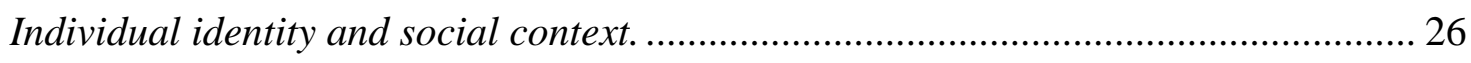

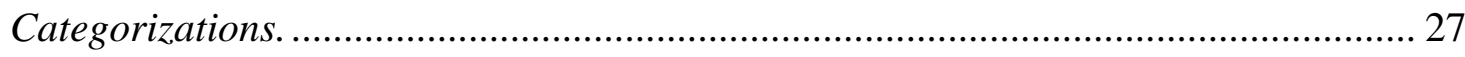

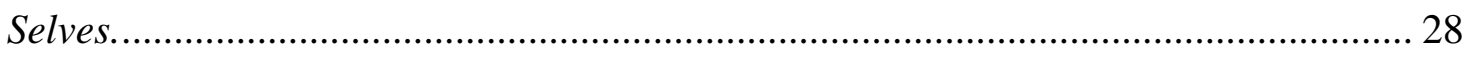

The male definition of beauty. ................................................................................ 29

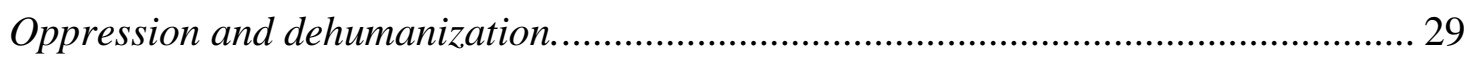

Males and their influences on the female image, role, and identity. ........................... 31

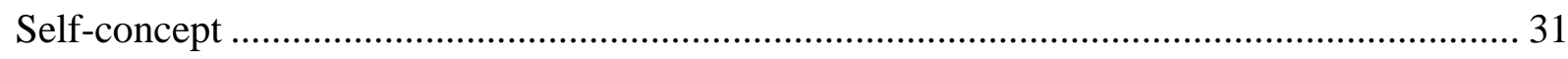

(Negative) self-concepts and (resulting) disorders. ................................................... 33

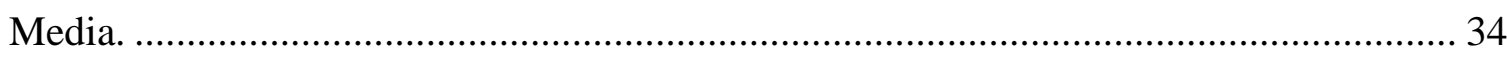

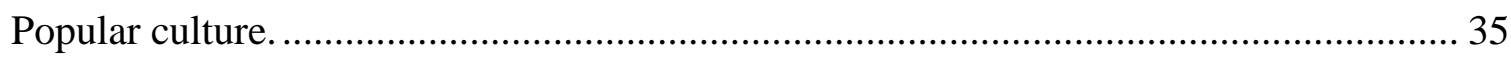

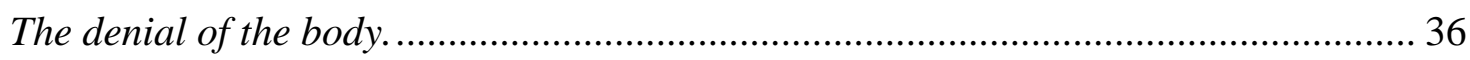

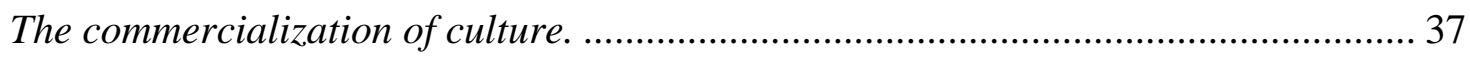

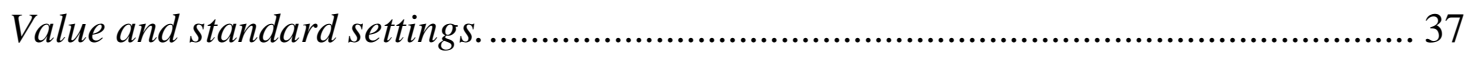

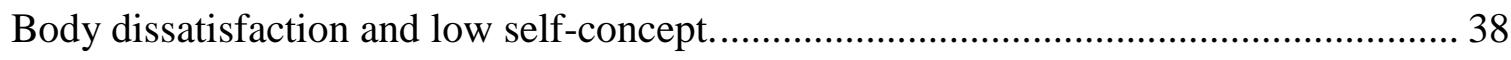

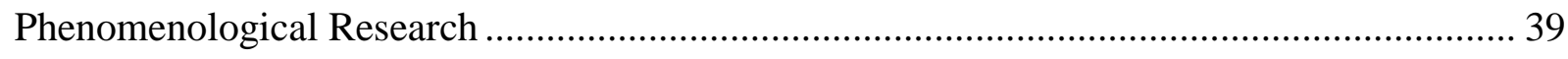

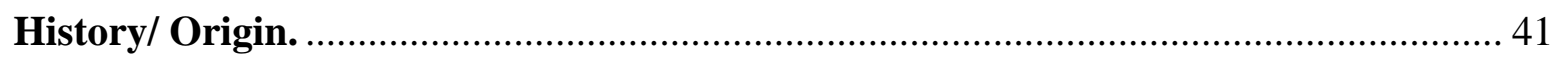


The phenomenological researcher.

The phenomenological analysis. 44

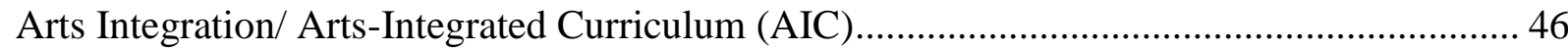

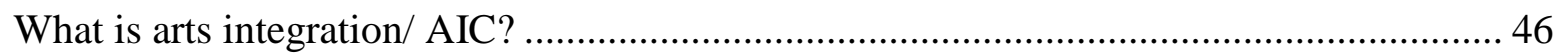

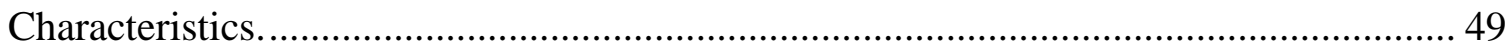

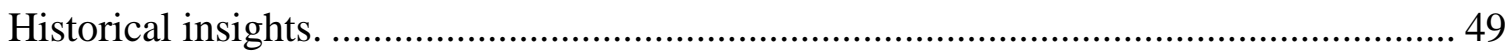

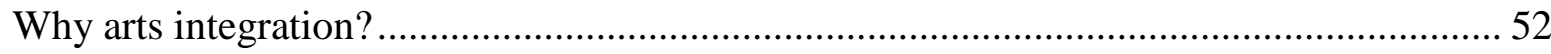

Research related to arts integration and the AIC. ...................................................... 52

Intrinsic motivation and flow experience in students. ............................................ 52

Chicago Art Partners in Education (CAPE) schools as fields of study....................... 54

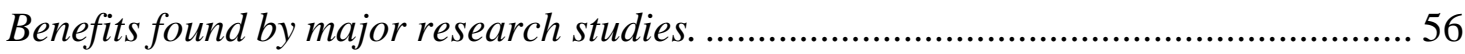

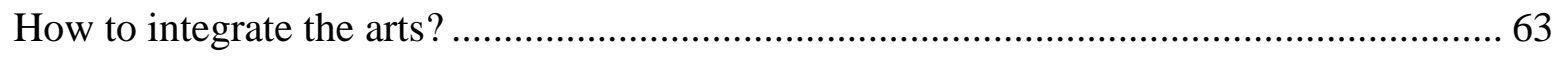

The "How" (big "H”) - a practical application of arts integration................................. 63

The "how" (small "h") - a possible effect of arts integration........................................ 65

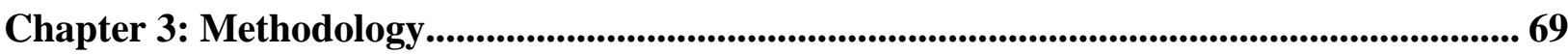

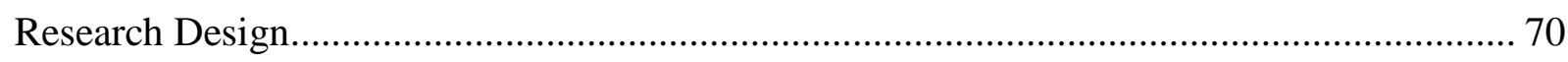

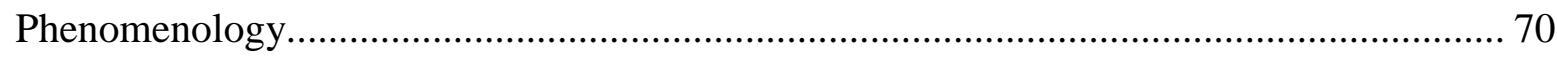

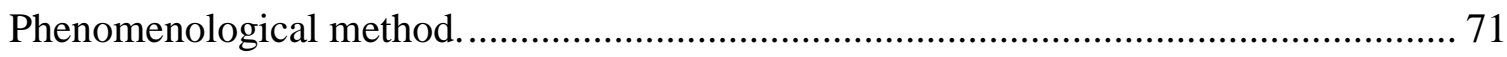

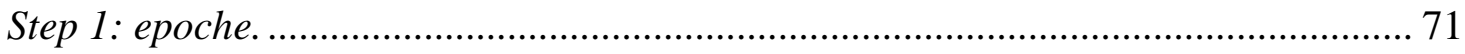

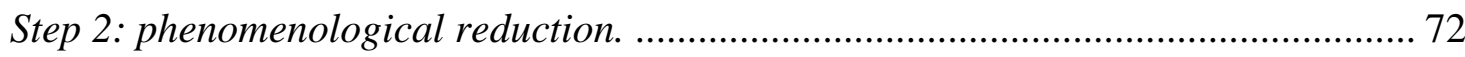

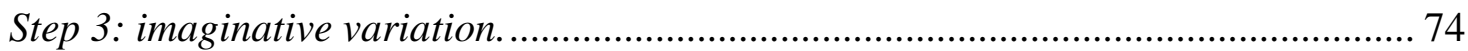




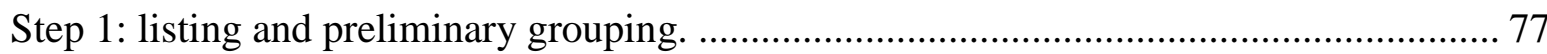

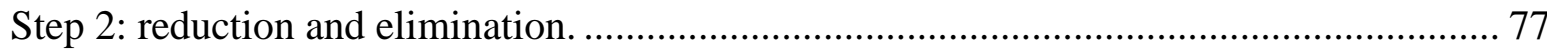

Step 3: clustering and thematizing the invariant constituents.......................................... 78

Step 4: final identification of the invariant constituents and themes (validation). .............. 78

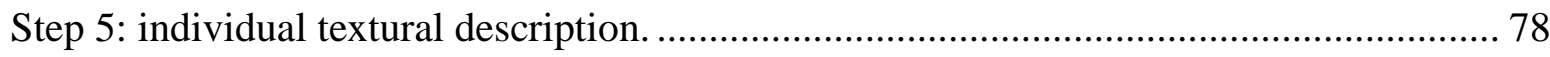

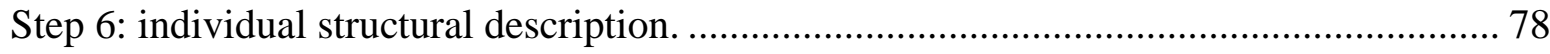

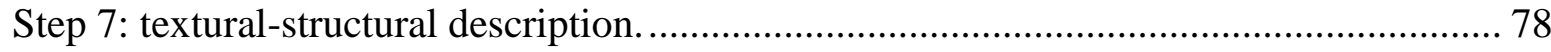

Step 8: integrated textural/ structural description. ........................................................... 78

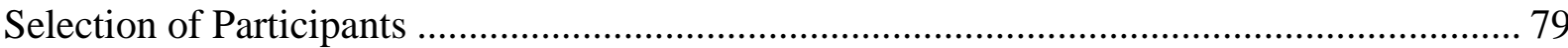

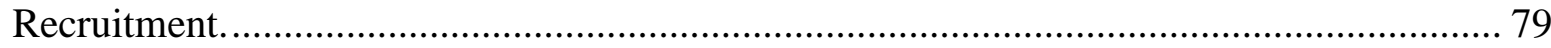

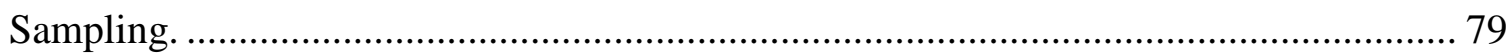

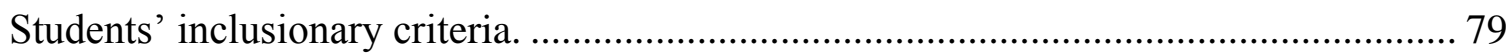

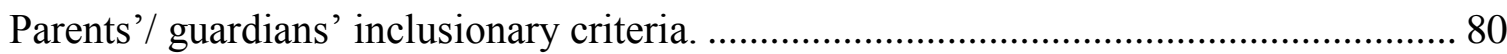

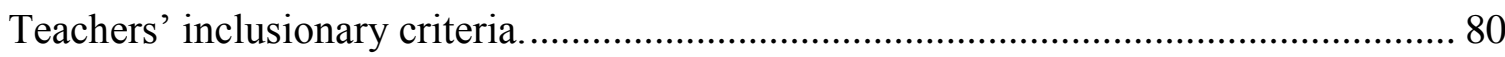

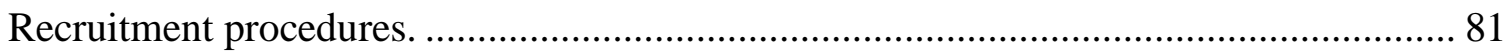

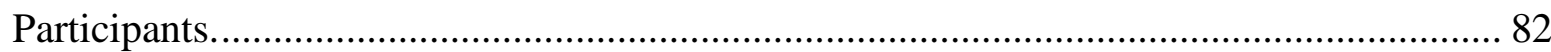

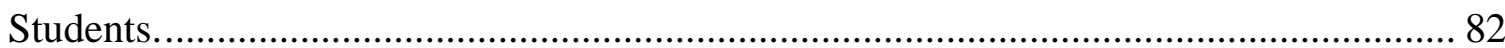

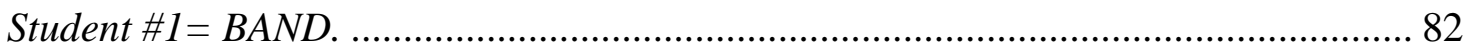

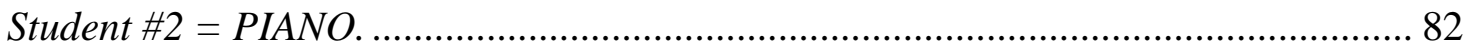


Student \#3 = FLUTE.

Student \#4 = DRAWING

Parents 84

Parent \#1 = BUSY 84

Parent \#2 = CARING. 84

Parent \#3= LOVING. 85

Teacher(s). 85

Teacher \#1 = INVOLVED. 86

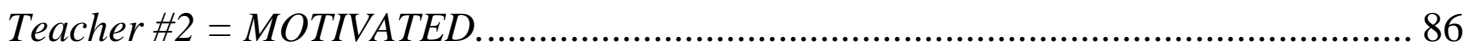

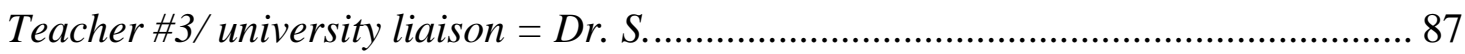

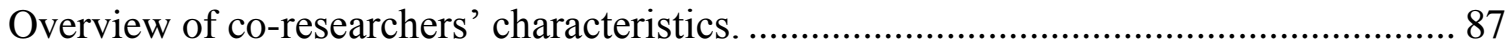

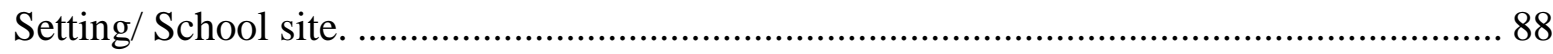

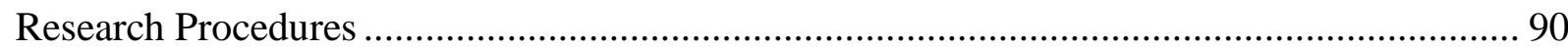

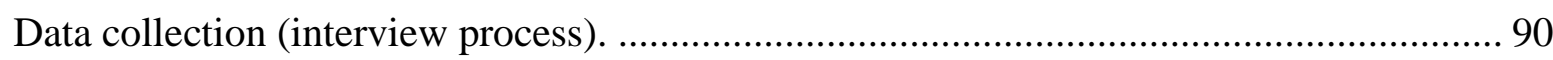

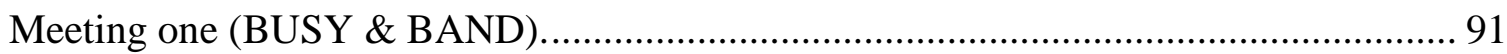

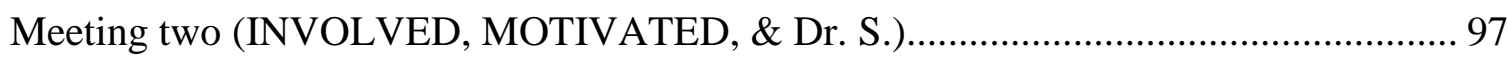

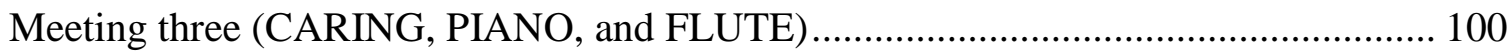

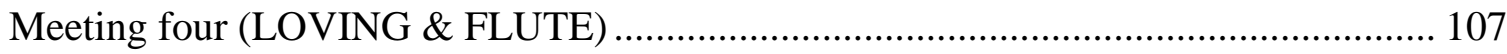

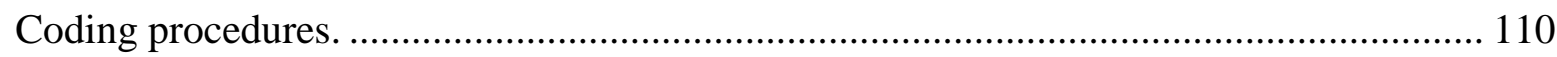

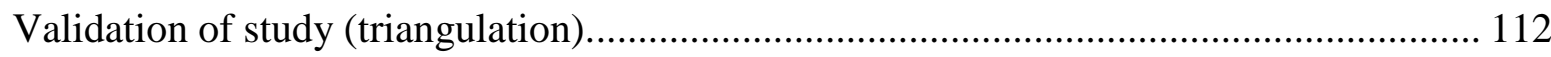

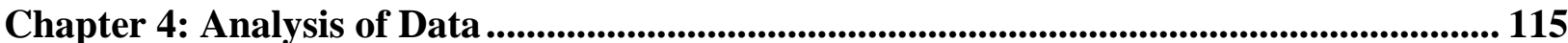


Step 1. Listing and Preliminary Grouping

Interview transcript (T-P-BUSY \& T-S-BAND-29.04.2011).

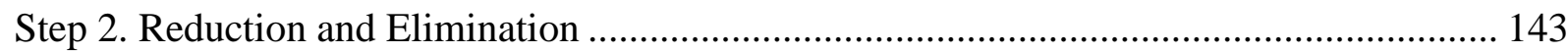

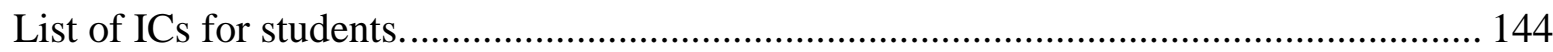

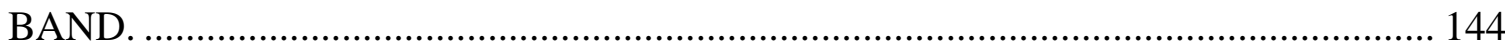

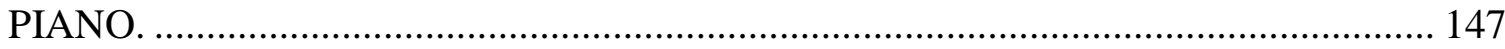

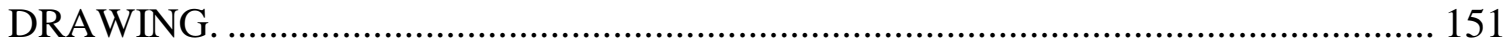

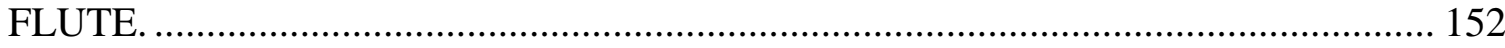

Step 3. Clustering and Thematizing the Invariant Constituents (ICs) .................................. 154

Step 4. Final Identification of the Invariant Constituents (ICs) and Themes (Validation) ..... 156

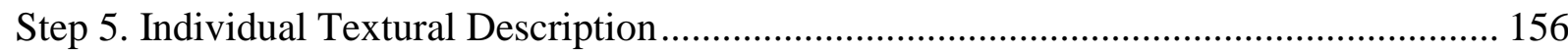

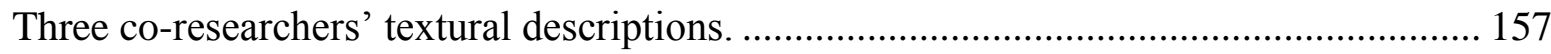

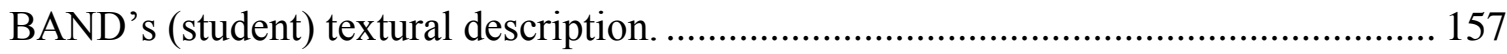

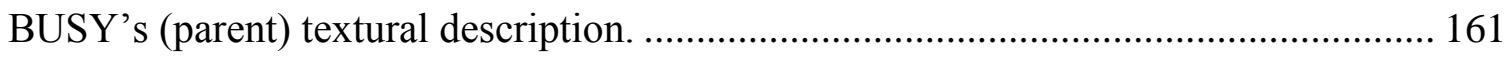

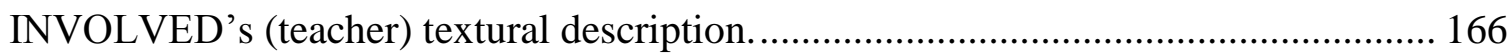

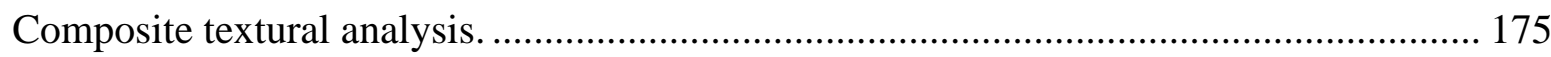

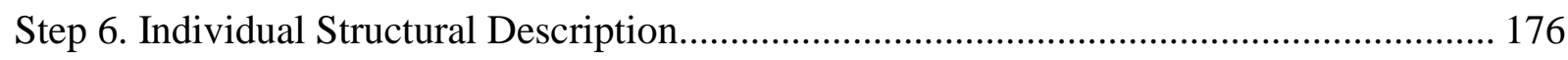

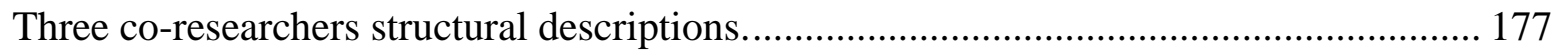

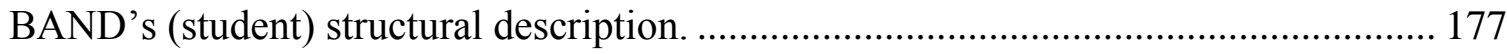

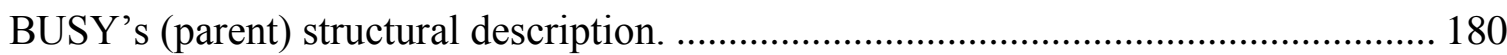

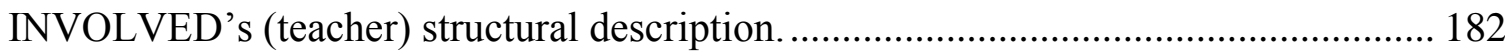


Comprehensive individual structural synthesis.

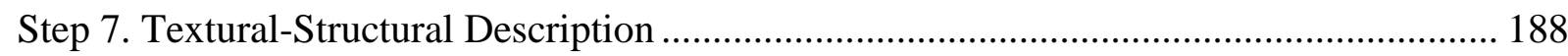

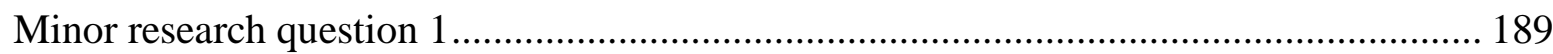

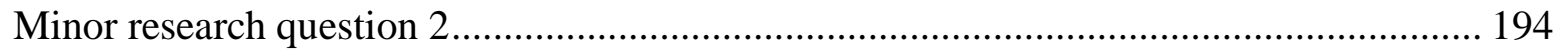

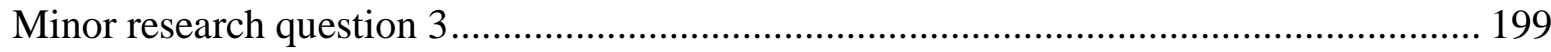

Step 8. Integrated Textural/ Structural Description ......................................................... 206

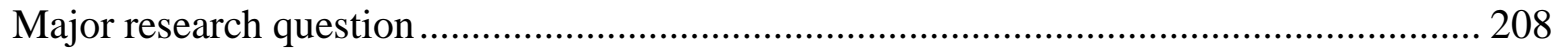

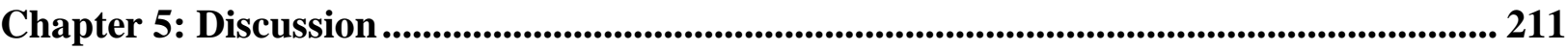

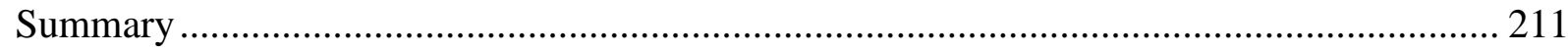

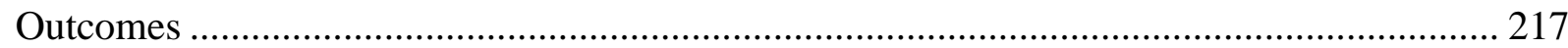

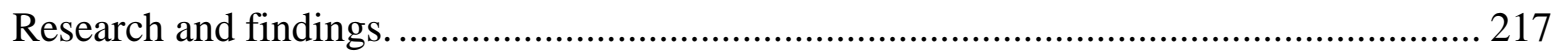

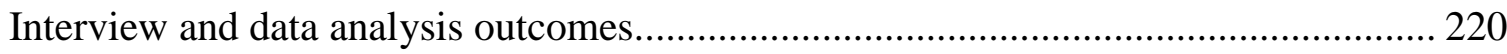

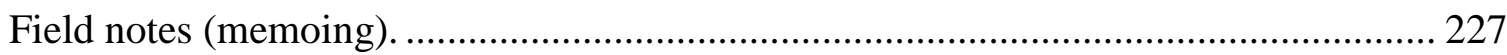

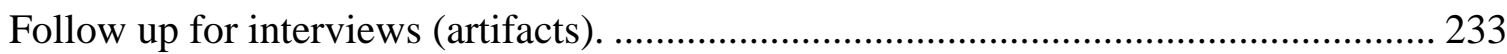

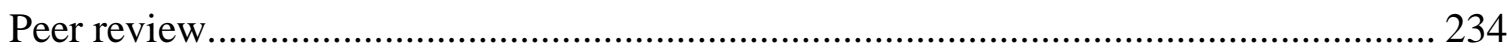

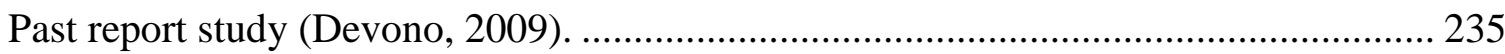

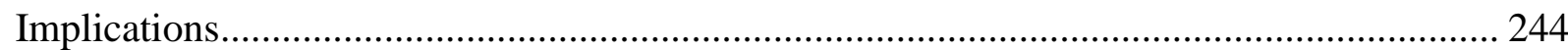

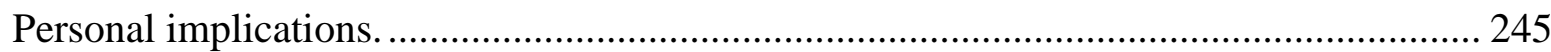

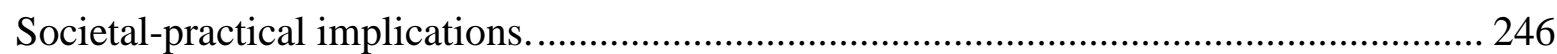

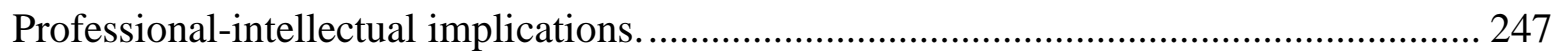

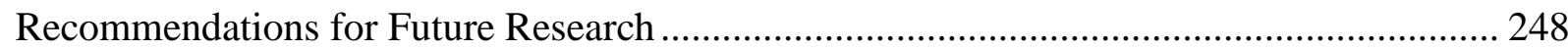


The male perspective.

AIC versus non-AIC.

Other possible influences 251

Closing Remarks 252

References 254

Appendix A: Quick Facts about Arts Integration by American for the Arts 268

Appendix B: Benefits of Arts Education - as simple as A-B-C 270

Appendix C: 10 Lessons the Arts Teach (Eisner, 2002a) 272

Appendix D: Six Distinctive Forms of Thinking (Eisner, 2002c) 274

Appendix E: Eight Ways that the Arts are included in Present U.S. School Settings (Davis, $1999 b)$ 275

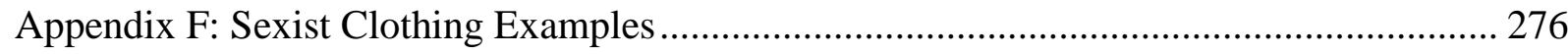

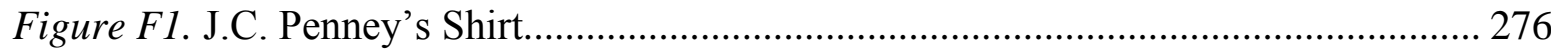

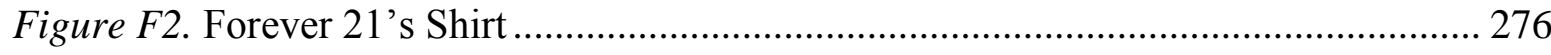

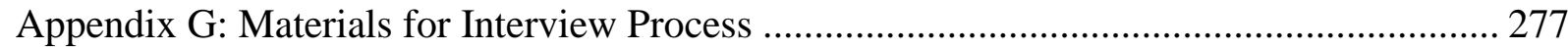

Appendix G1 - Email to Potential Participants (Parents and Students) ............................. 277

Appendix G2 - Email to Potential Participants (Teachers) .......................................... 278

Appendix G3 - Cover Letter Parent Participants ......................................................... 279

Appendix G4 - Cover Letter Parent-of-Student Participants ......................................... 281

Appendix G5 - Cover Letter Student Participants ..................................................... 283

Appendix G6 - Consent and Information Form ...................................................... 285

Appendix G7 - Parental or Guardian Consent and Information Form.............................. 290 
Appendix G8 - Assent Form

Appendix G9 - Cover Letter Teacher Participant ................................................................ 300

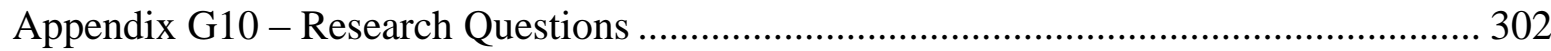

Appendix G11 - Expedited-IRB Protocol Approval ......................................................... 304

Appendix G12 - Demographic Questionnaire for Parents .............................................. 306

Appendix G13 - Demographic Questionnaire for Students ........................................... 309

Appendix G14 - Interview Questions for Parents ........................................................ 312

Appendix G15 - Interview Questions for Students ...................................................... 314

Appendix G16 - Email Request for Parents and Students to Validate the Data ................ 315

Appendix G17 - Interview Questions for Teachers: ..................................................... 317

Appendix G18 - Demographic Questionnaire for Teachers ............................................ 319

Appendix G19 - Email Request for Teachers to Validate the Data .................................. 323

Appendix H: Data Analysis Materials (Working Documents) …........................................ 325

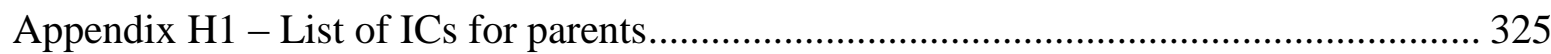

BUSY:

CARING:

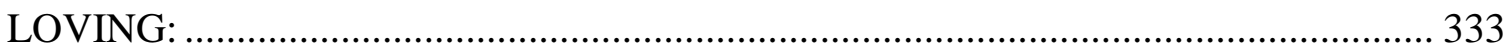

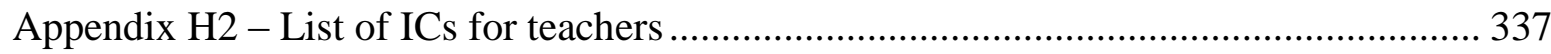

INVOLVED:

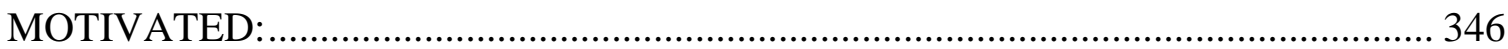

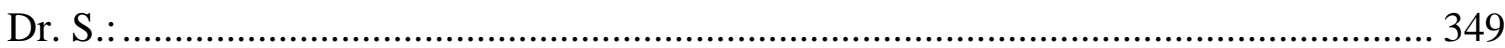


Appendix H3 - Students' themes

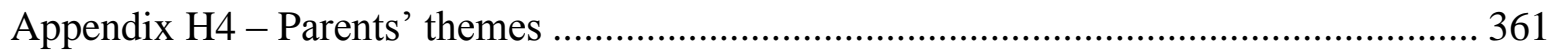

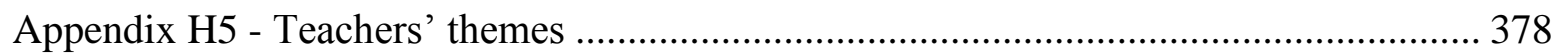

Appendix H6 - Summary cluster of themes for each of the three group .......................... 398

Appendix H7 - Common thematic portrayals of the AIC ............................................. 402

Appendix H7.1. Common thematic portrayals of the experience for STUDENTS: ...... 402

Appendix H7.2. Common thematic portrayals of the experience for PARENTS: ......... 404

Appendix H7.3. Common thematic portrayals of the experience for TEACHERS: ...... 406

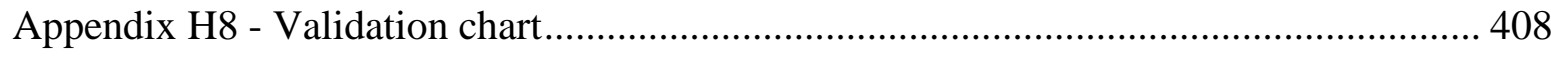

Appendix H9 - New common thematic portrayals of the experience after validation....... 410

Appendix H9.1. New Common thematic portrayals of the experience for STUDENTS

Appendix H9.2. New common thematic portrayals of the experience for PARENTS... 411

Appendix H9.3. New common thematic portrayals of the experience for TEACHERS 412 Appendix H10 - Summary chart for phenomena of all co-research groups and participants

Appendix H11 - Summary list of chart (Appendix H10) for phenomena of all co-research groups and participants 415

Appendix H11.1. Phenomena that are representing the groups as a WHOLE: ............. 415

Appendix H11.2. Phenomena that are representing only two of the groups: ................. 416

Appendix H11.3. Phenomena that are representing only one group ............................ 417 
Appendix I - Devono's (2009) and Douglas' accord with findings for teachers in comparison 


\section{List of Figures}

Figure 1. A digitized postcard on PostSecret (Motter, 2011, p. 43). 1

Figure 2. Four phenomenological processes (Moustakas, 1994; Patton, 2002) of Moustakas'

(1994) "Phenomenological Model” (p. 180).

Figure 3. Moustakas' "Modification of the Van Kaam Method of Analysis of Phenomenological

Data" (Moustaks, 1994, p. 120). .77

Figure 4. The data collection process. 91

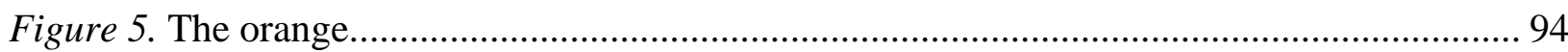

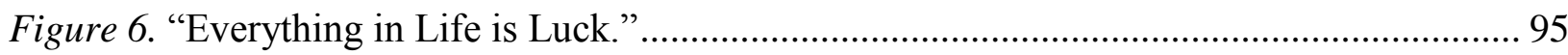

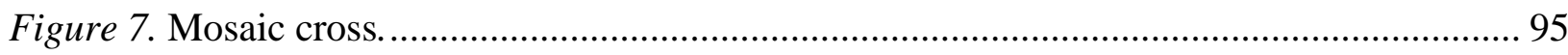

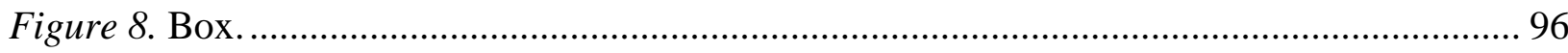

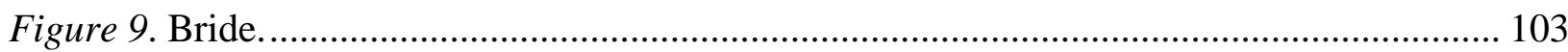

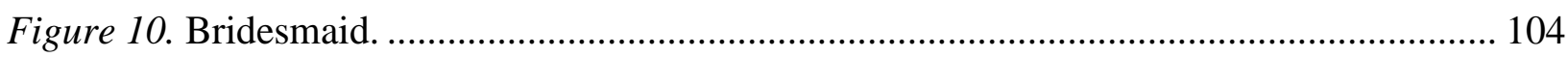

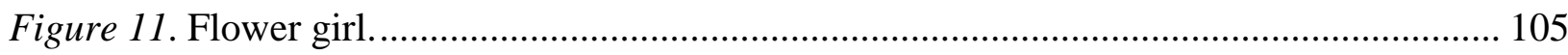

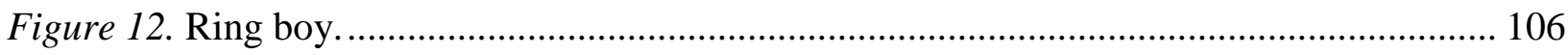

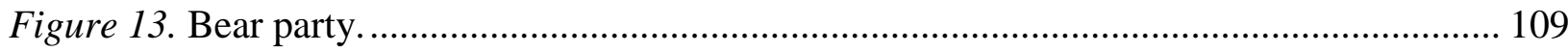

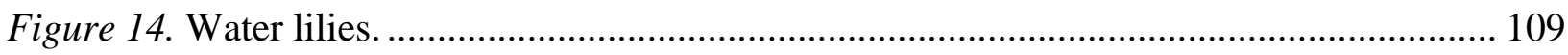

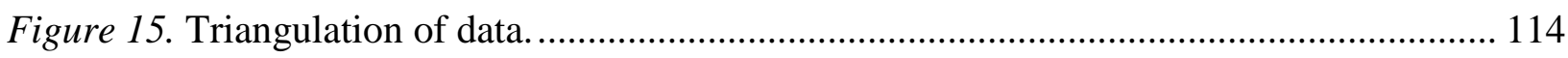

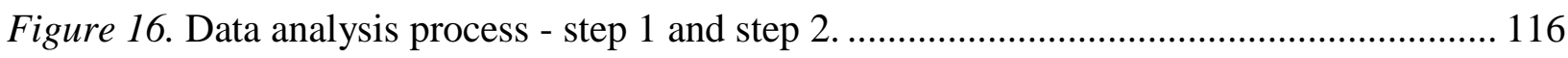

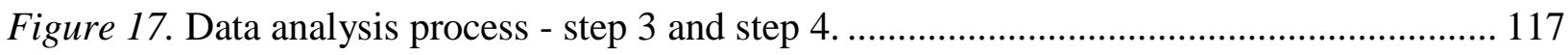

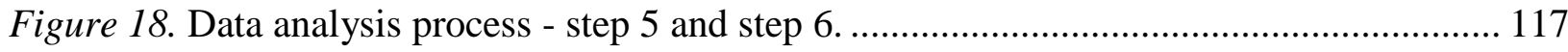

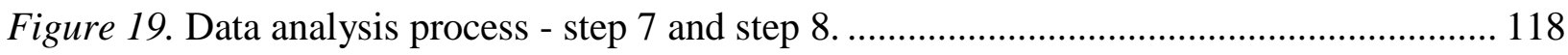

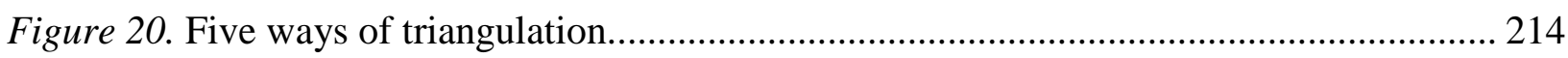

Figure 21. Implications of this study on a societal-practical, personal, and professional-........ 245

Figure 22. Recommendations for future research. ............................................................. 248 


\section{List of Tables}

Table 1. Summary Table of Co-researchers' Inclusionary Criteria .......................................... 79

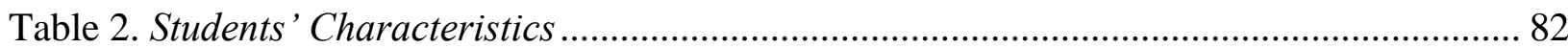

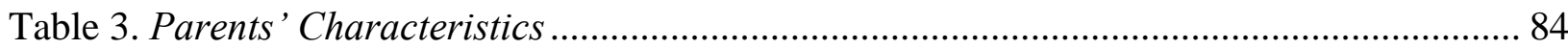

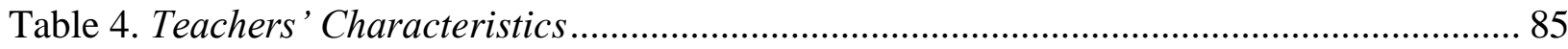

Table 5. Overview of Co-researchers' Characteristics ........................................................... 88

Table 6. Overview of Core Themes for Three Minor Research Questions .............................. 207

Table 7. Devono's (2009) and Douglas' (2011) Similarities of Arts Integration/ AIC Findings 236 


\section{Chapter 1: Introduction}

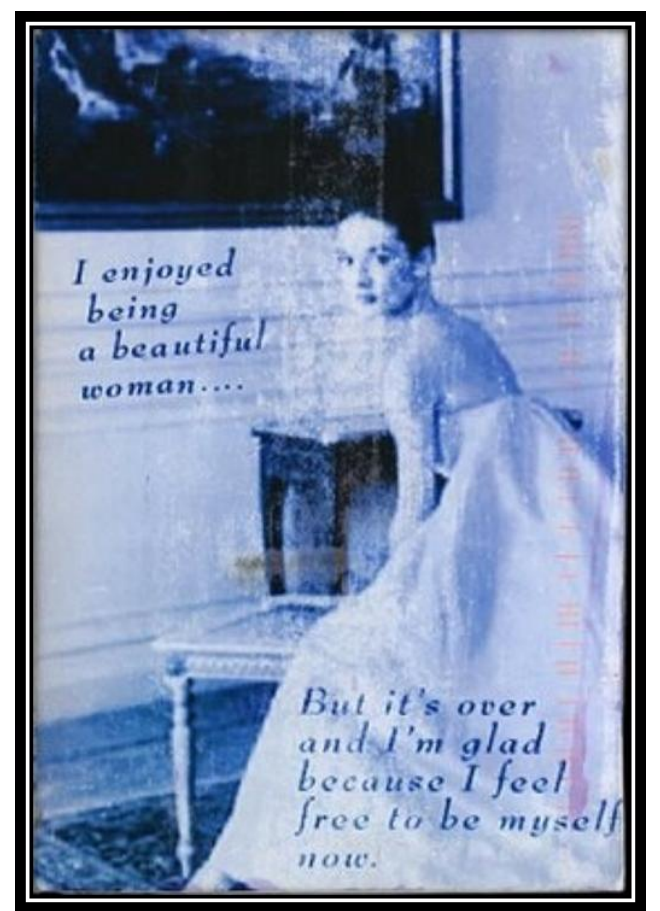

Figure 1. A digitized postcard on PostSecret (Motter, 2011, p. 43).

Females in Western culture are facing difficult issues related to unobtainable beauty standards. The text and the image of a postcard on PostSecret (see Figure 1) appears to support this conclusion with the following words: "I enjoyed being a beautiful woman... But it's over and I'm glad because I feel free to be myself now" (Motter, 2011, p. 43). What did the artist attempt to express with this statement? Does this mean one cannot be oneself if one is beautiful but determined by some other outside force? If so, who or what is this external influence?

Wendy Bantam appears to address the same problem in her art exhibit on women and appearance, noting that: "Every day in the life of a woman is a walking Miss America Contest" (as cited in Pipher, 1994, p. 55). It seems like over the last few decades, beauty standards have become more difficult to meet. Pipher for example (1994) mentions how "Miss Americas have become taller and slimmer over the years" and how "in 1951, Miss Sweden was 5 feet 7 inches tall and weighed 151 pounds," while "in 1983, Miss Sweden was 5 feet 9 inches tall and 109 pounds" (p. 56). It looks like these standards are mainly set by our media-driven society, causing 
what is accepted as culturally beautiful today to be achieved only with the help of artificial beautification- - "photo croppings, camera angles and composite bodies are necessary to get the pictures we now see of beautiful women" (p. 56). Even the stars cannot achieve these cultural ideals without great sacrifices. Famous women like Dolly Parton and Jamie Lee Curtis had to overcome body and self-esteem issues. Jane Fonda and Princess Diana struggled with eating disorders (p. 56).

Apparently females, especially adolescent girls, seem to be most affected. Is this a sign that we live in a male-driven, gendered society? Susan Bordo (2003) is convinced that "our language, intellectual history, and social forms are gendered; there is no escape from this fact and from its consequences on our lives" (Bordo, 2003, p. 242). She also notes that there is no gender-neutrality (p. 242) in this media-driven society which presents a major challenge for adolescent girls who strive to adhere to these rigid societal pressures.

The main goal of this study is to examine whether an Arts-Integrated Curriculum (AIC) can have a positive impact on the self-concept of adolescent girls and their perceptions of beauty. The AIC was chosen for this purpose because it supports three major perspectives that inform this study and that shed light on how girls develop a strong sense of self. These perspectives are the personal, the practical, and the intellectual (Maxwell, 2005).

My interest in the topic of how girls who are battling with self-concept and/or beauty issues can find new hope and support in the arts stems from personal experience. As an adolescent, I struggled with health issues that impacted my self-esteem, and I experienced firsthand the positive effect that working with the arts had on me. At the age of 16, I was diagnosed with a very serious scoliosis that required immediate treatment. Scoliosis is a lateral (side-toside) curvature in the spine commonly combined with a rotation of the vertebrae. Larger lateral curves often cause medical problems and postural imbalance, also leading to muscle fatigue and pain. More severe scoliosis can complicate breathing (Answers.com, 2011). 
Doctors agreed that spinal surgery was necessary, despite the risk of temporary or permanent paraplegia. My family and I refused to take this risk. Instead, I chose to be treated with a medical corset that I had to wear 20 to 24 hours a day for three years and that was accompanied by extended clinical stays as well as daily physiotherapy. Even though the corset was painful, the clinical stays difficult, and the daily physiotherapy exhausting, the matter that concerned me the most was my appearance. I could not wear the clothes I used to wear. The corset was too bulky to be hidden by the outfits I wore before the diagnosis. Comments by wellmeaning people about my change of appearance were difficult to hear. Inevitably, I began to struggle with my body image and self-concept. During this difficult time I began to paint, and this activity helped me through my struggles and gave me confidence. I no longer needed people to approve of my appearance. The arts, for example my paintings and drawings, gave me selfassurance. I participated in art shows and received recognition. My participation in drama classes and my performance in plays also strengthened my self-concept. I started to see people and my surroundings differently. The arts helped me realize that my life had value beyond how I looked, that self-worth need not stem from having the "perfect" body. I realized that inner values and internal beauty, like a special gift or a pleasant personality, were even more meaningful. I credit the arts for turning my self-perception from negative to positive. As a consequence, I am interested in finding out whether students who are struggling with low self-esteem, whether because of health-concerns or for other reasons, can develop a positive sense of self as a result of working in the arts. While an AIC could be particularly helpful for students who are struggling with issues of self-esteem, confident students could also benefit from this approach by having their perception of beauty challenged.

The second, practical goal focuses on why the arts and the AIC might be important to make society, especially those involved in education, aware of the fact that action could be taken in our schools to avoid or at least to minimize low self-esteem as it relates to body image. Taking 
time for self-reflecting exercises, group interactions, and practice of tolerance could be a good start. I also hope to show that integrating the arts in our schools' curricula will improve students' abilities to learn other subjects as well and hence, will help them build skills that they will need to succeed in life. This focus will clarify the benefits that the AIC holds for individual students, whether or not they are considered "at risk," as well as for society as a whole.

The third, intellectual goal of this study is to describe the AIC and the current research on its potential effects. I will summarize findings about the arts and the AIC reached by wellestablished researchers. Though some valuable research exists (Catterall \& Waldorf, 1990; Devono, 2009; Gullatt, 2008), more is needed. Present studies, such as Mary Devono's (2009) Teacher Decision Making and the Implementation of an Integrated Arts Curriculum, contribute valuable information and insights concerning the AIC, specifically in relation to teachers (see chapter five for more details). However, not enough research has been done that involves the actual participants in an AIC: the parents, students, and teachers. I hope this study will fill that gap. To help answer the research question What, if any, phenomena may be associated with the self-concept and perceptions of beauty of young ladies who completed an AIC?, I interviewed three students, at least one parent of each student, and two AIC teachers and their university liaison and asked them to describe their experiences with the AIC.

Before describing those interviews, outlining their transcripts, and analyzing the collected data, I will briefly review significant concepts that are integral to this study by relying on the work of well-known educators, researchers, and philosophers in (arts) education. Despite the inclusion of John Dewey's work and those of other well-known educators and philosophers in the field, I would like to clarify that this is not a philosophical study or discussion on their works but a phenomenological research study that examines female experiences in reference to the AIC. The following referenced works only serve as background information to provide the reader with the most important (art) education concepts. 
The ongoing change in arts and culture and its effects on beauty standards is described with the help of John Dewey's work, Art as Experience (1934/1980/2005). The idea that changes and "Multiple Realities" of culture demand "wide-awakeness," is presented by Maxine Greene (1978) in her book, Landscapes of Learning. Culturally complex signifying chains and (im)perfect body images are introduced by Judith Butler (1993) in her work, Bodies that matter. On the Discursive Limits of “Sex.” Elliot Eisner's (2002b) viewpoint on standardization instead of individualization of culture is clarified with the help of his book, The Arts and the Creation of Mind. The comparison factor, an impossible-to-achieve image struggle, is approached with the help of Susan Bordo (1993/2003) and her work, Unbearable Weight. Feminism, Western Culture, and the Body. Finally, the importance of the arts in public spaces and their support by political figures and educators is also considered.

\section{Arts and Beauty}

\section{Ongoing change in arts and culture affects beauty standards (Dewey, 1934/1980/2005).}

From John Dewey's (1934/1980/2005) point of view, the arts are not a reasonable or stable rule book. Instead, they are free, open-ended, and constantly changing. Their change is an ongoing process that reflects the shifting culture in which the arts are being produced and which includes evolving concepts of beauty. Denis Dutton (2006) also relies on this assumption but adds that concepts of beauty are not just shaped by culture but also the society of which they are a part. In his article, A Naturalist Definition of Art, he stresses that beauty models are "conditioned by the aesthetic issues and debates of their own times" (p. 367). These descriptions by Dewey and Dutton clearly conform to a number of adolescent girls that are struggling with their self-concept because of the latter described unobtainable beauty standards that are set by the society and culture of our times. 


\section{Changes and "multiple realities" of culture demand "wide-awakeness" (Greene, 1978).}

More than 30 years ago, Maxine Greene (1978) already stressed in her work Landscapes of Learning, that "changes have recently taken place in our culture - changes too pervasive and significant for a teacher educator to ignore" (p. 1). This quote still appears to apply today. It shows how fast moving our culture and society have become and how important it is for those employed in the educational field to stay up to date. Greene (1978) calls this phenomenon "new irrationalism" (p. 1). It is not just the "new irrationalism" concept of ongoing changes that causes trouble in our schools but especially the "multiple realities of our culture" (p. 1) that take over. Critical and self-reflective questions that presuppose a "wide-awakeness" (p. 42) on the part of individuals become indispensable. Consciousness and perception (pp. 14-15) are especially related to these multiple realities. Greene (1978) says that "multiple realties are available to human consciousness" (p. 16). Furthermore, she interconnects consciousness and perception as reciprocal concepts when she says that "consciousness as perspectival grasping is our way of encountering the natural and human world" (p. 15). As such, we cannot really perceive our world without consciousness. But what kind of consciousness are we, as individuals, supposed to demonstrate? What does it mean to be conscious of something or someone? When do we know that we are having a conscious perception? Can others tell that we are conscious or is it a characteristic that can only be intrinsically experienced? To perceive oneself in conscious relation to others, Green (1978) demands a "wide-awakeness" for the students, the teachers, and our society (p. 46) also in relation to artwork "because perceptive encounters with works of art can bring human beings in touch with themselves" (p. 165) and their social surrounding (p. 172).

Culturally complex signifying chains and (im)perfect body images (Butler, 1993). Judith Butler (1993) and others (Becker, 1999; Pipher, 1994; Silverman, 2010) discuss the problem of disorders, especially eating disorders, that are becoming more widespread in our 
schools and in society more generally. At the same time that such disorders are becoming more prevalent, the public as a whole is refusing to acknowledge that the problem exists, and that our youth is suffering from a condition that social forces, namely the creation of "impossible-toreach" and "unrealistic" beauty standards, have created.

In her work Bodies that matter. On the Discursive Limits of "Sex," Butler (1993) describes the difficulty of perceiving one's own body as an "idealized body," especially when one is surrounded by perfect images (Butler, 1993, p. 76). The image of the other appears always to be more perfect than the self. It is through this comparison with the perfect "Other" that the ego is constituted by its social surrounding (p. 76) and controlled by the public gaze. This body ego is no longer able to distinguish between imagination and reality. Instead, the borders between these two concepts are blurred. Butler (1993) calls this a "culturally complex signifying chain" (p. 90). The girls see themselves differently than they are perceived by others. To overcome this problem, it would be necessary to substitute the hegemonic imaginary with a new, more positive, alternative imaginary (p. 91). This is a claim, however, that proves to be almost impossible to reach for our medial and culturally influenced generations.

\section{Standardization, instead of individualization, of culture (Eisner, 2002b).}

Nowadays, everything seems to be predetermined by our media, society, and culture. Why is it that individuality does not seem to be appreciated anymore? Instead, everyone feels compelled to conform to socially acceptable standards. This trend is not just true anymore in society but also can be found in the educational system. This becomes especially obvious in the large number of standardized tests students are required to take.

An individual no longer perceives his or her surroundings from a unique perspective, but rather, from that of the society. Connections between an individual's experience and his or her cultural environment, hardly occur anymore. Today's society contrasts with the type of society that Elliot Eisner (2002b) imagines in his work, The Arts and the Creation of Mind. For him, the 
experience of the environment is "a process that continues throughout life; it's the very stuff of life. It is a process that is shaped by culture, influenced by language, impacted by beliefs, affected by values, and moderated by the distinctive features of that part of ourselves we sometimes describe as our individuality" (Eisner, 2002b, p. 1). His view addresses the interconnections between cultural and individual experience and how art education could help students learn how to apply the main ideas of culture, language, beliefs, values, and individuality to their own lives.

As such, the following questions must be addressed: What needs to be done to incorporate these concepts into our school curriculum in a way that allows students to apply them when studying their core subjects? How is a student expected to acquire knowledge about his or her societal environment (outside world) in school (inside world) when he or she does not get the chance to experience it first-hand? - Just by hearing about it or imagining it and talking about this "made-up" image or second-hand experience? Eisner (2002b) considers art education as a solution to this problem. He suggests that "art education should help students learn how to create and experience the aesthetic features of images and understand their relationship to the culture of which they are a part" (p. 43). But what if "the culture of which they are part" (p. 43) does not appreciate "original," "true," or "first-hand" experiences? What happens if students are mostly affected by "the widespread visual culture, especially TV programs (...) and TV advertisements" (Chan, as cited in Eisner, 2002b, p. 64)?

In articulating his theories, Elliot Eisner (2002b) refers very often to the importance of duality described by John Dewey. Eisner (2002b) specifically, refers to Art as Experience, in which Dewey (1934/1980/2005) stressed the dual connection of process and product. From Dewey's (2005) viewpoint, these two concepts are not supposed to stand alone, but to be interconnected and to complement each other. Like Eisner (2002b), Dewey (2005) thinks that human beings need the chance to experience both process and product, also referred to as theory 
and practice in the educational field. This thought should be transferred to theory and practice in students' work. More options should be provided for students to pursue both, theory and practice. Students should be encouraged to create theories that they can put into practice (or conversely, put practice into theory). Society refuses this dualistic viewpoint. De-individualizing standardized testing and measurement increase instead. Consequently, none of the students that are leaving the educational system are going to remember individual, first-hand experiences. They will not get the chance to reflect upon and learn from personal struggles they had to overcome to reach a goal. Individual feelings or unique experiences will not be built. Only second-hand experiences will be recalled.

The comparison factor - an impossible-to-achieve image struggle (Bordo, 2003).

Susan Bordo (2003) also considers a dualistic idea (Dewey, 2005) of beauty and ugliness in her work, Unbearable Weight. Feminism, Western Culture, and the Body. The dualism of which she speaks can also be called a "comparison factor" and is one of the main reasons for health disorders experienced by girls. Adolescent girls compare and contrast their developing bodies to perfectly slender images. Most often they develop eating disorders by trying to lose weight to look like their idol. The magazine Frontline asked "Alexandra Shulman, editor of British Vogue, if the fashion industry felt any responsibility for creating the impossible-toachieve images that young girls measure themselves against" (Bordo, 2003, p. XV). Shulman's answer was quite shocking. She stated that "not many people have actually said to me that they have looked at my magazine and decided to become anorexic" (Shulman, as cited in Bordo, 2003, p. XV). Her statement makes it quite obvious that Shulman and her magazine do not assume any responsibility for the escalating numbers of eating disorders like anorexia, nervosa, or bulimia.

In general, magazines, newspapers, and other media assume no responsibility for health issues like eating disorders that arise more and more frequently in our culture. But who then is to 
blame? Is there someone against whom one can make a charge? Is there someone or something responsible for this growing health concern? If the media are not the ones to blame, who or what is responsible for this issue?

Why is it that Ann Becker's (1999) research in Fiji, Body, Self and Society: The View from Fiji, is quoted in so many different professional journals that deal with eating disorders, self-image struggles, and their connection to media in our society? Becker's research (1999) discovered that up until 1995, the Fiji islands did not have access to television and no cases of eating disorders were reported (Becker, as cited in Bordo, 2003, p. XV). However, after the introduction of television in 1995 , only three years later " 11 percent of girls reported vomiting to control weight, and 62 percent of the girls surveyed reported dieting" (Bordo, 2003, p. XVi). Becker (1999) does not necessarily blame media for this development. She thinks the Fijians' vulnerability lies in the fact that "they were not sophisticated enough about media to recognize the television images were not 'real"' (Becker, as cited in Bordo, 2003, p. XVi).

But is this really the case? Does this apply to the society we live in, as well? Is our society in general and our young (female) students in specific unable to distinguish between real and unreal images? And if so, who in our society is sophisticated enough about media to perceive the perfect images as not "real?" Apparently, we all have to become more knowledgeable in reference to media literacy, as Robyn Silverman's example shows. In her work, Good Girls don't get fat, Silverman (2010) even supplies the reader with a whole list of "Best Bet Media Literacy Resources/ Programs" (p. 232). Media literacy is only rarely considered a useful topic in schools. One just takes it as something that is a given and understood.

Despite the fact that one cannot blame all the recent problems in our society on media students, parents, and teachers all should take responsibility and develop a broader sense for media literacy (Silverman, 2010). Also, other factors should be considered since "people's 
identities are not formed only through interaction with such images, powerful as they are" (Bordo, 2003, p. 62). Instead, other "unique configurations (of ethnicity, social class, sexual orientation, religion, genetics, education, family, age, and so forth) that make up each person's life" have a huge impact, too (p. 62). I agree that, besides media, all these issues influence younger generations. Nonetheless, one still has to be aware of the huge impact that media play in the lives of our young ones. Marie Hoskins (2002) stresses this thought as well in her work Girls' Identity Dilemmas: Spaces defined by Definitions of Worth, when she says that "although we cannot assume a simple causal relationship between television or other media and eating disorders, we can explore how these images are adopted by girls and how they fit with their overall system of meaning and identities" (Hoskins, 2002, p. 234). This system of meaning and identities might also be connected to the institutionalized system of values and practices of our society in which "girls and women - and, increasingly, men and boys as well - come to believe that they are nothing (...) unless they are trim, tight, lineless, bulgeless, and sagless" (Bordo, 2003, p. 32). The problem is that people fail too often to reach the almost unattainable goal of perfect slenderness and beauty. As a consequence, a negative self-concept can develop followed by a lack of self-esteem and body image problems (p. 57).

Considering the ideas outlined above, the following questions must be asked by an alarmed society: What can one do to enhance media literacy? How can one help young women minimize or even dispose of the "comparison factor" that causes so much trouble? How can one support the development of a positive self-concept and body image in young girls, women, and even young boys and men?

\section{The importance of the arts in public spaces and their support by political figures and educators.}

The arts are a perfect way to help school-aged children become more active, self-aware, and self-determined individuals. Through the arts, our youngsters can finally develop and show 
more self-esteem in various areas of their lives. Cornett (2011), Deasy (2002), Fiske (2002), Greene (1978), and The Arts Education Partnership (n.d.) all support this idea, as does the nation's leading nonprofit organization for advancing the arts in America-Americans for the Arts (n.d.) — which provides quick facts about arts education (see Appendix A). Included in these facts is one finding of the Business Circle for Arts Education in Oklahoma, Arts at the Core of Learning 1999 Initiative, which is significant for this study because it stresses the same basic idea of the arts on which Greene (1978) focused. It maintains that even businesses and firms understand that arts education can help all students to develop more appreciation and understanding of the environment and world around them (as cited in Americans for the Arts, (n.d.)). Claudia Cornett (2011) refers back to the same issue when she describes how arts can serve as an expression of the self: "Art is an extension of a person, an expression of who I am and what I am" (Sabe \& Harrison, as cited in Cornett, 2011, p. 147). In alignment with this quote, the remarks at the Arts Education Partnership National Forum by United States Secretary of Education Arne Duncan fit right in. He referred to First Lady Michelle Obama who said that "the arts are not just a nice thing to have or do if there is free time or if one can afford it... Paintings and poetry, music and design... they all define who we are as a people.” (Duncan, 2010). It becomes more obvious that the importance of the arts is not just a "made-up idea" by (art) educators to secure their jobs, when even political figures, like First Lady Michelle Obama, realize the need for the arts and stress their expansion. Arts define our character, define our personality, and influence who we are - not just as individuals but as a people.

Similarly, Prince William and Kate Middleton value the arts as an important part of children's personality, skills, and potential. During their royal visit in California, the duke and duchess visited Inner-City Arts. This program helps underprivileged children through arts to unlock their potential (James, 2011). 
Interestingly enough, integration of the arts is not only promoted in education or by political figures but also in public spaces. The United States General Service Administration (GSA) promotes the creation of art works for federal buildings. These artworks are supposed to "enhance the civic meaning of federal architecture and showcase the vibrancy of American visual arts" (GSA, U.S. General Services Administration, (n.d.)). Arts integration in this sense is not just used to unite a nation but also to make the people of the United States aware of the culture of which they are a part.

The United States House of Representatives even designated September 12-18, 2010 to be set aside to focus on the value of arts education around the nation and to celebrate the unique ways the arts contribute to student learning. Also, West Virginia students explored the benefits of music, dance, theater, and other arts.

Former West Virginia state superintendent Steven Paine commented on this occasion by saying that West Virginia has demonstrated its commitment to arts education as part of Global21: Students deserve it. The world demands it. He continued by recognizing that the arts help students to become knowledgeable decision makers instead of being mere consumers of mass culture. Finally, he stressed skills like “creativity, collaborative learning, global awareness, critical thinking and problem solving" that are needed by our students and taught daily in classrooms in and through the arts (Paine, 2010).

Also, West Virginia's current superintendent recognizes the values of the arts in her message: Invest now or pay later (Ruben, 2011). Jorea Marple stresses how we know from research and good practice that academic skills improve when children are actively engaged in the arts and exposed to technology. She adds, that they want to respect the developmental level at which each child is and that they want to see growth and improvement based upon their "ability to provide a holistic curriculum" (Ruben, 2011). 
These comments by political figures and educators, as well as the value of the arts in federal buildings, take us back to Maxine Greene (1978) and her ideas of "multiple realities of culture" and "wide-awakeness" that can bring human beings in touch with themselves (individual) and their surrounding (society and culture), referenced at the beginning of this chapter. Claudia Cornett (2011) builds on this point, saying that students gain confidence by using art concepts and skills to express themselves. They make new personal discoveries and gain self-confidence as art shows them different ways to deal with their environment (Cornett, 2011, p. 147). Edward Fiske (2002) stresses the same idea when he claims that the arts connect students not just to themselves but also to each other:

Creating an artwork is a personal experience. The student draws upon his or her personal resources to generate the result. By engaging his or her whole person, the student feels invested in ways that are deeper than "knowing the answer." Beyond the individual, Steve Seidel and Dennie Palmer Wolf show how effective arts learning communities are formed and operated. James Catterall also describes how the attitudes of young people toward one another are altered through their arts learning experiences. (Fiske, 2002, p. ix) Fiske stresses that students need to be personally engaged in the process of creating art. Developing relationships within one's community is also of great importance. These ideas are shared by the Arts Education Partnership, who view the arts as a means of promoting selfdevelopment and community involvement: "learning in the arts helped students develop the sense that they can be agents of their own learning (...). It contributed to their sense of selfefficacy and self-esteem” (The Arts Education Partnership, (n.d.), p. 1).

In their brochure, Making a Case for the Arts. How and why the Arts are Critical to Student Achievement and Better Schools, The Arts Education Partnership (n.d.) sums up in more detail how the arts benefit student achievement. In their opinion, this is possible because learning 
in the arts is academic, basic, and comprehensive, (see Appendix B) and therefore promotes a heightened awareness concerning the self and social relationships:

Art learning experiences contribute to the development of certain thinking, social and motivational skills that are considered basic for success in school, life and work. These fundamental skills encompass a wide range of more subtle, general capacities of the mind, self-perceptions and social relationships. (The Arts Education Partnership, (n.d.), p.

2)

Self-perception, self-concept, and self-esteem appear to play a major role in schools, education, and society. Nevertheless, one should not forget about the problem of actually "teaching" the arts, of carrying the theory into practice. One such form of integrating the arts into the classroom is provided by Visual Thinking Strategies (VTS). VTS is a research-based teaching method that is designated to improve critical thinking and language skills by discussing visual images. Throughout the discussion of painting by famous artists, "VTS encourages participation and self-confidence, especially among students who struggle. VTS is easy to learn and offers a proven strategy for educators to meet current learning objectives" (Visual Thinking Strategies, (n.d.)). VTS is just one means that educators can use to build self-confidence and selfesteem in their students.

The need to increase self-confidence and self-esteem in our youth, especially in girls, is becoming increasingly indispensable since children have to handle a countless number of influences from media, society, and culture. As mentioned by Greene (1978), students are having trouble relating to themselves, others, and outside influences. Instead, they often live in a self-created, unreal world that is defined by media images. Students compare and contrast their worlds, their lives, their images, and themselves, especially their bodies, to those unreal and artificial worlds and to the superficial images. The distinction between the "I" and the "outside world" ceases to exist. As a consequence, a broad range of disorders arise. 


\section{Statement of the Problem}

The concept of beauty plays an important role in our society. This becomes especially obvious when examining the lives of adolescent girls who develop a sense of identity that is at least partly constrained by a desire to embody the beauty standards of the society in which they are living. Unfortunately, the limitations that adolescent girls are under more frequently result in a poor self-concept than in a positive self-concept. Marie Hoskins (2002) states that eating disorders can have a major impact on girls' identity development. Consequently, she suggests that great effort should be put in understanding "how the symbols, images, and metaphors of a culture become integrated into girls' identities" (Hoskins, 2002, p. 232). The influences of media and television are especially obvious and well documented (Becker, 1999). Margaret Walshaw (2001) also stresses the influence of popular culture over time. Duncum (2005) and several other authors (Dutton, 2006; Korsmeyer, 2004; Pipher, 1994; Romanska, 2005; Steiner, 2004) place their focus on the considerable influence of culture on the human body and its beauty standards.

The dissatisfaction that girls experience with regard to their bodies and the resulting low self-esteem are starting to develop at earlier ages. Research shows that appearance schemas (Clark \& Tiggemann, 2007), diets (Muir, Wertheim, \& Paxton, 1999), and thinness (Dohnt \& Tiggemann, 2006) emerge as ideas that are all commonly linked. Appearance schemas are defined as the cognitive representation of someone's body image, including the beliefs that this individual holds about the way she appears and the value she attaches to appearance in life (Cash, 1996, in Clark \& Tiggemann, 2007).

\section{Purpose of the Study}

The main purpose of this study is to explore the main phenomenological research question of What, if any, phenomena may be associated with the self-concept and perceptions of beauty of young ladies who completed an AIC with the help of three minor research questions:

Q1. How do young ladies who completed an AIC describe their experience? 
Q2. How do parents of young ladies who completed an AIC describe their experience?

Q3. How do teachers of young ladies who completed an AIC describe their experience? These research questions attempt to find out whether an AIC could be used as a helpful tool to discover more about the self-concept of adolescent girls and whether it could have a positive impact on their self-concept and their perceptions of beauty.

Another goal of this dissertation is to determine to what extent changing cultural norms negatively influence female students and their self-concept and perception of beauty and, in turn, to gauge the effectiveness of the AIC in countering these negative influences. If the AIC is found to have a positive effect, an effort should be made to incorporate an AIC in our schools, i.e., to make it part of our regular school curriculum.

The final goal of this study is to answer the question of what exactly an AIC is and what the current state of research in this field is. Recent findings by well-established researchers in the field were used to answer this question.

\section{Significance of the Work}

A possible solution to the problems of our adolescent girls could be the fostering and the advocating of arts education in our educational systems. The arts, including all art forms (e.g. painting, drawing, sculpting, singing, dancing, playing an instrument, acting, etc.), arts education, and as a consequence the AIC are too often treated as unnecessary. On a regular basis, they are considered a waste of time for teachers and their students. Instead, preparing lessons for standardized tests is favored. Even though standardized testing cannot be eliminated from the curricula of American schools from one day to the next, educators and administrators must learn to recognize the positive effects that the arts can have on a student's individual development, academic learning, and, yes, even test scores.

James Catterall studied 25,000 students from eighth to tenth grade and discovered that the students who are "highly involved in arts programs" showed better results in other subjects 
and were "much less likely to drop out" of school (Catterall, as cited in Milner, 2000, p. 11). Catterall's research study also reveals that "students from low-income families who participate in arts experiences are more likely to do better academically than those who do not" (as cited in Milner, 2000, p. 11). Students appear to have better attitudes, display higher attendance, show greater abilities, and receive better grades when the arts become part of their school life. Catterall is also convinced that "arts education programs result in measurable gains in student motivation and achievement in reading, writing and mathematics" (Catterall, as cited in Milner, 2000, p. 11).

The listed research (Devono, 2009; Milner, 2000) clearly shows the positive effects of the arts, arts integration and the AIC on students' learning. Nevertheless, this study sees the need to go a step further by not just focusing on learning and improving test scores in and through the arts, but also by examining how students' sense of self-worth and their perception of beauty are affected by an AIC.

\section{Limitations of the Study}

1. I, the researcher, might be biased in favor of arts integration due to a lifetime of arts instruction. My parents were very interested in the arts, especially the visual arts. Art exhibitions were always on the agenda. Furthermore, my parents own a gallery and a frame store. My father is an artist and gives art lessons. I was also influenced by the early experience of music in school followed by piano lessons. In school, I was a member of the choir and theater group that formed part of the after school program. This artsintegrated education was followed by a Masters in Elementary Education with a focus on arts education and a Ph.D. program with a focus on arts integration.

2. Unique demographic data of participants, like gender, age, location, educational background, and experiences with the arts, could have had an influence on the results. The outcome of this phenomenological research study could have possibly been different if the interviewed parents would not have been exclusively female. Also, all the parents 
in the study were about the same age. There could be a difference in the research outcomes if the parents' ages were more diverse. If the geographical location of the interviewees were more spread out, this might also affect the results. Some parents or students could come from an arts-enriched area where the arts are part of the community life. Others could be from an area that does not foster the arts at all and does not place any significance on the arts. Correspondingly, the educational level of participants could differ and cause contradictory conclusions.

3. All participants (students, parents, and teachers), as well as myself (the researcher), were female. The selection of schools, and as a consequence, of parents and students who were interviewed, was very limited because of the lack of AIC in schools in surrounding areas.

4. For this study, the number of participants could have been higher in order to improve reliability.

5. Instead of limiting the design to interviewing the co-researchers in paired groups of mother-daughter or teacher-teacher, one could group the participants in parent-parent, student-student, and more teacher-teacher groups to support interaction and dialogue between them.

6. This investigation focused on girls. The study could have been more comprehensive by including boys, as well.

7. This research study was limited to students who experienced an AIC in Elementary School. Other school types and levels could be researched, as well. Consequently, AIC methods and experiences would not be limited to the Elementary School level but could also be transferred and applied to higher levels of schooling.

\section{Organization of Dissertation Chapters}

Chapter Two provides a review of the literature on this topic. At the beginning, definitions and descriptions of beauty, phenomenology, self-concept, and the AIC are provided. 
Questions about arts integration and the AIC (What is arts integration or the AIC?, Why arts integration?, and How to integrate the arts?) are raised and answered to the extent that the available literature allows. The question of how the arts relate to our present day society is explored by reviewing literature on problems and struggles of girls and women, the male definitions of beauty and their consequences, and the possible negative results for adolescent girls.

Chapter Three describes the methodology of this phenomenological research study. The selection of participants, the recruitment process, as well as the setting and the school site are described. Moustakas's (1994) phenomenological method, in combination with Patton's research method (2002/2005), are explained. Moustakas's (1994) “Modification of the Van Kaam Method of Analysis of Phenomenological Data" (p. 120) is applied to the data analysis of the collected interview data of 10 co-researchers. The five ways of triangulation for this study are explained in more detail.

Chapter Four displays the textures and structures of the phenomenological research findings. Core themes that determine and influence the AIC experiences of each group (parents, students, and teachers) are listed. The chapter concludes with the six phenomena that were identified by all three groups, with the self-concept and perceptions of beauty of young ladies who completed an AIC.

In the final chapter, research outcomes and findings about any phenomena that may be associated with changes in the self-concept and perceptions of beauty of adolescent girls who completed an AIC are summarized. Three implications (personal, societal-practical, and professional-intellectual) are listed. A comparison between findings of this and another study (Devono, 2009) on teachers and the arts integration/ AIC is made available. Research methods and procedures are critiqued, suggestions are listed for future studies, and closing remarks are provided. 


\section{Chapter 2: Literature Review}

This phenomenological research study focuses on the following question: What, if any, phenomena may be associated with the self-concept and perceptions of beauty of young ladies who completed Arts-Integrated Curriculum (AIC)? In answering this research question, it is the goal of this literature review section to provide the reader with essential definitions, important overviews, and background information for this research study: beauty, self-concept, phenomenology, and arts integration/ AIC.

Starting out with the concept of beauty, an overview is provided on the struggles of girls and women, including beauty standards, as well as the male definition of beauty and its influence on the female image, role, and identity. The following section focuses on the self-concept and its possible negative developments and resulting disorders, in reference to media and popular culture. Furthermore, a definition and an overview of phenomenological research is provided. A distinction is made between phenomenological research and phenomenology as a philosophical concept. The last part of this section focuses primarily on arts integration and the AIC. The questions of "What is arts integration/ AIC?," Why arts integration?," and "How to integrate the arts?" received closer attention.

We live in a world where it is necessary to have multiple differing perspectives. To see things from a different angle is now even more important than it was before. Ken Wilber (1997/1997, November) and Maxine Greene (1995) put an emphasis on this issue. Wilber (1997/1997 November) describes the different worldviews which exist in a postmodern revolution in philosophy, psychology, and sociology. Greene (1995) talks in her famous work, Releasing the Imagination. Essays on Education, the Arts, and Social Change, about how important multiple perspectives are in order to enhance our imagination. She stresses how art in specific supports the human mind to develop and to enhance characteristics which are necessary to achieve the goal of acquiring different world views. She says that for her, as well as others, 
"the arts provide new perspectives on the lived world" (p. 4). Through these statements, one can see that the idea of art and its support of multiple perspectives have not received notice by just one person. Wilber (1997/1997, November) and Greene (1995) represent only two experts in the field who mention this important aspect, which is necessary to enable people to handle change over time.

This constant change, "from epoch to epoch, and from culture to culture" (Wilber, 1997, November, p. 1), results in a constant struggle of girls and women, specifically in the areas of self-identity and body image in our present day culture. While one might consider this battle an end product of problems that plague our present day society, such an assumption entirely neglects the fact that this issue has been present for many centuries (Bordo, 2003).

Despite the ongoing change in our culture there are two constants concerning the aspect of beauty. On one hand there is a permanent struggle of women to meet an "official standard" of beauty. On the other hand, there is a constant shift of beauty standards in "adapting" to particular periods of time.

\section{Beauty}

The question arises of what exactly is beauty? Popova (2010) asks the same question in her article Denis Dutton's Provocative Darwinian Theory of Beauty. She even refers back to the "beauty is in the eye of the beholder" theory that many people consider to approach this difficult question. Despite considering the "beauty is in the eye of the beholder" theory, she immediately dismisses it by referring back to Denis Dutton (2009) and his work, The Art Instinct. Beauty, Pleasure, and Human Evolution. Dutton (2009) is convinced that beauty is not in the eye of the beholder but that it is in our minds. He thinks it is a gift that we received from the "intelligent skills and rich emotional lives of our most ancient ancestors." He describes it as "our powerful reaction to images, to the expression of emotion in art, to the beauty of music, to the night sky, 
will be with us and our descendants for as long as the human race exists" (Dutton, 2009, as cited in Popova, 2010).

This short extract of Dutton's (2009) and Popova's (2010) works show that the definition of beauty is not a simple one. It is difficult to find the one and only standard definition. Walter Stace (1997) for example describes the meaning of beauty in his work, Meaning of beauty a theory of aesthetics, as a concept that coincides with a perceptual field. Even though he says that it does not take place in our ordinary perceptive or cognitive acts, he is convinced that it "takes place in the experience of the beautiful, and constitutes the nature of beauty." He believes that "when perceptual concepts and categories are fused with sensations, the result is perception. When empirical non-perceptual concepts are fused with perceptions, the result is beauty" (Stace, 1997, p. 54).

The Andy Warhol Museum (2008) in Pittsburgh also approaches the definition of beauty on its website. One has to keep in mind that the museum defines the concept of beauty mainly in relation to aesthetics. The museum talks about the function of aesthetics and its meaning of "a particular taste for or approach to what is pleasing to the senses - especially sight" (The Andy Warhol Museum, 2008, slide 6). It furthermore describes the function of aesthetics in interconnection to beauty and defines it as "a branch of philosophy dealing with the nature of art and beauty" (slide 17). At the end, aesthetics is linked to the concept of beauty since it is "a particular theory or conception of beauty or art" (slide 24).

Even though various definitions beauty are available (e.g. Dutton, 2009, as cited in Popova, 2010; Stace, 1997; The Andy Warhol Museum, 2008) one must still consider the difficulty of finding a uniform definition for this illusive concept. One knows that the history of beauty has not always been one upon which everybody agreed. Shifts took place in society as well as in art, which highly influenced the discussion and development of the definition. As a 
consequence, the available definitions appear to be constantly shifting, depending on a special culture, at a particular period of time (Halsall et al., 2006; Dutton, 2006).

Because of this instability of defining beauty, I consider it necessary to provide the reader with a work definition of beauty. For this research study, I defined beauty as everything that the female students perceive as 'beautiful' - from an objective (meaning an object or another person) or subjective (including themselves or their body) perspective. The students also could refer to 'external' (the way one looks from the "outside") or 'internal' (the characteristics one carries “inside") beauty. This description was also provided to the research participants with the research questions handout (see Appendix G10) that they received during the interview process.

\section{Struggles of girls and women.}

The struggles that girls and women face nowadays are taken on by Mary Pipher (1994) in her work, Reviving Ophelia: Saving the Selves of Adolescent Girls. She discusses the difficulty of comparing challenges of her generation with the ones of today's generation. This becomes even more obvious when she recounts her own experiences as a psychologist with young girls of today: "I became aware of how little I really understood about the world of adolescent girls today" (Pipher, 1994, p. 11). The difference between her former world and the one girls face today seemed to be shocking to her. She said that she was shocked when she realized that she could not transfer her own experiences from adolescence in the early 1960s to draw conclusions. Instead, she is convinced that "girls [are] living in a whole new world" (p. 11). Her examples support the conclusion of a constant and ongoing change in society.

\section{Beauty standards.}

While Pipher (1994) and other authors (Bordo, 2003; Wilber, 1997/1997, November) place their focus on a specific culture or a particular period of time to describe the influences on the definition of beauty, there is a third perspective worth considering - the control of the male 
perception of beauty. This perception plays a major role in nearly every society because its level of satisfaction significantly impacts the formation of beauty standards.

This definition of beauty standards, which is mostly set by the males, oppresses girls and women all over the world. In evaluating this view of beauty, Pipher (1994) concludes that girls of today are much more oppressed than those of her generation (p. 12). She not only mentions the aspect of oppression but also accuses the media of being driven by male desires. Thus, Pipher (1994) indirectly blames our culture for the struggles of young women, especially when she argues that girls of today "are coming of age in a more dangerous, sexualized and mediasaturated culture" (p. 12). This characterization, in turn, represents the issue that so many women had to fight with in the past, have to deal with in the present, and will have to struggle with in the future. It is not just about standards of beauty but also about images, roles, and identities.

Pipher (1994) closely analyzes these pressures and oppressions for girls and women. She shares her concern not only for the "incredible pressures" that girls nowadays have to face to be "beautiful and sophisticated," but also with the "girl-poisoning culture" in which we live today (p. 12). From Pipher's (1994) point of view, our society has to rid itself of passivity. Instead, we have to become active and address this issue. She suggests that our whole culture has to become involved in this change "to build a culture that is less implicated and more nurturing, less violent and sexualized and more growth-producing"- a society in which all the gifts of our daughters "can be developed and appreciated" (p. 13).

Pipher (1994) is on the right track with her demand to change our society and, as a positive side effect, our culture. Nevertheless, this undertaking is not as easy as it seems. Such a task requires a lot of effort, labor, and willingness to change old patterns. The set goal is only achievable if one analyzes the existing problems honestly, accepts weaknesses in the system, and actively exerts the effort needed to change old paradigms. Apart from these upcoming "how" 
truths, the question arises how one can tackle the "where" truths. Where does one have to start in order to induce change? The theoretical answer might discern our educational institutions. However, the practical implementation is far more complicated because of the old patterns and paradigms in which our educational system seems to be caught.

\section{Image, role, and identity.}

\section{Schools and their influences.}

The institution of school defines who we are and affects the way our society appears. Accordingly, the educational system of schooling has an indirect impact on our personal image, role, and identity in the midst of a society. As Pipher (1994) already mentioned, one has to ask the question, especially in reference to identity and institutionalization, how and where individual actions can change society and, in turn, how and where society changes and influences individual actions. Furthermore, one has to try to get to the bottom of the question of what impact the individual identity has in a social context.

Pipher (1994) is also interested in finding an answer to these questions. She is especially fascinated by the correlation between culture and individual psychology, and wonders "why cultures create certain personalities and not others, in how they pull for certain strengths in their members, in how certain talents are utilized while others atrophy from lack of attention" (Pipher, 1994, p. 26). Moreover, she expresses concern for the potential impact cultures have on the development of individual disorders. Trying to please the expectations of their environment, society, and culture often results in an endeavor that seems impossible for girls to fulfill.

\section{Individual identity and social context.}

To help this problem, schools and education systems could try to adapt their paradigms and rules to the constantly changing world, instead of stubbornly clinging to their old patterns. An initial step in the right direction could be the effort to make it easier for girls, but also 
students or learners in general, to bring their individual identity and social context together. Unfortunately, these are very often kept disconnected from each other.

Latta Macintyre (2004) presents an interconnected approach as she describes the dualism of individual identity and social context in her article, Traces, Patterns, Texture: In Search of Aesthetic Teaching/Learning Encounters. She concentrates on the theme of the individual and his or her surrounding world. This viewpoint reflects one of the main concepts of the AIC (Dewey, 2005; Eisner, 2002b) and could be adopted by our schools.

\section{Categorizations.}

Gender-driven subjects should not exist in our modern-day schools. Despite the positive effort by our education systems to not place students into certain categories because of their gender, it appears that the view of society, in reference to gender categorization, has not changed. The clothes stores J.C. Penney and Forever 21 have recently created controversy by putting boys and girls into categories. Both companies appear to see a market for their "sexist clothing" that they put on their shelves (Credo action. More than a network. A movement., (n.d.)). J.C. Penney's shirt (see Appendix F, Figure F1) with the slogan "I'm too pretty to do homework so my brother has to do it for me" teaches girls that being smart and pretty are mutually exclusive (Krupnick, 2011). Forever 21's shirt for girls with the slogan "Allergic to Algebra" (see Appendix F, Figure F2) supports stereotype thinking and consequently drew major criticism $(\mathrm{Ng}, 2011)$.

These categorizations, which presume to know what is and is not proper for a particular gender, cause more problems in our society and for our children than one might expect. Pipher (1994) describes that "this gap between girls' true selves and cultural prescriptions for what is properly female creates enormous problems" (p. 20). Still, even in our society, too often choices are made by (female) students while depending on thoughts like "What must I do to please others?” (p. 20). Girls especially think about what others expect from them and what they could 
do in order to fulfill those expectations. Decisions are not made depending on individual preferences or gifts. Instead, students try to fill the gap in which they are supposed to belong.

Margaret Mead (1949) would like to put an end to this inflexible idea and describes the ideal culture as one in which "there is a place for every human gift" (Mead, 1949, as cited in Pipher, 1994, p. 22). Nevertheless, the reality looks different. In Mead's (1949) opinion, too "many gifts are unused and unappreciated. So many voices are stilled" (Mead, 1949, as cited in Pipher, 1994, p. 22). The society is not flexible enough to deal with the diversity of gifts and characteristics. Girls are forced into special kinds of roles that they only agree to play because they think they are supposed to and will be appreciated this way. As a consequence, selves develop that do not have anything to do with true and natural selves. But how can one distinguish, or avoid, the development of true and false selves?

\section{Selves.}

These true and false selves can be found in media images that appear to set the standards for beauty. More models in magazines or on television have undergone surgery than not, and even in our "regular" society, plastic surgery has become an every day theme and nothing special anymore (Bordo, 2003). This phenomenon of true and false selves reminds me of Kant's two types of beauty_-“natural beauty” and "artificial beauty" (Gasché, 2002, p. 105). These concepts of natural and artificial beauty, as well as the true and natural selves, put a lot of pressure on young children. Research (Silverman, 2010) shows that outside influences, like parents, can intensify this pressure even more. Alice Miller (1981) describes this aspect in more detail when she reflects on the weight that parents directly or indirectly place on their children. These pressures lead some young children "to deny their true selves and assume false selves to please their parents" (Miller, 1981, as cited in Pipher, 1994, p. 22). Pipher cautions that "this time the pressure comes not from parents but from the culture" (p. 22). 
Our children face a lot of pressures: please your parents and your peers, fulfill the role that the society and its culture expect, deny your identity and at the end even yourself. An interconnection of both culture and individual becomes necessary. At the same time, one has to make sure that this interrelation is an equal one, not one of power and oppression. Only if equality is assured can an individual develop an independent and strong identity. The end product is a desirable identity that parents and teachers should aspire for their offspring. Unfortunately, this aspiration rarely seems to come to fruition, especially not for girls or women.

\section{The male definition of beauty.}

The works of John Dewey (1934, 1938, 2005), Latta Macintyre (2004), and Mary Pipher (1994) reveal the three concepts of image, role, and identity and their interconnection with the male definition of beauty. Their ideas on these themes are so important because these three concepts appear to be mainly responsible for the struggles that women of our society have to face and to overcome. In real life, too often women feel dehumanized and oppressed by their male “oppressors.” Instead of “re-empowering,” recreating, and liberating themselves (Freire, 2007), they often remain under oppression.

\section{Oppression and dehumanization.}

Paulo Freire (2007) describes the phenomena of oppression and dehumanization in his work, Pedagogy of the Oppressed. He thinks the helplessness and passivity of the oppressed are consequences of the duality (e.g. trying to please own desires and requirements of others) in which the oppressed live. The oppressed seek to overcome this duality which already became part of their inner personality (Freire, 2007, p. 48) but are not always successful. They have to make the choice "of being wholly themselves or being divided" (p. 48). The oppressed has to become active in order to determine their destiny. Freire (2007) describes these situations of decision-making as the "tragic dilemma of the oppressed" and claims education responsible (p. 
48) for saving students by showing a sense of responsibility and by helping them overcome their challenges.

Freire (2007) even offers a new theory he calls "the pedagogy of the oppressed" (Freire, 2007, p. 48) in his book that can serve as a guide for responsible educators. He defines "the pedagogy of the oppressed" as "a pedagogy which must be forged with, not for, the oppressed (whether individuals or peoples) in the incessant struggle to regain their humanity" (p. 48). This definition shows the importance of the victim's (any oppressed) engagement (p. 48). Liberation cannot take place without self-involvement. It is necessary for the oppressed person to undergo a period of struggle under which he or she has to get drawn into a dialogue with the oppressor to negotiate. Passivity in contrast, can lead to dehumanization.

Dehumanization can be defined as oppression driven a step further. While one can be oppressed without being conscious of it, dehumanized individuals readily perceive their oppression. The personalities of the oppressed determine whether they become active or remain passive, meaning they decide if they are oppressed or dehumanized. To avoid this issue, our educational system should enable children to become active, involved, and critical. Students should not avoid dialogues and discussions.

The goal of an educator in today's generation should not be to create dehumanized, dependent, and uncritical individuals. Educators should be happy, not angry, to have students who enjoy engaging in discussions and dialogues, which could be viewed as liberating for both. This liberation does not just seem to be very important in accordance with the aspect of dehumanization but also in line with the concept of identity - especially in reference to the oppression of girls and women by the supremacy of the males in more areas than education. 


\section{Males and their influences on the female image, role, and identity.}

Males not only have a major impact on the female identity but also on other areas or concepts of the female life, the definition of beauty, and as a consequence on the female image, role, and identity. These influences are possible for two reasons.

First, male attitudes towards beauty seem to be much more influential on a society's value and standard settings than the female ones. Carolyn Korsmeyer (2004) also refers to this idea when she writes about the common aesthetic judgment rules in her work, Gender and Aesthetics: An Introduction. She argues that "although women were considered capable of developing fine taste, arguably the model of the ideal aesthetic judge, the arbiter of taste, was implicitly male, for men's minds and sentiments were considered to be more broadly capable than women's" (Korsmeyer, 2004, p. 46).

Second, value and standard settings of males, especially in reference to beauty, appeared to be significantly different from those of females. They also seemed to be more valued. Korsmeyer (2004) expands in more detail on this subject by arguing that "the combination of theoretical assumptions and social norms," produce the opinion that "higher mental powers are asymmetrically exercised in males and females" (p. 46). She continues by saying that the supposed greater mental facility of males even makes them more capable of "judgments of taste for complicated subjects" (p. 46). Females were attributed with lower mental powers and consequently excluded of the process of value and standard settings of beauty, which makes evolving self-concept issues in women even less surprising.

\section{Self-concept}

A short summary with definitions of self-concept will be given to provide the reader with a basic idea. Like the concepts of beauty or phenomenology, the self-concept is an idea that cannot be tackled easily. Throughout the centuries, various theories have developed. For this work, I would like to focus on two definitions of self-concept. One is provided by Seymour 
Epstein (1973) and his article, The Self-Concept Revisited: Or a Theory of a Theory. The other definition is provided by Anna Bardone-Cone et al. (2010) and their work, Aspects of SelfConcept and Eating Disorder Recovery. What does the Sense of Self look like when an Individual recovers from an Eating Disorder?

Epstein (1973), whose research falls into the field of an historical overview, provides an encyclopedic collection of theories regarding self-concept (James, 1910; Cocley, 1902; Mead, 1934; Sullivan, 1953; Lecky, 1995; Snugg and Combs, 1949; Hilgard, 1949; Rogers, 1951; Allport, 1955; Sarbin, 1952). The collection serves as an important framework for this study, including typical characteristics for self-concept:

1. It is a subsystem of internally consistent, hierarchically organized concepts contained within a broader conceptual system.

2. It contains different empirical selves, such as a body self, a spiritual self, and a social self.

3. It is a dynamic organization that changes with experience. It appears to seek out change and exhibits a tendency to assimilate increasing amounts of information, thereby manifesting something like a growth principle. (...).

4. It develops out of experience, particularly out of social interaction with significant others.

5. It is essential for the functioning of the individual that the organization of the selfconcept be maintained. When the organization of the self-concept is threatened, the individual experiences anxiety, and attempts to defend himself against the threat. If the defense is unsuccessful, stress mounts and is followed ultimately by total disorganization.

6. There is a basic need for self-esteem which relates to all aspects of the self-system, and, in comparison to which, almost all other needs are subordinate. 
7. The self-concept has at least two basic functions. First, it organizes the data of experience, particularly experience involving social interaction, into predictable sequences of action and reaction. Second, the self-concept facilitates attempts to fulfill needs while avoiding disapproval and anxiety (Epstein, 1973, p. 407).

Even though Epstein's (1973) article was written quite some time ago, his work still provides a groundwork of the main characteristics of self-concept. The traits he came up with are still valid in our time. Nevertheless, to give the reader an idea on how more recent research views selfconcept, a more "up-to-date" definition of self-concept will also be given.

Bardone-Cone, et al. (2010) assume that from a cognitive perspective, the "self-concept can be defined as 'a set of knowledge structures about the self' (Stein \& Corte, 2007, as cited in Bardone-Cone et al., 2010, p. 823) and encompasses a wide range of constructs" (Baumeister, 1999, as cited in Bardone-Cone et al., 2010, p. 823). Markus and Wurf (1978) add that "selfconcept represents a dynamic multifaceted construct, rather than a unitary undifferentiated structure" (Markus \& Wurf, 1978, as cited in Bardone-Cone et al., p. 823). Bardone-Cone et al. (2010) and Epstein (1973) agree upon three main structures. First, knowledge structures about the self and organizations of self-concept need to be maintained to avoid chaos. Second, a wide range of selves exists in individuals. Third, the self-concept is dynamic, multifaceted, and changes with experience and interactions with others.

\section{(Negative) self-concepts and (resulting) disorders.}

Self-concepts can develop in a positive or negative direction. This part of the study is supposed to focus mainly on negative self-concepts and resulting disorders. By now, it can be officially stated that the concept of beauty plays an important role in our society. We, as a society, have developed special sets of standards that are supposed to reflect characteristics of beauty. This process becomes even clearer in areas concerning the physical appearance of women. Adolescent girls appear to develop various disorders while attempting to meet these 
different requirements of model standards. Despite negative self-concepts, even eating disorders like anorexia nervosa, bulimia, or obesity are very common (Pipher, 1994; Silverman, 2010).

The questions arise why (because of which influences) and when (at what age) the phenomenon of a negative self-concept develops in girls. This is a question that is not easy to answer but an obstacle that should be tackled in order to cause change in our society, school culture, and, most importantly, in the lives of those adolescent girls whose self-worth and identity appear to be no longer found in themselves. Hoskins (2002) is one of the researchers who discusses these issues and wonders about the causes. She states that although the phenomenon of eating among adolescent girls has been the subject of thousands of research projects, an in-depth understanding of the complexity of this disorder has fallen short in many ways. We have yet to determine conclusively why some girls and not others succumb to anorexia nervosa and bulimia, what the best practices are for treatment, or how to design and implement effective prevention programs. (Hoskins, 2002, p. 231)

In order to prevent disorders in general or eating disorders in specific, it is important to find out what exactly it is that has such a major impact on the self-concepts of girls. The apparently ever-increasing influence of beauty should not just alarm doctors and researchers but also our schools and educational systems. Hoskins (2002) specifically stressed that "we have to more fully understand how the symbols, images, and metaphors of a culture become integrated into girls' identities" (Hoskins, 2002, p. 232).

\section{Media.}

Media appear to play a major role in creating the described problem. Children and adolescents are almost always surrounded by media that portray not only perfect objects, perfect families, and perfect life circumstances but also perfect bodies which match perfect beauty standards. Hoskins (2002) recognizes that television is not the only source of supporting these 
impossible to achieve ideals. Besides television, also "magazines, advertisements, billboards, and movies all perpetuate similar kinds of images that tend to be harmful for girls" (Hoskins, 2002, p. 234).

Ann Becker's (1999) famous research in Fiji, for example, provides some insight into television's major influence. She is convinced that "Western ideals for beauty have been presented through the introduction of television into Fijian society. Prior to exposure to these images, eating disorders were rare, and most adolescent Fijian girls seemed relatively satisfied with their bodies" (Becker, as cited in Hoskins, 2002, p. 234). Only after television was introduced and as a consequence, images of idealized North American body sizes took over, girls "began to reevaluate themselves, and body dissatisfaction increased. Dieting became a topic for discussion and girls began to monitor their eating behaviors" (p. 234). This research example shows clearly how immense the impact of television on the self-concept of adolescent girls can be, even on the ones that seemed relatively satisfied with their bodies beforehand.

\section{Popular culture.}

While Becker (1999) showed in her research the general influence of television on the identities of girls in later, adolescent stages, Margaret Walshaw (2001) stresses the influence of popular culture over time in her journal article, A Foucauldian Gaze on Gender Research: What do you do when confronted with the Tunnel at the End of the Light? In her opinion, popular culture plays a part in framing girls' "understanding of the world and of themselves, of gender relations and cultural symbols, values, and social power. To that end, cultural and media representation enable learning about gender to take place" (Walshaw, 2001, p. 488).

Unfortunately, this learning about gender is often characterized by unattainable cultural norms which cause adolescent girls or young women to neglect their bodies. 


\section{The denial of the body.}

The focus on the body appears to play an important role not just in today's society but also in every society and culture throughout the centuries. Paul Duncum (2005) concludes in his work, Visual Culture and an Aesthetics of Embodiment, that some of the past cultures even tried to deny the body. But it refuses to be denied (Duncum, 2005, p. 9). This is a truth that many girls who try to reject their bodies in various ways, including through eating disorders or other psychological dysfunctions, have to face.

Duncum (2005) finds that the denial can be understood in contradicting ways. It can be understood as the celebration of the body that characterized popular culture, especially in Europe, for many centuries. It is also about the curtailing of the body. In earlier times, the body played a major role in public discourse. This importance of "the body in public discourse is abundantly clear in considering carnival, perhaps the major form of popular culture” (p. 9). Through this notion, the substantial role of culture in reference to the body and the set of beauty standards becomes obvious. While this was especially true for the cultures of earlier times, nowadays society cannot deny similar customs and traditions. Carnival for example is not just a right that is long forgotten. Almost all over the world, one can find celebrations that worship the body, similar to carnival.

In addition to Duncum (2005) also several other authors (Dutton, 2006; Korsmeyer, 2004; Pipher 1994; Romanska, 2005; Steiner, 2004) place their focus on the considerable influences of culture on the human body and its beauty standards. Adolescent girls are often not able to see their bodies as a part of their personalities anymore. Instead, they treat their bodies as “an identificatory beauty model of desirable feminity" (Romanska, 2005, p. 486). Whereas Pipher (1994) still carries hope to change our media-driven and male-defined world, Duncum (2005) shares her idea on media without an ounce of hope for change. 


\section{The commercialization of culture.}

Duncum (2005) specifically critiques the commercialization of culture. From his point of view, our society has become more and more visceral. He does not appreciate that "fashion magazines use images of models that are anorexic, self-abusing or appear to have been beatenup" (Duncum, 2005, p. 16). The appeal to the senses becomes the most important thing, rather than to the mind. Media use this technique to influence and reach more people. Culture is more often driven by the consumer body, instead of the human (body). To please this consumer body, media "create a dream world of adolescent male fantasy in which all women are nymphomaniacs, hungry to satisfy their own and their partner's desires and in which 'No' invariably means 'Yes'” (p. 16). Sut Jhaly (1995), a producer, writer, and director, concludes that "this is the world where people are driven by deep, powerful, primary instincts" (Jhaly, 1995, as cited in Duncum, 2005, p. 16). Unfortunately, these are the instincts that not only rule today's society but also ruled the societies of earlier times, which becomes obvious in traditions like the previously-mentioned carnival that societies perpetually pass on to their descendants.

\section{Value and standard settings.}

Questions about generally accepted value and standard settings arise. Which values and set of traits must an individual have in order to meet the expectations? And at what age does an individual usually start to develop them? Mary Martin and Patricia Kennedy (1993) reported in their article, Advertising and Social Comparison: Consequences for Female Preadolescents and Adolescents, that even "girls between the ages of 8 and 11 compared themselves to fashion models and other media images and felt bad about the comparison" (Martin \& Kennedy, 1993, as cited in Clark \& Tiggemann, 2007, p. 77). Girls start to develop various disorders to punish themselves and to make up for unreachable goals at earlier ages. Unapproachable goals, which are most often caused by media like newspapers or television, transfer into the " "not good enough' syndrome" (Hoskins, 2002, p. 234) and are spread beyond girls and young women. 


\section{Body dissatisfaction and low self-concept.}

Frustration of girls with their bodies and low self-concepts are the consequence, even among very young age groups. The older these girls grow an increase in body dissatisfaction takes place. Kirsten Davison and Leann Birch (2002) conclude in their work, Processes linking Weight Status and Self-Concept among Girls from Ages 5 to 7 Years, that the "self- perception of girls as young as 5 and 7 years of age are influenced by their weight status; girls who are more overweight perceive themselves as less competent across multiple domains of self-concept" (Davison \& Birch, 2002, p. 746). Also elementary-school girls are affected, as shown by Levina Clark's and Marika Tiggemann's (2007) research, Sociocultural Influences and Body Image in 9to 12- year-old Girls: The Role of Appearance Schemas. They reported that "the use of magazines to obtain weight and beauty information" (Clark \& Tiggemann, 2007, p. 77) was related to poor body image in elementary-school girls. Sharynn Muir, Eleanor Wertheim, and Susan Paxton (1999) found that "more grade 10 girls (66\%) than grade 7 girls (43\%) having taking up dieting" which in their opinions prove that "many girls commence dieting in the years between grades 7 and 10” (p. 264). Philip Myers' and Frank Biocca's (1992) research mentions this phenomenon, too. They state in their work, The Elastic Body Image: The Effect of Television Advertising and Programming on Body Image Distortions in Young Women, that the preoccupation of girls with their bodies and their evaluation of themselves "against impossible body size ideals has resulted in 53\% of 15 -year-old girls being dissatisfied with their bodies, increasing to $78 \%$ when girls reach the age of 17' (Myers \& Biocca, 1992, as cited in Hoskins, 2002, p. 231). Additional research shows that appearance schemas (Clark \& Tiggemann, 2007), diets (Muir, Wertheim, \& Paxton, 1999), and thinness (Dohnt \& Tiggemann, 2006) emerge as ideas that are all commonly linked to the age and weight status of young girls.

In order to reverse this increase or at least keep the upcoming negative self-concept and body dissatisfaction at a minimal level, a thorough and detailed analysis and interpretation of the 
results of the various influences is necessary. With my research on arts integration and the AIC, I hope to provide a possible model to counteract the effects of self-concept disorders at an early age.

\section{Phenomenological Research}

This research is set up for the qualitative research method framework of phenomenology. Before starting out with definitions, I would like to note that this research study distinguishes phenomenological research and its various processes from phenomenology as a philosophical concept. Even though I shortly refer to phenomenology and is philosophical origins, the study focuses on phenomenology, as used by Moustakas' (1994) and his research method framework.

At the beginning of this phenomenological research study, I had to overcome the problem that there is no consistent definition of this concept available. Even Patton (2005), who appears to provide a very well-organized idea of phenomenology, stresses that the "initial clarity of this definition can fade rapidly because the term phenomenology has become so popular and has been so widely embraced that its meaning has become confused and diluted" (Patton, 2005, p. 104). From his point of view, phenomenology can refer to various concepts, like "philosophy (Husserl 1967), an inquiry paradigm (Lincoln 1990), an interpretive theory (Denzin and Lincoln 2000b:14), a social science and analytical perspective or orientation (Harper 2000:727; Schutz 1967, 1979), a major qualitative tradition (Creswell, 1998), or a research methods framework (Moustakas, 1994)" (Patton, 2005, p. 104).

Mary Johnson (2000) also acknowledges the difficulty of finding a standard definition of phenomenology in her work, Heidegger and meaning: Implications for Phenomenological Research. Nevertheless, she provides us with a basic idea what phenomenological research is: "Although there is not a consistent definition of phenomenology, phenomenological research can be distinguished from other qualitative methods in that the phenomenological researcher aims to 
describe and/ or understand the meaning of the participants' lived experiences" (Omery, 1983; Cresswell, 1998; as cited in Johnson, 2000, p. 240).

Donna Mertens (2005) focuses on the individual's subjective experience that Johnson (2000) described when referring to phenomenological research and Bogdan and Biklen (2003) in her book, Research and Evaluation in Education and Psychology. Integrating Diversity with Quantitative, Qualitative, and Mixed Methods. Mertens (2005) also cites Michael Patton's (2002/2005) well-known phenomenological research question: "What is the meaning, structure, and essence of the lived experience of this phenomenon for this person or group of people?" (Patton, 2005, p. 104).

Despite all the confusion, Patton (2005) concludes that all these phenomenographic approaches have one thing in common, namely that they "focus on exploring how human beings make sense of experience and transform experience into consciousness, both individually and as shared meaning" (p. 104). Patton (2005) believes that this commonality requires a thorough description of "how people experience some phenomenon [phenomena] - how they perceive it, describe it, feel about it, judge it, remember it, make sense of it, and talk about it with others" (p. 104). To gather such phenomenological data, as is the goal of this research study, "one must undertake in-depth interviews with people who have directly experienced the phenomenon of interest" (Patton, 2005, p. 104) - in this case, interviews about the experience of the AIC by students, parents, and teachers. That is, they have "lived experience" as opposed to "second-hand experience" (p. 104).

Valerie Bentz and Jeremy Shapiro (1998) focus on the same aspects of phenomenology as Patton (2005), when they refer to thinking, feeling, lived experience, and the study of consciousness in their work, Mindful Inquiry in Social Research. In their opinion, the intent of phenomenology "is to understand the phenomena in their own terms - to provide a description of 
human experience as it is experienced by the person herself”' (Bentz \& Shapiro, 1998, p. 96) and not from a second source.

Bentz and Shapiro (1998) and Mertens (2005) also mention the method of conversational analysis. This phenomenological method offers analytical tools to interpret and answer research questions because it "developed and refined techniques of analyzing transcripts of conversations in order to uncover universal patterns of relationships" (Bentz \& Shapiro, 1998, p. 101).

For this research study, the research methods framework by Clark Moustakas (1994), also mentioned and described in more detail by Patton (2005), was chosen because it not only provides detailed steps and processes on how to analyze data but it also explains how the data can be transferred and generalized from various individuals to a whole group.

\section{History/ Origin.}

The German philosopher, Edmund Husserl, is considered as the founder of phenomenology as a philosophical tradition (Patton, 2005; Bentz \& Shapiro, 1998). The work of Alfred Schutz (1899-1959) also "was an important influence in applying and establishing phenomenology as a major social science perspective" (Patton, 2005, p. 105). Patton (2005) adds that other important influences have been "Merleau-Ponty (1962), Whitehead (1958), Giorgi (1971), and Zaner (1970)." He says also that “more recently, phenomenology has become an important influence in certain approaches to psychotherapy (Moustakas 1988, 1995)” (p. 105).

Patton (2005) continues to describe how Husserl (1913) defines phenomenology as "the study of how people describe things and experience them through their senses" (Patton, 2005, p. 105). I agree with Husserl's most basic philosophical assumption that "we can only know what we experience by attending to perceptions and meanings that awaken our conscious awareness" (p. 106). Simple awareness is not good enough. We have to be conscious to perceive experiences that separate us as an individual from a whole group. 
As Patton (2005) and Bentz and Shapiro (1998) summarize, phenomenologists research in a way that allows us to figure out the phenomena that we experience so that they make sense to us and the world. Consequently, we develop a worldview. Accordingly, as it was indicated at the beginning of this section, the "subjective experience incorporates the objective thing and becomes a person's reality, thus the focus on meaning making as the essence of human experience" (Patton, 2005, p. 106). Patton (2005) describes two implications of this perspective that are often confused but of major interest to him, and this study, as well:

The first implication is that what is important to know is what people experience and how they interpret the world. This is the subject matter, the focus, of phenomenological inquiry. The second implication is methodological. The only way for us to really know what another person experiences is to experience the phenomenon as directly as possible for ourselves. This leads to the importance of participant observation and in-depth interviewing. (Patton, 2005, p. 106)

Patton's (2005) description of the "what" and the "how" of an experience for a person and a whole group, is important for this study. It is not enough to know "what" a person experienced, nor is it enough to know "how" they experienced it. Both inquiries have to go together and build a picture of the whole, shared experience. Furthermore, it is important to notice that even though every individual viewpoint might seem different, specific phenomena might evolve that are similar for the whole group. Patton's (2005) methodological implications of in-depth interviews were considered and build the main core of this study. The aspect of participant observation does not apply to this study.

\section{The phenomenological researcher.}

Mertens (2005), Patton (2002), and Bentz and Shapiro (1998) address the issues of the phenomenological researcher. I decided to include this section so that the reader can become acquainted with the struggles a phenomenological researcher has to face. From my point of view, 
any phenomenological researcher has a difficult role and must know what, how, and when to do things in order for his or her phenomenological research study to succeed.

While Mertens (2005) describes the phenomenological researcher simply as someone who "does not make assumptions about an objective reality that exists apart from the individual" and rather as someone whose "focus is on understanding how individuals create and understand their own life spaces" (Mertens, 2005, p. 240), Bentz and Shapiro (1998) more specifically stress two points of view, the empathic and the meaningful. They think that a researcher should neither be too passive nor too reflective. Instead, he or she should hold good social interaction skills and a pervasively empathic manner. On the other hand, they stress that phenomenological research is "one of the most resolutely analytical modes of inquiry, requiring a willingness to engage in dissection of and reflection on the nature of consciousness and meaning" (Bentz \& Shapiro, 1998, p. 102). The most detailed characterization of the phenomenological researcher is probably provided by Patton (2002), referring to Eichelberger (1989). From his point of view, phenomenologists are:

Rigorous in their analysis of the experience, so that basic elements of the experience that are common to members of a specific society, or all human beings, can be identified. This last point is essential to understanding the philosophical basis of phenomenology, yet it is often misunderstood. On the other hand, each person has a unique set of experiences which are treated as truth and which determine that individual's behavior. In this sense, truth (and associate behavior) is totally unique to each individual. Some researchers are misled to think that they are using a phenomenological perspective when they study four teachers and describe their four unique views. A phenomenologist assumes a commonality in those human experiences and must use rigorously the method of bracketing to search for those commonalties. Results obtained from a phenomenological study can then be related to and integrated with those of other phenomenologists studying 
the same experience, or phenomenon. (Eichelberger, 1989, p. 6, as cited in Patton, 2002, pp. 106-107)

This quotation by Eichelberger (1989, as cited in Patton, 2002) shows clearly that a simple collection and listing of individuals' experiences is not sufficient. Commonalities between individual experiences have to be found with the help of bracketing. Moustakas (1994) defines bracketing as a research process in which the whole attention is put on brackets. All other influences are put aside so that the whole process only focuses on the most important things, the topic and the question (p. 97). Once similarities are found between individuals that put them into specific groups, the shared experiences can be described as phenomena. In order to get to this point, a detailed phenomenological analysis has to take place.

\section{The phenomenological analysis.}

Even though the two applied phenomenological analysis processes called "Phenomenological Model" and "Modification of the Van Kaam Method of Analysis of Phenomenological Data" (Moustakas, 1994) are described in more detail in chapter three, a short overview and conceptual background of the processes is given in this section.

Throughout the analytical process, I frequently refer back to the four steps of Moustakas' (1994) “Phenomenological Model” (p. 180): 1) epoche, 2) phenomenological reduction, 3) imaginative variation, and 4) synthesis of texture and structure. Still, Moustakas' (1994) "Modification of the Van Kaam Method of Analysis of Phenomenological Data" (p. 120) with its seven-plus-one steps of data analysis builds the body of the data analysis process.

Patton (2002) thinks that it is "relatively meaningless to describe 'phenomenological analysis' as if it constituted a single approach or perspective" (Patton, 2005, p. 483). As a consequence, he decided to include in his work only two phenomenological approaches to analysis - the ones taken by Clark Moustakas and Bruce Douglass of The Union Institute Graduate College (Cincinnati, Ohio) and the Center for Humanistic Studies (Detroit, Michigan). 
In addition to these two phenomenological approaches, this research study also took Hycner's (1985) phenomenological process into consideration. However, I eventually discarded this process as it is not entirely up to date with the present day model of phenomenology suggested by Moustakas (1994). Hycner's (1985) model was criticized (Giorgi, 2006) because it does not describe fully the phenomenological reduction, consisting of (a) bracketing, (b) horizonalizing, (c) clustering horizons into themes, and (d) organizing the horizons and themes into a coherent textural description of the phenomenon (Moustakas, 1994, p. 97). Therefore, Hycner's model is more appropriately updated for this study (Giorgi, 2006, p. 309). As Giorgi writes, he got the part about "bracketing" correct, but he does not mention the notion that the "given" is only understood to be the way it is present to consciousness of the experiencer and not assumed to exist the way the experiencer perceives it. In other words, the phenomenal status of what is experienced is not at all mentioned by Hycner. (Giorgi, 2006, p. 309)

Since Hycner's phenomenological process has been criticized and refused by various researchers in the field, the phenomenological analysis defined by Moustakas (1994), which he also calls "Phenomenological Model" (Moustakas, p. 180), was used for this study. While Moustakas (1994) lists epoche, transcendental-phenomenological reduction, and imaginative variation as "the core processes that facilitate derivation of knowledge" (Moustakas, p. 33), Patton (2002) adds "synthesis of texture and structure" (p. 486) as a fourth process to the phenomenological process. All the processes listed by Moustakas (1994), as well as Patton's (2002) additional process, were used for this research study: 1) epoche, 2) phenomenological reduction, 3) imaginative variation, and 4) synthesis of texture and structure. In specific, the data analysis process for this study followed Moustakas' (1994) suggestion of the "Modification of 
the Van Kaam Method of Analysis of Phenomenological Data" (Moustakas, 1994, p. 120). During this process, seven-plus-one steps were conducted (Moustakas, 1994): 1) listing and preliminary grouping; 2) reduction and elimination; 3) clustering and thematizing the invariant constituents; 4) final identification of the invariant constituents (ICs) and themes (validation); 5) individual textural description; 6) individual structural description; 7) textural-structural description; and 8) integrated textural/ structural description. In addition, Copen's (1992) work on insomnia was also used as a model for the data analysis process. For more detailed information on these steps and processes, please see chapter three (methodology).

\section{Arts Integration/ Arts-Integrated Curriculum (AIC)}

\section{What is arts integration/ AIC?}

What is arts integration and how can one define it? Of course, as is the case with all important concepts, arts integration is a notion that is defined in various ways by researchers in the field. Jacob Mishook and Mindy Kornhaber (2006) even describe arts integration as a “contested and confusing term" (Mishook \& Kornhaber, 2006, p. 4). In their opinion, "this is not surprising given that arts educators do not have any shared agreement on what arts integration should look like, or even whether arts integration should be a goal of arts education" (Mishook \& Kornhaber, 2006, p. 4).

Because of this overflow of definitions several definitions will be cited in this study (Cornett, 2011; Mishook \& Kornhaber, 2006; Purnell, 2004; The John F. Kennedy Center for Performing Arts, 2008; Weiss, Lichtenstein \& Booth, 2008), but the focus will be on three definitions by Claudia Cornett (2011), Weiss et al. (2008), and The John F. Kennedy Center for Performing Arts (2008).

Mishook and Kornhaber (2006) generated a whole list of possible meanings for the term arts integration:

The use of project based learning to address community problems or issues (Wolk, 1994); 
thematic instruction (Ackerman and Perkins, 1989); multiple intelligences (Garner, 1983); transfer of knowledge across artistic and nonartistic disciplines (Darby and Catterall 1994; Fiske 1999; Hamblen 1993); the use of arts to enhance the study of academic disciplines (Catterall and Waldorf 1999; Fiske 1999); or interdisciplinarity among different arts forms, such as painting and music (Roucher and Lovano-Kerr 1995). (Mishook \& Kornhaber, 2006, p. 4)

Paula Purnell (2004), in contrast, uses a very simple working definition of arts integration, which she adopted from the Center for Applied Research and Educational Improvement (2003): “Arts integration: instruction in which arts-related concepts and activities are infused with other academic areas" (Purnell, 2004, p. 157).

Cornett (2011) adds to these definitions by referring to various terms like arts infusion, arts immersion, arts-based, and interdisciplinary, by which arts integration is also known. Furthermore, Cornett (2011) adds that some of these terms can be found in content standards for national arts standards, under interdisciplinary and inquiry learning, and in schools implementing multiple intelligences. Nevertheless, through time a general definition of arts integration developed, formulated by Cornett (2011): “Arts integration is the meaningful use of arts processes and content to introduce, develop, or bring closure to lessons in any academic area" (p. 17). She elaborated that the goal of arts integration is "to transform learning by using the arts to actively engage learners in problem solving that creates understanding and expands expressive communication abilities" (p. 17). Weiss et al. (2008) define arts integration as "arts in-te-gration" (Weiss, Lichtenstein, \& Booth, 2008, p. 00) that is divided into two sections. First, it is "an educational field that specializes essentially in relationships - between people, ideas, curricula, places, themes, and areas of study, with an emphasis on arts learning at the center of these relationships" (p. 00). Second, arts integration is described as "working with curricular intention to merge learning in the arts with learning in other academic subject areas" (p. 00). 
The John F. Kennedy Center for Performing Arts (2008) provides another, yet similar, definition of arts integration: "Arts Integration is an APPROACH to TEACHING in which students construct and demonstrate UNDERSTANDING through an ART FORM. Students engage in a CREATIVE PROCESS which CONNECTS an art form and another subject area and meets EVOLVING OBJECTIVES in both” (The John F. Kennedy Center for Performing Arts, 2008, pp. 1-4).

Though the above-mentioned definitions of arts integration (Cornett, 2011; Mishook \& Kornhaber, 2006; Purnell, 2004; The John F. Kennedy Center for Performing Arts, 2008; Weiss et al., 2008) three (Cornett, 2011; The John F. Kennedy Center for Performing Arts, 2008; Weiss and Lichtenstein, 2008) appear to be more closely related and to summarize very similar ideas. The following concepts stand out as common to their classifications: 1) the notion of communication; 2) the notion of relationships; and 3) the notion of connectedness. Cornett (2011) and The John F. Kennedy Center for Performing Arts (2008) appear to agree on one more concept than Weiss et al. (2008). The authors stress the idea of learning, transformed learning, and learning in other areas.

Furthermore, they include problem solving in connection with creative processes in their definitions. Understanding also seems to play a major role, as well as the active engagement of learners. In general, one gets the impression that Cornett (2011) and Weiss et al. (2008) provide a basic yet fundamental start for the definition of arts integration. Nevertheless, The John F. Kennedy Center for Performing Arts (2008) expounds upon the definition with more details and additional concepts.

As a consequence, the previously-quoted definition of The John F. Kennedy Center for Performing Arts (2008) will be used as a basic working definition for arts integration in this this dissertation. 


\section{Characteristics.}

In Enhancing Student Learning Through Arts Integration. Implications for the Profession

David Gullatt (2008) talks about how the arts are mainly treated like they are only emotionally, not cognitively valuable. In his opinion, it is ironic that "the arts are frequently dismissed as merely emotional, not cognitive" (Gullatt, 2008, p. 14). In this regard, he quotes Rabkin and Redmond (2006), arguing that it is this "very emotional and personal content of the arts" that "causes the arts to become cognitively powerful" (Rabkin \& Redmond, 2006, as cited in Gullatt, 2008, p. 14). DeMoss and Morris (2002) also observed special characteristics concerning arts integration. From their point of view, a classroom that is defined by arts integration shows clear activities, expectations, and outcomes. Furthermore, work habits, equal participation, and connected instruction are part of the lesson. Content integrity from the teachers' and the artists' sides also play an important role. Art concepts are usually applied and focus on academic contents, as for example math, science, reading, or writing. For DeMoss' and Morris' (2002) democratic inclusion, in which all students have active roles, is also very important (p. 7).

\section{Historical insights.}

Linda Nathan (2008) talks about how the arts and their link to education are not really a new idea or concept. Even "our ancestors knew the arts were synonymous with survival. We created art to communicate emotions: our passions, jealousies, and enduring conflicts" (Nathan, 2008, p. 177). In Nathan's (2008) opinion, “the performing and visual arts have been the foundation of our recorded existence," and she believes that "the arts are key to how we educate ourselves” (p. 177). Appel (2006) follows up on Nathan's (2008) idea of the arts as old concepts. She mentions in her article, Arts Integration across the Curriculum: Engagement in the Arts ignites Creativity and provides Students with Opportunities to critically interpret the World around Them, how even in Da Vinci's time "the arts were an integral part of education, 
community life and reflection upon the human condition; they were inseparable from the sciences and other academic disciplines" (Appel, 2006).

David Gullatt (2008) also presents a nice summary of advocating the arts in education by providing and epochal overview of the art education development. From his point of view, the art education journey started with Horace Mann in the late 1800s. Nevertheless, during the time of the social efficiency movement at the end of the first quarter of the $20^{\text {th }}$ century, a gap of the art education movement existed. Thorndike, Taylor, Cubberly, and others were able to gain support for their ideas of schools as manufacturing plants. Efficiency and standardization played a major role while Dewey's idea of art education was not even taken into consideration. At first sight, with the exception of a few independent schools, Thorndike had succeeded and Dewey lost (Eisner, 2002c, p. 3). Later on, even though it took him a while, Dewey was able to find a place for his ideas. As Gullatt (2008) mentions, while quoting Bresler (1995), "the roots of integration of the arts into the curriculum could be traced back as far as Dewey with his views on progressive education" (Bresler, 1995, as cited in Gullatt, p. 13).

Paula Purnell (2004) stresses Dewey's important role for arts education at this point in time, as well. From her point of view, Dewey’s book, Art as Experience (1934), “provided the foundation for the Aesthetic Education movement” (Purnell, 2004, p. 154). Nevertheless, as already indicated above, the times during the publication of Dewey's work were very unstable. Gulatt (2008), referring to Bresler (1995), added that in the 1930s and 1940s behaviorism played a major role in education. Nevertheless, this idea was challenged not only by Dewey but also by Lev Vygotsky, who "insisted that students constructed cognitive knowledge through the active process of learning, and that the arts were integral to that process" (Gullatt, 2008, p. 13). Kern (1985) thinks that by 1949 , "the integration of art into the general curriculum had reached a kind of zenith" (Kern, 1985, as cited in Purnell, 2004, p. 154). Purnell (2004) goes on to 1957 by quoting Berube (1999) and observing that "the launching of the Soviet satellite Sputnik in 1957 
introduced a new, competitive age in education. A counterrevolution against aesthetics education took place" (Berube, 1999, as cited in Purnell, p. 154). In his speech given at Amherst College in October 1963, President Kennedy said, "I see little of more importance to the future of our country, our civilization than full recognition of the place of the artists" (Wilson \& Harlan (Eds.), 1985, as cited in Purnell, 2004, p. 154). In the 1970s, Elliot Eisner began his research in educational outcomes of those students who participated in arts programs (Purnell, 2004). "In 1983, a government document titled A Nation at Risk: The Imperative for Educational Reform ignited an education reform movement which had no place for the arts in either its standards or its core curricula" (Purnell, 2004, p. 154). Purnell (2004) continues that in 1989, President George H. W. Bush's goals for education did not even include the arts. In 1984, John Goodland published a book named A Place Called School, in which he stated that "to grow up without the opportunity to develop such sophistication in arts appreciation is to grow up deprived" (Goodland, 1984, as cited in Purnell, 2004, p. 155). Purnell (2004) then observes that in 1992, twenty-eight states required the study of the arts from their students. Also, in 1994, the Clinton administration showed its favor of the arts in education, when it described the arts as "a core subject" (p. 155), in Goals 2000: Educate America. Purnell (2004) adds that in 2001, "President George W. Bush's education reform plan No Child Left Behind Act of 2001 (NCLB) presented three major areas of reform affecting arts education" (p. 155).

When considering the current state of arts integration, it must be acknowledged that during the last decade some schools tried to integrate and to use the arts as a catalyst in learning. Purnell (2004) acknowledges that various studies on arts integration have been conducted and that their findings have been compiled in two important compendia, the 1999 booklets Champions of Change: The Impact of the Arts on Learning and Critical Links (p. 155). Nevertheless, referring to our present-day situation, one has to know that the role of the arts in education is still "limited and sporadic" (p. 156). 
This idea becomes even more obvious if one considers Eisner's (2002c) comment about the values and visions that have driven education during the first quarter of the $20^{\text {th }}$ century and how they appear to be reappearing "with a vengeance today" (p. 5). From his point of view, there are three main points that still appear to be true today. First, we are trying to find "best methods;" second, the United States conducts more testing than any other country; and third, curriculum uniformity remains important. Another concept that Eisner (2002c) stresses, and that is also one of the main issues that this dissertation tries to tackle, is the idea of intrinsic versus extrinsic motivation. From Eisner's (2002c) point of view, “we pay practically no attention to the idea that engagement in school can and should provide intrinsic satisfactions, and we exacerbate the importance of extrinsic rewards" (p. 5).

\section{Why arts integration?}

This section explores some of the main aspects of the "why" of arts integration with the help of specific research about motivation (Csikszentmihalyi, 1990/1993/1997a/1997b; Csikszentmihalyi \& Csikszentmihalyi, 1988), Chicago Art Partners in Education (CAPE) schools (DeMoss \& Morris, 2002; Mishook \& Kornhaber, 2006), and benefits of arts integration found by major research studies (Why your child needs the arts, (n.d.); Ruppert, 2006; Nathan, 2008; McGowan,1988; Appel, 2006; Catterall, Henry, 2002, May 20; DeMoss \& Morris, 2002; Gullatt, 2008; Tunks \& Grady, 2003; Eisner, 2002b/2002c; Corrnett, 2011).

\section{Research related to arts integration and the AIC.}

Intrinsic motivation and flow experience in students.

In order to encourage students to develop a higher self-concept, arts integration could serve as a useful tool. Arts integration might allow students an opportunity to experience intrinsic motivation by being completely absorbed in a task. As such, the fostering of intrinsic motivation in young girls might be just one of the many reasons and examples of why arts integration could be important in our schools. 
Hand in hand with intrinsic motivation goes the concept of "flow," which Lori Custodero (2002) connects to the work of Mihalyi Csikszentmihalyi (1990, 1993, 1997a, 1997b) and colleagues (Csikszentmihalyi \& Csikszentmihalyi, 1988) who represent some of the most wellknown researchers in this area of study. Custodero (2002) describes flow as "a state of optimal enjoyment defined by the individual's perception of high skill and high challenge for a given activity" (Custodero, 2002, p. 3). She defines the criteria for flow experience as goals that are clear, feedback that is immediate, action and awareness that emerge, concentration that is deep, control that is possible, and self-consciousness that disappears. In my opinion, the disappearance of self-consciousness is the best indication of why the goal of flow is so important in arts integration and in trying to improve the self-concept of girls. Through the construct of flow, students are able to totally concentrate on the experience without being disturbed by their placement in their environment or their thoughts about themselves. Jazz flute performer Paul Horn, for example, describes his flow experience this way: "We are in that place of stillness, which is the 'absolute field,' but in playing [music], what I have transcended is my selfconsciousness, my mind drifting off to other things. I'm unaware of myself; I'm totally absorbed in the music" (Boyd, 1992, as cited in Custodero, 2002, p. 5). This account shows Horn as a student who is engaged, lost in the "flow" experience, and who is intrinsically motivated.

Thomas Houlihan, executive director of the Council of Chief State School Officers supports the idea that arts motivate students in a special way. He states in an article in USA Today that "he was impressed by the one study finding that 'arts motivate and reach certain students"” (Henry, 2002, May 20). DeMoss and Morris (2002) also describe how arts-integrated instruction creates "more independent and intrinsically motivated investments in learning" (DeMoss \& Morris, 2002, p. 1). Hence, what makes their study the most valuable to me and my research are their future research suggestions. Even though they found that intrinsic learning motivation encouraged the student to take over "more responsibility for their learning in their 
arts units than their non-arts units" (p. 22), they suggest that future research "could explore a more complete set of dimensions of achievement motivation that arts integration may activate intrinsic and extrinsic rewards, attributions for success or failure in school, cognitive engagement, and aspects of self-concept" (DeMoss \& Morris, 2002, p. 22). This suggestion by DeMoss \& Morris (2002) for future research shows clearly that there is a need for self-concept research upon which this dissertation focuses. Intrinsic and extrinsic motivation as well as aspects of self-concept are major study goals that require more research.

Chicago Art Partners in Education (CAPE) schools as fields of study.

Research in other areas of study has given additional reasons for "why" arts integration is important. Research by James Catterall and Lynn Waldorf (1990), as well as David Gullatt (2008) stress the importance of and the meaning for arts integration in reference to education, in general. DeMoss and Morris (2002) as well as Mishook and Kornhaber (2006) refer to Chicago Art Partners in Education (CAPE) schools as important fields of study, in specific. In Study: Arts Education has Academic Effect, Tamara Henry (2002, May 20) quotes James Catterall, an education professor at the University of California-Los Angeles. Catterall, who coordinated the research done by the Arts Education Partnership, said that "notions that the arts are frivolous add-ons to a serious curriculum couldn't be further from the truth" (Catterall, as cited in Henry, 2002, May 20, p. 1).

Thomas Houlihan, executive director of the Council of Chief State School Officers, adds that "many school superintendents, principals and teachers are unaware of the value of arts education" (Houlihan, as cited in Henry, 2002, May 20, p. 2). Surprisingly, this mindset about arts is still around in our days. So much research and work has been done that show the positive effects of arts integration, as one can see by Gullatt's (2008) argument: “Over the past 10 years prominent theorist and practitioners such as Catterall, Eisner, and Gardner have begun to argue 
that the arts are integral to the education of the "whole child"" (Catterall, 1998; Eisner, 1998; and Gardner, 1999a, as cited in Gullatt, 2008, p. 12).

These statements suggest that arts integration not only educates children on an emotional level, as it is often assumed, but also in other areas of student achievement, as for example standardized tests. This is especially the case for CAPE schools. Research that focuses on "the 1999 evaluation of arts integration that appears in Champions of Change found that Chicago Arts Partners in Education (CAPE) schools showed growth along several different measures of student achievement” (DeMoss \& Morris, 2002, p. 1).

This research also found that CAPE schools in specific "attained stronger standardized test score increases over time on the city's standardized test scores" (Catterall \& Waldorf, 1999, as cited in DeMoss \& Morris, 2002, p. 1). Mishook and Kornhaber (2006) also cite CAPE research studies by Catterall and Waldorf (1999), who offered the following goals as essential to effective arts integration:

1. Students should see connections and walk away with bigger ideas.

2. Students should take their work seriously.

3. The expression and activities in the arts should genuinely speak to important areas of the academic curriculum. This also means that the content is seen through more than the one form, for example, beyond the traditional written and spoken word.

4. The content lesson and the artistic lesson should be of equal importance.

5. The experience should have a planned assessment with rubrics and scoring guides.

6. The lesson plan should grow from state curriculum standards in both content areas and the arts (Catterall \& Waldorf, 1999, p. 58).

This review of relevant literature on CAPE schools and their interconnection to arts integration reveals two important points. Arts integration has become more important in today's educational system, and a list of bulletins with explanations of "why" arts integration begins to 
rise to the surface. The following section gives an overview of most important benefits of arts integration with the support of major research studies.

Benefits found by major research studies.

The following authors, researchers, and inquiry groups, all worked on, studied, and summarized the "why" of arts integration and have been reviewed for this literature review: Appel (2006), Arts Education Partnership (2000), Henry (2002, May 20), Corrnett (2011), DeMoss \& Morris (2002), Eisner (2002a, 2002c), Fieske (Ed.) (2002), Gullatt (2008), McGowan (1988), Nathan (2008), Ruppert (2006), Tunks and Grady (2003). All of these research studies contributed important knowledge to the arts integration field. Nevertheless, only the main findings receive focus and are listed below because an all-embracing summary would surpass the boundaries of this work.

This research overview for the arts in education and arts integration begins with a list of six major findings by a team of seven research groups that studied arts education programs (Art Education Partnership, 2000). This list is followed by four positive facts about the arts and arts integration (Ruppert, 2006). These report and compendium listings are followed by a chronological list of research done in the field. McGowan (1988) provides six justifications for the arts in education in her research. Henry (2002, May 20) describes how different art forms benefit students in different ways. De Moss and Morris (2002) share their four positive findings on arts-integrated instruction. Appel (2006) describes the positive effects of arts integration research. Nathan (2008) explains "why" the arts are important and "what" arts can do in reference to students' selves. Gullatt (2008) refers to Catterall's (1998) research while stressing arts education and its way of learning beyond memorization of information. Cornett's (2011) most recent rationale on arts integration was integrated in this review of literature, as well. At the end, all these findings were summarized by Cornett's (2011) and Eisner's (2000a, 2002c) works. 
The Arts Education Partnership (2000), formerly known as the Goals 2000 Arts Education Partnership, referred to a GE Fund/ MacArthur Foundation report called Champions of Change. The Impact of the Arts on Learning (1999), (Fiske, (Ed.), 2002). This report compiles the results of seven researchers and teams that "examined a variety of arts education programs using diverse methodologies" (p. IV). These seven researchers and teams are:

1. James S. Catterall of the Imagination Project at the University of California at Los Angeles;

2. Shirley Brice Heath of The Carnegie Foundation for the Advancement of Teaching and Stanford University, with Adelma Roach;

3. The Center for Arts Education Research at Teachers College, Columbia University, including the researchers Judy Burton, Rob Horowitz, and Hal Abeles;

4. James S. Catterall and the North Central Regional Educational Laboratory (NCREL);

5. Researchers at the National Center for Gifted and Talented at the University of Connecticut;

6. Steve Seidel and researchers from Harvard University's Project Zero;

7. Dennie Palmer Wold and researchers from the Performance Assessment

Collaboratives for Education (PACE) of Harvard's Graduate School of Education (Fieske, (Ed.), 2002).

These seven teams summarized six major findings:

- Students with high levels of arts participation outperform "arts-poor" students on virtually every measure.

- The arts have a measurable impact on students in "high-poverty" and urban settings.

- The arts in after-school programs guide disadvantaged youth toward positive behaviors and goals. 
- Learning through the arts has significant effect on learning in other domains.

- Arts experiences enhance "critical thinking" abilities and outcomes.

- The arts enable educators to reach students in effective ways (Arts Education Partnership, 2000).

These six major outcomes can be supported by Sandra Ruppert (2006) and her work, Critical evidence: How the Arts benefit Student Achievement. In this compendium, Ruppert (2006) lists four positive facts of support for the arts in education and names for each one a proper reference to main research literature in the field:

- In the federal No Child Left Behind Act, also known as NCLB, the arts share equal billing with reading, math, science, and other disciplines as "core academic subjects," which can contribute to improved student learning outcomes (U.S. Department of Education, No Child Left Behind Web site, http://www.nochildleftbehind.gov.).

- Forty-nine states have established content and/ or performance standards that outline what students should know and be able to do in one or more art forms; 43 states require schools or districts to provide arts instruction (Education Commission of the States (2005), State Policies Regarding Arts in Education. Denver, CO: ECS.).

- Schools [that] integrate the arts into the curriculum as part of a comprehensive education reform strategy are documenting positive changes in the school environment and improved student performance (Deasy, Richard J. (editor) (2002), Critical Links: Learning in the Arts and Student Achievement and Social Development, Washington, DC: AEP).

- The American public, by an overwhelming margin, believes the arts are vital to a wellrounded education; more than half rate the importance of arts education a "ten" on a scale of one to ten. Americans for the Arts (2005), "New Harris Poll Reveals That 93\% of Americans Believe That the Arts Are Vital to Providing a Well-Rounded Education," (News Release, http://www.artsusa.org), (Ruppert, 2006, p. 1). 
Surprisingly, the idea of arts integration in the school curriculum is long-established. Even twenty years ago, John McGowan (1988) was thinking about the importance of the arts in his work, A Descriptive Study of an Arts-In-Education Project, when he offered six justifications for the inclusion of the arts in the education of children:

1. The arts are a major discipline for study.

2. Arts education is a way to focus on self-awareness.

3. The involvement of students in the arts may be a means of developing creativity.

4. Concepts and skills learned through the arts may enhance learning.

5. Many students find the arts interesting and enjoyable.

6. The arts may give teachers alternative means, through various media and methods, to introduce and reinforce learning (McGowan, 1988, p. 47).

Henry (2002, May 20) stresses in her article that James Catterall and the Arts Education Partnership observed in their research that various art forms benefit students in different ways. They related each of the aspects they listed to one area of study:

* Drama. Helps with understanding social relationships, complex issues and emotions; improves concentrated thought and story comprehension.

* Music. Improves math achievement and proficiency, reading and cognitive development; boosts SAT verbal scores and skills for second-language learners.

* Dance. Helps with creative thinking, originality, elaboration and flexibility; improves expressive skills, social tolerance, self-confidence and persistence.

* Visual arts. Improve content and organization of writing; promote sophisticated reading skills and interpretation of text, reasoning about scientific images and reading readiness. * Multi-arts (combination of art forms). Helps with reading, verbal and math skills; improves the ability to collaborate and higher-order thinking skills (Henry, 2002, May 20). 
Out of these listed areas, dance is the one that mainly supports "self-confidence" (Henry, 2002, p. 2), an aspect on which this dissertation research primarily focuses.

Karen DeMoss and Terry Morris (2002) conclude in their work, How Arts Integration Supports Student Learning: Students Shed Light on the Connections, that arts-integrated instruction "created more independent and intrinsically motivated investments in learning" (DeMoss \& Morris, 2002, p. 1), an aspect that is very important for the development of the selfconcept in later ages, as we heard before from Csikszentmihalyi's (1993/ 1997a/ 1997b) "flow" studies. DeMoss and Morris (2002) added that arts-integrated instruction (a) "fostered learning for understanding as opposed to recall of facts for tests," (b) "transformed students' characterizations of 'learning barriers' into 'challenges' to be solved," and (c) "inspired students to pursue further learning opportunities outside of class" (p. 1).

Morgan Appel (2006) adopted a similar summary in her research, Arts Integration across the Curriculum: Engagement in the Arts ignites Creativity and provides Students with Opportunities to critically interpret the World around Them. She is convinced that arts integration research indicates and makes people aware of the fact that arts integration "enhances cognitive engagement among students; provides a better sense of ownership of learning; improves attention, engagement, attendance and perseverance among students; provides unique avenues for parent and community involvement; and inspires positive transformation of school community and culture" (Appel, 2006, p.15).

Linda Nathan (2008) summarizes very thoroughly in her article, Why the Arts make Sense in Education, "why" the arts are important. Specifically, Nathan (2008) explains "why" the arts are so important for strengthening and supporting personal development in students. From her point of view, there is ample literature available concerning the development of adolescents and the necessity of finding new forms of exploration and expression for these young people. She is convinced that "the arts provide opportunities to explore and interpret and react to a world that 
can seem cruel and distant and complicated," and that "the arts help young people develop a critical lens toward the world" (Nathan, 2008, p. 178). The last part of this quote deserves some special attention. As mentioned in earlier parts of this work, it is imperative that we encourage our children to interpret and evaluate our world, its culture, and its media in a critical way. On this point, Nathan (2008) is convinced that the arts are a good bridge to critical thinking: "The arts show that problems can have more than one solution. Through the arts, children learn to make good judgments about qualitative relationships" (p. 178). We will see later on in this work that this aspect of good judgments about relationships is also stressed by Eisner (2002c).

Nathan (2008) adds that as opposed to the curriculum "in which correct answers and rules dominate, judgment counts in the arts. The arts provide another language, a way of working together with disparate people and creating an experience that will be appreciated by others" (p. 179) . This way, students are able to experience and learn through the arts that one should judge and evaluate, instead of merely accepting things the way they appear. Students should learn to think critically and make positive or negative judgments. Nevertheless, the arts are not just an activity and challenge for our children, they also "provide that safe space, that structured time to be passionate, completely engaged, and taken seriously by both peers and adults" (Nathan, 2008, p. 179).

David Gullatt (2008) also stresses the role of the arts in education in his work, Enhancing Student Learning Through Arts Integration: Implications for the Profession. Specifically, Gullatt (2008) describes his idea by referring to Catterall's (1998) research, saying that "learning through the arts, such as the use of drama to re-enact historical events or the use of paintings to introduce and analyze life in historical periods, allows students to learn beyond the rote recall of information" (Catterall, 1998, as cited in Gullatt, 2008, p. 16). Furthermore, Gullatt (2008) adds an idea by Oddleifson (1994) that "the schools should be concerned with integrating 'head, heart, and hand"' (Oddleifson, 1994, p. 448, as cited in Gullatt, 2008, p. 22). The integration of these 
three channels makes it even more obvious that the "arts are considered communication systems" (p. 19). This notion is also considered by Claudia Cornett (2011), as one can see at the end of this section. Karolynne Gee (2000) even suggests that because the arts are considered communication systems "students are able to construct new knowledge and use the arts to express it" (Gee, 2000, as cited in Gullatt, 2008, p. 19).

Claudia Cornett's (2011) rationale on arts integration was cited by Jeanne Tunks and Patricia Grady (2003) in their work, Arts Infusion in University Courses. The Effect on Student Choice to infuse Art in Elementary Classes. Since Cornett's (2011) previous research appeared to play such a major role for their work in elementary education, her newest rationale received considerable attention for this work as well. From Cornett's (2011) most recent point of view in her book, Creating Meaning through Literature and the Arts. Arts Integration for Classroom Teachers, teachers should integrate visual art in their teaching because art:

1. Is a basic communication vehicle.

2. Engages emotions and motivates.

3. Develops aesthetic understanding.

4. Promotes attention to detail.

5. Develops higher order thinking.

6. Gives confidence to be unique.

7. Promotes respect for diversity.

8. Develops concentration, responsibility, and self-discipline.

9. Is a natural partner with other curricular areas.

10. Is a way to assess (Cornett, 2011, p. 145).

This list by Cornett (2011), Eisner's (2002a) catalog of 10 Lessons the Arts Teach (Appendix C) and Eisner's (2002c) six distinctive forms of thinking (Appendix D) provide the perfect summary of all the reasons "why" one should integrate the arts that were described by 
various educators and researchers (Appel, 2006; Arts Education Partnership, 2000; Henry, 2002, May 20; Corrnett, 1999, 2011; DeMoss \& Morris, 2002; Eisner, 2002a, 2002c; Fieske (Ed.), 2002; Gullatt, 2008; McGowan, 1988; Nathan, 2008; Ruppert, 2006; Tunks \& Grady, 2003). Though the "why" of arts integration is important, probably even more critical is "how" arts integration can be supportive for strengthening the self-concept of girls.

\section{How to integrate the arts?}

\section{The "How" (big “H”) - a practical application of arts integration.}

The "How" (big "H") refers to the practical application of arts integration. It can be seen as a practical tool that one uses to implement arts integration. In order to give the reader a better understanding of "How" arts integration can be used in the educational system, the classroom, and the curriculum, the approaches of several researchers will be outlined (Arts Education Partnership, 2000; Bresler, 1995; Davis, 1999a/1999b; Eisner, 1998, as cited in Gullatt, 2008).

David Gullatt (2008) gives a number of examples on "How" teachers and educators can integrate the arts by referring to major ideas of several well-known educators and researchers in the field (Davis, 1999a/1999b; Eisner, 1998, as cited in Gullatt, 2008; and Bresler, 1995). Davis (1999b) focuses on eight ways of how the arts are included in today's U.S. school settings. Eisner (1998) offers a three-tiered system of outcomes in art education. Bresler (1995) presents four styles of art integration in the classroom. Arts Education Partnership (2000) provides a rationale for why hands-on activities in arts are so important for students.

For example, Gullatt (2008) offers eight ways on how the arts are included in the present U.S. school settings suggested by Jessica Davis (1999b) and her work, Nowhere, Somewhere, Everywhere: The Arts in Education: (1) arts-based, (2) arts-injected, (3) arts included, (4) artsexpansion, (5) arts-professional, (6) arts-extra, (7) aesthetic education model, and (8) arts-cultura (Davis, 1999b, pp. 24-25). For more detailed information on each of these eight ways, please see Appendix E. 
Citing Eisner (1998), Gullatt (2008) suggests a three-tiered system of outcomes in arts education that could give a somewhat better insight into the "How" of arts integration. The first tier is arts-based outcomes, which were "used to assess the arts curriculum taught in an arts program." The second tier is arts-related outcomes, which "consisted of those aesthetic features that students transferred outside of the school day within their own environment." And the third tier is ancillary outcomes of arts education "that affected the general curriculum" (Eisner, 1998, as cited in Gullatt, 2008, p. 15).

Gullatt (2008) also referred to Bresler (1995), who proposed four styles of arts integration into the classroom, to describe how the arts are integrated into the classroom setting. The first style is the subservient approach. "Educators who endorse this approach used the arts as a 'spice' or an 'extra' for their curriculum." The second is the co-equal cognitive integration approach whereby "the arts [are] integrated with other aspects of the curriculum" and the students are required to "use higher order thinking skills and aesthetic qualities to gain further understanding of a particular academic concept." The third is the affective approach through which "students were immersed in the arts while the arts complimented the classroom curriculum." The fourth is the social integration and is performance-based. "This approach complemented the curriculum only insofar as it is used as a vehicle to increase participation in parental involvement activities" (Bresler, 1995, as cited in Gullatt, 2008, p. 16).

Arts Education Partnership (2000) stresses the importance of hands-on participation in the arts. From its point of view, children who receive an arts-focused education, like "children who receive an elementary foundation in the arts; ongoing, comprehensive, sequential education in music, drama, dance, and the visual arts; who are afforded opportunities for higher levels of achievement," will be those who "step into tomorrow's world with 'the arts advantage"” (Arts Education Partnership, 2000, p. 2). In my opinion, this "arts advantage" (p.2) is an advantage that is not just going to be of help for the students' outside, work world, but also for their 
personal life, their own world. The key elements that they acquire through arts education, like "a quick mind, focus, discipline, imagination, judgment, personal drive, experience in teamwork, attention to detail, grasp of the big picture, and an essential urge to continue learning" (p.2) are going to help them out in the "real world" but also in their "own world" that often seems disturbed by so many outside influences. The good news is that once the students acquire these skills through arts integration, they almost automatically are able to sort out things for their own life, too. Consequently, they have a chance to become confident young adults with a healthy selfconcept.

This list of possible arts integration approaches should provide the reader with an overview of available concepts for the "How" of arts integration and uncover an awareness of arts integration, as well.

\section{The "how" (small " $h$ ") - a possible effect of arts integration.}

The idea of the "how" (small "h") refers to whether the experience of arts integration, or more specifically an AIC, influences or shows any effect on the self-concepts of adolescent female students and their perceptions of beauty.

The major research question of, What, if any, phenomena may be associated with the selfconcept and perceptions of beauty of young ladies who completed an AIC?, is intended to help to find an answer to the "how" of arts integration and its possible effect. Before this main research question will be approached by this research study (see chapter three), a short overview on the present stage of available literature on this topic will be provided.

For each of the following main concepts of the research question, a definition and a review of literature will be provided in this chapter: phenomenology, self-concept, beauty, and arts integration (AIC). I was able to find various literature resources on each of these topics to provide this overview. 
However, it became more challenging, almost impossible, to provide a review of literature that included all areas of interest of my research question: beauty, self-concept, phenomenology, arts integration (AIC), and arts integration experiences of parents, students, and teachers. For example, two popular education research databases were accessed that could not provide the amount of literature necessary for a literature review on the topic: (1) Academic Search Complete and (2) ERIC (EBSCOhost). Academic Search Complete seems to serve as a good start to research "any topic, covering most areas of academic study and containing full text for 5,500 periodicals, including 4,600 peer-reviewed publications. In addition to the full text, this database offers indexing and abstracts for more than 9,500 journals" (West Virginia University Libraries). ERIC

is sponsored by the US Department of Education to provide extensive access to educational-related literature. The ERIC database corresponds to two printed journals: Resources in Education (RIE) and Current Index to Journals in Education (CIJE). Both journals provide access to some 14,000 documents and over 20,000 journal articles per year. In addition, ERIC provides coverage of conferences, meetings, government documents, theses, dissertations, reports, audiovisual media, bibliographies, directories, books and monographs. Comprehensive coverage of the journal and report literature in the field of education. Composed of indexing and abstracts for articles and reports. (West Virginia University Libraries)

I began my literature research with the Academic Search Complete Database and used various descriptor combinations to receive the best results. The first search with the descriptors of "Arts Integrated Curriculum (AIC)" and “(adolescent) girls" provided one result: Kimberly Oliver's and Rosary Lalik's journal article called The Body as Curriculum: Learning with Adolescent Girls. Despite the integration of concepts on which this research also focuses (e.g. body, curriculum, adolescent girls), the main goal of this research article was more related to the 
body as curriculum, instead of the AIC. The second search with the descriptors of "AIC", "(adolescent) girls," and "self-concept" did not provide any results. The third search with just the descriptor combination of "AIC" and "self-concept" resulted in three articles that did not relate in any way to my research interests. The fourth search with the descriptors of "AIC" and "perception of beauty" showed one article that also did not relate to this research study. The fifth search with the descriptors of "AIC" and "beauty" delivered three articles, two of which were already displayed in the former search trials. The final search with the descriptors of "Arts Integration" and "girls" resulted in eight findings that also did not relate in any way to this research study.

In order to receive more fruitful results, this literature research was continued with ERIC, another educational database. Similar to the research in Academic Search Complete, this search began with the descriptors of "AIC" and "Adolescent Girls." The two articles recovered did not relate to my research study. The descriptor combination of "AIC" and "girls" provided better results for this research database. Ten articles were provided. One of these articles was especially interesting: Building Self-Esteem through Visual Art: A Curriculum for Middle School Girls, by Ann Emerson (1994). Though this article provided the best match with this research study so far, it appeared to be somewhat outdated, written nearly 20 years ago. Furthermore, the component of parents', students', or teachers' experiences with the AIC are missing. The search was continued with the descriptors of "AIC," "girls," and "self-concept." Two articles were found, including one repeat. Quantitatively, the descriptors of "AIC" and "self-concept" provided the best results, finding 71 articles. Unfortunately, most of these articles did not consider any of the concepts of this research. The search was specified with the aim of narrowing the search down to articles that are of interest to this study. As a result, the database did not provide any articles that matched this advanced research. The descriptors of "AIC" and "perception of beauty" did not match any works. The search with "AIC" and "beauty" showed six articles, but none are helpful 
for this research. "Arts Integration" and "girls" also provided two articles that could not be taken into consideration for this study.

The two database research examples for relevant literature in the field show that a lack of research studies exists which include the concepts of beauty, self-concept, phenomenology, arts integration/ AIC and its experience by parents, students, and teachers. Consequently, this study makes the point that its research is necessary and relevant in the field to bring awareness and to provide new insights. 


\section{Chapter 3: Methodology}

The main purpose of this study is to determine whether an Arts-Integrated Curriculum (AIC) can be used as a helpful tool to discover more about the self-concept of young women and girls and if it can eventually have an impact on the self-concept of those girls and their perceptions of beauty. In order to address this issue, one major and three minor research questions were developed. The main research question is:

What, if any, phenomena may be associated with the self-concept and perceptions of beauty of young ladies who completed an AIC?

This main phenomenological research question will be explored with the help of three minor phenomenological research questions:

\section{Q1. How do young ladies who completed an AIC describe their experience?}

Q2. How do parents of young ladies who completed an AIC describe their experience?

Q3. How do teachers of young ladies who completed an AIC describe their experience?

With the purpose of addressing and answering these research questions, the possible arising phenomena were mainly researched through in-depth interviews with those who were or still are directly involved in the AIC, including three former AIC students and one non-AIC student, three parents, and two former teachers of those students, plus one university liaison.

As the literature review in chapter two already has shown very thoroughly, there are countless reasons "why" it is important to undertake this study. The factor that appears to be the most important, though, is the struggle of adolescent girls and women with beauty concepts, and as a consequence with their self-concept. As such, the goal of this dissertation is also to show that societal changes have occurred, are occurring, and will occur, while having a direct or indirect influence on female students and their self-concept and perception of beauty, which in turn could be impacted by an AIC. If this would prove true, more support for, and a greater representation of, the arts would appear to be necessary in our schools. Consequently, an effort 
could be made to claim more hours for the AIC in schools to make them a part of the "regular school curriculum.”

\section{Research Design}

One major and three minor research questions guided this search for phenomena that may be associated with the self-concept and perceptions of beauty of young ladies who completed an AIC. The way the research questions are stated shows that the nature of this research study is individual, personal, and private. Consequently, a phenomenological research design was chosen. From all of the available qualitative research methods and qualitative inquiries, e.g. ethnography, auto-ethnography, reality testing, constructionism/ constructivism, phenomenology, heuristic inquiry, ethnomethodology, symbolic interaction, semiotics, hermeneutics, narratology/ narrative analysis, ecological psychology, systems theory, chaos theory (nonlinear dynamics, grounded theory), orientational (feminist inquiry, critical theory, queer theory), etc. (Patton, 2002), the phenomenological approach, and more specifically, the transcendental phenomenological approach by Moustakas (1994) appeared to be the most appropriate one to answer the research questions.

\section{Phenomenology.}

This work focused on a phenomenological inquiry defined by Moustakas (1994), which he also calls "phenomenological model" (p. 180). This framework was selected because it provided detailed steps and processes on how to analyze the data. Furthermore, it explains how the data can be transferred and generalized from individuals to a group.

While Moustakas (1994) lists epoche, transcendental-phenomenological reduction, and imaginative variation as "the core processes that facilitate derivation of knowledge" (p. 33), Patton (2002) adds "synthesis of texture and structure" (p. 486) as a fourth process. Throughout the phenomenological data analysis process, which is described in detail in chapter four of this study, I relied mainly on Moustakas' (1994) and Patton's (1992) suggested steps. In addition, 
Copen's (1992) work on insomnia was used as a model for the data analysis process. I considered Copen's (1992) work already as an outstanding, phenomenological study that should serve as a model even before I found out that Moustakas had served as Copen's dissertation chair.

\section{Phenomenological method.}

To find answers to the research question(s), a phenomenological qualitative design, which combined the processes of Moustakas and Patton, was applied. I used Patton's (2002) overview of the steps of Moustakas' (1994) phenomenological analysis (see Figure 2):

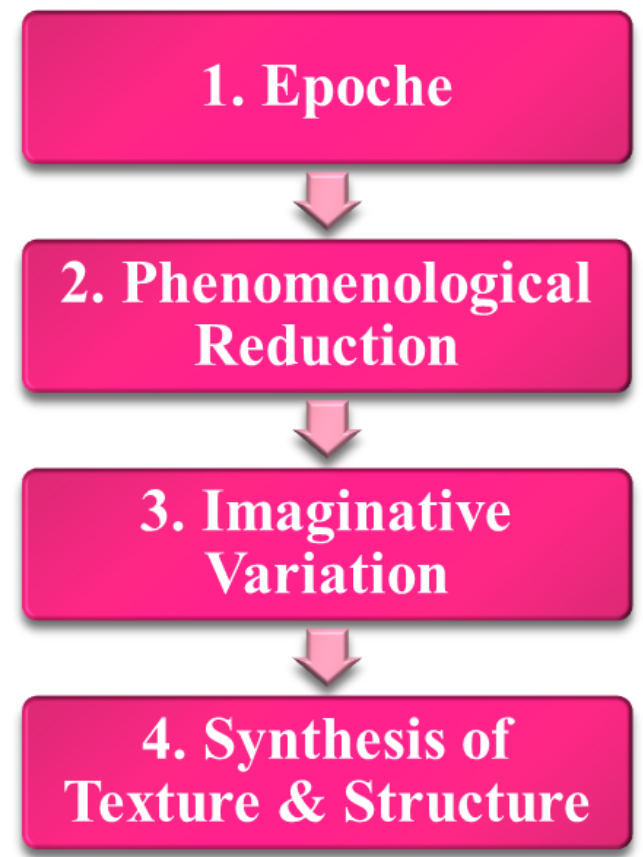

Figure 2. Four phenomenological processes (Moustakas, 1994; Patton, 2002) of Moustakas' (1994) "Phenomenological Model" (p. 180).

Step 1: epoche.

Patton (2002) calls the first step, epoche, which Moustakas (1994) describes as a Greek word meaning to refrain from judgment, to abstain from or stay away from the everyday, ordinary way of perceiving things (p. 33). He states that we assume that what we perceive is actually there and stays the way we observed it. Epoche in contrast, "requires a new way of 
looking at things, a way that requires that we learn to see what stands before our eyes, what we can distinguish and describe" (Moustakas, 1994, p. 33). Moustakas also thinks that in Epoche, "the everyday understandings, judgments, and knowings are set aside, and the phenomena are revisited, visually, natively, in a wide-open sense, from the vantage point of a pure or transcendental ego" (p.33).

Patton (2002) summarizes the ideas of several researchers (Moustakas, 1994; Katz, 1987; Ihde, 1979) by concluding that "epoche is an ongoing analytical process rather than a single fixed event" (p. 485). Furthermore, he adds that "the process of epoche epitomizes the databased, evidential, and empirical (vs. empiricist) research orientation of phenomenology" (Patton, 2002, p. 485). Epoche was applied to this study because it appears to be a necessary stage to free a researcher from prejudices and presumptions, to be open for "whatever or whoever appears in our consciousness," and to approach it with an openness to see "just what is there and allowing what is there to linger" (Moustakas, 1994, pp. 86-87). Furthermore, this step helps to silence outside forces to just encounter the phenomenon in its purest form (p. 88). Epoche seems to give me, as the researcher, the possibility to be open to new notions, ideas, and phenomena in reference to young girls, their self-concept, and the role that the AIC might or might not play in this context.

\section{Step 2: phenomenological reduction.}

The second step is called phenomenological reduction. Phenomenological reduction starts out with an analytical process called "bracketing." In this process, "the researcher "brackets out' the world and presuppositions to identify the data in pure form, uncontaminated by extraneous intrusions" (Patton, 2002, p. 485). Patton (2002) refers to Denzin (1989b) in order to describe bracketing in more detail:

In bracketing, the researcher holds the phenomenon up for serious inspection. It is taken out of the world where it occurs. It is taken apart and dissected. Its elements and essential 
structures are uncovered, defined, and analyzed. It is treated as a text or a document; that is, as an instance of the phenomenon that is being studied. It is not interpreted in terms of the standard meanings given to it by the existing literature. Those preconceptions, which were isolated in the deconstruction phase, are suspended and put aside during bracketing. In bracketing, the subject matter is confronted, as much as possible, on its own terms. (Denzin, 1989b, as cited in Patton, 2002, p. 485)

For Denzin (1989b), bracketing is a process that consists of five steps:

1. Locate within the personal experience, or self-story, key phrases and statements that speak directly to the phenomenon in question.

2. Interpret the meanings of these phrases, as an informed reader.

3. Obtain the subject's interpretations of these phrases, if possible.

4. Inspect these meanings for what they reveal about the essential, recurring features of the phenomenon being studied.

5. Offer a tentative statement, or definition, of the phenomenon in terms of the essential recurring features identified in step 4 (Denzin, 1989b, as cited in Patton, 2002, pp. 486487).

After this first stage of bracketing, the data are "horizonalized." This means "the data are treated with equal value" (Patton, 2002, p. 486). In this process, the data are first spread out for examination. Data that are irrelevant, repetitive, or overlapping are deleted "leaving only the horizons (the textural meanings and invariant constituents of the phenomenon)" (Moustakas, 1994, p. 97). During the next process, data are organized into meaningful clusters, which Moustakas (1994) calls “clustering the horizons into themes” (p. 97). In a final step, "organizing the horizons and themes into a coherent textural description of the phenomenon" (Moustakas, 1994, p. 97) takes place. The step of phenomenological reduction was applied because it seems to be relevant to bracket out data in its purest form, to treat data with equal value, to organize themes into meaningful chunks, and to organize these clusters and themes finally into a coherent 
textural description to get to the bottom of the question of the AIC and its possible impact on the self-concept of young women and their perceptions of beauty. As Moustakas (1994) says, "the task is that of describing in textural language just what one sees, not only in terms of the external object but also the internal act of consciousness, the experiences as such, the rhythm and relationship between phenomenon and self' (p. 90).

\section{Step 3: imaginative variation.}

At step three, I identified invariant themes, "to perform an 'imaginative variation' on each theme," which Douglass (1985) has described as “"moving around the statue' to see the same object from different views" (Patton, 2002, p. 486). Through this third step, the imaginative variation, "the researcher develops enhanced or expanded versions of the invariant themes" (p. 486). The goal is to arrive at structural descriptions of an experience, the answer to "how did the experience of the phenomenon come to what it is?" (Moustakas, 1994, p. 98). In order to achieve this goal, the researcher has to complete four steps of imaginative variation:

1. Systematic varying of the possible structural meanings that underlie the textural meanings;

2. Recognizing the underlying themes or context that account for the emergence of the phenomenon;

3. Considering the universal structures that precipitate feelings and thoughts with reference to the phenomenon, such as the structure of time, $(\ldots)$;

4. Searching for exemplifications that vividly illustrate the invariant structural themes and facilitate the development of structural descriptions of the phenomenon (Moustakas, 1994, p. 99).

Imaginative variation and its four steps have been chosen for this study because they appeared to be necessary steps to find out more about possible phenomena that are related to the self-concept of girls, their perception of beauty, and the AIC. With the help of the conducted interviews, textural structures and meanings were received. Nevertheless, it was necessary to use 
imagination to get to structural descriptions and meanings. Without the use of imagination, the textural structures would have been useless, as Moustakas (1994) also stated (p. 97). Another reason this step of phenomenology was chosen was the idea that structural descriptions, alone, are not enough. One has to find those structural descriptions that are common for all the participants, in this case the interviewees of this study (students, parents, and teachers). Only if one can narrow things down to common structural descriptions is it possible to talk about a phenomena. Moustakas (1994) used the color red as a possible exemplification:

In considering the red of individual objects we know that there is a generic redness as such. No matter how many variations we perceive in the color of red, all have the redness of red running through them. We can arrive at this intuition only through an imaginative integration of what is common in all the shadings of red. (Moustakas, 1994, p. 98) Step 4: synthesis of texture and structure.

At the final step, called synthesis of texture and structure, "the researcher moves to the textural portrayal of each theme (...). The textural portrayal is an abstraction of the experience that provides content and illustration, but not yet essence" (Patton, 2002, p. 486). Moustakas (1994) adds that a "phenomenological analysis then involves a 'structural description' that contains the 'bones' of the experience for the whole group of people studied, 'a way of understanding how the co-researchers as a group experience what they experience."' (p. 142). From Patton's (2002) standpoint, “in the structural synthesis, the phenomenologist looks beneath the affect inherent in the experience to deeper meaning for the individuals who, together, make up the group" (p. 486). Nevertheless, Moustakas (1994) comments that the final step requires "an integration of the composite textual and composite structural descriptions, providing a synthesis of the meanings and essence of the experience" (p. 144). This last addition appears to be of great importance, since Patton (2002) also stresses this quote in his summary on phenomenology. This last step has been applied in this study because it represents the intuitive integration of the basic 
textural and structural descriptions into a summary of the "essences of the experience of the phenomenon as a whole" (Moustakas, 1994, p. 100).

I chose the phenomenological approach for this research study to better understand whether an AIC can be used as a helpful tool to discover more about the self-concept of young women and girls and if it could have an impact on the self-concept of those girls and their perceptions of beauty.

Also Copen (1992) considers it important to go through all four of these processes in his phenomenological study because they "enable the investigator to complete an analysis of data in an organized and disciplined manner" (p. 25).

\section{Data Analysis}

During the data analysis process for this study, a special focus was placed on organizing, analyzing, and synthesizing data. Moustakas (1994) describes this process in more detail in his work, Phenomenological Researcher Methods. In specific, the data analysis process, which is displayed in detail in chapter four, followed Moustakas' (1994) suggestion of the "Modification of the Van Kaam Method of Analysis of Phenomenological Data" (Moustakas, 1994, p. 120). Individual textural and structural descriptions, textural and composite structural descriptions, and a synthesis of textural and structural meanings and essences of the experience, were generated for the collected data. Moustakas' (1994) modification consists of the following seven plus one steps (see Figure 3). For this methods chapter, only a short explanation for each step is available. A more detailed description and application of Moustakas' (1994) steps for this research study is provided in chapter four. 


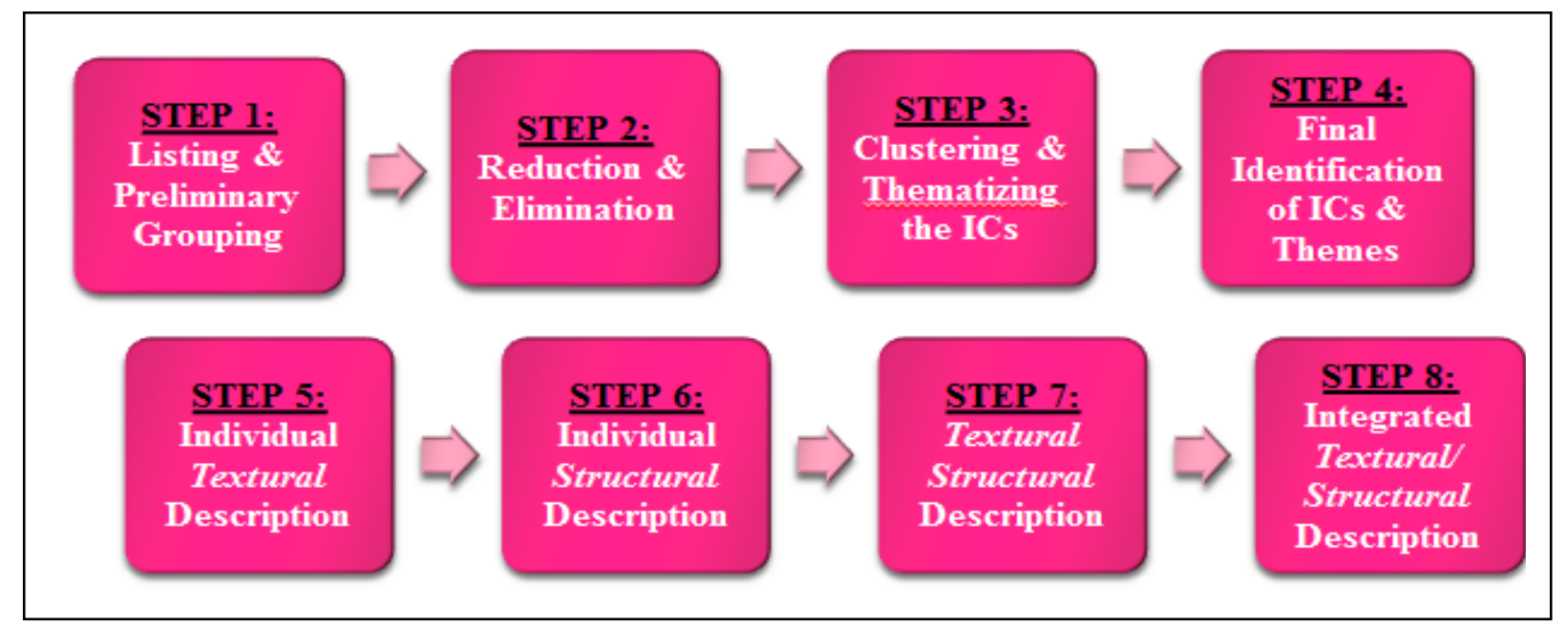

Figure 3. Moustakas' "Modification of the Van Kaam Method of Analysis of Phenomenological Data" (Moustaks, 1994, p. 120).

\section{Step 1: listing and preliminary grouping.}

During this step, I listed all the expressions relevant to the experience. This is also called "horizonalization" (Moustakas, 1994, p. 120).

\section{Step 2: reduction and elimination.}

During this step, each expression was tested for two requirements in order to determine invariant constituents (ICs):

a) Is there a moment of the experience that is a "necessary and sufficient constituent for understanding it" (p. 121)?

b) "Is it possible to abstract and label it? If so, it is a horizon of the experience." If this requirement is not met, the expressions will be eliminated. "Overlapping, repetitive, and vague expressions are also eliminated or presented in more exact descriptive terms." Remaining horizons are the "invariant constituents of the experience" (p. 121). 


\section{Step 3: clustering and thematizing the invariant constituents.}

During this step, I clustered the ICs of the experience that are related into a thematic label. Clustered and labeled constituents are core themes of the experience (p. 121).

Step 4: final identification of the invariant constituents and themes (validation).

During this step, ICs and their accompanying themes were checked against the total record of the research participant: “(1) Are they expressed explicitly in the complete transcription? (2) Are they compatible if not explicitly expressed? (3) If they are not explicit or compatible, they are not relevant to the co-researcher's experience and should be deleted" ( $\mathrm{p}$. 121).

\section{Step 5: individual textural description.}

During this step, an individual textural description of the experience was created by "using the relevant, validated invariant constituents and themes" (p. 121) for each co-researcher.

\section{Step 6: individual structural description.}

During this step, I created an individual structural description of the experience, "based on the individual textural description and imaginative variation" (p. 121).

\section{Step 7: textural-structural description.}

During this step, a textural-structural description of the meanings and the essences of the experiences was created for each research participant, "incorporating the invariant constituents and themes" (p. 121).

\section{Step 8: integrated textural/ structural description.}

In this last step I developed "a composite description of the meanings and essences of the experience, representing the group as a whole" (p. 121), based on the individual texturalstructural descriptions. 


\section{Selection of Participants}

\section{Recruitment.}

The possible phenomena that may be associated with the self-concept and perceptions of beauty of young ladies who completed an AIC refer to a specified group of female students, their parents, and teachers. A special sample was required.

\section{Sampling.}

Purposeful sampling, in specific criterion sampling (Patton, 2002), was applied to recruit student, parent, and teacher participants who would match definite inclusionary criteria.

Table 1. Summary Table of Co-researchers' Inclusionary Criteria

\begin{tabular}{|c|c|c|c|c|}
\hline & Criteria & Students & Parents & Teachers \\
\hline 1. & $\begin{array}{l}\text { Past participant of AIC } \\
\text { a) Elementary }(\mathrm{K}-12) \\
\text { b) Sunrise School }\end{array}$ & $\begin{array}{l}\square \\
\square \\
\square\end{array}$ & $\begin{array}{l}\square \\
\square \\
\square\end{array}$ & $\begin{array}{l}\square \\
\square \\
\square\end{array}$ \\
\hline 2. & $\begin{array}{l}\text { Willing to participate } \\
\text { (\& for daughter) }\end{array}$ & $\nabla$ & $\nabla$ & $\nabla$ \\
\hline 3. & $\begin{array}{l}\text { Forms of agreement } \\
\text { Consent form } \\
\text { Assent form }\end{array}$ & $\begin{array}{l}\nabla \\
\nabla\end{array}$ & $\nabla(2 x)$ & $\nabla$ \\
\hline 4. & Female gender & $\bar{\nabla}$ & & \\
\hline 5. & Adolescent & $\nabla$ & & \\
\hline 6. & Same age & $\bar{\nabla}$ & & \\
\hline 7. & Intense arts experience & $\nabla$ & & \\
\hline 8. & $\begin{array}{l}\text { Regularly exposed to } \\
\text { arts }\end{array}$ & $\bar{\nabla}$ & & \\
\hline 9. & Parent participating & $\square$ & & \\
\hline
\end{tabular}

These standards will be defined in more detail for each group of the participants. This description will be followed by the depiction of the recruitment procedures that were used to obtain the sample. Afterwards, the sample, each participant per se, will be presented.

\section{Students' inclusionary criteria.}

To be recruited, each student participant had to fit the following selection criteria (see also Table 1). First, the student had to be a past participant of the AIC at the Elementary school level (Kindergarten - third grade level) at Sunrise Primary Elementary School (Sunrise). Second, the student had to be willing to participate in this study. Third, the student had to be of female 
gender. Fourth, the student had to be in the developmental stage of adolescence during the time of the study. Fifth, the students had to be all about the same age to guarantee comparability. Sixth, the student had to have an intense and long experience in the arts. Seventh, the student had to be exposed to arts experiences on a regular basis. Eighth, at least one of the student's parents had to be willing to take part in this study. Finally, before being able to participate in this study and its research activities, the students had to sign a written assent form (see Appendix G8), stating their willingness to participate in this study. The parents of the students also had to sign a written consent form (see Appendix G7) for their daughters, confirming that they approved the participation of their child. This step was necessary because the students were all under the age of 18 .

\section{Parents'/guardians' inclusionary criteria.}

To be recruited, the parents/ legal guardians had to satisfy the following conditions (see also Table 1). They had to agree to participate, and as a consequence, to provide written consent for themselves. They also had to be a parent/ legal guardian of a potential student participant. Their female child had to have participated in an intensive AIC at the Elementary school level at Sunrise. The parents had to be willing to allow their adolescent daughter to participate in this study by providing a written consent for their daughter.

\section{Teachers' inclusionary criteria.}

The following inclusionary criteria were necessary for the teachers to be recruited (see also Table 1). The teachers had to be willing to participate in this study. They had to indicate this by signing a written consent form. They also had to be a current or former teacher at Sunrise and its intensive AIC at the Elementary School level. They had to be a current or previous teacher of at least one of the potential female students that were interviewed for this research study. 


\section{Recruitment procedures.}

During the recruitment process, I underwent five steps and was able to recruit a total of ten participants. The participants were selected in a specific, purposeful sampling manner (Patton, 2002). First, potential participants that matched the previously-mentioned inclusionary criteria for students, parents, and teachers, were recommended by faculty from the university. A member of the faculty suggested those parents, students, and teachers to me. She knew, based on her past experiences with those potential participants, that those parents, their children (serving as students), and the teachers were participants of an intensive AIC in the past at Sunrise. Second, contact information for all of these participants, email addresses and/ or phone numbers were provided by the faculty. Third, either phone calls were made and/ or requests or emails (see Appendices G1 and G2) were sent out to potential participants. Parents were asked formally to participate with their daughters in this research study. Also, teachers were formally asked to participate. Fourth, phone conversations took place and/ or reply emails and/ or phone calls were received. Times and places for meetings were coordinated. Fifth, a first meeting with the mothers and their daughters took place. During these meetings, the parents and their daughters, were provided with cover letters (see Appendices G3, G4, and G5), the necessary consent and information forms (see Appendix G6), parental or guardian consent and information forms (see Appendix G7), and the assent forms (see Appendix G8). In a sixth and final step, the parents, as well as the students, signed all the previously-listed and necessary forms for the research study. All the same steps were conducted for the teachers, except they only were provided with a cover letter (see Appendix G9) and the necessary consent and information forms (see Appendix G6), before the interview process. 


\section{Participants.}

\section{Students.}

Table 2 represents four of the students' characteristics: name, gender, ethnicity, and age (Goff, 2010).

Table 2. Students' Characteristics

\begin{tabular}{|l|l|l|l|}
\hline Students' Names & Gender & Ethnicity & Age \\
\hline BAND & female & White & 13 \\
\hline PIANO & female & White & 12 \\
\hline FLUTE & female & Latino & $11 / 12$ \\
\hline DRAWING & female & White & 11 \\
\hline
\end{tabular}

All of the female students attended Sunrise and were adolescent young ladies. Each of the students will be described in more detail below.

Student \#1=BAND.

BAND is a female, White student, who is 13 years old. She is originally from Morning Star. Now, BAND lives in Evening City. She attended Sunrise, where she received an AIC education. She received this special type of education for two years, from Kindergarten to first grade. Before she went to this school, she had not received any specific arts training. BAND has three siblings, two sisters and one brother. Her siblings went to Sunrise, as well. Currently, BAND goes to Sunrise Middle School and plays there in the school band. She plays the clarinet. BAND took art classes at Sunrise. In her introduction, she stated that she likes art because she has gotten recognition from it. I called her BAND because she stressed throughout the whole interview process how much she likes and enjoys to play in the school band and how much fulfillment she gets out of it.

Student $\# 2$ = PIANO.

PIANO is a female, White student, and twelve years old. She is originally from Evening City. She still lives there with her family. She attended Sunrise, where she received an AIC education. She received this special type of education for four years, from Kindergarten to third 
grade. Before she went to this school she did not receive any specific arts training. PIANO has one two-year-old brother, who did not attend an AIC school, yet. Nevertheless, the parents would like him to attend an AIC school, as well. PIANO takes part in theater with the Evening City Theater Company. She also participates in the school band, plays the saxophone, and takes piano lessons. I called her PIANO because she was very passionate about the piano. At the end of the interview, she even played a piece for me on the piano. One could tell that she really loves to play the piano.

Student \#3 = FLUTE.

FLUTE is a female, Latino student, and eleven/ twelve years old. She was adopted. FLUTE lives now with her adoptive parents in Evening City. She attended Sunrise, where she received an AIC education for one year, third grade. Before she went to this school, she received other specific arts training. She went to summer camps, where she participated in painting and drawing classes. She also partook in dancing classes. FLUTE has no siblings growing up with her. Right now, she attends Sunrise Middle School. I called her FLUTE because she is very passionate about playing the flute in the school band, as well as taking private flute lessons at home. She lit up as soon as she started to talk about playing the flute. She even would have played for me, but she had left the flute at school for a concert the next day.

Student \#4 = DRAWING.

DRAWING was an 11-year-old student, who is female and Caucasian. She was an only child originally from Sunny Town before moving to Evening City. DRAWING was the only student that did not go to Sunrise Elementary. At the time of the interview, DRAWING was in sixth grade at Sunrise Middle School. Even though DRAWING did not attend a school with an AIC education, she participated in pottery classes during the summer and played the violin. Furthermore, she enjoyed theater, painting, and drawing. She was especially interested in drawing. This was also the reason why I named her DRAWING for this interview. 


\section{Parents.}

Table 3 represents four of the parents' characteristics: name, gender, ethnicity, and age (Goff, 2010).

Table 3. Parents' Characteristics

\begin{tabular}{|l|l|l|l|}
\hline Mothers' Names & Gender & Ethnicity & Age \\
\hline BUSY & female & White & 47 \\
\hline CARING & female & White & 46 \\
\hline LOVING & female & White & 55 \\
\hline
\end{tabular}

All of the participating parents were female, even though the option existed for the students' fathers to be interviewed. Each mother will be described in more detail in the following.

Parent \#1 = BUSY.

BUSY is the mother of BAND, White, and 47 years old. BUSY is married and usually works seven to eight hours a day, away from home. She is originally from Evening City, where she also lives today. BUSY has four children, three girls and one boy. Her children have attended an AIC school for a total of 15 years. BUSY herself did not receive an AIC education. She owns a business in Evening City which keeps her very "busy." This is also the reason why I called her "BUSY." She appeared to be very busy with her business, her own children, as well as her willingness to conduct interviews with doctoral students. From what I can tell, she does a great job to keep all the "busyness" under control.

Parent \#2 = CARING.

CARING is the mother of PIANO. She is White and 46 years old. CARING is married and usually works eight hours a day. Her office and workspace is at home. She was born in Heavenly Sky, but lived in other places. She lived in Evening City from age eight to 21, and finally returned to Evening City at age 33, where she also lives right now. CARING has two children, one girl and one boy. CARING's daughter has attended an AIC school for a total of four years. CARING herself does not believe that she received an AIC education. CARING was 
always very interested in role playing and drama when she was a child. She played instruments, was part of the orchestra, and sang a lot. She enjoys drawing and loved it when they had a special person come into the school to do storytelling. She now works in the arts herself. I called her CARING because she appeared to care a lot about her daughter, her family, but also about others.

Parent \#3= LOVING.

LOVING is the mother of FLUTE. She is White and 55 years old. LOVING is married and usually works six hours a day. She works away from home. She is originally from Water Falls and lives right now, with her daughter and her husband, in Evening City. LOVING has one daughter, FLUTE. She is 12 years old. She has attended Sunrise for four years (K-3). Still, she received an AIC only for one year, during her third grade. LOVING herself did not receive an AIC education. I called her LOVING because of the way she treated and talked to her daughter. Even when she introduced herself, she focused on her daughter. She said "I am FLUTE's mom. That is what I do and what I am." Somehow, I could feel a sense of great love and pride toward her daughter. (Nevertheless, this should not give the impression that I did not sense this from the other mothers, but this characteristic was the one that stuck out to me immediately).

\section{Teacher(s).}

Table 4 represents four of the teachers'/ university liaison's characteristics: name, gender, ethnicity, and age (Goff, 2010).

Table 4. Teachers' Characteristics

\begin{tabular}{|l|l|l|l|}
\hline Teachers' Names & Gender & Ethnicity & Age \\
\hline INVOLVED & female & White & 68 \\
\hline MOTIVATED & female & White & 65 \\
\hline $\begin{array}{l}\text { Dr. S. } \\
\text { (university liaison) }\end{array}$ & female & White & - \\
\hline
\end{tabular}

Both teachers, INVOLVED and MOTIVATED, taught AIC at Sunrise. They both are retired now, but still were willing to participate in this study. Dr. S. was also involved with 
Sunrise. She served as a university liaison with Sunrise for many years. Each of these educators will be described in more detail in the following section.

Teacher \#1 = INVOLVED.

INVOLVED is a female, White, retired teacher, and 68 years old. She is originally from Ice Water, but lives now in Evening City. She received her teacher Education from Under the Stars and Windy Valley University. INVOLVED has been a teacher for 44 years. She taught grade levels K-6, but mostly third grade. She taught at Sunrise. All in all, she taught at five or six different schools. INVOLVED received specialized training like 4MAT, Foxfire, and Masters plus 45. She did not receive any prior arts training. INVOLVED taught at two different AICs at Windy Valley University and Sunrise. She taught AIC for more than 10 years. She does not hold any other certifications, but a Masters plus 45. This degree is also her highest level of degree and was received from Windy Valley University. INVOLVED did not serve on committees. When she introduced herself, she talked about how she taught at Sunrise for a long time, possibly 33 years. She said she could not remember for how long. This statement might have been one of the reasons that I called her INVOLVED. She appeared to be so INVOLVED in and excited about the arts and the AIC that one did not even realize that she was talking about her former work.

Teacher \#2 = MOTIVATED

MOTIVATED is a female, White, retired teacher, and 65 years old. She is originally from Clear Water, but lives now in Evening City. She received her teacher Education from Cold Snow University and Windy Valley University. MOTIVATED has been a teacher for 28 years. She taught grade levels 1-3, but mostly third grade. She taught at three different kinds of schools in Moonlight County, Windy Valley, one of which was Sunrise. During her career, she taught at three schools. MOTIVATED received specialized training and visited workshops for arts integration. She did not receive any prior arts training, but taught in an AIC for 14 years. During that time, her focus was third grade. MOTIVATED received a certification as a reading specialist 
(K-12), as well. She has a Bachelor in Elementary Education and a Master of Arts in Reading. Her highest level of degree is a Masters of Arts plus 45, which was received from Windy Valley University. MOTIVATED served on several committees at a school, county, and university level. By now, she has been retired from teaching for six years. Nevertheless, she never really retired from the arts. She now owns an art business. This is also one of the reasons why I called her MOTIVATED. Even as a retired teacher, she finds a way to exert herself. She is so motivated that she runs her own business after retirement, instead of breaking with the arts.

Teacher \#3/ university liaison $=$ Dr. S.

Dr. S. worked very closely together with INVOLVED and MOTIVATED. Nevertheless, she approached the AIC from a different angle. Dr. S. served as the university liaison for the teachers at Sunrise. She herself described her work with those teachers and her connection to Sunrise the following way:

And I am Dr. S. and I am the University liaison that worked with them during the artsintegration project for ten years and also know the extent of the work that these wonderful teachers, who created an amazing classroom environment and arts-focused environment that reaped benefits for all of the children in the room. They were the rock stars of the arts-integration world. (T-T\&L-INVOLVED, MOTIVATED, \& Dr. S.03.05.2011)

\section{Overview of co-researchers' characteristics.}

Table 5 represents an overview of the co-researchers' characteristics: name, gender, ethnicity, and age. 
Table 5. Overview of Co-researchers' Characteristics

Students'Characteristics
\begin{tabular}{|l|l|l|l|}
\hline $\begin{array}{l}\text { Students' } \\
\text { Names }\end{array}$ & Gender & Ethnicity & Age \\
\hline BAND & female & White & 13 \\
\hline PIANO & female & White & 12 \\
\hline FLUTE & female & Latino & $11 / 12$ \\
\hline $\begin{array}{l}\text { DRAWING } \\
\text { (non AIC) }\end{array}$ & female & White & 11 \\
\hline
\end{tabular}

\begin{tabular}{|l|l|l|l|}
\hline Parents' Characteristics \\
\hline Parents' Names & Gender & Ethnicity & Age \\
\hline BUSY & female & White & 47 \\
\hline CARING & female & White & 46 \\
\hline LOVING & female & White & 55 \\
\hline
\end{tabular}

Teachers'/University Liaison's Characteristics

\begin{tabular}{|l|l|l|l|}
\hline $\begin{array}{l}\text { Teachers' } \\
\text { Names }\end{array}$ & Gender & Ethnicity & Age \\
\hline INVOLVED & female & White & 68 \\
\hline MOTIVATED & female & White & 65 \\
\hline $\begin{array}{l}\text { Dr. S.(university } \\
\text { liaison) }\end{array}$ & female & White & - \\
\hline
\end{tabular}

\section{Setting/ School site.}

Sunrise was selected as the school site because it was the only school in Windy Valley that offered a fully developed AIC from 1991 to 2000. From 2000 to 2006 the AIC was heavily stressed in all third grade classrooms. During that time, teachers of the school received professional development in order to teach the AIC in an appropriate and professional way. For these reasons, I was referred to this school by a university faculty member who was actively involved in promoting and supporting the AIC at Sunrise. Even though I never entered the school, the recruitment of potential participants indirectly took place through the school. The same faculty member suggested two teachers who had taught all three of the potential student interviewees at Sunrise during the time of the AIC. 
The full immersion of students, teachers, and parents into an AIC across the school curriculum was not continued after 2006. Nevertheless, the school still favors the arts today (2011). It supports the arts across the curriculum, as stated on the school information website, "Instruction is further enhanced through the integration of the arts across the curriculum. Teachers help students learn through music, visual arts, movement, and drama" (Anonymous school web site, (n.d.)). Even though this full AIC immersion does not take place anymore, Sunrise still tries to integrate the arts in the daily teaching, which was a major factor for the selection of the school site.

Sunrise also has other advantages and specific characteristics, which led to its selection. First, it is located in the Moonlight County School district, whose Board of Education is known to offer one of the best public school systems in the United States. "Offspring and Expansion Management Magazines have rated the Moonlight County School System as one of the best 100 school systems in the country" (Anonymous school website, (n.d.)). On the Anonymous school website, one can find further facts about Moonlight Schools. Enrollment in the Moonlight County Schools totals 10,076 students, including 156 adult students. Second, the Moonlight County Schools served approximately 2,000 exceptional students during the 2004-2005 academic year, including approximately 470 gifted and 1,530 disabled students. As sources from 2005 show, the average daily school attendance (ADA) county-wide is $95 \%$ for elementary and middle schools. As of April 2005, the ADA for the county's high schools was 94\% (Anonymous school web site, (n.d.)). These data further support the fact that Sunrise appeared to be an appropriate school site, or better, recruitment area for this study.

The mission and vision of the school also appeared to be in alignment with my expectations. This is especially true for the close cooperation and teamwork of the school, the community, and the families. On October 13, 1998, the Moonlight County Board of Education adopted the mission to work cooperatively with families and the community to: 
- Help all students achieve their educational potential by recognizing and accommodating individual differences;

- Prepare all students to assume adult roles as responsible, productive citizens in a pluralistic society;

- Offer curricula to facilitate future employment and/or postsecondary education for all students; and

- Promote lifelong learning that addresses emotional, physical, social, character, and intellectual growth and development (Moonlight County Schools, 2009).

The vision of Moonlight County Schools, "to provide the community with an exemplary educational environment that promotes individual development, intellectual growth, and responsible citizenship" (Moonlight County Schools, 2009) is of particular importance to this study and indirectly reflects the ideas and the goals that an AIC serves.

\section{Research Procedures}

The best way to answer the research question(s) was to find out more about the perspectives of those who were directly and indirectly involved. First-hand interviews of parents, students, and teachers who participated in an AIC during their Elementary School years were conducted. This data collection process, from beginning to end, will now be described, in detail.

\section{Data collection (interview process).}

The data collection (interview process) started after West Virginia University's Office of Integrity and Research Compliance approved the Expedited-IRB Protocol on April 20, 2011 (see Appendix G11).

After this official Approval, the potential participants were contacted either by email (see Appendices G1 and G2) or phone. Contact information (email addresses and/ or phone numbers) for almost all of the participants (BUSY \& BAND, CARING \& PIANO \& DRAWING, INVOLVED \& MOTIVATED \& Dr. S.), with the exception of LOVING and FLUTE, were 
provided by the faculty. The contact information for LOVING and FLUTE, was provided by CARING. After the interview, CARING compiled a list of friends that she thought might be interested in participating in this study. She only selected candidates that fulfilled the requirements for this research study.

The data collection process for each interview meeting with all four groups consisted of the same steps (see Figure 4). The interview process started out with the demographic questionnaires and interview questions that represented the main portion of the process. Throughout the interviews, I took field notes (memoing) of what the interviewees said and how they reacted. After the transcriptions of the interviews, the co-researchers were provided with the transcripts to follow-up on them (artifacts) and validate the data.

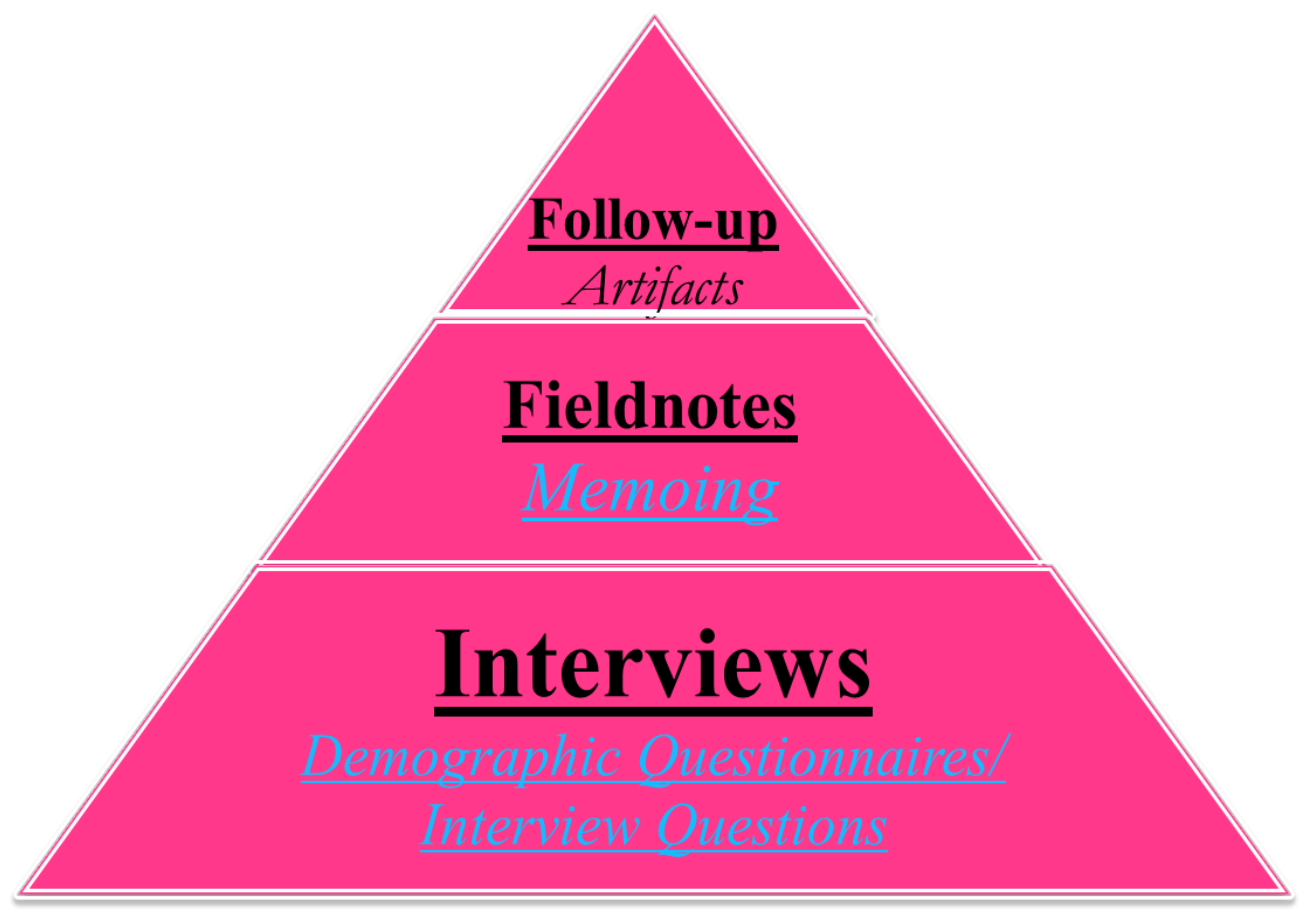

Figure 4. The data collection process.

Meeting one (BUSY \& BAND) - demographic questionnaire, interview, follow-up.

The first email to recruit potential participants was sent out to BUSY and BAND on April 22,2011 , with the request to participate in this study. An appointment for an interview was scheduled for April 29, 2011, at the house of the co-researchers (BUSY \& BAND). BUSY said it 
would be more convenient for her to sit for the interview at their house. She did not want her daughter to have to wait while I conducted her interview. I arrived a little bit earlier at the house. BAND just came back from school when I walked to the doorstep. She let me in and told me that her mother should be on her way back to the house from work. BUSY arrived and appeared to be very "busy" and multitasking with her kids while also helping me out. BUSY was under time pressure, since she had to go to an event in the evening. I asked her how she would like to do the interview. I told her that I had planned on interviewing her and her daughter separately, but that the interview might be quicker, if we do it together. She asked me to please do it together to save her some time. I agreed. BAND also appeared to be relieved and happy to be interviewed together with her mother. I started right away with the interview process, which consisted of the following steps.

First, forms had to be signed in order to start the interview. As the parent of BAND, BUSY had to sign the Parental or Guardian Consent and Information Form (see Appendix G7) for BAND to state her willingness to allow her daughter to participate in this study. In addition, BUSY also had to sign a Consent and Information Form (see Appendix G6) for herself, which states that she agrees to participate in the research study. BAND had to sign only one form, called Assent Form (see Appendix G8) to show her willingness to participate. It took BUSY and BAND no longer than 10 minutes to sign these forms. After they had handed the forms back to me, I checked the documents to make sure all the necessary information was provided and all the pages were signed and initialed.

Second, after all the release forms had been signed by both of the co-researchers, the official part of the interview process could begin. I provided BUSY and BAND with a demographic questionnaire (see Appendices G12 and G13). The demographic questionnaire for parents contains 13 questions, while the demographic questionnaire for students contains 11 questions. The purpose of the questionnaires is to give me a better idea of accompanying 
(demographic) data and factors that might have an influence on the interpretation and analysis of the collected data. BUSY and BAND filled out both of their questionnaires without any problems. This lasted approximately 5-10 minutes. From time to time BAND asked her mother or me some questions. She wanted to make sure she answered all the questions correctly. Once the participants were finished with the questionnaires, they handed them back to me.

Third, after the demographic questionnaires were completed by the participants, the digitally audiotaped interview process could start. I provided BUSY and BAND with the preestablished interview questions (see Appendices G14 and G15) before the interview to make sure they were comfortable and familiar with the questions. Before the interview officially started, I tested the digital audio-recorder to make sure it worked properly. After the test, I told BUSY and BAND that I would start the recorder in a few seconds and that I would introduce myself as the researcher at first and then ask each of them, as my co-researchers, to give a brief introduction. After the introductions, I started the interview with the first interview question (for students: How did you personally experience the arts-based learning classroom?; for parents: How did you personally experience your daughter participating in the arts-based learning classroom?). All in all, there were 10 pre-determined interview questions that I asked the participants. I tried to mix up the order in which I asked each new question, so that BUSY, as well as BAND, had to answer new questions first without being influenced by what the other one had said before. Even though 10 of the interview questions were pre-prepared, the topic or theme of the interview did not have to refer only to that single interview question. Whatever thoughts the question triggered in the participants' minds were well received. This is the nature of phenomenological research. For some questions, the participants responded directly to the exact interview question. For other questions, new themes and ideas developed that drifted away from the original question. Towards the end of the interview, I asked if there was anything else the co-researchers would like to add to the interview, topics for example that we did not touch on. BUSY and BAND were 
very open to this invitation and added new ideas. Since this interview with BUSY and BAND serves as a model in chapter four, the complete transcript of interview number one can be found in chapter four, step one: Listing and Preliminary Grouping. At the end of the interview, BAND was willing to share her artwork with me. She went to her room and got some works that she described to me in more detail. After BAND was finished with her explanations, she gave me permission to take a picture of her creations (Figures 5-8):

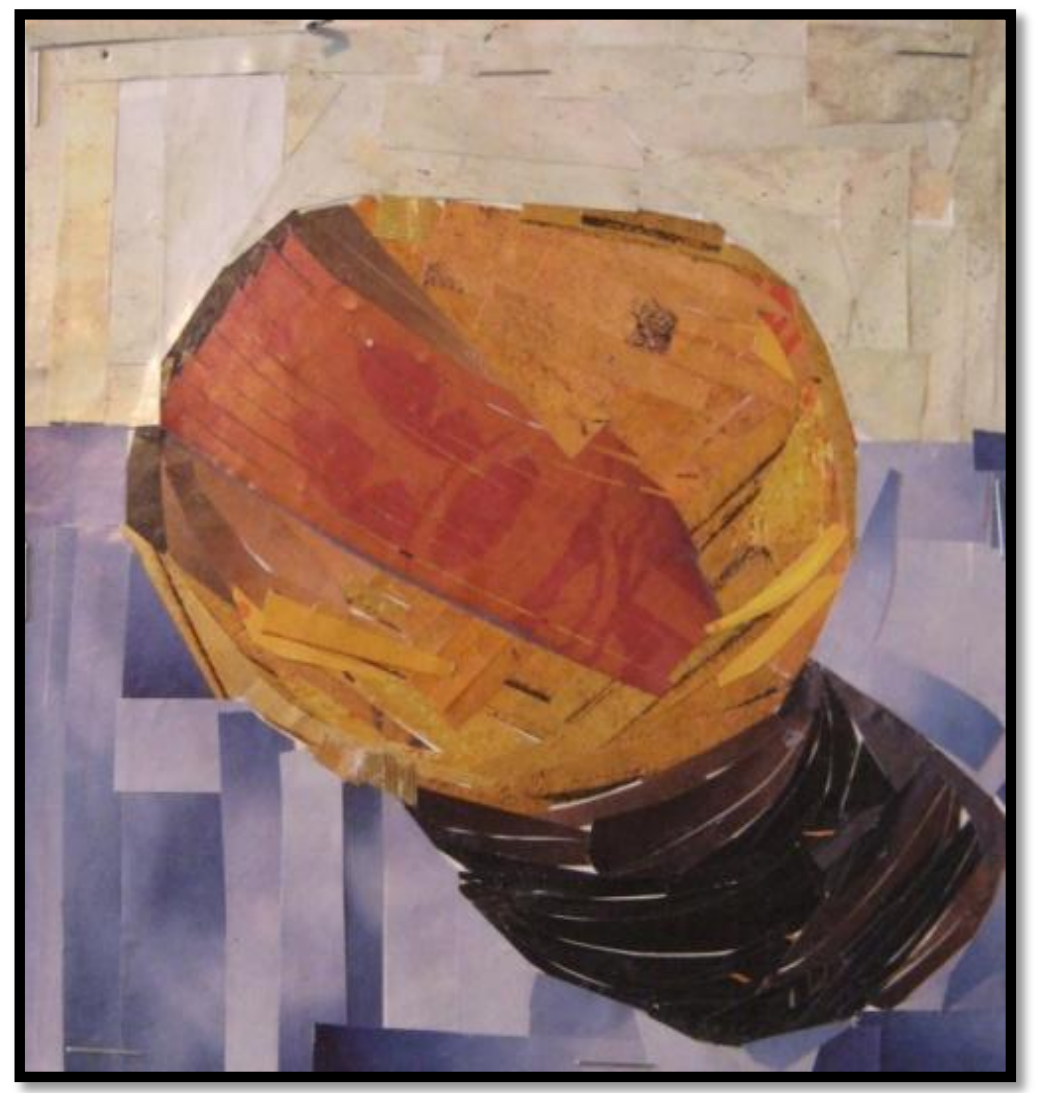

Figure 5. The orange.

A picture that was up on BAND's wall. She said: We could do a lemon, orange or pear or apple, but I chose an orange. And we had to take clippings of magazine and match the color... it was o.k. if it was different (T-P-BUSY \& T-S-BAND-29.04.2011). 


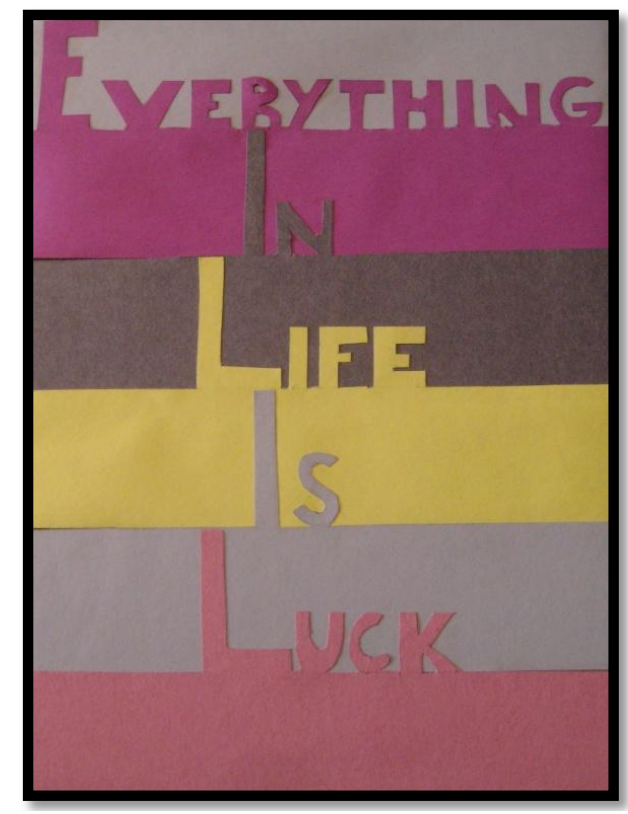

Figure 6. "Everything in Life is Luck."

BUSY says about this quote, "I do not think it is that true, some stuff in life is luck, but...." BUSY explains that she is not sure why she picked these colors. She says, "I guess they kind of went together. I didn't want to be like matchy, I wanted to be like, just colorful." (T-P-BUSY \& T-S-BAND-29.04.2011).

Figure 7. Mosaic cross.

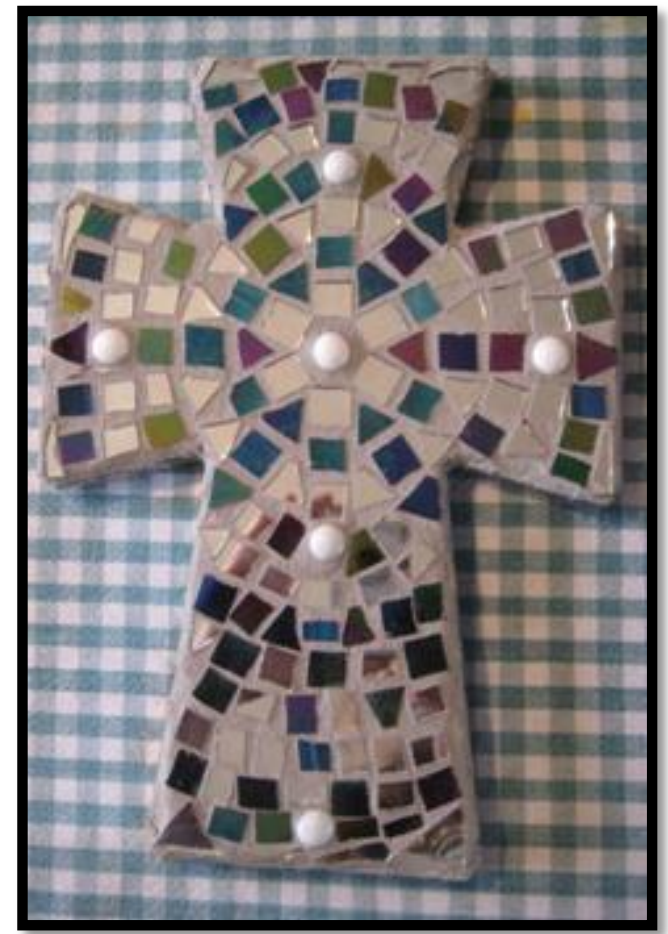

BAND had it hanging on her wall. She says about it: "I didn't make it at school but I made it at my mother's business.” (T-P-BUSY \& T-S-BAND-29.04.2011). 

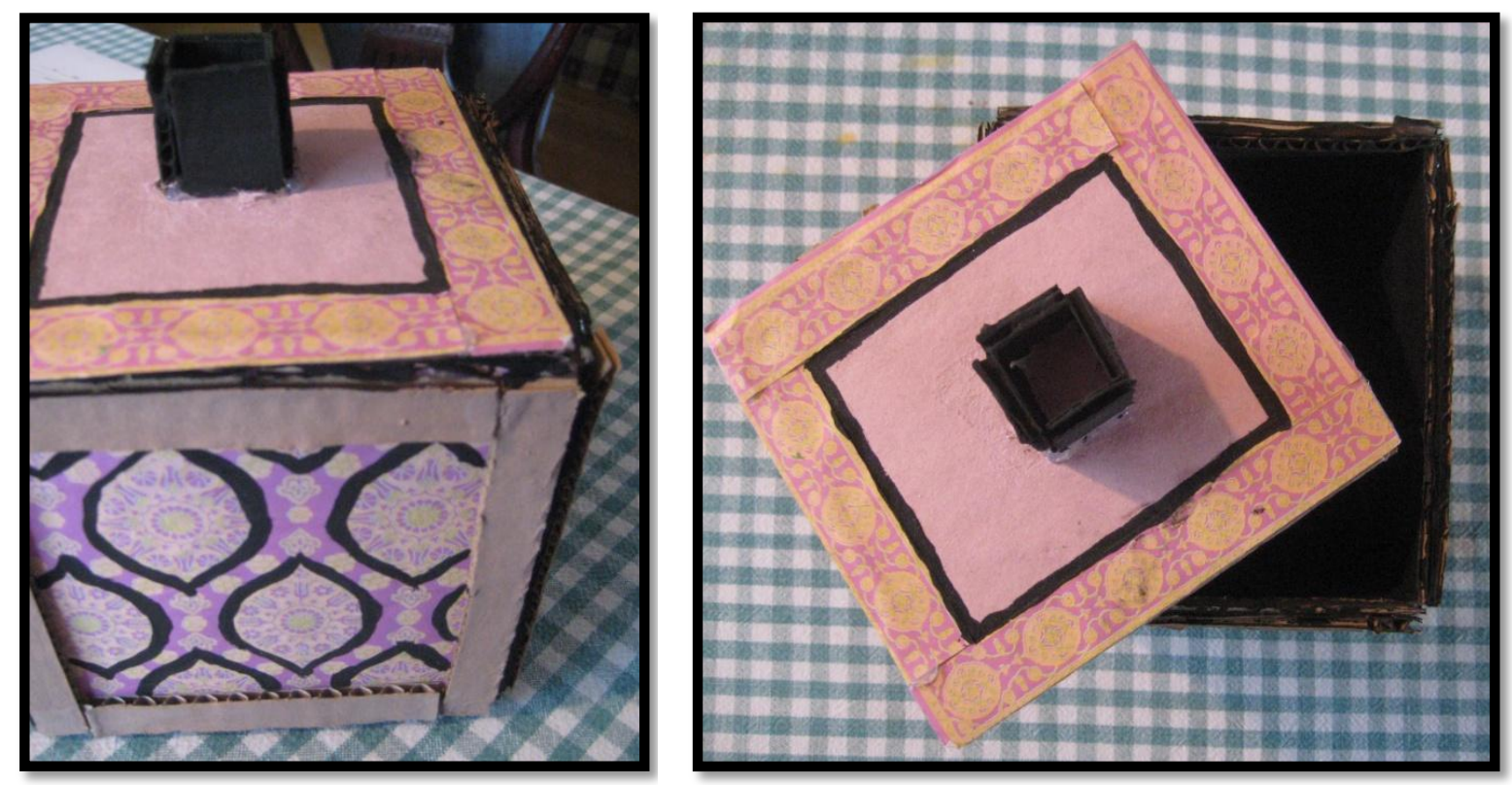

Figure 8. Box.

BAND says about the box, "we had to make it out of cardboard (...) from scratch (...). (...) when my teacher put out the paper that we could use, he said that these were patterns from around the world. There were a lot of different colors (...)." (T-P-BUSY \& T-S-BAND29.04.2011).

The interview lasted 43 minutes and 57 seconds. The whole interview process took approximately 60 minutes.

After BUSY's and BAND's interview, the data had been transcribed and were sent to them by email (see Appendix G16). I requested that they would validate the data. The participants were asked to review the final transcripts for accuracy. All of the co-researchers were also asked to add comments to the interview transcripts, in case some new or additional ideas came to mind during their review. This way, themes that emerged during the interview were checked, verified, and clarified by the interviewee. I also offered to send the transcripts per mail. This was suggested in case the co-researchers did not feel comfortable editing and adding comments to the transcript on the computer. The due date to send back reviewed transcripts was July 5, 2011 to make sure to have all of the paperwork back around the same time to be able to 
continue with the research study. BUSY's and BAND's reviewed documents were received on July 20, 2011. They stated that no changes or additions were necessary and that their experiences were captured entirely by my transcripts.

\section{Meeting two (INVOLVED, MOTIVATED, \& Dr. S.) - demographic questionnaire, interview, follow-up.}

The second email to recruit potential teacher participants was sent out to MOTIVATED on April 25, 2011 with the request to participate in this study. On April 27, I received a reply email from MOTIVATED. She denied her participation in the study because she felt like she did not have any data to provide that spoke directly to my research. Since I did not have an email address available for INVOLVED, I called her on the phone and was hoping that she would be willing to participate in my research study. INVOLVED declined to participate in the study, as well. She added that she thinks she will not be able to do justice to the study, since she has been retired for some years. She suggested a study that she has been interviewed for before in order to get the information I need. Since INVOLVED and MOTIVATED, as potential teacher participants, were supposed to play a larger part and provide some major insight to my study, I talked to Dr. S. about my problem. She sent an email to the two teachers on April 28, 2011, with the request to rethink their possible participation in my research study. Dr. S. was convinced that both teachers could contribute important knowledge and insights to my study, even though they had been retired for a few years. On the same day, April 28, 2011, I received an email from Dr. S. with the information that INVOLVED and MOTIVATED were willing to meet up with us (Dr. S. and me) on May 3, 2011 at a public coffee place, to conduct the interview. We met early in the morning at 8:30a.m., a time that seemed more convenient for the two co-researchers.

When I arrived at the meeting place, Dr. S. was already there. Shortly after I arrived, INVOLVED showed up and shortly after that MOTIVATED was there, too. Dr. S. introduced us, I got coffee for all the participants, and we prepared to conduct the interview. In total, four 
people participated in this interview (INVOLVED, MOTIVATED, Dr. S., and myself). MOTIVATED was somewhat under time pressure and wanted to start the interview process immediately without wasting any time. Step one (signing the forms) and step two (filling out the demographic questionnaires) were pushed back to the end of the interview process.

The digitally audiotaped interview process started right away and for meeting two, the same data collection steps were taken as in meeting one. I provided INVOLVED, MOTIVATED, and Dr. S. with the pre-established interview questions for teachers (see Appendix G17) before the interview. I tested the digital recorder to make sure it would work properly. INVOLVED, MOTIVATED, and Dr. S. introduced themselves. The interview started out with the first interview question (for teachers: How did you personally experience your female students participating in the arts-based learning classroom?). All in all, there were 10 predetermined interview questions that I asked the teacher participants (see Appendix G17), similar to the 10 interview questions for the parents and the students (see Appendices G14 and G15). I did not really direct the interview questions. They were asked in an open way. INVOLVED, MOTIVATED, and Dr. S. answered the questions, depending on which of them thought of an answer to the question first. Departures from the original theme or question were welcomed. Towards the end of the interview, I asked if there was anything else the co-researchers would like to add to the interview. MOTIVATED appeared to be in a hurry. She said that she could not think of anything else. She apologized, but said that she had to leave because of another appointment. MOTIVATED left early. INVOLVED stayed and added new things. I stopped the digital audio-recorder once it seemed like the conversation drifted more towards a private one. After the interview process was completed, I asked INVOLVED to fill out the Consent and Information Form (see Appendix G6), as well as the demographic questionnaire (see Appendix G18). Once INVOLVED finished the paperwork, I realized that MOTIVATED had left without signing any papers. INVOLVED offered to call MOTIVATED so I could arrange an 
appointment with her to complete the necessary paperwork. MOTIVATED had to sign and fill out the paperwork so that the collected data could be used for the dissertation. INVOLVED called MOTIVATED who suggested that I bring the forms to her business. I left our meeting place and drove immediately to MOTIVATED's business. She filled out the Consent and Information Form (see Appendix G6) as well as the demographic questionnaire for teachers (see Appendix G18). It took INVOLVED and MOTIVATED about 5 minutes to sign the Consent and Information Forms and approximately 10 minutes to fill out the demographic questionnaire that contained 20 questions. Once the two co-researchers were finished filling out the Consent and Information Forms and the questionnaires, they handed them back to me and interview number two was officially completed. The interview lasted 1 hour, 30 minutes, and 2 seconds. The whole interview process took approximately 2 hours.

INVOLVED's, MOTIVATED's, and Dr. S.'s data were transcribed after the interview. The final transcripts were sent to them by email (see Appendix G19). The participants were asked to validate the data, to review for correctness, and to add comments if necessary. INVOLVED did not have computer access to review the transcripts. I offered to send her the documents by mail. INVOLVED declined. She stated that MOTIVATED and herself had talked things through so that it was not necessary to send a paper version to her. MOTIVATED and INVOLVED made changes together. The reviewed document was received on June 21, 2011. The participants made the point that some of the comments were not attributed to the correct participant. Changes to the interview transcript were marked in red by the co-researchers. I accepted those changes. I realized that it was possible that in an interview with four female participants voices could get mixed up. 


\section{Meeting three (CARING, PIANO, and FLUTE) - demographic questionnaire, interview, follow-up.}

The only contact information that I had for CARING was her phone number. I called on April 27, 2011 to ask CARING if she and her daughter would be willing to participate in my dissertation research study. CARING was very nice on the phone and open to an interview, but she said that she and her family would be very busy for the next few weeks and that it would be best for them to do it on a weekend. She suggested May 14, 2011 as a possible interview date. I agreed, and we decided that I would come to their house to make it easier on them. The two coresearchers, CARING and her daughter PIANO, live in the country side and it was a beautiful drive to their house. On the way, I saw two girls with a dog walking on the country roads. They were very friendly and waved at me. Once I arrived at the house, CARING let me in the house and told me that PIANO had a friend over last night and that they are outside, on a walk with the dog. She said that they should be back in a few minutes. She asked me if I would like to have a coffee and fixed some coffee for me while we were waiting. A few minutes later, the girls arrived. PIANO and DRAWING were the two girls that I just had seen on my way to the house. Both girls were carrying caterpillars and told us about how they found the caterpillars on their walk and that they named them. CARING asked me if I would like to interview PIANO's friend, as well. CARING said that PIANO's friend did not go to Sunrise, but that she still is very much into the arts so that it might be interesting for me to see her point of view, as well. I told CARING that I would need the Consent Forms signed by PIANO's friend and her mother to be allowed to use the data. CARING said that she is convinced that the mom of PIANO's friend would be happy to see her daughter participating in this study. She suggested that she could get the consent from the mother of PIANO's friend and that they would send it to me. I left the Parental or Guardian Consent (see Appendix G7) and Information Form (see Appendix G6) for the mother of PIANO's friend with CARING. CARING promised that she would make sure I 
receive the signed forms back. It seemed like PIANO's friend DRAWING wanted to participate, but she was shy at the same time. I told her that she could stay during the interview and see if she would like to provide additional responses to my questions, if so inclined. It appeared that she was relieved and happy to be part of the interview. Even though I had not planned on interviewing two girls, especially not one that had not participated in an AIC during her Elementary School years, I was anxious to see what I would find out. Once everybody was sitting down at the table and felt comfortable, I started the interview process, which consisted of the same steps as meetings one and two.

The Parental or Guardian Consent (see Appendix G7) and Information Form (see Appendix G6) had to be signed by CARING. Also the mother of PIANO's friend had to sign the same forms. CARING took care of this issue, and I received the Parental or Guardian Consent and Information Form for DRAWING in the mail on May 17, 2011. CARING also had to sign a Consent and Information Form (see Appendix G6) for herself. PIANO and DRAWING only had to sign the Assent Form (see Appendix G8). It took CARING, PIANO, and DRAWING no longer than 10-15 minutes to sign these forms. After they had handed the forms back to me, I checked the documents.

Now, the interview process could officially begin. I provided CARING, PIANO, and DRAWING with a demographic questionnaire (see Appendices G12 and G13). The three of them filled out the questionnaires without any problems. PIANO and DRAWING sometimes checked back with me or CARING, to make sure they answered the questions as intended. This process lasted approximately 10 minutes. Once the co-researchers were finished with the questionnaires, they handed them back to me and waited for further instruction.

After the demographic questionnaires were completed by the participants, the digitally audiotaped interview process started. I provided CARING, PIANO, and DRAWING with the pre-established interview questions (see Appendices G14, G15) and tested the digital recorder. 
The four of us introduced ourselves, and I started the interview with the first interview question out of the total of 10 pre-determined interview questions. At the beginning of the interview, DRAWING was very involved and participated likewise in the interview. Later on, there were a few questions DRAWING did not want to answer. She started drawing on the sheet that contained my interview questions (after she had asked if she was allowed to do so). Also, she went to the bathroom and did not come back for a while. Even though, she sparingly offered comments or ideas, it did not seem like she wanted to participate in the interview till the end. I did not force her to participate and finished the interview. After the last interview question, I asked if there was anything else they wanted to add to the interview. At the end of the interview, I asked PIANO if she would be willing to share some of her artwork with me. She went to her room and got some drawings of her dream wedding. She had drawn the clothes that she, her bride's maids, her ring boy, and her groom are going to wear. She described and explained each of the individual drawings in great detail. After PIANO was finished with her explanations and I had turned off the digital audio-recorder, PIANO gave me permission to take pictures of her drawings (Figures 9-12): 


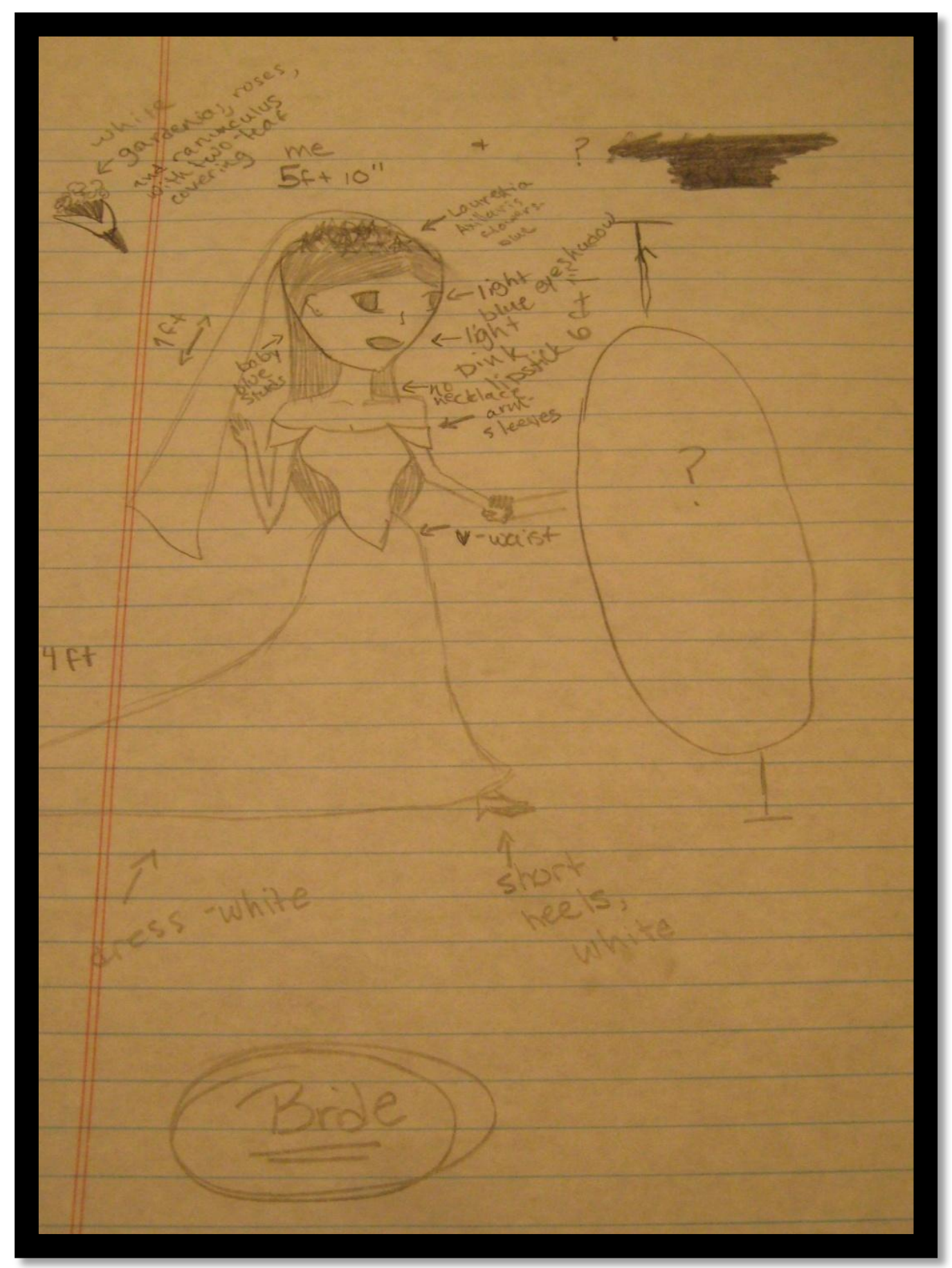

Figure 9. Bride.

PIANO describes: "Ok. So, this is my wedding plan. This is the flower girls, the ring bearer, the bridesmaid, and (...)." CARING asks: "And what is this question mark?" PIANO answers: "That is my dude. (...) He is less important when it comes down to it. It is really about the dress." (TS\&P-PIANO, DRAWING \& CARING-14.05.2011) 


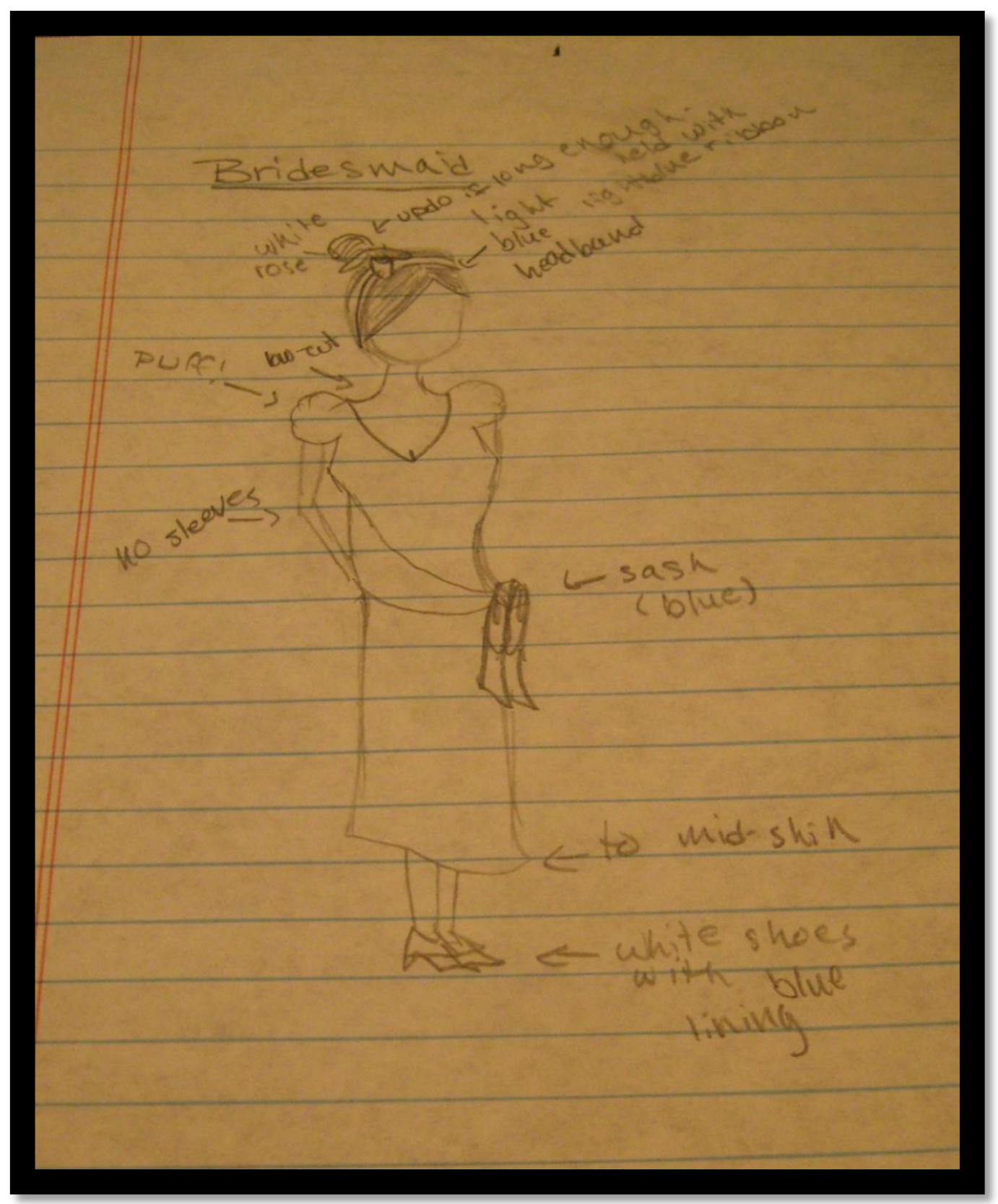

Figure 10. Bridesmaid.

PIANO says: "I designed stuff that I would like to have my guests wear, bridesmaids wear at my wedding” (T-S\&P-PIANO, DRAWING \& CARING-14.05.2011). 


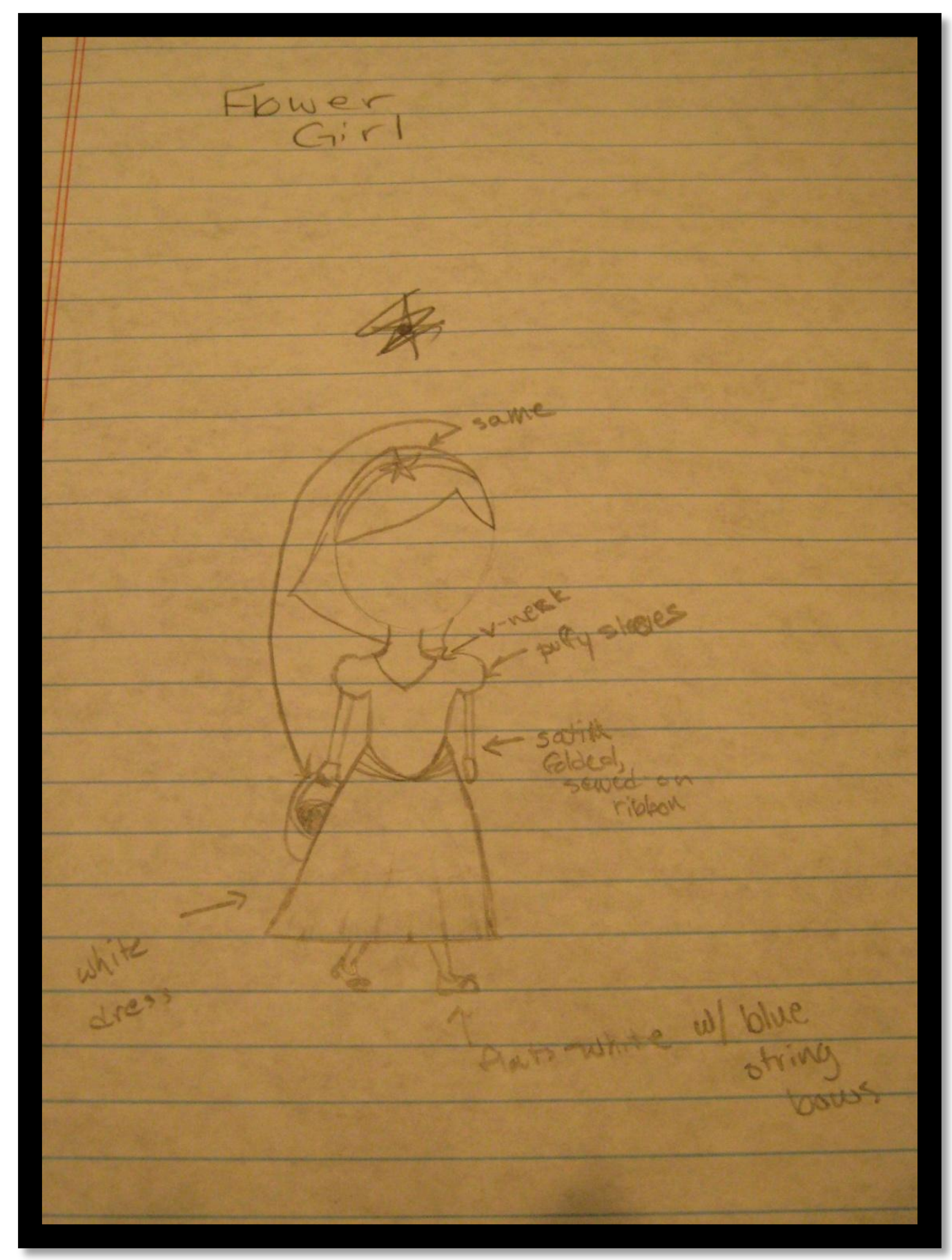

Figure 11. Flower girl.

PIANO says about her: "And here is my flower girl, she is adorable, I love her. She has a large cloth copy of a large axilaris flower in her hand and (...)" (T-S\&P-PIANO, DRAWING \& CARING-14.05.2011). 


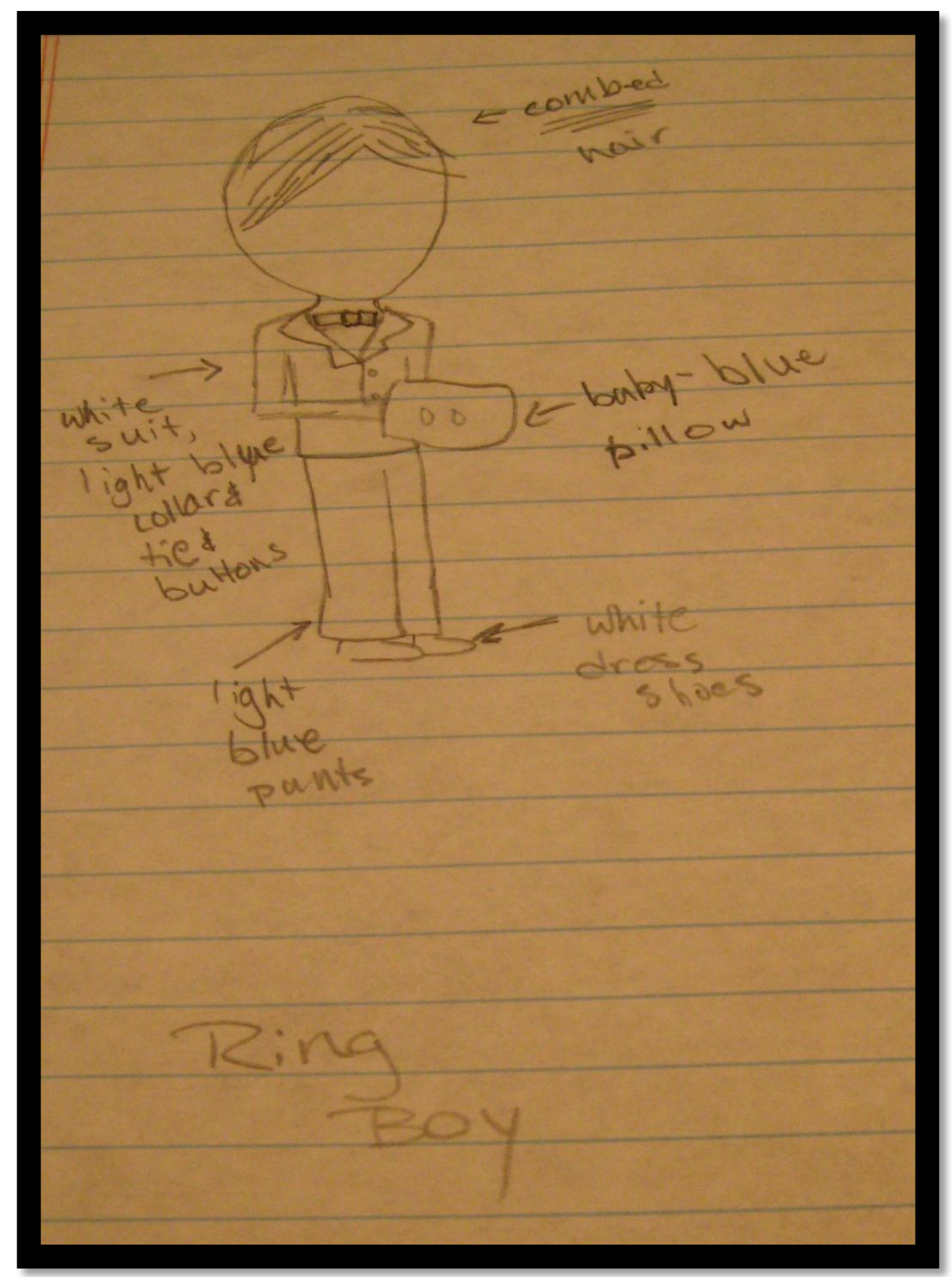

Figure 12. Ring boy.

PIANO says about him: "This is my ring boy or ring bearer, whichever you want to say. The hair has to be combed because if it is not, I will kick him out of the wedding." (T-S\&P-PIANO, DRAWING \& CARING-14.05.2011) 
CARING asked her daughter if she would play the piano for me. Even though PIANO was hesitant at first, she agreed to do so. She set one condition, though. She wanted to choose the piece to play. CARING consented and PIANO played a beautiful piece for us. This interview lasted 1hour, 1 minute, and 57 seconds. The whole interview process took approximately 1 hour and 30 minutes.

CARING's, PIANO's, and DRAWING's data were transcribed after the interview. The final transcripts were sent to CARING by email (see Appendix G16) on June 21, 2011. Like the other co-researchers, these participants were asked to validate the data, to review for correctness, and to add comments if necessary. An email by CARING was received on June 22, 2011. CARING stated that she would review the document and let me know by July 5, 2011, if changes were necessary. No email was received by July 5, 2011. This meant no changes were necessary.

Meeting four (LOVING \& FLUTE) - demographic questionnaire, interview, follow-up.

On May 19, 2011, an email was sent out to LOVING, with the request to participate in this study. I had received LOVING's and FLUTE's contact information from CARING. CARING had compiled some names and contact information of friends who had gone through the same AIC at Sunrise. She thought that her friends would be happy to participate in this study. As CARING predicted, LOVING was interested in participating and responded the same day to my email. We set up an interview for May 24, 2011. LOVING decided that it would be most comfortable for me to conduct the interviews at their place. When I arrived at their house, a nice dog welcomed me. LOVING asked if I would like to conduct the interview in the living room or if I would prefer the dining room table. I decided it might be more convenient to use the dining room table, since forms had to be completed. LOVING's husband was at the house, too. We had a short conversation with him about my dissertation research, future plans after the completion of the dissertation, and my cultural background. LOVING had called me on the phone with the 
intention to cancel our meeting. She changed her mind though, after she found out that I was already in town. Despite the fact that she must have been somewhat under time pressure, she still appeared to be quite relaxed and took her time to have a conversation, apart from the interview. Also this interview with LOVING and FLUTE consisted of the same steps as the previous ones.

The Parental or Guardian Consent and Information Form (see Appendix G7) had to be signed, as well as the Consent and Information Forms (see Appendix G6). FLUTE signed only the Assent Form (see Appendix G8). It took LOVING and FLUTE no longer than 10 minutes to sign these forms. After they handed the forms back to me, I checked the documents.

After all the release forms had been signed by both of the co-researchers, the interview process could begin. I provided LOVING and FLUTE with a demographic questionnaire (see Appendices G12 and G13). They had a few questions while filling out the questionnaires because of their special family situation (FLUTE had been adopted). In total, it took them about 10 minutes to fill out the questionnaires.

The digitally audiotaped interview process began after I had tested the recorder. LOVING and FLUTE had received the 10 pre-established interview questions (see Appendices G14 and G15). The questions were just starters for a conversation about a theme and did not intend to limit the participants to just one topic. Towards the end of the interview, I asked if FLUTE would be willing to share some artwork with me. LOVING and FLUTE asked whether it had to be visual art or if it could be music. I told them that any kind of art would be interesting, not just visual art. LOVING asked her daughter if she would be willing to play the flute for me. FLUTE slowly shook her head and said "no". LOVING and I did not want to pressure FLUTE and were about to accept that she did not want to play. Suddenly, she added that she would like to play but had left her flute at school. LOVING came up with the idea to show me one of the video-clips she took of her daughter, playing the flute. FLUTE agreed but disappeared while her mother played the video-clip for me. LOVING was worried that FLUTE had disappeared 
because she was embarrassed by the video. But FLUTE had been in her room trying to find visual artwork from her time at Sunrise. She brought down some of her artworks and showed and explained them to me. FLUTE even allowed me to take a picture of her oeuvres (Figures 13-14):

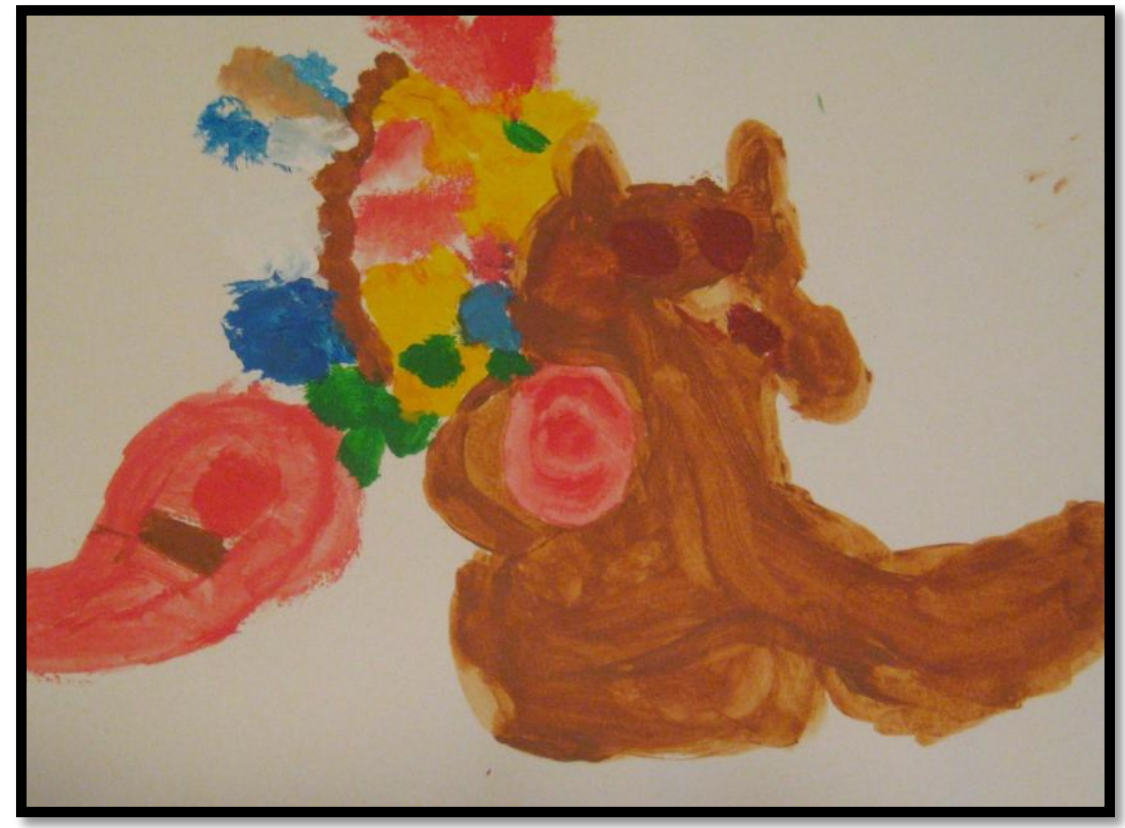

Figure 13. Bear party.

About this painting, FLUTE says: "Doing this was hard because you have to go through the flowers and then some of the flowers would go over it, so that was hard" (T-P\&S-LOVING \& FLUTE-24.05.2011).

Figure 14. Water lilies.

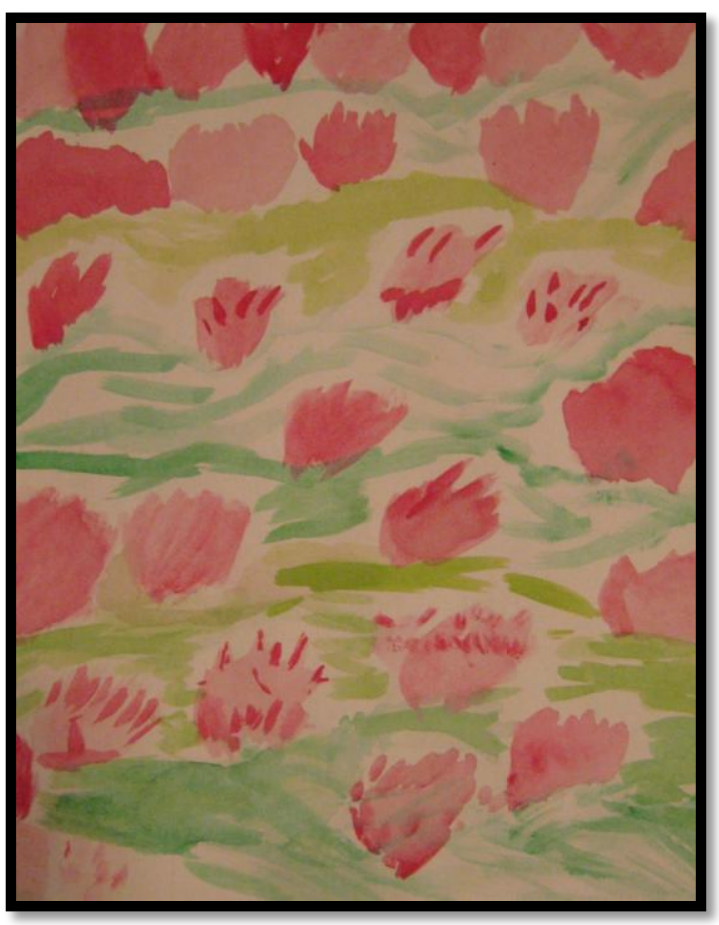

FLUTE describes the painting: "Here is a copy of the water lilies (...)" (T-P\&S-LOVING \& FLUTE-24.05.2011). 
The interview lasted 40 minutes and 25 seconds. The whole interview process took approximately 1 hour and 10 minutes.

After the interview, LOVING's and FLUTE's data were transcribed. These transcripts were sent to them by email (Appendix G16). LOVING and FLUTE were asked to validate the data, to review for correctness, and to add comments, if necessary. LOVING's and FLUTE's reviewed documents were received on the June 23, 2011. No changes were suggested by them.

\section{Coding procedures.}

Throughout this study, two kinds of coding procedures were used. First, the interviews and their transcripts were coded to make it easier to keep them organized, but anonymous at the same time. The original interviews were labeled with an "I." The transcripts were labeled with a "T."

This sign was followed by the code " $\mathrm{S}$ " for students, " $\mathrm{P}$ " for parents, " $\mathrm{T}$ " for teachers, or " $\mathrm{L}$ " for university liaison. This code was then followed by the fully coded name of the co-researchers (students: BAND, PIANO, DRAWING, FLUTE; parents: BUSY, CARING, LOVING; teachers: INVOLVED, MOTIVATED, and university liaison: Dr. S.). I provided a coded name for all of the co-researchers, names of places, and universities. This was necessary to keep the data confidential and anonymous. Next, the date of the interview, starting with the month followed by the day and the year will be noted. For example, a common code for an interview conducted with a student named BAND, on November 11, 2011, would be: (I-S-BAND-11.11.2011). A transcript for the same interview would be coded: (T-S-BAND-11.11.2011).

Second, the transcribed interviews and the narrative data were reviewed for emerging themes. These themes were color-coded or font-coded (see also Appendices H3, H4, H5, and I for more detailed information).

For the students' themes table (see Appendix H3), the following color codes apply:

1. Dark Red:

All four students (BAND, PIANO, FLUTE, and DRAWING) mentioned these units (ICs). 


\section{Medium Red:}

Only the three students who completed an AIC mentioned these units (ICs).

\section{Light Red:}

Only three students mentioned these units (ICs).

\section{Green:}

Only two out of four students mentioned these units (ICs).

5. Yellow:

Only the one student, who did not complete AIC, mentioned these units (ICs).

For the parents' themes table (see Appendix H4) the following color codes apply:

\section{Dark Red:}

All three parents (BUSY, CARING, \& LOVING) mentioned these units (ICs).

2. Medium Red:

Only two out of three parents mentioned these units (ICs).

\section{Light Red:}

Only one out of three parents mentioned these units (ICs).

For the teachers' themes table (see Appendix H5) the following color codes apply:

\section{Dark Red:}

All two teachers/ university liaison (INVOLVED, MOTIVATED, \& Dr. S.) mentioned these units (ICs).

2. Medium Red:

Only two out of two teachers/ university liaison mentioned these units (ICs).

\section{Light Red:}

Only one out of two teachers/ university liaison mentioned these units (ICs). 
With regard to the clustered themes chart for each of the three groups table (students, parents, and teachers, see Appendix H6) those themes are marked in bold font that were considered important during an interview by at least three out of four students, two out of three parents, and two out of two teachers/ university liaison,

As for the summary chart for all co-research groups' and participants' validation chart (see Appendix H10), the following codes apply:

1. Same colors in the table mark the same phenomena that arose for each group (parents, students, and teachers).

As for the summary list of chart/ Appendix H10, for phenomena of all co-research groups and participants, the following codes apply (Appendix H11):

1. Dark Grey Background table (Appendix H11.1):

themes that are representing the group as a whole (all three groups considered these important).

2. Medium Grey Background table (Appendix H11.2):

themes only relevant for two of the groups, instead of three.

3. White Background table (Appendix H11.3):

themes only relevant for one of the groups (will not be considered).

Validation of study (triangulation).

The first step of ensuring validity, trustworthiness, and credibility of this study is the official approval of the expedited IRB Protocol. This document was granted on April 20, 2011, by West Virginia University's Office of Integrity and Research (see Appendix G11). This approval was indispensable, since this study is a phenomenological study which consists of interviews with human beings.

In a second step, I contacted potential participants per email to ask them about their willingness to participate and to set up an interview meeting with them. 
Third, I met up with those students, parents, and teachers who agreed upon participating in this study to clarify requirements, to obtain written consent, and to conduct interviews.

In a fourth step, the validity of this study was ensured by conducting triangulation of data collection methods. Creswell (2007), to whom Sandra Schroder (2010) referred in her dissertation, lists several procedures that ensure validation:

Prolonged engagement and persistent observation, triangulation, peer review or debriefing, rich and thick description, negative case analysis, clarifying of researcher bias, member checks, and external audits. He suggests that researchers utilize at least two of the following procedures to add rigor to the study. (Creswell, 2007, as cited in Schoder, 2010, p. 63)

For the triangulation of my data collection methods, some of the procedures listed by Creswell (2007) and others (Devono, 2009; Gay, Mills, \& Airasian, 2009; Groenewald, 2004) were included (see Figure 15): (1) the actual interviews (Creswell, 2007), (2) memoing (Groenewald, 2004), (3) artifacts/ follow-ups on interviews (Gay, Mills, \& Airasian, 2009), (4) peer review (Creswell, 2007), and the (5) referencing of a past report study that focused on the teachers' perspective in relation to art integration (Devono, 2009). This process of triangulation that Joseph Maxwell (2005) describes in his work Qualitative Research Design: An Interactive Approach, was necessary because it "reduces the risk that your conclusions will reflect only the systematic biases or limitations of a specific source or method, and allows you to gain a broader and more secure understanding of the issues you are investigating” (Maxwell, 2005, pp. 93-94). 


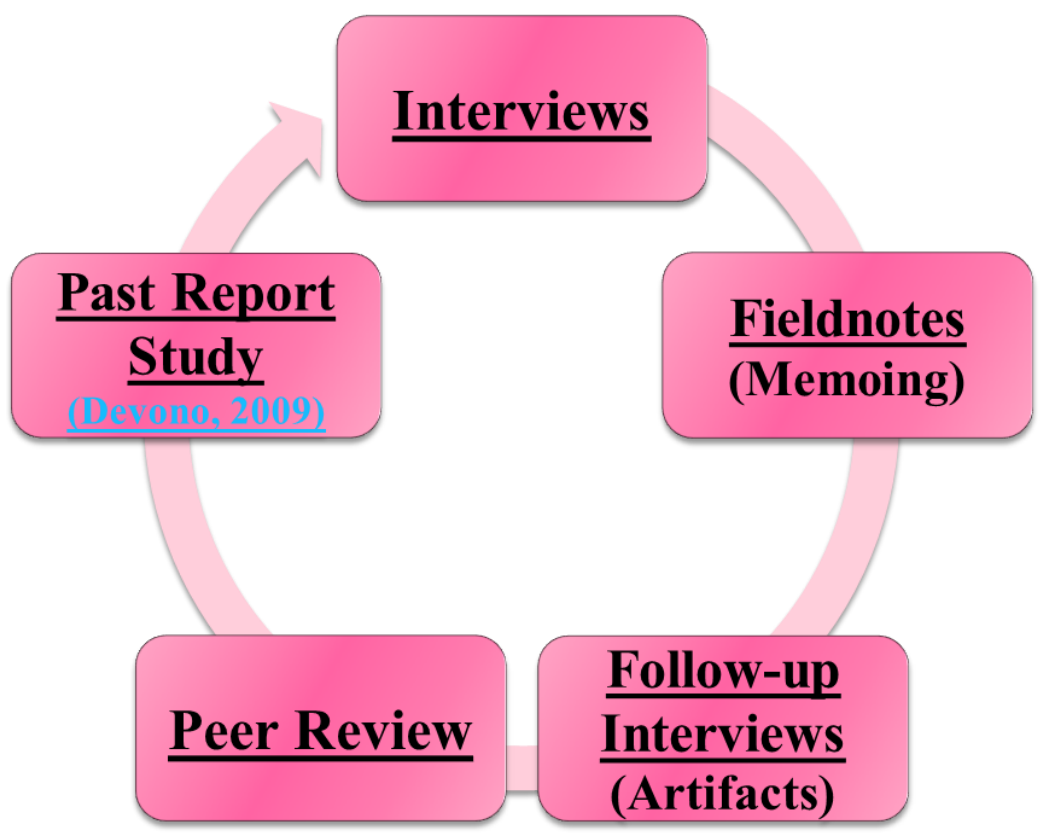

Figure 15. Triangulation of data.

Each of the previously listed individual steps for the triangulation process that was conducted is described in short in the following section. First, the data was collected through actual in-depth interviews with students, parents, and teachers. These three groups shared their experiences with an AIC and its possible influences on the female students' self-concepts and their perceptions of beauty. Second, fieldnotes (memoing) played an important role in this qualitative research study. Memoing presents in Groenewald's (2004) opinion those field notes of the researcher that demonstrate the hearing, seeing, experiencing, and thinking procedures of the researcher in the course of collecting and reflecting on the process. Third artifacts, in this case, follow-ups on the interviews, were used to validate the correctness of the conducted interviews. Fourth, a second reviewer was consulted to check the data in a test run and analyze the narrative for emerging themes. The independently-occurring themes between the second reader and myself were cross-checked for consistency and validity. Also, a past report and study with teachers' perspectives on arts-integration (Devono, 2009) was referenced, compared, and related to this study (see Table 7 and Appendix I). These five various ways of triangulation contributed to the validity, trustworthiness, and credibility of this study. 


\section{Chapter 4: Analysis of Data}

At the beginning of this chapter, I feel the need to clarify the selection of the name "analysis of data" for this section. I wish to inform the reader that I am aware of the ongoing discussions and negative connotations in the qualitative research field that are related to the term “analysis of data." Hycner (1999), for example, states that the term "“analysis' has dangerous connotations for phenomenology" because this term usually describes "a 'breaking into parts' and therefore often means a loss of the whole phenomenon... [whereas 'explicitation' implies an]... investigation of the constituents of a phenomenon while keeping the context of the whole" (Hycner, 1999, as cited in Groenewald, 2004, p. 17). For the longest time, I considered naming this chapter "explicitation of data," as Hycner (1999) suggests in Groenewald's (2004) article, A Phenomenological Research Design Illustrated. Even though I understand Hycner's (1999) and Groenewald's (2004) concern, I nevertheless decided to call this chapter "analysis of data" and to apply this term in my research study. The reasons for this decision were Clark Moustakas (1994) and his "Modification of the Van Kaam Method of Analysis of Phenomenological Data" (Moustakas, 1994, p. 120). Throughout his work on phenomenology, and even more specifically during the phenomenological research process, he refers to the "analysis of data" (p.120). As such, I finally decided to adopt the language Moustakas (1994) himself uses in order to stay true to his method and remain consistent in the wording.

This chapter presents the phenomenological data analysis process with a focus on the organizing, analyzing, and synthesizing of data by Moustakas" "Modification of the Van Kaam Method of Analysis of Phenomenological Data" (p. 120). During this process, individual textural and structural descriptions, textural-structural and integrated textural/ composite structural descriptions, and a synthesis of textural and structural meanings and essences of the experience, were generated for the collected data. 
The interview data from 10 co-researchers served as exemplary illustrations of their experiences with the Arts-Integrated-Curriculum (AIC) and its possible impact on the selfconcepts of adolescent girls and their perceptions of beauty. The collected data represent the experience of the whole group of 10 research participants. I sought to better understand how young ladies who completed an AIC at the Elementary school level, their parents, and their teachers describe their AIC experience. With the help of these three distinctive perspectives, I approached the major research question, "What if any, phenomena may be associated with the self-concept and perceptions of beauty of young ladies who completed an AIC."

As explained in chapter three, the data analysis method conducted in this chapter consists of horizonalization, delimiting, thematizing, validation, and various descriptions of the data of the experience of the AIC and its (possible) effect on the self-concept of adolescent girls and their perception of beauty.

The following section will display the seven plus one steps (see Figure 3 and Figures 1619) of Moustakas' "Modification of the Van Kaam Method of Analysis of Phenomenological Data" (p. 120) step-by-step (Figures 16-19):

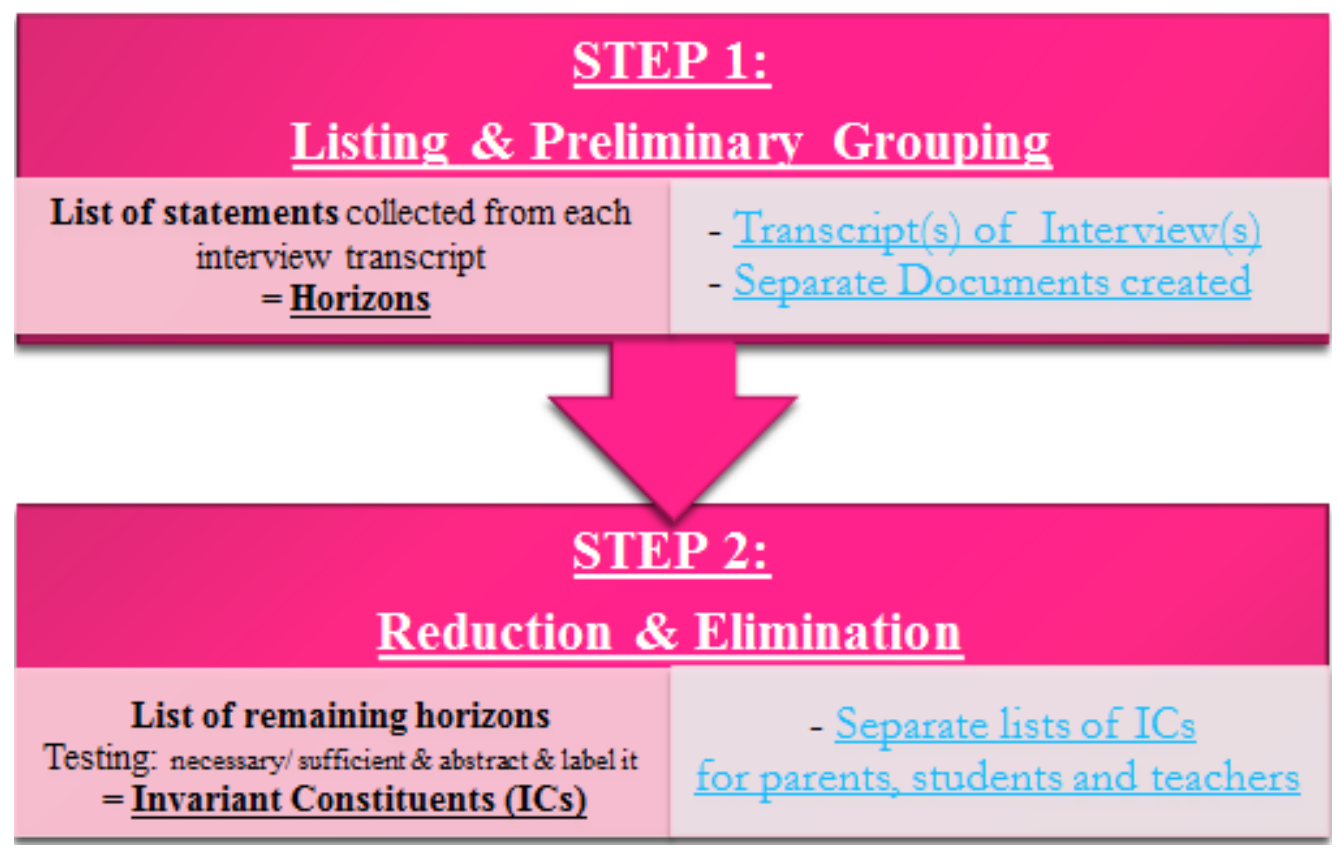

Figure 16. Data analysis process - step 1 and step 2. 


\section{STEP 3: \\ Clustering \& Thematizing the ICs}

ICs were clustered \& situated (into)

$=\underline{\text { Thematic } \text { Label }=\text { Core Themes }}$

- Separate IC/ Themes Charts:

a) Students; b) Parents; c) Teachers

- Summarr Cluster of Core Themes

\section{STEP 4:}

\section{Final Identification of ICs and Themes}

\section{ICs and Themes}

Checked: Explicit? Compatible?

$=\underline{\text { Validity check }}$ (Groenemald, 2004)

\section{- Validation Chart}

Figure 17. Data analysis process - step 3 and step 4.

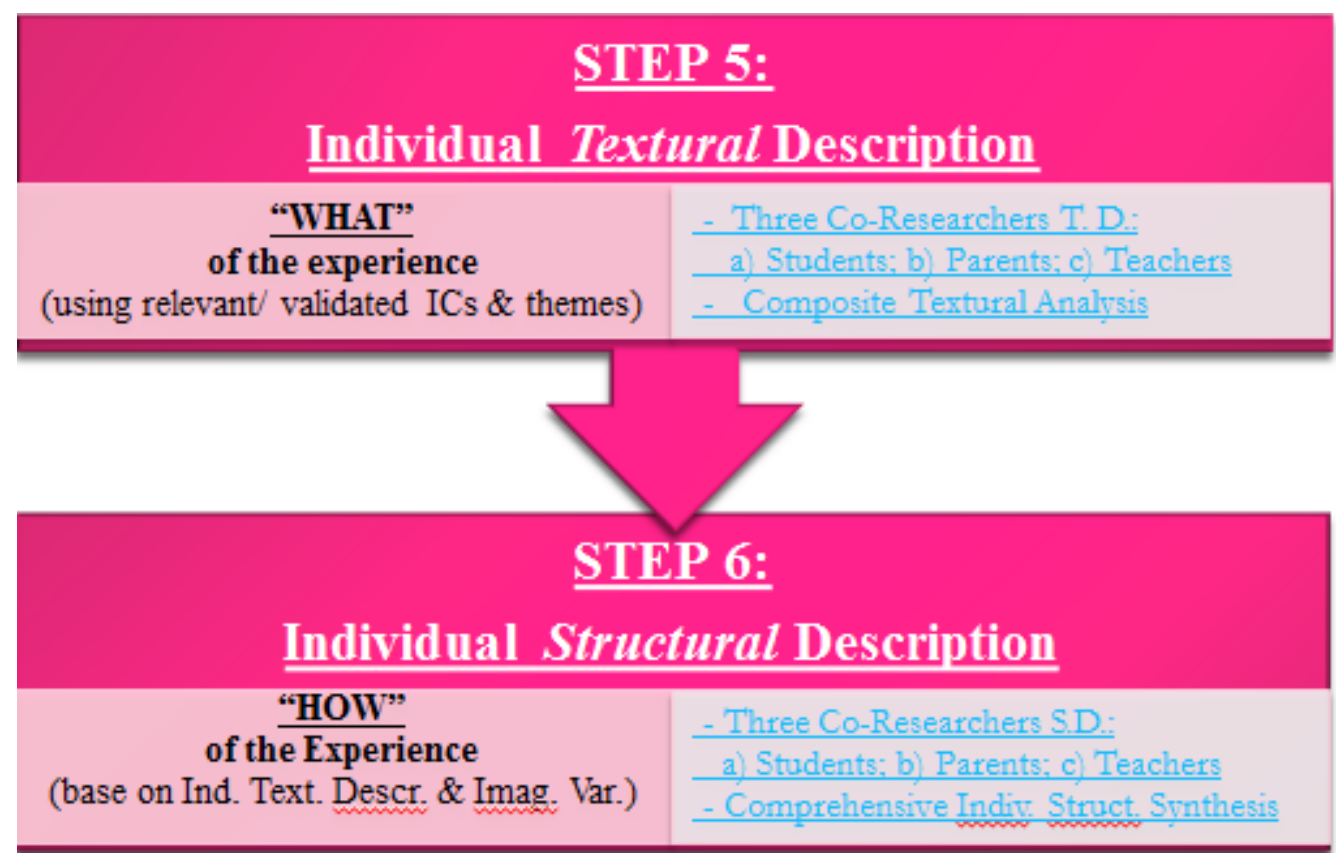

Figure 18. Data analysis process - step 5 and step 6. 


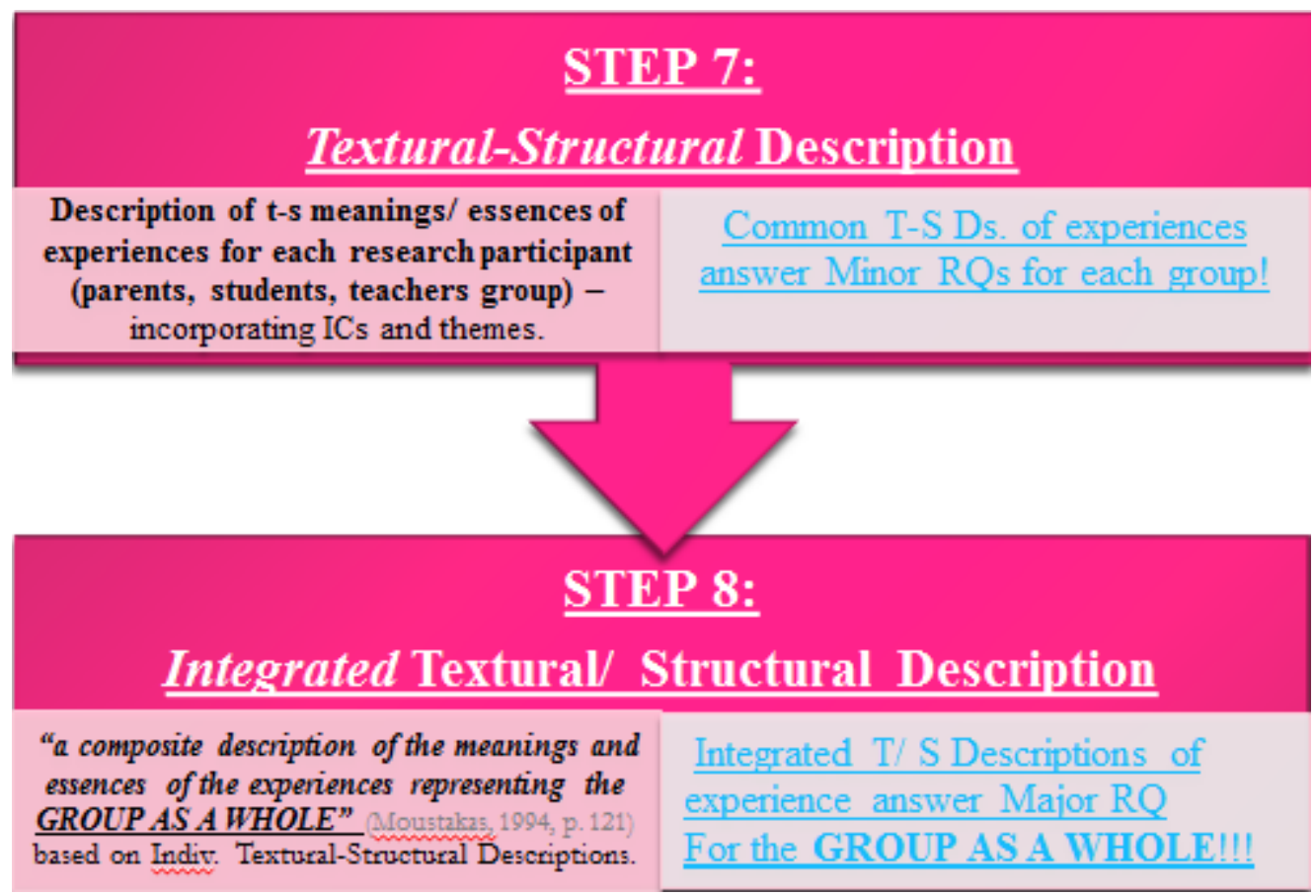

Figure 19. Data analysis process - step 7 and step 8.

\section{Step 1. Listing and Preliminary Grouping \{Horizonalization or Recognition\}}

For step one (see Figure 16), I followed Moustakas' (1994) suggestions and used Copen's (1992) work on insomnia as a model for the data analysis process. A list of statements containing an important moment of the experience and fulfilling the requirement of reduction and elimination was collected from each interview transcript. During this step, I listed all the expressions relevant to the experience of the AIC and its (possible) effect on the self-concept of adolescent girls and their perception of beauty.

Following the recommendation of Groenewald (2004), Holloway (1997), and Hycner (1999), I listened repeatedly to the audio recordings and studied the transcripts of each interview "to become familiar with the words of the interviewee/ information in order to develop a holistic sense, the 'gestalt'" (Holloway, 1997, Hycner, 1999, as cited in Groenewald, 2004, p. 18). 
Below, I have displayed a transcript of one of the four interviews conducted. This interview was chosen because it is representative of all the interviews undertaken for this study. It is an excellent embodiment of the style of investigation used. The first step of horizonalization, also called recognition (Copen, 1992), was carried out. In a separate document, which is not published in this dissertation for lack of space, every expression relevant to the experience was listed for all of the four interviews since "every statement has equal value" (Moustakas, 1994, p. 125).

\section{Interview transcript (T-P-BUSY \& T-S-BAND-29.04.2011).}

(Miriam Roth Douglas $=$ Researcher, Busy $=$ Co-researcher $($ mother $)$, Band $=$ Co-researcher (daughter)).

MIRIAM ROTH DOUGLAS: My name is Miriam Douglas and I am a doctorate student at West Virginia University and I am conducting my research on the arts-integrated curriculum (AIC) and its effect on the self-concept of girls and their perception of beauty.

Would you introduce yourself real quick?

BAND: I am BAND. I am 13 years old. I go to Sunrise Middle School. I am currently in the Sunrise Middle School band. I play the clarinet. I have taken art classes at Sunrise and I do like art because I have got like-I have gotten recognitions from it. And I enjoy band a lot, too.

MIRIAM ROTH DOUGLAS: Okay, thank you very much.

BAND: I am BUSY. I am BAND's mother and I own my own business and am from Evening City originally, have four children. MIRIAM ROTH DOUGLAS: Okay, thank you very much. I will start with the first research question. [test] 
Okay, the first, I'll ask you first and then you.

The first question is how did you personally experience the

arts-based-learning classroom?

BAND: I really—I really like the arts because it, like,

affected me up to now because now I am doing really good in my art classes and I really enjoy it. And before when I started band, I wasn't sure about it, but then

once I have gotten into it now, I feel like I am really good at it.

MIRIAM ROTH DOUGLAS: Thank you. So I assume that you like music the best

of the arts or is it the same with painting and drawing and dancing, or mainly music?

BAND: I think I like the music better just because, I don't

know, my mom has been involved with music and so has my grandfather and my sister and my brother. So-

MIRIAM ROTH DOUGLAS: Okay. That makes sense. Thank you. And if you think about it, how did you personally experience your daughter for the arts-based learning?

BUSY: So, I would hear about it. They would come home from school and talk about some things they would do in the classroom, not a whole lot, but, you know, they would come home and say, "Oh, we learned this song today. Or, we, in math we sang this song," or there was one time where they were doing some African music with painting, bare foot painting to African music and that was actually for an art class. But I remember where there were times where they would incorporate some different types of arts into even math or reading and things like that, and even today, yeah, I still hear about it with the little one.

MIRIAM ROTH DOUGLAS: They kind of brought it home and showed it to you, 
like the results, they were proud?

BUSY: Yes, if it was art, something they

painted or drew, you know, they would show me that, or they

learned a song or did something, they would either sing it for

me or tell me about it.

MIRIAM ROTH DOUGLAS: Okay. And how did you think the arts-based-learning classroom that your daughter went through is different from the school experiences of others who did not participate in it?

BUSY: Well, okay, I am a little biased with arts-based learning just because of influences you know within my own family because music was big part of our family. So I feel like they gain more when they are exposed to the arts in all different subjects. I think it just broadens their outlook, open to newer things and maybe show more of an interest in it.

MIRIAM ROTH DOUGLAS: What especially comes to your mind if you compare it, for example, with families who have kids, while the kids did not visit an arts-integrated curriculum, do you have a comparison?

BUSY: Yes, there were some other-let's see, do I know of other people? Yes, I know of some other families that have not gone to this school, and I think that, you know, like we will go out and seek-you know, we will go to concerts and we will-I just think we have more of an interest in the arts.

MIRIAM ROTH DOUGLAS: Involvement, also, like with the community, not just with the school?

BUSY: Oh, yes, definitely, Windy Valley University, especially, just because there are so many opportunities there.

MIRIAM ROTH DOUGLAS: Okay. And what about you and, like, how do you think 
the arts-based-learning classroom that you went through is different?

BAND: Well, I definitely think that it does make learning at school like more fun and stuff other than math and social studies. It makes it more exciting, but, yeah, children like from other schools who don't get that kind of art or learning from music and stuff, I don't think it would be as much entertaining to them.

MIRIAM ROTH DOUGLAS: And what do you mean with "exciting and entertaining," like do you have a special example, what you mean by that?

BAND: Well, I remember I would always, after math-I mean, it is not that I didn't like math, but after math I would probably go to art or music or something and I would be excited to go to it, because after learning math and stuff, it would get kind of tough and hard.

BUSY: Do you use anything in math, do you remember, to help with some of that? Did they use any writing or-

BAND: They did. There was songs that we used and drawings and stuff.

BUSY: Drawing in math?

BAND: Drawings. Yes, you have to figure out a problem, and once you got the problem, you got to color. It involved different stuff.

MIRIAM ROTH DOUGLAS: So when they involved that, that was more fun for you, you enjoyed math class better?

BAND: Uh-huh.

MIRIAM ROTH DOUGLAS: But in general, artsy classes like music or something?

BAND: Yes. 
MIRIAM ROTH DOUGLAS: Thank you. Do you feel like you notice a difference in learning in and through the arts?

BAND: Uh, it definitely taught me just, just how to be more like bright and more, I'm not sure.

MIRIAM ROTH DOUGLAS: Think in different ways, kind of?

BAND: Yeah, yeah, like think outside the box.

MIRIAM ROTH DOUGLAS: Uh-huh. And how was that for you? Did you notice a difference in your daughter's learning experiences?

BUSY: (Sights). You know, it is hard to pinpoint anything exactly, not knowing how it would have been if she hadn't.

MIRIAM ROTH DOUGLAS: Yeah.

BUSY: I just know, it is a big part of our lives. The arts are a big part of our lives and that could have come from, you know, being exposed to it.

MIRIAM ROTH DOUGLAS: Most likely. Yes.

Okay, do you feel like your daughter learned through the arts-based-learning experience how to look differently at things?

BUSY: Definitely, I think so. Just with the different, you know, she was talking about the coloring. I think even in math they would add numbers and then if the answer was such and such, she is doing it now, you know, color that in and when you colored in the different colors, it would form a picture and so it is like, oh, wow, you can do this with numbers. So, yes, I think it exposed them to different things that made them look at things in a different way.

MIRIAM ROTH DOUGLAS: Differently, and also in general like with nature or so? 
BUSY: Yes, I think so. She also had some—did you have a drawing class or something, at some point in time too?

BAND: I am pretty sure I did because I know-

BUSY: Like looking at perspectives of things? Yeah, I think so.

MIRIAM ROTH DOUGLAS: Yes, and how did you experience it? Do you feel like you look at things differently or do you think you probably would look-

BAND: Yes, because I know in math, I wasn't sure we were learning it, but once they explained that we would need it for when we get a job when we get older, and in social studies they taught us about, like, our country and how we would need that in the future. And the arts just like made us more, I don't know, I don't know how to explain it. It just made us be more like interactive and creative.

BUSY: Broaden these perspectives.

MIRIAM ROTH DOUGLAS: Do you feel like you learned through the arts-based-learning experiences to look different at people? Like, for instance, the way they dress, the way they look like in general?

BAND: I know I did because when my cousin came to teach us in art class, we were talking about how the African people danced and she did mention how they dressed. It is not similar to what we do dress here in America. And in general, I mean, it just - it did teach us how to look differently at people just because we know nobody is the same and we are all different.

MIRIAM ROTH DOUGLAS: Focus more on details and other things, or differences?

BAND: Uh-huh.

MIRIAM ROTH DOUGLAS: How do you feel about that? Do you feel she learned 
through the arts-based-learning experiences to look different at people?

BUSY: That is a tough one. To look differently at people, I

don't know that I can say that it was

specifically that, that it would make her look differently at

people. I think-I guess it could have an affect on that. I

also think it depends a lot on how you are raised, as well, your family environment, you know, how you are brought up. I mean, I guess it could have an impact on that. I can't tell you specifically.

MIRIAM ROTH DOUGLAS: Of the things, but in general you think?

BUSY: Yes.

MIRIAM ROTH DOUGLAS: Okay. Do you feel like the arts-based-learning

experience has had an influence on how your daughter sees herself, like the way she looks or dresses?

BUSY: Yes, I think so. I would say- (Pause) I don't know whether it is like the way you dress-I mean, she is very conscientious about how she looks and colors and matching and things like that. Maybe, you know, that could have been a result of the arts-based-learning. I guess some of the dancing and, you know, how to hold yourself, I know they did some dancing classes in grade school and how to sort of hold yourself and present yourself and things like that. So, yes, I think so.

MIRIAM ROTH DOUGLAS: And what about you, do you feel like those experiences had an influence on how you see yourself?

BAND: Yes, just because it taught me not to just be like- not to just be like everybody else. Like, it is okay to be different, it is okay to be more colorful. It did have an impact on how I dress because I think that back then, I wasn't-I 
don't know, I just wasn't so good with clothes and stuff, but, I mean, because I grew up, it had an influence on the way that I look and dress.

MIRIAM ROTH DOUGLAS: Okay, thank you. If you think about it, what would you consider as an example for what you think is beautiful? You can mention whatever comes to your mind, an object, a thing, something in nature, a human being, another human being, yourself, what do you find beautiful on yourself or others, just in general, whatever comes to your mind.

BAND: Thinking about objects or things, I don't know, a rose popped into my mind just because, I mean, they are just like very pretty and there are others like them. And then-

BUSY: Is that the first thing that popped in?

BAND: Yes. That is the very first thing.

BUSY: A flower?

BAND: A flower, they are very simple, but they are beautiful.

Humans, thinking about them, I don't know. I don't know how to describe someone like beautiful, but, I mean, I guess people on television are thought of more as, like, role models and people to look up to.

MIRIAM ROTH DOUGLAS: If you think of a singer, an actor or actress, who comes to your mind who you think is beautiful?

BAND: I don't know, because some of them, they were an influence, but then they went in the wrong direction.

(Laughter)

MIRIAM ROTH DOUGLAS: Okay. What do you think about that question, what your daughter thinks is beautiful? 
BUSY: Okay, what she thinks is beautiful. I think it is more that she thinks objects are beautiful. She mentioned a rose. That is interesting because, okay, so I have a buisness, which is a creative place, she sometimes will come in and do some things. She made a flower out of clay and had petals and everything, and was all, it was beautifully done and she painted it and everything. And so I think for her it is more nature and things like that, that she thinks are beautiful.

MIRIAM ROTH DOUGLAS: If you think about role models or people that she might think are beautiful, is there something that might pop into your mind?

BUSY: Not anyone in particular, but I think she is more-oh, I mean, gosh, I think it is more of a natural beauty and the nicer personality that she would think of as beautiful.

MIRIAM ROTH DOUGLAS: So in a way, you mean it is more the internal beauty than external?

BUSY: Yes, I think so. Well, I think there still has to be a physical beauty about it, too, but not the real made up kind of person, all of the real striking, stark makeup, but it is more of a natural.

MIRIAM ROTH DOUGLAS: Do you agree with that?

BAND: Yes.

MIRIAM ROTH DOUGLAS: Okay. Then you as a mom, how would you define self-concept, yourself?

BUSY: Myself? How do you define self-concept?

MIRIAM ROTH DOUGLAS: Yes.

BUSY: Well, self-concept would be how you think of yourself, right? I mean, do you want me to- 
MIRIAM ROTH DOUGLAS: There is no wrong or right answer, just whatever you think, whatever it is for you.

BUSY: Self-concept, I will say like how confident you are of yourself and how are you as a person, whether it is how you relate as a mom, you know, am I a good mother, am I a good business owner or, you know, what kind of image do I portray to other people.

MIRIAM ROTH DOUGLAS: Okay. And what is it for you, if you hear self-concept, how would you describe it?

BAND: I don't know, I don't regularly hear that, but I guess it is just the way that you do think of yourself. Like I do think sometimes if I'm being like a good friend to my friend, if they are like stuck in a situation or if I help them out, or with my siblings, too, I mean, if they have a problem or they need me to help them, I mean, I will. So-

MIRIAM ROTH DOUGLAS: So that is also the way you think about yourself. Like if I mention self-concept, do you think you have a good—or what kind of self-concept do you have? How would you-

BAND: I just think I am very-

MIRIAM ROTH DOUGLAS: How do you think about yourself? It might be easier.

BAND: I think I'm a role model to my sisters because I know my little sister likes to like hang out with me, like she is my friend. And then to my friends, I don't know, I am a nice person to them and they come to me when they have problems, too. And I don't cause problems a lot. And if I do, it is mostly with my siblings.

MIRIAM ROTH DOUGLAS: Okay. And how would you as a mother describe her 
self-concept?

BUSY: She is a very caring person, always willing to help out, very responsible. She does laundry. She cooks. She takes care of... She is like a second mother to the little one. She is - she is somewhat shy, like she is not a real outgoing, flamboyant type of person. She is a good friend, to her friends, did I say good listener? She is a good listener when it comes to them. She will listen, but not pass judgment, you know.

MIRIAM ROTH DOUGLAS: And if you think about yourself, are you happy with yourself or would you like to be different or somehow different?

BAND: I don't know, when I see some people at school, I mean, they have all of the new clothes and stuff and I just think, "Well, I am not going to get that," so I mean, why change? But, I mean, sometimes I mean if I make a mistake, I mean, I will get mad at myself, but I mean everybody makes mistakes, but, otherwise, I am happy with myself.

MIRIAM ROTH DOUGLAS: And you are also happy with yourself in reference to, for example, how tall you are, your hair color, eye color, things like that, like the perception of yourself?

BAND: Yeah, with my friends, I know it is —we just joke about the height that we are because I have three other friends at school and, I mean, one of them is way taller than us...

BUSY: Who is taller?

BAND: (Clarifying... difficult to hear).... and then we will we joke about the other girl, Jennifer, because she was way taller than us, but I guess we kind of grew up to her. And then my hair color, I think I like my hair, but other times I get mad. 
(Laughter- "Like everybody")

But, yeah, I mean everybody in our family has got the dark hair, so.

MIRIAM ROTH DOUGLAS: Do you feel the same way, like when you think about the way she might think about herself, do you feel like she-

BUSY: I think for the most part, she is happy with herself. There are times she is trying on clothes or trying to figure out what to wear, oh, this doesn't look right, oh man, and every so often we will start to talk about healthy eating and exercise and things like that, and try to focus more on that in the family.

MIRIAM ROTH DOUGLAS: If you start to talk about that, why do you start talking about that? Like is it something in-Yeah, why?

BUSY: Usually, (laughing) like, for me I am trying clothes on that don't fit and I really need to make more time to get outside and get some exercise or if it is you, well, like, we were bathing suit shopping. "You know, I don't think I want to wear a two-piece bathing suit because I know my belly will show," but, you know, it is okay. And I think we are comfortable with where we are at. It would be nice to be a little thinner, but, she always looks nice in whatever she wears and she is clean and well-kept and she takes care to do her makeup and everything.

MIRIAM ROTH DOUGLAS: Where do you think it comes from, for example, that you think, well, it would be good to be thinner or maybe-

BAND: Definitely, healthwise, high blood pressure, high

cholesterol runs in the family, and, in fact, I just had a doctor's appointment two days ago and they had one of those body mass index charts up there and I looked mine up, I was like, okay. 
(Laughter)

But it is more of a healthy type, nothing from

MIRIAM ROTH DOUGLAS: But it is more of a healthy type, nothing from the media or so-

BUSY: From me, no. You go ahead, you answer that one.

BAND: When I see people on TV, it is normal that they take-like they choose thinner people and now on TV, they are like showing like- there is a show on Disney Channel, it is like a little thing. It is talking about healthy eating and stuff, all these people, they are like thin, but I guess America has like a lot of large percentage of large people.

BUSY: And where did you hear that?

BAND: That is what - I heard it.

BUSY: Like news or hear it on TV?

BAND: And school, too, yeah.

MIRIAM ROTH DOUGLAS: Okay. What memories and situations come to your mind when you think of your experiences with the arts-based-learning curriculum in elementary school? I know we already talked a little bit about it, but is there something specific that comes up?

BAND: I definitely think of my friends when I think of that because they were there with me, but I do remember the art and stuff. I remember when my cousin came to teach us and I remember how she showed us how to paint with our feet. We had paint on our feet and she taught us how to do that. And then I do remember the teachers, how they taught us, some of their methods, yeah. 
MIRIAM ROTH DOUGLAS: And what about you, like when you think about it,

Other special experiences or events or situations that come to your mind?

BUSY: Okay, so my memory is bad (laughter), I just know—well, you know, when they first did, like they would have periodic — they would have like an introductory meeting at the beginning of the year. And the teachers would talk about their concepts and how they go about teaching and how they would use arts and music and I think maybe some like physical movements to integrate that into their curriculum. I just thought it was a great idea. A specific instance or situation, I don't remember too much, but I know that every so often they would come home and talk about, you know, what they did and, you know. I can sort of remember her saying, "Well, that was pretty neat. That is neat how they incorporated that," but specifically, I can't.

MIRIAM ROTH DOUGLAS: Like a show or an exhibit or something like that they did, or remember a painting or-

BUSY: Well-

MIRIAM ROTH DOUGLAS: A music performance?

BUSY: There were quite a few, but that wasn't the curriculum, actually. The parents volunteered. We put on a musical, 101 Dalmatians.

MIRIAM ROTH DOUGLAS: Nice.

BUSY: It was an after-school program that we did with the kids and I was involved in that.

MIRIAM ROTH DOUGLAS: How did it come together? It kind of developed out of the curriculum?

BUSY: No, it was the parents. (whispering) 
MIRIAM ROTH DOUGLAS: Okay, well, tell me more about that.

BUSY: It was an individual, CARING is her name, and she

is - I think maybe she had experienced that as a child, as well. And so she had suggested we put on a production and have people - you know, the kids actually tried out for parts because there are how many puppies? You know, we purchased the play from Disney, the actual script and music and everything and kids signed up. They tried out for the main parts, but everybody that wanted to be a part of it was in it. They would sing, they would practice songs, they would do choreography. There was - the school did support it. They helped arrange for after-school practices and things like that, we used the building and everything. I thought it was a great experience.

MIRIAM ROTH DOUGLAS: But the initiative kind of came from the parents?

BUSY: Yes, it was the parents.

MIRIAM ROTH DOUGLAS: How do you remember that?

BAND: I just remember, I remember trying out for a part, but I didn't get it (BUSY laughing), the maid of the house. But I remember being-I don't remember which dog I was, but I remember practicing constantly. Like I would go home and practice my part because I thought it was just like the best thing. I thought it was so fun. And I just remember the costumes and the backgrounds, the set stuff.

MIRIAM ROTH DOUGLAS: And how do you remember to handle that you didn't get the role that you wanted?

BAND: I know after I thought about it, I thought there is probably going to be more people who will try out for this part. I was confident at first and I was like well, there is going to be other people and I didn't think I did so well. 
MIRIAM ROTH DOUGLAS: Why did you think you didn't do very well?

BAND: I'm not sure, I guess I was just nervous. But, I mean, everybody gets nervous.

MIRIAM ROTH DOUGLAS: Okay. Do you wish, maybe BAND you first, other people like your friends, family members, siblings, should have the chance to go through such a curriculum and why or why not?

BAND: Yes, I do. I definitely think they should because once they do go through it, I mean, they can go home and tell their parents, like friends can, and younger siblings. Once they go through that class, they will be coming home to tell my parents and I will overhear them, and I will say, "Oh, yeah, I remember that," and just like pass down. And I just think like it is a better experience if you do because with the art, it is just more like a-I don't know how to explain that.

MIRIAM ROTH DOUGLAS: You can just describe it in maybe more words, if it is difficult.

BAND: Well, with friends I remember mentioning that, what we did in art class. During school we would discuss during lunch time what we did. So then we would be talking about like what we were going to do the next day for that class.

MIRIAM ROTH DOUGLAS: Like you had something to talk about, something to plan on and look forward to that you were excited about?

BAND: It gave us stuff to talk about like in our free time and during lunch, so-

MIRIAM ROTH DOUGLAS: Okay. What do you think about that? Do you wish other people could experience it?

BUSY: Well, other people, definitely. When we were moving-I 
moved away for about 16 years and then came back and when we were

looking where to live, I knew that Sunrise was a school that integrated the arts. And so I wanted to live

in this area for that reason. So I would definitely recommend-I always recommend that school, knowing, you know, what they use in the curriculum, to other people. I just think it just exposes the kids to so much more, that they might-you know if they are not brought up in a family where arts is emphasized, then they might not ever be exposed to something like that.

I also think it helps them learn, when they come home and they are chanting or singing a song, they come home and sing the days of the week or months of the year and things like that, I mean it helps them definitely remember. They still, you know, they can still sing it to this day.

BAND: Mmmh (Confirming).

BUSY: I mean, I even use it, I remember like when we are practicing spelling words, you know, hard words right now, we will sing-song it, you know, to try to remember. I mean, I would do it with you when you were in school. We would sort of sing-song it. Try it this way. It might be easier to remember.

MIRIAM ROTH DOUGLAS: It works better.

BUSY: It does (laughter).

MIRIAM ROTH DOUGLAS: So in general, I have the bunch of questions so far. Is there something else that comes to your mind that we didn't really touch on, but you think is still important or that comes to your mind after we went through all of these questions, something in addition that might not necessarily have to do with the arts or anything, just something you want to add?

BUSY: I just think it has been a positive experience and I think 
knowing - I think the school, this particular school that we went to because they used that, I think it has a good reputation for that. And that, you know, it sort of, you know, they do a little bit more than - it is a different environment maybe than some other schools that don't

and that is a good thing, you know. I mean, I was very happy with it and enjoyed, you know, hearing, you know, what was going on and what I saw. I would volunteer at the school, so I would see some of the things that were going on, as well.

MIRIAM ROTH DOUGLAS: So you would do it all over again?

BUSY: Oh, absolutely, no regrets, yes. Definitely.

MIRIAM ROTH DOUGLAS: What about you BAND, what comes to your mind, something we didn't mention or that you would like to add?

BAND: I just would, arts and stuff, I just-I don't know, I remember coming home and just being excited to tell my mom what we did at school. It was just-I mean it would give us something to talk about, like I know now like when my parent asked me what did I do at school, it is just, like, it is just another school day. Back then, I mean it was like the beginning of school, you were starting school. So, of course, you were excited to go home and tell your parents about all you did in school.

MIRIAM ROTH DOUGLAS: Somehow I hear that you kind of are saying that the school where you are right now, it — arts might be a part of it, but not such a big focus and that is why you don't really have to talk about so much as you did before?

BAND: I think it is that, because I think more of the schools are focused on like the after-school activities, like all of the sports because I know when I come home from track, I just — I am just tired and stuff, but I do still tell my parents what I do after school, if 
I have homework or not. But, I mean, back then it was just like all-I-Ihat is all I was doing.

MIRIAM ROTH DOUGLAS: Excited about it?

BAND: Uh-huh.

MIRIAM ROTH DOUGLAS: Now, you mentioned that after school and at home you talked about the arts and you told your parents about the arts and in school you had the possibility-you had something to talk about with your friends. How is that now? What are you talking about now?

BAND: I am-I still at lunch with my friends just to talk about something, we do talk about our classes because not all of us, like back in the elementary school and in primary school, we all had our classes together. They weren't split up or anything. There was just two classes in one grade and they would just rotate with each other to the next classes, but in one room. And that is what I remember from back then, but now like all of the kids are split up. There is like multiple classes. You don't — it is like — it is hard to sign up with a class with your friend because you are not supposed-you can't really do that because the teachers have the class assignment for you. So then when we go to talk about it with each other at lunch, we tell each other what they are going to do in that class because maybe one of us have already had that class. And with arts-based-learning class right now, I have been taking health, but my other friend has been taking like creative writing and I think my other friend has general music right now, so we can't really talk about that. But it just gives us a lot to talk about during lunch, though.

BUSY: I hear more from her about her art class that she had And band.

MIRIAM ROTH DOUGLAS: Than the others? 
BUSY: Yes. She comes home and shows me what she has done. Really nice.

MIRIAM ROTH DOUGLAS: Like what, for example?

BUSY: Go get some of your stuff.

MIRIAM ROTH DOUGLAS: Yes.

BAND: Okay.

(Laughter)

BUSY: She has told me that the teacher has a couple of things from her class, so I think she is proud of that.

MIRIAM ROTH DOUGLAS: She can be proud, she put a lot of work and time and effort.

BUSY: I think she is all good with that. In general, the art just, you know-I danced as child. I did 12 years of dancing. We always took art classes. We were always involved in music.

BAND: (Showing her artwork). Okay, this was a cross-I didn't make it at school but I made it at the my mom's business. And it was a mosaic cross and I had it hanging up on my wall. This was up on my wall, too. We had to create like a fruit, like we could do a lemon, orange or pear or apple, but I chose an orange. And we had to take like clippings of magazine and match the color. Like it was o.k. if it was different. The oranges are not all of the same in my picture, by it just—like blended it a little bit.

MIRIAM ROTH DOUGLAS: Why did you choose an orange?

BAND: I don't really know why. I think because if you did a smaller, you had to do two. If it was bigger, you could do one. You had to do less work. I still had to do a shadowing of it.

MIRIAM ROTH DOUGLAS: How did you choose the colors, the other colors besides the orange? 
BAND: Besides the orange? I was really indecisive about that because I wasn't sure what I should make the wall color in the background or the table color, but it kind of turned out to be a little bit of a mixture. The wall looks like textury and the table looks more like a blended blue. So that is that — and then for this we had to pick a saying like a

quote. I never have any quotes in mind, so I just looked up on the computer, it says, "Everything in life is luck." I was like indecisive at that time, so I just chose that. I do not think it is that true, some stuff in life is luck, but.... And then we had to choose our colors and then layer it. And you could choose like multiple colors that you wanted. I am not sure why I chose these. I guess they kind of went together. I didn't want to be like matchy, I wanted to be like, just colorful.

\section{MIRIAM ROTH DOUGLAS: Nice.}

BAND: And then I made this in my free time. It is kind of like the quilt that I had, but I made it out of - I traced this one, but my quilt that I made before, I did that free handed. I did this in my free time, so it wasn't like a project.

BUSY: What about your box?

BAND: My box, that is in my room, too. Hold on.

(Pause)

MIRIAM ROTH DOUGLAS: Very nice. Do you feel like when she does these things, what do you see in her or what do you realize when she does these things?

BUSY: She is talented and I like that she has an interest in it and she enjoys it.

BAND: This is my box, we had to make it out of cardboard like 
from scratch and I was so confused when we started making it. Because my teacher is just like " O.k., you are going to get four pieces of cardboard, hot glue them together and then make a lid for it and design it". I am just like, "okay", she threw it out there. But my friend was actually going to use this pattern, like this was-it was from like when my teacher put out the paper that we could use, he said that this was like patterns from around the world. There were like a lot of different colors in there and-

MIRIAM ROTH DOUGLAS: Why did you choose these?

BAND: Well, I chose this because when I was looking for a pattern to use, I couldn't find anything because either there wasn't enough paper or I just didn't like it. My friend was going to use this pattern, but then she decided to free paint hers, so I then said, "Okay, that is pretty," so I used like this pink and orange stuff. Then for my lid, I made a little like grip handle and I painted the inside, the solid black and then on all of the edges we had to add cardboard just to make it look good.

MIRIAM ROTH DOUGLAS: And you said that the pattern and the colors are pretty. What is it that makes it pretty for you?

BAND: I think the pink.

MIRIAM ROTH DOUGLAS: The color?

BAND: Yes, mostly the color and the pattern. I mean, I wouldn't have been able to do that free handed if I tried, I don't know, but... And I just added on some black because it just didn't stand out that much to me. And 
then I put the border on the top of the box.

MIRIAM ROTH DOUGLAS: So, if you are involved in doing something like that, how do you feel?

BAND: Well, actually in that art class, I feel like we don't get enough time just because, I mean, I am just so into it. And I feel like if the class was longer, I would like that, but I mean I could do it all day, but it just—it just—it is not like rushing or anything, it is a matter of time and, like, process and it is just fun because you know what you are going to do next, you hot glue the cardboard together and pick out your pattern, then add your own-add your on taste on to it and then I painted the inside of it.

MIRIAM ROTH DOUGLAS: So it is you enjoy it because you kind of know what the next step is.

BAND: Uh-huh.

MIRIAM ROTH DOUGLAS: And you get satisfaction out of it?

BAND: Uh—huh, and when I show it to my teacher, I am hoping he will give a positive comment. I know he will because in the past projects I have done, he has done that a lot. He said he was pleased with this one.

MIRIAM ROTH DOUGLAS: And that makes you feel good?

BAND: Yes.

MIRIAM ROTH DOUGLAS: What happens if it is not as good as expected, like when the comments are not as good as expected?

BAND: I don't know, no one has ever told me, "Oh, my gosh, I don't like that." If they did say that, I would say maybe it is not their kind of taste, maybe they would prefer something else. I mean, this is what I like, but my friends always ask me, "Oh, I will probably 
do this." I am like, "Oh, that is a good idea." I will tell them like, "That looks really good," so just like back and forth feedback.

MIRIAM ROTH DOUGLAS: So when you get a good comment, a positive feedback, how does that make you feel?

BAND: It makes me feel good, makes me feel like I can do more, like I have accomplished what I needed to do.

MIRIAM ROTH DOUGLAS: Okay, well, thank you very much.

BUSY: You are welcome.

MIRIAM ROTH DOUGLAS: I really appreciate you taking your time.

BAND: Sure.

(The recording concluded) 


\section{Step 2. Reduction and Elimination [Delimiting of Meaning, Meaning Units, or Horizons result in List of Invariant Constituents (ICs) \}}

During the second step (see Figure 16), a list of units containing relevant meanings was extracted from each of the interviews (Groenewald, 2004). This list represented the core qualities of the AIC and its effect on the self-concept of adolescent girls and their perception of beauty (Copen, 1992).

Following Moustakas' (1994) approach, each of the selected expressions was verified for two requirements to find invariant constituents (ICs). First, the expressions were tested for a moment of the experience that is a "necessary and sufficient constituent for understanding it" (Moustakas, 1994, p. 121). Second, I determined whether it was "possible to abstract and label" (p. 121) the expression. If so, the expression was called "a horizon of the experience" (p. 121). If not, the expressions was eliminated. "Overlapping, repetitive, and vague expressions are also eliminated or presented in more exact descriptive terms" (p. 121). Remaining horizons are the "invariant constituents of the experience" (p. 121), which I called ICs. Lists of the remaining ICs from the transcripts of the interviews were created. Summaries of the ICs were generated separately for students, parents, and teachers. This way, I avoided lists that would contain parents' and students' opinions mixed together.

Below, the list of the students' ICs (BAND, PIANO, DRAWING, FLUTE) is displayed. It appeared to be a good representative for all co-researchers' IC lists. The remaining lists of parents' and teachers' ICs will not be displayed in this chapter, but they can be reviewed in the Appendix (see Appendices $\mathrm{H} 1$ and $\mathrm{H} 2$ ). 


\section{List of ICs for students.}

BAND.

"I am BAND. I am 13 years old. I am currently in the Sunrise Middle School band. I play the clarinet."

"I have taken art classes at Sunrise and I do like art because I have got like-I have gotten recognitions from it."

"And I enjoy band a lot, too."

"I really-I really like the arts because it, like, affected me up to now because now I am doing really good in my art classes and I really enjoy it. And before when I started band, I wasn't sure about it, but then once I have gotten into it now, I feel like I am really good at it."

"I think I like the music better just because, I don't know, my mom has been involved with music and so has my grandfather and my sister and my brother."

"Well, I definitely think that it does make learning at school like more fun and stuff other than math and social studies. It makes it more exciting, but, yeah, children like from other school who don't get that kind of art or learning from music and stuff, I don't think it would be as much entertaining to them."

"(...) after math I would probably go to art or music or something and I would be excited to go to it, because after learning math and stuff, it would get kind of tough and hard."

"There was songs that we used and drawings and stuff."

"Drawings. Yes, you have to figure out a problem, and once you got the problem, you got to color."

“(...) it definitely taught me just, just how to be more like bright (...)."

"Yeah, yeah, like think outside the box."

"I am pretty sure I did [learning in and through the arts] because (...) the arts (...) made us be more like interactive and creative."

"I know I did [learned through the arts-based-learning experiences to look differently at people] (...) we were talking about how the African people danced and she did mention how they dressed. It is not similar to what we do dress here in America. (...) it did teach us how to look differently at people just because we know nobody is the same and we are all different."

"Yes, (...) it taught me (...) not to just be like everybody else. Like, it is okay to be different, it is okay to be more colorful. It did have an impact on how I dress because I think that back then 
(...), I just wasn't so good with clothes and stuff, but (...) it had an influence on the way that I look and dress."

"Thinking about objects or things (...) a rose popped into my mind just because, I mean, they are just like very pretty and there are others like them."

"A flower, they are very simple, but they are beautiful."

"Humans, thinking about them (...) I don't know how to describe someone like beautiful, but, I mean, I guess people on television are thought of more as, like, role models and people to look up to."

"[Definition of self-concept] (...) I guess it is just the way that you do think of yourself. Like I do think sometimes if I'm being like a good friend to my friend, if they are like stuck in a situation or if I help them out, or with my siblings (...) if they have a problem or they need me to help them, I mean, I will."

"I think I'm a role model to my sisters because I know my little sister likes to like hang out with me, like she is my friend. And then to my friends, I don't know, I am a nice person to them and they come to me when they have problems, too. And I don't cause problems a lot."

"I don't know, when I see some people at school, I mean, they have all of the new clothes and stuff and I just think, "Well, I am not going to get that," so I mean, why change? (...) if I make a mistake, I mean, I will get mad at myself, but I mean everybody makes mistakes, but, otherwise, I am happy with myself."

“ (...) with my friends, (...) we just joke about the height that we are because I have three other friends at school and, I mean, one of them is way taller than us... and then we will we joke about the other girl, Jennifer, because she was way taller than us, but I guess we kind of grew up to her. And then my hair color, I think I like my hair, but other times I get mad."

"But, yeah, I mean everybody in our family has got the dark hair, so."

"When I see people on TV, it is normal that they take-like they choose thinner people and now on TV, they are (...) showing (...) a show on Disney Channel, it is like a little thing. It is talking about healthy eating and stuff, all these people, they are like thin, but I guess America has like a lot of large percentage of large people."

"That is what-I heard it."

"And school, too, yeah."

"I definitely think of my friends when I think of that because they were there with me, but I do remember the art and stuff. (...) I remember how she showed us how to paint with our feet. We had paint on our feet and she taught us how to do that."

"And then I do remember the teachers, how they taught us, some of their methods, yeah." 
“(...) I remember trying out for a part, but I didn't get it. (...) I don't remember which dog I was, but I remember practicing constantly. Like I would go home and practice my part because I thought it was just like the best thing. I thought it was so fun. And I just remember the costumes and the backgrounds, the set stuff."

"I know after I thought about it, I thought there is probably going to be more people who will try out for this part. I was confident at first and I was like well, there is going to be other people (...)."

"I definitely think they should because once they do go through it (...) they can go home and tell their parents, like friends can, and younger siblings. Once they go through that class, they will be coming home to tell my parents and I will overhear them, and I will say, "Oh, yeah, I remember that," and just like pass down. And I just think like it is a better experience if you do (...)."

"With friends I remember mentioning that, well we did in art class. During school we would discuss during lunch time what we did. So then we would be talking about like what we were going to do the next day for that class."

"It gave us stuff to talk about like in our free time and during lunch."

"I remember coming home and just being excited to tell my mom what we did at school. (...) it would give us something to talk about, like I know now like when my parent asked me what did I do at school, it is just, like, it is just another school day."

"But, I mean, back then it was (...) all I was doing."

(Showing her artwork):

“(...) this was a cross - I didn't make it at school but I made it at my mother's buisness. And it was a mosaic cross and I had it hanging up on my wall. This was up on my wall, too. We had to create like a fruit (...) and we had to take like clippings of magazine and match the color. Like it was o.k. if it was different. The oranges are not all of the same in my picture, by it just-like blended it a little bit."

"I was really indecisive about that [the color] because I wasn't sure what I should make the wall color in the background or the table color, but it kind of turned out to be a little bit of a mixture. The wall looks like textury and the table looks more like a blended blue."

"(...) we had to pick a saying like a quote. I never have any quotes in mind, so I just looked up on the computer, it says, "Everything in life is luck." I was like indecisive at that time, so I just chose that."

"And then we had to choose our colors and then layer it. (...) I didn't want to be like matchy, I wanted to be like, just colorful." 
“(...) I made this in my free time. It is kind of like the quilt that I had, but I made it out of-I traced this one, but my quilt that I made before, I did that free handed. I did this in my free time, so it wasn't like a project."

"This is my box, we had to make it out of cardboard like from scratch and I was so confused when we started making it. Because my teacher is just like "O.k., you are going to get four pieces of cardboard, hot glue them together and then make a lid for it and design it". I am just like, "okay," she threw it out there."

"(...) so I then said, "Okay, that is pretty," so I used like this pink and orange stuff. Then for my lid, I made a little like grip handle and I painted the inside, the solid black and then on all of the edges we had to add cardboard just to make it look good."

"I think the pink [makes it pretty]."

"[And] mostly the color and the pattern. I mean, I wouldn't have been able to do that free handed if I tried, (...). And I just added on some black because it just didn't stand out that much to me."

“(...) in that art class, I feel like we don't get enough time just because, I mean, I am just so into it. And I feel like if the class was longer, I would like that, but I mean I could do it all day, but it (...) is not like rushing or anything, it is a matter of time and, like, process and it is just fun because you know what you are going to do next, you hot glue the cardboard together and pick out your pattern, then add your own (...) taste on to it and then I painted the inside of it."

“(...) and when I show it to my teacher, I am hoping he will give a positive comment. I know he will because in the past projects I have done, he has done that a lot. He said he was pleased with this one."

"I don't know, no one has ever told me, "Oh, my gosh, I don't like that." If they did say that, I would say maybe it is not their kind of taste, maybe they would prefer something else. (...) I am like, "Oh, that is a good idea." I will tell them like, "That looks really good," so just like back and forth feedback."

"[Positive comment/ feedback] makes me feel good, makes me feel like I can do more, like I have accomplished what I needed to do."

PIANO.

"I am 12 years old and am in sixth grade (...) I take part in theater with Evening City Theater Company and I take piano lessons with Melissa Cag and I am part of my school band, I play also saxophone (...)."

"The biggest thing that comes to mind is third grade and Mrs. INVOLVED's classroom we did a whole painting unit where we had to do a project on a specific painter. I had Claude Monet."

"I had Claude Monet, and actually what I remember best, is one day, when we were coming in school, she had us wait outside the door and when everybody was there, she opened the door and 
she had all of the gorgeous paintings all around the room by all these Renaissance painters and she had music playing and it was just, I loved it."

"That was different."

"One time we had to do a thing where we pretended to be someone completely different. (...) And I don't remember who I was, but we just picked someone. We imagined them up, and then we came into class one day dressed as that person and pretended to be them."

"I had to do that [self-portrait] once."

"That [self-portrait] was when my Montessori preschool actually did that once. They were very focused on the arts. We had a special time set apart for each day to just draw and one time she had us draw - she gave us all little mirrors and she had us draw what we thought we looked like. I remember mine was pretty much just a stick figure."

"It would have been Aladdin, maybe?"

"Bugsy Malone was my first."

"The only thing I did was dance in the finale."

"Anabell."

"They just needed to get up on the stage."

"I remember having a really good time dancing up there."

"No. [Not afraid to dance at that point in time]. I don't know [why]."

"Anabell was right there next to me and dancing with me. And Anabell was just - she was pretty much the person who got me to not be afraid of the audience. I mean, and from then on, the audience has been like a friend to me. I just... ."

"I think so, yes. [Difference in learning in and through the arts]. Because (...) it is just before I was, as she said, really shy and I don't think that I was as sociable, really, because before I started these shows, I would always play with myself at recess, never really talked to anybody. I would, basically, just — and I didn't have very many friends (...)."

"You can find this guy [shows a caterpillar]."

"Well, if you have done things where you have to include detail, like arts classes, you really notice more of the smaller things in your life. Like I noticed yesterday in the drawing that DRAWING had done that she had included people of all races. And before, I mean if I hadn't taken the arts, if I hadn't been involved in the arts, I probably wouldn't have noticed that. And I just thought that that was cool. Like, I think I pay much more attention to detail now." 
"I have gotten much better pitch now."

"I definitely am not as shy. I am less focused on what people will think of me."

"I think it "[effect of arts] is in between positive and negative. There are some negative things, like I think it makes me think about - I think it makes me think about how I look, more, a little bit, but there is also the positive thing that I know that I am my own person and I can do whatever I want."

"I think a little more negatively about myself."

"Yes, I criticize the way I look and criticize more the way that I do things (...)."

"I think maybe seeing how other people who are better at that particular kind of art or theater does it, makes me criticize myself."

"The positive effect is, well, I know more, I think now that I can do things that I want to do if I just try."

"Well, I have always thought this, pretty much since I was born, and I think partly it is because of where I live. But I think that the most beautiful thing that could possibly ever be is our earth. (...) nature, trees, caterpillars, animals, (...) I love it. I mean, without nature, I don't think I could really keep going."

"It [proportions] is what makes babies cute and what makes older people beautiful or handsome or ugly."

"Well, mostly I just love her hair."

"I love her hair. I don't know, I just-."

"Yes, well, it is dirty blond."

"I just love it, okay."

"Well, you don't like your hair color."

"You don't like your natural hair color."

"So you are not used to the way you look."

"Dang it, I wish I would have remembered my balloon, I made that in third grade, but it is at dad's office."

"Oh Jesus. My wedding plan thing."

"I designed stuff that I would like to have my guests wear, bridesmaids wear at my wedding." "It is like a baby blue and white theme. I can go get it if you want. I mean, it is just right upstairs."

"(...) this is my wedding plan (...) the flower girls, the ring bearer, the bridesmaid, and (...)." 
"So I have Laurentia flowers, Laurentia axilaris flowers, they are little star-shaped flowers and I am going to have little blue ones on a wreath on the head, and my veil is going to be attached to that. It is going to be a one foot veil, fairly short."

"A four foot train, also fairly short, compared to Diana. Baby blue studs for earrings, nothing too fancy. Probably like a light blue eye shadow, barely visible, still there, light pink lipstick, also barely visible. Necklace, sort of arm sleeves, look like it is going to be sleeveless, but it goes out here, and V waist, short white heels, white dress and this is my bouquet, white gardenias, roses and with a two leaf covering with a thing that goes whoops, whoops, like a V."

"I was just bored one night and I started to draw and I decided I would draw myself at my wedding, so I did."

"I knew I wanted star-shaped blue flowers so I looked them up on the Internet."

"I tried to look up cute flower girl dress, but that didn't work."

"This is my bridesmaid, a baby blue sash tied in a Bow on this hip (shows the hip). No sleeves past here, puffy sleeve here and no sleeves past that. Sort of a $V$ cut neck line of, a blue head band, a white rose on the head band, and then if her hair is long enough, in an up do, if not, just down. In an up do, held with a light blue ribbon and the dress comes to the mid shin, and white shoe with blue lining, because it is a white and blue theme, and that is all I have for my bridesmaid really. I am very detailed, as you can see. This is my ring boy or ring bearer, (...)."

"No, actually my favorite color is lime green, but I just had this idea and I liked it."

"Blue and white just seemed to fit, you know."

"[Worked on it] A couple of hours."

"Not too long."

"I really liked it. It was fun."

"I was just bored and I decided to plan my wedding."

"When I am bored, I try to either start writing a story or drawing something."

“(...) it [AIC] is a wonderful experience. I only wish Mrs. INVOLVED still taught because I was in her last class, but very much so. I hope that they can have this chance because I just feel like I am a much better person for it."

"Only about 200 people a time in there."

"Well, we are not going to let it die." 
"And all of the people there are so nice. I mean, there is no real problems (...) nobody really gets in huge trouble ever. (...) Nothing bad ever really happens at Sunrise Primary, just a great little school."

"I wish I could still be there."

"I am so glad that you chose that one [Sunrise Elementary School].

DRAWING.

"I am DRAWING. I am PIANO's friend. I go to school also in Sunrise Middle, in the sixth grade, I am 11 years old. My connection with the arts, I take regular pottery classes during the summer, enjoy painting and drawing, especially drawing, and I play violin in the school orchestra."

"My parents are both actors."

“(...) we had a Spanish teacher, who also was an art teacher. And sometimes she would bring in paintings and sometimes even during Spanish class we would make it art class instead and stuff like that. It was very interesting."

"I remember (...) we did this one thing where we had to do a self-portrait where we had to look in a mirror at ourselves and then we had to draw what we saw in the mirror, that was like a selfportrait of what we think of ourselves."

"Yeah, it is really interesting, a lot of great things. We did — Lara Stick, she was a really good teacher, she was great."

"It [self-portrait] was really neat because we just had to draw free hand. It was interesting learning how to draw exactly what we saw. It was a good exercise."

"Can I draw on the back of this paper?"

"I think the arts are a wonderful experience. Like CARING said that acting can make you braver. If you have to stand in front of a bunch of people, obviously, it is a lot harder than just speaking to your friends. They can make you braver, they can make you better, you know."

“(...) say you are looking for something to paint or draw outside, you can do-you can have a greater interest to detail."

"Obviously, our view of people changes over time (...)."

"I think the key to beauty is proportion in anything. You know, in nature, the Fibonacci code is the thing that makes up, let us say it is like three, five, eight, I forget exactly how it goes (...)." 
"The sequence, yeah, but it determines the proportions and the different numbers of things on flowers and other plants and that is one of the keys of nature, and then, of course, humans have proportions, too, that determine how beautiful they are (...)."

"Lime green is kind of an unusual wedding color."

\section{FLUTE.}

"It was very fun. (...) there was a lot of hands-on activities. We watched a lot of movies, we spray painted paper to make hats and we painted a lot. We went outside and we painted what we saw with different colors and it was fun, but strict at the same time."

"Mrs. INVOLVED would come around and she watched what we were doing and if we were doing something wrong, she would, well- (...) It was mostly for me helpful criticism, like you can add the color green in, too, and yellow, so she just helped out with different techniques I could use."

"It [schools without AIC] probably wasn't as fun. And not as much hands-on activities, mostly paperwork and just watching videos. So, it was just different in the way we learned compared to the way they learned."

"It was just funner sitting at a desk and writing notes down on paper and it was just funner getting to go around and paint and draw and have fun."

"I liked painting - (...) I liked painting the best because I like getting messy and having fun."

"Just talking and working at the same time, and drawing what you saw was fun because there might be something different every day as small as like maybe like a little caterpillar on the ground, so it was - there was something new to draw every day. So a lot of our pictures took like a week to draw. (...) And paint."

"My favorite one was the flower painting because we each picked a flower from our garden and we would sit by that flower every day and draw and paint what we saw. And that took about a week and by the end of the week it was very pretty and colorful with a lot of different things. Yeah."

"I was excited a little, happy that I knew what it [painting in Art Museum] was, made me feel smart."

"WELL! I don't really know if this is arts, but for class activities, for English and social studies, we would have mini plays where we would have a script and act out what like happened, when was the civil war? And it was just about a family during that time, so we acted that out."

"And in sixth grade we have Greek day where you perform."

"And my class did the Odyssey. And we learned a lot about ancient Greek-“" 
"We learned about the temples and we made fake temples out of paper and we had to limit our supplies. And my group's temple won the award for best temple because it stood up sturdily and it was sturdy and we did everything right."

"Well, when someone else is playing the part of something, you can see how dramatic and in an accident how it can be and how it can change the world. And it can help you determine if it should ever happen again or if it shouldn't. So when we were doing plays with the civil war, we would act it out and it was very dramatic and it helped to see that this should not happen again."

"Also, in third grade we read Number the Stars."

"We did a small play on that and we learned that this should never and hopefully will never happen again."

"Ms. Christell does mini plays, we all will go up to the white board and she gives us a script, assigns us a part and we just go through the script and act it out as best as we can. (...) Junior Scholastic will sometimes - which are magazines that we get for school, will have mini plays in it and one was Brown versus Board of Education. I forget what part I had in that."

"Well, I don't look at them that differently because the media, they might be famous, but they are still people and just because they are famous doesn't mean that you have to be exactly like them when you get older."

"I had a lot of friends."

"And also camp. (...). The performing arts."

"And now we have the public speaking unit in school. (...) That is okay. It is hard because I am shy and quiet, so getting up on a podium in front of the whole class is hard, especially when you are the first one to go up.

"Well, I played on stage before and danced and all that, it is easy to do that."

"I think everyone is beautiful in their own way from how they look to their personality because you don't have to be slim and tall and have beautiful features to be popular. You can be short and not the prettiest, but still have a lot of friends. And your personality helps a lot with that because some of my friends may depend on clothes and like the way they look instead of their personalities to make them more popular and more pretty."

"My music (...). I think it is beautiful because the sound of the flute and having the whole band come together and playing a concert is fun. And the sound we all make as one is beautiful. (...) whenever a song, like it is a story about how the music department at one school got shut down because of budget cuts, so you can like tell how they would - it is like telling a story. And it is very pretty because in the beginning it starts out slow and then it gets fast and then at the end it just stops."

"I like the way I look." 
"I like some of my hobbies that I do and activities that I do. I do like track, so it gives me something to do. And it helps you stay fit (...)."

"When you are running, there is something about it that just makes you feel better. Like if you are sad or depressed, something like that, and you do something that you like, you can get your feelings out through it. Like, music, you can get your feelings out through music. And I think maybe some famous composers did that, like Beethoven, so-it just makes you feel better about yourself."

"I think they should because it is a fun way to learn and there is a lot of activities, so it just makes learning funner."

FLUTE shows her artwork:

"Here is a copy of the water lilies and this one, she gave us a picture, just a random picture and we had to copy it."

"Doing this was hard because you have to go through the flowers and then some of the flowers would go over it, so that was hard."

"That did not turn out so well."

"[So you knew exactly what you had to do]. Yes."

"Well, she taught us a lot about the different brush strokes and like dabbing and just like painting. I paid a lot of attention to that."

Step 3. Clustering and Thematizing the Invariant Constituents (ICs) \{Units, Themes, or Common Categories\}

During this step (see Figure 17), I clustered the ICs of the experience and situated them into a thematic label. Statements that were overlapping and repetitive were removed (Moustakas, 1994, p. 118). Three separate charts were created, one for each group: students: BAND, PIANO, DRAWING, FLUTE; (see Appendix H3); parents: BUSY, CARING, LOVING; (see Appendix H4); teachers: MOTIVATED, INVOLVED, DR. S.; (see Appendix H5). 
These charts show the various steps that I undertook to cluster the ICs into themes for each individual and their group. The charts organize the outcomes in a simpler way to review and interpret the data.

Since this research study focused on the three co-researcher groups, a separate summary chart was provided for each group to simplify the overview for the reader (see Appendix H6). These charts were compiled by thoroughly and repeatedly reviewing and analyzing the interviews. After each group was analyzed and summarized separately (see Appendices H3, H4, and H5), I created a separate summary cluster of themes for each group (see Appendix H6). Moustakas (1994) describes these clustered and labeled constituents as the core themes of the experience (p. 121).

The following common thematic portrayal of the AIC experience for the three coresearchers' groups provides an overview and illuminates the overlap of the core themes that exist in each of the main groups.

Common thematic portrayals of the AIC experience for students, parents, and teachers:

1. Everything related to the arts or the AIC

2. Positive effects, characterizations, and experiences of the arts or the AIC

3. Skills acquired through the AIC

4. Influences of the teachers, the school, and/ or the environment

5. Things related to the self-concept

6. Beauty (internal/ external), also in relation to clothes and dressing up

7. Various forms of involvement (e.g. family involvement)

8. Requests and wishes in relation to the arts and the AIC 
For a detailed and separated listing of how these categories came into existence for each individual group, see Appendix H7.

\section{Step 4. Final Identification of the Invariant Constituents (ICs) and Themes (Validation)}

At step four (see Figure 17), ICs and their accompanying themes were checked against the total record of the research participant, while considering the following inquiries (Moustakas, 1994): “(1) Are they expressed explicitly in the complete transcription? (2) Are they compatible if not explicitly expressed? (3) If they are not explicit or compatible, they are not relevant to the co-researcher's experience and should be deleted" (p. 121). Groenewald (2004) calls this a 'validity check' (Groenewald, 2004, p. 20).

Groenewald's (2004) 'validity check' of the ICs and the themes was conducted. All the clustered themes were checked individually for each of the four students, each of the three parents, and each of the two teachers/ university liaison. A theme passed the 'validity check' (Groenewald, 2004) only if at least three out of four students, two out of three parents, and two out of two teachers/ university liaison considered it important during interview.

The themes that were not explicit or compatible were determined to be irrelevant to the co-researchers experience and thus deleted (Moustakas, 1994).

\section{Step 5. Individual Textural Description}

During this step, an individual textural description of the experience was created by using the relevant, validated ICs and themes (Moustakas, 1994, p. 121). Copen (1992) calls this textural description the "what" of the experience, which "presents the feel or shape of the experience, just 'what' appears in consciousness" (Copen, 1992, p. 57). With the help of the ICs and the core themes, which were described by Copen (1992) as "the building blogs of a textural portrait" (p. 58), the phenomena that may be associated with the self-concept and perceptions of 
beauty of young ladies who completed an AIC are presented in the following textural descriptions. For this process, various data were selected, like the analysis of horizons, the ICs, and the themes of three co-researchers experiences. The data of each of the three co-researchers' serve as an exemplary representation for each of the three groups (students, parents, teachers):

BUSY - (T-P\&S-BAND\&BUSY-29.04.2011)

BAND - (T-P\&S-BAND\&BUSY-29.04.2011)

INVOLVED - (T-T\&L-INVOLVED, MOTIVATED, \& Dr. S.-03.05.2011)

BAND represents the students' group, BUSY the parents' group, and INVOLVED the teachers' group.

\section{Three co-researchers' textural descriptions.}

\section{BAND's (student) textural description.}

For BAND, her experience of the AIC in Elementary school is connected to her interest in music. She mentioned that she was " in the Sunrise Middle School band," that she played "the clarinet," and that she enjoyed "band a lot." She experienced the AIC not just through her involvement in music but also through the visual arts. Painting in school was equally important to her because she received "recognitions from it."

Positive descriptions and characterizations of art came generously from BAND. She said, "I really - I really like the arts because it, like, affected me up to now because now I am doing really good in my art classes and I really enjoy it." Even though it seemed like there was insecurity at the beginning about the arts, BAND eventually gained confidence in her artistic capabilities. She developed a positive self-concept through the arts, as illustrated when she said "I am happy with myself." 
BAND said family has also influenced her interest in music. "I think I like the music better just because, I don't know, my mom has been involved with music and so has my grandfather and my sister and my brother."

BAND described learning through the AIC and its connection to interdisciplinary learning as being "FUN." "I definitely think that it does make learning at school like more fun and stuff other than math and social studies." Problem solving was considered a "fun" game rather than "work." The concepts of thinking differently, being creative, and using your imagination were used as a matter of course. The AIC taught her to be "bright" and to think "outside the box."

The experience of the AIC was also associated with interactive and social connections with friends, since experiences are shared together. BAND also mentioned clothes, the way one dresses, and how people are different. For her, the arts fostered an awareness of more than one viewpoint. For example, the way of dressing differently in other cultures was mentioned. "We were talking about how the African people (...) dressed. It is not similar to what we do dress here in America."

Perspectives were broadened through the AIC: "It did teach us how to look differently at people just because we know nobody is the same and we are all different." The challenge "not to just be like everybody else" was introduced.

BAND considered natural things like a rose or a flower as beautiful because they were very simple, but beautiful. In reference to people, she observed that “(...) people on television are thought of more as, like, role models and people to look up to." Thinness played a role in BAND's consideration of beautiful people and TV, because to her, it seems like thinner people are chosen for TV. 
BAND's self-concept was mainly positive. She described herself as a good friend, helper, and someone who is confident. She even considered herself a role model to others, for example to her sister because she liked to hang out with her, "she is my friend." Even though height and hair color were seen as beauty issues for BAND, they did not bother. Though BAND did not consider her height and hair color "perfect," she found positive ways to describe herself: "Everybody in our family has got the dark hair, so."

The AIC school appeared to have an influence on the image and the self-concept of BAND by providing her with specific artistic knowledge, like showing her how to paint with her feet or some other methods. Through these examples, the teachers' positive influences became visible.

Time was mentioned in two different contexts. Once, it was stated in reference to the frequency of practice: "I remember practicing constantly." On another occasion, it was related to the fact that there is not enough time for the arts: "I feel like we don't get enough time just because, I mean, I am just so into it (...) it is a matter of time (...)."

The idea of learning to live with competition and possible failure was also developed through the AIC. BAND remembered unsuccessfully trying out for a part in a school play. She learned through her acting experience that she did not exist in a vacuum, that she was not the only one trying to get the role for a play, and that she might be unsuccessful. This way, real life skills were developed through the arts.

BAND also found it important to share her experiences in the AIC with others. She enjoyed telling her parents about what she had learned at school: "I remember coming home and just being excited to tell my mom what we did at school. (...) it would give us something to talk about." Her involvement in the AIC also served as a conversation starter with friends: "I 
remember mentioning that, what we did in art class. During school we would discuss during lunch time what we did." According to BAND, her longing to talk about what she did in school subsided after moving to a non-AIC. When her parents asked her what she did at school, she responded that "it is just another school day," nothing special anymore. After the move, there was nothing to look forward to anymore, unlike during her AIC times, when "we would be talking about like what we were going to do the next day for that class."

Nevertheless, BAND's passion for art remained. She continued her artwork, even in her free time and worked on artistic projects, even if they were not required for the school curriculum.

Importantly, BAND found pride and self-esteem in her artwork because her work received value by being displayed or praised by the teacher. He said he was pleased with this one [artwork]." The possibility of negative feedback did not seem to bother her. She said "no one has ever told me, 'Oh, my gosh, I don't like that'." Positive feedback motivated her to continue and gave her pleasure and enjoyment in what she already accomplished.

The computer and media in general were used by BAND as resources for information that she herself did not have readily available. "I never have any quotes in mind, so I just looked it up on the computer."

BAND placed great value on the ability to draw something free-handed. As such, it seemed even more important that teachers instruct their students on methods and techniques of how to draw. As a positive consequence, students usually developed a positive self-concept.

Things were also supposed to "look good” or "pretty” for girls BAND's age. Sometimes special artistic characteristics, like color or shape, can have that effect. "I think the pink [makes it pretty]." "[And] mostly the color and the pattern." BAND 's involvement in, and enjoyment of, 
the AIC was also visible by her describing details when she talked about her artistic work: "Then for my lid, I made a little like grip handle and I painted the inside, the solid black and then on all of the edges we had to add cardboard just to make it look good." Even though she was very much into the method of how she created her artwork, the aesthetic component of "looking good or pretty" still was an important factor in her description.

BAND found security in her knowing which step to do next. "You know what you are going to do next, you hot glue the cardboard together and pick out your pattern, then add your own (...) taste on to it and then I painted the inside of it." The fear and insecurity of not knowing what to do or how to do it seemed to disappear for BAND.

Considering these descriptions, one can conclude that BAND's experience with the AIC and its possible influence on her self-concept and her perceptions of beauty consisted of six main textural themes: 1) her interest in the arts and their positive effects and characterizations; 2) a positive self-concept through the arts caused by positive feedback; 3) enjoyment of interdisciplinary learning/ AIC and its way of problem solving (creative and different way of thinking); 4) the concept of a role model (herself for others and others for herself); this role model concept is interactive at the same time by sharing things with family, friends, or involving the family; 5) the concept of beauty in reference to nature or natural things; 6) The concept of time that is necessary for the arts to develop (it is a process). As such, the phenomena of the AIC and their influences on BAND's self-concept and her perceptions of beauty can be described as positive contributions to the development of BAND's "self" and "being" during adolescence.

\section{BUSY's (parent) textural description.}

BUSY's link to the AIC as a parent is her interest in the arts in general and specifically in her workplace. She owns her own art business. This ownership of an artistic business has 
influenced her daughter's interest in the arts. Perhaps more importantly, BUSY herself was always involved in the arts. For example, she "did 12 years of dancing," as a child. She also was "always involved in music." BUSY also "always took art classes." Her family was always into the arts and she and her siblings were used to being surrounded by the arts. This history of her own arts-based learning makes her a little bit "biased," because of influences within her own family_ “music was a big part of our family." BUSY feels that she has successfully passed on the family tradition by exposing her children at an early age to the arts. "The arts are a big part of our lives and that could have come from (...) being exposed to it." She just thinks that "we have more of an interest in the arts."

BUSY also described how she experienced the arts through her daughter, by hearing about the AIC. Her children "would come home from school and talk about some things they would do in the classroom. (...) They would come home and say, 'Oh, we learned this song today. Or (...) in math we sang this song,.”

For BUSY, it goes without saying that children gain more when they are exposed to the arts or an AIC at school "in all different subjects." They learn to be more "open to newer things and maybe show more of an interest in it." According to BUSY, children's perspectives are broadened by the AIC.

BUSY was convinced that the way BAND looked at herself and other people has been heavily influenced by the AIC. BAND seemed to be more conscious about things, such as the way colors and clothes match. Furthermore, BUSY thought that there were other positive results of arts-based learning experiences in reference to the self-concept of children, like for dancing, "how to hold yourself and present yourself." 
BUSY was also impressed with BAND's interest in the visual arts and was proud of what her daughter could do. She described her daughter as "talented." BUSY liked that BAND had an interest in the arts and that she enjoyed it. BUSY used very positive descriptions and characterizations of the AIC: "how they would use arts and music and (...) physical movements to integrate that into their curriculum (...) it was a great idea." Based on these positive experiences, BUSY found that BAND never really struggled to motivate herself for (AIC) activities.

Eventually, this positive side-effect also had to do with the recognition, the confirmation, and the positive feedback that BAND received from artistic activities. Even the teacher showed that he valued her artwork by keeping it for display and BAND was very proud of that.

The AIC also influenced learning and memory in a positive way. Songs and other art forms were described as easier ways to recall and actually retain information for a longer period of time than with more traditional learning styles: "It helps them learn, when they come home and they are chanting or singing a song, they come home and sing the days of the week or months of the year (...) I mean it helps them definitely remember. They still, you know, they can still sing it to this day." The best characteristic in BUSY's opinion was that this learning style not only works for adolescents but also for adults.

Interdisciplinary learning in an AIC exposed students to so many different subject areas, "it just exposes the kids to so much more." From BUSY's viewpoint, this was especially true when students were not able to receive an artistic input from their family environments. "Then, they might not ever be exposed to something like that."

In general, BUSY believed that BAND was "happy with herself," her appearance and her clothes. Nevertheless, there are times when BAND was trying to figure out what to wear and not 
happy with herself: "Oh, this doesn't look right, oh man.” Even though BUSY and BAND discussed body appearance, healthy eating, and exercise throughout the interview, the focus was never just on BAND, but the whole family.

BUSY described BAND's view on beauty as connected to objects that are somehow natural in their being, like a flower or a rose. "I think it is more that she thinks objects are beautiful." In reference to beauty in human beings, BUSY referred to BAND's comment on television's preference for thin people and that she appeared to admire people on television because of their thin body type. BUSY also found that BAND favored the natural beauty instead of "the real made up kind of person, all of the real striking, stark makeup." Besides physical beauty (thinness), BUSY found that BAND believed that beauty also consisted of inner beauty, "a natural beauty and the nicer personality that she would think of as beautiful."

For the most part, BUSY described BAND's self-concept as positive. To BUSY, BAND was a very caring, responsible person that was "always willing to help out." She was also a "good friend, to her friends" and she was a "good listener." BUSY reinforced BAND's positive self-concept by talking very positively about BAND's appearance. "She always looks nice in whatever she wears and she is clean and well-kept and she takes care to do her makeup and everything." BUSY herself was also comfortable with her self-concept. Though BUSY thought "it would be nice to be a little bit thinner" she and her daughter "are comfortable with where [they] are."

Parent involvement and integration played a big role for the AIC. Parents met at the beginning of the year with the teacher to talk about AIC concepts. The parents did not just hear about what was going on in the curriculum throughout the year, but also became active and volunteered in an initiative for an after-school-program: "We put on a musical, 101 Dalmatians." 
The interest in that musical was great. "Everybody that wanted to be a part of it was in it." Surprisingly, not the teachers, but the parents were behind this initiative. Nevertheless, "the school did support it." The parents would practice songs and choreography with the students. No one was excluded. Everyone was welcome to participate. For these reasons and more, BUSY highly recommended the school to others. She thinks that everyone "[should experience AIC], definitely."

Students' images and self-concepts appeared to be influenced positively by the school. The students learned how to be patient and show endurance during their practices in various types of arts. Students who completed an AIC, for example, often continued their artwork during their free time. This showed their passion for the arts and the work they are doing.

Because of their involvement, their enthusiasm, and the positive feedback, kids freely shared and displayed their artwork. They were proud of what they had done and value it. Of course, BAND was willing to share her artwork. She proudly presented some of her artworks to me: a painting (see Figure 5), a collage (see Figure 6), a mosaic cross (see Figure 7), and a box (see Figure 8).

The AIC school received a very positive evaluation from BUSY. She even mentioned that she moved to the school district so that BAND could attend the school. The curriculum also received positive recommendations: "I think the school, this particular school that we went to because they used that, I think it has a good reputation for that [AIC]. The AIC school is different from a "traditional" school in that "they do a little bit more than —it is a different environment maybe than some other schools that don't and that is a good thing."

BUSY's experience with the AIC and its possible influence on the self-concept and the perceptions of beauty, in relation to her adolescent daughter, consisted of seven main textural 
themes: 1) an interest and connection to the arts and their positive effects/ characterizations through work, as well as her own past experiences; 2) experiencing the arts through her daughter sharing it; 3) positive influence of the AIC (recognition/ positive feedback) supports positive self-concept; 4) beautiful things and beautiful people are characterized by "something natural"; 5) involvement of family, parents (especially volunteering parents), and students; 6) AIC and interdisciplinary learning support learning and memory; 7) AIC schools positive evaluations. Also, BUSY's descriptions of the effects of the AIC on BAND's self-concept and perceptions of beauty can be interpreted as a positive influence on BAND's "self" and "being” during adolescence.

\section{INVOLVED's (teacher) textural description.}

INVOLVED has strong connections to the AIC, being one of the few teachers that specialized in the AIC. She taught third grade at Sunrise Primary School for a long timepossibly 33 years. She has been retired for three years.

The research question was a difficult one for INVOLVED because she and her colleague, MOTIVATED, tried to break down gender and I was trying to identify it for this study. So the teachers struggled with that. Nevertheless, INVOLVED conceded that there was at least one exception. "One year I remember more girls were identified gifted than boys." But still, she and her colleague argued that it was more of a "TAG thing" than a "gender thing." The same was the case with selecting boys over girls or the other way around. INVOLVED commented that the labels that were favored in schools did not really work in the AIC classroom: "You could not pick out the kids who were ADHD, LD, BD," or "the talented and gifted." In the AIC classroom, kids were not labeled. She stressed that one "would not have found the kids that are ADHD," nor 
could one select kids with discipline or other problems. Equally, one also could not distinguish between artwork based on gender.

Children that were identified as talented or gifted could take tests very well. This meant, they were very traditionally and academically gifted. Unfortunately, "kids who are really artistic, truly artistic" did not qualify for TAG, yet—“they are not talented and gifted." INVOLVED stressed that one has to be "academically inclined to be in the gifted program or the talented and gifted program." In this manner, the talented and gifted qualification was just a one-way road. But even if these children were evaluated as academically talented and gifted, they faced problems of being limited to only two answers, the right or wrong one. The AIC tried to help kids overcome this problem.

There was no difference found between boys and girls and the way they perceived the AIC, their artwork, and their final product. But, there was a difference in the way they approached their artwork. Boys struggled more with the activities of the AIC than girls. They struggled and needed help from the teacher or the girls. Especially for dressing up, "it was easier for girls to find stuff." Boys struggled because they did not have as many options as the girls. Consequently, boys more often than girls received help from the teacher: "We really had to coax them along in giving them ideas." INVOLVED also found some small differences between boys and girls concerning the way they observed art. She found that "the girls were easier to focus in on a piece and stay longer," while "boys probably scanned. And then they wandered more." Girls also wanted to teach others, they wanted to be a teacher when they grew up. Boys usually would not say that. In contrast, boys were not as demanding as girls in reference to their body image: "Boys, oh, well, it fits fine, what the heck. But girls, you know, they want to look, that image of looking cute." Girls also seemed to care more about clothes. Nevertheless, boys also would be 
into clothes. From INVOLVED's viewpoint, they did all the things girls did, it just "took them a little bit longer."

Problem-solving skills were one of the most important skills that boys and girls acquired through the AIC. The teachers encouraged students to take risks in coming to a solution. With the help of the arts, for example by observing and analyzing a picture, they learned to focus on the details. This capability came in quite handy, especially for test taking, when the students analyzed questions as if they were examining art. As a positive consequence, the teachers noticed that the students scored higher. They were willing to take risks. They knew to look at all of the questions of a test form looking at a piece of art. So when they saw a written question on a test, they were able to do the same thing on that test. They looked at all of the questions and analyzed the different parts of the questions. As a result, they often tested higher. Throughout this process, teachers provided assistance, rather than just exercising authority. The teachers were part of the solution, but did not give the answers away. These problem-solving skills applied socially and academically. If there was a problem in class, the students were part of the solution, by holding a class meeting. Also academically or practically, the students often had problems to solve. On one occasion they had to build a structure. An architect came in to show them how to build. Then, the kids had to apply what they had learned and sometimes had to ask for help. But often, when the teachers tried to confirm that they really needed help, the students decided to solve the problems themselves. At the same time that they had to draw and make plans for a future structure, they also had to take care of other related tasks. For instance, they had to keep track of their bank account. "Everything had a price to it. And so they had to keep a journal of how much they had and how much they spent." They took turns in order to keep this map log. Each of the students had to take responsibility at some point. At the end of the project, they acquired knowledge in 
many areas. "They knew how to add or subtract, they knew how to keep a ledger (...) we knew so much at the end of that project."

The AIC enabled students to be free, to be creative, and to develop their own viewpoints and solutions for problems they had to solve. For example, they thought of the idea to have a bargain table to have things for sale. So, they built their own discount store.

No barriers were accepted in the AIC classroom. There was always an atmosphere of tolerance. INVOLVED stressed that everyone was considered unique "in that we all had different gifts and we looked at them differently than they looked at them at some schools." Barriers to groups were broken down. Students were not considered smart or dumb, small or big, light or dark. Children made comments, but did not tease.

Discrimination was not accepted at any time. Children of color were treated the same by everyone. Differences and individuality were accepted as the norm. One student, for example, had to use different languages to teach his parents in a project. Depending on the proficiency level, INVOLVED and MOTIVATED "looked at everybody's piece differently."

Life, social, and soft skills also were part of the AIC teaching. The school environment and the classroom were used as an "art gallery" to do so. 500 hundred paintings were all over the room. Students learned through rules and by role playing how to behave in a public place, like an art gallery. The art was kept in the hall and the teachers explained the rules that would apply at an art gallery.

As part of the "art gallery" experience, the students also learned critical thinking skills. It was not enough to take a quick look at a painting and continue their walk through the "museum." "They had to look beyond the painting. Critical thinking skills were developed as a byproduct of looking at a painting, analyzing, and thinking about it. Their test scores got better, almost 
automatically." But not just their scores on tests like the SAT got better. "Their creative writing was better, too."

Discipline problems were never an issue. "Kids were everywhere. They were never in their seats - they were in their seats, but they were not structured, okay, this is an English class, sit down and pay attention, here is the page." The kids could move around wherever they wanted, though they were aware of the boundaries. The children and their self-concepts developed towards an independence that influenced their decision processes in a positive way. INVOLVED described how "they were independent that way," and how she and MOTIATED taught more of that independence: "'You are big now, you can take care of yourself,' rather than, 'Okay, you can't paint until I get everything out for you.'” Team spirit was important among the students, but also between the teachers and their classrooms. The teachers worked very well together, and the students knew in whose room the paintings would be that they were looking for. So, "they could go easily in her room and back (...), they didn't even have to excuse themselves, they would just go in and would walk in. They were walking back and forth all the time."

The results of arts-based learning experiences were very positive. The students took initiatives, were autonomous, and were "in charge." They blossomed immensely in all kinds of ways and subjects. Non-readers became interested in reading. Math skills were enhanced.

Discussions were stimulated and test scores were improved by the teachers "sneaking things in" through the arts. Attention to details was stressed by observing more thoroughly. Students acted in different, more positive ways as an effect of roleplaying and dressing up. The self-image of students developed in a positive direction. They were proud of their work and wanted to show off their good results. 
The AIC received positive descriptions and characterizations from both teachers and students. Teachers stressed that the AIC made the kids free to express themselves. They learned how to think things through and, as a consequence, scored higher on tests: "I think we consistently had high test scores." But also positive physical effects on kids became apparent. A very good example is one boy who transferred from a parochial school. He stated: "My goodness, my headaches are gone."

The feedback from students was very important for the teachers, who enjoyed discovering how the students felt. The students mentioned time for reflection and observation, which were seen as some of the most important concepts in the AIC. Even as adults, "we don't spend enough time" reflecting. INVOLVED and MOTIVATED gave them that opportunity to reflect on what happened and how they could have done it differently.

INVOLVED also found that a positive self-concept was developed through the arts. For instance, students cultivated leadership skills by pursuing their goals and standing up for their ideas. This was shown by three boys who wanted to play ball. They took over leadership and told everyone how they felt. Consequently, the whole group had to problem-solve. The class decided that "they would let them play," that "they could have three turns," and that they would help them. The students themselves had solved the problem while the teachers just served as facilitators, instead of ruling authorities.

Self-identity also was developed throughout the AIC process. It was made clear to all the students that it was okay to be different or to see things differently in an AIC. To explain, INVOLVED chose the theme of obesity. She stressed that from the teachers' viewpoint, obese students should also be involved in the AIC practices. She mentioned the two obese twins who had two different teachers. While one teacher said, “'I wouldn't do that because that would 
embarrass her,"' the other teacher made the student's sister do all of those things. It is not just okay to be different in reference to obesity, but also in relation to other things. INVOLVED picked language as another example. In the AIC, it is not a problem if a student speaks a different language than everyone else. There was a little boy who taught his parents a lesson in English and his grandparents a lesson in Spanish. Like other kids, this little boy was still able to express himself through art. There were no set standards on how or through which process the goal had to be achieved, as long as it is achieved.

Family involvement and parental integration also played an important role. Whole families were affected and influenced by their children's interest in the arts. Sometimes, when they planned a summer trip, they had to go to an art museum. Discussions about family values were also triggered through the AIC. The teachers prepared questions for students to ask their parents. "That was a good chance for family discussions at home." Parental support was needed to achieve and realize these goals and efforts. Often, parents even provided monetary support to teachers because they realized how much they invested to make the AIC possible. The teachers did not just spend time, but also money. One parent even came and wrote the teachers a check because they thought this had to cost a lot of money.

The students learned in different ways through the AIC. Interdisciplinary learning was present in various areas, like music, math, arts, and sports. The students studied learning styles and student-based learning. No subject was neglected or too difficult to integrate the arts. It was, however, more difficult to put the AIC into practice. The requirements of the standardized testing just do not allow it anymore, which frustrates teachers and students. INVOLVED reported that some of her colleagues from other schools complain that they do not do fun units anymore because they have to teach for the test. This resulted in frustration: "It was fun, but the sad part is 
nobody appreciated it in a county that they picked up on." All the officials wanted to know the secrets of the good test results, but they did not want to listen, learn, or watch.

Instead of taking traditional written tests, students had shown through teaching what they had learned and acquired. "They taught their unit to their parents so they became the teacher."

Of course, this way of teaching and testing was not acquired through traditional ways. The teachers had to be willing to serve as role-models and mentors. INVOLVED found that "modeling is [was] so important" to support the kids in their learning process. A good example of how the students learned from their role-models, the teachers, was the mirror illustration for self-portraits. The teachers decided that the students had to have a model. The teachers had a mirror, looked at the mirror, and would talk out loud: "I said, 'I am thinking out loud, so bear with me.' I would talk it through. Then they would know what to look for." INVOLVED found that one of the most serious problems in our schools was that many of the teachers are not willing to model for their students. They just do not feel comfortable enough.

The self-portrait was another one of those invaluable projects. The teachers, again, took the time to model for their students. The teachers would not let them start because they were supposed to learn patience. They were meant to learn to focus by looking at the mirror. Through this exercise, they learned to see different things, the uniqueness of a human being, and the reality. When they drew a portrait of themselves, they made comments like, "I didn't know my nose was that big." At the end of the project, it became obvious through all the individual portraits how students expressed themselves and their perceptions of themselves through the visual arts. The portraits were characterized by individuality. One even could determine nationality by looking at the portraits. 
From the teachers' viewpoint, the school had a major impact on the image and the selfconcept of boys. The older boys became, the more they wanted to be taller to play ball. Girls want to be smaller, “'Oh, you are so tiny or you are so big or you are so tall or you are so short."” Also, the teachers had a significant influence in their function as role-models, their involvement, motivation, and their engagement. A former student who was going to graduate as an engineering professor wanted to teach even with an engineering and a math degree. INVOLVED wondered from where this motivation came. His mother told INVOLVED that she was his favorite teacher and INVOLVED asked the mother: "Why do you think that?" His mother responded: “Because you let him express himself." All of INVOLVED's or MOTIVATED's students had to express themselves in one way or another. No exceptions were made.

It was important in school to make time for the arts. In reference to the teachers' projects, for instance, people criticized the time they spent on one theme. INVOLVED clarified that people did not realize that through the arts, the students learned more than one thing. INVOLVED was convinced that they learned several things that they would not have learned in a "regular" curriculum.

The AIC school always received positive evaluations and recommendations by students and parents. Other things that the teachers considered as pro art were the high test scores that students achieved, while participating in the AIC. Consequently, "no one interfered too much or came down on us and said, 'You are going off the curriculum here,' because we really weren't. We were just doing it in a different way, but our teaching was always very purposeful.” Different learning styles and student-based learning developed parallel with the AIC. Students experienced many different areas, not just one single part of the curriculum. 
INVOLVED's experience with the AIC and its influence on her students' self-concepts and their perceptions of beauty consisted of seven main textural themes: 1) the connection to the arts through their work and interests; 2) the positive results, effects, and characterizations of the arts and the arts-based learning experiences; 3 ) the positive self-concept through the arts and the resulting leadership roles, adopted by students; 4) learning in different ways through the AIC and interdisciplinary learning are FUN; 5) the AIC and its effects are not limited to girls, even though some gender differences exist; 6) teachers have a positive influence on students as role-models and mentors; 7) the small AIC school receives positive evaluations by students, parents, and teachers. As such, the consequences of the AIC and its influence on INVOLVEMENT's students' self-concept and their perceptions of beauty (boys and girls!) can be described as positive contributions to the development of the student's "self" and "being" throughout adolescence.

\section{Composite textural analysis.}

For all three of the co-researchers (BUSY representing the parents; BAND representing the students; INVOLVED representing the teachers), the phenomena that may be associated with the self-concept and perceptions of beauty of young ladies who completed an AIC appear to be similar. Their interest in the arts and their strong connections to the AIC appear to cause positive effects. The AIC and the arts in general were described and characterized in a very positive manner:

BAND: "I do like art (...). And I enjoy band a lot, too."

BUSY: "I just think it has been a positive experience." INVOLVED: "It was very exciting (...)." 
This influence of the AIC indirectly had a positive effect on the self-concept of girls (and boys!). Students were not as critical of themselves. They were more content concerning their bodies and their appearances:

BAND: "I am happy with myself."

BUSY: "I think for the most part, she [BAND] is happy with herself."

INVOLVED about a boy: “He didn't like math, he didn't like reading, he didn't like any of that, but he excelled in art. That is where he finally found himself."

The AIC and its interdisciplinary learning style supported learning in a very positive way. Co-researchers described this kind of learning as "fun" and not as boring as the learning in other subjects and areas:

BAND: "I thought it [AIC] was just like the best thing. I thought it was so fun."

BUSY: "She is talented and I like that she has an interest in it and she enjoys it [AIC]." INVOLVED: "It was fun."

Another positive effect, mentioned by the participants, was the desire to share and show the results of the work completed throughout the AIC. The involvement of students, teachers, parents, and whole families was also stressed. AIC schools and their positive evaluations were mentioned, as well. A pool of different kinds of concepts and assumptions exist that appear to be related to the AIC and its positive effect on the self-concept of adolescent girls and their perception of beauty. Nevertheless, the previously-mentioned phenomena appear to be the major ones for the individual textural description for all three co-researcher groups.

\section{Step 6. Individual Structural Description}

During this step, an individual structural description of the experience was created "based 
on the individual textural description and imaginative variation" (Moustakas, 1994, p. 121).

Copen (1992) describes this step as "the dynamic that enables the researcher to understand 'how' something comes about." At this step, more than just transcript text is important. Beyond appearance, "imaginative variation, reflection and analysis," are supposed to bring to light the "real meanings or essences of the experience" (Copen, 1992, p. 65). Moustakas (1994) also describes this step in one concise sentence: "How did the experience of the phenomenon come to be what it is?" (p. 98).

\section{Three co-researchers structural descriptions.}

In the following section, the individual structural descriptions of the participants that served as exemplary co-researchers were analyzed.

\section{BAND's (student) structural description.}

The structures that defined BAND's connection to her AIC experience and its possible (positive) effect on her self-concept and her perception of beauty were time, meaning her age (adolescence); her relationship to others, in connection to bodily concerns; her motivation, positive feedback and approval by others; and her positive self-concept.

In particular, this means that BAND's self, in reference to time, was affected by her age. At the time of the interview, she was an adolescent thirteen-year-old teenager. At this age, children often become more sensitive about the way they look and the impression they make on others. BAND compared herself to others in appearance and behavior. She was torn between fulfilling the "beauty standard" that her peer-group and the media required of her identity, her personality, and her individuality. Clothes and the way she or others dressed were some of her main concerns. "I don't know, when I see some people at school, I mean, they have all of the new clothes and stuff and I just think, 'Well, I am not going to get that,' so I mean, why 
change?" Even though she was affected by these thoughts of comparison, she usually found her own way of solving these problems. She eventually became comfortable with herself and her sometimes different but individual personality. She did not want to depend on clothes and her appearance, like some of her friends.

BAND described her relationships to others as mainly determined by media or school. In reference to media, thinness played a major role. She referred to television shows that she watched, heard about, or discussed in school. She considered the people on television, who she believed were supposed to serve as role-models, as "perfect," especially their weight. She referred to a show on Disney Channel, in which the people talked about healthy eating. She said these people were thin. Even though her concern about weight and her worries about not being perfect in relation to "thinness" were obvious, she still seemed to be confident in herself and realized that there were many people not as "perfect" as those on television. She appeared to understand that "thinness" was more of a fashion trend, with which she not necessarily agreed. She felt comfortable with her appearance.

This comfort in her-self, her body, and her appearance seemed to result from her family support. BAND felt very close to her family. And even though she thought she and her family did not match the "perfect" norms that the society, the media, or her peers asked for, she found strength in the fact that she was not alone. She had her family as a support group. This gave her a feeling of comfort and strength. As long as she belonged to the group of "her family" and matched their "standards," she did not worry about the way she looked or the pressure of fulfilling standards that were set by other outside forces.

BAND's self in relation to recognition, confirmation, and positive feedback was related to her motivation. She enjoyed receiving positive recognition and confirmation from others, 
especially her teachers. It gave her a positive attitude towards her work. The positive feedback she received for her finished artworks especially enhanced her intrinsic motivation. She was not just proud of what she accomplished, but also felt encouraged that she could do much more. When the artworks became more complicated, her extrinsic motivation was enhanced even more. BAND often received the positive feedback she sought in the form of her teacher displaying her artwork. She never received any negative feedback but even if so, BAND would have found a way to make negative feedback positive. "I don't know, no one has ever told me, 'Oh, my gosh, I don't like that.' If they did say that, I would say maybe it is not their kind of taste, maybe they would prefer something else." This comment shows how, on the one hand, she was extrinsically motivated by receiving positive feedback. On the other hand, she was ready for and knew how to deal with a possibly negative comment.

BAND's openness and eagerness to share and even display her artwork demonstrated her positive self-concept. She trusted in herself. She was convinced that she was producing good work and that people would likely find it interesting and might even provide her with positive feedback. She was willing to show me some of her artwork (see Figures 1-4) and described it in great detail. In this way, BUSY defined herself and her body image through her art work. Positive feedback caused her to feel good about herself and to be proud.

Yet, BAND's self-concept was not just defined by her artwork or her body image. She also described self-concept as the way one thinks of oneself. This definition shows that she also considered the thinking process. For her, the self-concept was not just defined by the external way one thinks about oneself, but also by the internal way of thinking. Consequently, personal and internal thinking processes became involved, processes that could reflect the correct image, but sometimes could be disturbed by false images (see chapter two for more detail). 
Another definition BAND gave of self-concept was one, independent of external beauty, which can be referred to as the inner self-concept. BAND described it as the way one thinks about oneself in relation to others (e.g. friends or siblings). As such, BAND described selfconcept as a way of showing friendship, closeness, or a positive relation to others. A positive self-concept for her was defined by being sociable and did not necessarily have to be related to a bodily self-concept, though both ways of approaching the definition of self-concept were not necessarily mutually exclusive.

BAND's main individual structural descriptions of time or age, her relationship to others, her motivations, positive feedback, and positive self-concept were all influenced by her distinction between internal and external concepts. BAND was not just defined by the one or the other. For her, the internal and the external were always interwoven. There was never a time she only focused on one. Beauty, reflected by "medial thinness" was on her mind, but she found her own way of "being beautiful." Self-concept for her was connected not only to the body, but also to relationships. BAND was able to find balance between these two ideas.

\section{BUSY's (parent) structural description.}

The structures that defined BUSY's connection to her AIC experience and its positive effect on her daughter's self-concept and perception of beauty were determined by the concept of time, her comparison to others in connection to bodily concerns (healthy eating, exercise, thinness), motivation in various ways, the definition of beautiful things/ people, and her excitement to see her daughter being proud to show her artwork to others (positive self-concept).

BUSY's description of time consisted of two parts. First, she discussed how BAND lost track of time when working with the arts. A special kind of motivation arose in her daughter that let her forget the time. BAND gave the impression that she could continue working on her art 
project for hours without any interruption. This state of mind can be called "flow," an idea introduced by Csíkszentmihályi (1990) that was described in chapter two. Second, BAND experienced an interest in arts in her free time. Even away from school and school projects, she worked on art projects. In the absence of school-related pressures, her motivation remained.

BUSY's thoughts of herself and others were defined by beauty and bodily concerns. Weight was an especially important issue for BUSY and her daughter BAND. Both of them expressed that they wanted to lose weight. BUSY claimed that she mainly wanted to drop weight because of health reasons. She found out in the waiting room at the doctor's office that her BMI was higher than normal. But, even though she stressed that the main concern would be health, BUSY also mentioned appearance. She talked about how she had gone bathing suit shopping and felt uncomfortable with some of the bathing suits. Apparently, both health and appearance influenced BUSY's and BAND's desire to lose weight. Despite these environmental influences including the perfect "supposed-to-be" role-models shown on television, BUSY and BAND were still content with themselves. "I think we are comfortable with where we are at" (BAND).

Here, the "we" and the "unity" of the mother and the child are important to consider. The mother did not talk just about her daughter, her weight, and that she thought her daughter should lose weight. She included herself, even the whole family, in this endeavor. This way, the weight loss was not just focused on one family member, but the whole family. Nobody had to feel excluded because of weight issues. Weight loss was seen as a goal for the whole family, not just one member.

The self-concept and self-image was also determined by beautiful things or beautiful people. It seemed that two kinds of beauty were distinguished by BUSY and her daughter BAND. First, physical beauty was described, which also can be regarded as external beauty. 
Second, they referred to inner beauty and a pleasant personality, which also can be seen as internal beauty. Even though both types of beauty were mentioned, BUSY was of the opinion that her daughter preferred the inner, more natural beauty. Still, BUSY thought her daughter was also affected by the "perfect, external beauty" that was favored in the media and thought "there still has to be a physical beauty about it, too." But, there was a distinction between physical beauty that was naturally beautiful and the artificial kind of person that BAND did not favor. She was "more of a natural." Here again, one can see how mother and daughter did not close their eyes to the media. Instead, they were able to distinguish between their natural "real world" and the made-up "media world." Both were able to draw an educated distinction.

Regarding the self-concept of her daughter, BUSY was convinced that the positive feedback from art caused intrinsic motivation in BAND. She loved to work on her own projects and artworks and was proud to show around her final product. She gained a lot of positive feedback. BAND was always willing to show and share. This was a sign of her being selfconfident, believing in herself, and enjoying the things she did.

BUSY's positive thoughts and structural themes on the AIC and the AIC experiences of her daughter reflected the concept of time, the positive effect of the AIC, and the final result of an increase in motivation. A positive self-concept was also visible. BAND was not hesitant to share her finished artwork. Structural themes that were more negative in nature were those that included comparisons to people in the media. However, one can conclude that BUSY was convinced that for BAND people or things considered "beautiful" must be of a "natural beauty."

\section{INVOLVED's (teacher) structural description.}

The structures that defined INVOLVEMENT's connection to the AIC experience and its possible positive effect on the self-concept of her female students and their perception of beauty 
were characterized by time, specifically taking time for reflection; space for individual development; the relationship of her students' self to others, especially in reference to selfportraits and self-images, body image, and dieting; and positive self-concepts of students through teaching.

INVOLVED's self in reference to time was defined by her willingness to take time for the students and their problems. For INVOLVED, it was more important to take the time to reflect, than just rushing through the requirements, especially because this was a problem that so many adults also face in our society. No one takes time to reflect or think about things anymore and what one could have done differently. This way, students were enabled to learn from and thus avoid repeating past mistakes. Reflection might have also been responsible for the enhancement of test scores for students. Allowing time to reflect on tests or to review, and learn from past mistakes was an important step to scoring higher on tests. Time for problem-solving and community learning was a high priority. Learning tolerance, diversity, and problem-solving was more important than just focusing on the subject. Time pressure was not considered a major issue because the test scores were high. INVOLVED noted that as long as the test scores are high, she was given great flexibility concerning her style of instruction.

The structure of space was also included in the AIC teaching. The students were permitted to be free and open for independence. The students knew their boundaries and took over responsibility for the space that was offered to them. Trust also played a role in the success of this approach. INOVOLVED was convinced that if a teacher trusts the students, they would be able to handle situations responsibly. The students were enabled, even encouraged, to use their space freely, both actual and figurative space. 
Regarding self, in relationship to others, INVOLVED described her students as very social. They always wanted to be part of the group. No one was excluded. If there was a problem, they helped out each other. This was also the case with boys and girls. They blossomed through each other. While boys were more into the "being part of the group thing," related to sports, whereas girls were that way about beauty. Nevertheless, the most important thing for both was that they wanted to be with their peers. They wanted to be involved and integrated, instead of being excluded. Boys, for example, wanted to play ball because "all of the sports kids played ball and they wanted to be a part of that." For girls, it was more about appearance, beauty, and fashion. It was more about "who is the girly one, who is not the girly one."

These structures show that gender was not determinative. Girls might have been more enthusiastic about the arts at the beginning, and more quickly than boys. But still, boys and girls ended up being equally involved in the AIC. The difference existed that girls were more into clothes and boys more into sports. Nevertheless, both approaches were image related. Both genders were very involved in the AIC, just in different ways. There was not really a gender stigma at hand. Roleplaying, for example, resulted in positive effects on the self-images of both genders. Boys and girls enjoyed taking over other roles and having freedom to express feelings and experiences that were not directly related and connected to them and their actual personality. They enjoyed being free in their role and trying out things they could not experience if they would be themselves.

Students also enjoyed it very much to express and experience themselves and their personalities through the self-portrait exercise. They learned to take a very close look at themselves. They found out how different they were from what they expected to see. With the help of the self-portrait observations, the various and diverse selves of students were uncovered. 
INVOLVED mentioned that one could even determine the nationality of the students from their self-portrait. From an art and cognitive developmental perspective (Sims, 2011), one says that kids who fill out their self-portraits with details, instead of just painting a stick figure, are further advanced in their developmental stage than others. The way they draw can reflect their image, their self, and its developmental stage.

With the help of their progressed developmental stage of self-image, the students showed a positive self-concept by teaching their own parents. The students were proud of their knowledge and enjoyed teaching their parents, passing on their knowledge. This behavior reflected a sign of leadership, of taking over responsibility, and of being self-confident.

INVOLVED, like her students, was very aware of her body and self-image. Weight loss and dieting was a big theme between the three interviewed teachers, even before the interview officially started. Various types of dieting were discussed. Even though snacks were offered throughout the interview, none of the teachers partook because (all three of them!) were either on a diet or wanted to cut back on eating. Consequently, teachers (and parents) appeared to be as much into body image and dieting as their youngsters. Why is this the case? What influenced the parents and teachers themselves? It is not the main purpose of this study to find an answer to these questions. But, the question arises whether negative self-concepts and body images in students might be caused, or at least influenced, by the negative self-concepts and body images of their role models - parents and teachers.

In sum, INVOLVED's thoughts and structural themes of the AIC and the AIC experiences of her students were defined by various concepts: time and its reflective characteristics; space and its connections to independence; social interactions in specific groups; self-portraits that express the self-concept stage of students who teach their parents and show 
leadership through this activity; and weight-loss and dieting problems that do not just relate to students, but in the same way to parents and teachers.

\section{Comprehensive individual structural synthesis.}

In sum, the 10 participating co-researchers discussed their AIC experiences and their possible (positive) effect on the self-concept of adolescent girls and their perception of beauty. The main structures experienced by all 10 of the co-researchers were as follows: self and selfconcept in relation to time and in relationship to others, internal and external motivation, positive feedback and approval by others, positive self-concept, beautiful things and people, and space for individual development. These structures caused mainly positive thoughts about the AIC and a positive effect on the self-concept and the body image of adolescent girls.

All three groups of co-researchers sacrificed a lot of time for, and exerted much effort into the arts and the AIC. Parents took time to listen, watch, and experience the things their children provided them. Teachers used time out of their curriculum to support the AIC, despite the fact that they sometimes did not really have enough time to do so. Students took the initiative to continue artwork across the boundaries of school.

Also, the self of the students in relationship to others was always experienced in various ways. No efforts were spared to build up on relationships with others, to create a group, and build teams. Parents were involved in what was going on in school and tried to volunteer and support the teachers as much as possible. Students tried to solve issues on their own. They wanted to be a group that worked well together and encouraged each other to improve weaknesses. Teachers also teamed up to expand their students' experiences of the arts.

Motivation was one of the underlying core themes without which the AIC and its positive influences never would have been possible. Internal and external motivation had to be combined 
to cause a positive final result. Parents supported their children by showing pride and interest in their work. They took the time out of their busy schedules to come to school. They watched their children teaching and provided them with positive feedback. Students were motivated externally by the fact that teachers chose their artwork for display or by receiving positive comments from classmates, teachers, or other people from outside the school. The internal motivation was caused indirectly by their deep involvement in their artwork. They felt like they could continue their artwork without stopping, without a break.

Since this motivation was awarded with positive feedback and approval by others, students developed a positive self-concept. They were proud of their artwork and enjoyed sharing it. Parents and teachers found this willingness of sharing as a very positive effect on their children's and students' self-concepts.

The definition of beautiful things or beautiful people also presents this positive influence of the AIC on the self-concept of adolescent girls. Parents, students, and teachers likewise regarded the media and television as the "role-model" that presents thinness as ideal. Nevertheless, the whole group of co-researchers was able to come to the educated conclusion that "thinness" is not enough to be "beautiful." Students decided that external beauty, meaning the way one looks and presents oneself, is not most significant. Internal beauty, including a more pleasant personality and inner values, was also considered very important.

The participants' freedom to create their own space was strongly related to individual development. Students assumed leadership roles that they had not previously experienced. As a result, they blossomed in unexpected ways through the AIC. Parents enjoyed watching their kids grow in unanticipated directions. Shyness was overcome and parents could follow very closely how positive self-concepts developed in their own child. Teachers educated and encouraged their 
students to become self-confident and independent individuals. Decision-making-skills, as well as social skills, were acquired.

In summary, these experiences guided the female students into a positive direction. The self-concept of girls (and as this research shows, also boys) were nurtured in various ways through the AIC. However, it was not just the AIC that affected the students positively. Also other things, like student-based learning, tolerance, and diversity were taught at the same time and made a contribution.

\section{Step 7. Textural-Structural Description}

Moustakas (1994) has described this step as a textural-structural description of the meanings and the essences of the experiences for each research participant, "incorporating the invariant constituents and themes" (Moustakas, 1994, p. 121). Applying this concept to this research study, a textural-structural description of the meanings and essences of the experiences for each research participant group was created by summarizing and clustering the experiences for each group into common themes (see Appendices H3, H4, and H5). From these ICs themes charts, charts of clustered themes were created for the student, the parent, and the teacher group (see Appendix H6). For these charts, all ICs and themes (STUDENTS': no. 1.-40.; PARENTS': no. 1.-68.; TEACHERS': no. 1.-64.) were included.

At the next step, only relevant themes were included. Relevant themes were those mentioned during the research interview by at least three of the four students, two of the three parents, and two of the two teachers/ university liaison. Themes that fulfill these requirements were also consistent with the validated ICs and themes for the students: no. 1.-18.; parents: no. 1.-21.; and teachers: no. 1.-28.; (see Appendix H8). New common thematic portrayals of the 
experiences for each of the participating groups were created from these themes and by including the ICs (see Appendix H9).

The following descriptions of the AIC experiences for each of the co-researcher groups answered the three minor research questions which, in turn, helped to answer the major research question of this study in the final step.

\section{Minor research question 1: how do young ladies who completed an art-integrated curriculum (AIC) describe their experience?}

All four of the students mentioned six core themes (see also Table 6) that mainly determined and influenced their AIC experiences:

1. Everything related to the arts or the AIC caught their interest;

2. Positive effects, characterizations, and experiences of the arts or the AIC;

3. The various skills that were acquired through the AIC;

4. The positive influences of the teachers, the school, and the environment;

5. The various topics related to the self-concept;

6. Beauty, both internal and external;

All four of the students were interested in almost everything that was in some way related to the arts or the AIC. All of them liked the visual arts, to draw, and to paint. Some of the coresearchers preferred it to take visual arts classes in school, while others preferred pottery, painting, or drawing classes. Performing arts and theater classes were also on the list. The attention to music was outstanding, as well. Often, the students' interest was sparked because of their family involvement, for instance in music. Others developed a natural interest in music and enjoyed playing an instrument. All four of the interviewed students played at least one instrument (BAND: clarinet; PIANO: piano; DRAWING: violin; FLUTE: flute). FLUTE even 
described her music as one of the most beautiful things. She did not describe a celebrity as beautiful, as one might expect from an adolescent girl, but her music, "because the sound of the flute and having the whole band come together and playing a concert is fun. And the sound we all make as one is beautiful" (FLUTE).

Interdisciplinary learning was also mentioned by all of the interviewed students. BAND talked about her experience with Math and how the arts were integrated into calculating. DRAWING mentioned her experience with the Spanish teacher, "who also was an art teacher." She described how "sometimes she would bring in paintings and sometimes even during Spanish class we would make it art class instead" (DRAWING). FLUTE remembered her AIC experiences from English and Social Studies classes: "For English and social studies, we would have mini plays where we would have a script and act out what like happened" (FLUTE). She stressed how students learned about the civil war and historical events through the arts. This way of learning was definitively more fun than juggling numbers and dates around to try to remember them. Instead, the students could better recall historical events by learning about them through the arts. In fact, all of the AIC students defined learning through the AIC as FUN and enjoyable.

Each co-researcher found that the arts and the AIC had positive effects on them and their self-concepts. Experiences were defined very positively by all four students: "I like art;" "arts are a wonderful experience;" "lot of great things;" "exciting." In specific, the experience of going outside to take in the environment with the help of the arts was perceived as very special. This way of learning opened up a new learning space for the students. Despite the different approaches to learning, this cannot be seen as "fluff" or "laissez-faire style," as one would refer to Summerhill, a model serving alternative education in the U.S. (Gardner, 2010). Quite the 
contrary is true. Students even described the AIC experience as strict: "We went outside and we painted what we saw with different colors and it was fun, but strict at the same time" (FLUTE).

In addition to the positive effects on learning, the AIC also supported friendships and helps students become more interactive and social. There was no opportunity for hiding behind shyness. PIANO especially stressed how the AIC caused a difference in her self-concept. Before she encountered the arts, she said she was really shy and anti-social. She would always play alone at recess and never really talk to anybody. She did not have very many friends. As such, the friendships that were established through the AIC were very important for the adolescent students. But even the students that stayed at home and did not want to have many friends around could keep themselves busy with the arts. Their solution was oftentimes creativity: "I was just bored and I decided to plan my wedding." "When I am bored, I try to either start writing a story or drawing something" (PIANO).

With the help of the AIC, the students acquired problem-solving skills that did not only help them during their daily lives and routines in school and at home but often caused them to score higher on tests. In both areas, the students realized that they could solve problems themselves. PIANO, for example, talked about how she could not find the dress that she was looking for on the internet. Instead of getting frustrated, she spontaneously drew the dress herself. For BAND, the AIC helped her overcome indecisiveness and find solutions for problems. But also practical problems, like building sturdy structures or copying random pictures, had to be solved. The students had to have enough self-confident to at least try to problem-solve. Sometimes they had to find solutions as individuals, sometimes as a whole group. Different ways of thinking, being creative, and using imagination were practiced every day. Students could not get settled in to one way of thinking. As BAND described it, "the arts (...) 
made us be more like interactive and creative." Endurance, especially in practice, was encouraged by the arts. The students learned that a task could take longer than just one school day. They experienced that one might have to return to tasks to finish them and that they might look differently. As a consequence, the students learned that it might be necessary to approach things in a different way. This became especially obvious during drawing practice. Students had to be patient and return repeatedly to the objects. As FLUTE described it: "There was something new to draw every day. So a lot of our pictures took like a week to draw. (...) And paint."

These young ladies were also impacted by the teachers, the school, and the environment. Support by role-models was mentioned quite often as a theme. Teachers and older students considered mentors important for the girls' development. Anabel, an older, more experienced student, served as a very important role-model for PIANO: "She was pretty much the person who got me to not be afraid of the audience. I mean, and from then on, the audience has been like a friend to me." As these examples show, teachers and other role-models had a major influence on the development of a positive self-concept of these girls.

BAND, PIANO, and FLUTE also described their self-concepts in mainly positive ways. From their point of view, this positive attitude came from the AIC and their experiences with the arts. BAND described herself as a good friend and a helper. She also thought that she is confident. PIANO raved about herself "having a good time dancing" on stage. She thought very positively and confidently about herself. She had a positive self-concept. "I know that I am my own person and I can do whatever I want." She knew that she only had to try. "I can do things that I want to do if I just try." FLUTE talked about how the arts can help people get their feelings out and feel better about themselves. She connected this experience to sports, music, and specific artists: "When you are running, there is something about it that just makes you feel better." This 
showed what a great way the AIC is to help students feel better about themselves and to increase their self-concepts through the arts.

Beauty was also mentioned in this study, both internal and external. For these students internal beauty related more to the personality of a human being. In contrast, external beauty for them was defined by the body. From BAND's perspective, nature was beautiful. A flower or a rose was beautiful for her. But at the same time, she also referred to people on television. She thought they were beautiful and should be role-models. The theme of nature and natural beauty was also taken up by PIANO. "I think that the most beautiful thing that could possibly ever be is our earth. (...) nature, trees, caterpillars, animals, (...) I love it." FLUTE referred to two kinds of beauty when she analyzed beautiful things or people. She believed that peoples' internal and external beauty had to work together to make each individual beautiful in his or her own way. For her, there was no single all-determining beauty standard:

I think everyone is beautiful in their own way from how they look to their personality because you don't have to be slim and tall and have beautiful features to be popular. You can be short and not the prettiest, but still have a lot of friends. And your personality helps a lot with that because some of my friends may depend on clothes and like the way they look instead of their personalities to make them more popular and more pretty.

\section{(FLUTE)}

These statements by FLUTE showed how self-confident she was and how her attitude also had a positive influence on her self-concept. This was also true in her approach to media. She did not look differently of people in the media. She did not consider them role-models. She was aware of the fact that these people are normal people. This way of thinking might have been influenced by FLUTE's mother. She used to work in the media business and taught her daughter 
to be aware of this fact. In addition to beauty in human beings, FLUTE also considered "things" or "objects" beautiful. She loved music and described the sound of music as beautiful. Her attitudes about internal and external beauty were surprising for an adolescent girl.

It was interesting to see that more than external beauty played a role for the girls who completed an AIC. These girls thought deeper, were critical, and judged in an educated way. It was not just the façade or the outside view that counted for them. Instead, they recognized that internal values can cause feelings and emotions.

\section{Minor research question 2: how do parents of young ladies who completed an art- integrated curriculum (AIC) describe their experience?}

The three parents of the female students mentioned five core themes (see also Table 6) that described their AIC experience the best:

1. Everything related to the arts or the AIC;

2. Positive effects, characterizations, and experiences of the arts or the AIC;

3. Various things related to the self-concept of their daughters;

4. Internal and external beauty, specifically related to clothes and dressing up;

5. Various forms of involvement;

From the beginning of the interview the parents showed, that they had a special connection to the arts. BUSY mentioned her ownership of an art business. CARING noted her and her husband's involvement in the arts, as well. LOVING defined herself mainly as FLUTE's mother and stressed that her whole family was into the arts. The majority of the parents themselves took dance classes or art classes. All of them were in some way involved in visual arts, music, drama, or other kinds of arts. CARING grew up like many girls, being very interested in role playing and drama. She also played instruments, took lessons, played in an 
orchestra and band, and sang a lot. She also enjoyed drawing and listening to stories. The arts appeared to be part of her and her family's whole live. Attending concerts, listening to music, singing in a choir, or playing in a band were also mentioned by the mothers. BUSY practiced 12 years of dancing and took several art classes. She was constantly involved in music. Since the parents were so involved in and excited about the arts, the interdisciplinary learning approach of the AIC was perceived very positively. Each parent was able to describe an example of her daughter's interdisciplinary learning experiences. The parents listed math, reading, music, arts, sciences, literature, and history as examples. BUSY: "I remember where there were times where they would incorporate some different types of arts into even math or reading." CARING: “(...) there was music playing and, the kids were very knowledgeable, all of them." LOVING: "Yes, I remember her doing that play on Number the Stars about the holocaust (...) and history."

The AIC school received a very good evaluation from the parents. All of them recommended the school as one of the "best schools." Some of them even moved to the school district to be able to send their kids to Sunrise Elementary: “(...) I knew that Sunrise was a school that integrated the arts. And so I wanted to live in this area for that reason" (BUSY). Others transferred their daughter to this school because of the AIC. The parents described the school environment as nurturing, as well as encouraging of parent involvement.

The parents were also inspired by the positive effects, characterizations, and experiences of the arts and the AIC. All of them talked about how they experienced the arts through their daughters. They highlighted the fact that their children brought art products home from school to share with the family: "There have been lots of little things where PIANO would do something and bring it home" (CARING). This way, the parents were able to hear about or to see what their 
kids were doing in school. They experienced the AIC through their daughters, becoming indirect participants.

The parents' AIC experience was mainly characterized by positive descriptions. Independently from each other, BUSY, CARING, and LOVING described the AIC as "a great idea," "positive experience," "really nice," "wonderful," "really unique," "impressive," "amazing," "awesome," and others as "fabulous," or "phenomenal.” From their viewpoint, selfmotivation played a major role, especially intrinsic motivation. The students learned that if they were willing to try, their efforts would be rewarded. The parents were convinced that positive developmental steps were also supported by the AIC. "And the fact that she got up there on that stage with all of these other little Dalmatians and sang and danced her little heart out was a big step for her, a big developmental step for her in a very positive direction. And that was through the arts" (CARING). Self-concepts were strengthened through the arts. Recognition, confirmation, and positive feedback caused intrinsic motivation, which played a vital role. Also the ability to learn and recall was from the parents' viewpoint supported by the AIC. The artsintegrated teaching methods made it easier for students to learn and to remember in a playful way, without even realizing it. "I also think it helps them learn, when they come home and they are chanting or singing a song, they come home and sing the days of the week or months of the year and things like that, I mean it helps them definitely remember" (BUSY). Life-long learning was evoked by the AIC, instead of short-term memory. "They can still sing it to this day" (BUSY). Friends were an important component of the AIC experience. Children were able to either share their experiences with their peers or make new friends through the arts: "Exactly, you had a lot of friends and it worked out, but it was an arts-based thing, it wasn't that you were 
playing sports with a lot of friends" (LOVING). The arts caused the kids to be more interactive and social.

Furthermore, the parent co-researchers related the AIC in a number of ways to the positive self-concept of their daughters. CARING referred to her daughter as "confident" and "not as shy anymore as she used to be" before she experienced the AIC. The students also appeared to recognize their newly-developed qualities. However, they also faced the truth that they were not perfect. "I think she knows that she is a very talented and beautiful young woman, but I think that she also thinks it doesn't match up to exactly an ideal" (CARING). The attitude of seeing your strengths, but also realizing your weaknesses, was a very important experience on the adolescents' way to developing a healthy self-image and self-concept. In the real world, no one is perfect. Complete perfection is only possible on television and in the media. The mothers were convinced that their daughters had developed positive self-concepts because of their artwork being displayed or shared. For the girls, it did not really matter how the artwork was shared. An actual audience just made it more real for them. For example, the teachers often displayed the students artwork in the hallway. "And any time we came, they had lots of open houses, PIANO would bring us around and show us everything that was hers and she was very proud of it" (CARING). The student participants were willing, even excited, to show me their artwork. This was also true for stories that they had written. BAND showed me her collages (Figures 5 and 6), her mosaic cross (Figure 7), and her box (Figure 8). PIANO showed me her wedding plan drawings: the bride (Figure 9), the bridesmaid (Figure 10), the flower girl (Figure 11), and the ring boy (Figure 12). She also performed on her piano. FLUTE also showed me some of her artwork: the bear party (Figure 13) and water lilies (Figure 14). In addition, her mother showed me a clip of FLUTE playing the flute. She was very proud of her daughter. 
Internal and external beauty were also considered central to the students' AIC experiences. The parents connected the concept of beauty for their daughters to clothes and dressing up. The girls considered objects beautiful, especially in nature. One of the mothers mentioned that she thought her daughter considered the ocean or a landscape beautiful. It was surprising to me how adolescent girls that experienced the AIC leant towards nature and objects to describe beauty. One would have expected that they would rely more on human role-models in the media. For human beings, inner beauty appeared to be favored by the girls before the external one. Even if they mentioned external beauty, they praised a natural external beauty. With these characterizations and descriptions of beauty by the participants, it became obvious that beauty was not a static concept that relates only to human beings and the way they looked, even though this is often the case in the media.

The parents also referred to many forms of involvement as outstanding concepts for the description of the AIC experience of adolescent girls. Not just the participating girls, but often their whole families were involved in the arts. One of the interviewed mothers owned an art business that undoubtedly influenced her daughter. Other participating parents showed a very strong interest in selecting the school for their daughter. The parents toured all the potential schools to finally choose the perfect one. Entire families became excited about the AIC approach and applied it in their leisure time. For example, families visited art museums together. Parent involvement and integration were high priorities for participating parents. The mothers appreciated that AIC teachers called meetings at the beginning of the year to inform the parents about their ideas. The parents even established a PTG (Parent Teacher Group). Indeed, the parents were always welcome at the school. In fact, special events were organized so that parents would come in to experience what their children had learned. Parents did not just come in to see 
the results of the teachers' and students' efforts. Often, they became active themselves. As an example, all three parent co-researchers mentioned the musical of the 101 Dalmatians. BUSY: “The parents volunteered. We put on a musical, 101 Dalmatians." CARING: "We did, 101 Dalmatians and literally, more than half of the school, more like two-thirds of the school was involved in the show." LOVING: "There was a play they did 101 Dalmatians." The involvement was outstanding. Parents, students, and teachers were all integrated and part of the school—one collective body.

\section{Minor research question 3: how do teachers of young ladies who completed an art- integrated curriculum (AIC) describe their experience?}

The teachers mentioned seven core themes (see also Table 6) that described their experiences as teachers of young ladies who completed an AIC:

1. Things related to the arts or the AIC, specifically in schools;

2. Positive effects, characterizations, and experiences of the arts or the AIC;

3. Skills that were attained by the students through the AIC;

4. Influences of the teachers, the school, and the environment on the students;

5. Things related to the self-concept;

6. Numerous forms of involvement;

7. Internal and external beauty, including clothes and dressing up.

First, the teachers talked about their own connections to the AIC. All three of them taught for many years in an AIC. They mainly trained students at the third-grade level and had many years of experience. Dr. S. also worked together with these teachers for many years. As the university liaison, Dr. S. always knew what was going on at the school and even helped the teachers develop the curriculum. Although INVOLVED and MOTIVATED were retired, they 
were still very interested in the arts. INVOLVED mentioned that she would like to start a writing club during the summer and MOTTIVATED related that she owns an art business. This interest in the arts, even in retirement, showed that teaching the AIC was not just a job for them. They considered it a mission. After working in this field for so many years, the teachers noticed that the success of the AIC was not gender. For them, there was no boys-versus-girls gender issue involved. Actually, the found that there was too much focus on this "gender thing" in education. The teachers themselves experienced that the arts and the AIC crossed gender lines and were inclusive, not exclusive. The teachers especially stressed having difficulties with stereotypes, such as that girls do not like math: “(...) you always hear about math, especially girls don’t like math. (...) again that is that gender thing. Well, what we were trying to do is break that (...)" (INVOLVED). With this topic, INVOLVED and MOTIVATED raised an issue that is clearly present in our culture. For example, campaigns by Credo Action have invited people to join them in stopping JC Penney and Forever 21 "from putting more sexist clothing on their shelves" (Credo action. more than a network. a movement. , (n.d.)). Two recent articles, J.C. Penney Shirt Teaches Girls That Being Smart \& Pretty Are Mutually Exclusive (Krupnick, 2011) and Forever 21's 'Allergic to Algebra' Shirt Draws Criticism (Ng, 2011), have provided more information on this problem. These teachers worked really hard to break these false assumptions. It was their goal neither to put boys nor girls in specific categories. Instead, they consciously and subconsciously made efforts to not have anything aligned by gender.

The teachers also dedicated time to interdisciplinary learning. While the arts were integrated in all disciplines, they were not the only themes with which the students were dealing. INVOLVED, for example, worked on one unit with her students in which they supported and developed reading and math skills. They had to learn how to count and how to manage their 
money in order to buy books they were interested in reading. The students progressed immensely in each of these two subjects. MOTIVATED also mentioned that they always had more than one task or exercise going on. Concepts were interconnected and taught across disciplines. This interconnection of learning many things at one time and higher test score results caused a positive attitude towards the AIC. Even school officials were impressed by the results. The interconnection of the AIC, different learning styles, and student-based learning enabled all children to participate, not just the kids that scored high on standardized tests. Individual gifts were cultivated and valued. Everyone could contribute something in one way or another. The students complemented and supported each other with their area of specification. The AIC school received positive evaluations for the way they approached students' learning. MOTIVATED described the school as "non-traditional" because the students did not always realize how much they were learning. One male student, for example, said: “'Well, I learned more than I wanted to." Even the kids realized that this school was different. A boy who had transferred from a traditional school asked: "When are we going to have normal school?" Another boy even ascribed positive physical effects to the AIC. He said: "My goodness, my headaches are gone." This comment is a sign that the instruction that students experience could have a positive influence on them - psychologically and physically. The little boy had been transferred from a parochial school. Still, one must remember that the teachers did not depart from the curriculum. They just approached things a different, non-traditional way. Objectives and goals remained unchanged.

The teachers' experiences included positive effects and characterizations of the arts and the AIC. The interest of the students in the arts especially stood out to the teachers. Students maintained their interest in the arts, even after they had finished the AIC. They still were 
interested in the arts even outside of school. Teachers were surprised by this phenomenon. They found it interesting that students chose art over figures of the day for display in their room. "You don't see that so often, the girl that picked out the Monet for her room rather than the figure of the day" (Dr. S.). The arts were positively described by teachers. The positive influence of the AIC on adolescent girls, but also students in general, was underscored. Students were freer to express themselves and think through things with the help of the arts. Methods and strategies that they learned by reviewing a painting, looking at details, and finding differences were applied to think things through. Even struggling students seemed to be enabled to be academically and socially successful and finally accepted. Arts like dance or drama helped students to overcome their shyness and become brave enough to at least try. Students became fearless and courageous enough to at least try.

From the teachers' experiences, students acquired helpful skills through the AIC. Problem-solving skills were some of the most important ones. Risk-taking was also very significant and modeled by teachers themselves. Students were encouraged by the teachers to take risks. The students did not have to be afraid of negative outcomes resulting immediately in a bad grade. It was just part of a problem-solving process. Students were always supposed to be part of the solution. The teacher never took total control. This concept applied academically and socially in the classroom. The students also had to take over responsibility. This is a skill that they not only needed in school, but also would use outside the classroom. Existing barriers were removed and tolerance was strengthened. The students learned to accept differences: INVOLVED: "So we really looked at those differences and accepted that." MOTIVATED: "One of the things is that just tolerance meant to respect each other's differences." Dr. S.: The artworks, "they all looked very different from one another. It was never cookie-cutter art, never. 
It was never all replicating Barbie image. It looked like the people that were in the room." Each student was considered a distinct individual. Since there were so many different individuals, various learning methods were encouraged in the AIC. The students often learned by teaching others what they had themselves just learned. This was applied as a different kind of testing. The teachers assumed that students only could teach what they had learned and really knew. If the students had failed to acquire new knowledge, they would not be able to teach. This way, the students had to take over a leadership role. Both teachers and the university liaison commented on this phenomenon: INVOLVED: "At the end of our unit, they taught their unit to their parents so they became the teacher, another way of teaching leadership." MOTIVATED: "She would take on like a leadership — she would want to do more. (...) so instead of fading into the background, she really (...) [blossomed]." Dr. S.: "Both of you described these were girls that were struggling in some way when they came to you, but what you saw they blossomed into a leadership position given the opportunities that you were providing." The students became the teacher. They themselves were responsible for passing on knowledge. They had to solve problems alone, if necessary. This responsibility also contributed to the development of the students' self-concept. They had to rely on themselves. They could not just depend on adults, like their teachers or their parents.

The influences of the teachers, the school, and the environment were also considered as having an effect on the students and their self-concepts. Gifted and talented students were usually identified academically. Students that were not gifted academically, but in other areas, were not considered "gifted." This way of identifying "gifted" students could have had a very negative effect on students that were not academically gifted, but skilled in other areas. No one will ever find out because they attended a traditional school. They might have been lost in the 
system. However, this AIC school paid attention to other areas of talent. This did not mean that one should neglect academic encouragement. Nevertheless, one should seek to discover other gifts. By seeing that students could be gifted in different ways, teachers could have a positive influence on students and their future. Teachers could involve students in various processes that motivated them in different ways. All the stimuli through the arts that teachers were able to provide were very helpful to motivate students: "We were writing about the bubbles so we always - a lot of times before the writing experience, we would provide the motivation or the background for writing through an artistic experience" (MOTIVATED).

According to the teachers, serving as role-models or mentors was also important for a vivid AIC. Unfortunately, not all teachers were willing, free, and confident enough for this approach. Students needed someone to show them "how" to do things. They needed to watch in order to be free and brave enough to do the same thing or to understand what exactly they were supposed to do. By providing students with a positive self-concept through such guided observations and modeling, the teachers and the school were enabled to have some influence on the image and the self-concept of students (boys and girls). Interestingly enough, the girls who completed the AIC did not show shyness, fear, or hesitation. Teachers also never had to search for volunteers. The school environment was always one in which the students were positively influenced. It was described as safe and fun. The teacher stated that they created this environment as much for themselves as for the children because they also wanted to have a good time in the classroom. Fear was never an option for teachers or students.

The self-concept was also considered by the teachers. A positive self-concept was possible with the help of the arts. Students were confident enough to identify problems and to take over the initiative to solve problems. All three of the teachers described an occasion when 
three boys, who were not allowed to participate in sports, identified that issue and set out to solve their problem. INVOLVED: "We are always at the end of the line and never play." MOTIVATED: “(...) if you don't want me on your team because I can't play, then help me learn how to play so that I can be -.” Dr. S.: “(...) The kids had to solve the problem about, who wants the basketball and how can we be fair about sharing the basketball." Everyone was part of the solution. At first, students had to learn how to problem-solve together. They needed to be willing to take over responsibility and risk.

Self-identity was also related to the self-concept of students. They realized through the AIC that it was okay to be different. In the AIC classroom, it did not matter if one had to speak a different language to teach the parents the lesson or if one was obese. All that mattered was that one could express oneself through the arts. This attitude allowed students to develop their selfidentities independently from set standards that might have limited them at the beginning. All artwork "even if not perfect" was shared and displayed. Valuing the students' finished products gave them a positive feeling and supported the development of a positive self-concept. The students proudly showed their families what they had been working on.

Family involvement was mentioned as an important part of the AIC experience. More specifically, the involvement of parents, students, and teachers supported the atmosphere of being one big family. Everyone felt responsible when things were not going well. Everyone worked together and was reliable, which was possible because of the small size of the school.

In reference to beauty, the teachers mentioned the four characteristics that appeared to be important for female students: weight, height, seize, and beauty. The teachers never made exceptions because of weight. The teachers had obese students in their classes who had to participate in the same activities as all the other students. Height was considered equally 
important for boys and for girls. Boys worried about height because of sports and girls because of "looks." Even though height arose as a theme, the teachers did not consider it a problem. In reference to size, comments like "you are so little or you are so big" were the order of the day, a type of comment which the teachers believed "bothers [bothered] girls more than boys at that age" (INVOLVED). So, size was definitively more of an issue for girls than boys. This was also the case for beauty. Girls appeared to pay more attention to beauty than boys. Boys did not really care that much about clothes and their appearances. In contrast, girls cared very much about clothes and if they fit, especially during adolescence. Overall, teachers found very little difference between boys and girls concerning the AIC and its effects. Clearly, however, there was a difference in what was important for each gender. As a consequence, teachers had to make sure to mix up AIC exercises. They had to find some that matched boys and some that were good for girls. Teachers had to take this difference into consideration to provide equal ground for girls and boys to totally experience the AIC their way and to develop a positive and healthy selfconcept.

\section{Step 8. Integrated Textural/ Structural Description \{Composite Description\}}

In this last step, "a composite description of the meanings and essences of the experience, representing the group as a whole" (Moustakas, 1994, p. 121) was developed. This final phase was based on the individual textural-structural descriptions of all 10 co-researchers. Collected interview data were reviewed and analyzed from various angles and viewpoints. Based on these findings, the following integrated textural and structural description, also called a composite description, was created. The validation chart (see Appendix H8) was used as a starting point. The themes that are listed in this chart are those that were already preselected. The pre-selection took place with the help of themes that at least three of four students, two of three parents, and 
two of two teachers and university liaison considered important during the interview process.

The summarized themes for each group (students: BAND, PIANO, DRAWING, FLUTE;

parents: BUSY, CARING, LOVING; teachers: INVOLVED, MOTIVATED, DR. S.) were

compared and contrasted in order to identify common themes that represented the group as a

whole (see Appendix H10). With the help of this summary chart, the major research question

was approached.

Table 6. Overview of Core Themes for Three Minor Research Questions

\begin{tabular}{|c|c|c|c|}
\hline $\begin{array}{l}\frac{\text { Minor Research }}{\text { Ouestions } 1-3:} \\
\text {... who completed } \\
\text { AIC describe their } \\
\text { AIC experience? }\end{array}$ & $\begin{array}{l}\text { MROuestion 1: } \\
\text { How do young } \\
\text { Ladies... }\end{array}$ & $\begin{array}{l}\text { MROuestion 2: } \\
\text { How do parents of } \\
\text { young ladies... }\end{array}$ & $\begin{array}{l}\text { MRQuestion 3: } \\
\text { How do teachers of } \\
\text { young ladies... }\end{array}$ \\
\hline Core Themes: & $\underline{6}$ & $\underline{5}$ & $\underline{7}$ \\
\hline $\begin{array}{l}\text { Things related to } \\
\text { the arts/ AIC }\end{array}$ & $\nabla$ & $\nabla$ & $\nabla$ \\
\hline $\begin{array}{l}\text { Positive effects/ } \\
\text { characterizations/ } \\
\text { experiences of the } \\
\text { arts/ AIC }\end{array}$ & $\nabla$ & $\nabla$ & 甲 \\
\hline $\begin{array}{l}\text { Things/ topics } \\
\text { related to self- } \\
\text { concept }\end{array}$ & $\nabla$ & $\nabla$ & $\nabla$ \\
\hline $\begin{array}{l}\text { Internal/ external } \\
\text { beauty }\end{array}$ & $\bar{\nabla}$ & $\bar{\nabla}$ & 可 \\
\hline $\begin{array}{l}\text { Skills acquired } \\
\text { through the AIC }\end{array}$ & $\nabla$ & - & V \\
\hline $\begin{array}{l}\text { Positive influences } \\
\text { of teachers/ school/ } \\
\text { environment }\end{array}$ & $\bar{\nabla}$ & - & V \\
\hline $\begin{array}{l}\text { Forms of } \\
\text { involvement }\end{array}$ & - & $\nabla$ & $\nabla$ \\
\hline
\end{tabular}




\section{Major research question: what, if any phenomena may be associated with the self- concept and perceptions of beauty of young ladies who completed an arts- integrated-curriculum (AIC)?}

The analysis of the integrated textural/ structural descriptions of the self-concepts and perceptions of beauty of young girls who completed an AIC presented six main phenomena that were highlighted by the groups' (parents, students, teachers) experience:

1. Interest in music and art;

2. Positive descriptions and characterizations of art;

3. Positive self-concept through arts;

4. Interdisciplinary learning;

5. Beautiful things;

6. Self-concept in general (see Appendix H11.1.).

The overall interest in the arts, specifically music, was stressed. The students were described as being fascinated by music. All of them played an instrument and were members of the school band, orchestra, or chorus. Student co-researchers took art classes and were involved in the arts, even outside of school. A positive attitude towards the arts and a support by the family was common.

Each group of interviewees gave positive descriptions and characterizations of art. Students: "I like art." Parents: "wonderful” and "positive experience.” Teachers: "very interesting." There was no exception. Everyone was fascinated by the positive effect of the arts and the AIC.

The positive self-concept that appeared to develop in students was another phenomenon in this study. All the participants talked at some point about the positive self-concept that 
students established with the help of the arts. The interviewees described how the students blossomed in various, unexpected ways. Often, they took over leadership roles. The students either established or cultivated confidence.

Interdisciplinary learning was another phenomenon that was connected to the selfconcept and perceptions of beauty of young ladies who completed an AIC. Interdisciplinary learning referred not only to different subjects that were connected throughout the learning process, but also to social and soft skill acquirement like tolerance or problem-solving. These skills enabled the girls to trust in themselves, to be confident, and to display a positive selfconcept.

The concept of beauty played an important role, as well. While one would assume that beauty in connection to adolescent girls would refer mainly to physical beauty, this was not the case. These young ladies' perception of beauty was not just focused in one direction but described in various ways. For example, beauty was linked to music and arts, or artwork in general. The beauty of the nature and the environment was also a major theme described in detail. The love and necessity of nature was clearly expressed: "I love it. I mean, without nature, I don't think I could really keep going" (PIANO). Even when the conversations finally touched upon human beauty, natural beauty was favored over artificial beauty. Even though people on television were considered beautiful role-models because of their thinness, the main focus was not on the people in the media.

The self-concept itself developed as a phenomenon in the conversations by all participants. It was mainly described as positive, even though it sometimes was described as critical, but not overly-critical. Though shyness and retentiveness had to be overcome, the selfconcept of AIC students was basically considered as very positive. 
The above-mentioned themes presented the six main phenomena described by the whole group of co-researchers. They defined the self-concept and perceptions of beauty of young ladies who completed an AIC from the perspective of the parents, the students, and the teachers.

The following 12 themes were also mentioned but will not be considered in detail because they represent phenomena that were only relevant for two of the three groups: 1) took art classes; 2) role-model (support); 3) teachers' positive influence; 4) learning through the AIC is FUN; 5) problem solving; 6) friends (interactive/ social); 7) learning and recall are supported by the AIC; 8) (family) involvement; 9) (parent) involvement, integration, and volunteering; 10) displayed or shared artwork; 11) positive evaluation of the AIC school; 12) connection to arts; (see Appendix H.11.2.).

Themes that were only significant for one of the three groups will not be considered in more detail in this part of the research study, but can be followed up on independently by the reader (see Appendix H11.3).

After this presentation of the analysis of data and the actual phenomena that were associated with the self-concept and perceptions of beauty of young ladies who completed an AIC, the next chapter contains the summarized findings of the whole study. The outcomes are described and interpreted in reference to prior studies, including Devono's (2009) research. Possible future research projects are discussed, as well as improvements of the study. Implications for this research study (Copen, 1992) are listed, as well. 


\section{Chapter 5: Discussion}

\section{Summary}

In chapter one, I provided a short introduction to my research themes: perceptions of beauty, self-concept, and the AIC. I connected these research themes with related concepts like beauty standards and change, "multiple realities" and "wide-awakeness," body images, standardization, comparison factors, and the importance of the arts. Furthermore, I provided basic thoughts (by no means a philosophical study) on these ideas by well-known philosophers and educators in the field (Dewey, 1934/1980/2005; Greene, 1978; Butler, 1993; Eisner, 2002b; Bordo, 2003), to provide a short overview for the reader. I described various issues, including the unreachable beauty standards that females are facing. I talked about how these standards appear to be set by our media-driven and gendered society and present major challenges for adolescent girls. My interest in this topic and its challenges was personal. I, myself, was affected by body image and self-concept struggles as an adolescent girl. I talked about how my scoliosis had a negative impact on my whole life but more specifically on my self-concept and my perception of beauty. The arts and the AIC came into play when I described my own experience with the arts, particularly drama and visual arts, and how they gave me confidence and strengthened my selfconcept. These art experiences taught me to not just focus on external but also on internal beauty. The idea of transferring and applying my positive experiences with the arts to the school curriculum (AIC) and adolescent girls led me to my research question: What, if any, phenomena may be associated with the self-concept and perceptions of beauty of young ladies who completed an AIC?

In chapter two, I carefully researched and reviewed the available literature for my field of interest. At the beginning, I focused on the definition and description of beauty (The Andy 
Warhol Museum, 2008), phenomenology (Moustakas, 1994; Patton, 2005), and self-concept (Bardone-Cone et al. 2010; Epstein, 1973). A special focus was put on my research method of phenomenology. I mentioned how my research study is based on Moustakas' (1994) phenomenological research method framework for the analysis of data and what kind of role this plays for this work. Questions about arts integration and the AIC concluded this section: "What is arts integration or the AIC?" (Mishook \& Kornhaber, 2006; The John F. Kennedy Center for Performing Arts, 2008), “Why arts integration?” (Catterall, 1990; Cornnett, 2011; Csikszentmihalyi, 1990, 1993, 1997a, 1997b; Csikszentmihalyi and Csikszentmihalyi, 1988; Gullatt, 2008;), and "How/ how to integrate the arts" (Arts Education Partnership, 2000; Gullatt, 2008). Unfortunately, I learned that related research was absent from the field. I inferred from this discovery that there was a need for this research study on possible phenomena that might be associated with the self-concept and perceptions of beauty of young ladies who completed an AIC. The arts and their relation to society, school culture, and beauty standards were explored more in depth. Problems and struggles of girls and women (Pipher, 1994) were described, like beauty standards, image, role, and identity (Latta Macintyre, 2004; Silverman, 2010). Also, the male definitions of beauty and their consequences (Dewey, 2005; Pipher, 1994) were touched upon, as well as the negative results that these notions can have for adolescent girls (Hoskins, 2002; Becker, 1999; Duncum 2005; Clark \& Tiggemann, 2007).

Chapter three described the methodology that I applied in this phenomenological research study. At the beginning, I talked about the selection of the parent, student, and teacher participants, their recruitment, and the selection of the setting/ school site of this study. This section was followed by the research design. The major and the three minor research questions were posed, which made a phenomenological research design indispensible. Moustakas' (1994) 
phenomenological method, in combination with Patton's (2002), served as a basic four-step framework that included

1. Epoche;

2. Phenomenological reduction;

3. Imaginative variation;

4. Synthesis of texture and structure (Moustakas, 1994, pp. 84-102).

After the description of the research procedures, the data analysis followed. The data collection with the interview process was displayed, as well. Moustakas' (1994) "Modification of the Van Kaam Method of Analysis of Phenomenological Data" (p. 120) was applied to the collected interview data of the 10 co-researchers. These seven plus one steps (Moustakas, 1994; Patton, 2002) consisted of:

1. Listing and preliminary grouping;

2. Reduction and elimination;

3. Clustering and thematizing the invariant constituents;

4. Final identification of the invariant constituents and themes (validation);

5. Individual textural description;

6. Individual structural description;

7. Textural-structural description;

8. Integrated textural/ structural description (Moustakas, p. 120 ).

In order to validate these phenomenological methods of description and data analysis, I chose five ways of triangulation (see also Figure 20): (a) interviews, (b) memoing, (c) artifacts, (d) peer-review, and (e) referencing of a past report study (Devono, 2009). 


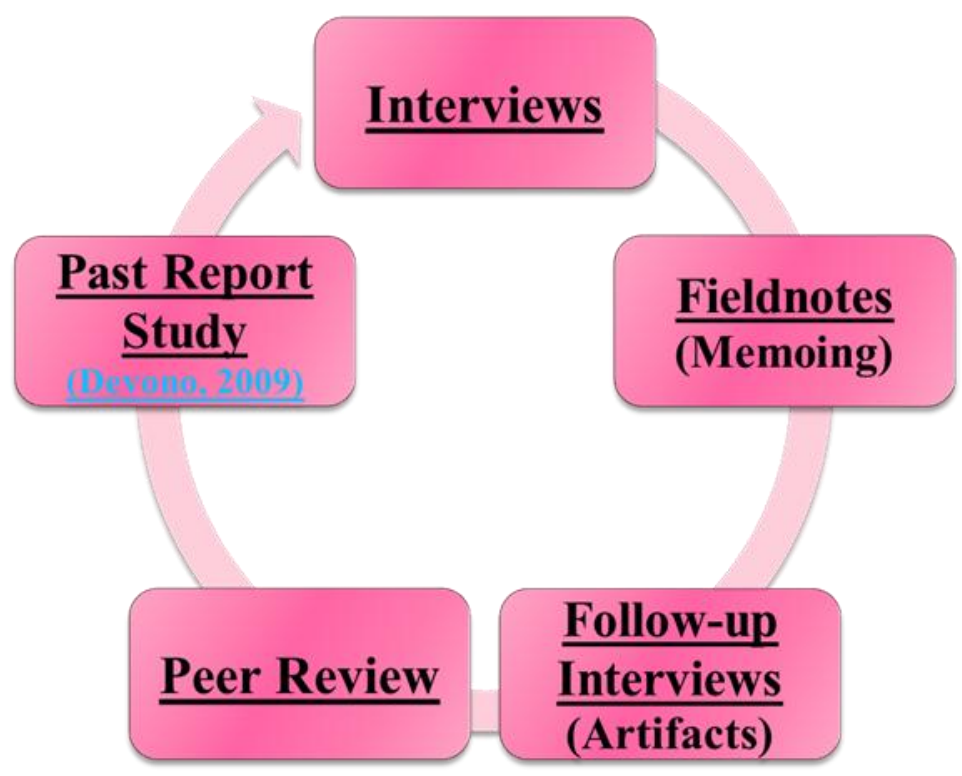

Figure 20. Five ways of triangulation.

Chapter four displayed the textures and structures of my phenomenological research findings. Core themes that determined and influenced the AIC experiences of parents, students, and teachers were revealed (see also Table 6). I discovered universal structures for each of the co-researcher groups. The female students who completed an AIC expressed their experiences through six core themes:

1. Everything related to the arts or the AIC caught their interest.

2. Positive effects, characterizations, and experiences of the arts or the AIC were mentioned.

3. The various skills that were acquired through the AIC were considered important.

4. The positive influences of the teachers, the school, and the environment caught their attention.

5. The various topics related to the self-concept contained meaning.

6. Beauty, both internal and external, was central. 
The parents of young ladies who completed an AIC described their experience with the help of five core themes:

1. Everything related to the arts or the AIC caught their interest.

2. Positive effects, characterizations, and experiences of the arts or the AIC were mentioned.

3. Various things related to the self-concept of their daughters were listed.

4. Internal and external beauty were significant, specifically related to clothes and dressing up.

5. Various forms of involvement were significant for them.

The teachers of young ladies who completed an AIC focused on seven core themes, while describing their experiences:

1. Things related to the arts or the AIC specifically in schools were noticed.

2. Positive effects, characterizations, and experiences of the arts or the AIC were listed.

3. Skills that were attained by the students through the AIC had meaning.

4. Influences of the teachers, the school, and the environment on the students were taken into consideration.

5. Things related to the self-concept seemed important to them.

6. Numerous forms of involvement were found.

7. Internal and external beauty, including clothes and dressing up, had special meaning for them.

Through these core theme listings, it became readily apparent that the different groups considered some of the same core themes important. The very nature of phenomenological research became evident. Referring back to chapter two, Patton's (2005) well-known 
phenomenological research question illustrated this perfectly: "What is the meaning, structure, and essence of the lived experience of this phenomenon for this person or group of people?" (Patton, 2005, p. 104). At first, one starts out with one person's experience, since the intent of phenomenology is "to understand the phenomena in their own terms - to provide a description of human experience as it is experienced by the person herself" (Bentz \& Shapiro, 1998, p. 96) and after this step, one moves on to possibly discover the phenomena that a whole group of people had in common. For this research study, I found six phenomena that were associated with the self-concept and perceptions of beauty of young ladies who completed an AIC by all three of the groups - the parents, the students, and the teachers:

1. Interest in music and art.

2. Positive descriptions and characterizations of art.

3. Positive self-concept through arts.

4. Interdisciplinary learning.

5. Beautiful things.

6. Self-concept in general.

In this final chapter of my dissertation, I summarized the research outcomes and findings that I discovered about the experience of any phenomena that may be associated with the selfconcept and perceptions of beauty of young ladies who completed an AIC. I also discussed personal implications that this study had for myself (student perspective), societal-practical implications (parental and community perspective), and professional-intellectual implications (schools or teachers perspective; e.g. Devono, 2009) that are relevant to the field of education. A comparative listing (see Table 7) was displayed and discussed. It showed similarities between the findings of this study that focused on the AIC experiences of parents, students, and teachers and 
Devono's (2009) study, which mainly focuses on the implementation of an integrated arts curriculum and the teachers' decision making. Research methods and procedures were critiqued. Recommendations were provided for future studies and possible future research projects were briefly outlined. At the end, closing remarks were displayed.

\section{Outcomes}

It was the goal of this research study to discover the themes, meanings, and essences of the experience of "what, if any, phenomena may be associated with the self-concept and perceptions of beauty of young ladies who completed an AIC." The experiences were related by 10 co-researchers (three parents, three students, and two teachers/ one university liaison) and were described in the everyday language used by the participants. The data collection took place through in-depth face-to-face interviews with the research participants. The research design and methodology, including the phenomenological analysis of the collected data, provided an insight into six phenomena that were associated with the self-concept and the perceptions of beauty of adolescent girls who completed an AIC.

\section{Research and findings.}

During the last few years, debates have arisen about the importance of arts integration in our educational system and its curriculum (American for the Arts, (n.d.); Ruppert, 2006). Nevertheless, instruction in the arts is being slowly eliminated in our schools in favor of the allegedly more important "core subjects" (Findlay, 2011) that are being analyzed in standardized testing. Despite this fact, some parents still favor the arts and would like their children to experience an arts education. These parents have become frustrated with the budget cuts the arts are facing in our educational system. As a consequence, they have come together in parental initiatives to "save" the arts in our schools. The National Art Education Association, for 
example, writes that they receive calls every week or emails "from parents asking for help and materials that can help them advocate keeping visual arts programs in their schools" (Association, 2003). Oftentimes, when parents feel like they cannot do anything about the elimination of the arts from the system, they do not have any other choice than to privately enroll their children in community-based art programs. Interestingly enough, these programs and centers are highly in vogue for parents and their children. It appears that those parents have a reason to aid their children's participation in the arts. In my opinion, parents intuitively know that the arts can have a positive influence on their children. Research studies (Henry, 2002; McGowan, 1988) show that the arts can be helpful in the development of various skills in children. Specifically, in Henry's (2002) research study, the positive academic effect the arts can have on students was stressed.

Even though there is ample research available that supports the idea of a positive influence of the arts on children (Gullatt, 2008), specifically in reference to academics, this research study attempted to drive the research on arts integration a step further. The integrity of the arts should be kept in mind and the idea of the "art for the art's sake" (l'art pour l'art) should not be too easily abandoned because of improved test scores. Instead, they should be embraced by investigating any (possibly positive) effects the AIC and the arts in general can have on the self-concept and perceptions of beauty of adolescent girls who completed an AIC. This attempt called for the major research question of this study: What, if any, phenomena may be associated with the self-concept and perceptions of beauty of young ladies who completed an AIC?

The review of literature for this study did not result in any findings of research that explored the experience of the AIC in reference to the self-concept of adolescent girls and their 
perceptions of beauty. Therefore, at this time, very little is known about the textural qualities and the structural dynamics of the AIC experience of adolescent girls and their perceptions of beauty.

Accordingly, the literature review for this study proved to be difficult. The literature search only disclosed information on research addressing some of the main aspects of this study individually but not in connection with each other (e.g. self-concept (Epstein, 1973); beauty (The Andy Warhol Museum, 2008). The literature review was expanded by referring three questions concerning arts integration/ AIC: (1) What is arts integration/ AIC? (The John F. Kennedy Center for Performing Arts, 2008), (2) Why arts integration? (Why your child need the arts, (n.d.)), and (3) How to integrate the arts? (Arts Education Partnership, 2000; Gullatt, 2008). Literature was also found on the arts and multiple perspectives in a culture of change (Greene, 1995; Pipher, 1994; Wilber, 1997/ 1997, November), referring back to themes like the struggles of girls and women, beauty standards, and image, role, and identity (Pipher, 1994). Themes like the male definition of beauty (Dewey, 2005; Latta Macintyre, 2004; Pipher, 1994), the negative self-concept and resulting disorders (Pipher 1994; Silverman, 2010), media (Hoskins, 2002), culture (Duncum, 2005; Walshaw, 2001), body dissatisfaction, and low self-esteem (Clark \& Tiggemann, 2007) provided plenty of research literature.

Still, all these writings that were available for the literature review provided only a general insight into the aspects of the AIC, beauty (including its standards), self-concept, and struggles adolescent girls have to face. They did not disclose any information on the individual experiences of those that were involved in the AIC, the parents, the students, and the teachers.

This is how my research and its findings can be distinguished from the ones in the literature review. I attempted to drive the research further by intertwining the notions of adolescent girls, beauty, self-concept, and the AIC in my investigation of any phenomena. 
These research findings underwent triangulation (Creswell, 2007) - see also Figure 20 and were listed in the following order. First, results from the conducted interviews and their data analysis outcomes (Copen, 1992; Moustakas, 1994) were listed. Second, findings of the field notes were shown, a process which is also called "memoing" (Groenewald, 2004). Third, the follow-up process for interviews and its most important results were described, which can also be referred to as the artifacts (Gay, Mills, \& Airasian, 2009). Fourth, the outcomes of the peerreview (Creswell, 2007) were revealed. Finally, the comparison findings of this research study with a past report study (Devono, 2009) were outlined.

\section{Interview and data analysis outcomes.}

For the interview process and the data analysis outcomes of the experiences of adolescent girls who completed an AIC, the voices of those involved in the AIC (the parents, the students, and the teachers) were considered and summarized in core themes that were common for each of the co-researcher groups.

The student-co-researcher group described their experiences through six core themes. The first core theme for students included everything related to the arts or the AIC. They especially referred to their interest in music. All three of the students played an instrument (BAND - clarinet; PIANO - piano and saxophone; DRAWING - violin; FLUTE - flute) and took art classes (BAND - painting; PIANO - theatre; DRAWING - pottery, painting, drawing; FLUTE - performing arts). Interdisciplinary learning, such as combining a foreign language with visual arts or social studies with drama, played a major role for all of them. They also described learning through the AIC as "fun."

The second core theme included positive effects, characterizations, and experiences of the arts or the AIC. The students labeled the AIC as: "exciting" (BAND), "wonderful 
experience" (PIANO), "lot of great things" (DRAWING), and "very fun" (FLUTE).

Experiencing an environment outside the classroom was rated very highly. The interactive and social aspect of the AIC, especially in reference to friendships, was considered of great value: "I had a lot of friends" (FLUTE). Furthermore, the arts were identified as a helpful tool against boredom: "It gives me something to do" (FLUTE).

In the third core theme, various skills that were acquired through the AIC were considered important. For example, problem-solving was one of these skills: "Once you got to the problem, you got to color" (BAND). The students stressed how the AIC helped them think differently, to be creative, and to use their imaginations. Endurance in practice was also mentioned by all of them. They enjoyed it to take one week to work on an art project. It was a new and exciting experience for them to sit by a flower every day for a whole week and draw it from different perspectives.

The fourth core theme captured the positive influences of the teachers, the school, and the environment. The students enjoyed having a role-model for support. It did not matter to the students who the role-model was, whether it was a teacher or an older student who mentored them.

In the fifth core theme, students described to the self-concept, in specific a positive selfconcept that was acquired through the arts: "I am happy with myself" (BAND), "I am a much better person for it [arts]" (PIANO), "They can make you better" (DRAWING), "[arts] made me feel smart" (FLUTE). Despite the fact that a negative self-concept was not completely absent, e.g., "that did not turn out so well" (FLUTE), the positive self-concept predominated.

Finally, in the sixth core theme, beauty (both internal and external) was central, especially in relation to clothes and dressing up. The students mentioned that some classmates 
depended on clothes instead of personalities, which should not have been the case. For the students, internal beauty was at least as important as external beauty. Beautiful things were mainly found in nature (BAND - flower, rose; PIANO - our earth; FLUTE - flower painting) instead of media.

The parent-co-researcher group described their experiences through five core themes. The first core theme included everything related to the arts or the AIC. All three parents mentioned that they were connected to the arts through their work (BUSY - owns her own art business; CARING - she and her husband work in the arts field; LOVING - used to work in the arts/ media business). The three mothers were interested in the arts and some even took art classes at some point (BUSY - danced for 12 years, took art classes, and was involved in music; CARING - took drawing classes, played an instrument, and sang in the choir). Interdisciplinary learning that was linked to the AIC was fundamental for all the parents (BUSY - songs in Math; CARING - arts in sciences; LOVING - music in story telling). Sunrise Elementary received a very positive evaluation from all the mothers as an outstanding AIC school.

The second core theme summarized positive effects, characterizations, and experiences of the arts or the AIC. All the parents enjoyed experiencing the arts through their daughters by hearing about the different things they did in school. The mothers also mentioned things that their children brought home from school or how they were invited at the end of a unit. The parents used positive descriptions and characterizations to describe the AIC: "great idea," "wonderful experience" (CARING), and "phenomenal" (LOVING). Also, the positive influence of the AIC was related to self-motivation (BUSY), benefits of self-esteem (CARING), and developmental steps in positive direction (LOVING). Intrinsic motivation was mentioned and linked to recognition, confirmation, and positive feedback from art. Furthermore, the parents 
were convinced that learning and recalling were supported by the AIC. But not just the academic effect, also the interactive and social one was ascribed to the arts: "[FLUTE] had a lot of friends (...) but it was an arts-based thing, it wasn't that you [FLUTE] were playing sports with a lot of friends" (LOVING).

The third core theme referred to certain characteristics related to the self-concept. The mothers described their daughters' self-concepts as positively influenced through the arts. They stressed how much their daughters enjoyed displaying and sharing their artwork, how proud their daughters were of their work, and how much value they would ascribe to it.

The fourth core theme discussed internal and external beauty, also in relation to clothes and dressing up. The parents described things that they thought their daughters found beautiful. Interestingly enough, none of the parents mentioned celebrities or models. Instead, natural objects like a rose, a flower, or the ocean were mentioned.

The fifth core theme stressed various forms of involvement, such as in their families and the school. Family involvement was shown in various ways. One parent owned an art business, while others showed involvement by visiting several kinds of schools to select the best one for their child. Others involved their whole family by visiting art museums. The parents also enjoyed their welcomed involvement at school. They mentioned meetings at the beginning of the school year, parent teacher groups that were formed, open houses for parents, or units to which the parents were invited. Their willingness to volunteer received attention, as well. All three of the parents talked about the musical 101 Dalmatians, which was a parent-initiated after school program, which involved students, parents, and teachers. The students' involvement in the arts outside of the school also received attention. Parents shared how their children asked for artwork in their rooms and how they showed interest in the arts (for instance art museums). 
The teacher-co-researcher group described their experiences through seven core themes. The first core theme referred to everything related to the arts or the AIC. The two teachers (INVOLVED and MOTIVATED) and the university liaison (Dr. S.) stressed their own connections to the arts. INVOLVED and MOTIVATED taught in an AIC at a third grade level for many years. Dr. S. had the unique experience of being involved for ten years in the AIC project, serving as a university liaison. From the beginning, the teachers stressed that the AIC was not a "gender thing." From their point of view, it was not boys versus girls, even though small differences existed. Interdisciplinary learning was also a major theme for the two teacher co-researchers and the university liaison. Simultaneous learning (e.g. tolerance, student-base learning, and others) was especially emphasized. Clearly, the teachers expressed a pro-AIC point of view. Their positive opinions were not just supported by high standardized test scores but also by positive social and individual outcomes of different learning styles, student-based learning, the participation of all children, as well as many ways for the students to show intelligence and to be gifted. This positive effect was mainly ascribed to the school's non-traditional approach and its small size, which enabled the school to reach across different skill sets. Learning was approached in a different way. Still, this did not mean that the teachers neglected the required curriculum. The teachers just looked at things differently for students. The three educators agreed that learning through the AIC was fun, at least as long as units were not eliminated to teach for the test.

The second core theme included positive effects, characterizations, and experiences of the arts/ AIC. It was stressed that the students maintained their interest in the arts even after they had completed the AIC. The teachers mentioned that the arts encouraged students to freely express themselves and to carefully process problems. The educators highlighted how the arts 
brought out unique gifts of students, how they benefited all students, and what a positive physical effect they could have on students.

In the third core theme, skills acquired through the AIC received special attention (e.g. problem-solving). The teachers talked about how they encouraged the students to take risks. Instead of exerting too much control over the students, the teachers assisted and helped the students find the solution for a problem. Students were part of the solution and learned to solve both social and academic problems. Responsibility had to be taken over by all students.

Tolerance was also acquired. Barriers to certain groups were eliminated. Discrimination was not accepted. Instead, differences, individuality, all comments, and all cultures were accepted and validated. Also, leadership roles had to be taken over by all students. Instead of taking a test, the students had to teach a significant other about the arts to show what they had learned.

In the fourth core theme, the school, and/ or the environment received attention. The teachers expressed their concern that gifted and talented students are usually academically identified, which put the artistically gifted children at a disadvantage. The educators also stressed the importance of role-models and mentors, but were concerned that teachers were often not willing to serve as such. All three of the educators mentioned beauty as a concern for girls. From their point of view, girls paid more attention to beauty than boys and worried a lot about weight, how tall they are, and the size of clothes they wore. The three co-researchers agreed that schools could have a major impact on the images and the self-concepts of girls AND boys. They wanted to be tall to be good in playing sports while girls wanted to be little to look cute. Nevertheless, the students did not hesitate to volunteer or to fulfill a task, neither girls nor boys, big or small. What the students were most worried about was being part of the group. They did not want to be left out. The educators also stressed a positive influence that a teacher can have on the students, 
namely the power to involve, motivate, and engage students. The educators were convinced that a safe and fun environment for students and teachers was the key for the AIC and its positive effects to blossom.

The fifth core theme related to the self-concept. The teachers thought that a positive selfconcept could be acquired through the arts when confidence and comfort level were supported. The arts gave students a chance to build up confidence and to be proud of their achievements. Self-identity was also mentioned by the teachers. From their point of view, the students learned through the AIC that it was okay to be different or to see things differently. Also, the displaying and the sharing of artwork had a positive influence on the students. They saw that their artwork was valued, which in turn affected their self-concept positively.

The sixth core theme covered internal and external beauty in relation to clothes and dressing up. From the educators' perspective, girls desired to look pretty and cute and were more concerned about size than boys (INVOLVED).

In the seventh core theme, various forms of involvement emerged. The educators specifically mentioned teacher, parent, student, and family involvement. In their opinion, all the concerned parties had to be involved for an AIC to be successful.

Even though the AIC experiences of the parent, student, and teacher co-researchers were slightly different from each other, common themes for all three groups could be recognized. These overlapping themes represented the six main phenomena of this research study that were associated with the self-concept and perceptions of beauty of young ladies who completed an AIC:

1. All three of the groups stressed an interest in music and art.

2. Positive descriptions and characterizations of the arts and the AIC were listed. 
3. A positive self-concept, developed through the arts was discovered.

4. Interdisciplinary learning was ranked highly by all three participating groups.

5. Things were more often considered beautiful than people.

6. The self-concept in general achieved high attention.

\section{Field notes (memoing).}

The field notes that were taken for each of the four interviews reflected many of the core themes that were found for the parent, student, and teacher co-researchers individually, or as a whole group. The themes and phenomena that recurred in the field notes were summarized for each of the four interviews.

The field notes from my first parent and student interview (I-P-BUSY \& I-S-BAND29.04.2011) showed that BUSY enjoyed hearing about her daughter's arts experiences in school and that she was disappointed by the fact that her daughter did not have an AIC in school anymore. From BUSY's viewpoint, the absence of the AIC resulted in her daughter not having so much to talk about anymore when she returned from school. BUSY loved to see the art products that her daughter brought home. In reference to beauty, BUSY thought that BAND more often considered objects as beautiful, instead of human beings. The musical that was offered by parental volunteers as an after school program also appeared to be of major interest to BUSY, especially because she pointed out that she was a volunteering parent in school. Her involvement and excitement about the arts was not just shown in her voluntary work at school and in her arts business, but also in the way she and her family chose the area in which they now live. They selected the area because of the AIC school. BUSY also stressed that her family, especially her father who has an arts and music background, might have influenced her interest in the arts. Concerning self-concept and body image, my field notes showed that I thought it was 
of interest to mention that BUSY was convinced she should go on a diet because of her BMI, which was unhealthily high. My field notes also illustrate that BUSY smiled when BAND mentioned that she felt like a role-model to her sister and friends.

My notes for BAND showed that she enjoyed it very much to be a role-model for her friends and family, since friends and social contacts were of major interest to her. My notes also showed that BAND considered the AIC more fun than the curricula through which her friends went. Concerning beauty, I noted that BAND at first only considered objects as beautiful. Later on, she mentioned human beings as well but only referred to the ones on TV. She also mentioned people on TV who were trying to lose weight, but she did not relate this idea in any way to herself and her body. Still, it seemed like she was aware of weight loss. It also was special how motivated BAND was to show me her artwork. She stressed that she liked the approval she got out of her artwork. She mentioned twice that she had some artwork she would like to show me but did not get it back from the teacher, yet. I also considered it noteworthy that she stressed to me that it was okay to be different and that one did not have to match what the society predetermined.

The field notes for my teacher and university liaison interview (I-T\&L-INVOLVED, MOTIVATED, \& Dr. S.-03.05.2011) also revealed important information. After this meeting, I took notes of what had happened before the actual interview. The two teachers, the university liaison, and I met at a coffee house. Before the official interview began, the three co-researchers used the remaining time for catching up. I had not even mentioned the theme of my interview yet, when one of the three participants noticed that the other one had lost some weight. She complimented her and stated that she looked great. Immediately, a whole conversation started about dieting. Two of the educators mentioned how much they liked the new 17-day diet that 
they were doing and that they already had lost quiet some weight. Another one of the participants mentioned that she and her husband were planning on doing this diet, as well. Also, shortly before the interview started, I wanted to get some coffee and a snack for the participants. Uniformly, all of the educators stated that they did not want anything to eat, just coffee and tea. As these pre-interview notes show, not just students are into dieting and face self-concept and body image struggles but also their educators. Throughout the rest of the interview, I took other field notes that I considered important. For example, the teachers appeared to be very motivated and excited about the arts and their arts classroom. They also felt strongly about the same kind of involvement of girls and boys in reference to the AIC. The teachers stressed how much they refused to distinguish between genders. They did not want any gender stigma to arise in their classroom. It was the teachers' goal to work against any kind of gender stereotypes. I noted that both emphasized that girls and boys were both equally involved in the AIC, just in different ways. I also sensed that the teachers were frustrated about the fact that test scores of AIC students were always high but no one thought to consider why this was the case. The teachers also stated their concern that an AIC educator could not expect from all colleagues the type of involvement that was necessary in the AIC classroom. From their viewpoint, an AIC teacher could not be easily embarrassed or too shy to make a fool out of him or herself. One of the teachers also raised the question of age. She mentioned how her granddaughter talked about "girly girls" and "non girly girls." She wondered whether this thought process of her granddaughter had to do with age and the stage of adolescence she was in at that time.

The field notes for my second parent and student(s) interview (I-S\&P-PIANO, DRAWING \& CARING-14.05.2011) also contained important information about the main themes and phenomena that were discovered in this research study. Before the interview started, 
the two students came in the house and showed us very proudly three caterpillars that they had found outside on their walk through the fields. PIANO and DRAWING even had named the caterpillars, and kept them inside during our interview. This showed their apparent love and excitement for nature. I asked the girls how they managed to know which caterpillar had which name since they seemed to look all the same. Interestingly, PIANO answered that they actually all looked very different and that it was easy for her to distinguish them. This showed how much attention the girls payed to details. I also noted that the mother, CARING, had beautiful lily flowers on her kitchen table. I wondered if that might have been a sign that the family valued nature. CARING stressed that she and her husband were both in the arts. From CARING's point of view, PIANO's interest and fascination for the arts could result from their family involvement in the arts. I further noted that CARING talked about losing weight and emphasized that it would be for health reasons, even though she had mentioned a concern and dissatisfaction about her weight. So far, it has to be noted that all the parents and all the educators mentioned that they would have liked to lose weight. The concern about beauty, weight, and self-concept issues did not appear to affect just adolescent girls but also their mothers and educators. CARING moved on by describing the moment when PIANO lost her fear of being on stage. It was special how CARING talked about before and after "it happened." CARING was convinced that PIANO only lost her fear of being on stage because of her mentor. The mentor, an older female student, was able to convince PIANO to go out on stage and dance while CARING, as her mother, was not. This showed the importance of role-models for children. PIANO did not seem very shy, but selfconfident. She was only shy and reserved when it came to her looks and things she considered beautiful. She actually mentioned during the interview that she felt uncomfortable with questions relating to looks and beauty. It was the same case with DRAWING, who showed her discomfort 
by occupying herself with drawing. The activity of drawing appeared to be a way for her to escape from reality. At the end of the interview, CARING asked if PIANO would be willing to play the piano for me. She agreed and played a beautiful piece on the piano, and I noticed that she was proud of herself afterwards. I also want to add in this section that CARING took me to my car. On the way, she told me how important she found my research. She even came up with an example to support this statement. She told me that she was not sure about what exactly it was, the age, the environment, media, culture, or the peers but to her it seemed like as soon as her daughter started to attend middle school, she changed. CARING thought that PIANO started to pay more attention to the way she looked. She grew her hair out and even wanted to take showers now, when she sometimes refused to do so before. From her point of view, this change happened in such a short period of time. CARING felt so strongly about this that she went back in the house to get a picture of PIANO for me. The photograph, not very old, showed that CARING was right. PIANO had changed a lot. CARING described PIANO's development as a change from a tomboy to a young woman. In the picture, PIANO's hair was short and her postures were very "boyish." Singe beginning middle school, however, CARING said that PIANO had grown her hair out and had started to look like a young woman. Major changes had taken place that CARING enjoyed. Nevertheless, she asked herself why these changes had taken place. She wondered whether it is just the age of adolescence that made youngsters change? Do the media, the culture, and the environment have an influence? Is it the "male driven society" that starts to have an influence and a meaning for these young girls? CARING stated that she had not found an answer for these questions yet, but she expressed her hope that this research would result in greater funding for the arts so that instead of being undervalued the arts should be considered as important as all the other subjects. 
The field notes for the third and final parent and student interview (I-P\&S-LOVING \& FLUTE-24.05.2011) also referred to themes and phenomena that had arisen during the data analysis process. My field notes stated that FLUTE repeatedly used the word "fun" in connection to her AIC experiences. She really liked the fact that the AIC was fun and hands-on, but her mother LOVING did not really share FLUTE's opinion. LOVING raised the concern that Mathematics education was lacking in the AIC. As a reaction to her mother's comment, FLUTE continued the interview by expressing a "well," followed by a long pause, and long listing of all the things she thought she learned through the arts. It was only after FLUTE's statement that LOVING realized how much her daughter really had acquired through the arts. LOVING started to remember things that she had forgotten. It almost seemed to me like the mother had a very different definition of learning at the beginning of the interview. From her perspective, learning Mathematics from a book, not through the art, was learning.

Before the interview, LOVING privately had expressed some concern of FLUTE being at that age where she might not answer all my questions or might answer most with "I do not know." Despite this fear, my field notes showed that FLUTE answered all my questions in a very polite and detailed manner. She did not say a single time, "I do not know." FLUTE described herself as shy and so did her mother. I am not really convinced this is the case. In my opinion, she definitively did not just go with the flow. FLUTE, for example, talked about how some of her classmates were all about clothes and that they defined themselves through what they wore. She added that she thought she was not that way and criticized them. She did not feel like she had to do what the group did, which showed a strong self-concept. FLUTE's passion for her flute also caught my attention. She was so enthusiastic when she talked about music and playing the flute in a band. She also became excited when talking about how beautiful it was when the band 
played together as a group. She even would have been willing to play the flute for me, but she had left it in school. It appeared that her mother really wanted me to see her play and asked if I would like to see a video of her playing the flute. I agreed. FLUTE did a great job playing the flute, and I noticed that LOVING was very proud of her daughter. I also highlighted in my field notes that the father, who was also in the office when his wife played the video clip for me, turned around and watched the video with us. He did not say anything but I noticed that he was very proud of his daughter, as well. While we were watching the video, FLUTE went upstairs to collect some of her visual artwork to show me. She described her paintings very thoroughly. It was obvious that she enjoyed sharing her works of art. It also seemed like she got motivation and recognition out of presenting them to me.

\section{Follow up for interviews (artifacts).}

After the four interviews had been concluded, the data were transcribed. These documents were sent to the participants by email (see Appendices G16 and G19). The participants were asked to review these final transcripts for accuracy. The co-researchers were asked to add comments, if some new or additional ideas came to their minds. This way, themes that had emerged during the interview were checked, verified, and clarified by the interviewees.

BUSY's and BAND's reviewed documents from interview number one (T-P-BUSY \& TS-BAND-29.04.2011) were received on July 20, 2011. From their point of view, no changes or additions were necessary. They found that their experiences were captured entirely by my transcripts.

INVOLVED's, MOTIVATED's, and Dr. S.'s reviewed documents from interview number two (T-T\&L-INVOLVED, MOTIVATED, \& Dr. S.-03.05.2011) were received on June 21, 2011. The participants made the point that some of the comments were not attributed to the 
correct participant. Changes to the interview transcript were marked in red by the co-researchers. I accepted all of their changes.

CARING's and PIANO's reviewed documents from interview number three (T-S\&PPIANO, DRAWING \& CARING-14.05.2011) were received on June 22, 2011. CARING stated that she would review the document and let me know by July 5, 2011, if changes were necessary. No email was received by July 5, 2011. Evidently, no changes were necessary.

LOVING's and FLUTE's reviewed documents form interview number four (T-P\&SLOVING \& FLUTE-24.05.2011) were received on June 23, 2011. No changes were suggested by these two co-researchers.

In sum, the majority of the researchers did not have any changes for the follow-up of the interviews (artifacts). Only minor changes had been suggested by the teachers and university liaison group. These modifications were accepted and taken into consideration.

\section{Peer review.}

A peer review was conducted by a second reviewer to triangulate and validate the data. The basic invariant constituent units for all participating groups, parents (see Appendix H1), students (see chapter four, step two: reduction and elimination), and teachers (see Appendix H2) were sent out for review on September 2, 2011. The original interview transcripts were also provided to the peer reviewer. The typed results of the reviewer were received on October 5, 2011. The second reviewer created a list with themes that she independently had filtered out of the provided material for each of the participating groups. The reviewer offered that I could follow up with her on themes that might needed clarification. The list provided by the peer reviewer was compared to my findings. It matched my results almost identically. Despite the fact that a follow-up was not really necessary because of the unambiguous agreement, a conversation 
was sought with the second reviewer. This discussion confirmed once more the validity of the themes I had defined for this study.

\section{Past report study (Devono, 2009).}

The results of this research study were contrasted with a past report study, called Teacher Decision Making and the Implementation of an Integrated Arts Curriculum, conducted by Mary Devono (2009). The comparison showed that both studies agree almost identically on the themes for the experiences of teachers in reference to arts integration/ AIC.

The seven core themes for teachers that were identified in this study (see Appendix H9.3.), which also conform with the six main phenomena (see Appendix H11.1.), and Devono's (2009) findings were compared. The results that also can be found in Table 7 and are displayed in more detail and in a narrative way in the next section. The focus of this comparison were the seven main themes for teachers that were found in this study, even though there were additional themes that these two studies agree upon (see Appendix I). 
Table 7. Devono's (2009) and Douglas' (2011) Similarities of Arts Integration/ AIC Findings

\begin{tabular}{|c|c|c|}
\hline (Devono, 2009) & \multicolumn{2}{|c|}{ (Douglas, 2011) } \\
\hline $\begin{array}{c}\text { Teacher decision } \\
\text { making and } \\
\text { the implementation } \\
\text { of } \\
\text { an integrated arts } \\
\text { curriculum }\end{array}$ & \multicolumn{2}{|c|}{$\begin{array}{l}\text { The Arts-Integrated Curriculum (AIC) and its possible impact } \\
\text { on } \\
\text { the self-concepts of adolescent girls and their perceptions of } \\
\text { beauty }\end{array}$} \\
\hline $\begin{array}{l}\text { Devono's (2009) } \\
\text { findings that match } \\
\text { Douglas' (2011) teacher } \\
\text { findings }\end{array}$ & $\begin{array}{c}\text { Douglas (2011) } \\
7 \text { core themes for } \\
\text { Teachers experience with the AIC } \\
(=\mathrm{RQ} 2)\end{array}$ & $\begin{array}{c}\text { Douglas (2011) } \\
6 \text { main phenomena for } \\
\text { group (parents, } \\
\text { students, teachers) as a } \\
\text { whole }\end{array}$ \\
\hline 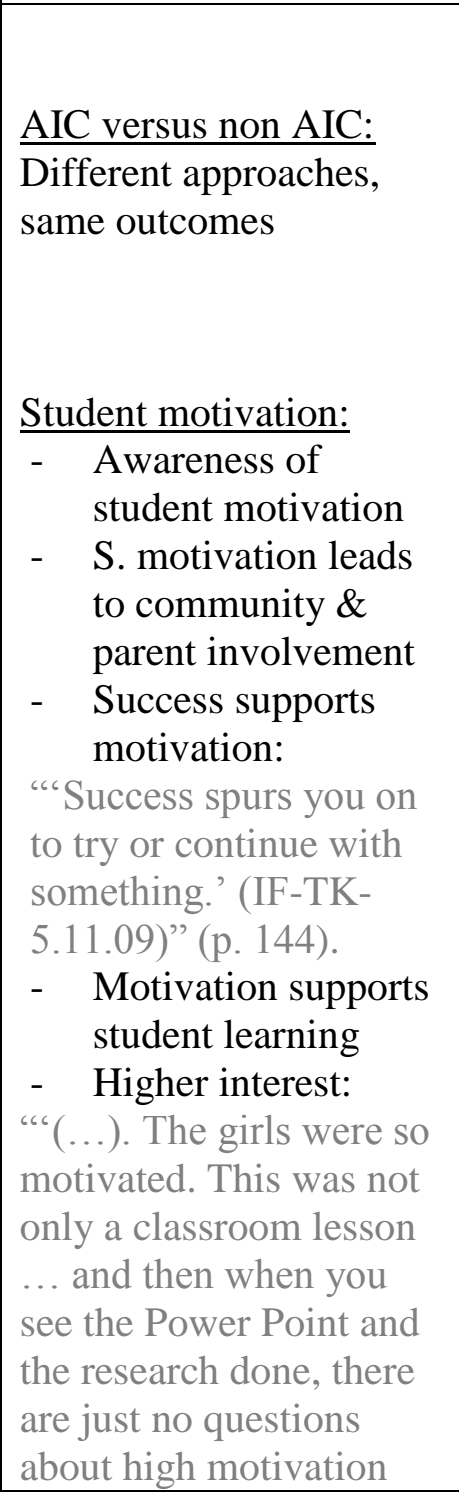 & $\begin{array}{l}\text { 1) things related to the arts or the } \\
\text { AIC, specifically in schools; } \\
\text { AIC versus non AIC: } \\
\text { INVOLVED: } \\
\text { "We were just doing it in a different } \\
\text { way, but our teaching was always very } \\
\text { purposeful." } \\
\text { Student motivation: } \\
\text { INVOLVED: } \\
\text { "All of a sudden, these kids were } \\
\text { reading and then we were writing to } \\
\text { these authors and asking for an } \\
\text { autographed copy for our classroom. } \\
\text { [Intrinsic Motivation]" }\end{array}$ & $\begin{array}{l}\text { 1) interest in music and } \\
\text { art; }\end{array}$ \\
\hline
\end{tabular}




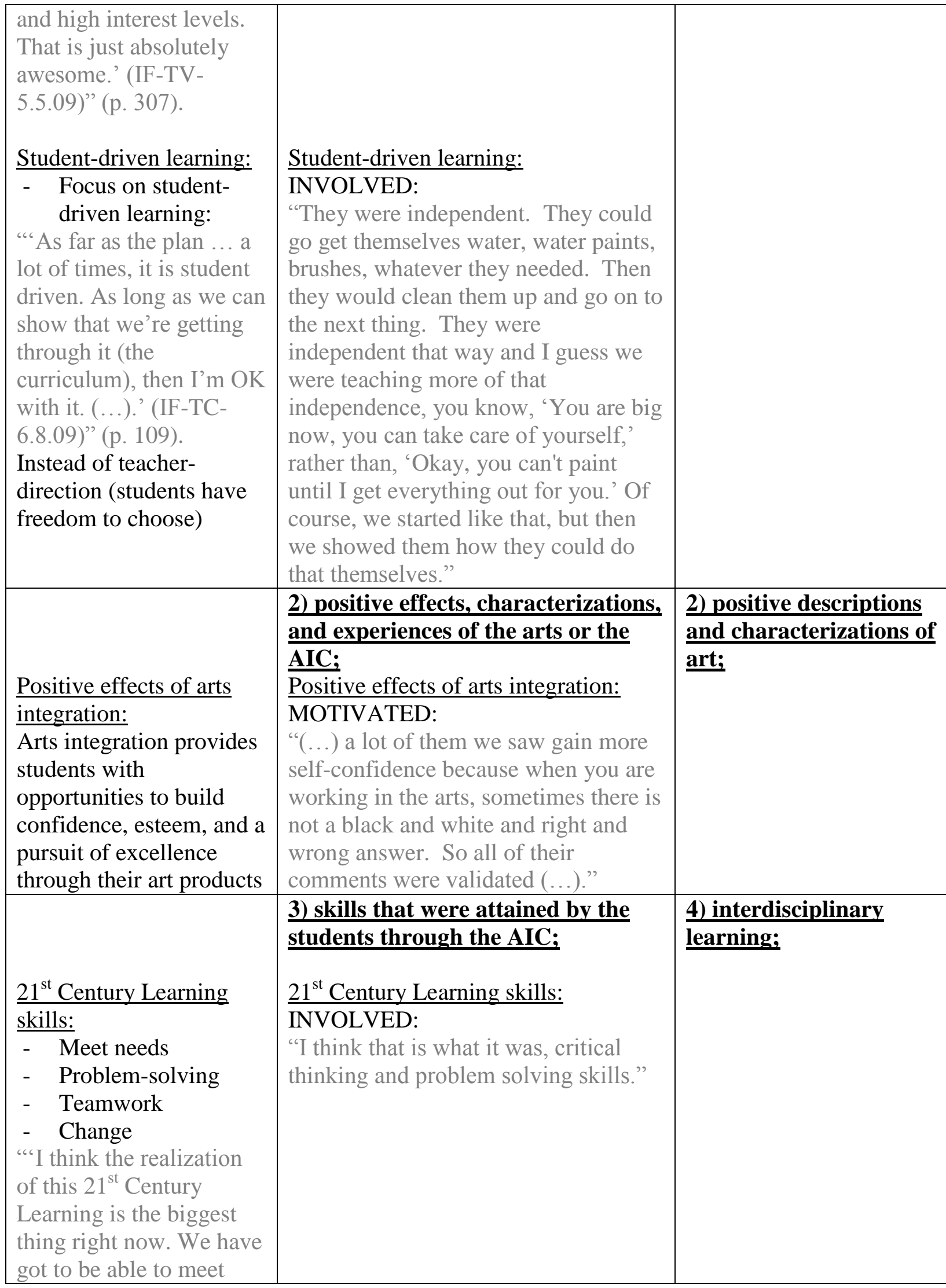




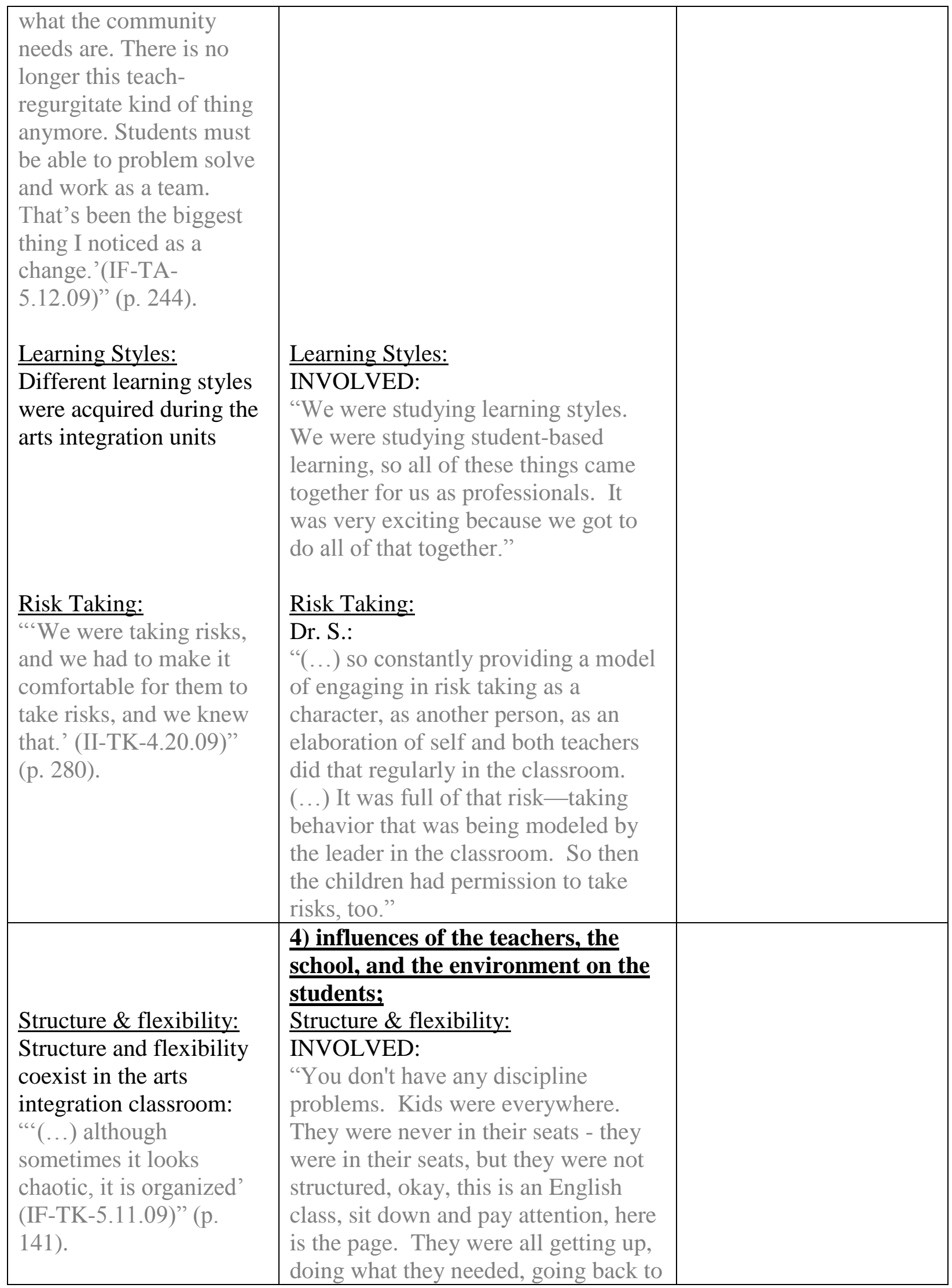




\begin{tabular}{|c|c|c|}
\hline & $\begin{array}{l}\text { their seats, proceeding to do what they } \\
\text { needed to do." }\end{array}$ & \\
\hline $\begin{array}{l}\text { Self-confidence: } \\
\text { Help students build self- } \\
\text { confidence }\end{array}$ & $\begin{array}{l}\text { 5) things related to the self-concept; } \\
\text { Self-confidence: } \\
\text { Dr. S.: } \\
\text { "So when you talk about what is a } \\
\text { leader, this is a child who had some } \\
\text { confidence and I would think that } \\
\text { might be connected to their self- } \\
\text { concept." }\end{array}$ & $\begin{array}{l}\text { 3) positive self-concept } \\
\text { through arts; } \\
\text { 6) self-concept in } \\
\text { general }\end{array}$ \\
\hline $\begin{array}{l}\text { Display artwork: } \\
\text { Arts integration products } \\
\text { that are evidence of } \\
\text { learning are put out for } \\
\text { all to see }\end{array}$ & $\begin{array}{l}\text { Display artwork: } \\
\text { Dr. S.: } \\
\text { "They had their portfolios and they } \\
\text { would take to the hallway displays } \\
\text { where the art was celebrated and } \\
\text { labeled like an art gallery with their } \\
\text { name and their title. They would walk } \\
\text { them out there, 'Here is my example } \\
\text { of my self-portrait. Here is my } \\
\text { example of extending the } \\
\text { landscaping,' and here is my example } \\
\text { of ..., and things that weren't } \\
\text { displayed were in their portfolio. And } \\
\text { they would take them to their } \\
\text { portfolios and pulled out their art and } \\
\text { show them different examples." }\end{array}$ & \\
\hline $\begin{array}{l}\frac{\text { Community (parent) }}{\text { involvement: }} \\
\text { (go beyond classroom): } \\
\text { "Every unit has some } \\
\text { sort of introduction to } \\
\text { parents.' (II-TC-5.1.09)" } \\
\text { (p. 160). } \\
\text { - Parent newsletters } \\
\text { - Volunteers } \\
\text { - Invitations to special } \\
\text { learning events } \\
\text { - At-home read } \\
\quad \text { together assignments } \\
\text { - Opportunities for } \\
\text { parents to become } \\
\text { involved at school } \\
\text { and at home } \\
\text { "Once they start }\end{array}$ & $\begin{array}{l}\text { 6) numerous forms of involvement; } \\
\text { Community (parent) involvement: } \\
\text { INVOLVED: } \\
\text { "(...) so we had kind of guided the } \\
\text { questions for them to talk about, you } \\
\text { know, is he telling the truth? Is it } \\
\text { okay to protect a dog if you think he is } \\
\text { being abused? Is that right or wrong? } \\
\text { That is not my thinking to say for you, } \\
\text { but your family needs to. So it kind of } \\
\text { brought family together (...)." }\end{array}$ & \\
\hline
\end{tabular}




\begin{tabular}{|l|l|l|}
\hline $\begin{array}{l}\text { learning more and get } \\
\text { more involved with their } \\
\text { puppet and report, the } \\
\text { excitement grows. } \\
\begin{array}{l}\text { Parents get involved with } \\
\text { their children. (...).' (II- } \\
\text { TC-5.1.09)" (p. 306). }\end{array}\end{array}$ & \\
\hline $\begin{array}{l}\text { Teachers taught in } \\
\text { dresses/ costumes to } \\
\text { engage and motivate } \\
\text { their students }\end{array}$ & $\begin{array}{l}\text { (7) internal and external beauty, } \\
\text { including clothes and dressing up; } \\
\text { "They also imitated us (...), because } \\
\text { we would dress up. They could tell } \\
\text { when they walked in in the morning, } \\
\text { we often are dressed - gave them clues } \\
\text { about what was going to happen that } \\
\text { day." }\end{array}$ & 5) beautiful things; \\
\hline
\end{tabular}

Note: All of Douglas' (2011) quotes for findings are taken from the transcript for interview number two (T-T\&L-INVOLVED, MOTIVATED, \& Dr. S.-03.05.2011).

The first phenomenon of the AIC experience for teachers found in this study are things related to the arts or the AIC, specifically in schools. In more general terms, this also can be described as the AIC versus the non-AIC experience in the classrooms. In her study, Devono (2009) referred to the AIC approach when she talked about various methods that arts integration requires in teaching. Devono (2009) stressed that despite various arts integration approaches, in comparison to "regular" approaches, the same outcomes were achieved. INVOLVED, one of the teachers in my study, described her AIC experience in a similar way: "We were just doing it in a different way, but our teaching was always very purposeful” (T-T\&L-INVOLVED, MOTIVATED, \& Dr. S.-03.05.2011). Devono (2009) added how one has to be aware of the students' motivation and how it could lead to community and parent involvement. She noted that motivation supported student learning and spurs higher interest in students: ““(...). The girls were so motivated. This was not only a classroom lesson ... and then when you see the Power 
Point and the research done, there are just no questions about high motivation and high interest levels. That is just absolutely awesome.' (IF-TV-5.5.09)" (Devono, 2009, p. 307). This study focused on the same finding. Student motivation was of high priority for the students' learning from INVOLVED's viewpoint: “"All of a sudden, these kids were reading and then we were writing to these authors and asking for an autographed copy for our classroom. [Intrinsic Motivation]" (T-T\&L-INVOLVED, MOTIVATED, \& Dr. S.-03.05.2011). Both studies emphasized a focus on student-driven learning, instead of teacher-direction: “"As far as the plan ... a lot of times, it is student driven. As long as we can show that we're getting through it (the curriculum), then I'm OK with it. (...).' (IF-TC-6.8.09)" (Devono, 2009, p. 109). INVOLVED said about the students: "They were independent. They could go get themselves water, water paints, brushes, whatever they needed. Then they would clean them up and go on to the next thing” (T-T\&L-INVOLVED, MOTIVATED, \& Dr. S.-03.05.2011).

The second phenomenon of the AIC experience for teachers in this study was defined by positive effects, characterizations, and experiences of the arts or the AIC. In specific, MOTIVATED described these effects with the help of self-confidence: “(...) a lot of them we saw gain more self-confidence because when you are working in the arts, sometimes there is not a black and white and right and wrong answer. So all of their comments were validated (...)" (T-T\&L-INVOLVED, MOTIVATED, \& Dr. S.-03.05.2011). Devono (2009) also mentioned these positive effects of arts integration on the students' self-concept. She talked about how arts integration provided students with opportunities to build confidence and self-esteem and encouraged a pursuit of excellence through their art products.

The third phenomenon, described for teachers by this study, was the set of skills that students attained through the AIC. Devono's (2009) and my study agreed on three of these: (1) 
$21^{\text {st }}$ century learning skills, (2) learning styles, and (3) risk taking. INOLVED talked more particularly about $21^{\text {st }}$ century learning skills: "I think that is what it was, critical thinking and problem solving skills" (T-T\&L-INVOLVED, MOTIVATED, \& Dr. S.-03.05.2011). Devono's (2009) participant stressed the same idea about $21^{\text {st }}$ century learning skills: “'I think the realization of this $21^{\text {st }}$ Century Learning is the biggest thing right now (...). Students must be able to problem solve and work as a team. That's been the biggest thing I noticed as a change' (IF-TA-5.12.09)" (Devono, 2009, p. 244).

Next, Devono (2009) mentioned that different learning styles were acquired during the arts integration units. Similarly, INVOLVED referenced the different learning styles: "We were studying learning styles. We were studying student-based learning, so all of these things came together for us as professionals" (T-T\&L-INVOLVED, MOTIVATED, \& Dr. S.-03.05.2011). Finally, risk taking was a major theme for both studies. Devono (2009) quoted a comment by one of her teacher participants: “We were taking risks, and we had to make it comfortable for them to take risks, and we knew that.' (II-TK-4.20.09)" (p. 280). In this study, Dr. S. stressed the idea of risk taking by mentioning how teachers constantly were "providing a model of engaging in risk taking as a character, as another person, as an elaboration of self" and how "both teachers did that regularly in the classroom." She also talked about how teaching was "was full of that risk-taking behavior" and how it was constantly "modeled by the leader in the classroom." So then, "the children had permission to take risks, too" (T-T\&L-INVOLVED, MOTIVATED, \& Dr. S.-03.05.2011).

The fourth phenomenon was defined by influences of the teachers, the school, and the environment on the students. My study and Devono's (2009) both mainly stressed structure and flexibility. From Devono's (2009) viewpoint, both concepts coexisted in the arts integration 
classroom. She was convinced that ““(...) although sometimes it looks chaotic, it is organized' (IF-TK-5.11.09)" (p. 141). INVOLVED expressed the same phenomenon: "You don't have any discipline problems. Kids (...) were never in their seats - they were in their seats, but they were not structured (...). They were all getting up, doing what they needed, going back to their seats" (T-T\&L-INVOLVED, MOTIVATED, \& Dr. S.-03.05.2011).

As a fifth phenomenon, things related to the self-concept were stressed in this study, as well as Devono's (2009). From her perspective, arts integration helped students build selfconfidence. In this study, Dr. S. agreed: "So when you talk about what is a leader, this is a child who had some confidence and I would think that might be connected to their self-concept" (TT\&L-INVOLVED, MOTIVATED, \& Dr. S.-03.05.2011).

Numerous forms of involvement were found as a sixth phenomenon for teachers and their AIC experience. This study and Devono's (2009) both stressed community and parent involvement. INVOLVED talked about how the teachers prepared guided questions for the students to take home to discuss with their parents. An example could be, "is it okay to protect a dog if you think he is being abused? Is that right or wrong? That is not my thinking to say for you, but your family needs to. So it kind of brought family together (...)" (T-T\&L-INVOLVED, MOTIVATED, \& Dr. S.-03.05.2011). Devono's (2009) findings also considered this kind of parent involvement important. “'Every unit has some sort of introduction to parents.' (II-TC5.1.09)" (p. 160).

For a seventh and final phenomenon, internal and external beauty, including clothes and dressing up, were identified for this research study. Devono (2009) discussed how teachers dressed up and even taught in costumes to engage and motivate their students. The same was true in this study. MOTIVATED described how the students imitated the teachers, "because we 
would dress up. They could tell when they walked in in the morning, we often are dressed - gave them clues about what was going to happen that day" (T-T\&L-INVOLVED, MOTIVATED, \& Dr. S.-03.05.2011).

All these listed findings show how identical the outcomes are that this study and Devono's (2009) detected, in reference to the teachers' perspectives and experiences on arts integration/ AIC. Despite these similarities, the two studies differed in results related to other participants. This study drove research on arts integration and the AIC a step further by including everyone involved in the AIC. The voices of parents, students, AND teachers were heard, since this study's focus was not limited to teachers and their experiences, as it was the case in Devono’s (2009).

\section{Implications}

After the listing and summarizing of the most important outcomes and findings for this study, insights and understandings emerged that were valuable on a (a) personal, (b) societal, and (c) professional level. These arising implications (see Figure 21) also conformed with the personal, practical, and intellectual goals that were listed in chapter one of this work. Furthermore, each of the listed implications ( $\mathrm{a}-\mathrm{c}$ ) could be linked to one of the co-researcher groups. The personal implications could be related to the students. The practical-societal implications could be connected to the parents. The intellectual-professional implications could be associated with the teachers. The following paragraph expanded on this idea. 


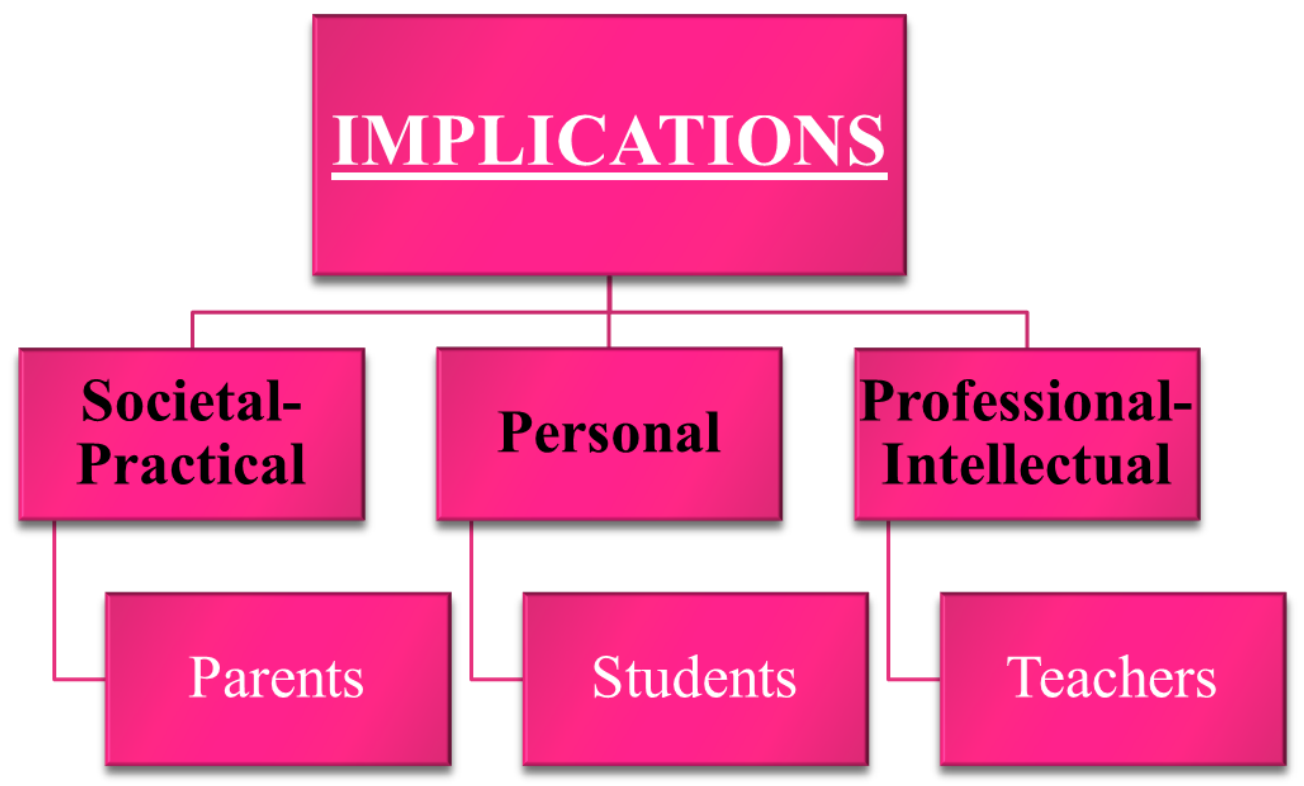

Figure 21. Implications of this study on a societal-practical, personal, and professionalintellectual level.

\section{Personal implications.}

The personal implications of this study (see also Figure 21) were of special value for me. It was my personal goal for this study to determine whether, and if so how, girls battling with self-concept and/ or beauty issues can find new hope and support in the arts/ AIC. This desire arose from my own arts experiences and the positive effects they had on me when I was an adolescent girl struggling with health issues. As a consequence, the possibility of communicating with parents, students, and teachers about their AIC experience was very enlightening to me. I learned that even if there were no specific health issues involved, as it was the case for me, self-concept struggles can arise in healthy students. The kind of issues that the adolescent girls of my study had to face were different from the one with which I struggled (scoliosis). Nevertheless, the main problem, which often consists of self-concept struggles and skewed perceptions of beauty, remained the same for these adolescent girls, as well. As a result, I thought it might be a good approach to relate the arts directly to education in order to possibly 
provide a support system for girls that struggle with self-concept and beauty issues. The idea of this study was born. As it turns out, this study was able to illustrate positive experiences of parents, teachers, and, more specifically, students in relation to the arts/ AIC. Phenomena were found that had positive effects on the self-concepts of adolescent girls and their perceptions of beauty, similar to the way the arts have impacted my personal growth in relation to self-concept and perceptions of beauty. However, I realized that the phenomena I was studying were more than "just" arts integration and could not be limited to the AIC. These phenomena were not supposed to lead conclusions about arts integration or the AIC but to describe experiences that speak for themselves. DRAWING gave a very good description for these experiences: "I think the arts are a wonderful experience. (...) acting can make you braver. If you have to stand in front of a bunch of people, (...). They [arts] can make you braver, they can make you better, you know. (T-S\&P-PIANO, DRAWING \& CARING-14.05.2011)

\section{Societal-practical implications.}

Societal-practical implications (see also Figure 21) arose for this study, as well.

Throughout the study, it became clear that the society has a major influence on our children and schools. Not always, but often, self-concept struggles of adolescent girls can be linked to culture. Societal beauty standards, for example, do not stop in front of our school doors — disorders can develop as a consequence. However, this study indicated that the self-concept of adolescent girls possibly could be strengthened by the AIC, whether for currently-struggling or at-risk students. Teachers of the AIC emphasized that students were free to express themselves and to develop a positive self-concept in the AIC - boys and girls. Experiences and reactions of students to the AIC were not different for the two genders. MOTIVATED stated very clearly that she had a hard time isolating the girls' reaction from the boys' because the effects crossed gender lines. To her, 
it seemed that "both boys and girls were freer in the classroom to express themselves and to act out in different ways when we would do, you know, role playing or dressing up and, you know, it was just very free" (T-T\&L-INVOLVED, MOTIVATED, \& Dr. S.-03.05.2011). As such, this study also had a practical implication for our schools. It stated that the positive effects of the AIC were not dependent on gender but that coeducation in an AIC even fostered collaboration and teamwork of boys and girls in the classroom. In accordance with these findings, parents were convinced of the advantages of an AIC and made known their desire that more schools would implement this approach because their children acquired life skills that were considered equally important to the ones they acquired in the so-called "core-subjects." CARING, for example, expressed her opinion about the arts being undervalued. From her point of view, the arts "are as important as learning the times tables." She continued by adding that "all of these things are important to becoming a well-rounded individual who can live in a very complex society." Furthermore, she stated her hope that "somehow we can turn that around and get people to understand that the arts are just as important as anything else, if not even more so, for the development of the human being" (T-S\&P-PIANO, DRAWING \& CARING-14.05.2011).

\section{Professional-intellectual implications.}

Professional-intellectual implications (see also Figure 21) must be mentioned in the context of current research, like Devono's (2009) study on Teacher Decision Making and the Implementation of an Integrated Arts Curriculum. As described earlier in the outcomes section, this study and Devono's (2009) agreed on the positive findings for arts integration/ the AIC from the teachers' viewpoint and concluded that the arts are essential in our schools and for the development and learning outcomes of our children. Consequently, the arts and the AIC should be more often applied in all subjects. Despite this suggestion, I would like to clarify that the 
integration of the arts in all subjects does not require that the arts specialists be replaced. Instead, arts specialists could serve as models and inspirations for their colleagues who try to integrate the arts in their subjects and classrooms. Also, it is understood that the introduction of an AIC in schools is not a solution for everyone- but it could make a difference for some, as the phenomena tended to illustrate.

\section{Recommendations for Future Research}

I believe that further phenomenological research is needed in the area of phenomena that may be associated with the self-concept and perceptions of beauty of adolescent girls who completed an AIC, in order to describe the arising phenomena in more detail. Furthermore, I would like to propose three additional phenomenological studies that could be conducted in the future to expand the research (see also Figure 22).

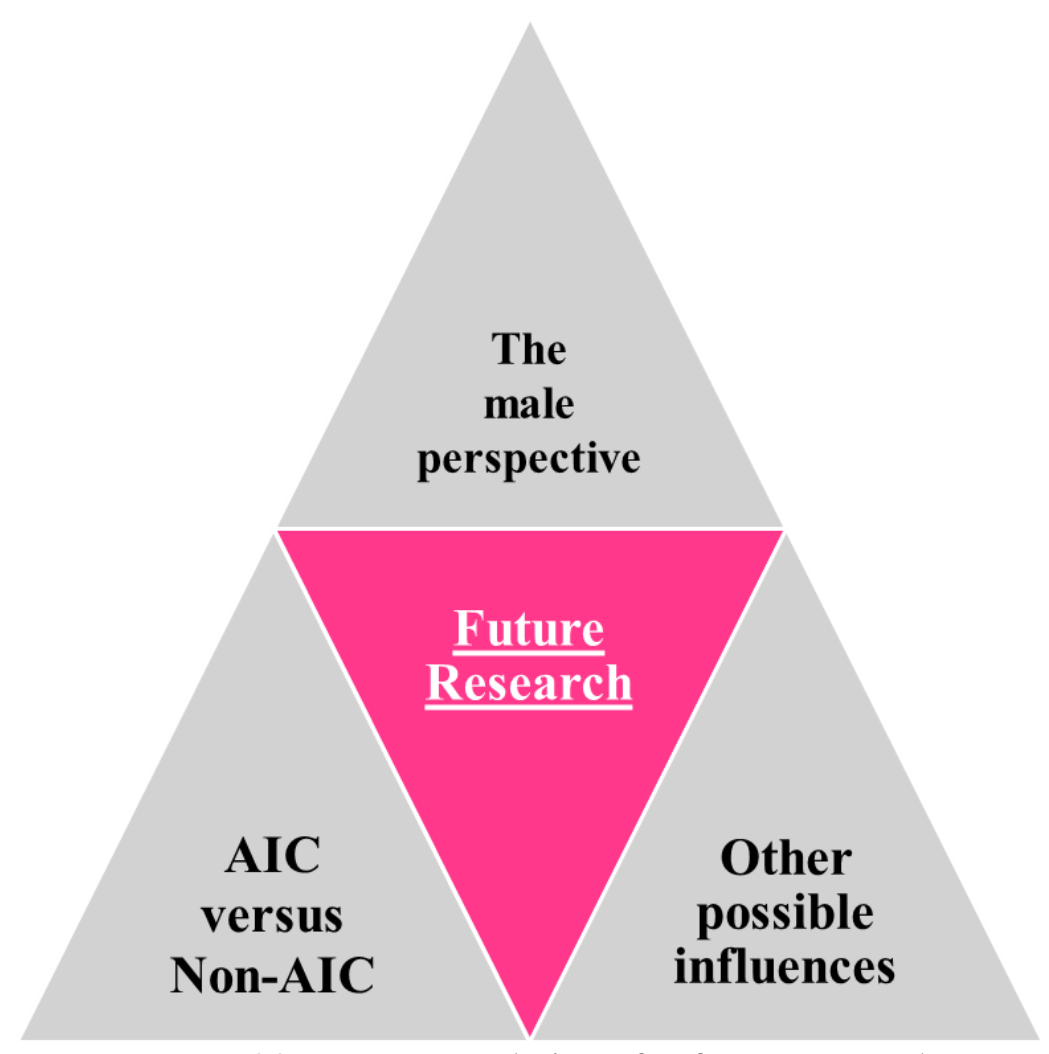

Figure 22. Recommendations for future research. 


\section{The male perspective.}

The inclusion of a male perspective (see also Figure 22) on the phenomena could provide a new direction. The design of this study featured a female perspective, since all 10 coresearchers and I were female. Though this exclusivity does not necessarily have to be seen as a disadvantage, it might have influenced the participants' perception and not necessarily expressed the overall perception of the phenomena because of the absence of the male input. Consequently, additional examination of the male's experience could provide further insight of the phenomena that are related to the AIC and its possible influence on the self-concept of adolescent girls or boys and their perception of beauty. Male teachers, male parents, and boys could be interviewed. This way, the perspective of beauty standards and the requirement of girls looking cute, being slim, and being tall, could be expanded or even transferred to a male perspective. Oftentimes, we do not consider that boys might have similar issues but express themselves in a different way. While girls are worried about beauty standards in relation to the way they look, boys worry about standards in relation to their ability to be successful in sports. This issue became obvious throughout the interview process. Public voices have also begun to acknowledge this problem.

During a recent visit to the Andy Warhol Museum in Pittsburgh, I had the opportunity to see an art exhibition called Mixed Signals: Artists consider Masculinity in Sports. Christine O’Toole (2011) wrote in her article Mixed Signals about the display and how it "digs deeper into the complex relationship between athletes and fans" (O'Toole, 2011). She then explained this exhibition of works by well-known contemporary artists "draws imagery from the American sports scene to examine what we really admire—or overlook—beneath those team colors" (O'Toole, 2011). Eric Shiner, the acting director of The Warhol, was quoted by O'Toole (2011): "We're hoping that this show turns the camera on the ideal of masculine beauty and youth as a 
way to rethink gender norms" (Shriner (n.d.), as cited in O'Toole, 2011). This quote features many similarities that are known from issues and struggles of adolescent girls. While these issues of gender, self-concept, and beauty standards were analyzed in more detail in this study from a female perspective, it might be time to focus on the same concepts from a male perspective. In a future study for boys, sports could replace the role that beauty plays for girls. This becomes even more obvious when Joey Nolfi (2011) described in his article, Warhol Exhibit questions Sports Industry's Idea of Masculinity, that sports are as essential to mainstream ideals of masculine identity as a certain part of the male anatomy (Nolfi, 2011). The claim for more research on this topic of the male gender, identity, and sports is also stressed by the Middlebury College Museum of Art (2011). In an article titled, Museum Exhibit Wrestles with the Concept of the Male Athlete, the museum emphasized how despite all the changes of identity politics "one American stereotype still remains particularly entrenched: that of the aggressive, hyper-competitive, emotionally undemonstrative, heterosexual male athlete" (Bedford, 2009, p. 6; Middlebury College Museum of Art, 2011). Similar to this study, the museum also raised the concern that "this subject has, until recently, been overlooked (...)" (Middlebury College Museum of Art, 2011). Thus, I believe it is time to consider the male perspective on issues like self-concept, body image, and gender stereotyping. The voices should of male parents, students, and teachers should be heard. The main research question could be changed to: What, if any, phenomena may be associated with the self-concept and perceptions of sports of adolescent boys who completed an AIC?

\section{AIC versus non-AIC.}

Even though this study focused on what, if any, phenomena may be associated with the self-concept and perceptions of beauty of young ladies who completed an AIC, an additional 
perspective could be provided by including experiences of those who did not go through an AIC (see also Figure 22). A new aspect might develop by comparing and contrasting the experiences of students, parents, and teachers who experienced an AIC versus those who did not. Outcomes might or might not be totally different.

\section{Other possible influences.}

There also might be other directions (see also Figure 22) for the understanding of the phenomena that may be associated with the self-concept and perceptions of beauty of young ladies who completed an AIC. Geographical data, for example, might change the outcomes. Depending on the area and geographical location, the parents, students, and teachers might have the possibility of being exposed or not being exposed to the arts or the AIC. The environment of friends or peers, including the family background, also might play a significant role. Depending on the socio-economic background of friends and family, the findings could also vary. This study focused on middle class co-researchers. For future studies, lower or upper class research participants could be included. Cultural differences also might have an effect on the outcomes. It might play a role if co-researchers are selected from different cultural groups. AfricanAmerican, Asian, or Latino populations might describe their experiences with the AIC and its possible influences quite differently. The arts might play different roles depending on the cultural backgrounds of the participants.

A combination of my own research with the three suggestions of possible future studies, (a) the male perspective, (b) AIC versus non-AIC, and (c) other possible influences, could offer a broader perspective of the phenomena that may be associated with the self-concept and perceptions of beauty of young ladies who completed an AIC, as well as the perception of boys who completed an AIC. 


\section{Closing Remarks}

As this phenomenological investigation comes to an end, there are many personal, professional, and general insights and understandings that I have gained through this study. Because of my family background, I have been exposed to the arts my whole life. The arts have even helped me to overcome some of the most difficult times of my life. Out of all these experiences, the goal emerged to find out if the arts also could be supportive and beneficial to others, whether or not they are struggling with a specific problem. By interviewing my coresearchers in a phenomenological manner and making sense out of the collected data, I feel like I reached this goal. Regardless, I would like to stress that it was not my intention to portray arts integration or the AIC as a perfect solution that works for anyone-but it could be for some.

Throughout the whole research process, I acquired personal growth, as well as professional knowledge. On a personal level, I have learned that almost everyone struggles at some level with self-concept, body image, and beauty standards: parents, students, or teachers; adults or children; slim or not so slim; tall or not so tall and that each of us has to overcome these challenges individually. On a professional level, I have learned that we, as educators, have the responsibility to support our students in the process of working through these struggles in any way we can-for some students it might be the AIC, for others a different approach that helps them to see their strengths and become more self-confident. Again, as stressed previously, the arts and the AIC might not be the ultimate solution or for all of our students, but at least they are a good start and might make a difference for some (e.g. in a school environment where bullying is involved). In addition, I also understand that the arts/ AIC are not just important for students' learning because they can be related to a cause and effect in academic outcomes, like test scores. 
They also have a value on their own in the phenomena that they cause, which were touched upon with this research study.

In addition to my personal and professional learning outcomes, I am also delighted to have acquired the knowledge for conducting a qualitative, phenomenological research study. Even though it was sometimes a difficult learning process "to describe an experience rather than explain or analyze it” (Moustakas, 1994, p. 175), there are so many lessons I have learned. I hope that this research study is only the beginning of further phenomenological studies in the area of the AIC, the self-concepts of adolescent girls, perceptions of beauty, and other concepts related to these research interests.

At the end, I would like to note that ...

... this phenomenological research study was born out of my personal experiences with selfconcept struggles, the arts, and the connection of both.

... this phenomenological, qualitative research process developed with the help of the descriptions of lived experiences of those that were directly involved in the AIC.

... the resulting phenomena that were associated with the self-concept and perceptions of beauty of young ladies who completed an AIC portray more than arts integration and the AIC, but the phenomena that I studied.

... the goal of this phenomenological study was not to draw conclusions by using arising phenomena but to describe arts experiences in a way that they speak for themselves. 


\section{References}

Americans for the Arts. (n.d.). Retrieved from The arts ask for more. Arts education facts. Quick facts. Americans for the arts. [Website]: http://www.americansforthearts.org/public_awareness/artsed_facts/001.asp

Anonymous school web site. (n.d.). Retrieved 05 04, 2011, from anonymous

Answers.com. (2011). Retrieved 09 06, 2011, from Gale Encyclopedia of Children's Health:

Scoliosis: http://www.answers.com/topic/scoliosis

Arts Education Partnership. (2000). Retrieved 09 23, 2011, from Why your child needs the arts.

Advantage and how you can gain it [pdf]: http://www.aep-

arts.org/files/publications/WhyYourChildNeedstheArts.pdf?PHPSESSID=88dd2652c29a 07afdc01f69ec8f3e 52c

Appel, M. P. $(2006,11)$. Arts integration across the curriculum: engagement in the arts ignites creativity and provides students with opportunities to critically interpret the world around them. Retrieved 09 13, 2011, from The CBS interactive buisness network : http://findarticles.com/p/articles/mi_m0HUL/is_2_36/ai_n27073104/?tag=mantle_skin;c ontent

Association, N. A. (2003, 10). Art education. Tips for parent advocacy. Retrieved 09 27, 2011, from art educators: http://www.arteducators.org/advocacy/TipsforParent.pdf

Bardone-Cone, A. M., Schaefer, L. M., Maldonado, C. R., Fitzsimmons, E. E., Harney, M. B., Lawson, M. A., et al. (2010). Aspects of self-concept and eating disorder recovery. What does the sense of self look like when an individual recovers from an eating disorder? Journal of Social and Clinical Psychology, 29(7), 821-846. 
Becker, A. (1999). Body, self and society: The view from Fiji. Philadelphia, PA: University of Pennsylvania.

Bedford, C. (2009). Mixed signals: Artists consider masculinity in sports. New York, NY: Independent Curators International.

Bentz, V. M., \& Shapiro, J. J. (1998). Mindful inquiry in social research. Thousand Oaks, CA: Sage.

Berube, M. R. (1999). Arts and education. Clearing House, 72(3), 150-153.

Bogdan, R. C., \& Biklen, S. K. (2003). Qualitative research for education. Boston, MA: Allyn \& Bacon.

Bordo, S. (2003). Unbearable weight. Feminism, Western culture, and the body. Berkeley, CA: University of California Press.

Bresler, L. (1995). The subservient, co-equal, affective, and social integration styles and their implications for the arts. Arts Education Policy Review, 5, 31-37.

Business Circle for Arts Education in Oklahoma (n.d.). Arts at the Core of Learning 1999 Initiative. Retrieved from http://www.americansforthearts.org/public_awareness/artsed_facts/001.asp

Butler, J. (1993). Bodies that matter. On the discursive limits of "sex". New York, NY: Routledge.

Catterall, J. S., \& Waldorf, L. (1990). Chicago arts partnerships in education: Summary evaluation. In E. B. Fiske (Ed.). Champions of Change: The Impact of the Arts on Learning. (pp. 47-62). Washington, DC: The Arts Education Partnership.

Catterall, J. S., \& Waldorf, L. (1999). The Chicago arts partnerships in education evaluation. In E. B. Fiske (Ed.). Champions of Change: The Impact of the Arts on 
Learning. (pp. 47-62). Washington, DC: Chief Council of State School Officers.

Clark, L., \& Tiggemann, M. (2007). Sociocultural influences and body image in 9- to 12- yearold girls: The role of appearance schemas. Journal of Clinical Child and Adolescent Psychology, 36(1), 76-86.

Copen, R. G. (1992). Insomnia: A phenomenological investigation. ProQuest Dissertations and Theses (PQDT).

Cornett, C. E. (2011). Creating meaning through literature and the arts. Arts integration for classroom teachers. New York, NY: Pearson.

Credo action. more than a network. a movement. . (n.d.). Retrieved 09 18, 2011, from Stop JC Penney and Forever 21 from putting more sexist clothing on their shelves : http://act.credoaction.com/campaign/sexist_shirts/?r=231259\&id=27146-2720527DpOd9Kx

Creswell, J. W. (1998). Qualitative inquiry and research design: Choosing among five traditions. Thousand Oaks, CA: Sage.

Creswell, J. W. (2007). Qualitative inquiry and research design: Choosing among five approaches. Thousand Oaks, CA: Sage.

Csíkszentmihályi, M. (1990). Flow: the psychology of optimal experience. New York : Harper \& Row.

Csikszentmihalyi, M. (1993). The evolving self. New York, NY: Harper Collins.

Csikszentmihalyi, M. (1997a). Finding flow: The psychology of engagement in everyday life. New York, NY: Basic Books.

Csikszentmihalyi, M. (1997b). Assessing aesthetic education. Measuring the ability to ward off chaos. Arts Education Policy Review, 1, 33-38. 
Csikszentmihalyi, M., \& Csikszentmihalyi, I. S. (Eds.). (1988). Optimal experience.

Psychological studies of flow in consciousness. New York, NY: Cambridge University Press.

Custodero, L. A. (2002). Seeking challenge, finding skill. Flow experience and music education. Arts Education Policy Review, 103(3), 3-9.

Davis, J. (1999a). Design's inherent interdisciplinarity. The arts in integrated curricula. Arts Education Policy Review, 101(1), 8-13.

Davis, J. (1999b). Nowhere, somewhere, everywhere: The arts in education. Arts Education Policy Review, 100(5), 23-28.

Davison, K. K., \& Birch, L. L. (2002). Processes linking weight status and self-concept among girls from ages 5 to 7 years. Developmental Psychology, 38(5), 735-748.

Deasy, R. J. (Ed.). (2002). Critical links: Learning in the arts and student academic and social development. Washington, DC: Arts Education Partnership. Retrieved from http://www.aep-arts.org/resources/toolkits/criticallinks/criticallinks.pdf

DeMoss, K., \& Morris, T. (2002). How arts integration supports student learning. Students shed light on the connections. Unpublished manuscript, University of New Mexico, Albuquerque, NM. Retrieved from http://artseveryday.berndtgroup.net/uploadedFiles/Cultural_Organizations/How\%20Arts \%20Integration\%20Supports\%20Student\%20Learning.pdf

Denzin, N. K. (1989b). Interpretive interactionism. Newbury Park, CA: Sage.

Devono, M. K. (2009). Teacher decision making and the implementation of an integrated arts curriculum (Doctoral dissertation). Retrieved from 
http://wvuscholar.wvu.edu:8881//exlibris/dtt/d3_1/apache_media/22977.pdf.

$(000002342)$

Dewey, J. (1934/1980/2005). Art as experience. New York, NY: Penguin Group.

Dewey, J. (1938). Experience and education . New York, NY: Collier.

Dohnt, H., \& Tiggemann M. (2006). The contribution of peer and media influences to the development of body satisfaction and self-esteem in young girls: A prospective study. Developmental Psychology, 42(5), 929-936.

Douglass, B., \& Moustakas, C. (1985). "Heuristic inquiry: The internal search to know.". Journal of Humanistic Psychology, 25(3), 39-55.

Duncan, A. (2010, April 9). The well-rounded curriculum [speech]. U.S. Department of Education. Retrieved from http://www2.ed.gov/news/speeches/2010/04/04092010.html

Duncum, P. (2005). Visual culture and an aesthetics of embodiment. International Journal of Education through Art, 1(1), 9-19.

Dutton, D. (2009). The art instinct. Beauty, pleasure, and human evolution. New York, NY: Bloomsbury Press.

Dutton, D. (2006). A naturalist definition of art. The Journal of Aesthetics and Art Criticism, 64, $367-377$

Education Commission of the States. (2005). State Policies Regarding Arts in Education. [pdf]. Denver, CO: ECS. Retrieved from http://www.ecs.org/clearinghouse/63/92/6392.pdf

Eichelberger, R. T. (1989). Disciplined inquiry: Understanding and doing educational research. White Plains, NY: Longman. 
Eisner, E. W. (2002a). 10 lessons the arts teach. Reston, VA: NAEA. Retrieved from http://www.arteducators.org/advocacy/10-lessons-the-arts-teach

Eisner, E. W. (2002b). The arts and the creation of mind. New Haven, CT: Yale.

Eisner, E. W. (2002c). What can education learn from the arts about the practice of education? Retrieved 09 13, 2011, from infed: http://www.infed.org/biblio/eisner_arts_and_the_practice_of_education.htm

Emerson, A. (1994). Building self-esteem through visual art: A curriculum for middle school girls. Retrieved from http://www.eric.ed.gov/PDFS/ED423173.pdf

Epstein, S. (1973). The self-concept revisited: Or a theory of a theory. American Psychologist, 404-416. Retrieved from http://www.humsci.auburn.edu/classes/hdfs8040/Readings/epstein_1973.pdf

Fiske, E. B. (Ed.). (2002). Champions of change. The impact of the arts on learning [pdf]. In Deasy, R. J. (Ed.), Critical Links: Learning in the arts and student academic and social development (pp. iv-98). Retrieved from http://artsedge.kennedy center.org/champions/pdfs/ChampsReport.pdf

Findlay, J. E. (2011, 06 01). The art of education: Comparing classroom dynamic influences on the learning process. Retrieved 10 12, 2011, from Trinity College Digital Repository: http://digitalrepository.trincoll.edu/cgi/viewcontent.cgi?article=1027\&context=theses\&se iredir=1\&referer=http $\% 3 \mathrm{~A} \% 2 \mathrm{~F} \% 2 \mathrm{Fwww}$. google.com $\% 2 \mathrm{Fsearch} \% 3 \mathrm{Fsource} \% 3 \mathrm{Dig} \% 26 \mathrm{~h}$ 1\%3Den\%26rlz\%3D\%26\%3D\%26q\%3Darts\%2Bget\%2Bcut\%2Bdown\%2Bfor\%2Bcore $\% 2$ Bsubjects $\% 26$ oq $\% 3$ Darts $\% 2 B g e t \% 2$

For the Advancement of Teaching, Americans for the Arts Monograph. (November, 
1998). YouthARTS Development Project, 1996, U.S. Department of Justice, National Endowment for the Arts, and Americans for the Arts. Retrieved from http://www.ncjrs.gov/pdffiles1/ojjdp/186668.pdf

Freire, P. (2007). Pedagogy of the oppressed. New York, NY: Continuum.

Gardner, W. (2010, 09 19). Laissez-faire education . Retrieved 09 18, 2011, from Education Week : http://blogs.edweek.org/edweek/walt_gardners_reality_check/2010/09/laissezfaire_education.html

Gasché, R. (2002). The theory of natural beauty and its evil star: Kant, Hegel, Adorno. Research in Phenomenology, 32, 103-122.

Gay, L. R., Mills, G. E., \& Airasian, P. (2009). Educational research. Competencies for analysis and applications. Columbus, $\mathrm{OH}$ : Pearson.

Gee, K. (2000). Visual arts as a way of knowing. Bangor, ME: Stenhouse.

Giorgi, A. (2006). Concerning variations in the application of the phenomenological method. The Humanistic Psychologist, 34(4), 305-319.

Goff, C. D. (2010). The Black self-determination experience. (Doctoral dissertation). Retrieved from Proquest Dissertations and Theses Fulltext (PQDT). (UMI Number: 3409152).

Greene, M. (1978). Landscapes of learning. New York, NY: Teachers College Press. Greene, M. (1995). Releasing the imagination. Essays on education, the arts, and social change. San Francisco, CA: Jossey-Bass.

Groenewald, T. (2004). A phenomenological research design illustrated. International Joural of Qualitative Methods, 3(1) 1-26.

GSA, U.S. General Services Administration. (n.d.). Art in Architecture Guidelines 
[website]. Retrieved from

http://www.gsa.gov/graphics/pbs/Design_Excellence_Policies_and_Procedures_Chapter8 .$p d f$

GSA, U.S. General Services Administration. (n.d.). Art in Architecture Program [website]. Retrieved from http://www.gsa.gov/portal/content/104456

Gullatt, D. E. (2008). Enhancing student learning through arts integration. Implications for the profession. The High School Journal, April/ May, 12-25.

Halsall, F., Jansen, J. \& O'Connor, T. (2006). Editorial introduction. Aesthetics and its objects challenges from art and experience. Journal of Visual Art Practice, 5(3), 123-126.

Henry, T. (2002, May 20). Study. Arts education has academic effect. USA Today.

Access number: J0E288516674102. Retrieved from

http://web.ebscohost.com.www.libproxy.wvu.edu/ehost/detail?vid=4\&hid=14\&sid=7718

$511 b-c 5 c e-45 e 7$

aaa809b10bcb5488\%40sessionmgr11\&bdata=JnNpdGU9ZWhvc3QtbGl2ZQ\%3d\%3d\#d $\mathrm{b}=\mathrm{a} 9 \mathrm{~h} \& \mathrm{AN}=\mathrm{J} 0 \mathrm{E} 288516674102$

Hoskins, M. (2002). Girls' identity dilemmas. Spaces defined by definitions of worth. Health Care for Women International, 23, 231-247.

Husserl, E. (1913). Ideas. London, England: George Allen.

Hycner, R. H. (1985). Some guidelines for the phenomenological analysis of interview data. Human Studies, 8, 279-303.

Ihde, D. (1979). Experimental phenomenology. New York, NY: Putnam.

Jhaly, S. (Producer/Writer/Director) (1995). Dreamworlds 2. Desire/Sex/Power in music video (Motion picture). (Available from Media Education Foundation, 60 
Masonic Street, Northampton, MA. 01060).

James, M. S. (2011, 07 08). abc NEWS/ Entertainment. Retrieved 09 06, 2011, from Kate Middleton and Prince William arrive in California for royal visit: http://abcnews.go.com/Entertainment/kate-middleton-prince-william-california-royalvisit/story?id=14019103\&page $=2$

Johnson, M. E. (2000). Heidegger and meaning: Implications for phenomenological research. Nursing Philosophy, 1, 134-146.

Katz, L. (1987). The experience of personal change. (Unpublished doctoral dissertation). The Union Institute, Cincinnati.

Kern, E. (1985). The purposes of arts education in the United States from 1870-1980. The History of Art Education. Wilson and Hoffa (Ed.). The Pennsylvania State University College of Arts \& Architecture School of Visual arts, 40-53.

Korsmeyer, C. (2004). Gender and aesthetics: An introduction . New York, NY: Routledge.

Krupnick, E. (2011, 08 31). J.C. Penney Shirt Teaches Girls That Being Smart \& Pretty Are Mutually Exclusive . Retrieved 09 18, 2011, from The Huffington Post : http://www.huffingtonpost.com/2011/08/31/jc-penneys-girls-shirt_n_943349.html

Latta Macintyre, M. A. (2004). Traces, patterns, texture: In Search of aesthetic teaching/ learning encounters. In Pérez Callejo, D. M., Fain, S. M., \& Slater, J. J. (Eds.), Pedagogy of place: Seeing space as cultural education (79-96). New York, NY: Peter Lang. Retrieved from http://digitalcommons.unl.edu/cgi/viewcontent.cgi?article=1016\&context=teachle arnfacpub 
Martin, M. C., \& Kennedy, P. F. (1993). Advertising and social comparison: Consequences for female preadolescents and adolescents. Psychology and Marketing, 10, 513-530.

Maxwell, J. A. (2005). Qualitative research design. An interactive approach. Thousand Oaks, CA: Sage Publications.

McGowan, J. J. (1988). A descriptive study of an arts-in-education project. JRME, 36(1), 47-57.

Mead, M. (1949). Men and women. New York, NY: Morrow.

Mertens, D. M. (2005). Research and evaluation in education and psychology. Integrating diversity with quantitative, qualitative, and mixed mehtods. Thousand Oaks, CA: Sage.

Middlebury College Museum of Art. (2011, 01 24). Retrieved 10 11, 2011, from Museum Exhibit Wrestles with the Concept of the Male Athlete: http://museum.middlebury.edu/news/archives/2010-2011/node/516

Miller, A. (1981). The drama of the gifted child. New York, NY: Basic Books.

Milner, Jenifer (2000). Arts impact. Research supports arts in education. Performing Arts \& Entertainment in Canada, 33(2), 11. Retrieved from http://web.ebscohost.com.www.libproxy.wvu.edu/ehost/pdfviewer/pdfviewer?vid=5\&hid $=113 \& \operatorname{sid}=\mathrm{cd} 2 \mathrm{~d} 4 \mathrm{c} 59-4 \mathrm{de} 5-4578-92 \mathrm{cb}-\mathrm{d} 414 \mathrm{ec} 819335 \% 40$ sessionmgr 112

Mishook, J. J., \& Kornhaber, M. L. (2006). Arts integration in an era of accountability. Arts Education Policy Review, 107(4), 3-11.

Moonlight County Schools. (2009, 06 16). Retrieved 04 05, 2011, from anonymous

Motter, J. (2011). Public pedagogy via PostSecret. A transitional space where private and public coincide. Journal of Art Education, 64(5), 43-48.

Moustakas, C. (1994). Phenomenological Research Methods. Thousand Oaks, CA: SAGE Publications. 
Muir, S. L., Wertheim, E. H., \& Paxton, S. J. (1999). Adolescent girls' first diets: Triggers and the role of multiple dimensions of self-concept. Eating Disorders, 7, 259-270.

Myers, P. N., \& Biocca, F. A. (1992). The elastic body image: The effect of television advertising and programming on body image distortions in young women. Journal of Communication, 42(3), 108-133.

Nathan, L. (2008). Why the arts make sense in education. The Phi Delta Kappan, 90(3), 177181.

Ng, C. (2011, 09 12). Forever 21's 'Allergic to Algebra' Shirt Draws Criticism. Retrieved 09 18, 2011, from abcNEWS: http://abcnews.go.com/blogs/headlines/2011/09/forever-21sallergic-to-algebra-shirt-draws-criticism/

Nolfi, J. (2011, 07 20). post-gazette.com A\&E/ Art \& Architecture. Retrieved 10 11, 2011, from Warhol exhibit questions sports industry's idea of masculinity: http://www.postgazette.com/pg/11201/1161510-437.stm

Oliver, K. L., \& Lalik, R. (2001). The body as curriculum: learing with adolescent girls. J. Curriculum Studies, 33(3), 303-333.

Omery, A. (1983). Phenomenology: A method for nursing research. Advances in Nursing Science, 5, 49-63.

O'Toole, C. H. (2011). Mixed Signals. Retrieved 10 11, 2011, from Carnegie Museums of Pittsburgh: http://www.carnegiemuseums.org/cmag/article.php?id=271

Paine, S. (2010, September 13). W.Va. schools to celebrate arts in education [speech]. West Virginia Department of Education. Retrieved from: http://wvde.state.wv.us/news/2158/ 
Patton, M. Q. (2002/2005). Qualitative research \& evaluation methods. Thousand Oaks, CA: Sage.

Pipher, M. (1994). Reviving Ophelia. Saving the selves of adolescent girls. . New York: Riverhead Books.

Popova, M. (2010, 11 17). Denis Dutton's Provocative Darwinian Theory of Beauty. Retrieved 11 22, 2011 from http://www.brainpickings.org/index.php/2010/11/17/denis-duttondarwinian-theory-of-beauty/

Purnell, P. (2004). A place for the arts. The past, the present and teacher perceptions. Teaching artists journal, 2(3), 153-161.

Rabkin, N., \& Redmond, R. (2006). The arts make a difference. Educational Leadership(February), 60-64.

Romanska, M. (2005). Ontology and eroticism: Two bodies of Ophelia. Women's Studies, 34, 485-513.

Ruben, M. (2011, 03 23). The State Journal. Retrieved 09 07, 2011, from W.Va. Superintendent Jorea Marple's message — Invest now or pay later: http://statejournal.com/story.cfm?func=viewstory\&storyid=96451

Ruppert, S. S. (2006). Critical evidence: How the arts benefit student achievement. Washington, DC: National Assembly of State Arts Agencies (NASAA) \& Arts Education Partnership (AEP).

Schoder, S. (2010). Language learning in three early childhood programs in Austria, Germany and the United States (Doctoral dissertation). Retrieved from http://wvuscholar.wvu.edu:8881//exlibris/dtl/d3_1/apache_media/23550.pdf. $(000002565)$ 
School Information . (n.d.). Retrieved 04 05, 2011, from anonymous

Silverman, R. J. (2010). Good girls don't get fat. How weight obsession is messing up our girls and how we can help them thrive despite it. New York: The Stonesong Press.

Sims, C. (2011). [Themes, schema, and scaffolding: art and cognitive development]. Unpublished doctoral dissertation article.

Stace, W. T. (1997). Meaning of beauty a theory of aesthetics. Kila, MT: Kessinger Publishing.

Steiner, D. M. (2004). Aesthetics between philosophy and pedagogy. Journal of Education, 184(1), 39-56.

The Andy Warhol Museum (2008). Power point presentations - resources for teachers. Aesthetics response [Power point presentation]. Retrieved from http://edu.warhol.org/20c_ppt.html

The Arts Education Partnership (n.d.). Making a case for the arts. How and why the arts are critical to student achievement and better schools. Art Education Partnership. Retrieved from http://aep-arts.org/files/publications/MakingaCaseforarts.pdf

The John F. Kennedy Center for Performing Arts (2008). Artists as educators - laying a foundation. Defining arts integration [Seminar]. Materials developed by Silverstein, L.B.\& Layne, S. for the Partners in Education Program of National Partnerships in the Education Department of the John F. Kennedy Center for the Performing Arts. Washington, DC.

The World Book Encyclopedia. (2001). Beauty. [Definition], p. 746. Chicago, IL: World Book Encyclopedia.

Tunks, J., \& Grady, P. M. (2003). Arts infusion in university courses. The effect on student choice to infuse art in elementary classes. Curriculum and Teaching Dialogue, 5(1), 61- 
70.

Van Manen, M. (1990). Researching lived experience: Human science for an action sensitive pedagogy. New York, NY: State University of New York.

Visual Thinking Strategies (n.d.). Retrieved from http://www.vtshome.org/

Walshaw, M. (2001). A Foucauldian gaze on gender research: What do you do when confronted with the tunnel at the end of the light? Journal for Research in Mathematics Education, $32(5), 471-492$.

Weiss, C.; Lichtenstein, A. L. ; Booth, E. (2008). AIM print. New relationships in the arts and learning. Chicago, IL: Columbia College.

West Virginia University Libraries. (n.d.). Retrieved 09 25, 2011, from Databases: http://www.libraries.wvu.edu/databases/database.php?id=50

Why your child needs the arts. (n.d.). Gaining the arts advantage [pdf]. Retrieved from http://aep-arts.org/files/publications/WhyYourChildNeedstheArts.pdf

Wilber, K. (1997). To see a world - art and the I of the beholder. Journal of Transpersonal Psychology, 29(2), 143-149.

Wilber, K. (1997, November). Ken Wilber online. To see a world—art and the I of the beholder [Website article]. Retrieved from http://wilber.shambhala.com/html/misc/tosewo.cfm

Wilson, B., \& Harlan, H. (. (Eds.). (1985). The history of education. The Pennsylvania State University College of Arts \& Architecture School of Visual Arts. 


\section{Appendix A: Quick Facts about Arts Integration by American for the Arts}

\section{Did You Know?}

Young people who participate in the arts for at least three hours on three days each week through at least one full year are:

* 4 times more likely to be recognized for academic achievement

* 3 times more likely to be elected to class office within their schools

* 4 times more likely to participate in a math and science fair

* 3 times more likely to win an award for school attendance

* 4 times more likely to win an award for writing an essay or poem

Young artists, as compared with their peers, are likely to:

* Attend music, art, and dance classes nearly three times as frequently

* Participate in youth groups nearly four times as frequently

* Read for pleasure nearly twice as often

* Perform community service more than four times as often

('Living the Arts through Language + Learning: A Report on Community-based Youth Organizations," Shirley Brice Heath, Stanford University and Carnegie Foundation For the Advancement of Teaching, Americans for the Arts Monograph, November 1998)

The facts are that arts education...

* makes a tremendous impact on the developmental growth of every child and has been proven to help level the "learning field" across socio-economic boundaries 
(Involvement in the Arts and Success in Secondary School, James S. Catterall, The UCLA Imagination Project, Graduate School of Education \& Information Studies, UCLA, Americans for the Arts Monograph, January 1998)

* has a measurable impact on at-risk youth in deterring delinquent behavior and truancy problems while also increasing overall academic performance among those youth engaged in afterschool and summer arts programs targeted toward delinquency prevention

(YouthARTS Development Project, 1996, U.S. Department of Justice, National Endowment for the Arts, and Americans for the Arts)

Businesses understand that arts education...

* builds a school climate of high expectation, discipline, and academic rigor that attracts businesses relocating to your community

* strengthens student problem-solving and critical thinking skills, adding to overall academic achievement and school success

* helps students develop a sense of craftsmanship, quality task performance, and goalsetting — skills needed to succeed in the classroom and beyond

* can help troubled youth, providing an alternative to destructive behavior and another way for students to approach learning

* provides another opportunity for parental, community, and business involvement with schools, including arts and humanities organizations

* helps all students develop more appreciation and understanding of the world around them

* helps students develop a positive work ethic and pride in a job well done (Business Circle for Arts Education in Oklahoma, "Arts at the Core of Learning 1999 Initiative"). 


\section{Appendix B: Benefits of Arts Education - as simple as A-B-C}

One convenient way to sum up how study of the arts benefits student achievement is the recognition that learning in the arts is academic, basic and comprehensive. It is as simple as A-BC.

A

Learning in the Arts is Academic.

Learning experiences in the arts contribute to the development of academic skills, including the areas of reading and language development and mathematics.

\section{B}

Learning in the Arts is Basic.

Arts learning experiences contribute to the development of certain thinking, social and motivational skills that are considered basic for success in school, life and work. These fundamental skills encompass a wide range of more subtle, general capacities of the mind, self-perceptions and social relationships. 
C

Learning in the Arts is Comprehensive.

The arts help create the kind of learning

environment conducive to teacher and

student success by fostering teacher innovation,

a positive professional culture, community

engagement, increased student attendance,

effective instructional practice and school identity. 


\section{Appendix C: 10 Lessons the Arts Teach (Eisner, 2002a)}

1. The arts teach children to make GOOD JUDGMENTS about qualitative relationships. Unlike much of the curriculum in which correct answers and rules prevail, in the arts, it is judgment rather than rules that prevail.

2. The arts teach children that problems can have MORE than ONE solution and that questions can have more than one answer.

3. The arts celebrate multiple PERSPECTIVES. One of their large lessons is that there are many ways to SEE and INTERPRET the world.

4. The arts teach children that in complex forms of problem solving purposes are seldom fixed, but change with circumstance and opportunity. Learning in the arts requires the ABILITY and a WILLINGNESS to surrender to the unanticipated possibilities of the work as it unfolds.

5. The arts make VIVID the fact that neither words in their literal form nor numbers exhaust what we can KNOW. The limits of our language do not define the limits of our COGNITION.

6. The arts teach students that SMALL DIFFERENCES can have LARGE EFFECTS. The arts traffic in subtleties.

7. The arts teach students to think through and within a material. All art forms employ some means through which IMAGES become REAL.

8. The arts help CHILDREN LEARN to say what cannot be said. When children are invited to disclose what a work of art helps them FEEL, they must reach into their POETIC CAPACITIES to find the words that will do the job. 
9. The ARTS ENABLE us to have EXPERIENCE we can have from no other source and through such experience to DISCOVER the range and variety of what we are capable of FEELING.

10. The arts' position in the school curriculum symbolizes to the young what adults BELIEVE (Eisner, 2002a). 


\section{Appendix D: Six Distinctive Forms of Thinking (Eisner, 2002c)}

1. Experiencing qualitative relationships and making judgments.

2. Flexible purposing.

3. Form and content is most often inextricable.

4. Not everything knowable can be articulated in propositional form.

5. Looking to the medium.

6. The aesthetic satisfactions that the work itself makes possible (Eisner, 2002c). 


\section{Appendix E: Eight Ways that the Arts are included in Present U.S. School Settings (Davis, 1999b)}

1. Arts-based involved the arts as a required core subject in the school curriculum. Using this medium, skills taught through the arts are transferred to skills in other academic areas.

2. Arts-injected (or infused) included those arts activities that are integrated into the general curriculum in order to enhance a particular study with no requirements of specific content studied.

3. Arts included (...) involved arts instruction being offered alongside other subjects with or without integration into these subjects.

4. Arts-expansion (...) through this process, the arts were taken outside the school setting. Students explored the arts at museums, performances, or concerts.

5. Arts-professional was designed to train students who are seriously preparing for careers in arts.

6. Arts-extra (...) referred to the use of the arts in an ancillary role. Students who participated in extra-curricular arts activities such as school plays or piano lessons were involved in the arts-extra approach.

7. Aesthetic education model referred to the type of instruction where the arts were viewed as ways of knowing and where students were encouraged to construct their knowledge.

8. Arts-cultura was based on the premise that the arts connect the cultures of the world (...) students can be empowered to create their own meaning of content through the arts. This model further encouraged risk-taking, critical thinking, and diligence (Davis, 1999a, as cited in Gullatt, 2008, p. 14). 


\section{Appendix F: Sexist Clothing Examples}

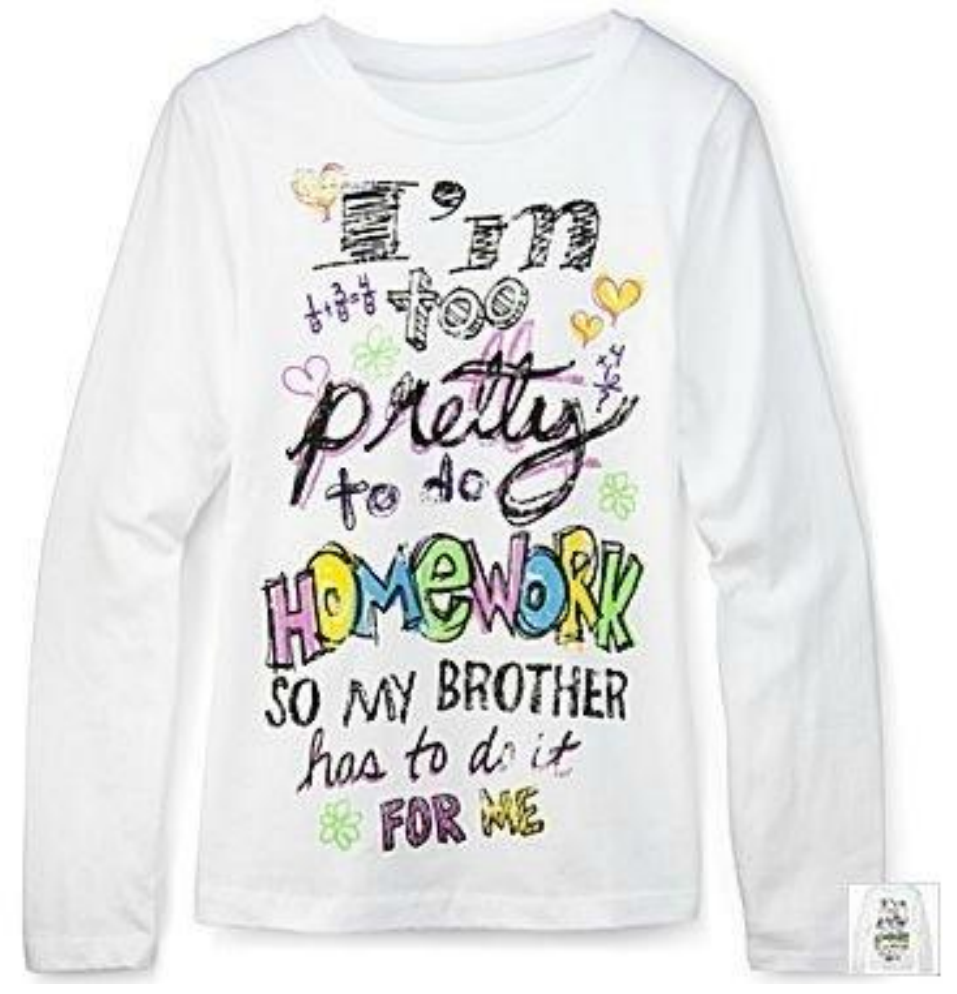

Figure F1. J.C. Penney's Shirt with the Slogan "I'm too pretty to do Homework so my Brother has to do it for me."

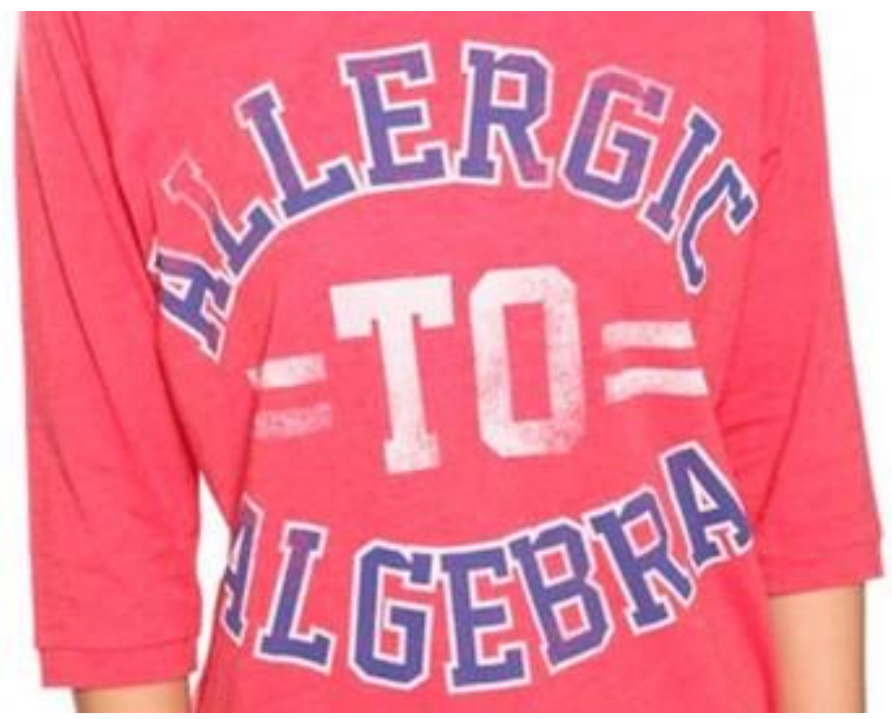

Figure F2. Forever 21's Shirt for Girls with the Slogan "Allergic to Algebra." 


\section{Appendix G: Materials for Interview Process \\ Appendix G1 - Email to Potential Participants (Parents and Students)}

Dear

My name is Miriam Roth Douglas, and I am a doctoral student of Dr. Joy Faini Saab, who provided me with your contact information. She also suggested that I contact you in reference to participating in the research of my dissertation. My dissertation research will be on arts based learning experiences and their possible impact on the self-concepts of adolescent girls and their perceptions of beauty. The purpose of this study is to learn more about participants' elementary school experiences with the arts (e.g. painting, drawing, sculpting, singing, dancing, playing an instrument, acting, etc.). Specifically, this study focuses on how you and your daughter experienced the incorporation of the arts in the classroom for all subjects (e.g. English, Mathematics, ...) and how these art forms (painting, drawing, sculpting, ...) possibly influenced your daughter in different ways, e.g., her self-concept. Dr. S. recommended you and your daughter to me as possible interviewees because of your past experiences with the arts. The purpose of this email is to tell you about my future research and to ask if you and your daughter would be willing to participate in my dissertation study.

If you have any further questions about the project or would like to talk to me in person, please feel free to call me at Xxx-Xxx-Xxxx or send an email:

$\underline{\text { mrd.odgi@gmail.com or mroth2@ mix.wvu.edu }}$

Sincerely,

Miriam 


\section{Appendix G2 - Email to Potential Participants (Teachers)}

Dear

My name is Miriam Roth Douglas, and I am a doctoral student of Dr. Joy Faini Saab. Dr. S. provided me with your contact information. She also suggested that I contact you in reference to participating in the research of my dissertation. My dissertation research will be on arts based learning experiences and their possible impact on the self-concepts of adolescent girls and their perceptions of beauty. The purpose of this study is to learn more about participants' elementary school experiences with the arts (e.g. painting, drawing, sculpting, singing, dancing, playing an instrument, acting, etc.). In specific, this study focuses on how you experienced the incorporation of the arts in the classroom for all subjects (e.g. English, Mathematics, ...) and how these art forms (painting, drawing, sculpting, ...) possibly influenced your female students in different ways, e.g., their self-concepts. Dr. S. recommended you to me as possible interviewees because of your past experiences with the arts based learning experiences. The purpose of this email is to tell you about my future research and to ask if you would be willing to participate in my dissertation study.

If you have any further questions about the project or would like to talk to me in person, please feel free to call me at Xxx-Xxx-Xxxx or send an email:

$\underline{\text { mrd.odgi@gmail.com or mroth2@mix.wvu.edu }}$

Sincerely,

Miriam 


\section{Appendix G3 - Cover Letter Parent Participants}

Dear Parent-Participant,

This letter is a request for you to take part in a research project to assess what, if any, phenomena may be associated with young ladies', who completed Arts-Integrated Curriculum, self-concept and perceptions of beauty. This research study is being conducted by Miriam Roth Douglas, MA with supervision of Joy Faini Saab, Ed.D., Chair of the Department of Curriculum \& Instruction/ Literacy Studies and Director or the Office for Diversity \& Global Initiatives in the College of Human Resources and Education at West Virginia University, for an Interdisciplinary

Ph.D. in Education. Your participation in this project is greatly appreciated and our interview(s) should last about 30-60 minutes. With your permission this interview will be audiotaped in order to accurately represent what you say.

Your involvement in this project will be kept as confidential as legally possible. All data will be reported in the aggregate. Your name will not be attached to notes from this interview or to transcribed data, and the audio will be erased once transcription is complete. At no time will your name be revealed during reporting. You must be 18 years of age or older to participate. I will not ask any information that should lead back to your identity as a participant. Your participation is completely voluntary. You may skip any question that you do not wish to answer and you may discontinue at any time. Your job status will not be affected if you decide either not to participate or to withdraw. West Virginia University's Institutional Review Board acknowledgement of this project is on file. 
I hope that you will participate in this research project, as it could be beneficial in understanding what, if any, phenomena may be associated with young ladies', who completed Arts-Integrated Curriculum, self-concept and perceptions of beauty. Thank you very much for your time. Should you have any questions about this letter or the research project, please feel free to contact Miriam Roth Douglas at xxx-xxx-xxxx or by e-mail at mroth2@mix.wvu.edu or miri.roth@ gmail.com.

Thank you for your time and help with this project.

Sincerely,

Miriam Roth Douglas

mroth2@mix.wvu.edu

$\mathrm{XXX}-\mathrm{XXX}-\mathrm{XXXX}$

Joy Faini Saab, Ed.D.

joy.saab@mail.wvu.edu

304-293-4084 


\section{Appendix G4 - Cover Letter Parent-of-Student Participants}

Dear Parent-of-Student-Participant,

This letter is a request for you to agree on your daughter participating in a research project to assess what, if any, phenomena may be associated with young ladies', who completed ArtsIntegrated Curriculum, self-concept and perceptions of beauty. This research study is being conducted by Miriam Roth Douglas, MA with supervision of Joy Faini Saab, Ed.D., Chair of the Department of Curriculum \& Instruction/ Literacy Studies and Director or the Office for Diversity \& Global Initiatives in the College of Human Resources and Education at West Virginia University, for an Interdisciplinary Ph.D. in Education. Your daughter's participation in this project is greatly appreciated. The interview(s) with her should last about 30-60 minutes. With your permission this interview will be audiotaped in order to accurately represent what your daughter says.

Your daughter's involvement in this project will be kept as confidential as legally possible. All data will be reported in the aggregate. Your daughter's name will not be attached to notes from this interview or to transcribed data, and the audio will be erased once transcription is complete. At no time will her name be revealed during reporting. You must be 18 years of age or older to participate. I will not ask any information that should lead back to her identity as a participant. Her participation is completely voluntary. She may skip any question that she does not wish to answer and she may discontinue at any time. Her class standing or grades will not be affected if she decides either not to participate or to withdraw. West Virginia University's Institutional Review Board acknowledgement of this project is on file. 
I hope that you will approve your daughter's participation in this research project, as it could be beneficial in understanding what, if any, phenomena may be associated with young ladies', who completed Arts-Integrated Curriculum, self-concept and perceptions of beauty. Thank you very much for your and your daughter's time. Should you or your daughter have any questions about this letter or the research project, please feel free to contact Miriam Roth Douglas at xxx-xxxxxxx or by e-mail at mroth2@ mix.wvu.edu or miri.roth@gmail.com.

Thank you for yours and your daughter's time and help with this project.

Sincerely,

Miriam Roth Douglas

mroth2@mix.wvu.edu

$\mathrm{xxX}-\mathrm{XxX}-\mathrm{XxXx}$

Joy Faini Saab, Ed.D.

joy.saab@mail.wvu.edu

304-293-4084 


\section{Appendix G5 - Cover Letter Student Participants}

Dear Student-Participant,

This letter is a request for you to take part in a research project to assess what, if any, phenomena may be associated with young ladies', who completed Arts-Integrated Curriculum, self-concept and perceptions of beauty. This research study is being conducted by Miriam Roth Douglas, MA with supervision of Joy Faini Saab, Ed.D., Chair of the Department of Curriculum \& Instruction/ Literacy Studies and Director or the Office for Diversity \& Global Initiatives in the College of Human Resources and Education at West Virginia University, for an Interdisciplinary

Ph.D. in Education. Your participation in this project is greatly appreciated and our interview(s) should last about 30-60 minutes. With your permission this interview will be audiotaped in order to accurately represent what you say.

Your involvement in this project will be kept as confidential as legally possible. All data will be reported in the aggregate. Your name will not be attached to notes from this interview or to transcribed data, and the audio will be erased once transcription is complete. At no time will your name be revealed during reporting. I will not ask any information that should lead back to your identity as a participant. Your participation is completely voluntary. You may skip any question that you do not wish to answer and you may discontinue at any time. Your class standing or grades will not be affected if you decide either not to participate or to withdraw. West Virginia University's Institutional Review Board acknowledgement of this project is on file. 
I hope that you will participate in this research project, as it could be beneficial in understanding what, if any, phenomena may be associated with young ladies', who completed Arts-Integrated Curriculum, self-concept and perceptions of beauty. Thank you very much for your time. Should you have any questions about this letter or the research project, please feel free to contact Miriam Roth Douglas at xxx-xxx-xxxx or by e-mail at mroth2@mix.wvu.edu or miri.roth@gmail.com.

Thank you for your time and help with this project.

Sincerely, Miriam Roth Douglas mroth2@mix.wvu.edu $\mathrm{XXX}-\mathrm{XXX}-\mathrm{XXXX}$

Joy Faini Saab, Ed.D. joy.saab@mail.wvu.edu 304-293-4084 


\title{
Appendix G6 - Consent and Information Form
}

\section{WestVirginiaUniversity.}

Office of Research Compliance

\section{CONSENT AND INFORMATION FORM}

\author{
OMR ICF - Parent \& Teacher - Participants
}

Principal Investigator: Saab, Joy

Department: HUMAN RESOURCES \& EDUCATION - Curriculum \& Tracking Number: Instruction/Literacy Studies (C\&I/LS) $\mathrm{H}-23154$

\section{Study Title:}

The Arts-Integrated Curriculum (AIC) and its possible impact on the selfconcepts of adolescent girls and their perceptions of beauty.

\section{Co-Investigator(s):}

Miriam Roth Douglas

\section{Sponsor}

NA

\section{Contact Persons}

If you have any questions, concerns, or complaints about this research, you can contact Dr. Joy Faini Saab at 304/ 293-4084 or Miriam Roth Douglas at

For information regarding your rights as a research subject, you may contact the Office of Research Compliance at 304/293-7073.

\section{Introduction}

In addition if you would like to discuss problems, concerns, have suggestions related to research, or would like to offer input about the research, contact the Office of Research Integrity and Compliance at 304293-7073.

You, have been asked to participate in this

\begin{tabular}{lllll}
\hline Tracking \#: & H-23154 & Page 1 of 5 & \\
Approved On: & $04 / 20 / 2011$ & & & Initials \\
Valid Through: & $04 / 19 / 2012$ & & & \\
Last Amended: & N/A & & &
\end{tabular}


Tracking \#: $\quad \mathrm{H}-23154$

research study, which has been explained to you by Miriam Roth Douglas, M.A. This study is being surveilled by Joy Faini Saab, Ed.D. and conducted by Miriam Roth Douglas, M.A. in the Department of Human Resources \& Education - Curriculum \& Instruction/ Literacy Studies (C\&I/ LS) at West Virginia University. This research is being conducted to fulfill the requirements for a doctoral dissertation in Curriculum, Literacy, and Cultural Studies (C\&I/ LS) in the Department of Human Resources \& Education - Curriculum \& Instruction/ Literacy Studies (C\&I/LS) at West Virginia University, under the supervision of Joy Faini Saab, Ed.D.

\section{Purposes of the Study}

The purpose of this study is to learn more about the participant's (students', parents', teacher's) learning experiences with the arts (e.g. painting, drawing, sculpting, singing, dancing, playing an instrument, acting, etc.) during their time/ involvement in Elementary School. In specific, this study focuses on the incorporation of the arts in the classroom for all subjects (e.g. English, Mathematics, and the possible influence of these art forms on female students. The study wants to find out if various art forms, integrated in all subjects of the curriculum, might guide the way female students think about themselves and how they see themselves and their bodies. WVU expects to enroll approximately seven to ten subjects.

\section{Description of Procedures}

This study involves a short demographic questionnaire for all participants, which asks questions about the participants' demographic background, like "How old are you?, What is your gender?", or "Where are you originally from?". After the completion of the demographic questionnaire, one to two interviews will be conducted. Interview \#1 contains questions about the learning experiences of the participants with the arts (e.g. painting, drawing, sculpting, singing, dancing, playing an instrument, acting, etc.) in Elementary School. Some of the questions will be about the incorporation of the arts in the classroom for all subjects and how these art forms possibly influenced female students and the way they think about themselves and their bodies s (e.g. in a positive way, a negative way, or they had no impact at all). Some interview questions, as for example "What did you notice about your daughter/ student when she was engaged in arts based learning experiences" and "What do you yourself consider

\begin{tabular}{lll}
\hline Tracking \#: & H-23154 & Page 2 of 5 \\
Approved On: & $04 / 20 / 2011$ & \\
Valid Through: & $04 / 19 / 2012$ & \\
Last Amended: & N/A
\end{tabular}


Tracking \#: $\quad$ H-23154

the most valuable experience(s) as a parent/ teacher of a student participating in arts based learning experiences", will be predetermined. Other, similar questions might develop during the interview process. The interview and its questions will be structured, but at the same time flexible and open-ended so that the participant will have a chance to talk instead of just answering "yes" or "no." The interview's main themes will include firstly, experiences of all the participants (students, parents, teacher) with the integration of the arts (e.g. painting, drawing, sculpting, singing, dancing, playing an instrument, acting, etc.) in the classroom/ all subjects (English, Mathematics, secondly, the way the girls think about themselves because of their learning experiences with the arts, and thirdly, what female students consider as "beautiful". A Follow-up Interview \#2 may take place, in case it is necessary to clarify arising themes from Interview \#1. The completion of the demographic questionnaire form will take approximately 5-15 minutes, and the interview(s) should last about 30-90 minutes. You do not have to answer all the questions. You will have the opportunity to see the questionnaire before signing this consent form. With your permission the interview(s) will be digitally audiotaped in order to accurately represent what you say.

\section{Risks and Discomforts}

There are no known or expected risks from participating in this study, except for the mild frustration associated with answering the questions.

\section{Alternatives}

You do not have to participate in this study.

Alternatives that could be considered in your case include not to participate in this study.

\section{Benefits}

You may not receive any direct benefit from this study. The knowledge gained from this study may eventually benefit others. The data could inform the research area of Arts-Integrated Curriculum (AIC) and its possible impact on the self-concepts of adolescent girls and their perceptions of beauty.

$\begin{array}{lll}\text { Tracking \#: } & \mathrm{H}-23154 & \text { Page } 3 \text { of } 5 \\ \text { Approved On: } & 04 / 20 / 2011 & \\ \text { Valid Through: } & 04 / 19 / 2012 & \\ \text { Last Amended: } & \text { N/A }\end{array}$


Tracking \#: $\quad \mathrm{H}-23154$

\section{Financial Considerations}

NA

\section{Confidentiality}

Any information about you that is obtained as a result of your participation in this research will be kept as confidential as legally possible. Your research records may be subpoenaed by court order or may be inspected by federal regulatory authorities without your additional consent.

Audiotapes will be kept locked up and will be destroyed as soon as possible after the research is finished.

In any publications that result from this research, neither your name nor any information from which you might be identified will be published without your consent.

\section{Voluntary Participation}

Participation in this study is voluntary. You are free to withdraw your consent to participate in this study at any time. Refusal to participate or withdrawal will not affect you in any way and will involve no penalty to you. In the event new information becomes available that may affect your willingness to participate in this study, this information will be given to you so that you can make an informed decision about whether or not to continue your participation. You have been given the opportunity to ask questions about the research, and you have received answers concerning areas you did not understand.

\begin{tabular}{lll}
\hline Tracking \#: & H-23154 & Page 4 of 5 \\
Approved On: & $04 / 20 / 2011$ \\
Valid Through: & $04 / 19 / 2012$ \\
Last Amended: & N/A
\end{tabular}

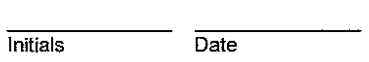

Valid Through: 04/19/2012

N/A 
Upon signing this form, you will receive a copy.

I willingly consent to participate in this research.

$\begin{array}{lll}\begin{array}{l}\text { Signature of Subject or } \\ \text { Subjects Legal Representative }\end{array} & \overline{\text { Pate }} & \overline{\text { Timed Name }}\end{array}$

The participant has had the opportunity to have questions addressed. The participant willingly agrees to be in the study.

$\begin{array}{lll}\begin{array}{l}\text { Signature of Investigator or } \\ \text { Co-Investigator }\end{array} & \text { Date } & \text { Primted Name }\end{array}$

\begin{tabular}{lllll}
\hline Tracking \#: & $\mathrm{H}-23154$ & Page 5 of 5 & \\
Approved On: & $04 / 20 / 2011$ & & & Date \\
Valid Through: & $04 / 19 / 2012$ & & & \\
Last Amended: & N/A & &
\end{tabular}




\title{
Appendix G7 - Parental or Guardian Consent and Information Form
}

\section{- West VirginiaUniversity. \\ Office of Research Complinance}

\section{PARENTAL OR GUARDIAN CONSENT AND INFORMATION FORM}

\author{
OMR - Parental Consent - Parents - of - Students
}

Principal Investigator: Saab, Joy

Department: HUMAN RESOURCES \& EDUCATION - Curriculum \&

Tracking Number: Instruction/Literacy Studies (C\&I/LS)

$\mathrm{H}-23154$

Study Title:

The Arts-Integrated Curriculum (AIC) and its possible impact on the selfconcepts of adolescent girls and their perceptions of beauty.

Co-Investigator(s):

Miriam Roth Douglas

Sponsor

NA

\section{Contact Persons}

If you have any questions, concerns, or complaints about this research, you can contact Dr. Inv Faini Saab at 304/ 293-7073 or Miriam Roth Douglas at

For information regarding your rights as a research subject, you may contact the Office of Research Compliance at 304/293-7073.

Introduction

In addition if you would like to discuss problems, concerns, have suggestions related to research, or would like to offer input about the research, contact the Office of Research Integrity and Compliance at 304293-7073.

You, have been asked to allow your child to participate in this study, which has been explained

Tracking \#: $\quad \mathrm{H}-23154$

Approved On: $\quad 04 / 20 / 2011$

Valid Through: $\quad$ 04/19/2012

Last Amended: N/A
Page 1 of 6

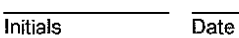


Tracking \#: $\quad$ H-23154

to you and your child by Miriam Roth Douglas, M.A. This study is being surveilled by Joy Faini Saab, Ed.D. and conducted by Miriam Roth Douglas, M.A. in the Department of Human Resources \& Education - Curriculum \& Instruction/ Literacy Studies (C\&I/ LS) at West Virginia University. This research is being conducted to fulfill the requirements for a doctoral dissertation in Curriculum, Literacy, and Cultural Studies in the Department of Human Resources \& Education - Curriculum \& Instruction/ Literacy Studies (C\&I/ LS) at West Virginia University, under the supervision of Joy Faini Saab, Ed.D.

\section{Purposes of the Study}

The purpose of this study is to learn more about the participant's (students, parents, teacher) learning experiences with the arts (e.g. painting, drawing, sculpting, singing, dancing, playing an instrument, acting, etc.) during their time/ involvement in Elementary School. In specific, this study focuses on the incorporation of the arts in the classroom for all subjects (e.g. English, Mathematics, and the possible influence of these art forms on female students. The study wants to find out if various art forms, integrated in all subjects of the curriculum, might guide the way female students think about themselves and how they see themselves and their bodies. WVU expects to enroll approximately seven to ten subjects.

\section{Description of Procedures}

This study involves a short demographic questionnaire for all participants, which asks questions about the participants' demographic background, like "How old are you?, What is your gender?", or "Where are you originally from?". After the completion of the demographic questionnaire, one to two interviews will be conducted. Interview \# 1 contains questions about the learning experiences of the participants with the arts (e.g. painting, drawing, sculpting, singing, dancing, playing an instrument, acting, etc.) in Elementary School. Some of the questions will be about the incorporation of the arts in the classroom for all subjects and how these art forms possibly influenced female students and the way they think about themselves and their bodies s (e.g. in a positive way, a negative way, or they had no impact at all). Some interview questions, as for example " What did you notice about your daughter/ student when she was engaged in arts based learning experiences" and " What do you yourself consider

\begin{tabular}{lllll}
\hline Tracking \#: & $\mathrm{H}-23154$ & Page 2 of 6 & & \\
Approved On: & $04 / 20 / 2011$ & & Initials & Date \\
Valid Through: & $04 / 19 / 2012$ & & & \\
Last Amended: & N/A & &
\end{tabular}


Tracking \#: $\quad \mathrm{H}-23154$

the most valuable experience(s) as a parent/ teacher of a student participating in arts based learning experiences", will be predetermined. Other, similar questions might develop during the interview process. The interview and its questions will be structured, but at the same time flexible and open-ended so that the participant will have a chance to talk instead of just answering "yes" or "no." The interview's main themes include firstly, experiences of all the participants (students, parents, teacher) with the integration of the arts (e.g. painting, drawing, sculpting, singing, dancing, playing an instrument, acting, etc.) in the classroom/ all subjects (English, Mathematics, secondly, the way the girls think about themselves because of their learning experiences with the arts, and thirdly, what female students consider as "beautiful". A Follow-up Interview \#2 may take place, in case it is necessary to clarify arising themes from Interview \#1. The completion of the demographic questionnaire form will take approximately 5-15 minutes, and the interview(s) should last about 30-90 minutes for your child to complete. Your child does not have to answer all the questions. You will have the opportunity to see the questionnaire before signing this consent form. With your permission, the interview(s) will be digitally audiotaped in order to accurately represent what your child says. The study will be performed at a public space. Approximately seven to ten subjects are expected to participate in this study.

\section{Risks and Discomforts}

There are no known or expected risks to your child from participating in this study, except for the mild frustration associated with answering the questions.

\section{Alternatives}

Your child does not have to participate in this study.

Alternatives that could be considered in your child's case include your child not participating in this study.

\section{Benefits}

Your child may not receive any direct benefit from this study. The knowledge gained from this study may eventually benefit others. The data

\begin{tabular}{lllll}
\hline Tracking \#: & $\mathrm{H}-23154$ & Page 3 of 6 & & \\
Approved On: & $04 / 20 / 2011$ & & & Date \\
Valid Through: & $04 / 19 / 2012$ & & & \\
Last Amended: & N/A & & &
\end{tabular}


Tracking f: $\quad H-23154$

could inform the research area of Arts-Integrated Curriculum (AIC) and its possible impact on the self-concept of adolescent girls and their perceptions of beauty.

Financial Considerations

No payments will be made for participating in the study.

\section{Confidentiality}

Any information about your child that is obtained as a result of participation in this research will be kept as confidential as legally possible.

Your child 's research records may be subpoenaed by court order or may be inspected by federal regulatory authorities without your additional consent.

Audiotapes will be kept locked up and will be destroyed as soon as possible after the research is finished.

In any publications that result from this research, neither your child's name nor any information from which your child might be identified will be published without your consent.

\section{Voluntary Participation}

Participation in this study is voluntary.

You or your child may refuse to participate in this study. You or your child may withdraw from this study at any time.

Refusal to participate or withdrawal will not affect your child in any way and will involve no penalty to you or your child.

In the event new information becomes available that may affect your willingness to allow your child to participate in this study, this information will be given to you so that you can make an informed decision about whether or not to continue your child's participation.

You have been given the opportunity to ask questions about the research, and have received answers concerning areas you did not understand.

\begin{tabular}{lllll}
\hline Tracking \#: & $\mathrm{H}-23154$ & Page 4 of 6 & & \\
Approved On: & $04 / 20 / 2011$ & & & Date \\
Valid Through: & $04 / 19 / 2012$ & & & \\
Last Amended: & N/A & &
\end{tabular}


ASSENT: (Statement of person conducting assent discussion)

1. I have explained all aspects of the research to the subject to the best of his or her ability to understand.

2. I have answered all the questions of the subject relating to this research.

3. The subject agrees to be in the research.

4. I believe the subject's decision to enroll is voluntary.

5. The study researcher and the study staff agree to respect the subject's physical or emotional dissent at any time during this research when that dissent pertains to anything being done solely for the purpose of this research.

Signature of Person Conducting Assent Discussion

Date

\section{Statement of Parent/Guardian:}

My child appears to understand the research to the best of his or her ability and has agreed to participate.

$\overline{\text { Signature of Parent/Guardian }}$

Date

\begin{tabular}{lll}
\hline Tracking \#: & $\mathrm{H}-23154$ & Page 5 of 6 \\
Approved On: & $04 / 20 / 2011$ & \\
Valid Through: & $04 / 19 / 2012$ & \\
Last Amended: & N/A &
\end{tabular}


Upon signing this form, you will receive a copy.

I willingly consent to allow my child to participate in this research.

Signature of Parent or Guardian

Printed Name

Date

Time

The parent/guardian has had the opportunity to have questions addressed. The parent/guardian willingly agrees to allow his/her child to be in the study.

$\begin{array}{lll}\begin{array}{l}\text { Signature of Investigator or } \\ \text { Co-Investigator }\end{array} & \text { Printed Name } & \text { Time }\end{array}$

\begin{tabular}{lll}
\hline Tracking \#: & H-23154 & Page 6 of 6 \\
Approved On: & $04 / 20 / 2011$ & \\
Valid Through: & $04 / 19 / 2012$ & \\
Last Amended: & N/A &
\end{tabular}

$\overline{\text { Initials }} \overline{\text { Date }}$




\title{
Appendix G8 - Assent Form
}

\section{- West VirginiaUniversity. \\ office of Research Compliance}

\section{ASSENT FORM}

\author{
OMR-Assent - Girls - Student - Participants
}

Principal Investigator: Saab, Joy

Department:

HUMAN RESOURCES \& EDUCATION - Curriculum \&

Tracking Number:

Instruction/Literacy Studies (C\&I/LS)

$\mathrm{H}-23154$

Study Title:

The Arts-Integrated Curriculum (AIC) and its possible impact on the selfconcepts of adolescent girls and their perceptions of beauty.

Co-Investigator(s):

Miriam Roth Douglas

Sponsor

NA

\section{Contact Persons}

If you have any questions, concerns, or complaints about this research, you can contact Dr. Joy Faini Saab at 304/ 293-4084 or Miriam Roth Douglas at

For information regarding your rights as a research subject, you may contact the Office of Research Compliance at 304/293-7073.

\section{Introduction}

In addition if you would like to discuss problems, concerns, have suggestions related to research, or would like to offer input about the research, contact the Office of Research Integrity and Compliance at 304293-7073.

You, have been asked to be in this research study, which has been explained to you by Miriam Roth Douglas.

\begin{tabular}{lllll}
\hline Tracking \#: & $\mathrm{H}-23154$ & Page 1 of 4 & \\
Approved On: & $04 / 20 / 2011$ & & & Date \\
Valid Through: & $04 / 19 / 2012$ & & & \\
Last Amended: & N/A & & &
\end{tabular}




\section{Purposes of the Study}

You have been told that the purpose of this study is to learn more about your Elementary School learning experiences with the arts (e.g. painting, drawing, sculpting, singing, dancing, playing an instrument, acting, etc.). In specific, this study focuses on how you experienced the incorporation of the arts in the classroom for all subjects (e.g. English, Mathematics, and how these art forms (painting, drawing, sculpting, possibly influenced you in different ways. The study wants to find out if the various art forms, integrated in different subjects, changed the way you think about yourself, how you see your body, and what you think is beautiful.

\section{Description of Procedures}

This study will be done at a public space. You will be asked to come and see the researcher, Miriam Roth Douglas, who will ask you questions about your arts based learning experiences. This study involves the completion of a short questionnaire, which includes questions like "How old are you?, What is your gender?", or "Where are you originally from?". After the completion of the questionnaire, one to two interviews will be conducted. Interview \# 1 contains questions about your learning experiences with the arts (e.g. painting, drawing, sculpting, singing, dancing, playing an instrument, acting, etc.) in Elementary School. Specifically, you will be asked about how these art forms (painting, drawing, sculpting, possibly influenced you in different ways or not at all. The study wants to find out if the various art forms, integrated in different subjects, changed the way you think about yourself, how you see your body, and what you think is beautiful. Examples of possible questions are "What did you notice about yourself when you were engaged in art forms like painting or drawing in subjects like English or Mathematics?" or "What do you consider your best experience(s) as a (former) participant in arts based learning experiences that integrated art forms like painting or drawing?". A Follow-up Interview \#2 might take place, in case it is necessary to clarify things from our conversation from Interview \#1. The completion of the demographic questionnaire form will take you approximately 5-15 minutes, and the interview(s) should last about 30-90 minutes. You may see the questions before signing this page. You do not have to answer all of the questions. With your permission the interview(s) will be digitally audiotaped in order to accurately represent what you say.

\begin{tabular}{lllll}
\hline Tracking \#: & H-23154 & Page 2 of 4 & & \\
Approved On: & $04 / 20 / 2011$ & & Dnitials & \\
Valid Through: & 04/19/2012 & & & \\
Last Amended: & N/A & &
\end{tabular}




\section{Discomforts}

Some of the questions will be difficult, may cause discomfort, and you may not enjoy trying to answer them.

\section{Benefits}

This study may not help you, but what they learn from the study may help other people.

\section{Confidentiality}

We promise that anything we learn about you in this study will be kept as secret as possible. Digital interview audiotapes, interview transcripts, demographic research data, and all other information obtained during this research study will be securely maintained in a locked file cabinet and/ or a password-secured private computer, accessible only to the committee chair and doctoral candidate. Confidentiality will be assured through the use of pseudonyms and a coding system that replaces individual names. Once the research is completed, digital audiotapes will be erased and transcriptions, as well as all other collected data will be shredded or deleted three years after the completion of the study.

\section{Voluntary Participation}

You do not have to do this. No one will be mad at you if you refuse to do this or if you decide to quit. You have been allowed to ask questions about the research, and all of your questions were answered.

Page 3 of 4

Initiais Date 
I willingly agree to be in this research.

Signature of Subject

The child has had the opportunity to have questions addressed. The child willingly agrees to be in the study.

Signature of Investigator or

Printed Name

Date

Time

Co-Investigator

\begin{tabular}{lll}
\hline Tracking \#: & $\mathrm{H}-23154$ & Page 4 of 4 \\
Approved On: & $04 / 20 / 2011$ & \\
Valid Through: & $04 / 19 / 2012$ & \\
Last Amended: & N/A &
\end{tabular}




\section{Appendix G9 - Cover Letter Teacher Participant}

Dear Teacher-Participant,

This letter is a request for you to take part in a research project to assess what, if any, phenomena may be associated with young ladies', who completed Arts-Integrated Curriculum, self-concept and perceptions of beauty. This research study is being conducted by Miriam Roth Douglas, MA with supervision of Joy Faini Saab, Ed.D., Chair of the Department of Curriculum \& Instruction/ Literacy Studies and Director or the Office for Diversity \& Global Initiatives in the College of Human Resources and Education at West Virginia University, for an Interdisciplinary Ph.D. in Education. Your participation in this project is greatly appreciated and our interview(s) should last about 30-60 minutes. With your permission this interview will be audiotaped in order to accurately represent what you say.

Your involvement in this project will be kept as confidential as legally possible. All data will be reported in the aggregate. Your name will not be attached to notes from this interview or to transcribed data, and the audio will be erased once transcription is complete. At no time will your name be revealed during reporting. You must be 18 years of age or older to participate. I will not ask any information that should lead back to your identity as a participant. Your participation is completely voluntary. You may skip any question that you do not wish to answer and you may discontinue at any time. Your job status will not be affected if you decide either not to participate or to withdraw. West Virginia University's Institutional Review Board acknowledgement of this project is on file. 
I hope that you will participate in this research project, as it could be beneficial in understanding what, if any, phenomena may be associated with young ladies', who completed Arts-Integrated Curriculum, self-concept and perceptions of beauty. Thank you very much for your time. Should you have any questions about this letter or the research project, please feel free to contact Miriam Roth Douglas at xxx-xxx-xxxx or by e-mail at mroth2@mix.wvu.edu or miri.roth@gmail.com.

Thank you for your time and help with this project.

Sincerely, Miriam Roth Douglas mroth2@mix.wvu.edu $\mathrm{XXX}-\mathrm{XXX}-\mathrm{XXXX}$

Joy Faini Saab, Ed.D.

joy.saab@mail.wvu.edu

304-293-4084 


\title{
Appendix G10 - Research Questions
}

\section{Major Research Question:}

What, if any, phenomena may be associated with the self-concept ${ }^{1}$ and perceptions of beauty $^{2}$ of young ladies who completed Arts-Integrated Curriculum (AIC) ${ }^{3}$ ?

\section{Minor Research Questions:}

\section{Q1. How do young ladies who completed Arts-Integrated Curriculum (AIC) ${ }^{3}$}

\begin{abstract}
${ }^{1}$ Self-concept described for participants: The way the female students think about themselves and perceive themselves (including their body-perception). Definition of Self-concept by Bardone-Cone et al. (2010):

Assumption that, coming from a cognitive perspective, the "self-concept can be defined as 'a set of knowledge structures about the self' (Stein \& Corte, 2007, quoted in Bardone-Cone et al., 2010, p. 823) and encompasses a wide range of constructs" (Baumeister, 1999, quoted in Bardone-Cone et al., 2010, p. 823). Bardone-Cone et al. (2010) furthermore add, according to Markus and Wurf (1978), that "self-concept represents a dynamic multifaceted construct, rather than a unitary undifferentiated structure" (p. 823).

${ }^{2}$ Beauty described for participants: Everything that the female students perceive as "beautiful" - an objective or subjective (including their body) perspective. The students also could refer to "external" (the way one looks from the "outside") or "internal" (the characteristics one carries "inside") beauty. Definition of Beauty by The World Book Encyclopedia's (2001):
\end{abstract}

Beauty as our desire to make and enjoy beautiful things. But others prefer to use the word beauty in a fuller sense. They say that the sheer satisfaction we feel when we perceive a complex but balances design and, the profound satisfaction we experience in understanding complex but clear meaning, both contribute to beauty. Even in the fuller sense, whether a thing is beautiful does not depend on its being useful. Many paintings (...) have no use apart from their value as works of art (...). Yet, some of these works are considered art and displayed in museums because they illustrate something of aesthetic importance in their design. (The World Book Encyclopedia, 2001, p. 746)

3 Arts-Integrated Curriculum (AIC) described for participants: Integrating the Arts, e.g. painting, drawing, sculpting, singing, dancing, playing an instrument, acting, etc., in the school curriculum/ all subjects (English, Mathematics, ...). Definition of Arts Integration by the John F. Kennedy Center for Performing Arts (2008): Arts Integration is an APPROACH to TEACHING in which students construct and demonstrate UNDERSTANDING through an ART FORM. Students engage in a CREATIVE PROCESS which CONNECTS an art form and another subject area and meets EVOLVING OBJECTIVES in both. 
describe their Arts-Integrated Curriculum (AIC) ${ }^{3}$ experience?

Q2. How do parents of young ladies who completed Arts-Integrated Curriculum (AIC) $^{3}$ describe their Arts-Integrated Curriculum (AIC) ${ }^{3}$ experience?

Q3. How do teachers of young ladies who completed Arts-Integrated Curriculum (AIC) $^{3}$ describe their Arts-Integrated Curriculum (AIC) ${ }^{3}$ experience? 


\title{
Appendix G11 - Expedited-IRB Protocol Approval
}

\author{
V. West VirginiaUniversity. \\ Office of Research Compliance
}

\section{Expedited-IRB Protocol-Approval}

\author{
To: $\quad$ Saab, Joy \\ From: $\quad$ WVU Office of Research Compliance \\ Date: Wednesday, April 20, 2011 \\ Subject: Approval Letter
}

Tracking \#: $\mathrm{H}-23154$

Title: $\quad$ The Arts-Integrated Curriculum (AIC) and its possible impact on the self-concepts of adolescent girls and their perceptions of beauty.

The research study referenced above was reviewed by the West Virginia University Instituional Review Board (IRB) via expedited review procedures and was approved in accordance with 46 CFR 46.101(b).

This protocol was reviewed using the following:

Initial Review ChecklistSubcommittee/Contingent Approval Checklist (210d)

The following documents have been approved and validated for use in this study and are available in the BRAAN system:

Surveys, Questionnaires, Interviews Attachments Demographic Questionnaire for Parents - Miriam Roth Douglas - 03-10-11 - 1.doc Demographic Questionnaire for Parents (Miriam Roth Douglas) Demographic Questionnaire for Students Miriam Roth Douglas - 03-10-11 - 1.doc Demographic Questionnaire for Students (Miriam Roth Douglas) Demographic Questionnaire for Teacher - Miriam Roth Douglas - 03-10-11 - 1.doc Demographic Questionnaire for Teacher (Miriam Roth Douglas) InterviewQuestions-Parents-MiriamRothDouglas.doc Interview Questions Parents - Miriam Roth Douglas InterviewQuestions-StudentsMiriamRothDouglas.doc Interview Questions Students - Miriam Roth Douglas interviewQuestions-Teacher-MiriamRothDouglas.doc Interview Questions Teacher - Miriam Roth Douglas Research Questions - Major and Minor - Míriam Roth Douglas - 03-10-11 - 1.doc Research Question(s) (Miriam Roth Douglas) Consent Form(s) Forms OMR ICF - Parent \& Teacher - Participants OMR Parental Consent - Parents - of - Students OMR-Assent - Girls - Student Participants Miscellaneous Attachments Attachments Email-parents\&students - 
MiriamRothDouglas.doc Email to Parents \& Students - Miriam Roth Douglas Email-teacher-MiriamRothDouglas.doc Email to Teacher - Miriam Roth Douglas

The expedited category is 7. The approval period is from April 20, 2011 through April 19, 2012.

Thank you.

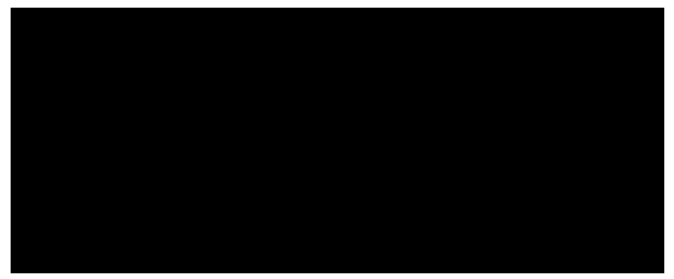

Once you begin your human subject research, the following regulations apply:

1. Unanticipated or serious adverse events/side effects encountered in this research study must be reported to the IRB within five (5) days.

2. Any modifications to the study protocol or informed consent form must be reviewed and approved by the IRB prior to implementation.

3. You may not use a modifíed informed consent form until it has been approved and validated by the IRB. 


\section{Appendix G12 - Demographic Questionnaire for Parents}

Parent (coded):

Date:

1. What is your gender?

2. What is your ethnicity?

3. How old are you?

4. Are you married or divorced?

5. Are you a single parent?

6. Do you work? 
7. How many hours a day do you usually spent at work (away from home)?

8. Where are you originally from?

9. Where do you live now?

10. How many children (boys/girls) do you have?

11. How old are your children? 
12. For how long has/ have your child/ children attended an Arts-Integrated Curriculum $(\mathrm{AIC})^{4}$ school?

13. Did you yourself receive an Arts-Integrated Curriculum (AIC) ${ }^{1}$ education?

4 Arts-Integrated Curriculum (AIC) described for parents: Integrating the Arts, e.g. painting, drawing, sculpting, singing, dancing, playing an instrument, acting, etc., in the school curriculum/ all subjects (English, Mathematics, ...).

Definition of Arts Integration by the John F. Kennedy Center for Performing Arts (2008): Arts Integration is an APPROACH to TEACHING in which students construct and demonstrate UNDERSTANDING through an ART FORM. Students engage in a CREATIVE PROCESS which CONNECTS an art form and another subject area and meets EVOLVING OBJECTIVES in both. 


\section{Appendix G13 - Demographic Questionnaire for Students}

Student (coded):

Date:

1. What is your gender?

2. What is your ethnicity?

3. How old are you?

4. Where are you originally from?

5. Where do you live now? 
6. To which school did you go when you received an Arts-Integrated Curriculum (AIC) ${ }^{5}$ education?

7. For how many years did you receive an Arts-Integrated Curriculum (AIC) ${ }^{1}$ education?

8. Did you receive any Arts training (e.g. painting, drawing, sculpting, singing, dancing, playing an instrument, acting, etc.) before you went to this Arts-Integrated Curriculum $(\mathrm{AIC})^{1}$ school ?

5 Arts-Integrated Curriculum (AIC) described for students: Integrating the Arts, e.g. painting, drawing, sculpting, singing, dancing, playing an instrument, acting, etc., in the school curriculum/ all subjects (English, Mathematics, ...).

Definition of Arts Integration by the John F. Kennedy Center for Performing Arts (2008): Arts Integration is an APPROACH to TEACHING in which students construct and demonstrate UNDERSTANDING through an ART FORM. Students engage in a CREATIVE PROCESS which CONNECTS an art form and another subject area and meets EVOLVING OBJECTIVES in both. 
9. Do you have siblings? If so, how many brothers or sisters do you have?

10. How old are your siblings?

11. Do or did any of them go to an Arts-Integrated Curriculum (AIC) ${ }^{1}$ school ? 


\section{Appendix G14 - Interview Questions for Parents}

1.) How did you personally experience your daughter participating in the arts based learning experiences?

2.) How do you think the arts based learning experiences that your daughter went through is different from the school experience of others who did not participate in an arts based learning experiences (e.g. friends of your daughter, who did not attend the same school or some of your other children)?

3.) Do you feel like you noticed a difference in your daughter's learning experience "in" and "through" the arts?

4.) Do you feel like your daughter learned through the arts based learning experiences how to look differently at things (e.g. notice details she did not pay attention to before)?

5.) Do you feel like your daughter learned through the arts based learning experiences to look differently at people (e.g. the way they are dressed, the way they look like in general, etc.)?

6.) Do you feel like the arts based learning experiences had an influence on how your daughter sees herself (e.g. the way she looks, the way she dresses, her body, etc.)?

If so, did it change it in a positive or negative way?

7.) What would you consider an example of what your daughter thinks is beautiful (e.g. object/ thing, human being/ other, human being/ self)?

8.) (A) How do you define self-concept?

(B) How would you describe your daughter's self-concept?

9.) What memories or situations come to your mind when you think of your daughter's experiences with the arts based learning experiences in Elementary School? 
10.) Do you wish other people (e.g. your friends' children, younger siblings of your daughter, etc.) should have the opportunity to go through arts based learning experiences, as well?

Why or why not? 


\section{Appendix G15 - Interview Questions for Students}

1.) How did you personally experience the arts based learning experiences?

2.) How do you think the arts based learning experiences that you went through are different from the school experience of other children (e.g. if you think of what your friends, who did not go to the same school as you, tell you about their school experience)?

3.) Do you feel like you notice a difference in learning "in" and "through" the arts?

4.) Do you feel like you learned through the arts based learning experiences how to look differently at things (e.g. notice details you did not pay attention to before)?

5.) Do you feel like you learned through the arts based learning experiences to look differently at people (e.g. the way they are dressed, the way they look like in general, etc.)?

6.) Do you feel like the arts based learning experiences had an influence on how you see yourself (e.g. the way you look, the way you dress, your body, etc.)? If so, did it change it in a positive or negative way?

7.) What would you consider an example of what you think is beautiful (e.g. object/ thing, human being/ other, human being/ self)?

8.) How would you describe your self-concept, or the way you think about yourself?

9.) What memories or situations come to your mind when you think of your experiences with the arts based learning experiences in Elementary School?

10.) Do you wish other people (e.g. your friends, younger siblings, etc.) should have the chance to go through arts based learning experiences, as well?

Why or why not? 


\section{Appendix G16 - Email Request for Parents and Students to Validate the Data}

Dear Parent Participant,

Thank you (and your daughter

) for meeting with me in an extended interview

and sharing your Arts-Integrated-Curriculum (AIC) experiences in reference to the self-concepts of adolescent girls and their perceptions of beauty. I appreciate you and your daughter's willingness to share your unique and personal thoughts, feelings, events, and situations. I very much enjoyed our interview and all your contributions and new insights that you and your daughter provided to me.

I have enclosed a transcript of our interview. Would you (and ) please review the entire document? Could you please make sure to ask yourself if this interview has fully captured your experience of the Arts-Integrated Curriculum (AIC) and its possible impact on the self-concepts of adolescent girls and their perceptions of beauty?

After reviewing the transcripts of the interview, you (and/or ) may realize that an important experience(s) was neglected or you would like to further clarify one of your comments. Please feel free to add comments that would further elaborate your experience(s). Please do not edit for grammatical corrections. The way you and told your story is what is critical.

When you have reviewed the verbatim transcript and have had an opportunity to make changes and additions, please return the transcript per email, if possible, the latest by the $5^{\text {th }}$ of July, to mrd.odgi@gmail.com. 
I would appreciate it if you and could use a different type of font or a differently colored font to make your changes, additions, and comments. This way, it is easier for me to distinguish who of you added which comments.

In case you do not feel comfortable to use the computer (e.g. the "Microsoft word program" or the "insert comments function") to add any comments or make any changes to the document, please let me know. I also could send a hardcopy of the interview by mail to your house so you and Giulia could edit it by hand.

I have greatly valued your participation in this research study and your willingness to share your experience. If you have any questions or concerns, do not hesitate to call me. I can be reached at $\mathrm{XXX}-\mathrm{XXX}-\mathrm{XXXX}$.

Have a great summer!

With warm regards,

Miriam

P.S./ FYI: Names of schools, places, or people have been changed to fictional names in order to make this document anonymous so that names, places, and people cannot be identified. , you will find your comments with the fictional name of and will find her comments labeled with In case you do not like the acronym that I used for your real names, please feel free to come up with new suggestions and I will be happy to change them. 


\section{Appendix G17 - Interview Questions for Teachers:}

1.) How did you personally experience your female students participating in the arts based learning experiences?

2.) How do you think the arts based learning experiences that your female students went through are different from the school experience of others who did not experience arts based learning experiences (e.g. friends of your female students, who did not attend the same school, etc.)?

3.) Do you feel like you noticed a difference in your female students' learning experiences "in" and "through" the arts?

4.) Do you feel like your female students' learned through the arts based learning experiences how to look differently at things (e.g. notice details they did not pay attention to before)?

5.) Do you feel like your female students learned through the arts based learning experiences to look differently at people (e.g. the way they are dressed, the way they look like in general, etc.)?

6.) Do you feel like the arts based learning experiences had an influence on how your female students see themselves (e.g. the way they look, the way they dress, their body, etc.)?

If so, did it change it in a positive or negative way?

7.) What would you consider an example of what your female students thinks is beautiful (e.g. object/ thing, human being/ other, human being/ self)?

8.) (A) How do you define self-concept?

(B) How would you describe your female students' self-concept?

9.) What memories or situations come to your mind when you think of your female students' experiences with the arts based learning experiences in Elementary School?

10.) Do you think other students (e.g. students who were unable to attend your school, etc.) should have the possibility/opportunity to go through arts based learning experiences, as well? 
Why or why not? 


\section{Appendix G18 - Demographic Questionnaire for Teachers}

Teacher (coded):

Date:

1. What is your gender?

2. What is your ethnicity?

3. How old are you?

4. Where are you originally from?

5. What city do you live in now?

6. From which institution(s) did you receive your teacher education? 
7. For how may years have you been a teacher (years of experience)?

8. What grade levels do/ did you teach?

9. At which schools did you teach?

10. At how many different schools did you teach?

11. Did you receive any specialized training?

12. Did you receive any prior Arts training? 
13. Did you teach in an Arts-Integrated Curriculum (AIC) ${ }^{6}$ ?

14. If so, for how long did you teach in an Arts-Integrated Curriculum (AIC) ${ }^{1}$ and which grade levels?

15. Do you hold any other certifications?

16. Which degree(s) total do you hold?

17. What is your highest level of degree?

6 Arts-Integrated Curriculum (AIC) described for teacher: Integrating the Arts, e.g. painting, drawing, sculpting, singing, dancing, playing an instrument, acting, etc., in the school curriculum/ all subjects (English, Mathematics, ...).

Definition of Arts Integration by the John F. Kennedy Center for Performing Arts (2008): Arts Integration is an APPROACH to TEACHING in which students construct and demonstrate UNDERSTANDING through an ART FORM. Students engage in a CREATIVE PROCESS which CONNECTS an art form and another subject area and meets EVOLVING OBJECTIVES in both. 
18. From which institution did you receive your degrees (in specific your highest level of degree)?

19. Do or did you serve on committees?

20. What is your income (range)? 


\section{Appendix G19 - Email Request for Teachers to Validate the Data}

\section{Dear MOTIVATED and INVOLVED,}

(MOTIVATED, could you please forward this to INVOLVED, as I mentioned before, I do not have her emailaddress. Thank you!)

Thank you for meeting with me in an extended interview and sharing your Arts-IntegratedCurriculum (AIC) experiences in reference to the self-concepts of adolescent girls and their perceptions of beauty. I appreciate your willingness to share your unique and personal thoughts, feelings, events, and situations. I very much enjoyed our interview and all your contributions and new insights that both of you provided to me.

I have enclosed a transcript of our interview. Would you please review the entire document? Could you please make sure to ask yourself if this interview has fully captured your experience of the Arts-Integrated Curriculum (AIC) and its possible impact on the self-concepts of adolescent girls and their perceptions of beauty?

After reviewing the transcripts of the interview, you may realize that an important experience(s) was neglected or you would like to further clarify one of your comments. Please feel free to add comments that would further elaborate your experience(s). Please do not edit for grammatical corrections. The way you told your story is what is critical.

When you have reviewed the verbatim transcript and have had an opportunity to make changes and additions, please return the transcript per email, if possible, the latest by the $5^{\text {th }}$ of July, to mrd.odgi@gmail.com.

I would appreciate it, if you could use a different type of font or a differently colored font from the original interview transcript one, to make your changes, additions, and comments. This way, it is easier for me to distinguish where you added comments.

In case you do not feel comfortable to use the computer (e.g. the "Microsoft word program" or the "insert comments function") to add any comments or to make any changes to the document, 
please let me know. I also could send a hardcopy of the interview by mail to your house so you could edit it by hand.

I have greatly valued your participation in this research study and your willingness to share your experience. If you have any questions or concerns, do not hesitate to call me. I can be reached at $x x x-x x x-x x x x$.

Have a great summer!

With warm regards,

Miriam

P.S./ FYI: Names of schools, places, or people have been changed to fictional names in order to make this document anonymous so that names, places, and people cannot be identified. MOTIVATED, you will find your comments with the fictional name of "MOTIVATED" and INVOLVED, you will find your comments labeled with "INVOLVED". Please, also feel free to exchange or switch your names in front of a quote, if you feel like you did not say that, but somebody else did (sometimes it was kind of difficult to distinguish between the four female voices). In case you do not like the acronyms that I substituted for your real names, please feel free to come up with new suggestions and I will be happy to change them. 


\section{Appendix H: Data Analysis Materials (Working Documents) \\ Appendix H1 - List of ICs for parents}

\section{BUSY:}

"I am BAND's mother and I own my own business (...)."

"I would hear about it. They would come home from school and talk about some things they would do in the classroom, not a whole lot, but, you know, they would come home and say, "Oh, we learned this song today. Or, we, in math we sang this song," or there was one time where they were doing some African music with painting, bare foot painting to African music and that was actually for an art class. But I remember where there were times where they would incorporate some different types of arts into even math or reading and things like that, and even today, yeah, I still hear about it with the little one."

"Yes, if it was art, something they painted or drew, you know, they would show me that, or they learned a song or did something, they would either sing it for me or tell me about it."

"Well, okay, I am a little biased with arts—-based learning just because of influences you know within my own family because music was big part of our family. So I feel like they gain more when they are exposed to the arts in all different subjects. I think it just broadens their outlook, open to newer things and maybe show more of an interest in it."

“(...) we will go out and seek (...) we will go to concerts and we will_— I just think we have more of an interest in the arts."

"I just know, it is a big part of our lives. The arts are a big part of our lives and that could have come from, you know, being exposed to it."

“(...). I think even in math they would add numbers and then if the answer was such and such, she is doing it now, you know, color that in and when you colored in the different colors, it would form a picture and so it is like, oh, wow, you can do this with numbers. So, yes, I think it exposed them to different things that made them look at things in a different way."

"Broaden these perspectives."

"I also think it depends a lot on how you are raised, as well, your family environment, you know, how you are brought up. (...)."

“(...) she is very conscientious about how she looks and colors and matching and things like that. (...) that could have been a result of the arts - based learning. I guess some of the dancing and, you know, how to hold yourself, I know they did some dancing classes in grade school and how to sort of hold yourself and present yourself and things like that. So, yes, I think so."

"A flower?." 
"I think it is more that she thinks objects are beautiful. She mentioned a rose. That is interesting because, okay, so I have a buisness, which is a creative place, she sometimes will come in and do some things. She made a flower out of clay and had petals and everything, and was all, it was beautifully done and she painted it and everything. And so I think for her it is more nature and things like that, that she thinks are beautiful."

"(...) I think it is more of a natural beauty and the nicer personality that she would think of as beautiful."

“(...) I think there still has to be a physical beauty [external beauty] about it, too, but not the real made up kind of person, all of the real striking, stark makeup, but it is more of a natural."

"Self - concept, I will say like how confident you are of yourself and how are you as a person, whether it is how you relate as a mom, you know, am I a good mother, am I a good business owner or, you know, what kind of image do I portray to other people."

"She is a very caring person, always willing to help out, very responsible. She does laundry. She cooks. She takes care of... She is like a second mother to the little one. She is — she is somewhat shy, like she is not a real outgoing, flamboyant type of person. She is a good friend, to her friends, did I say good listener? She is a good listener when it comes to them. She will listen, but not pass judgment, you know."

"I think for the most part, she is happy with herself. There are times she is trying on clothes or trying to figure out what to wear, oh, this doesn't look right, oh man, and every so often we will start to talk about healthy eating and exercise and things like that, and try to focus more on that in the family."

“(...) for me I am trying clothes on that don't fit and I really need to make more time to get outside and get some exercise or if it is you, well, like, we were bathing suit shopping. "You know, I don't think I want to wear a two-piece bathing suit because I know my belly will show," but, you know, it is okay. And I think we are comfortable with where we are at. It would be nice to be a little thinner, but, she always looks nice in whatever she wears and she is clean and well-kept and she takes care to do her makeup and everything."

"And where did you hear that? (...). Like news or hear it on TV?"

"They would have like an introductory meeting at the beginning of the year. And the teachers would talk about their concepts and how they go about teaching and how they would use arts and music and I think maybe some like physical movements to integrate that into their curriculum. I just thought it was a great idea. (...) I know that every so often they would come home and talk about, you know, what they did. I can sort of remember her saying, "Well, that was pretty neat. That is neat how they incorporated that (...)."

"The parents volunteered. We put on a musical, 101 Dalmatians."

"It was an after - school program that we did with the kids and I was involved in that." 
"No, it was the parents."

"It was an individual, CARING is her name (...) I think maybe she had experienced that as a child, as well. And so she had suggested we put on a production and have people the kids actually tried out for parts because there are how many puppies? (...) we purchased the play from Disney, the actual script and music and everything and kids signed up. They tried out for the main parts, but everybody that wanted to be a part of it was in it. They would sing, they would practice songs, they would do choreography. (...) the school did support it. They helped arrange for after-school practices and things like that, we used the building and everything. I thought it was a great experience."

"Yes, it was the parents."

"Well, other people [should experience AIC], definitely. (...) I knew that Sunrise was a school that integrated the arts. And so I wanted to live in this area for that reason. (...) I always recommend that school, knowing, you know, what they use in the curriculum (...). I just think it just exposes the kids to so much more (...) if they are not brought up in a family where arts is emphasized, then they might not ever be exposed to something like that. I also think it helps them learn, when they come home and they are chanting or singing a song, they come home and sing the days of the week or months of the year and things like that, I mean it helps them definitely remember. They still, you know, they can still sing it to this day."

"I mean, I even use it, I remember like when we are practicing spelling words, you know, hard words right now, we will sing — song it, to try to remember. (...) We would sort of sing — song it. Try it this way. It might be easier to remember."

"I just think it has been a positive experience and I think knowing__ I think the school, this particular school that we went to because they used that, I think it has a good reputation for that. (...) they do a little bit more than_— it is a different environment maybe than some other schools that don't and that is a good thing. (...) I was very happy with it and enjoyed hearing, what was going on and what I saw. I would volunteer at the school, so I would see some of the things that were going on, as well."

"[You would recommend a school with AIC]. Oh, absolutely, no regrets, yes. Definitively."

"I hear more from her about her art class that she had. And band. (...). She comes home and shows me what she has done. Really nice."

"She has told me that the teacher has a couple of things from her class, so I think she is proud of that."

"I think she is all good with that. In general, the art just, you know-I danced as child. I did 12 years of dancing. We always took art classes. We were always involved in music."

"She is talented and I like that she has an interest in it and she enjoys it." 


\section{CARING:}

"I am CARING. I am PIANO's Mom. (...)."

"I grew up, you know, I think like many girls, being very interested in role playing and drama and that kind of thing. So I was involved in drama as a kid, especially in high school. I played instruments, a few different instruments, took lessons and also played in orchestra and band and sang a lot. I sang in the church choir, I sang in choirs at school and I sang also solos at various places and things like that. So I think that is about it. I also enjoy drawing (...). And I loved it when we had classes at school or when we had a special person come into the school and do story-telling (...), some of the things that we might not associate as being part of the arts any more, but are wonderful arts that people share with others."

"And I work in the arts now."

"Well, yes, film making is considered an art."

"And maybe we should say that PIANO's dad is a graphic designer."

"Oh, it was wonderful, and I think Mrs. INVOLVED in particular was very focused on the arts, especially visual arts, I would say, but all sorts of things. And so I felt like PIANO was being exposed to things. I mean, she is exposed to things here at home with us, but in the classroom, it becomes more legitimate, almost, you know what I mean? You know, when you do things at home, kids can kind of think, "Oh well, that is just us. Our family does that," when you start to see it in a more institutionalized way, it I think makes more of an impact on them in the sense of, wow, this is something that our entire culture values, you know, our society values. So, it was wonderful. I will never forget during this impressionist unit, Mrs. INVOLVED had all of usall of the parents come in. (...) but each child took their parents around and taught them about impressionism and taught them about the various artists and showed them the techniques and things like that. We went to different stations. (...)."

"Yeah, that was a different unit, so many great things. She did one thing where it was kind of like some Renaissance thing. I can't remember what it was, but they did big floppy hats."

"So it was great. It was really a wonderful thing. (...) there was music playing and, the kids were very knowledgeable, all of them. It was a wonderful way to learn because I think because it was integrated and it was real, there in the classroom, it made more of an impression on them."

"You mean instead of projecting what you think you looked like, you had to actually draw [selfportraits] what you really looked like?"

“(...) she didn't have any real drama classes in school, which I wish they did have. I wish there was more of an opportunity at early ages for kids to sort of get up in front of people (...) everyone was so focused on the arts, we had a committee there called $\mathrm{A}$ is for the Arts, which was a PTG, parent teacher group committee. And we brought in a lot of different arts groups for the kids to see, musical groups and dance groups (...). But, we also put on a play there one year as a part as the $\mathrm{A}$ is for Arts, we did, 101 Dalmatians and literally, more than half of the school, more like two - thirds of the school was involved in the show. And I think to me that says what a hunger there is for kids to do that kind of thing at that age. (...) it was just packed and we told 
everyone that if you audition, you will have a part. So we had like 101 Dalmatians and we had about 120 people involved and it was unbelievable. So, you know, we broke up parts so that people shared them and things like that because we wanted everybody to have a speaking part. And I can tell you that for PIANO (...) because of her involvement in drama, she is not afraid to speak out. She is not afraid to stand up in front of people and do things and often, I think, to take a leadership role in things. Whereas before she got involved in that kind of thing, she was very, very, very shy. (...) I think her experiences at school, but, also, I think her experiences in this children's drama group in town here (...), really made a huge, huge difference in her (...) the ability to get out in front of people and know that that is a comfortable place to be, that that is okay. She gained a lot of comfort level, I guess, from that. (...) I think experiences like in Mrs. INVOLVED's class where she did have to do presentations, she did have to educate us about the impressionist and that kind of thing (...) got her (...) more deeply involved in the subject; because when you have to teach something, you really have to know it, but it also, I think, gave her confidence that she could know something well enough to be able to teach it or be able to explain it to other people."

"Oh, definitely, before her involvement with the arts and after? Absolutely, there is no question about it. We saw the moment when it happened, too. It was so funny, my husband and I, we were watching, I think it was your second or third show maybe."

"No, it was Bugsy Malone."

"No, I thought Fiddler on the roof was your first."

"It was her very first show and she was very fortunate to have a couple of high school girls who sort of took her under their wings. (...) it sort of is a K through 12 group, which is great. The older kids mentor the younger ones. And she was actually given a part (...) a little speaking part and she was too shy to do it. She would not go out on stage when it was time, so they gave it to somebody else to do. Then she wouldn't go out during, like for the numbers-like they were all supposed to march on stage and sing a little song."

"But then in the finale when they were doing curtain calls, one of these young women picked her up, (...) brought her out onto the floor and everybody was applauding and she started dancing with her and PIANO just looked out at the audience and her eyes lit up and she started laughing and smiling and my husband and I were, there it is. She has gotten over it, that I think many people live with forever, public speaking and that kind of thing, have a lot of anxiety." "Got rid of it just like that. It took a little while, it is not like it was instant. But yeah, it was a great, wonderful experience."

"I think it is because of Anabell."

"(...) I think, was she was a wonderful role model in the sense that she was not afraid of anything. She was very embracing of life, I would say, really fun, young woman. And she also had a great sense of self, I think from the acting a little bit, too. She was a terrible singer. I hate to say this about her because she is a wonderful kid. She has gotten better because she has had a lot of lessons. She was off key a lot. She would belt it out anyway, and she would sing, ahah-ah, and it was great, I mean, who cares, right? 
And she also played boys a lot. She would play the comedic role, which was a boy because there were not enough boys involved in theater. Some girls always have to play boys. Some of the best parts are boy parts."

"I think that was great, that she was not afraid to play nontraditional roles, too. (...) she played a lot of those kinds of roles. That made a big difference.

"I think that (...) when you incorporate these things into sort of who you are, you know, if you self-identify as an artist or as an actor (...) you learn those skills I think more because of who you start to feel it is who you are. And it becomes, I don't know how to explain that."

"Yes, and I think music, too. She has been doing piano for five years now, and I think it has helped her ear also. You know, she can hear things and appreciate them that she wasn't able to before. She can_like singing, playing the piano has helped with singing, too. She is able to hear harmonies."

“(...). I think it just gives you a greater appreciation, you know, for the complexity and the diversity and the beauty of what is around us."

"I'm not sure, maybe through acting because there is a lot of (...) stereotypes that are on stage because it is funnier and that is the way it seems to be. But there is also a lot of, with any kind of storytelling, I think, you learn about people's emotions and you learn about their motivations and you learn, I think that we are all alike in a certain level. Many of the classic stories that become theater, become, things that you study in the arts and even artist's lives, tend to be about the underdog, the person who is not understood, who feels like they are outside, the outsider, you know, who feels bad about themselves in one way or another. And I think reading and acting and learning about the people's lives, you get more of an insight into how these people are not really different and how, you know, I felt that way before, too, kind of thing. So you have more compassion, I think, more empathy for the outsider. Because artists often are outsiders, people who are drawn to the arts sometimes are outsiders or feel themselves to be."

"I do not know. That is interesting. I am sure there have been many studies. I don't know, maybe they feel because what they feel inside is not being expressed in the society around them, that they need to express it. You know, they need to express who they are and what they feel because they don't see it being shown back to them enough. You know, it is like when you can't be heard, you speak louder, you know, because you want to be heard. You want people to recognize that you are a valid person, even though you feel differently or you look at the world like Van Gogh or somebody looks at the world differently from the way other people did. He thinks, because of that, wanted other people brought in to experience that with him. Maybe he didn't feel quite as alone that way, you know, which is normal. Nobody wants to feel alone."

"You are not as shy as you used to be."

"Do you mean you are more critical of yourself?" 
"Well, I think mostly positive. I think that a lot of the critical stuff is normal, that is just the age. There is a lot of, this is not good enough and I am not pretty enough and I am not X, Y, or Z enough, even. It is hard to avoid that I think at this stage in life. But, I think that by and large it had a very positive effect on her self-image, her ability, like I say, to be fearless about standing in front of people (...). So, yes, I think it has been very positive overall."

"I think she is critical of herself. I don't think she is overly critical of herself."

“(...) I think she knows that she is a very talented and beautiful young woman, but I think that she also thinks it doesn't match up to exactly an ideal in sixth grade of (...). Another person, if there is someone who is prettier, better at something, I think PIANO takes that a little bit hard."

“(...) I think it [self-concept] is probably a lot like PIANO. I

think I am a fairly capable person. I know that I am good at certain things, but then I also recognize my weaknesses in other areas in terms of life skills and being able to do things. I think I look okay. I don't think I am gorgeous or anything like that, but I look the way I would like to look right now (...). I feel I am used to the way I look. I don't know how to describe it exactly. I am familiar with myself."

"Well, yes. I am switching hairdressers or I have."

"Yes, I do dye my hair. I do."

"No, it has been a long time, since I was about 20, has been 26 , almost seven years that I have had this color hair."

"I would like to lose a little weight. Partly that is because of the way I look and partly just for fitness reasons. (...). I guess I would like to have more of a chin like PIANO's that is well defined.

"I have the turkey neck kind of thing. (...). Yes, I mean, I don't complain. I am not going to complain too much because I think that I feel fine with the way I look. You know, as I get older, I would like my teeth to be whiter, but my dentist has told me as you get older, there is no amount of bleaching that you can do to your teeth that will get them bright. (...). Dang, I will never have those bright, white teeth. (...) there are people in my family who have said to me (...) "Oh, you need to get this mole removed because it is so distracting."”

"(...) I love my mole. You know, it is who I am, it is what I have seen since I was eight years old or whatever when I looked in the mirror. That is an example of I would not change too much, because it is who I am, it is what I am used to. It is a good feeling. It is like family, like I am my own family. I look in the mirror and there I am."

"Right, well, there was the one thing I told you about with the dancing on stage. And the wonderful experience with the impressionist unit, that was wonderful."

"There have been lots of little things where PIANO would do something and bring it home or go into the class. One of the things we did at Sunrise Primary a lot, they would put artwork out in the halls that the kids had done. And any time we came, they had lots of open houses, PIANO 
would bring us around and show us everything that was hers and she was very proud of it. Same things with stories she had written, that is one thing that I think you didn't mention that you do, PIANO does a lot of writing. She loves to write. And, you know, just stories that she had written in school that would be up on the wall, too, just like one page kind of things. You know, it was really neat, nice to see that and to see it being valued in that way."

"Just all of the time, there were things all of the time that were really great."

"She did a whole thing about the balloon for science."

"You have some things up in your room, drawings and things like that. My wedding plan thing."

“(...). I don't know if she would want to play for you, but watching her progress in that. (...) piano that has been a great thing for PIANO in the sense that, and I think any artistic discipline, once you get into really going deeply into it, you learn how to work. And that is so important, you know. Because you can be the smartest person in the world and if you don't like to work, you are doomed, you know. I have known lots of very smart people who are flipping burgers right now because they have no motivation. So that has been a wonderful thing with arts that she is doing. You learn that if you want to achieve results and you can see the results whenwhether you are a visual artist or musician, you will see that your will pays off. You might not get that in a normal school because you just feel like you are going on and you are not seeing it in the same way that if you sit down and you work really hard at a piano concerto, you can play it. And everybody else is, like that is great." "If you go to school, you might get A's on tests, but it is not quite the same palpable thing.[Intrinsic motivation]."

"You don't see it in the same way, the progress, I think. "Normal school education" is just——it just keeps going. (...). And it is hard to know_—it is hard to think back in the same way of- gosh, I wasn't able to do this math five years ago. You don't ever sit back and reflect on it in the same way you might with a skill, like a musical skill or artistic skill. I think it is different."

"He is less important when it comes down to it. It is really about the dress."

"She draws and writes when she is bored."

"It is a great little school in general, the focus on the arts.

"I guess, it is going to be the last one, the last small school in the county."

"We will try. So, in fact, we transferred PIANO into that school. This is not the right school district for Sunrise Primary. It was partly that the arts_-integrated learning__ “

"It is a good little school."

"It is hard to get in."

"Well, first of all, because we are a very arts—oriented family and we see the benefits of being involved in the arts for the children's self-esteem, primarily. And, also, we wanted her to go to a small school. (...) because we also know the importance of the nurturing environment. So, we went to every school. We went to every school in the county and - every elementary school and observed in the kindergartens, and just in the classrooms in general." 
"We had like I think four that we had narrowed it down to that we went to. It was the best, you know. There was a lot of parent involvement."

"So, it was (...) also (...) a culture of respect (..) kids didn't get into a lot of trouble because everyone knew them, everybody knows each other."

"I hope that it results in greater funding for the arts in schools, it is pitiful."

"[AIC]. I think it (...) is undervalued. And also other extracurricular things getting involved in sports and things like that, being able to go outside every day, you know, for recess and that kind of thing, I think are as important as learning the times tables. I mean, obviously, you cannot learn your times tables__ I mean, people would argue that maybe, but you know, all of these things are important to becoming a well-rounded individual who can live in a very complex society that we live in. So I hope that somehow we can turn that around and get people to understand that the arts are just as important as anything else, if not even more so, for the development of the human being."

\section{LOVING:}

"I am LOVING and I am FLUTE's mom. That is what I do and what I am-_."

"Well, I guess the only way that I personally experienced it is we - the parents were invited in at the end of this unit that she did about the impressionists, right. And it was wonderful. We were invited in and your child would - and there were stations. And your child would just take you around to each station, one station was like watching a video about one impressionist artist and they made hats, like Mary Cassatt, and they did a bunch of things (...). That is how I experienced it."

"I would say that really that was all that this arts-based-learning curriculum was, it was a unit on the impressionists (...)."

“(...) I can say I was really blown away by it. It was really unique. It was very impressive and not only was I impressed, but you could just tell the kids were utterly engaged. It was really pretty amazing.

I should add, in all honesty, that when these kids went through that unit, they didn't do anything else."

"I think it was just like intense. It was wonderful. I mean, it was unique and fabulous (...)."

"Just the family went to the Pittsburgh Art Museum and we turned a corner and lo and behold, there was Monet's (...) water lilies. (...) FLUTE knew what it was right away. She said, "Look, it is water lilies." And she says, "Is it the real one?" Sure enough, it is the real one in Pittsburgh. (...) it was Monet's water lilies and she was so thrilled and impressed and we sent pictures to Mrs. INVOLVED. But that is pretty cool."

"To be that excited about it, to recognize it, that is pretty awesome." 
"I remember you didn't realize how big it was."

“(...) It is way, several walls, so that was kind of cool. Then we went to look for the Van Gogh's and everything, we saw some Van Gogh's, she knew she wanted to see Van Gogh's. So. That is pretty cool. She is only in third grade."

"We have Van Gogh's wild flowers, just a print, just a poster over her bed."

"Right, that is an example [for AIC] totally."

"They did The Odyssey."

"So that is a good example of learning through the arts, is doing a play, that is great."

"And you did a play on that [Number the Starts], too?(...).

"Number the Starts. That was also Mrs. INVOLVED. That is a perfect example, again, literature, and then she had them do a play about the book. So it was (...) literature and history."

"Yes, I remember her doing that play on Number the Stars about the holocaust, so it wasn't just that they read the book and talked about it, they acted it out, too. And I think that really put them in the character's place. So, again, literature and history through the arts that was a good example. I had forgotten all about that. She was very good about that, doing plays."

"Are you familiar with Brown?"

"Brown versus Board of Education was a 1954 Supreme Court decision that, basically, desegregated American schools."

"There was a play they did 101 Dalmatians and I can tell you that, you probably don't remember it, but I saw it as your mom, that it—she was always, you know, a shy child. And the fact that she got up there on that stage with all of these other little Dalmatians and sang and danced her little heart out was a big step for her, a big developmental step for her in a very positive direction. And that was through the arts."

"Do you remember at first like you didn't want to be in the play and then Sandra invited you and then you were glad you were?"

"Exactly, you had a lot of friends and it worked out, but it was an arts—based thing, it wasn't that you were playing sports with a lot of friends"

"She did a summer camp in New York where we go in the summer. It is a performing arts camp and, boy, that was_— the fact that she just got up there and I couldn't convince her to do it, but a counselor convinced her that, you know, what do they say? You never say no in the theater, you try everything, and she did. It was great."

"Performance anxiety is performance anxiety, but if she didn't have that opportunity to address it at that early age through the arts, she would have been (...) more nervous maybe." 
"What does FLUTE think is beautiful? That is a good Question. Maybe the ocean? And as I say, she appreciates a painting that she thinks is beautiful."

"Oh, my, hello. FLUTE plays the flute and she is in the band and so I have to take a second to brag because her teacher is always saying that she has got natural talent."

"But, seriously, she picked it up and started playing right away. You know how some people struggle to get a sound out of it. Not FLUTE, she picked it up and she played it right away. She sight reads. Her teacher just adores her. Her music is beautiful, it really is. It is very impressive."

"So it tells a story through the music?"

"And you can hear like the frustration and the sadness?"

"I can't wait to hear it. Her band concert is tomorrow."

"I am pretty confident. I had a pretty rough childhood and because of that, I have had to and because of that and because of other unusual circumstances in my life, I have had to develop a real strong sense of self, who I am, and that served me well, I think, in life."

"Well, you know, I am 55 years old. Sometimes I look in the mirror, sometimes I don't even recognize myself, I mean it. I'm serious. It happened just this spring, I kid you not. I am like, "O my God, who is that? It doesn't look like me." (...) As a matter of fact, I used to be a TV news anchor and (...) it was all about how you looked, 110 percent about how you looked. And I ended up leaving that profession when I was 40 because I just didn't want to age on the air. I just didn't want to fight that battle. Why? (...). And so I walked off the set for the very last time and they make you wear a ton of makeup and I had it in this big, plastic thing. And as I walked out of that studio, I just dropped it in the trash can. I am done with this. And ever since then, I am not very much for makeup. And I think that having_ that because of that beauty being so much a part of my profession, in that profession, I kind of went the other way and said, "Now that I am doing something else, what I look like isn't all that important." And I am just now kind of like the pendulum is swinging back the other way where I am starting to care_— what does an older person_— what does an attractive, older person look like? I am trying to invent that as I go. Does that answer your question?"

"It is the way of the world. It is the way of the world. You want to look at a pretty girl. You want to look at a handsome man, you just want to. Whether you think you do or not, you do. That is just the way it is."

"Television is only two dimensional and, you know, because it is only two dimensional, you really don't_—you literally cannot see past appearances.

So, it is superficial by definition. Television is superficial by definition, so if you are not pretty, you are not nothing. It is just the way it is, a horrible way to make a living, horrible. I did it while I could, but it was a conscious decision to stop doing it, when I knew, you are 40 things are going to start happening, don't freak out about it, just do something else, you know. And I am really glad I did. Some people stay and fight and get the face lifts. I see the woman I used to 
work with doing all of that and I kind of_- they look terrific, they look wonderful, but I kind of feel sorry for them, though. It is just the way it is. It is just the way it is."

"Don't bet on it. Don't bet on it. It is human nature. I think so. I think so."

"Yes, we did, it [AIC] was really phenomenal. It was just great."

"Well, you know, it is just so sad to see the arts being cut and cut and cut and cut and I wish everyone, every kid could experience the arts. The more, the better. (...)."

"Do you want to play the flute?"

"I happen to have a clip I can play on the computer."

"It will be just a second, and that will be what she will share." 


\section{Appendix H2 - List of ICs for teachers}

INVOLVED:

"I am INVOLVED. I have taught third grade at Sunrise Primary School for a long time, possibly 33 years, I can't remember. And right now I have been retired for three years."

“(...) At one year I remember more girls were identified gifted than boys and the nice thing about that was that that would be one way that you could have seen maybe a little bit of difference, but the brighter the kids were, especially the girls, I think the harder it was for them to be free and to be creative because there is always a right and wrong answer. And we noticed that —we didn't look at it and say that is a gender thing. We noticed it and said that is definitely a TAG thing. The brighter they were, the more restrictive they were in letting out their feelings out by let's say, drawing or painting or whatever."

"(...) we did a lot of dress up. We had a huge dress - up closet and kids brought clothes to dress up for the different occasions, so it was easier for girls to find stuff. And so they were more free in bringing in things. Boys struggled because they could bring in — as a dress up for a boy- that was more difficult. We really had to coax them along in giving them ideas, but girls were very free about just, "I could bring in my mom's old prom dress," or my_— they always had lots of ideas, where boys didn't. Sometimes the girls would have to jump in and help, you know, have that conversation about what kind of things they could bring."

"As well as the environment, we hung a flag, (...) outside our classroom which

MOTIVATED and I would make, and that also would identify what was happening in the third-grade wing."

“(...) I was talking to a teacher there yesterday and it just broke my heart. She said, "I used to do fun units, like medieval units and rain forest, but I don't do any units because we have to teach for the test, so no units." And we found it contra. The more we taught and let kids be free to express, they were able to think through a question on a test. So, you know, that is one thing we noticed is that they scored higher, but they were willing to take risk and they knew to look at all of them because when you look at a piece of art, we gave them plenty of time to look at it and study it and to examine all angles of it so when they saw a written question on a test, they were able, then, to do that same thing on that test, look at all of the questions, what are some of the parts of the question? And so I think they tested higher. And I think those teachers who say, "I can't because I have to teach to the test," are missing a big piece of what teaching is all about. We also had the environment at our school because it was a small school. (...) we were allowed to do what we did because I think we consistently had high test scores. So (...) no one interfered too much or came down on us and said, "You are going off the curriculum here," because we really weren't. We were just doing it in a different way, but our teaching was always very purposeful. We knew what our goals were and it was_one of the big goals was getting kids to think about things."

"Yes, I do remember one girl specifically who was a nonreader. She really struggled (...) she was liked by kids, but teachers always struggled with her (...). I had a whole group that didn't want to read. We said, "What do you want to read?" Of course, they had ideas. I said, "If we would buy the books you want, would you read them?" Of course. They had to read the books to decide whether or not they were going to go in our class." 
"You read this book and tell me whether it deserves to be in this library. It had to meet criteria. (...). All of a sudden, these kids were reading and then we were writing to these authors and asking for an autographed copy for our classroom [Intrinsic Motivation]. So, they also were on their writing skills. This little girl really took it upon herself, to do this, she was in charge. Then we did a fund raiser of some sort. And she was in charge of the money. So she then tookshe and her mom went to the bank and she kept a register and she would report every so often how much money there was. And then the more money we had, then the more books we could buy. Then, of course, we had to decide again which books. And she did very well. I mean, she really blossomed. She became a very good reader, of course, her math skills became stronger because of the money experience."

"We cut the barrier to the groups, you are the smart and dumb ones."

"(...) we did the art gallery (...) we had maybe 500 paintings all over the room. They would walk in and they were entering the art gallery. And we talked a little bit about we accepted normal recess times, when they came in, this was a new experience. We kept them in the hall and we talked about, "You are going to the art gallery and these are some of the rules," so they knew how to behave in a formal situation. Then they were to just for 20 minutes or so as they were listening to really beautiful classical music, they were going to be observing this art. I think I would have to say that the girls were easier to focus in on a piece and stay longer. I think boys probably scanned, "There is my friend. I will stand right there by him," and this one and this one, then they wandered more. It took them longer to settle in on a painting where the girls would, I think, focus quicker."

"Another one at the end of our unit, they taught their unit to their parents so they became the teacher, another way of teaching leadership. (...) instead of giving them a written test, okay, now, this is how much do you know, they had to teach the whole unit to their parents. (...) all did a really good job, but I think I would have to say the girls were (...) they seem to be eager to do that. You know, "I am going to be a teacher when I grow up." Boys don't usually say that, so I think girls were (...) resistive, boys don't resist, they think they are either going to be a star athlete or rock star."

“(...) I have a granddaughter who is ten. I said to her, "I would like to start a writing club this summer. And it is going to be exclusive. Only special people can get in. And it is going to be "I was thinking of all of the things that we did in writing. She said, "Oh, Grandma, you can't do that." I said, "How come?" She said, "Call it a summer camp, they will come. Don't call it writing." (...) her stigma would be, "Oh, they would never come to do a writing camp, but they would come if it was a summer camp. Call it what needs to be called". "

"(...) oftentimes, it influenced their whole family because they would say when they planned a summer trip, they always had to go to an art museum."

"We wouldn't let them start..." [had to learn patience].

"Look in the mirror for 15 minutes. At first you see yourself and then you begin to see all of the different things, uniqueness about me that is different than you and you start looking at your nose 
and you eye lashes and eyebrows and lips and they start really examining it. So then when you draw, "I didn't know my nose was that big.""

"You don't have any discipline problems. Kids were everywhere. They were never in their seats_- they were in their seats, but they were not structured, okay, this is an English class, sit down and pay attention, here is the page. They were all getting up, doing what they needed, going back to their seats, proceeding to do what they needed to do. They were independent. They could go get themselves water, water paints, brushes, whatever they needed. Then they would clean them up and go on to the next thing. They were independent that way and I guess we were teaching more of that independence, you know, "You are big now, you can take care of yourself," rather than, "Okay, you can't paint until I get everything out for you." Of course, we started like that, but then we showed them how they could do that themselves."

“(...) you always hear about math, especially girls don't like math. (...) again that is that gender thing. Well, what we were trying to do is break that gender (...)."

"(...) she said__ I just may happen to remember the oldest one I had, the one that came to mind and she said he is an engineer, he is going to graduate this year as an engineering professor. He wants to teach, anyway, but an engineering degree and a math degree. And she said I was his favorite teacher. I said, "Why do you think that?" She said, "Because you let him express himself." I think those are things that, you know, he obviously had to be more left brain than right brain. And that is everything we study. You know, in addition to integrating the arts we had a lot of other things going on at that same time. We were studying learning styles. We were studying student - based learning, so all of these things came together for us as professionals. It was very exciting because we got to do all of that together."

"When we talk about talented and gifted, forget the talented part, it is gifted. That means I can take a test, very traditional, academically gifted. Kids who are really artistic, truly artistic, do not qualify for TAG. They are not talented and gifted. You have to be able to be academically inclined to be in the gifted program or the talented and gifted program."

"Oh yes, these kids they used to identify, and I am thinking of Alessandro, who really_ — there wasn't much he liked. He didn't like math, he didn't like reading, he didn't like any of that, but he excelled in art. That is where he finally found himself. (...) he joined the (...) the honors (...) every now and then I see his mom and she says, "He is still painting. He is still painting, I don't know if that is what he will do." But... "“

"I remember the twins (...). One was more obese than the other. And the other teacher who replaced MOTIVATED was not into this fluff that we did - skill and drill. And she had this little girl and her comment was, when we were talking about the twins because I had one of the twins and she had the other twin and she said, "I wouldn't do that because that would embarrass her." And that was the end of that. Now, her sister, did all of those things. (...) And she was heavy, the one I had. They were both heavy. (...)."

"The teacher said we won't do that and that was her excuse because that would embarrass her (the child) because she was much heavier. She weighed more than all of us at the table here. 
She was really obese. She needed to_ for her age. She was tall also, but she was just big, a big girl."

"The other thing, too, we noticed about girls, is that size, you know, oftentimes you say you are so little or you are so big. I mean it was, there was discriminations in all kinds of ways and I think we broke that down. That bothers girls more than boys at that age. At middle school, that changes when if the boys want to play ball and they are only this tall and the other boy is this tall; but the one thing I do remember the girls, you know, they would say, "Oh, you are so tiny or you are so big or you are so tall or you are so short."”

"I think it was more of a comment, I don't think it was a tease, it was like when they were dressing up, they would say, "This isn't going to fit you because you are so little. We need to find something else, or you are so big or tall" or so. I don't think it was a tease, I think they just, it was more this as a fact, it would fit you, you know..."

"We had lots of meetings with kids because of issues, but that never came up, as I remember."

"Well, only in that they mentioned it. (...) maybe because they were more into dressing up that they noticed it. Boys, oh, well, it fits fine, what the heck. But girls, you know, they want to look, that image of looking cute or whatever, more of a girl thing maybe."

"Yes, and boys would be into it, they did all of those things."

"But it took them a little bit longer (...)."

“"My goodness, my headaches are gone."”

"(...) when they did their self-portraits, you could tell their nationality."

"By looking at the painting, you could tell their nationality."

"And the other thing, too, was that when we talk about people of color they are black, but not one black child painted himself black."

"But I think maybe if you gave a first grader a picture and said, "That little boy is black," he would have used a black crayon. But because you are black, you don't see that. The other thing they notice is, "My face isn't all of the same color," so they would know to shade different things or highlight different things. So they didn't use just one color and they noticed their head wasn't a circle. The kids would draw a circle and then they put hair on it, stick it out here somewhere. They didn't do that. They notice their hair, bangs that were kind of sideways, and they had to figure out how to draw."

"(...) modeling is so important. Maybe the girl in us is that we decided, they had to have a model. So we would have a mirror and I would look at the mirror and I would talk out loud. I said, "I am thinking out loud, so bear with me." I would talk it through. Then they would know what to look for. Otherwise, sometimes they don't know if you don't_— so maybe that is the gender in us that says, "Okay, we need to be more sensitive to helping," I don't know, or is that the teacher in us? I'm not sure what that is." 
"And I think not all teachers are willing to do that. I think the teacher I mentioned, she wasn't comfortable-."

"We were good at making fools of ourselves."

"And parents, (...) one parent I remember (...) they came and wrote you a check because they said, "This had to cost you a lot of money."”

"It did. [cost a lot of money]."

"Then there is (...) a little boy, too who had to speak different languages to teach his parents. Because his parents came in and he taught them a lesson in English and his grandparents came in and he taught them Spanish."

“(...) my granddaughter (...) is in fourth grade. Every day it is an issue about they are girly or they are not girly or they think they are just special because they got a new skirt or it is always, I mean — I don't know if that is a fourth grade thing, but girls really bicker about that kind of stuff. That drives me crazy in fourth grade."

"I don't know. I don't know if it is an age thing or not, but it is just about "who is the girly one, who is not the girly one"."

"We assisted them in the problem solving part, the solution."

"(...) the three boys were really unusual. They were called the Three Musketeers. (...). They didn't look the part of the popular boys. Anyway, they always played by themselves, but they wanted to play ball because all of the sports kids played ball and they wanted to be a part of that. (...) We decided to have a meeting. They are all sitting there. (...) they decided that they take over. And they would say, "We want to play, but you don't let us." They say, "We let you". They said "But, yeah, you and you and you, you would never let us be the first in line. We are always at the end of the line and never play. We are on the team but we never play. So, how are we supposed to be good?" And they let them have it."

"(...) how they had proceeded to take leadership and proceeded to tell everybody how they felt. So then the group had to problem-solve. The girls were a part. They were all part and they decided that they would let them play and then they decided that they could have three turns. Yes, but they said, "Even then, we will strike out right a way and we will never be able to play." They said, "We will give you three tries and we will help you." So they proceeded to help these three boys. We often had class meetings to discuss. We were just in the habit of having the children help to solve, rather than just to dictate, there were occasions when we would remind them that it was not a democracy, just do what I say."

"Usually we would make them a part of the solution so we would have a class meeting. And if that meant that math got cut short or social studies didn't happen until the next day, then we did. That was a luxury that we had that was critical, I think, was that our testing was different. We didn't test the same way. Like I said, we taught. They had to teach this lesson and that was their 
grade. Oftentimes, if I knew that, like, you were very bright and I wasn't, I would expect more on your piece than mine. We looked at everybody's piece differently. So if you wrote one sentence, I would send you back and have you put more adjectives or more whatever it is I am looking for from you. Where, at the other hand, if somebody writes a simple sentence, I am really pleased I got a sentence out of that person. So we really looked at those differences and accepted that. You know, I didn't— — they couldn't just stay with that one sentence, but eventually that is good for now. Let's look again. But, so I think we looked at everybody. Again, I don't think we could pick a girl from a boy."

"I am thinking of this other little girl who, her mother was a stripper, her dad owned a bar and her language reflected it and her actions reflected it. (...). She was a real tough kid both in the classroom and out. She could tear you down as quick as not. And she said one day, "I would like to be a teacher, but my dad says I should do what Mom does because I could make more money." I thought, "I bet you could," but I know she is volunteering in classrooms. She has gotten her life turned around. Her dad finally quit the bar. Mom is out of the picture. Mom does not get to see her at all and she is now volunteering in classrooms (...)."

"I told her she would be a good teacher and then she said, "I would be better than you." I said, "I bet you would." She always had a comment. (...). We did conflict resolution and the policeman came and taught it for 13 weeks or something like that. So, the policeman comes in his usual garb, the gun and all. He has everything on that policemen would wear and he came in and talked. (...). She always had an answer. And when she left, I said, "How did that go for you?" He said, "I will see her down in the jail some day," and I don't think he will. I don't think he will."

"(...) she is finally beginning to see that there is a different life out there that could be more positive;"

"You could not have picked her out of the class to say that is the one. (...). The other thing, too, is you would not have walked in a classroom and said, "That kid is a discipline problem." You would not have found one kid. Or you would not have found the kids that are ADHD. You might have found autistic kids (...) but most you would not find (...) you could not pick out the kids who were ADHD, LD, BD. You would not pick out_—all of the labels we give kids now (...) or you could not pick out the talented and gifted either."

"(...) everybody was unique in that we all had different gifts and we looked at them differently than they looked at them at some schools."

“(...) there was this little boy who was very sports__ was not a good student in terms of academics. (...). We were playing ball and I was looking at a book. (...). He came to me and said, "What are you looking at?" And I said, "I am looking at this painting." He said, "Can I have it? Can I look at it?" So I gave him the book. He said, "Oh that is cool. That is cool." You could tell his little brain was thinking, then he went back and played ball. We go back in the classroom and he remembered that painting and he said, "Could I see that book? There is something in there." It was I think the color wheel is maybe what he was looking at. He was trying to figure out how to blend the colors together. He was looking at 
that wheel. (...). Here you are playing ball, stopping to come over and look at a piece of art and then go back."

"He was an interesting little boy, too, that just saw things differently. But that is okay. (...). Curls, he had little curls and little bows in his hair. (...) he had a little pink ruffle, but that was a boy thing at the time. And so they all thought it was girl, when they found out it was a boy, this cool dude, this professional ball player says, "I could see me doing that." (...) . He said, "Well, I am going to wear a pink shirt tomorrow," because pink at that time, boys didn't wear pink. And he went and he bugged his mom and bugged her until he got a cool pink shirt. And if he could wear pink, then so could all of the other kids wear pink. Here, I mean, you know, when you are the cool dude and you make a positive influence, that also effects them."

"I think girls are different in that they always wore jeans and they were more, you know, unisex, I guess in the sense of dress than boys would be."

"We said we were breaking down this gender and you are trying to identify it, so we are struggling with that (...)."

"The girl did this painting, I remember this one. There was a painting (...) there was a fence. This girl was faced backwards. (...). She was faced backwards and the instructions were, "Turn her around or paint the front of her. What does the front of her look like?" One little girl turned her around and looked at her, the fence behind her. The other little girl walked herself around and looked at her straight with the fence in front of her. (...)."

"It is how they looked at things differently."

"Oftentimes they had to look beyond the painting. (...) I think if anything else, we gave them critical thinking skills. That is why they did so well on tests. (...) I would love to see their SAT's and stuff where you really have to think through. Their creative writing was better, too. And that is why_ I am just saddened that they don't write, kids do not know how to write and they don't write."

"These kids need to write. They have got so many ideas and they are not doing a darn thing with it, every day."

"Oh yeah, they had to write - they had to read - (...) our goal was they would learn to like to read and learn about more genres of kinds of reading. And so, they had to read 15 minutes. (...) they had to take like a paragraph or a couple sentences and write what they read. So they were summarizing and that is another thing that kids don't know how to do is summarize. You know, when you hear kids tell the story, they tell it, listen to kids tell a movie. They start at the beginning and they say, "He said and she said and he said," and four hours later I know what the movie is about. We were trying to break that."

“(...) there was a little person who was trying to write about the Pittsburgh Steelers and he didn't know any information, so he couldn't write. So the thing is you have got to read, have got to be able to understand what is going on to write. So I said, "Where are they living?" He said, "I don't know." He did not know Pittsburgh was the city in which they lived. He just thought they 
were called that. And I said, "That is the town they live in." Oh, that is new information. He didn't know that they lived in Pittsburgh. That is the other thing that the exposure and that is our job is to make sure kids learn all of that and they get it by reading, anyway. They have to write."

“(...) Shiloh is a book written about a West Virginia family, very poor family, fun to read. (...) So one of the things we did, then, was had those kinds of questions to talk to their parents. What is lying? I am not going to tell you if that is lying or not. You guys talk about that at home and talked about values. So that was a good chance for family discussions at home. So we had kind of guided the questions for them to talk about, you know, is he telling the truth? Is it okay to protect a dog if you think he is being abused? Is that right or wrong? That is not my thinking to say for you, but your family needs to. So it kind of brought family together and they could - - I am sure they had different opinions and things."

“(...) MOTIVATED and I modeled for them how to teach. (...). She was the student and I was the teacher or vice versa and we would show them what happens when, all of a sudden, you two come in and you are best friends and you start talking and I am trying to teach you and you are talking to a friend. So we showed them how to get the parent away, you know, without being rude. (...). We would actually model. Or the other thing is, what is going to happen to you when they are ahead of you on the lesson plan and they want to see something else, say, "Yes, I will get there later, but right now I want to show you this"."

"So we really modeled for them how to go about doing that. (...). So they loved teaching that. They would teach that over and over again and then the next day they responded by, "How did it feel to be in charge?" So you always_—we don't spend enough time even as adults reflecting. I think we gave them that opportunity to reflect on what happened, how they could have done it differently."

"If they didn't have someone [to teach to], we made sure they had someone."

“(...) we struggled with, your brothers and sisters cannot come. This is your time to shine." (...). They had to teach it to whoever came."

"And the packet had a variety of things, one being some art things. So they could go and get paint and paint the painting if they wanted to for part of it. They weren't always stopped doing paper and pencil stuff."

"For several years we had the parents, a questionnaire for the parent and they had to respond to what they thought their child did. So it was a parent response (...)."

"The kids responded to what they thought to the unit and that was also our evaluation of ourselves, if this worked, this didn't work. This is what, you know, kids saw that was successful. So we kind of used that as our own evaluation (...) the whole day was set up so that you could come at any time during the day and there might be four parents in there and kids are teaching those four, and I was only their facilitator, so if they needed a piece of music or they needed a piece of art down from up there that they wanted to see closer, my job was just to run around and help them. The other kids were all busy doing this packet and it might be things like paint a painting, do a written response, do_— paint out of a math book, could be anything." 
"Some would even come, they said, "I didn't know it was going to take this long." The kid really got INVOLVED so they would go and come back. (...) when they had time and the kid would pick up where they left off and finish it. Some parents stayed for a long time, several came at 1:00 o'clock and stayed all of the way until the end of the day. (...). Some of these parents came and they decided they wanted to paint. So (...) the kids and they would sit down on the floor and they would paint."

"[We] teamed very well together, and so the kids knew that the paintings would be in her room. They knew exactly where all of the paintings were. We did not have the same things. So they could go easily in her room and back, you know, they didn't even have to excuse themselves, they would just go in and would walk in. They were walking back and forth all the time."

"It was fun, but the sad part is nobody appreciate it in a county that they picked up on — - they wanted to know what our secret was, why we had such high test scores. And one day I just said to the assistant superintendent, I said, "We cheated. What else do you want me to say? For heaven's sake, come and look what we are doing."'

"(...) they knew we were good, but they never said, "Oh, that should be around the county," or "Let's try it somewhere else."”

"You have to be committed to that. And I think it is a big commitment. It was a lot of work."

"Right, math is a good example where we had a unit. (...)."

"I think that is what it was, critical thinking and problem solving skills."

"I made them pretend money, a million dollars. And you are to build a structure. So, first of all, they have to plan it. They have to have a blueprint. So we had an architect come in and he came in with his blueprints and he showed how they used those to build. (...). Then the kids drew theirs and then they proceeded to build. And we had stuff, I mean just stuff everywhere. (...). Everything had a price to it. And so they had to keep a journal of how much they had and how much they spent and how much... Then the kids came up with the idea, which came from them, "Well, let's have a bargain table and we will have for sale. (...). So they built their own discount store (...). If they had a question for us, they had to pay. (...) we gave them the instructions and we gave them one consulting fee. If they wanted to talk to the art decorator, there were two guys that came in, two dads. I can't remember, one was an architect and one was maybe a builder. They came_ _ If you want to talk to them again, you have to pay a fee for that. It was some exorbitant amount. Or if you have a question for me that has to do with architectural building or design, you have to pay me, too. But, I was, of course, cheaper because I didn't have the experience. They could ask me first and I said, "Do you really want that question answered?" "No, we will solve the problem ourselves." (...) they were keeping this map log all of the time of the expenses how much and took turns and there were four in a group. So today you were the secretary and you were the treasurer and we all had a job and then we kept switching after a week or so. That was a five-week project. People said that was an awful lot of time spent on one thing. But I tell you, they knew had to add or subtract, they knew how to keep a ledger (...) we knew so much at the end of that project." 
"We taught many things and other people were going to get in the math book, but I looked at the book and said_—looked at the objectives, said, "This is what needs to be taught. I can do it that way." So we sought, you know— so that was another fun project. That was written up in the paper here."

\section{MOTIVATED:}

"I am MOTIVATED. I am retired also from Sunrise Primary, third grade, teaching Integrating the Arts Across the Curriculum, and I have been retired for six years and now have a machine quilting business."

"I am having a hard time just isolating the girls' reaction because the effects just crossed the gender line. And it seemed that, you know, both boys and girls were freer in the classroom to express themselves and to act out in different ways when we would do, you know, role playing or dressing up and, you know, it was just very free. So I think that what happened was, you know, a safe, fun environment was created. And we created it as much for ourselves, I think, as for the children because we wanted to have a good time in the classroom."

"(...). We have to remember how they were identified. That means they tested well on those tests (...) what our program began to do was just to see how other children were gifted in different ways."

"They also imitated us (...), because we would dress up. They could tell when they walked in in the morning, we often are dressed__ gave them clues about what was going to happen that day. Now, this did not happen every day, but it happened frequently."

"I always remember the one time, one of the other teachers, they weren't all INVOLVED to the extent that we were, but the superintendent was visiting the building and they came to warn us. (...). "The superintendent is in the building."”

"That is the part we tricked them a little bit, how do you know if you really want this book unless you read it?"

"(...) a lot of them we saw gain more self — confidence because when you are working in the arts, sometimes there is not a black and white and right and wrong answer. So all of their comments were validated, you know. We accepted them all and we taught our kids. We did lots and lots of brain storming when we were in these projects. And, you know, we taught them that, you know, all responses were accepted and, you can sort them out for yourself later, but it is okay to agree. And there is no right or wrong. So that was one of the big things I think when we are dealing with the arts, because it was more subjective. Everyone's opinion or comment was accepted, so children were freer to participate because a lot of the children that did not score on a standardized test really had insight to some things."

"And I remember another time when a school official visited. This was the state superintendent and he came by and you know, he was (...) happy with our test scores. (...) And what I said to him was, "We are more proud of what is not on the test, what you don't see on the test, the learning that is beyond the test." (...) Well, our test scores were already high. What do you want 
from us? And I feel when you do this, teach to the test, it is a very narrowed window and we broaden that window, which is hard to measure. We know we did it, but, you know."

"(...). She was a bright little girl. She was not what you would say one of the popular kids, but she was very bright and then we learned about how artistic she was. (...). We were writing about the bubbles so we always_ a lot of times before the writing experience, we would provide the motivation or the background for writing through an artistic experience. They were writing a poem about bubbles, so we filled the room with bubbles and we looked at them and talked about them. And... She was just, you could see her eyes. (...) I remember, she was holding her imaginary bubble. She was one that I think really did blossom in that way because she could offer so much. And we also saw when we did group work, lots of times traditionally when kids are choosing groups, they would either choose their good friends or choose the quote unquote smartest kids in the class. They started to recognize the gifts in each other. So some of the children who weren't always chosen for a group would say, "Well, you know, let's choose so and so because they can draw really well or they can paint or, you know, they are really good at words" or something."

"We knew there were lots of ways to be smart that the kids were beginning to recognize there were lots of ways than just..."

"(...). Drama was a little girl who had some learning issues. She had surgery as a baby and which caused a stroke. So she didn't walk just right and she had, you know_ — but she just ate this up, you know."

"(...) she really got into this. And she then loved the dressing up and the acting and she would take on like a leadership___ she would want to do more. (...) so instead of fading into the background, she really__ “

"I am guessing it would be her self-concept. Confidence is certainly a part of your selfconcept, but I know that she maintained that interest in the arts after the third grade because for her birthday she wanted a Monet painting or something, something that we had used in class. And she wanted a big poster of it. She still has that interest. (...).”

"I don't know how much of that is just that age, developmentally. We are talking about eight and nine year olds here. (...) some of that is maybe developmental, but in the other ways at that young age they are already starting to present the preadolescent kind of things in their actions and their bodies and so there is that going on."

"The things that the arts allowed us to do, it was like we said before about the books, we sneaked in a lot of stuff. You know, that they didn't realize was because it was such a nontraditional school, they didn't always realize how much they were learning as one of the children wrote in their journal. (...) they commented on the activities of the day and almost always they were positive. But this little person said, "Well, I learned more than I wanted to."'

"That was one thing that we noticed, is that observation is really a key thing." 
"One time (...) the kid ran out of the page, he was drawing and drawing, he said, "Oh, my goodness," he used more paper."

"We had lots of visitors in our classrooms and almost always they would say, they are so engaged in what they are doing. They are all doing what they are supposed to be doing."

"Those kinds of things I am sure affected girls and boys just teaching them to be independent and responsible, (...)."

"But INVOLVED just mentioned something that is one of the things why it might be hard for us to think about this as girls, is that we were trying to be so inclusive and cross those lines because what we saw was really for every girl we mention, we could mention a boy, too. You know, I mean, like how many nine-year-old boys would be comfortable coming in and dressing up like grandma and sitting in a rocking chair? Because I did it when I was reading "Troubled River," I would get out of a chair and before I knew it during recess, one of the boys was in there with my bonnet and cane and doing (...) we were consciously and unconsciously making efforts to not have anything (...) gender-based aligned."

"I don't think we ever stopped to consider that, because of her size. I think we would have-if anything, I would have made sure I had a gown that fit her."

"And we made sure doing the dress up thing."

"It never was an issue. We never had those problems."

"The one boy who moved here (...) and we were doing some of these things and he asked, "when are we going to have normal school?". "Why", I said, "isn't this a normal school”? I said, "Well, this is normal for here." The other one (...) this little boy said, "I don't have headaches anymore." He was transferred from a parochial school."

"Some of that was the guided observations that they did."

"Because, how are they supposed to know how to do it if we don't show them?"

"Yeah, we never lacked for volunteers for a role."

"(...) is probably rare, that we truly were multi-cultural because of where we are in the community, we had children from many countries, and not all West Virginia schools can say that. (...). We, again, we always made sure we were inclusive. So, you know, children knew, we wanted them to be proud of their heritage and know and we wanted them to respect each other."

"And yet they were all painted."

"There were so many things going on at that time. In addition to the arts integration, we were also teaching tolerance."

"We constantly had some facet of that going on, you know, simultaneously with everything else." 
"One of the things is that just tolerance meant to respect each other's differences, I think that is how we put it. (...) we had posters of kids demonstrating tolerance. (...). They were from the National, no, Southern Poverty Law Center, something, and beautiful posters, they were multicultural posters, too. We were living it because we had this population that we did. It was just anywhere (...) just like the arts. It was just permeated what we were doing. You couldn't always separate one thing from another.

"And after we did that for a while, then the kids started doing it themselves almost." "(...) if you don't want me on your team because I can't play, then help me learn how to play so that I can be_.."

\section{Dr. S.:}

"I am Dr. S. and I am the University liaison that worked with them during the arts - integration project for ten years and also know the extent of the work that these wonderful teachers, who created an amazing classroom environment and arts-focused environment that reaped benefits for all of the children in the room. They were the rock stars of the arts-integration world.

"They were identified as gifted."

"Bring it on. It is a great time for him to be here, come on in."

"But your observations as teachers are extremely valid and based on the knowledge of the child and that is why it is really important to get what your observations were related to the selfconcept thing. What you described in that student, she assumed leadership. I bet she had never done that before. She was in charge of the money, so then you are talking about the selfconcept, confidence and every contribution is valuable."

"(...). Both of you described these were girls that were struggling in some way when they came to you, but what you saw they blossomed into a leadership position given the opportunities that you were providing. (...). So when you talk about what is a leader, this is a child who had some confidence and I would think that might be connected to their self-concept."

“(...) You don't see that so often, the girl that picked out the Monet for her room rather than the figure of the day."

"You had them do self - portraits, too. (...). Do you remember anybody in particular, struggling terribly with that or really excellent at that? Because they studied themselves for a while, didn't they?"

"But, what I noticed about your self-portraits as opposed to others I had seen in the other schools, your self - portraits after all that observation of the mirrors and using the picture, filled the page, filled the page."

"That shows how involved they are." 
"The other thing you talked about, (...), is the idea of level. You know, some were coming in very good at traditional stuff and some weren't and, obviously, you were reaching across different abilities, too, and trying to include."

"So, it is a (limited) definition."

"[Obese girl] was not embarrassed."

"And she didn't have any trouble.

"The type of environment you set, you didn't have that hesitation whether it was boys, girls, big, small."

"Do you think they were paying attention to the aesthetics more, trying to design a fit that would work and would work for that body? (...). Where the boys, if it was too big, it didn't matter, they didn't pay attention to the aesthetics as much."

"You talked about the observation time you spent, critical observing and you said the girls could focus for a long time on the beauty and the aesthetics that they were examining, so it sounds like maybe that came out in the costuming as well."

"With their self - portraits, it struck me so much, their examination. You said they examined for a really long time before they drew. So, they noticed freckles and you know that this eye has a speck in it, and this eyebrow is a little different arch than this one and this kind of thing. What I noticed, that their portraits were huge. They filled the page. I would go to other schools and I would see little tiny stick figures down in the corner, you know, dark colors, no elaboration, no detail. And of course, in looking at art development, you are supposed to be able to tell a lot about the development of the child. And over time and their concept of themselves and all, they put time in. It was a full figure, it was a fully developed figure with all dimensionality to it, filling the page, not diminished, full of color. We have photos of them, too."

"You shared your critical examination of yourself out loud, so that whole thinking process was exposed to them before they did it. They saw that as what you were thinking through."

"These ladies [teachers - INVOLVED \& MOTIVATED] were very comfortable with themselves."

"They tap danced on the picnic table before school or on the reading table and would dance their way into the school on the first day. And they would wear costumes almost every day and they would use voices and they would use mannerisms and they would become a character in a book and bring it to life, so constantly providing a model of engaging in risk taking as a character, as another person, as an elaboration of self and both teachers did that regularly in the classroom. I observed that. I saw it firsthand. It was full of that risk - taking behavior that was being modeled by the leader in the classroom. So then the children had permission to take risks, too."

"Never was there a cookie cutter art up on the wall where there were like 30 Barbie doll pictures, you know. They didn't all have the common standard of beauty across their art. They had their 
own unique perspective of themselves and it was fully developed because they filled the page and made it very elaborate and full. But they all looked very different from one another. It was never cookie - cutter art, never. It was never all replicating Barbie image. It looked like the people that were in the room."

"Yes, it was celebrated in the hall, they were all published in the hall."

"You said you were teaching about tolerance."

"Everyone was accepted and you wouldn't allow them in your group_ because I saw that you would never allow them to be discriminatory against one another. In fact, you had conferences after recess, disagreements where the kids had to solve the problem about, who wants the basketball and how can we be fair about sharing the basketball."

"It was nonathletic and the solution had to come from everybody."

"They [the kids] called their own meeting."

"They recognized the problem and then they brought it in and solved it in the classroom. You had an environment where they could solve the problem."

"And she is already volunteering in middle school or high school?"

"She turned it around. And I wonder about the influence of your experiences on her to help her see that."

"Interesting, wow. Volunteering in school, what a remarkable progress for her, wow."

"How was her body image? She was confident, obviously."

"The types of experiences that you created in your classroom gave everybody a way to contribute."

"Did you see girls crossing lines like that, too?"

"A lot of processes that were in place I think that influenced the way these groups worked together and across lines and accepted one another and that is really what you describe, it is such a unique situation. Well, the self-portraits really showed, they were so full and rich compared to the other groups I saw."

"Those were accepted as being right."

“(...) book response and writing every night (...). They took their notebook home and they would have a minimum, they needed to write per night." 
"Oftentimes the books would be built into the arts - integrated unit in the classroom. While they would be reading at home and doing some responses and then they may come in to do the integrated unit."

"You were modeling appropriate interpersonal skills for them."

"But they all had to be briefed with a significant adult. They made sure they had somebody, everybody had somebody."

"You rearranged the schedule for the whole day so the parents could come at any point in the day to have that full hour with their own child, boy or girl to get debriefed. So if they can only come in the morning, there is a work packet for the rest of the group. That student could take the parent when they got there or the grandparent or the aunt or the uncle, whoever it was that came at any time of the day, they could debrief them about their feelings about the art and what they liked and what they didn't like and what they learned. (...)."

"I observed that time and for those events and what I saw is that the children were very confident and very proud of their work and they knew so much about each product that they had studied that the parent was asking questions and learning from the child because the child really knew deeply about all of the work that was being displayed. They had their portfolios and they would take to the hallway displays where the art was celebrated and labeled like an art gallery with their name and their title. They would walk them out there, "Here is my example of my self - portrait. Here is my example of extending the landscaping," and here is my example of ..., and things that weren't displayed were in their portfolio. And they would take them to their portfolios and pulled out their art and show them different examples."

"Here is Monet's bridge that we studied and it is over the lily pond, these are the water lilies and they knew if they knew they were hanging up in MOTIVATED's room they would be freebecause both classrooms were working together as a group, they would be free to taking the parent over there and say, "This is the Monet we studied."'

"I think they knew they couldn't impose that on everybody because not everybody could pull that [AIC] off."

"Yes, it was [a lot of work]. We would meet at 7:00 in the morning to plan and after school and weekends, late to the evening, we would be meeting and planning together. And I would bring my students to their classroom to observe so that the children knew that it was important what they were doing because our university students who wanted to be teachers would come and study in that classroom."

"Very interesting. You have a lot of good information. (...). The whole background and the way you describe is very important for her context of understanding, so when she is describing it, she knows the environment that you set that allowed the girls to do this and be this in your classroom." 


\section{Appendix H3 - Students' themes}

\begin{tabular}{|c|c|c|c|c|}
\hline Themes & BAND & PIANO & $\begin{array}{l}\text { DRAWING } \\
\text { (non AIC } \\
\text { school) }\end{array}$ & FLUTE \\
\hline Adolescent & $\begin{array}{c}\square \\
\text { (13 years old) }\end{array}$ & $\begin{array}{c}\square \\
\text { (12 years old) }\end{array}$ & $\begin{array}{c}\square(\text { pre }) \\
(11 \text { years old })\end{array}$ & $\begin{array}{c}\square \\
(12 \text { years } \\
\text { old) }\end{array}$ \\
\hline $\begin{array}{l}\text { Interest in Music } \\
\text { (plays an } \\
\text { instrument \& } \\
\text { is in (school) band) }\end{array}$ & $\begin{array}{c}\square \\
\text { clarinet } \\
\square \\
\text { (band) }\end{array}$ & $\begin{array}{c}\square \\
\text { piano \& } \\
\text { saxophone } \\
\square \\
\text { (band) }\end{array}$ & $\begin{array}{c}\square \\
\text { violin } \\
\square \\
\text { (orchestra) }\end{array}$ & $\begin{array}{c}\square \\
\text { flute } \\
\square \\
\text { (band) }\end{array}$ \\
\hline Took arts classes & $\begin{array}{c}\square \\
\text { painting (in } \\
\text { school) }\end{array}$ & $\begin{array}{c}\square \\
\text { theatre } \\
\text { (company) }\end{array}$ & $\begin{array}{l}\text { च } \\
\text { pottery, } \\
\text { painting, } \\
\text { drawing }\end{array}$ & $\begin{array}{l}\square \\
\text { performing } \\
\text { arts camps }\end{array}$ \\
\hline $\begin{array}{l}\text { Positive } \\
\text { descriptions/ } \\
\text { characterizations } \\
\text { of art }\end{array}$ & $\begin{array}{c}\square \\
-\quad \text { I like art } \\
-\quad \text { exciting } \\
\text { entertaining }\end{array}$ & $\begin{array}{c}\square \\
\text { - } \quad \text { different } \\
\text { - } \quad \text { wonderful } \\
\text { experience }\end{array}$ & $\begin{array}{c} \\
\text { - } \quad \text { interesting } \\
\text { - } \quad \text { wonderful } \\
\text { experience } \\
\text { - lot of great } \\
\text { things } \\
\text { - neat }\end{array}$ & $\begin{array}{c}\square \\
\text { - } \quad \text { very fun } \\
-\quad \text { exciting } \\
\\
\\
\text { - getting } \\
\text { messy } \\
\text { different } \\
\text { things }\end{array}$ \\
\hline $\begin{array}{l}\text { Recognition/ } \\
\text { confirmation/ } \\
\text { positive feedback } \\
\text { from art } \\
\text { (intrinsic } \\
\text { motivation) } \\
\end{array}$ & $\nabla$ & & & $\begin{array}{c}\square \\
\text { "made me } \\
\text { feel smart" }\end{array}$ \\
\hline $\begin{array}{l}\text { At first insecurity } \\
\text { about arts, now } \\
\text { good at it }\end{array}$ & $\nabla$ & $\nabla$ & & \\
\hline $\begin{array}{l}\text { Positive self- } \\
\text { concept through } \\
\text { arts }\end{array}$ & $\begin{array}{c}\square \\
\text { "I am happy with } \\
\text { myself" }\end{array}$ & $\begin{array}{c}\square \\
\text { “I am a much } \\
\text { better person }\end{array}$ & $\begin{array}{c}\square \\
\text { "they can make } \\
\text { you better" }\end{array}$ & $\begin{array}{c}\square \\
\text { "made me } \\
\text { feel smart" }\end{array}$ \\
\hline
\end{tabular}




\begin{tabular}{|c|c|c|c|c|}
\hline & & for it [arts]" & & $\begin{array}{l}\text { "I like the } \\
\text { way I look" }\end{array}$ \\
\hline $\begin{array}{l}\text { Family } \\
\text { involvement } \\
\\
\text { in special arts } \\
\text { forms ( } \rightarrow \text { interest } \\
\text { in it) }\end{array}$ & $\begin{array}{c}\square \\
\text { mother, } \\
\text { grandfather, } \\
\text { sister, and } \\
\text { brother. } \\
\text { music }\end{array}$ & $\begin{array}{c}\square \\
\text { mother - } \\
\text { filmmaker, } \\
\text { dad - graphic } \\
\text { designer }\end{array}$ & $\begin{array}{c}\square \\
\text { both parents are } \\
\text { actors }\end{array}$ & \\
\hline $\begin{array}{l}\text { Learning } \\
\text { through AIC= } \\
\text { FUN }\end{array}$ & $\square$ & $\square$ & & $\nabla$ \\
\hline $\begin{array}{l}\text { Interdisciplinary } \\
\text { Learning }\end{array}$ & $\begin{array}{c}\square \\
-\quad \text { songs } \\
-\quad \text { drawings }\end{array}$ & $\begin{array}{c}\square \\
\text { songs playing } \\
\text { drawings (art } \\
\text { show) }\end{array}$ & $\begin{array}{c}\square \\
\text { Spanish teacher } \\
\text { who was art } \\
\text { teacher \& mixed } \\
\text { it }\end{array}$ & $\begin{array}{c}\square \\
\text { - English/ } \\
\text { social } \\
\text { studies } \\
\text { - History- } \\
\text { civil war }\end{array}$ \\
\hline $\begin{array}{l}\text { Problem Solving } \\
\text { a) (enhanced } \\
\text { through arts)/ } \\
\text { extrinsic } \\
\text { motivation) } \\
\text { b) indecisive } \\
\text { (find solution) }\end{array}$ & $\begin{array}{c}\square \\
\text { "once you got to } \\
\text { the problem, you } \\
\text { got to color" } \\
\square\end{array}$ & $\begin{array}{c}\square \\
\text { (drew dress she } \\
\text { could not find) }\end{array}$ & & $\begin{array}{c}\square \\
\text { (build sturdy } \\
\text { temples) } \\
\\
\square \\
\text { (copy } \\
\text { random } \\
\text { picture) }\end{array}$ \\
\hline $\begin{array}{l}\text { Think differently/ } \\
\text { be creative/ } \\
\text { imagination }\end{array}$ & $\begin{array}{l}\square \\
\square\end{array}$ & $\begin{array}{c}\square \text { (color) } \\
\square \\
\square\end{array}$ & & $\begin{array}{c}\square \\
\text { (saw s.th. } \\
\text { different } \\
\text { every day) } \\
\end{array}$ \\
\hline $\begin{array}{l}\text { Friends } \\
\text { (interactive/ social) } \\
\text { audience }\end{array}$ & $\bar{\nabla}$ & $\begin{array}{l}\square \\
\square\end{array}$ & & $\begin{array}{c}\square \\
\text { "I had a lot } \\
\text { of friends" }\end{array}$ \\
\hline Clothes & $\nabla$ & $\begin{array}{c}\square \\
\text { (dress up) }\end{array}$ & & $\begin{array}{c}\square \\
\text { (some } \\
\text { depend on } \\
\text { clothes } \\
\text { instead of } \\
\text { personalities) }\end{array}$ \\
\hline $\begin{array}{l}\text { O.K. to be } \\
\text { different }\end{array}$ & $\begin{array}{c}\square \\
\begin{array}{l}\text { (e.g. colorful, } \\
\text { clothes) }\end{array}\end{array}$ & & & $\begin{array}{c}\square \\
\text { (different } \\
\text { learning } \\
\text { style) }\end{array}$ \\
\hline
\end{tabular}




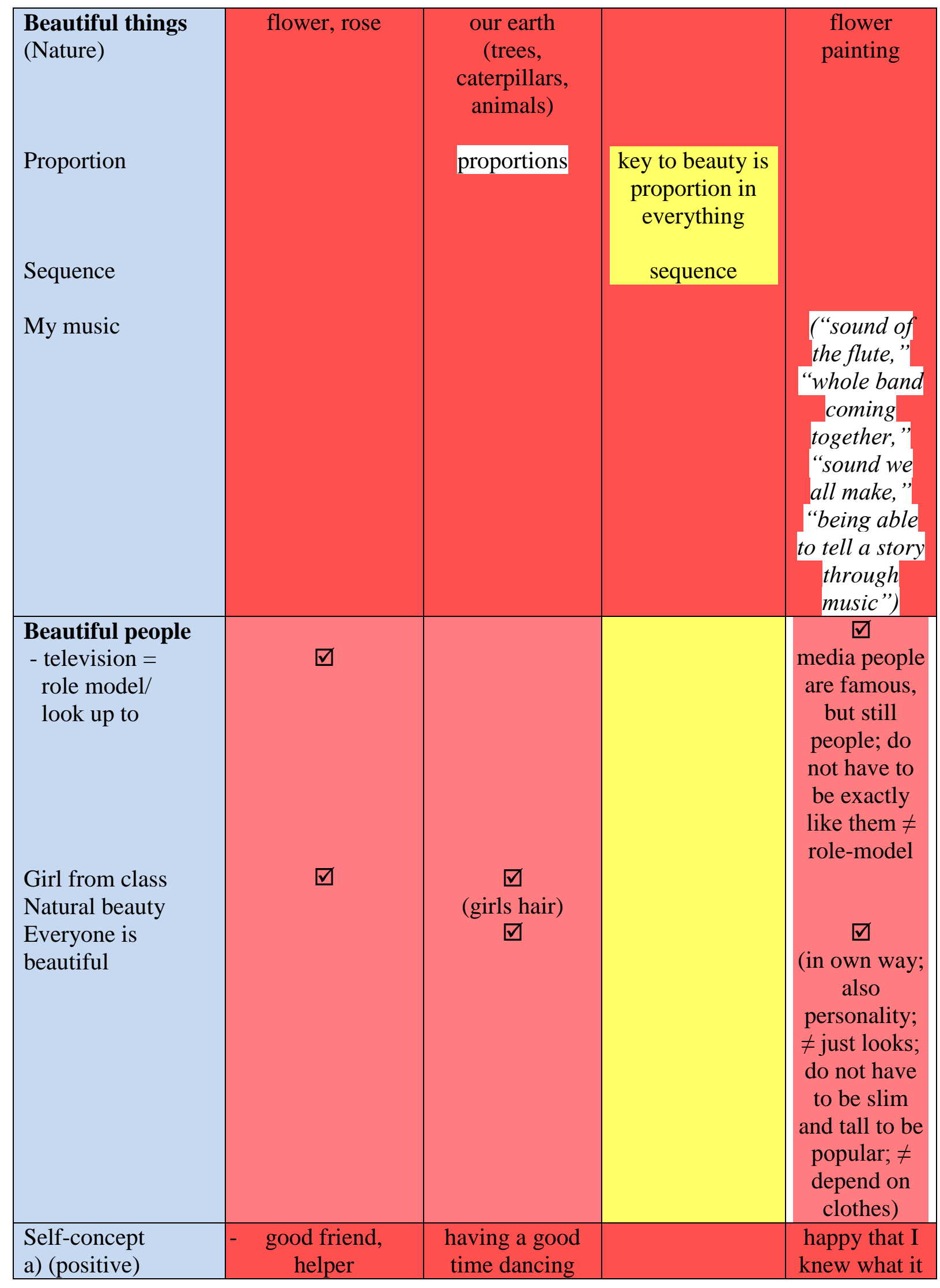




\begin{tabular}{|c|c|c|c|c|}
\hline & 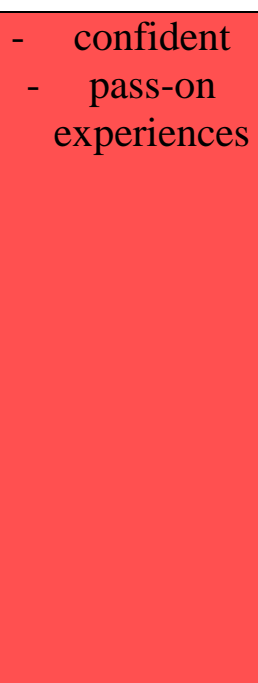 & $\begin{array}{l}\text { can do what I } \\
\text { want } \\
\text { can do it - just } \\
\text { have to try }\end{array}$ & & $\begin{array}{l}\text { [painting] } \\
\text { was } \\
\text { can get your } \\
\text { feelings out } \\
\text { through arts } \\
\text { "So it just } \\
\text { makes you } \\
\text { feel better } \\
\text { about } \\
\text { yourself;" } \\
\text { "Some } \\
\text { famous } \\
\text { composers } \\
\text { did that") }\end{array}$ \\
\hline b) (negative) & $\nabla$ & $\begin{array}{l}\text { more critical } \\
\text { about myself/ } \\
\text { my looks }\end{array}$ & & $\begin{array}{l}\text { "that did not } \\
\text { turn out so } \\
\text { well" }\end{array}$ \\
\hline $\begin{array}{l}\text { [compare \& } \\
\text { contrast] }\end{array}$ & & $\begin{array}{c}\square \\
\text { (compared to } \\
\text { Diana) }\end{array}$ & & \\
\hline $\begin{array}{l}\text { Role-model } \\
\text { (support) }\end{array}$ & $\nabla$ & $\begin{array}{c}\square \\
\text { (Anabell) }\end{array}$ & $\begin{array}{c}\square \\
\text { (teacher) }\end{array}$ & $\nabla$ \\
\hline $\begin{array}{l}\text { Beauty issues: } \\
\text { a) height } \\
\text { b) hair color }\end{array}$ & $\begin{array}{l}\square \\
\square\end{array}$ & $\begin{array}{c}\square \\
\text { (hair) }\end{array}$ & & \\
\hline $\begin{array}{l}\text { People on } \\
\text { television } \\
\text { thinner }\end{array}$ & $\nabla$ & & & \\
\hline $\begin{array}{c}\text { Healthy eating/ } \\
\text { Weight loss }\end{array}$ & $\square$ & & & \\
\hline $\begin{array}{l}\text { School = influence } \\
\text { on image/ self- } \\
\text { concept }\end{array}$ & $\nabla$ & & & \\
\hline $\begin{array}{l}\text { Learn how to } \\
\text { paint/ draw, ... } \\
\text { methods/ } \\
\text { techniques }\end{array}$ & $\nabla$ & & & $\begin{array}{c}\square \\
\text { (positive } \\
\text { criticism) } \\
\square\end{array}$ \\
\hline $\begin{array}{l}\text { Teachers } \\
\text { (positive) influence }\end{array}$ & $\nabla$ & $\nabla$ & $\nabla$ & $\nabla$ \\
\hline $\begin{array}{l}\text { Endurance } \\
\text { (in practice) }\end{array}$ & $\nabla$ & $\nabla$ & & $\begin{array}{c}\square \\
\text { (one week to } \\
\text { draw; sit by } \\
\text { it every day) }\end{array}$ \\
\hline
\end{tabular}




\begin{tabular}{|c|c|c|c|c|}
\hline $\begin{array}{l}\text { Learning to life } \\
\text { with competition } \\
\text { (and possible } \\
\text { failure) }\end{array}$ & $\nabla$ & & & \\
\hline $\begin{array}{l}\text { Share experience } \\
\text { with others } \\
\text { parents } \\
\text { friends (during } \\
\text { lunch time) } \\
=\text { communication } \\
\text { starter (talk) }\end{array}$ & $\begin{array}{l}\square \\
\square \\
\square\end{array}$ & & & \\
\hline $\begin{array}{l}\text { Something to look } \\
\text { forward to }\end{array}$ & $\nabla$ & & & \\
\hline $\begin{array}{l}\text { Artwork } \\
\text { continued in free- } \\
\text { time } \\
\text { (not a project)= } \\
\text { passion for art }\end{array}$ & $\nabla$ & $\begin{array}{c} \\
\text { (my wedding } \\
\text { plan, writing) }\end{array}$ & & \\
\hline $\begin{array}{l}\text { Artwork } \\
\text { displayed } \\
\text { value } \\
\text { proud }\end{array}$ & $\begin{array}{l}\square \\
\square \\
\square\end{array}$ & $\begin{array}{c}\square \\
\text { (at father's } \\
\text { office) }\end{array}$ & & \\
\hline $\begin{array}{l}\text { Computer/media } \\
\text { used as resources }\end{array}$ & $\begin{array}{c}\square \\
\text { (for saying) }\end{array}$ & $\begin{array}{c}\square \\
\text { (to find flower, } \\
\ldots \text {..) } \\
\end{array}$ & & \\
\hline $\begin{array}{l}\text { "Free-handed" } \\
\text { drawing }\end{array}$ & $\nabla$ & & $\begin{array}{c}\square \\
\text { "neat because } \\
\text { we had to draw } \\
\text { free-handed" }\end{array}$ & \\
\hline $\begin{array}{l}\text { "Make it } \\
\text { 'look good' or } \\
\text { 'pretty"' }\end{array}$ & $\begin{array}{l}\square \\
\square\end{array}$ & & & \\
\hline $\begin{array}{l}\text { Not enough time } \\
\text { for arts } \\
\text { (is a process) }\end{array}$ & $\nabla$ & $\begin{array}{c}\square \\
\text { (special time set } \\
\text { apart) }\end{array}$ & & \\
\hline Involvement & $\begin{array}{c}\square \\
\text { wanted to show } \\
\text { her art }\end{array}$ & $\begin{array}{c}\square \\
\text { wanted to show } \\
\text { her art }\end{array}$ & $\begin{array}{c}\square \\
\text { wanted to draw } \\
\text { during interview }\end{array}$ & \\
\hline $\begin{array}{l}\text { Feel comfortable } \\
\text { to know what to } \\
\text { do next }\end{array}$ & $\nabla$ & & & $\nabla$ \\
\hline $\begin{array}{l}\text { Familiarity with } \\
\text { artist \& art }\end{array}$ & & $\begin{array}{c}\square \\
\text { (Claude } \\
\text { Monet)/ }\end{array}$ & & \\
\hline
\end{tabular}




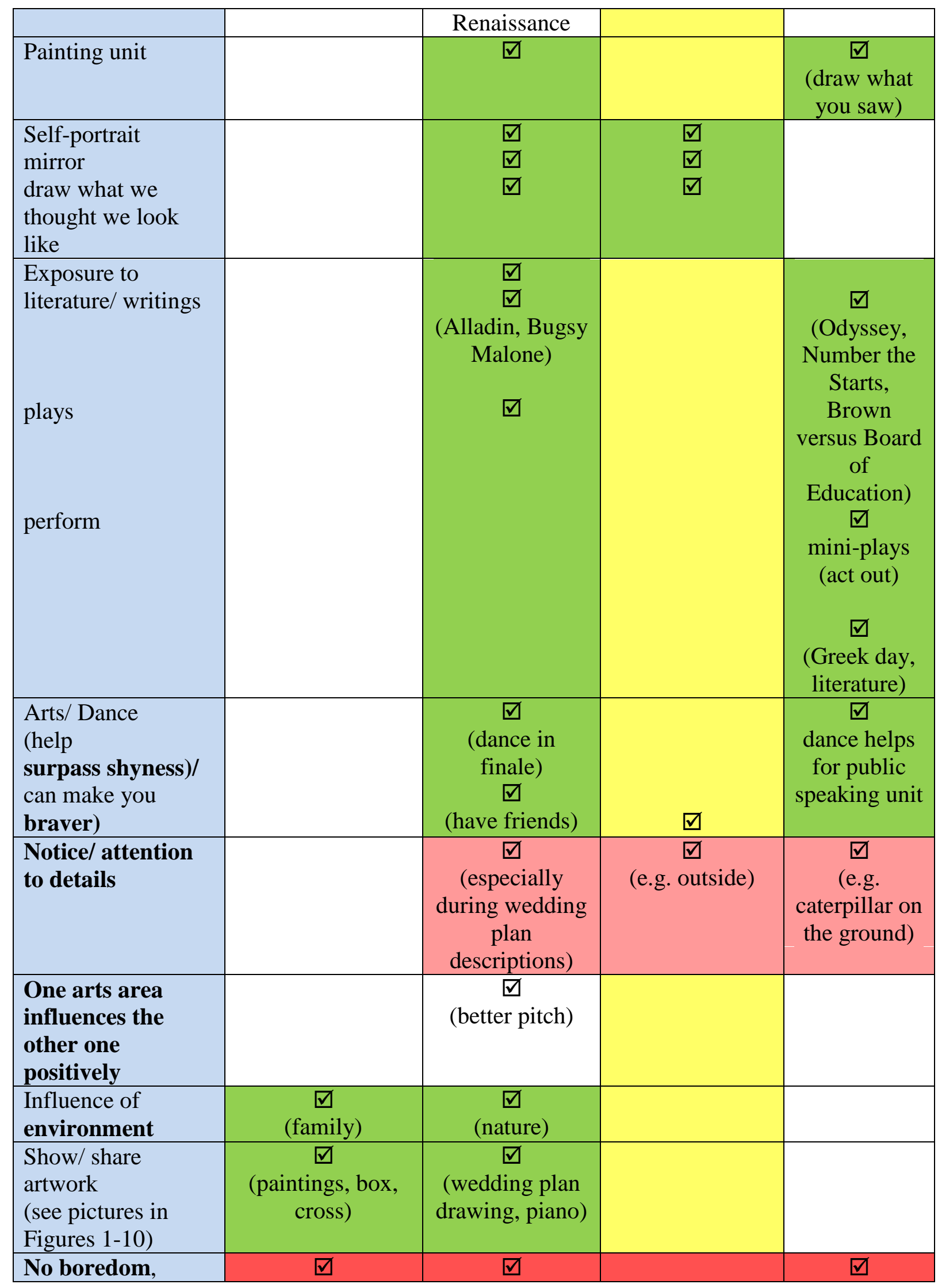




\begin{tabular}{|c|c|c|c|}
\hline arts help out & $\begin{array}{c}\text { "drew my } \\
\text { wedding or } \\
\text { write" }\end{array}$ & & $\begin{array}{l}\text { "it gives me } \\
\text { s.th. to do" }\end{array}$ \\
\hline Seems to "fit" & $\begin{array}{c}\square \\
\text { (colors Blue \& } \\
\text { White) }\end{array}$ & $\begin{array}{c}\square \\
\text { (Lime Green is } \\
\text { an unusual } \\
\text { wedding color = } \\
\text { does not fit) }\end{array}$ & \\
\hline $\begin{array}{l}\text { Small school is } \\
\text { - } \text { positive } \\
\text { - } \text { try to save it } \\
\text { - } \text { nice people } \\
\text { - } \text { no problems } \\
\text { - } \text { wish could still } \\
\text { be there } \\
\text { - good choice }\end{array}$ & $\begin{array}{l}\square \\
\square \\
\square \\
\square \\
\square \\
\square \\
\square\end{array}$ & & \\
\hline Outside (positive) & $\nabla$ & $\nabla$ & $\nabla$ \\
\hline Hands-on activities & & & $\nabla$ \\
\hline Independence & & & $\begin{array}{c}\square \\
\text { (go around; } \\
\text { talking and } \\
\text { working at } \\
\text { same time) } \\
\end{array}$ \\
\hline Empathy & & & $\begin{array}{c}\square \\
\text { (learn } \\
\text { through } \\
\text { experiences/ } \\
\text { actions from } \\
\text { others) }\end{array}$ \\
\hline
\end{tabular}

Notes: Color-coding information:

\section{Dark Red:}

All four students (BAND, PIANO, FLUTE, and DRAWING) mentioned this unit (IC).

\section{Themes:}

1.) Adolescent

2.) Interest in music (plays an instrument $\&$ is in (school) band)

3.) Took arts classes

4.) Positive descriptions/ characterizations of art

5.) Positive self-concept through arts

6.) Interdisciplinary learning

7.) Role-model (support)

8.) Teachers positive influence

9.) Outside (positive) 


\section{Medium Red:}

Only the three students that went through an AIC curriculum mentioned this unit (IC). Themes:

10.) Learning through AIC = fun

11.) Problem solving;

12.) Think differently/ be creative/ imagination

13.) Friends (interactive/ social)

14.) Clothes

15.) Beautiful things (nature)

16.) Self-concept a) positive; b) negative

17.) Endurance (in practice)

18.) No boredom, arts help out

\section{Light Red:}

Only three students (in general) mentioned this unit (IC).

Themes:

19.) Family involvement

20.) Involvement

21.) Notice/ attention to details

\section{Green:}

Only two of all four students mentioned this unit (IC).

\section{Yellow:}

Only one student, the one who did not go through an AIC mentioned this unit (IC). 
Appendix H4 - Parents' themes

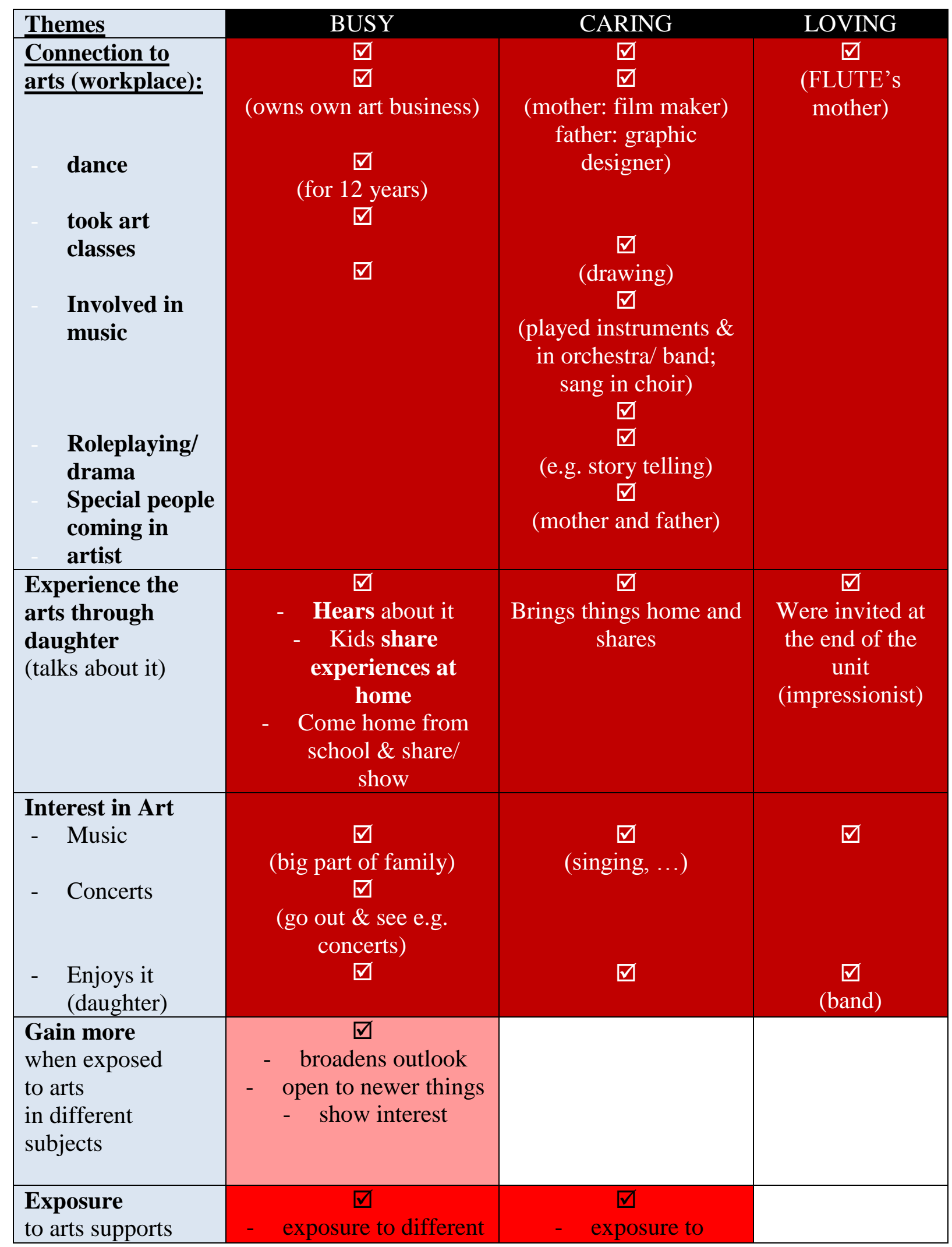




\begin{tabular}{|c|c|c|c|}
\hline $\begin{array}{l}\text { interest in arts } \\
\text { arts in school more } \\
\text { legitimate than } \\
\text { just at home }\end{array}$ & $\begin{array}{l}\text { things } \rightarrow \text { look at } \\
\text { them differently }\end{array}$ & $\begin{array}{c}\text { especially (visual) } \\
\text { arts } \\
\square\end{array}$ & \\
\hline $\begin{array}{l}\text { Arts (broaden) } \\
\text { perspectives } \\
\text { - } \quad \text { be open to } \\
\quad \text { things }\end{array}$ & $\begin{array}{l}\square \\
\square\end{array}$ & & \\
\hline Focus on arts & & $\begin{array}{c}\square \\
\text { everyone: } \\
\text { parents, students, } \\
\text { teachers); } \\
\text { PTG (parent teacher } \\
\text { groun }\end{array}$ & $\begin{array}{c}\square \\
-\quad \text { focus on } \\
\text { AIC unit } \\
-\quad \text { intense }\end{array}$ \\
\hline $\begin{array}{l}\text { Arts = equally } \\
\text { important }\end{array}$ & & $\begin{array}{c}\square \\
\text { necessary to become } \\
\text { "well-rounded } \\
\text { individual" in complex } \\
\text { society } \\
\square \\
\text { (for development of } \\
\text { human-being) }\end{array}$ & \\
\hline Looks & $\begin{array}{c}\square \\
\text { (daughter is } \\
\text { conscientious about it: } \\
\text { colors/ matching) }\end{array}$ & $\begin{array}{c}\square \\
\text { knows that she is } \\
\text { talented/ beautiful } \\
\text { young women, but also } \\
\text { her weaknesses }\end{array}$ & \\
\hline 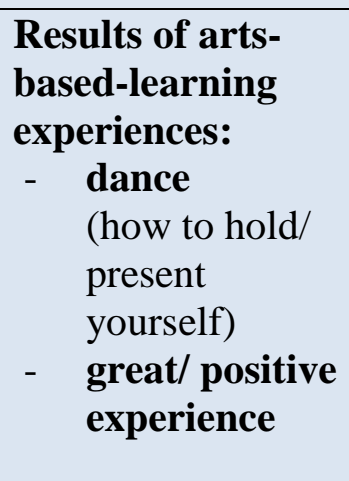 & $\begin{array}{c}\square \\
\square \\
\square \\
\square \\
\text { (sing, practice songs, } \\
\text { choreography) }\end{array}$ & $\begin{array}{c}\square \\
\text { made a difference } \\
\text { (not as shy anymore) }\end{array}$ & \\
\hline - $\quad$ story telling & & $\begin{array}{c}\square \\
\text { (learn about emotions, } \\
\text { motivation, and that we } \\
\text { are alike) }\end{array}$ & \\
\hline
\end{tabular}




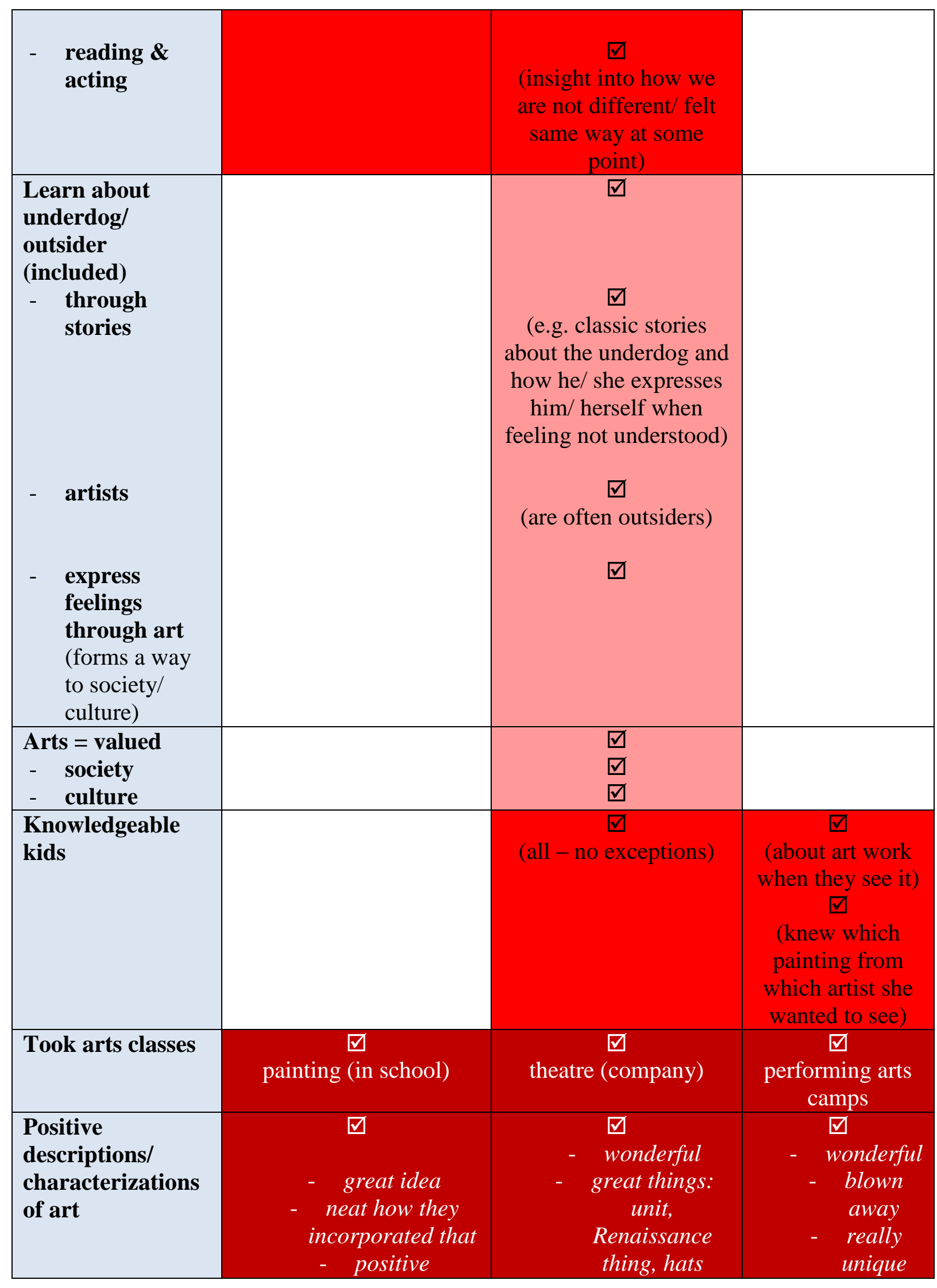




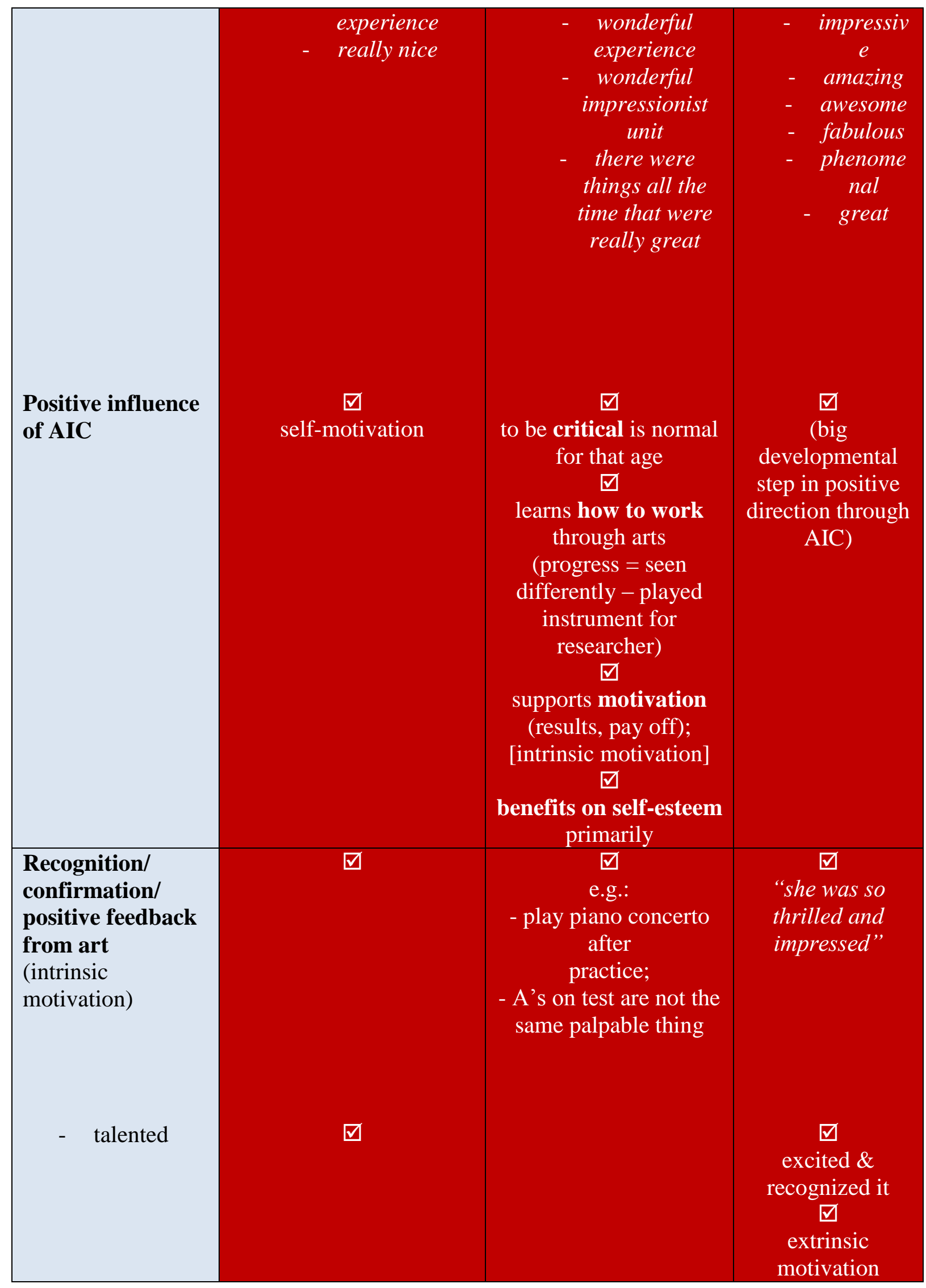




\begin{tabular}{|c|c|c|c|}
\hline & & & $\begin{array}{l}\text { through role- } \\
\text { model } \\
\square \\
\text { teacher said she } \\
\text { got talent to play } \\
\text { the flute }\end{array}$ \\
\hline $\begin{array}{l}\text { At first insecurity } \\
\text { about arts } \\
\text { (now she likes it) }\end{array}$ & & & $\begin{array}{l}\square \\
\text { (first did not } \\
\text { want to be in } \\
\text { play) }\end{array}$ \\
\hline $\begin{array}{l}\text { Positive self- } \\
\text { concept through } \\
\text { arts }\end{array}$ & $\begin{array}{c}\square \\
\text { teacher kept some of her } \\
\text { things }\end{array}$ & $\begin{array}{c}\square \\
\text { confidence \& comfort } \\
\text { level } \\
\square \\
\text { got over anxiety } \\
\text { through } \\
\text { art } \\
\square \\
\text { great sense of self } \\
\text { through acting } \\
\square \\
\text { positive effect on self- } \\
\text { image } \\
\square \\
\text { benefits of AIC for } \\
\text { self-esteem }\end{array}$ & $\begin{array}{c}\square \\
\text { big } \\
\text { developmental } \\
\text { step } \\
\square \\
\text { got over } \\
\text { performance } \\
\text { anxiety through } \\
\text { arts }\end{array}$ \\
\hline $\begin{array}{l}\text { Family } \\
\text { involvement } \\
\text { in special arts } \\
\text { form } \\
(\rightarrow \text { interest in it) }\end{array}$ & $\begin{array}{c}\square \\
\text { owns her own art } \\
\text { business }\end{array}$ & $\begin{array}{c}\square \\
\text { mother - filmmaker, } \\
\text { father - graphic } \\
\text { designer } \\
\text { interest in school - } \\
\text { visited schools to pick } \\
\text { from }\end{array}$ & $\begin{array}{c}\square \\
\text { whole family } \\
\text { went to art } \\
\text { museum }\end{array}$ \\
\hline $\begin{array}{l}\text { Learning/ } \\
\text { recall } \\
\text { are supported by } \\
\text { AIC }\end{array}$ & $\nabla$ & $\begin{array}{c}\square \\
\text { makes impression on } \\
\text { them }\end{array}$ & $\begin{array}{l}\text { प } \\
\text { play }\end{array}$ \\
\hline $\begin{array}{l}\text { Interdisciplinary } \\
\text { learning (AIC) }\end{array}$ & $\begin{array}{cc} & \square \\
\text { - } & \text { songs/ coloring/ } \\
\text { painting in Math } \\
\text { - } \quad \text { art in Reading } \\
\text { - } \quad \text { African music \& } \\
\text { painting }\end{array}$ & $\begin{array}{c}\square \\
-\quad \text { music playing } \\
-\quad \text { arts \& science } \\
\text { (balloon for } \\
\text { science) }\end{array}$ & $\begin{array}{c}\square \\
\text { - play/ literature } \\
\text { (Odyssey) } \\
\text { - literature \& } \\
\text { history } \\
\text { - story told }\end{array}$ \\
\hline
\end{tabular}




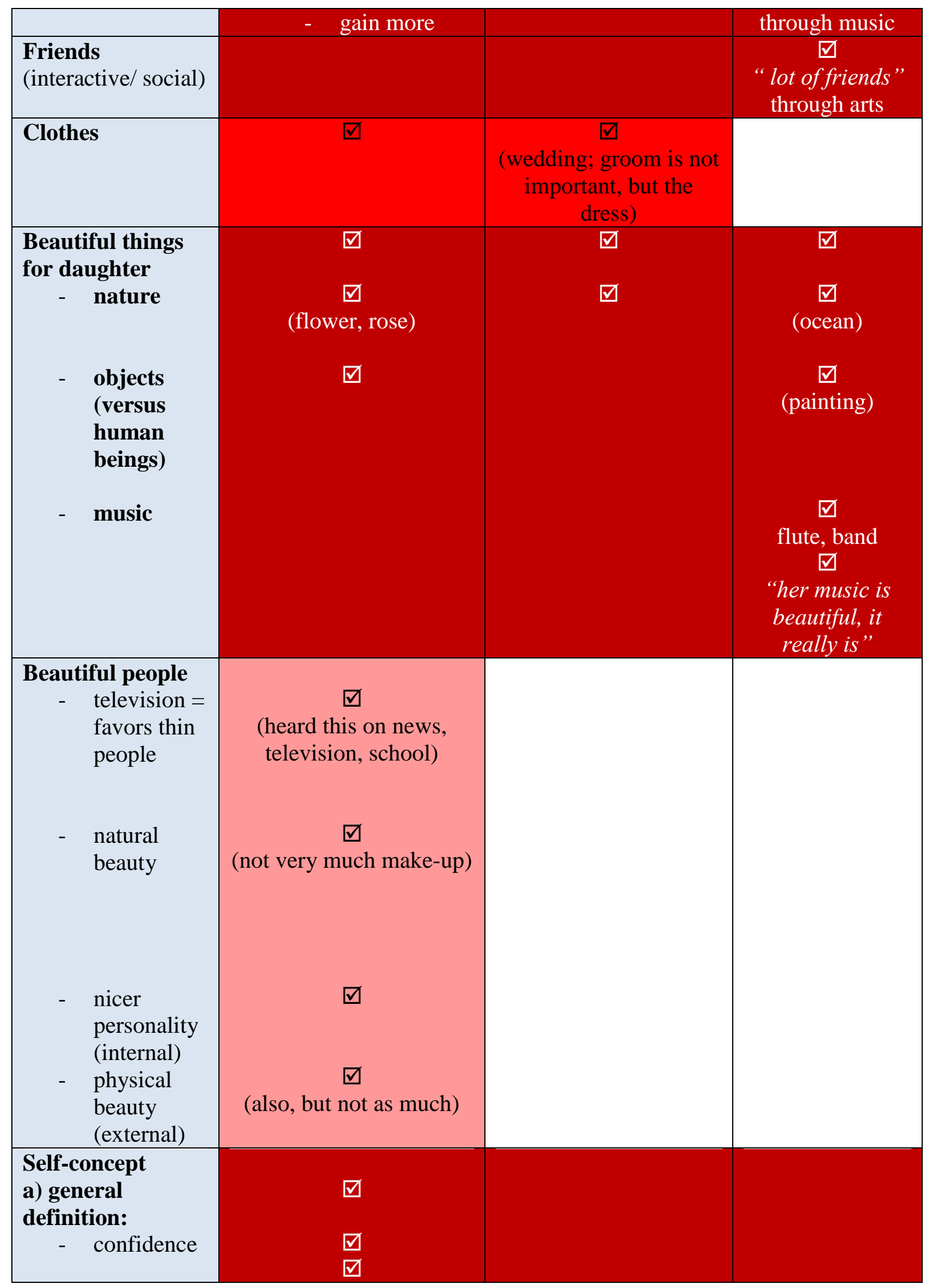




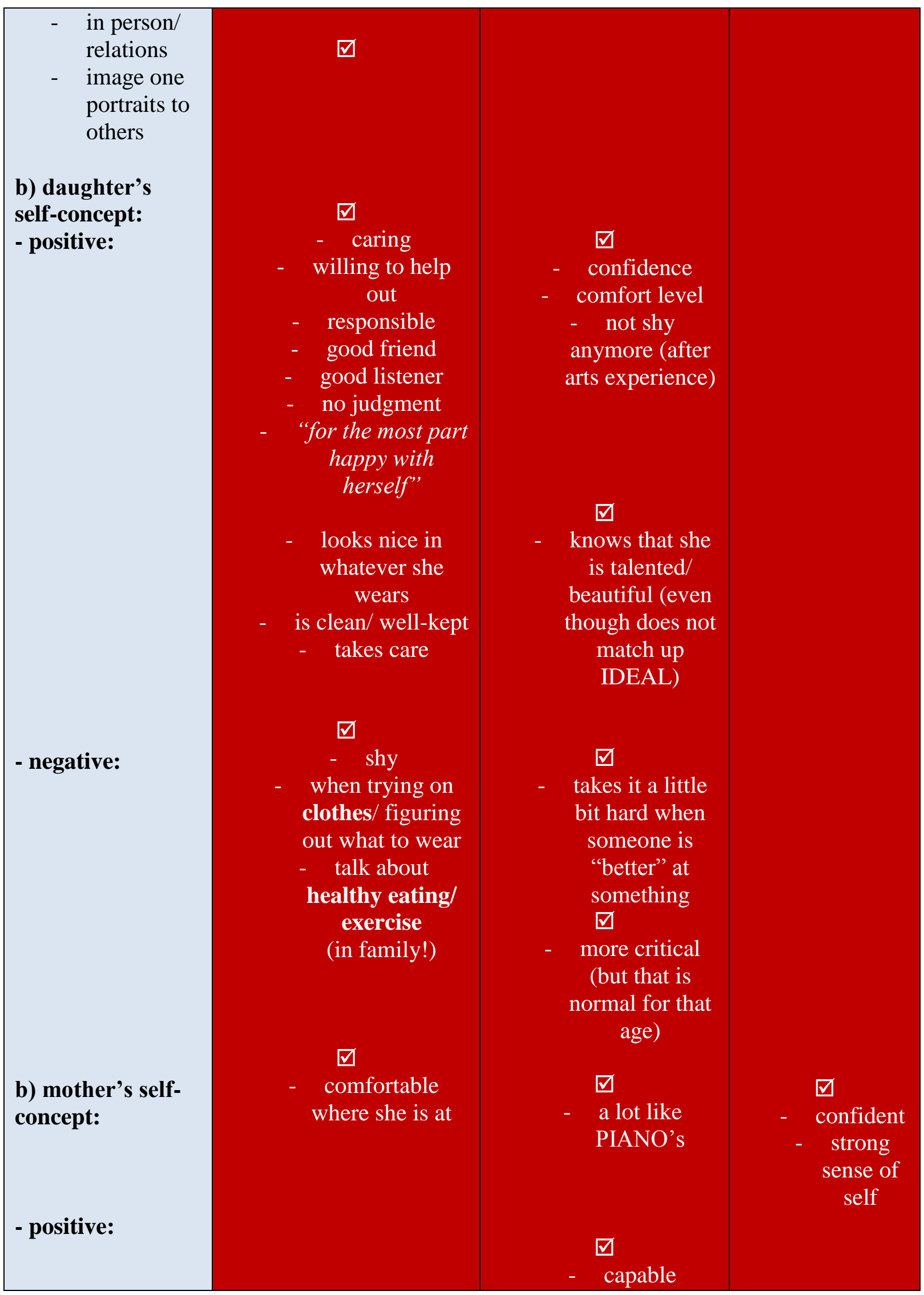




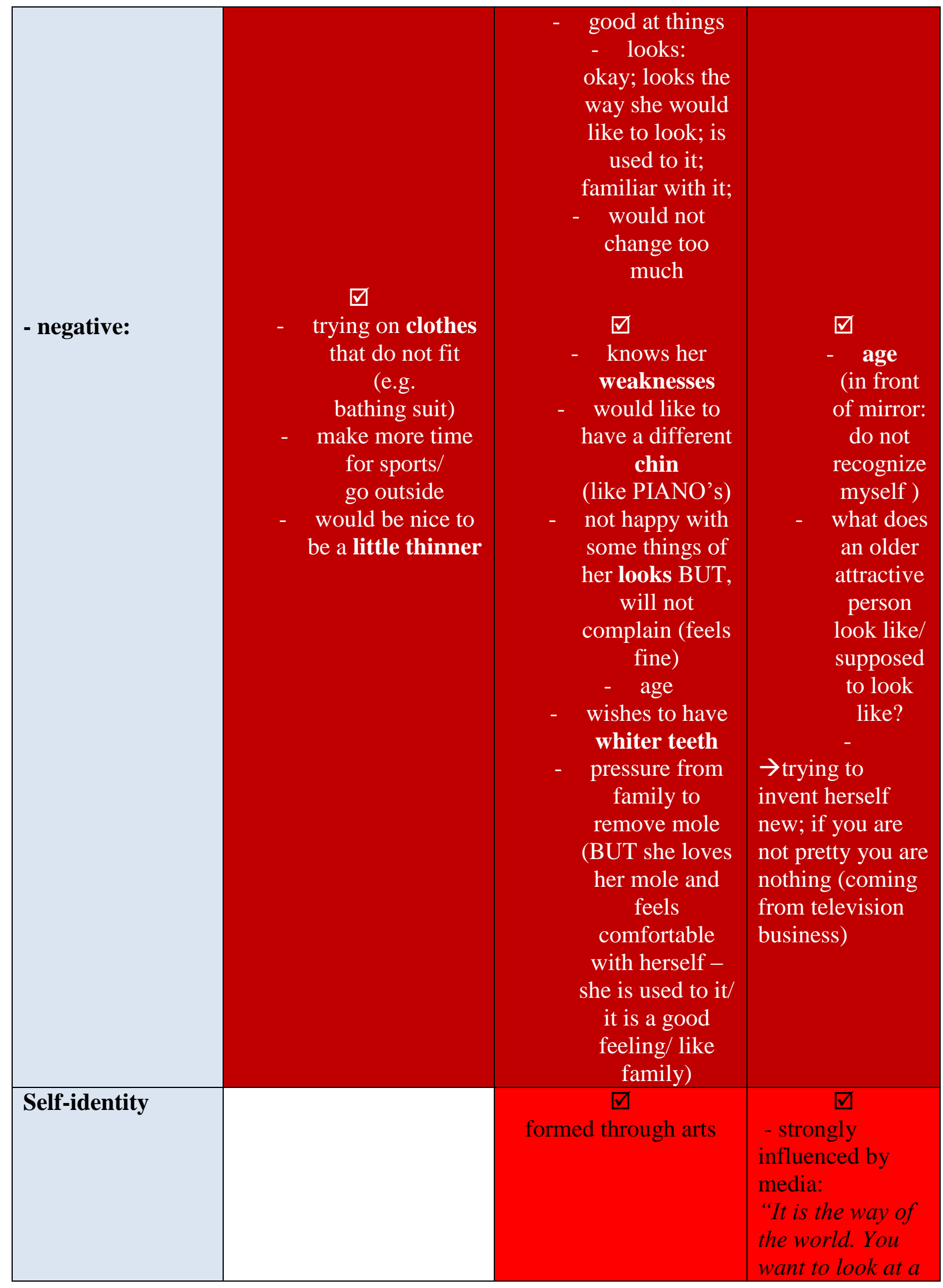




\begin{tabular}{|c|c|c|c|}
\hline & & & $\begin{array}{l}\text { pretty girl" } \\
\text { - If you are not } \\
\text { pretty you are } \\
\text { nothing } \\
\text { (television/ } \\
\text { media) }\end{array}$ \\
\hline $\begin{array}{l}\text { Parent } \\
\text { involvement/ } \\
\text { integration }\end{array}$ & $\begin{array}{c}\square \\
-\quad \text { meeting at } \\
\text { beginning of year } \\
-\quad \text { talk about } \\
\text { concepts }\end{array}$ & $\begin{array}{cc} & \square \\
\text { - } & \text { impressionist } \\
\text { unit; parents } \\
\text { came in } \\
\text { - } \text { PTG } \\
\text { (parent teacher } \\
\text { group) } \\
\text { - } \quad \text { open houses for } \\
\text { parents } \\
\text { - visited schools } \\
\text { before choosing }\end{array}$ & $\begin{array}{l}\square \\
\text { - } \quad \text { impressio } \\
\text { nist unit; } \\
\text { parents } \\
\text { came in }\end{array}$ \\
\hline $\begin{array}{l}\text { Parents } \\
\text { volunteered }\end{array}$ & $\begin{array}{c}\square \\
\text { - } \quad \text { put on a musical } \\
\text { (101 Dalmatians }) \\
-\quad \text { after-school- } \\
\text { program } \\
-\quad \text { initiative }\end{array}$ & $\begin{array}{c}\square \\
-\quad \text { musical } \\
\text { (101 Dalmatians) } \\
\text { - } \quad \text { PTG (parent } \\
\text { teacher group) } \\
\text { - } \quad \text { brought in arts/ } \\
\text { opportunities }\end{array}$ & $\begin{array}{l}\square \\
\text { - } \\
\text { play }(101 \\
\text { Dalmatia } \\
\text { ns })\end{array}$ \\
\hline $\begin{array}{l}\text { Interest } \\
\text { (in musical) } \\
\text { (in arts at home) } \\
\text { (in arts - public } \\
\text { space) }\end{array}$ & $\begin{array}{c}\square \\
\text { (many tried out) }\end{array}$ & $\begin{array}{c}\square \\
\text { (2/3 almost whole } \\
\text { school involved) } \\
\square \\
\text { (artwork in her room) }\end{array}$ & $\begin{array}{c}\square \\
\text { (artwork in her } \\
\text { room) } \\
\square \\
\text { (Art Museum) }\end{array}$ \\
\hline $\begin{array}{l}\text { Nobody excluded/ } \\
\text { all can participate } \\
\text { (but, had to try out) }\end{array}$ & $\nabla$ & $\nabla$ & \\
\hline Support by school & $\begin{array}{c}\square \\
\text { (building,...) }\end{array}$ & & \\
\hline $\begin{array}{l}\text { Leadership role } \\
\text { taken-over by } \\
\text { students }\end{array}$ & & $\nabla$ & \\
\hline Pro AIC & $\begin{array}{c}\square \\
\text { (others should experience } \\
\text { it) }\end{array}$ & $\nabla$ & \\
\hline $\begin{array}{l}\text { Parents taught } \\
\text { by children } \\
-\quad \text { knowledge } \\
\end{array}$ & & $\begin{array}{c}\square \\
\text { (impressionist unit) } \\
\square\end{array}$ & $\begin{array}{c}\square \\
\text { (impressionist } \\
\text { unit) } \\
\end{array}$ \\
\hline
\end{tabular}




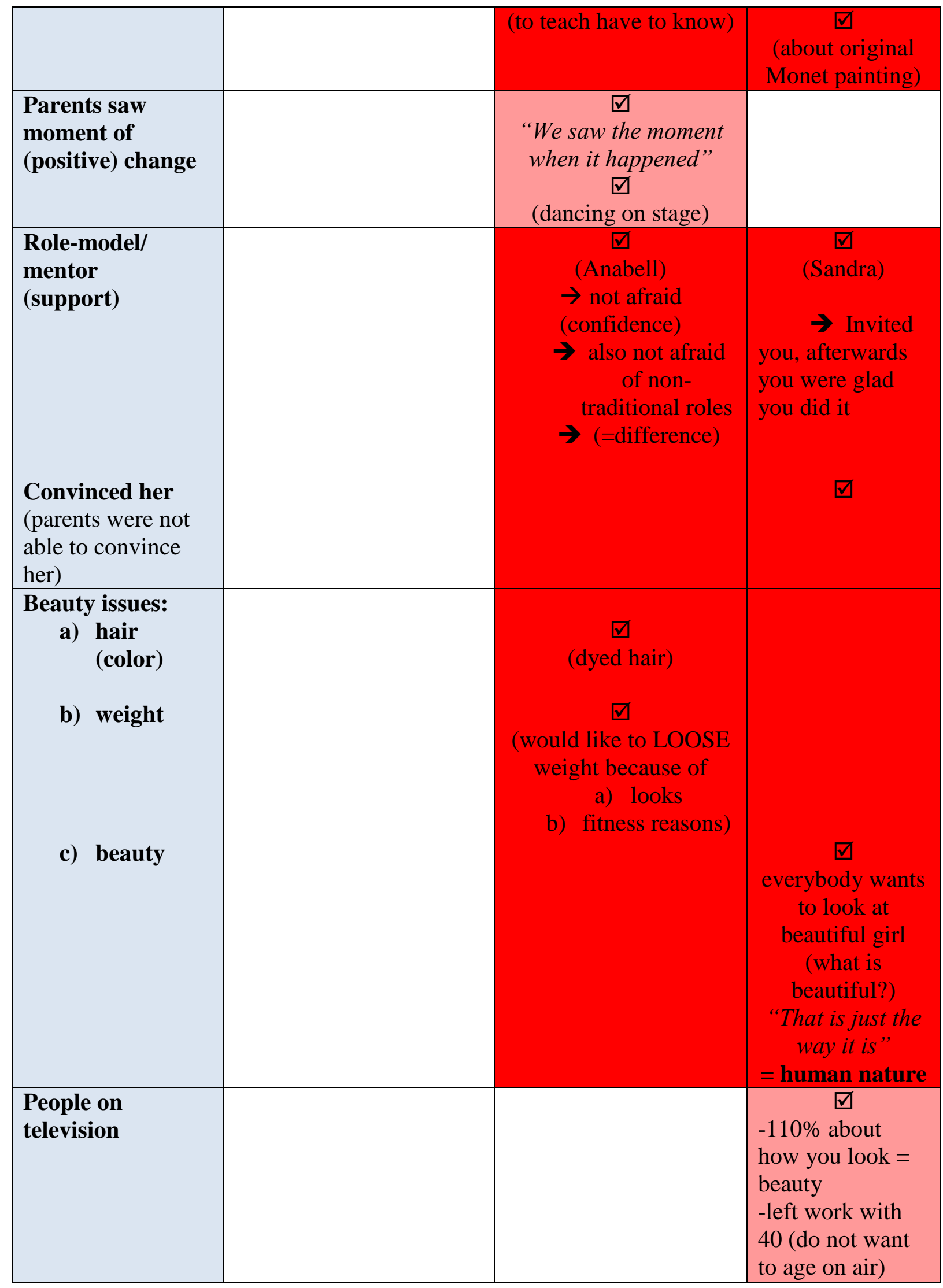




\begin{tabular}{|c|c|c|c|}
\hline television & & & $\begin{array}{l}\text {-ton of make-up } \\
\text {-beauty big part } \\
\text { of that profession } \\
\text {-looks = very } \\
\text { important } \\
\qquad \square \\
\text {-television = } \\
\text { two-dimensional } \\
\text { = cannot see past } \\
\text { appearance } \\
\text {-superficial by } \\
\text { definition } \\
\text {-if you are not } \\
\text { pretty you are } \\
\text { nothing }\end{array}$ \\
\hline $\begin{array}{l}\text { Healthy eating/ } \\
\text { weight loss }\end{array}$ & $\nabla$ & $\nabla$ & \\
\hline $\begin{array}{l}\text { School = influence } \\
\text { on image/ self- } \\
\text { concept }\end{array}$ & $\nabla$ & $\nabla$ & \\
\hline $\begin{array}{l}\text { Teachers } \\
\text { (positive) influence }\end{array}$ & & $\nabla$ & $\begin{array}{c}\square \\
\text { (sent pictures to } \\
\text { teacher) } \\
\square \\
\text { (positive } \\
\text { feedback) }\end{array}$ \\
\hline $\begin{array}{l}\text { Endurance } \\
\text { (in practice) }\end{array}$ & $\nabla$ & $\nabla$ & \\
\hline $\begin{array}{l}\text { Learning to life } \\
\text { with competition } \\
\text { (and possible } \\
\text { failure) }\end{array}$ & & $\nabla$ & \\
\hline $\begin{array}{l}\text { Share experience } \\
\text { with others: } \\
\text { artists } \\
=\text { communicate } \\
\text { with art }\end{array}$ & & $\begin{array}{c}\square \\
\square \\
\text { artist want to share } \\
\text { experiences with others } \\
\text { to not feel alone }\end{array}$ & \\
\hline $\begin{array}{l}\text { Artwork } \\
\text { continued in free- } \\
\text { time } \\
\quad \rightarrow \text { not a } \\
\quad \text { project }\end{array}$ & $\nabla$ & $\begin{array}{c}\square \\
\text { (my wedding plan, } \\
\text { writing) }\end{array}$ & \\
\hline
\end{tabular}




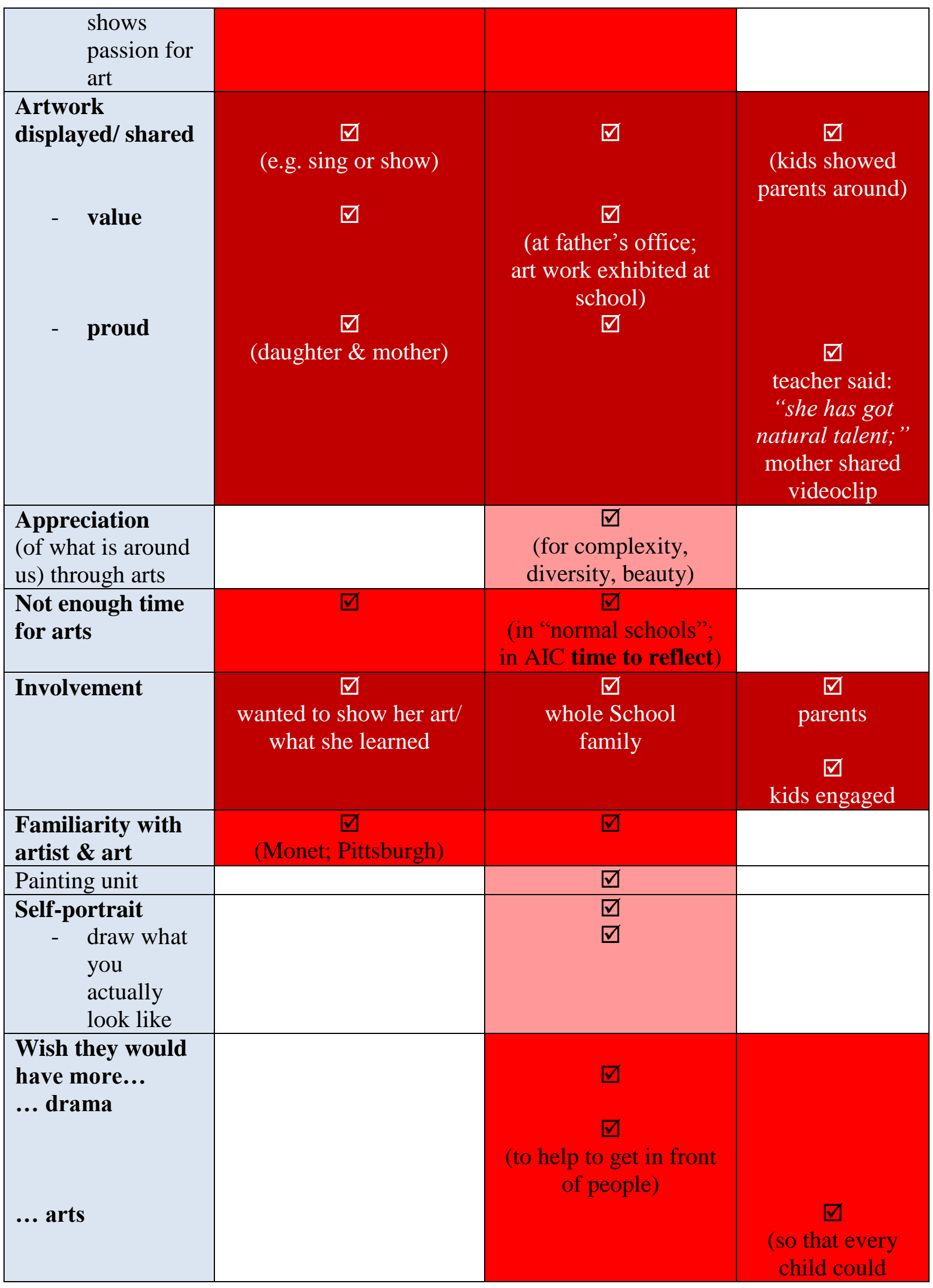




\begin{tabular}{|c|c|c|c|}
\hline & & & $\begin{array}{l}\text { experience it } \\
\text { [AIC]; } \\
\text { "the more, the } \\
\text { better") }\end{array}$ \\
\hline $\begin{array}{c}\text { Exposure to } \\
-\quad \begin{array}{l}\text { literature/ } \\
\text { writings }\end{array} \\
\text { - plays }\end{array}$ & & $\begin{array}{c}\square \\
\square \\
\text { (Bugsy Malone, Fiddler } \\
\text { on the Roof) } \\
\square\end{array}$ & $\begin{array}{c}\square \\
\text { (Odyssey, } \\
\text { Number the } \\
\text { Starts, Brown } \\
\text { versus Board of } \\
\text { Education) } \\
\square \\
\text { mini-plays } \\
\text { (act out; talk } \\
\text { about it) }\end{array}$ \\
\hline - perform & & & $\begin{array}{c}\square \\
\text { (Greek day, } \\
\text { literature) }\end{array}$ \\
\hline $\begin{array}{l}\text { Arts/ dance/ } \\
\text { drama } \\
\text { help to } \\
\text { surpass shyness)/ } \\
\text { can make you } \\
\text { braver }\end{array}$ & & $\begin{array}{c}\square \\
\text { (Dance!) } \\
\square \\
-\quad \text { not afraid to } \\
\text { speak } \\
\text { - } \quad \text { not afraid to be } \\
\text { in front of } \\
\text { people } \\
\Rightarrow \text { before arts } \\
\text { experiences } \\
\text { very shy }\end{array}$ & $\begin{array}{c}\square \\
\square \\
\begin{array}{c}\text { (on stage, sang, } \\
\text { danced) }\end{array}\end{array}$ \\
\hline $\begin{array}{l}\text { fearless } \\
\text { try }\end{array}$ & & $\begin{array}{l}\square \\
\text { (to stand in } \\
\text { front of people) }\end{array}$ & च \\
\hline $\begin{array}{l}\text { One area of arts } \\
\text { influences the } \\
\text { other one } \\
\text { positively }\end{array}$ & & $\begin{array}{c}\square \\
\text { (helped ear; singing; } \\
\text { better harmonies) }\end{array}$ & \\
\hline $\begin{array}{l}\text { Influence of } \\
\text { environment } \\
-\quad \text { family }\end{array}$ & $\begin{array}{c}\square \\
\square \\
\text { how you are raised/ } \\
\text { brought up) } \\
\square\end{array}$ & $\begin{array}{l}\square \\
\square\end{array}$ & \\
\hline
\end{tabular}




\begin{tabular}{|c|c|c|c|}
\hline - $\quad$ school & $($ environment $=$ different $)$ & $\begin{array}{c}\text { (small school nurturing } \\
\text { environment) }\end{array}$ & \\
\hline $\begin{array}{l}\text { Show/ share } \\
\text { artwork } \\
\text { (see Figures 1- } \\
\text { 10) } \\
\text { daughter } \\
\text { mother }\end{array}$ & $\begin{array}{c}\square \\
\text { (paintings, box, cross) }\end{array}$ & $\begin{array}{c}\square \\
\text { (wedding plan drawing, } \\
\text { piano) }\end{array}$ & $\begin{array}{l}\square \\
\text { (wanted to play } \\
\text { flute for the } \\
\text { researcher but } \\
\text { flute was at } \\
\text { school) } \\
\square \\
\text { (showed clip of } \\
\text { daughter playing } \\
\text { the flute to } \\
\text { researcher) }\end{array}$ \\
\hline $\begin{array}{l}\text { No boredom, arts } \\
\text { help out (continues } \\
\text { artwork in free- } \\
\text { time) }\end{array}$ & & $\begin{array}{c}\square \\
\text { (drew her wedding and } \\
\text { loves to write in free- } \\
\text { time) }\end{array}$ & \\
\hline Seems to "fit" & & $\begin{array}{c}\square \\
\text { (colors Blue \& White) }\end{array}$ & \\
\hline $\begin{array}{l}\text { AIC school } \\
\text { positive } \\
\text { evaluation/ } \\
\text { recommendation }\end{array}$ & $\begin{array}{c}\square \\
-\quad \begin{array}{c}\text { moved to school } \\
\text { district for school }\end{array} \\
\square\end{array}$ & $\begin{array}{c}\square \\
-\quad \text { small school } \\
\text { with nurturing } \\
\text { environment }\end{array}$ & $\begin{array}{c}\square \\
\text { unit on } \\
\text { impressionism } \\
\square\end{array}$ \\
\hline good reputation & & $\nabla$ & \\
\hline "Best School" & $\begin{array}{c}\square \\
\text { (do a little bit more) }\end{array}$ & $\begin{array}{l}\square \\
\text { (lot of parent } \\
\text { involvement) }\end{array}$ & \\
\hline $\begin{array}{l}\text { Small AIC } \\
\text { different from } \\
\text { "normal" school }\end{array}$ & & $\begin{array}{c}\square \\
\text { ("might not get that in } \\
\text { a normal school") } \\
\square \\
\text { (time to reflect on } \\
\text { skill, e.g. musical or } \\
\text { artistic skill) } \\
\square \\
\text { (nurturing } \\
\text { environment) }\end{array}$ & \\
\hline Good little school & & $\begin{array}{c}\square \\
\text { (with focus on arts) } \\
\text { - last one of its kind? } \\
\text {-will try to save it } \\
\text {-transferred daughter }\end{array}$ & \\
\hline
\end{tabular}




\begin{tabular}{|c|c|c|}
\hline & $\begin{array}{l}\text { because of AIC } \\
\text {-hard to get in } \\
\text {-good reputation }\end{array}$ & \\
\hline $\begin{array}{l}\text { Culture of respect } \\
\text { \& familiarity } \\
\text { (community) }\end{array}$ & $\begin{array}{c}\square \\
\text { (everyone knows } \\
\text { everyone) }\end{array}$ & \\
\hline Empathy & $\begin{array}{c}\square \\
\text { (for outsider; } \\
\text { learn more about their } \\
\text { viewpoints) }\end{array}$ & $\begin{array}{c}\square \\
\text { (be put in } \\
\text { character's place) }\end{array}$ \\
\hline emotions & & $\begin{array}{c}\square \\
\text { emotions } \\
\text { expressed } \\
\text { through music }\end{array}$ \\
\hline $\begin{array}{l}\text { Arts in schools } \\
\text { need more } \\
\text { support } \\
\text { (are undervalued) }\end{array}$ & $\begin{array}{c}\square \\
\text { (situation is pitiful) }\end{array}$ & च \\
\hline $\begin{array}{ll}- & \text { also } \\
& \text { extracurric } \\
& \text { ular things } \\
- & \text { sad }\end{array}$ & $\nabla$ & $\begin{array}{c}\square \\
\text { ( sad to see the } \\
\text { arts being cut) }\end{array}$ \\
\hline $\begin{array}{l}\text { Artist have } \\
\text { different points of } \\
\text { view } \\
\quad \rightarrow \text { experience }\end{array}$ & $\begin{array}{l}\square \\
\square\end{array}$ & \\
\hline
\end{tabular}

Notes: Color-coding information:

Dark Red: All three parents (BUSY, CARING, and LOVING) mentioned these units (ICs). Themes:

1.) Connection to works of art

2.) Experience the arts through daughter

3.) Interest in arts

4.) Took art classes

5.) Positive descriptions/ characterizations of art

6.) Positive influence of AIC

7.) Recognition/ confirmation/ positive feedback from art (intrinsic motivation)

8.) Positive self-concept through arts

9.) Family involvement

10.) Learning/ recall is supported by AIC

11.) Interdisciplinary learning (AIC)

12.) Friends (interactive/ social)

13.) Beautiful things for daughter

14.) Self-concept 
15.) Parent involvement/ integration

16.) Parents volunteered

17.) Interest

18.) Artwork displayed/ shared

19.) Involvement

20.) Show/ share artwork

21.) AIC school positive evaluation

Medium Red: Only two of the three parents mentioned these units (ICs) Themes:

1.) Exposure to arts supports interest in arts

2.) Focus on arts

3.) Looks

4.) Results of arts-based-learning experiences

5.) Knowledgeable kids

6.) Clothes

7.) Self-identity

8.) Nobody excluded/ all can participate

9.) Pro AIC

10.) Parents taught by children

11.) Beauty issues

12.) Role-model/ mentor (support)

13.) Healthy eating/ weight loss

14.) School = influence on image/ self-concept

15.) Teachers (positive) influence

16.) Endurance (in practice)

17.) Artwork continued in free-time

18.) Not enough time for arts

19.) Familiarity with artist \& art

20.) Wish they would have more...

21.) Exposure to ...

22.) Arts/ dance/ drama (help surpass shyness)

23.) Influence of environment

24.) Empathy/ emotions

25.) Arts in schools need more support

Light Red: Only one of the three parents mentioned these units (IC) Themes:

1.) Gain more

2.) Arts (broaden) perspectives

3.) Learn about underdog/ outsider (included)

4.) Arts = valued

5.) At first insecurity about arts

6.) Beautiful people

7.) Support by school 
8.) Leadership role

9.) Parents saw moment of (positive) change

10.) People on television

11.) Learning to life with competition

12.) Share experience with others

13.) Appreciation

14.) Painting unit

15.) Self-portrait

16.) One arts area influences the other one positively

17.) No boredom

18.) Seems to "fit"

19.) Small AIC different from "normal" school

20.) Good little school

21.) Culture of respect $\&$ familiarity (community)

22.) Artists have different points of view (experience) 
Appendix H5 - Teachers' themes

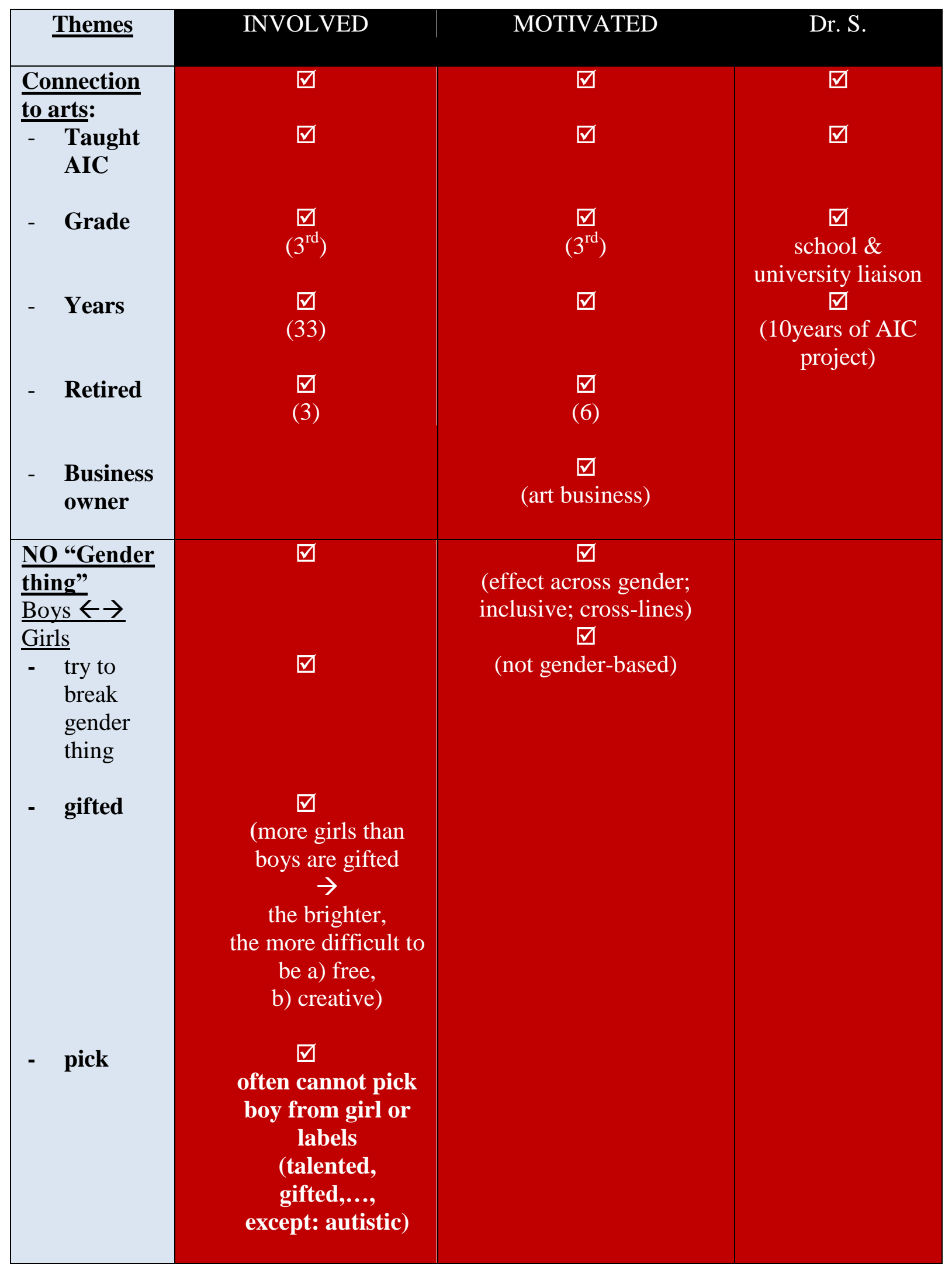




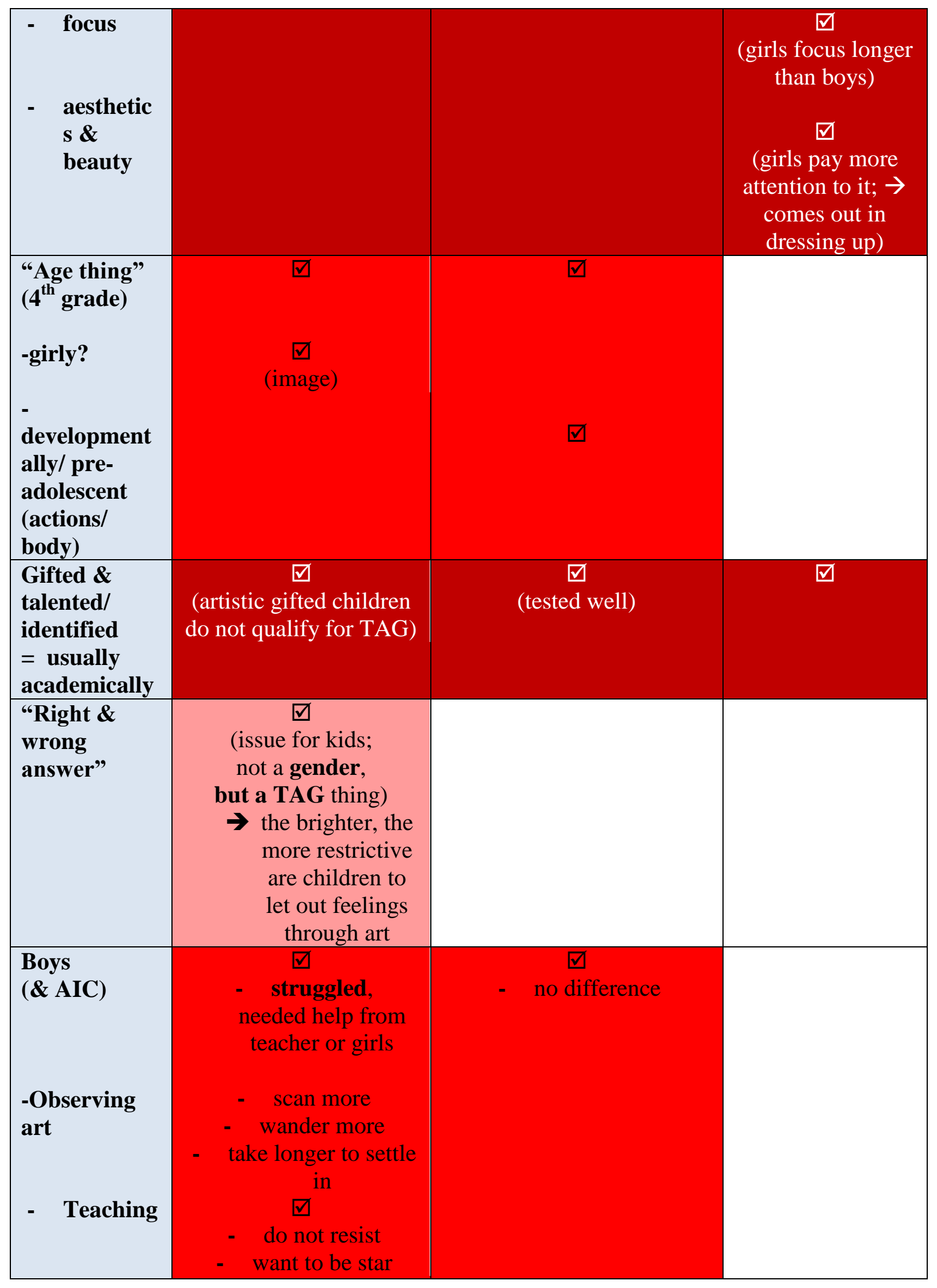




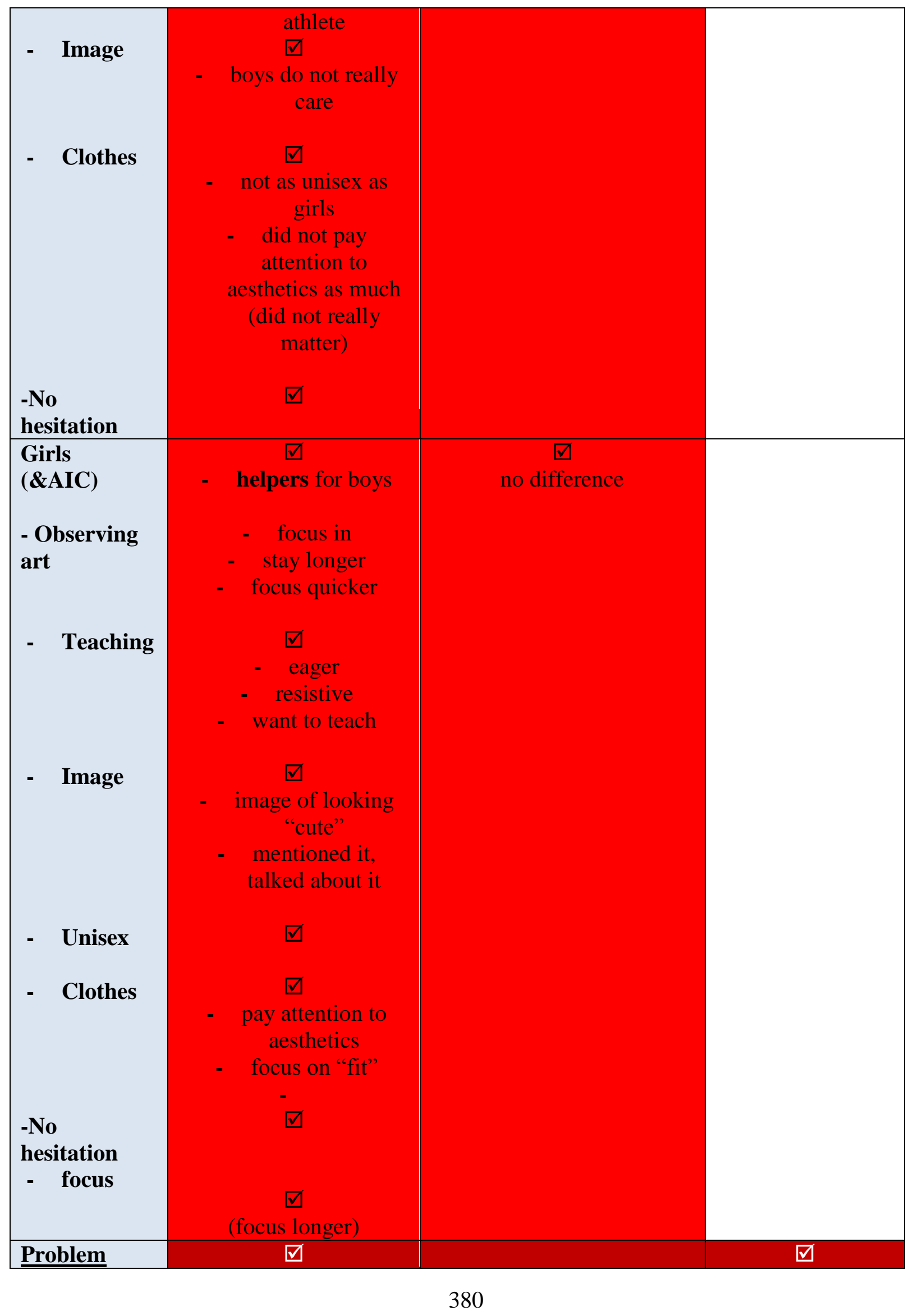




\begin{tabular}{|c|c|c|c|}
\hline $\begin{array}{l}\text { Solving } \\
\text { (skills) } \\
\text {-Assistance/ } \\
\text { help (do not } \\
\text { dictate) }\end{array}$ & $\begin{array}{c}-\quad \text { take risks } \\
-\quad \text { look at all } \\
\text { questions } \\
\text { - "how to draw" } \\
\square \\
\text { (= solution) }\end{array}$ & & $\begin{array}{c}\text { teachers } \\
\text { modeled } \\
\text { risk-taking } \\
=\text { permission } \\
\text { to take risks }\end{array}$ \\
\hline - Students & $\begin{array}{c}\square \\
\text { (part of solution) }\end{array}$ & & $\begin{array}{c}\square \\
\text { (not just teacher) }\end{array}$ \\
\hline $\begin{array}{l}\text { - } \quad \text { Socially } \\
\& \\
\text { academic } \\
\text { ally }\end{array}$ & $\square$ & & $\nabla$ \\
\hline $\begin{array}{ll}\text { - } & \text { Attempts } \\
\text { from } \\
\text { weaker } \\
\text { students }\end{array}$ & 口 & 口 & $\nabla$ \\
\hline 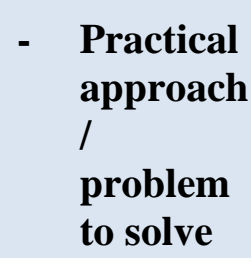 & $\begin{array}{c}\square \\
\text { (e.g. build a structure, } \\
\ldots \text { ) }\end{array}$ & & $\begin{array}{c}\square \\
\text { (as a class) }\end{array}$ \\
\hline $\begin{array}{ll}\text { - } & \text { Take } \\
\text { over } \\
\text { responsi } \\
\text { bility }\end{array}$ & $\begin{array}{c}\square \\
(\text { role })\end{array}$ & & $\nabla$ \\
\hline $\begin{array}{l}\text { - Independ } \\
\text { ent from } \\
\text { teacher }\end{array}$ & & & $\nabla$ \\
\hline $\begin{array}{l}\text { - Recogniz } \\
\text { ed } \\
\text { problem }\end{array}$ & & & $\nabla$ \\
\hline Creativity & $\begin{array}{c}\square \\
\text { (different viewpoints, } \\
\text { solutions) }\end{array}$ & $\begin{array}{c}\square \\
\text { (encouraged with clues } \\
\text { through dress-up) }\end{array}$ & \\
\hline
\end{tabular}




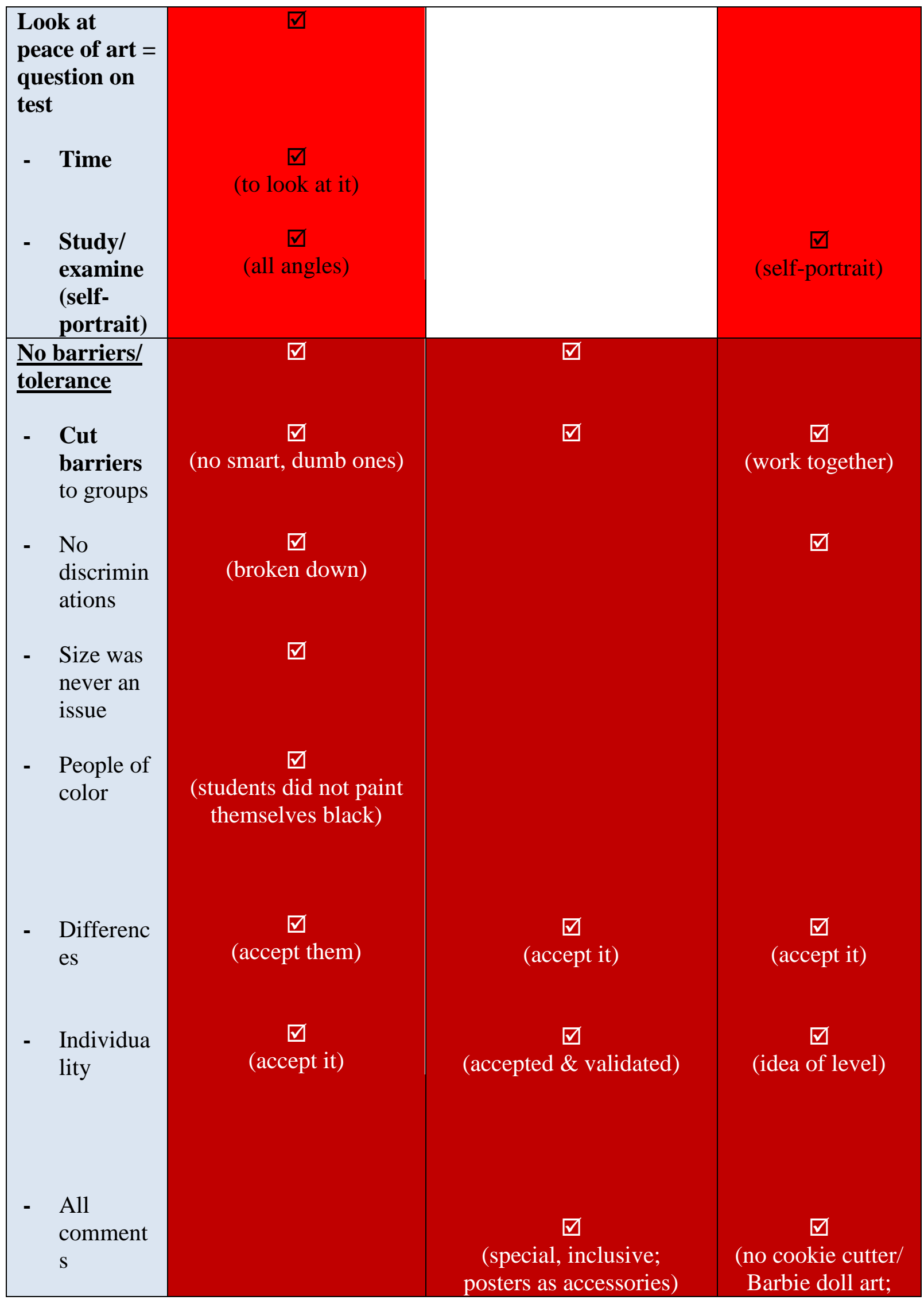




\begin{tabular}{|c|c|c|c|}
\hline $\begin{array}{ll}\text { - } & \text { Multi- } \\
\text { cultural }\end{array}$ & & & $\begin{array}{c}\text { all individual, } \\
\text { free) } \\
\square \\
\text { (accepted \& } \\
\text { validated) }\end{array}$ \\
\hline $\begin{array}{l}\text { Real life/ } \\
\text { social/ soft } \\
\text { skills }\end{array}$ & $\begin{array}{c}\square \\
\text { (art gallery) }\end{array}$ & & $\begin{array}{c}\square \\
\text { (interpersonal } \\
\text { skills modeled by } \\
\text { teachers) }\end{array}$ \\
\hline $\begin{array}{l}\text { Rules/ social } \\
\text { behavior }\end{array}$ & $\nabla$ & & \\
\hline $\begin{array}{l}\text { Critical } \\
\text { thinking } \\
\text { skills }\end{array}$ & $\begin{array}{c}\square \\
\text { (develop them) }\end{array}$ & & \\
\hline $\begin{array}{l}\text { Naming/ } \\
\text { label }\end{array}$ & $\begin{array}{c}\square \\
\text { (important!) }\end{array}$ & & \\
\hline $\begin{array}{l}\text { No discipline } \\
\text { problems }\end{array}$ & $\nabla$ & & \\
\hline $\begin{array}{l}\text { Independenc } \\
\text { e }\end{array}$ & $\begin{array}{c}\square \\
\text { - } \quad \text { Own self } \\
\text { - making decisions }\end{array}$ & च & \\
\hline $\begin{array}{ll}\text { - } & \text { decision } \\
\text { process } \\
\text { - } & \text { team }\end{array}$ & $\begin{array}{c}\square \\
\square \\
\text { (walk from room to } \\
\text { room) }\end{array}$ & $\stackrel{\square}{\square}$ & \\
\hline $\begin{array}{l}\text { Interest in } \\
\text { art } \\
-\quad \text { students }\end{array}$ & $\nabla$ & $\begin{array}{c}\square \\
\text { (maintained after AIC; } \\
\text { poster, ...) }\end{array}$ & $\begin{array}{l}\square \\
\text { (pick Monet, } \\
\text { rather than figure } \\
\text { of the day) }\end{array}$ \\
\hline $\begin{array}{l}\text { Exposure } \\
\text { to arts } \\
\text { enhances } \\
\text { necessary } \\
\text { information }\end{array}$ & $\begin{array}{c}\square \\
\text { (e.g. for reading \& } \\
\text { writing) }\end{array}$ & $\begin{array}{c}\square \\
\text { (e.g. tricked them to read) }\end{array}$ & • \\
\hline $\begin{array}{l}\text { Arts } \\
\text { (broaden) } \\
\text { perspectives } \\
-\quad \text { be open } \\
\text { to } \\
\quad \text { (differen) } \\
\quad \text { things }\end{array}$ & & $\begin{array}{c}\square \\
\square \\
\text { (allowed them things to do) }\end{array}$ & \\
\hline $\begin{array}{l}\text { Results of } \\
\text { arts-based- }\end{array}$ & $\nabla$ & $\nabla$ & $\nabla$ \\
\hline
\end{tabular}




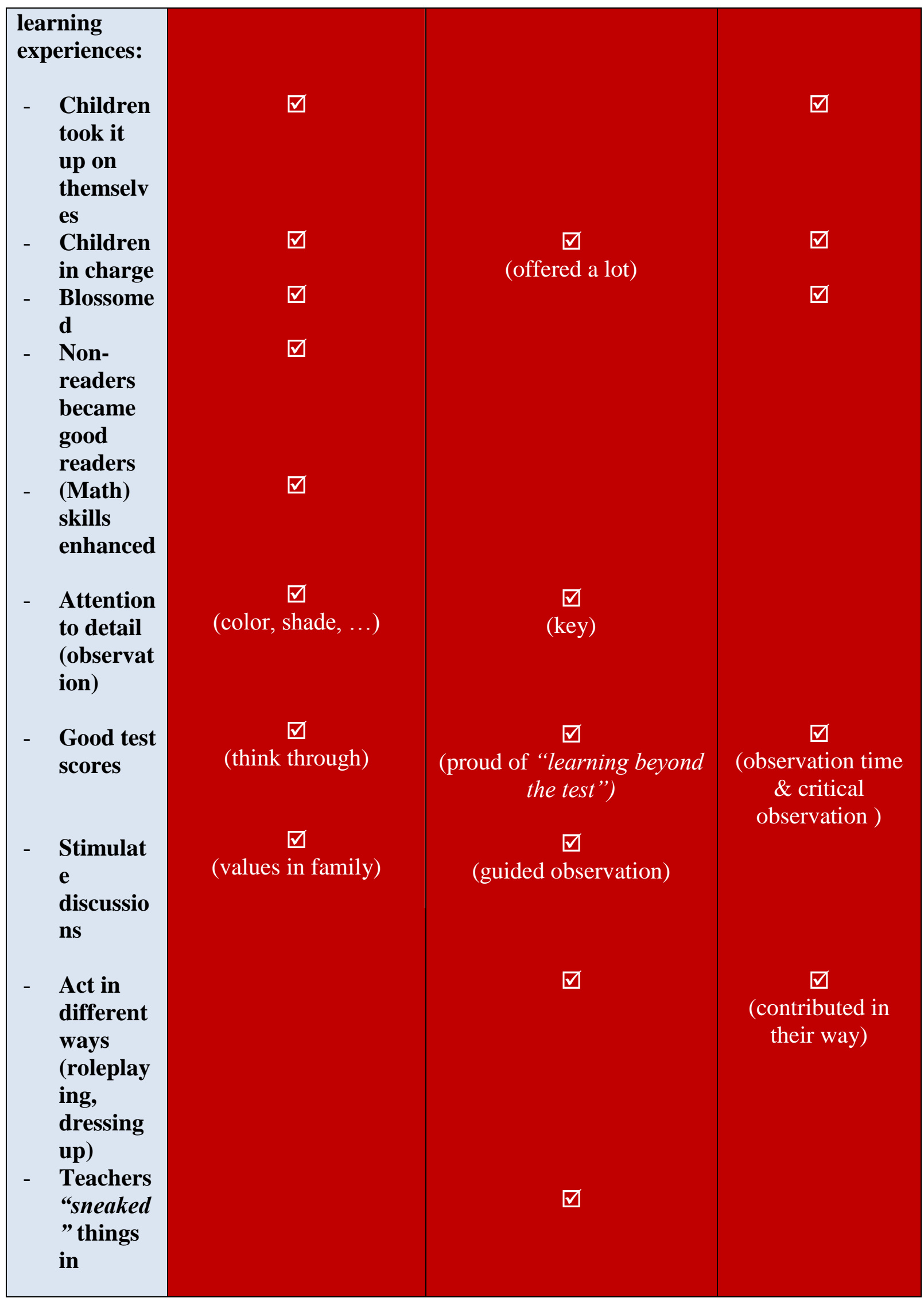




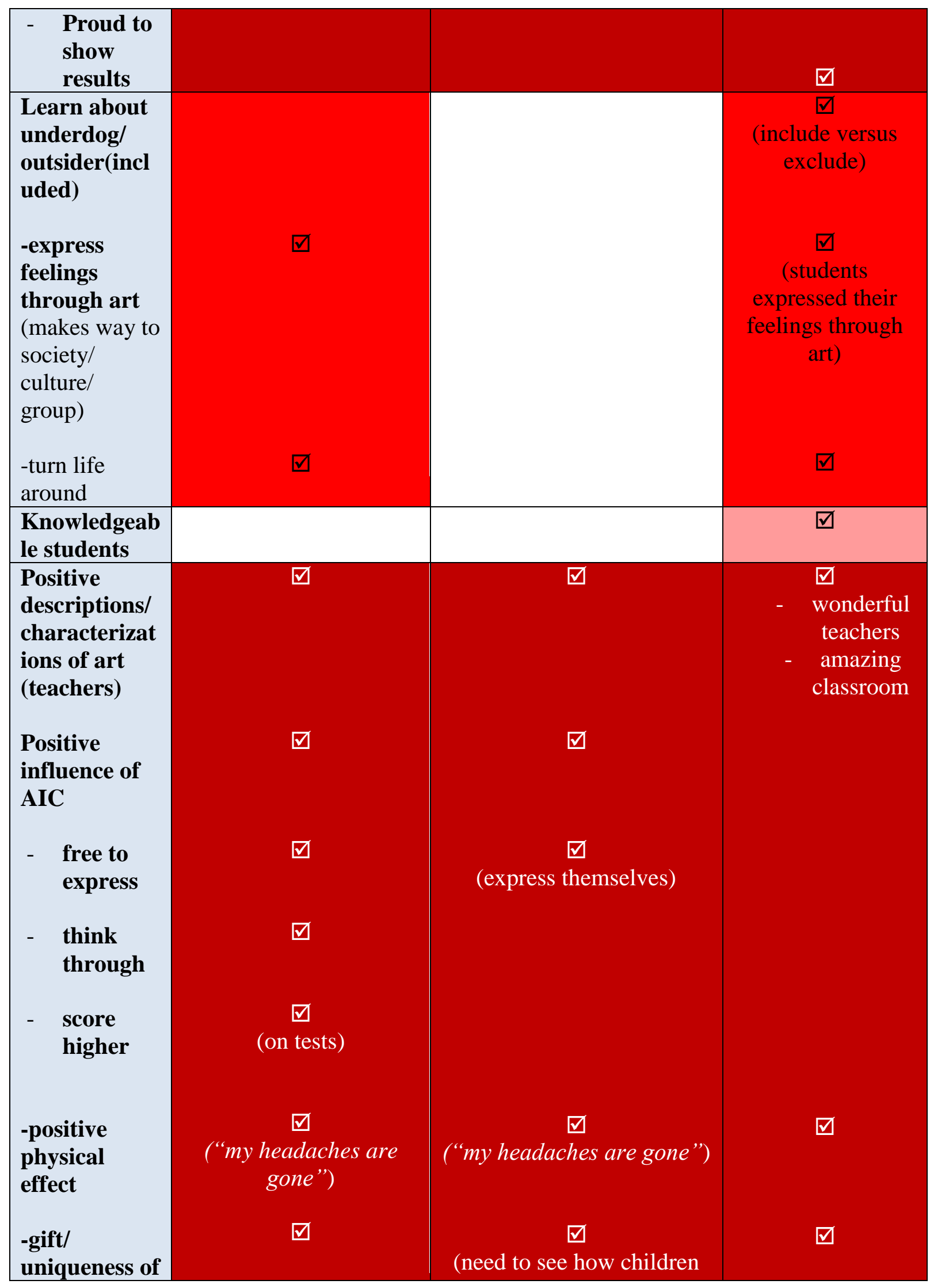




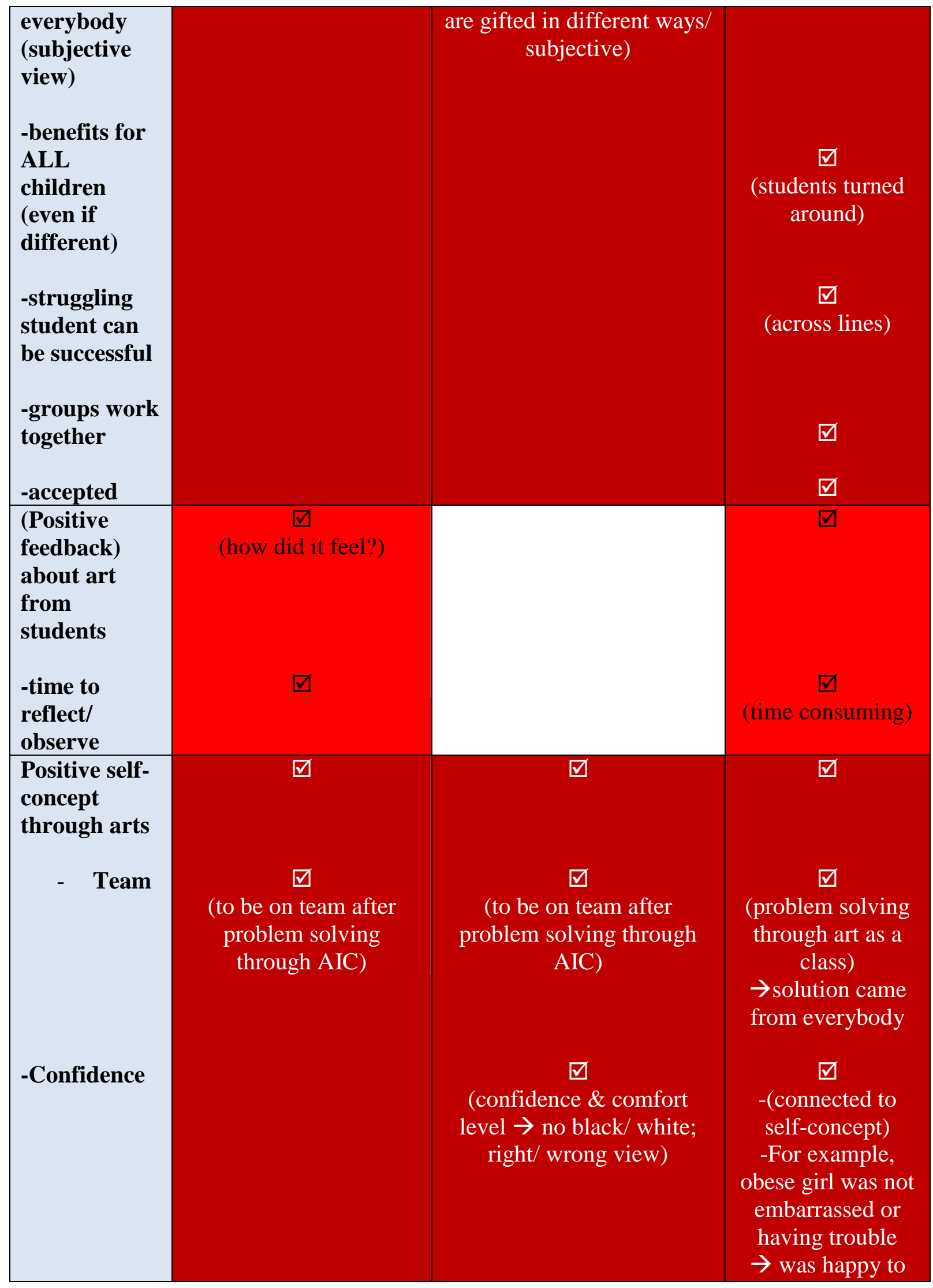




\begin{tabular}{|c|c|c|c|}
\hline -Details (self) & & & $\begin{array}{c}\text { participate/ } \\
\text { be integrated } \\
\square \\
\text { (development) }\end{array}$ \\
\hline $\begin{array}{l}\text { Family } \\
\text { involvement }\end{array}$ & $\begin{array}{c}\square \\
\text { (whole families went to } \\
\text { art museum) }\end{array}$ & & $\begin{array}{c}\text { (came to be taught } \\
\text { by their children) }\end{array}$ \\
\hline -discussions & $\begin{array}{c}\square \\
\text { (e.g. about values) }\end{array}$ & & \\
\hline $\begin{array}{l}\text { Learn in a } \\
\text { different way } \\
\text { through AIC } \\
\end{array}$ & $\nabla$ & $\nabla$ & $\nabla$ \\
\hline $\begin{array}{l}\text { Interdisciplin } \\
\text { ary learning } \\
\text { (AIC) }\end{array}$ & $\begin{array}{c}\square \\
-\quad \text { music } \\
-\quad \text { art } \\
-\quad \text { art \& sports } \\
-\quad \text { math }\end{array}$ & $\begin{array}{l}\qquad \square \\
\text { (multiple things } \\
\text { simultaneously) }\end{array}$ & $\begin{array}{c}\square \\
-\quad \begin{array}{c}\square \\
\text { read, write, } \\
\text { book } \\
\text { responses }\end{array}\end{array}$ \\
\hline $\begin{array}{l}\text { Friends } \\
\text { (interactive/ } \\
\text { social) }\end{array}$ & $\begin{array}{c}\square \\
\text { (want to be part of the } \\
\text { group) }\end{array}$ & & $\begin{array}{c}\square \\
\text { (want to be part of } \\
\text { the group) }\end{array}$ \\
\hline $\begin{array}{l}\text { Clothes/ } \\
\text { dress-up }\end{array}$ & $\begin{array}{c} \\
\text { - } \quad \text { huge closet } \\
\text { easier for girls } \\
\text { (more free) } \\
\text { - girls were into it/ } \\
\text { noticed it } \\
\text { - } \quad \text { boys do not care } \\
\text { but are also into } \\
\text { it (took them } \\
\text { longer) }\end{array}$ & $\begin{array}{l}\square \\
-\quad \begin{array}{l}\text { kids acted } \\
\text { differently }\end{array}\end{array}$ & \\
\hline $\begin{array}{l}\text {-find identity } \\
\text {-special } \\
\text { because of } \\
\text { clothes }\end{array}$ & $\square$ & & \\
\hline $\begin{array}{l}\text { Self-concept } \\
\text { a) teachers }\end{array}$ & $\begin{array}{c}\square \\
\text { - } \quad \text { (positive self- } \\
\text { concept)"we } \\
\text { were good at } \\
\text { making fools of } \\
\text { ourselves" }\end{array}$ & & $\begin{array}{c}\square \\
\text {-positive self- } \\
\text { concept } \\
\text { “comfortable with } \\
\text { themselves" } \\
\text { (danced, wore } \\
\text { costumes, } \\
\text { characters, voices, }\end{array}$ \\
\hline
\end{tabular}




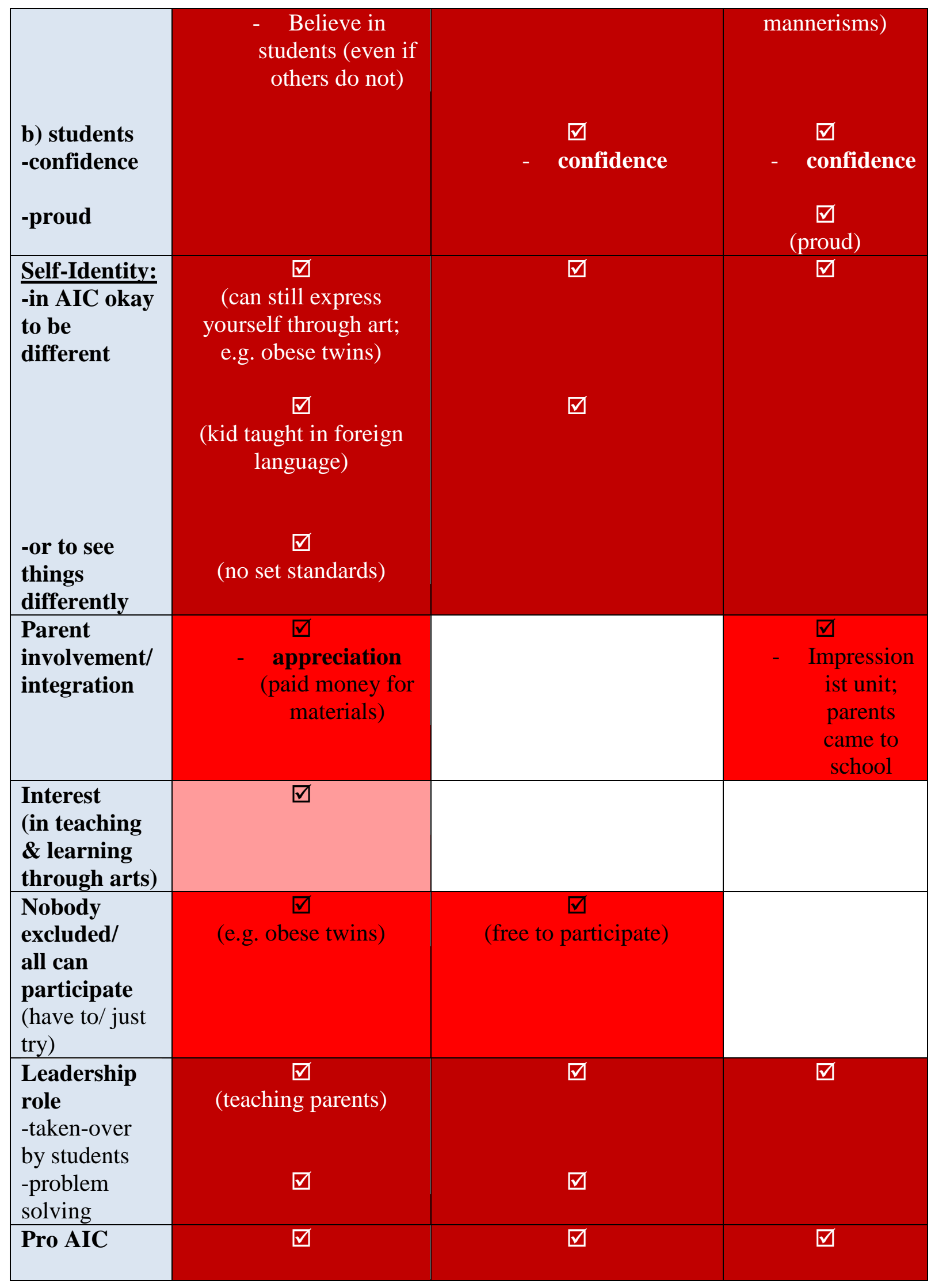




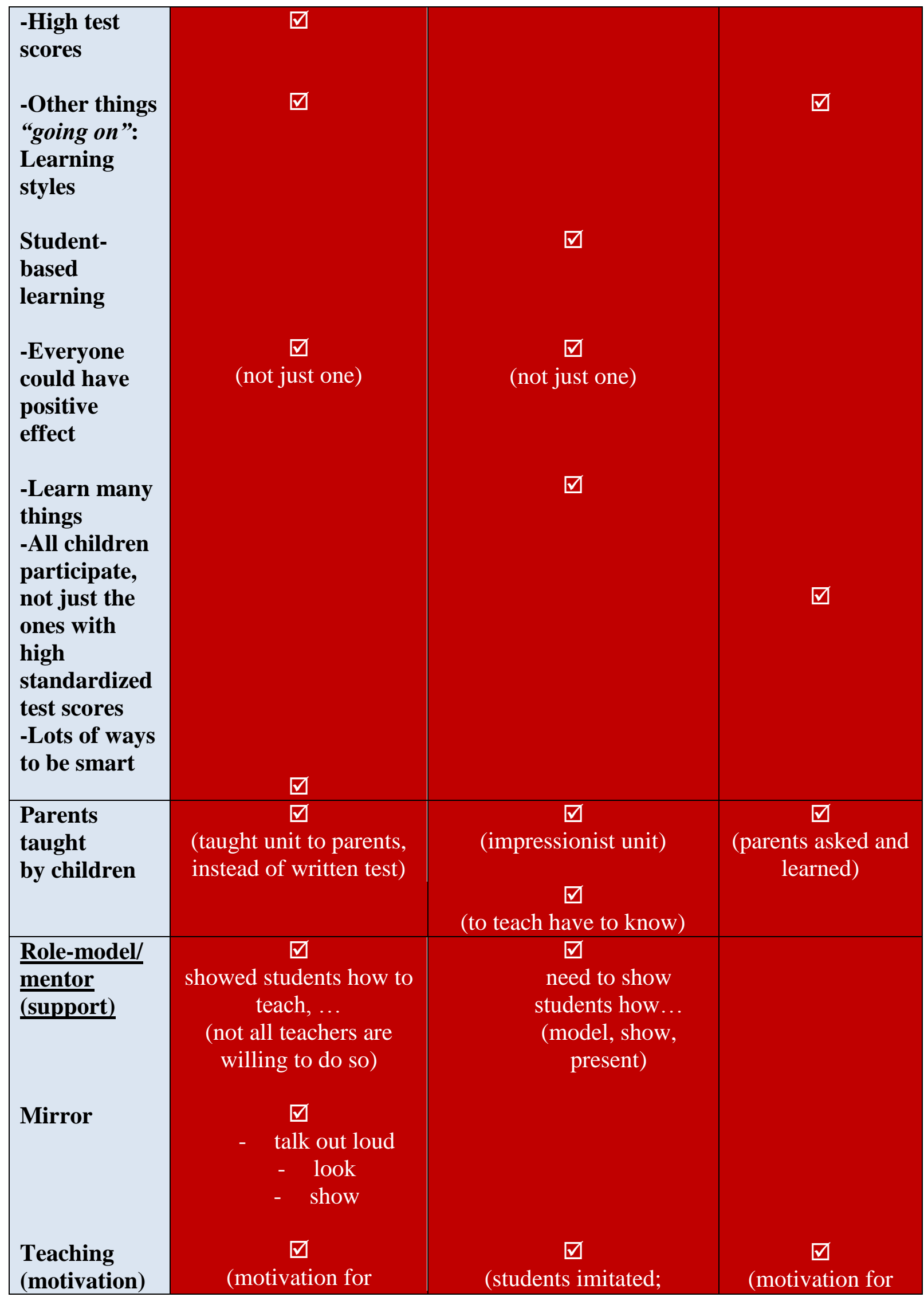




\begin{tabular}{|c|c|c|c|}
\hline $\begin{array}{l}\text { learned from } \\
\text { them }\end{array}$ & profession; how to do it) & $\begin{array}{c}\text { teachers }=\text { inspiration) } \\
\square\end{array}$ & $\begin{array}{c}\text { profession/ } \\
\text { volunteering) } \\
\square \\
\text { (teachers show } \\
\text { thinking process/ } \\
\text { critical } \\
\text { examination of } \\
\text { self = exposed) }\end{array}$ \\
\hline $\begin{array}{l}\text { Beauty: } \\
\text { a) } \\
\text { weight }\end{array}$ & $\begin{array}{c}\square \\
\text { - teachers mention } \\
\text { that obese } \\
\text { students should } \\
\text { lose weight; }\end{array}$ & $\begin{array}{l}\square \\
\text { (still participate in AIC, } \\
\text { even if over-weight) }\end{array}$ & \\
\hline b) tall & $\begin{array}{c}\square \\
\text { (obese/ heavy, } \\
\text { BUT tall) }\end{array}$ & $\begin{array}{c}\square \\
\text { (no teasing) }\end{array}$ & \\
\hline c) size & $\begin{array}{c}\square \\
\text {-plays a big role for } \\
\text { girls ("so little", "so } \\
\text { big") } \\
\text {-girls are more bothered } \\
\text { by it than boys } \\
\text {-becomes important } \\
\text { later for their self- } \\
\text { concept when boys want } \\
\text { to play sports } \\
\text {-was more a comment/ } \\
\text { fact than a tease ("you } \\
\text { are little, big, tall") }\end{array}$ & & \\
\hline $\begin{array}{l}\text { d) aesthe } \\
\text { tics }\end{array}$ & & & $\begin{array}{l}\text { (girls pay more } \\
\text { attention to beauty } \\
\text { and aesthetics than } \\
\text { boys) }\end{array}$ \\
\hline e) $\operatorname{art}$ & & & $\begin{array}{c}\square \\
\text { no common } \\
\text { beauty standard } \\
\text { (individual } \\
\text { standards, }\end{array}$ \\
\hline
\end{tabular}




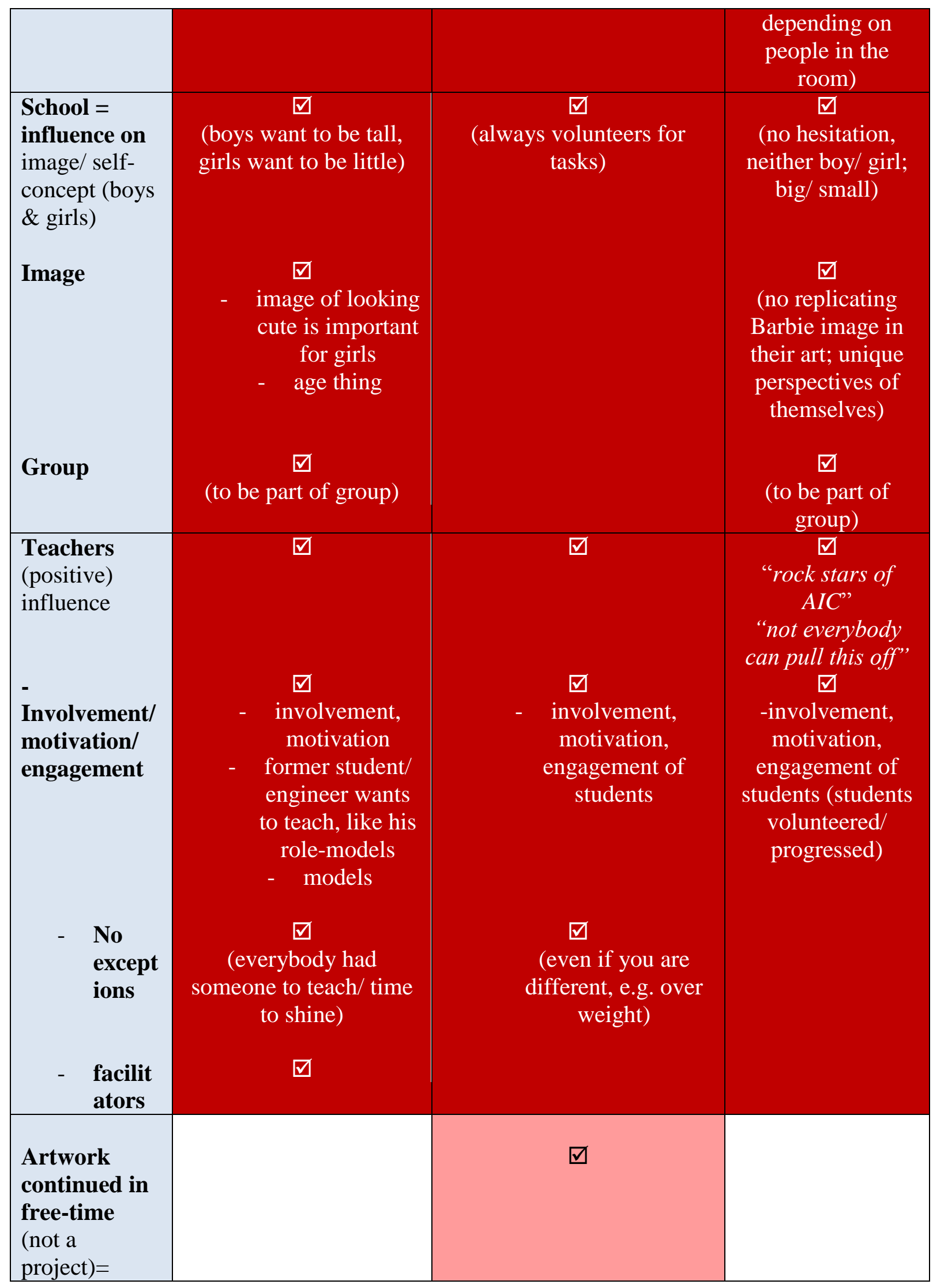




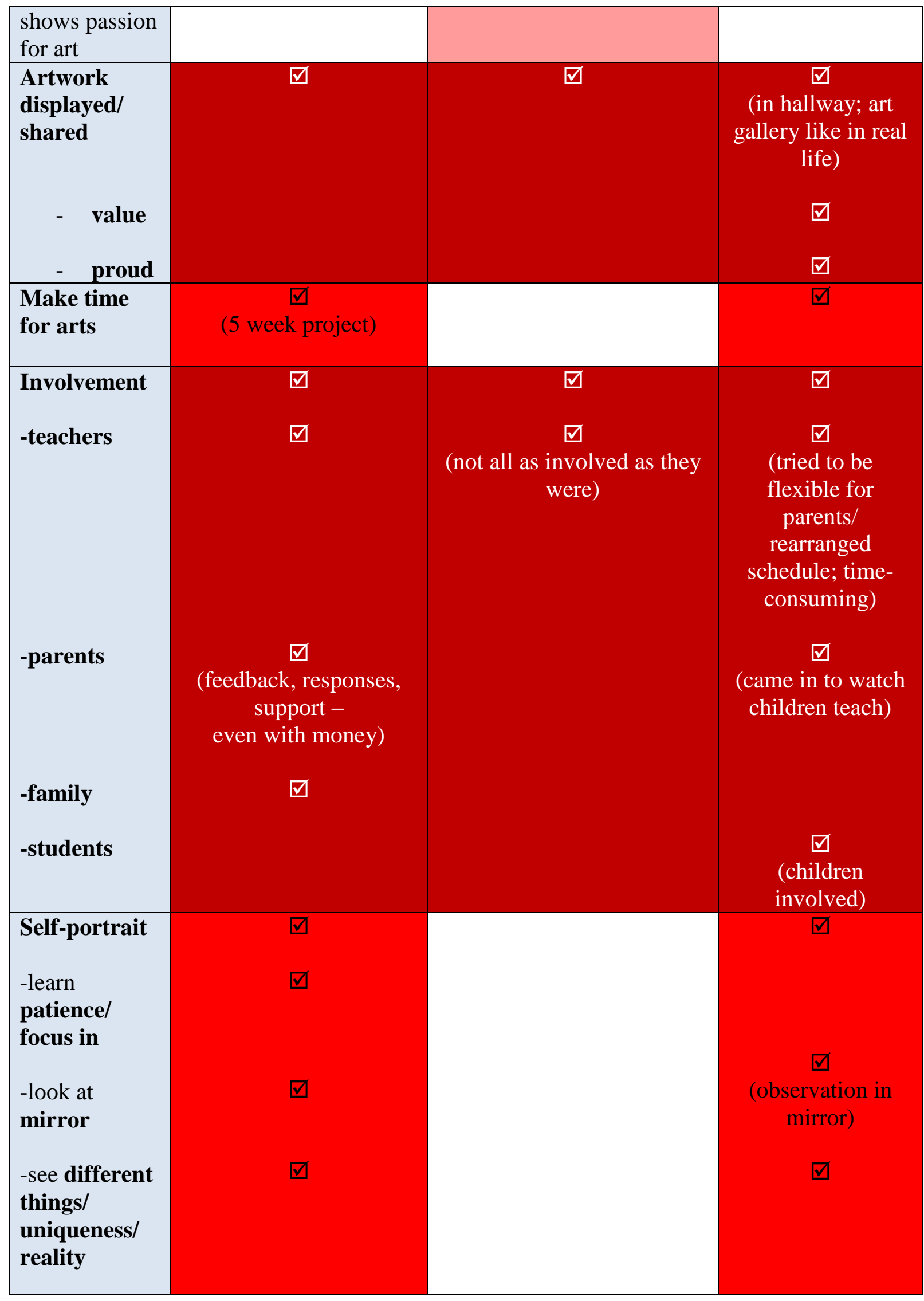




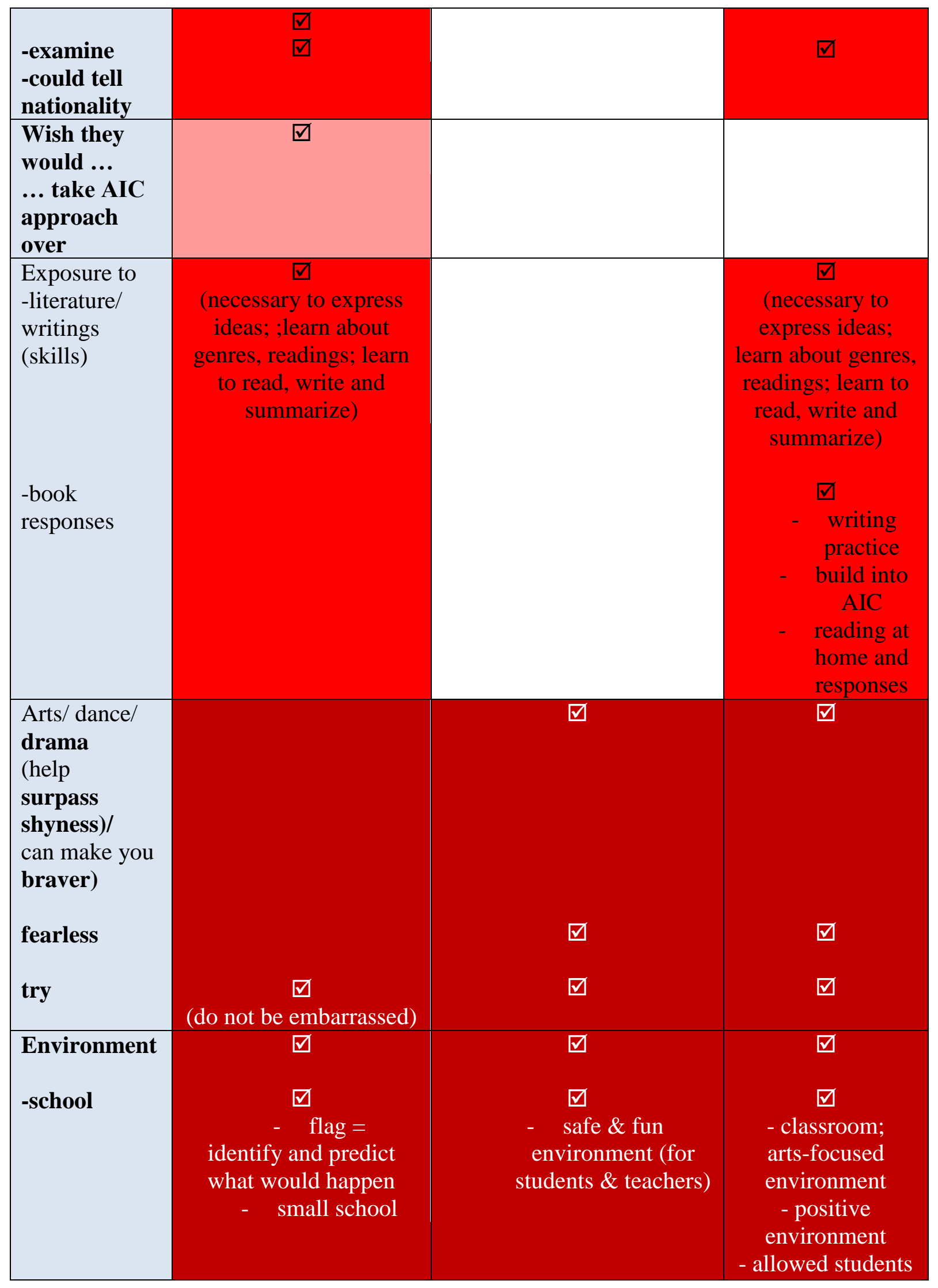




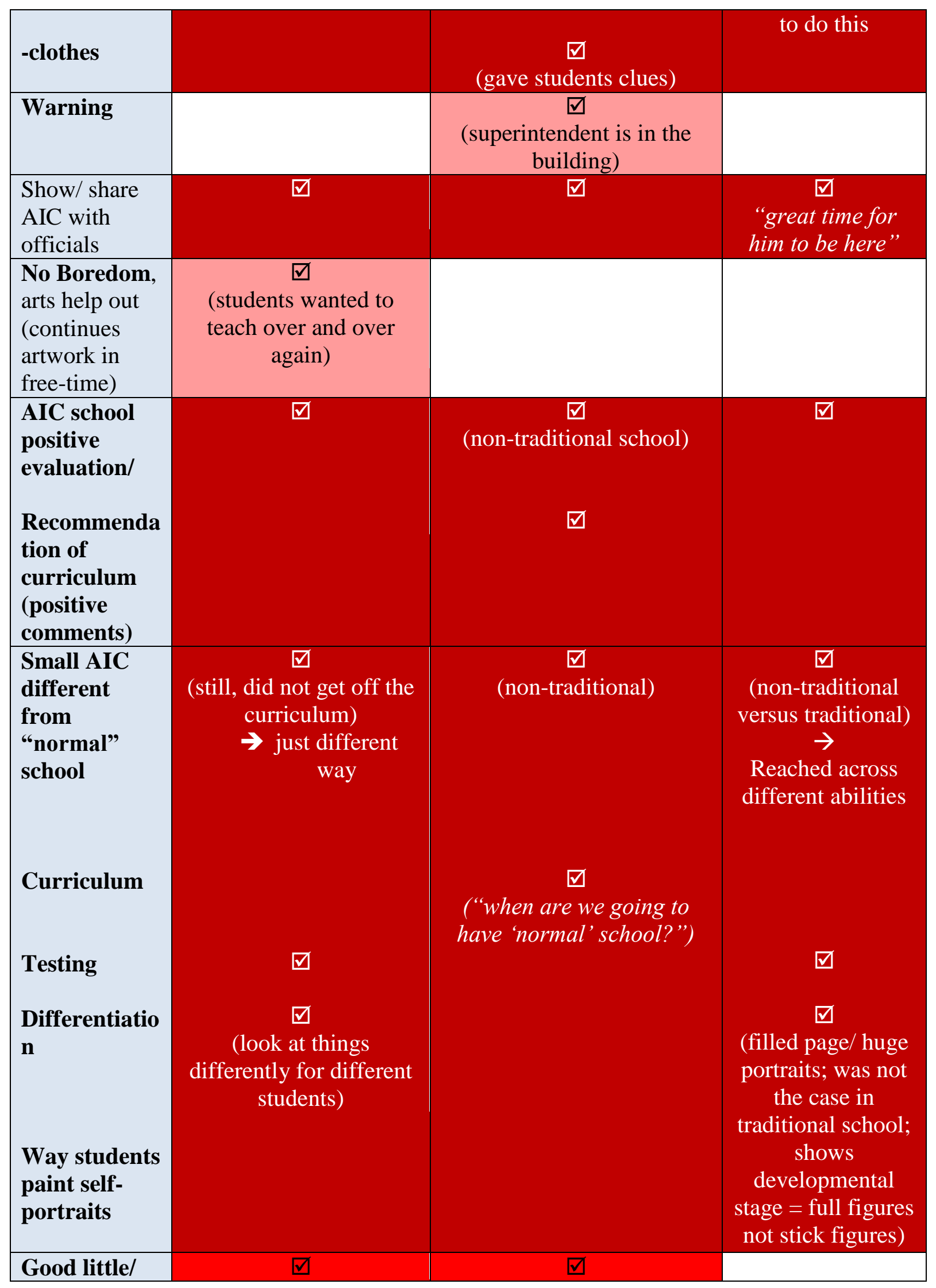




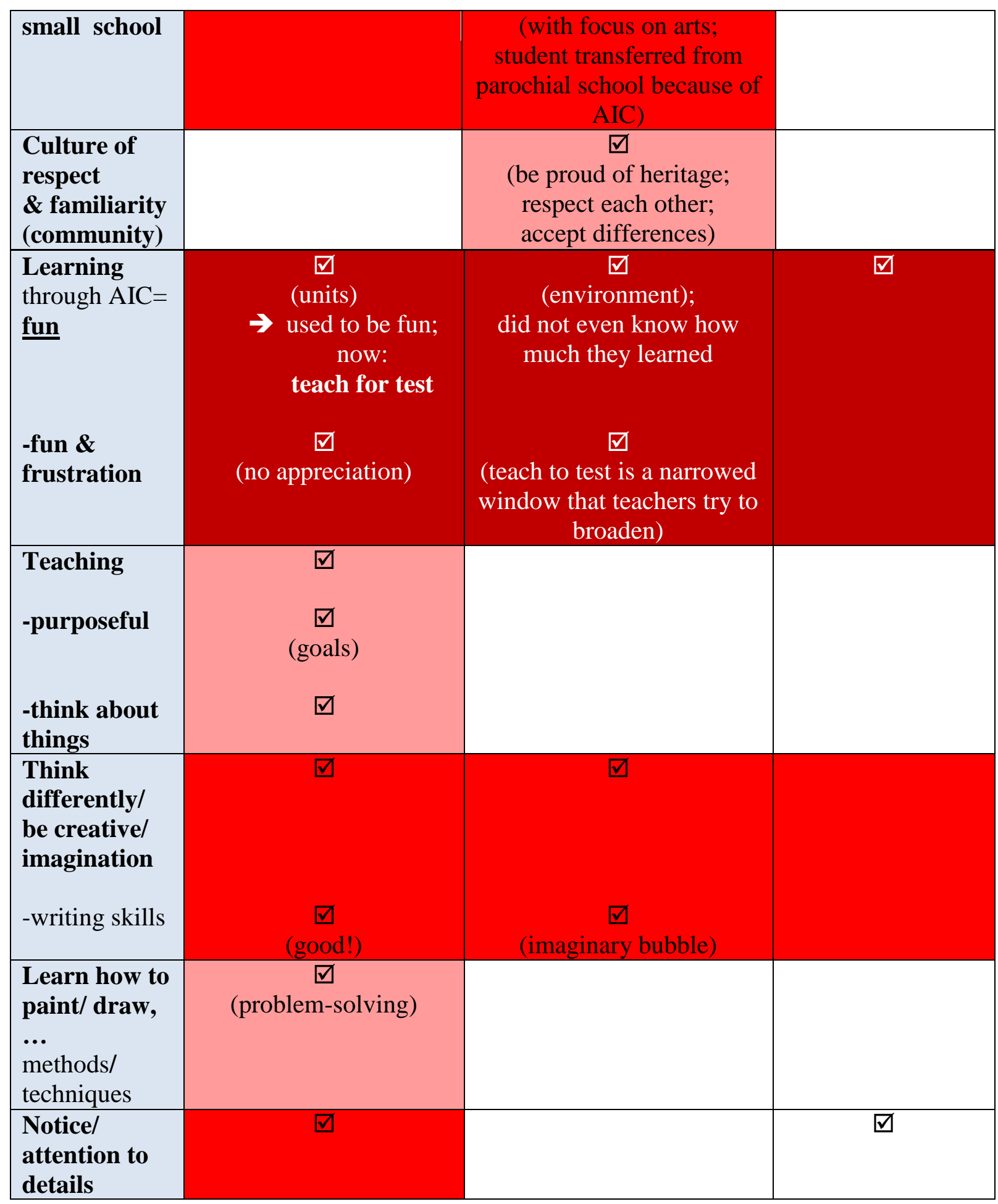

Notes: Color-coding information:

Dark Red:

All two teachers/ university liaison (INVOLVED, MOTIVATED, and Dr. S.) mentioned these units (ICs).

Themes:

1.) Connection to arts 
2.) No gender thing (boys versus girls)

3.) Gifted \& talented/ identified = usually academically

4.) Problem -solving (skills)

5.) No barriers/ tolerance

6.) Interest in art

7.) Results of arts-based learning experiences

8.) Positive descriptions/ characterization of arts/ positive influence of AIC

9.) Positive self-concept through arts

10.) Learn in different way through AIC

11.) Interdisciplinary learning (AIC)

12.) Self-concept

13.) Self-identity (in AIC okay to be different)

14.) Leadership role (taken over by students)

15.) Pro AIC

16.) Parents taught by children

17.) Role-model/ mentor

18.) Beauty (weight, height, ...)

19.) School = influence on image/ self-concept (boys and girls)

20.) Teachers $=$ positive influence

21.) Artwork displayed/ shared

22.) Involvement

23.) Arts/dance/ drama (help surpass shyness)/ can make you braver)

24.) Environment

25.) Show/ share AIC with officials

26.) AIC school positive evaluation

27.) Small AIC different from "normal" school

28.) Learning through AIC = fun

\section{Medium Red:}

Only two of the two teachers/ university liaison mentioned these units (ICs)

\section{Themes:}

1.) "Age thing"

2.) Boys and AIC

3.) Girls and AIC

4.) Creativity

5.) Look at piece of art = question on test

6.) Real life/ social/ soft skills

7.) Independence

8.) Exposure

9.) Arts (broaden) perspectives

10.) Learn about underdog/ outsider (included)

11.) (Positive feedback) about art from students

12.) Family involvement

13.) Friends (interactive/ social)

14.) Clothes/ dress-up

15.) Parent involvement/ integration 
16.) Nobody excluded/ all can participate

17.) Make time for arts

18.) Self-portrait

19.) Exposure

20.) Good little/ small school

21.) Think differently/ be creative/ imagination

22.) Notice/ attention to details

Light Red:

Only one of the two teachers/ university liaison mentioned these units (ICs)

Themes:

1.) Right \& wrong answer

2.) Critical thinking skills

3.) Naming/ label

4.) No discipline problems

5.) Knowledgeable children

6.) Interest (in teaching \& learning through arts)

7.) Artwork continued in free-time (not a project) $=$ Passion for art

8.) Wish they would... take AIC approach over

9.) Warning

19.) No boredom, arts help out (continues artwork in free-time)

20.) Culture of respect \& familiarity (community)

21.) Teaching

22.) Learn how to paint, draw, ... 
Appendix H6 - Summary cluster of themes for each of the three groups (students, parents, and teachers)

\begin{tabular}{|c|c|c|}
\hline $\begin{array}{l}\frac{\text { Students }}{\text { (BAND, PIANO, DRAWING, }} \\
\text { FLUTE) }\end{array}$ & $\frac{\text { Parents }}{\text { (BUSY, CARING, LOVING) }}$ & $\begin{array}{l}\frac{\text { Teachers }}{\text { (INVOLVED, MOTIVATED, }} \\
\text { Dr. S.) }\end{array}$ \\
\hline 1.) Adolescent & $\begin{array}{l}\text { 1.) Connection to arts/ } \\
\text { work }\end{array}$ & 1.) Connection to arts \\
\hline 2.) Interest in music & $\begin{array}{l}\text { 2.) Experience the arts } \\
\text { through daughter }\end{array}$ & $\begin{array}{l}\text { 2.) No gender thing (not } \\
\text { boys versus girls) }\end{array}$ \\
\hline 3.) Took art classes & 3.) Interest in art & $\begin{array}{l}\text { 3.) Gifted \& talented/ } \\
\text { identified = usually } \\
\text { academically }\end{array}$ \\
\hline $\begin{array}{l}\text { 4.) Positive descriptions/ } \\
\text { characterizations of art }\end{array}$ & 4.) Took art classes & 4.) Problem-solving (skills) \\
\hline \begin{tabular}{|l|} 
5.) Positive self-concept \\
through arts
\end{tabular} & $\begin{array}{l}\text { 5.) Positive descriptions/ } \\
\text { characterizations of art }\end{array}$ & 5.) No barriers/ tolerance \\
\hline $\begin{array}{l}\text { 6.) Interdisciplinary } \\
\text { learning }\end{array}$ & 6.) Positive influence of AIC & 6.) Interest in art \\
\hline 7.) Role-model (support) & $\begin{array}{l}\text { 7.) Recognition/ } \\
\text { confirmation/ positive } \\
\text { feedback from art } \\
\text { (intrinsic motivation) }\end{array}$ & $\begin{array}{l}\text { 7.) Results of arts-based } \\
\text { learning experiences }\end{array}$ \\
\hline $\begin{array}{l}\text { 8.) Teacher's positive } \\
\text { influence }\end{array}$ & $\begin{array}{l}\text { 8.) Positive self-concept } \\
\text { through arts }\end{array}$ & $\begin{array}{l}\text { 8.) Positive descriptions/ } \\
\text { characterizations of arts/ } \\
\text { positive influence of AIC } \\
\end{array}$ \\
\hline 9.) Outside (positive) & 9.) Family involvement & $\begin{array}{l}\text { 9.) Positive self-concept } \\
\text { through arts }\end{array}$ \\
\hline $\begin{array}{l}\text { 10.) Learning through } \\
\text { AIC is fun }\end{array}$ & $\begin{array}{l}\text { 10.) Learning/ recall is } \\
\text { supported by AIC }\end{array}$ & $\begin{array}{l}\text { 10.) Learn in different way } \\
\text { through AIC }\end{array}$ \\
\hline 11.) Problem solving & $\begin{array}{l}\text { 11.) Interdisciplinary } \\
\text { Learning (AIC) }\end{array}$ & $\begin{array}{l}\text { 11.) Interdisciplinary } \\
\text { Learning (AIC) }\end{array}$ \\
\hline $\begin{array}{l}\text { 12.) Think differently; } \\
\text { be creative; } \\
\text { imagination }\end{array}$ & $\begin{array}{l}\text { 12.) Friends } \\
\text { (interactive/ social) }\end{array}$ & 12.) Self-concept \\
\hline $\begin{array}{l}\text { 13.) Friends } \\
\text { (interactive/ social) }\end{array}$ & $\begin{array}{l}\text { 13.) Beautiful things } \\
\text { (for daughter) }\end{array}$ & $\begin{array}{l}\text { 13.) Self-identity } \\
\text { (in AIC okay to be } \\
\text { different) }\end{array}$ \\
\hline 14.) Clothes & 14.) Self-concept & $\begin{array}{l}\text { 14.) Leadership role } \\
\text { (taken over by students) }\end{array}$ \\
\hline $\begin{array}{l}\text { 15.) Beautiful things } \\
\text { (nature) }\end{array}$ & $\begin{array}{l}\text { 15.) Parent involvement/ } \\
\text { integration }\end{array}$ & 15.) Pro AIC \\
\hline $\begin{array}{l}\text { 16.) Self-concept } \\
\text { (positive/ negative) }\end{array}$ & 16.) Parents volunteered & $\begin{array}{l}\text { 16.) Parents taught by } \\
\text { children }\end{array}$ \\
\hline 17.) Endurance (in practice) & 17.) Interest & 17.) Role-model/ mentor \\
\hline \begin{tabular}{|l|} 
18.) No boredom \\
(arts help out)
\end{tabular} & $\begin{array}{l}\text { 18.) Artwork displayed/ } \\
\text { shared }\end{array}$ & 18.) Beauty (weight, tall,...) \\
\hline 19.) Family involvement & 19.) Involvement & $\begin{array}{l}\text { 19.) School has influence on } \\
\text { image/ self-concept }\end{array}$ \\
\hline
\end{tabular}




\begin{tabular}{|c|c|c|}
\hline & & (boys and girls!) \\
\hline 20.) Beautiful people & 20.) Show/ share artwork & $\begin{array}{l}\text { 20.) Teachers have } \\
\text { positive influence }\end{array}$ \\
\hline 21.) Involvement & $\begin{array}{l}\text { 21.) AIC school positive } \\
\text { evaluation }\end{array}$ & $\begin{array}{l}\text { 21.) Artwork displayed/ } \\
\text { shared }\end{array}$ \\
\hline $\begin{array}{l}\text { 22.) Notice/ attention to } \\
\text { details }\end{array}$ & $\begin{array}{l}\text { 22.) Exposure to arts supports } \\
\text { interest in arts }\end{array}$ & 22.) Involvement \\
\hline $\begin{array}{l}\text { 23.) Recognition, } \\
\text { Confirmation, } \\
\text { positive feedback from } \\
\text { art } \\
\text { (intrinsic motivation) }\end{array}$ & 23.) Focus on arts & $\begin{array}{l}\text { 23.) Arts, dance, drama } \\
\text { (help surpass shyness/ } \\
\text { can make you braver) }\end{array}$ \\
\hline $\begin{array}{l}\text { 24.) At first insecurity about } \\
\text { arts, now good at it }\end{array}$ & 24.) Looks & 24.) Environment \\
\hline 25.) Okay to be different & $\begin{array}{l}\text { 25.) Results of } \\
\text { arts-based-learning } \\
\text { experiences }\end{array}$ & $\begin{array}{l}\text { 25.) Show/ share } \\
\text { AIC with officials }\end{array}$ \\
\hline 26.) Beautiful people & 26.) Knowledgeable children & $\begin{array}{l}\text { 26.) AIC school positive } \\
\text { evaluation }\end{array}$ \\
\hline 27.) Beauty issues & 27.) Clothes & $\begin{array}{l}\text { 27.) Small AIC different } \\
\text { from "normal school" }\end{array}$ \\
\hline $\begin{array}{l}\text { 28.) Learn how to paint/ } \\
\text { draw } \\
\text { (methods/ techniques) }\end{array}$ & 28.) Self-identity & $\begin{array}{l}\text { 28.) Learning through AIC } \\
\text { is fun }\end{array}$ \\
\hline $\begin{array}{l}\text { 29.) Artwork continued in } \\
\text { free-time }\end{array}$ & $\begin{array}{l}\text { 29.) Nobody excluded/ } \\
\text { all can participate }\end{array}$ & 29.) "Age thing" \\
\hline 30.) Artwork displayed & 30.) Pro AIC & 30.) Boys and AIC \\
\hline $\begin{array}{l}\text { 31.) Computer/ media } \\
\text { used as resources }\end{array}$ & $\begin{array}{l}\text { 31.) Parents taught by } \\
\text { children }\end{array}$ & 31.) Girls and AIC \\
\hline $\begin{array}{l}\text { 32.) Not enough time for } \\
\text { arts (is a process) }\end{array}$ & 32.) Beauty issues & 32.) Creativity \\
\hline $\begin{array}{l}\text { 33.) Feel comfortable to } \\
\text { know what to do next }\end{array}$ & $\begin{array}{l}\text { 33.) Role-model/ mentor } \\
\text { (support) }\end{array}$ & $\begin{array}{l}\text { 33.) Look at piece of art is } \\
\text { question on test }\end{array}$ \\
\hline 34.) Painting unit & $\begin{array}{l}\text { 34.) Healthy eating/ weight } \\
\text { loss }\end{array}$ & $\begin{array}{l}\text { 34.) Real life/ social/ soft } \\
\text { skills }\end{array}$ \\
\hline 35.) Self-portrait & $\begin{array}{l}\text { 35.) School has influence on } \\
\text { image/ self-concept }\end{array}$ & 35.) Independence \\
\hline 36.) Exposure to & $\begin{array}{l}\text { 36.) Teachers (positive) } \\
\text { influence }\end{array}$ & 36.) Exposure \\
\hline $\begin{array}{l}\text { 37.) Arts/ dance help } \\
\text { surpass shyness/ } \\
\text { can make you braver }\end{array}$ & 37.) Endurance (in practice) & $\begin{array}{l}\text { 37.) Arts (broaden) } \\
\text { perspectives }\end{array}$ \\
\hline $\begin{array}{l}\text { 38.) Influence of } \\
\text { environment }\end{array}$ & $\begin{array}{l}\text { 38.) Artwork continued in } \\
\text { Free-time }\end{array}$ & $\begin{array}{l}\text { 38.) Learn about underdog/ } \\
\text { outsider (included) }\end{array}$ \\
\hline 39.) Show/ share artwork & 39.) Not enough time for arts & $\begin{array}{l}\text { 39.) (Positive feedback) about } \\
\text { art from students }\end{array}$ \\
\hline \multirow[t]{2}{*}{ 40.) Seems to "fit" } & $\begin{array}{l}\text { 40.) Familiarity with artist } \\
\text { and art }\end{array}$ & 40.) Family involvement \\
\hline & 41.) Wish they would have & 41.) Friends (interactive/ \\
\hline
\end{tabular}




\begin{tabular}{|c|c|c|}
\hline & more. & social) \\
\hline & 42.) Exposure to.. & 42.) Clothes/ dress-up \\
\hline & $\begin{array}{l}\text { 43.) Arts/ dance/ drama } \\
\text { (help surpass shyness) }\end{array}$ & $\begin{array}{l}\text { 43.) Parent involvement/ } \\
\text { integration }\end{array}$ \\
\hline & 44.) Influence of environment & $\begin{array}{l}\text { 44.) Nobody excluded/ } \\
\text { all can participate }\end{array}$ \\
\hline & 45.) Empathy/ emotions & 45.) Make time for arts \\
\hline & $\begin{array}{l}\text { 46.) Arts in school need more } \\
\text { support }\end{array}$ & 46.) Self-portrait \\
\hline & 47.) Gain more & 47.) Self-portrait \\
\hline & $\begin{array}{l}\text { 48.) Arts (broaden) } \\
\text { perspective }\end{array}$ & 48.) Exposure \\
\hline & $\begin{array}{l}\text { 49.) Learn about underdog/ } \\
\text { outsider (included) }\end{array}$ & 49.) Good little/ small school \\
\hline & 50.) Arts are valued & $\begin{array}{l}\text { 50.) Think differently/ } \\
\text { be creative/ } \\
\text { imagination }\end{array}$ \\
\hline & $\begin{array}{l}\text { 51.) At first child insecure } \\
\text { about arts }\end{array}$ & $\begin{array}{l}\text { 51.) Notice/ attention to } \\
\text { details }\end{array}$ \\
\hline & 52.) Beautiful people & 52.) Right and wrong answer \\
\hline & 53.) Support by school & 53.) Critical thinking skills \\
\hline & 54.) Leadership role & 54.) Naming/ label \\
\hline & $\begin{array}{l}\text { 55.) Parents saw moment of } \\
\text { positive change }\end{array}$ & 55.) No discipline problems \\
\hline & 56.) People on television & 56.) Knowledgeable children \\
\hline & $\begin{array}{l}\text { 57.) Learning to live with } \\
\text { competition }\end{array}$ & $\begin{array}{l}\text { 57.) Interest (in teaching in } \\
\text { and learning through } \\
\text { arts) }\end{array}$ \\
\hline & $\begin{array}{l}\text { 58.) Share experiences with } \\
\text { others }\end{array}$ & $\begin{array}{l}\text { 58.) Artwork continued in } \\
\text { free-time (not a project) } \\
\text { shows passion for art }\end{array}$ \\
\hline & 59.) Appreciation & $\begin{array}{l}\text { 59.) Wish they would... take } \\
\text { AIC approach over }\end{array}$ \\
\hline & 60.) Painting unit & 60.) Warning \\
\hline & 61.) Self-portrait & $\begin{array}{l}\text { 61.) No boredom, arts help } \\
\text { out (continue artwork in } \\
\text { free-time) }\end{array}$ \\
\hline & $\begin{array}{l}\text { 62.) One area of arts } \\
\text { influence the other one } \\
\text { positively }\end{array}$ & $\begin{array}{l}\text { 62.) Culture of respect and } \\
\text { familiarity (community) }\end{array}$ \\
\hline & 63.) No boredom & 63.) Teaching \\
\hline & 64.) Seems to "fit" & $\begin{array}{l}\text { 64.) Learn how to paint/ draw } \\
\text { (techniques) }\end{array}$ \\
\hline & $\begin{array}{l}\text { 65.) Small AIC different from } \\
\text { "normal school" }\end{array}$ & \\
\hline & 66.) Good little school & \\
\hline & $\begin{array}{l}\text { 67.) Culture of respect and } \\
\text { familiarity (community) }\end{array}$ & \\
\hline & $\begin{array}{l}\text { 68.) Artists have different } \\
\text { points of view }\end{array}$ & \\
\hline
\end{tabular}


\begin{tabular}{|c|}
\hline (experience) \\
\hline Note. Bold text: Themes that at least three of four students, two of three parents, and two of two
\end{tabular} teachers/ university liaison considered as important during interview. 
Appendix H7 - Common thematic portrayals of the AIC experience for students, parents, and teachers

\section{Appendix H7.1. Common thematic portrayals of the experience for STUDENTS:}

\section{Everything related to the arts or the AIC:}

2.) Interest in music

3.) Took art classes

6.) Interdisciplinary learning

10.) Learning through AIC $=$ fun

2. Positive effects, characterizations, and experiences of the arts or the AIC:

4.) Positive descriptions/ characterizations of art

9.) Outside (positive)

[10.) Learning through AIC $=$ fun] -1 .

13.) Friends (interactive/ social)

18.) No boredom (arts help out)

36.) Exposure to ...

\section{Skills acquired through the AIC:}

11.) Problem solving

12.) Think differently, be creative, imagination

17.) Endurance (in practice)

22.) Notice/ attention to details

28.) Learn how to paint/ draw (methods/ techniques)

31.) Computer/media used as resources

34.) Painting unit

40.) Seems to "fit"

4. Influences of the teachers, the school, and/ or the environment:

7.) Role-model (support)

8.) Teacher's positive influence

29.) Artwork continued in free-time

38.) Influence of environment

5. Things related to the self-concept:

1.) Adolescent

5.) Positive self-concept through arts

16.) Self-concept (positive/ negative)

23.) Recognition, confirmation, positive feedback from art (intrinsic motivation)

24.) At first insecurity about arts, now good at it

25.) Okay to be different

30.) Artwork displayed

33.) Feel comfortable to know what to do next

35.) Self-portrait

37.) Arts/dance help surpass shyness and/ or can make you braver 
39.) Show/ share artwork

6. Beauty (internal/ external) also in relation to clothes and dress up:

[4.) Positive descriptions/ characterizations of art] - see 2.

14.) Clothes

15.) Beautiful things (nature)

20.) Beautiful people (in general)

26.) Beautiful people (on television)

27.) Beauty issues

7. Various forms of involvement (e.g. family involvement):

19.) Family involvement

21.) Involvement (in general)

\section{Requests and wishes in relation to the arts and the AIC:}

32.) Not enough time for arts (is a process and needs more time)

Note. The numberings, 1.)-40.), relate to the numbers in the clustered themes chart for each of the three groups (students, parents, and teachers), see Appendix H6. 


\section{Appendix H7.2. Common thematic portrayals of the experience for PARENTS:}

\section{Everything related to the arts or the AIC:}

1.) Connection to arts/ work

3.) Interest in art

4.) Took art classes

11.) Interdisciplinary Learning (AIC)

21.) AIC school positive evaluation

23.) Focus on arts

30.) Pro AIC

42.) Exposure to...

59.) Appreciation

65.) Small AIC different from "normal school"

66.) Good little school

2. Positive effects, characterizations, and experiences of the arts or the AIC:

2.) Experience the arts through daughter

5.) Positive descriptions/ characterizations of art

6.) Positive influence of AIC

7.) Recognition/ confirmation/ positive feedback from art (intrinsic motivation)

10.) Learning/ recall is supported by AIC

12.) Friends (interactive/ social)

22.) Exposure to arts supports interest in arts

25.) Results (positive) of arts-based-learning experiences

29.) Nobody excluded/ all can participate

38.) Artwork continued in free-time

45.) Empathy/ emotions

47.) Gain more

48.) Arts (broaden) perspective

55.) Parents saw moment of positive change

63.) No boredom

[65.) Small AIC different from "normal school"] - see also 1.

3. Skills acquired through the AIC:

[25.) Results (positive) of arts-based-learning experiences] - see also 2.

26.) Knowledgeable kids

31.) Parents taught by children

37.) Endurance (in practice)

40.) Familiarity with artist \& art

49.) Learn about underdog/ outsider (included)

54.) Leadership role

57.) Learning to live with competition

60.) Painting unit

4. Influences of the teachers, the school, and/ or the environment:

33.) Role-Model/ Mentor (support) 
35.) School = influence on image/ self-concept

36.) Teachers (positive) influence

44.) Influence of environment

53.) Support by school

62.) One arts area influences the other one positively

64.) Seems to "fit"

67.) Culture of respect \& familiarity (community)

68.) Artists have different points of view (experience)

\section{Things related to the self-concept:}

8.) Positive self-concept through arts

14.) Self-concept

18.) Artwork displayed/ shared

20.) Show/ share artwork

28.) Self-identity

32.) Beauty issues

[35.) School = influence on image/ self-concept] - see 4 .

43.) Arts/ dance/ drama (help surpass shyness)

50.) Arts = valued

51.) At first child insecure about arts, no good at it

54.) Leadership role

58.) Share experiences with others

61.) Self-portrait

6. Beauty (internal/ external), also in relation to clothes and dressing up:

13.) Beautiful things (for daughter)

24.) Looks

27.) Clothes

[32.) Beauty issues] - see 5.

34.) Healthy eating/ weight loss

52.) Beautiful people

56.) People on TV

7. Various forms of involvement (e.g. family involvement):

9.) Family involvement

15.) Parent involvement/ integration

16.) Parents volunteered

17.) Interest

19.) Involvement (in general)

8. Request and wishes in relation to the arts and the AIC:

39.) Not enough time for arts

41.) Wish they would have more...

46.) Arts in school need more support

Note. The numberings, 1.)-68.), relate to the numbers in the clustered themes chart for each of the three groups (students, parents, and teachers), see Appendix H6. 


\section{Appendix H7.3. Common thematic portrayals of the experience for TEACHERS:}

\section{Everything related to the arts or the AIC:}

1.) Connection to arts

2.) AIC is not a gender thing (not boys versus girls)

11.) Interdisciplinary learning (AIC)

15.) Pro AIC

26.) AIC school positive evaluation

27.) Small AIC different from "normal school"

28.) Learning through AIC $=$ fun

30.) Boys and AIC

31.) Girls and AIC

36.) Exposure

45.) Make time for arts

54.) Naming/ label

55.) No discipline problems

2. Positive effects, characterizations, and experiences of the arts or the AIC:

6.) Interest in art

[7.) Results of arts-based learning experiences] - see 1 .

8.) Positive descriptions, characterizations of arts, positive influence of AIC

23.) Arts, dance, drama (help surpass shyness and/ or can make you braver)

[28.) Learning through AIC = fun] -1 .

37.) Arts (broaden) perspectives

38.) Learn about underdog/ outsider (included)

39.) (Positive feedback) about art from students

41.) Friends (interactive/ social)

48.) Exposure

58.) Artwork continued in free-time (not a project) shows passion for art

60.) Warning

3. Skills acquired through the AIC:

4.) Problem-solving (skills)

5.) No barriers/ tolerance

10.) Learn in different way through AIC

[11.) Interdisciplinary learning (AIC)] - see 1 .

14.) Leadership role (taken over by students)

16.) Parents taught by children

32.) Creativity

33.) Look at piece of art is question on test

34.) Real life, social, soft skills

35.) Independence

50.) Think differently, be creative, imagination

51.) Notice/ attention to details

52.) Right and wrong answer

53.) Critical thinking skills 
56.) Knowledgeable kids

61.) No boredom, arts help out (continue artwork in free-time)

62.) Culture of respect \& familiarity (community)

63.) Teaching

64.) Learn how to paint/ draw (techniques)

4. Influences of the teachers, the school, and/ or the environment:

3.) Gifted and talented students identified are usually identified academically

17.) Role-model/ mentor

18.) Beauty (weight, tall,...)

19.) School has influence on image/ self-concept (boys and girls!)

20.) Teachers have positive influence

24.) Environment

49.) Good little/ small school

57.) Interest (in teaching and learning through arts)

5. Things related to the self-concept:

9.) Positive self-concept through arts

12.) Self-concept

13.) Self-identity (in AIC okay to be different)

[14.) Leadership role (taken over by students)] -3 .

21.) Artwork displayed/ shared

[23.) Arts, dance, drama (help surpass shyness/ can make you braver)] -2 .

29.) "Age thing"

46.) Self-portrait (practical; students learn how to paint)

47.) Self-portrait (theoretical)

6. Beauty (internal/ external) also in relation to clothes and dressing up:

[18.) Beauty (weight, tall,...)] - 4.

[29.) "Age thing"] -5.

42.) Clothes/ dress-up

7. Various forms of involvement (e.g. family involvement):

22.) Involvement

40.) Family involvement

43.) Parent involvement/ integration

44.) Nobody excluded/ all can participate

\section{Request and wishes in relation to the arts and the AIC:}

59.) Wish they would... take AIC approach over

Note. The numberings, 1.)-64.), relate to the numbers in the clustered themes chart for each of the three groups (students, parents, and teachers), see Appendix H6. 
Appendix H8 - Validation chart

\begin{tabular}{|c|c|c|}
\hline $\begin{array}{l}\text { Students } \\
\text { (BAND, PIANO, DRAWING, } \\
\text { FLUTE) }\end{array}$ & $\frac{\text { Parents }}{\text { (BUSY, CARING, LOVING) }}$ & $\begin{array}{l}\text { Teachers } \\
\text { (INVOLVED, MOTIVATED, } \\
\text { Dr. S.) }\end{array}$ \\
\hline 1.) Adolescent & $\begin{array}{l}\text { 1.) Connection to arts } \\
\text { including work }\end{array}$ & 1.) Connection to arts \\
\hline 2.) Interest in music & $\begin{array}{l}\text { 2.) Experience the arts } \\
\text { through daughter }\end{array}$ & $\begin{array}{l}\text { 2.) No gender thing } \\
\text { (not boys versus girls) }\end{array}$ \\
\hline 3.) Took art classes & 3.) Interest in art & $\begin{array}{l}\text { 3.) Gifted and talented } \\
\text { students } \\
\text { are usually identified } \\
\text { academically }\end{array}$ \\
\hline $\begin{array}{l}\text { 4.) Positive descriptions/ } \\
\text { characterizations of art }\end{array}$ & 4.) Took art classes & 4.) Problem-solving (skills) \\
\hline $\begin{array}{l}\text { 5.) Positive self-concept } \\
\text { through arts }\end{array}$ & $\begin{array}{l}\text { 5.) Positive descriptions/ } \\
\text { characterizations of art }\end{array}$ & 5.) No barriers/ tolerance \\
\hline $\begin{array}{l}\text { 6.) Interdisciplinary } \\
\text { Learning } \\
\end{array}$ & 6.) Positive influence of AIC & 6.) Interest in art \\
\hline 7.) Role-model (support) & $\begin{array}{l}\text { 7.) Recognition, } \\
\text { confirmation, } \\
\text { positive feedback from } \\
\text { art (intrinsic motivation) }\end{array}$ & $\begin{array}{l}\text { 7.) Results of arts-based } \\
\text { learning experiences }\end{array}$ \\
\hline $\begin{array}{l}\text { 8.) Teacher's positive } \\
\text { influence }\end{array}$ & $\begin{array}{l}\text { 8.) Positive self-concept } \\
\text { through arts }\end{array}$ & $\begin{array}{l}\text { 8.) Positive descriptions, } \\
\text { characterizations of } \\
\text { arts, positive influence } \\
\text { of AIC }\end{array}$ \\
\hline 9.) Outside (positive) & 9.) Family involvement & $\begin{array}{l}\begin{array}{l}\text { 9.) Positive self-concept } \\
\text { through arts }\end{array} \\
\end{array}$ \\
\hline $\begin{array}{l}\text { 10.) Learning through AIC } \\
\text { is fun }\end{array}$ & $\begin{array}{l}\text { 10.) Learning/ recall is } \\
\text { supported by AIC }\end{array}$ & $\begin{array}{l}\text { 10.) Learn in different way } \\
\text { through AIC } \\
\end{array}$ \\
\hline 11.) Problem Solving & $\begin{array}{l}\text { 11.) Interdisciplinary } \\
\text { Learning (AIC) }\end{array}$ & $\begin{array}{l}\text { 11.) Interdisciplinary } \\
\text { Learning (AIC) } \\
\end{array}$ \\
\hline $\begin{array}{l}\text { 12.) Think differently, be } \\
\text { creative, imagination }\end{array}$ & $\begin{array}{l}\text { 12.) Friends (interactive/ } \\
\text { social) }\end{array}$ & 12.) Self-concept \\
\hline $\begin{array}{l}\text { 13.) Friends } \\
\text { (interactive/ social) }\end{array}$ & $\begin{array}{l}\text { 13.) Beautiful things } \\
\text { (for daughter) }\end{array}$ & $\begin{array}{l}\text { 13.) Self-identity } \\
\text { (in AIC okay to be } \\
\text { different) }\end{array}$ \\
\hline 14.) Clothes & 14.) Self-concept & $\begin{array}{l}\text { 14.) Leadership role } \\
\text { (taken over by } \\
\text { students) }\end{array}$ \\
\hline $\begin{array}{l}\text { 15.) Beautiful things } \\
\text { (nature) }\end{array}$ & $\begin{array}{l}\text { 15.) Parent involvement/ } \\
\text { integration }\end{array}$ & 15.) Pro AIC \\
\hline $\begin{array}{l}\text { 16.) Self-concept } \\
\text { (positive/ negative) }\end{array}$ & 16.) Parents volunteered & $\begin{array}{l}\text { 16.) Parents taught by } \\
\text { children }\end{array}$ \\
\hline $\begin{array}{l}\text { 17.) Endurance (in } \\
\text { practice) }\end{array}$ & 17.) Interest & 17.) Role-model/ mentor \\
\hline 18.) No boredom & 18.) Artwork displayed/ & 18.) Beauty (weight, tall,...) \\
\hline
\end{tabular}




\begin{tabular}{|c|c|c|}
\hline (arts help out) & shared & \\
\hline & 19.) Involvement & $\begin{array}{l}\text { 19.) School has influence on } \\
\text { image/ self-concept } \\
\text { (boys and girls!) }\end{array}$ \\
\hline & 20.) Show/ share artwork & $\begin{array}{l}\text { 20.) Teachers have } \\
\text { positive influence }\end{array}$ \\
\hline & $\begin{array}{l}\text { 21.) AIC school positive } \\
\text { evaluation }\end{array}$ & $\begin{array}{l}\text { 21.) Artwork displayed/ } \\
\text { shared }\end{array}$ \\
\hline & & 22.) Involvement \\
\hline & & $\begin{array}{l}\text { 23.) Arts, dance, drama } \\
\text { (help surpass shyness } \\
\text { and/ or can make you } \\
\text { braver) }\end{array}$ \\
\hline & & 24.) Environment \\
\hline & & $\begin{array}{l}\text { 25.) Show/ share AIC with } \\
\text { officials }\end{array}$ \\
\hline & & $\begin{array}{l}\text { 26.) AIC school positive } \\
\text { evaluation }\end{array}$ \\
\hline & & $\begin{array}{l}\text { 27.) Small AIC different } \\
\text { from "normal school" }\end{array}$ \\
\hline & & $\begin{array}{l}\text { 28.) Learning through AIC } \\
\text { is fun }\end{array}$ \\
\hline
\end{tabular}

Note. This chart shows the final identification of the invariant constituents (ICs) and themes (validation) which result into the validated ICs and themes for the students', parents', and teachers' groups. 
Appendix H9 - New common thematic portrayals of the experience for STUDENTS, PARENTS, and TEACHERS after validation

Appendix H9.1. New Common thematic portrayals of the experience for STUDENTS after validation:

1. Everything related to the arts or the AIC:

2.) Interest in music

3.) Took art classes

6.) Interdisciplinary learning

10.) Learning through AIC is fun

2. Positive effects, characterizations, and experiences of the arts or the AIC:

4.) Positive descriptions/ characterizations of art

9.) Outside (positive)

13.) Friends (interactive/ social)

18.) No boredom (arts help out)

3. Skills acquired through the AIC:

11.) Problem solving

12.) Think differently, be creative, imagination

17.) Endurance (in practice)

4. Influences of the teachers, the school, and/ or the environment:

1.) Adolescent

7.) Role-model (support)

8.) Teacher's positive influence

5. Things related to the self-concept:

5.) Positive self-concept through arts

16.) Self-concept (positive/ negative)

6. Beauty (internal/ external) also in relation to clothes and dressing up:

14.) Clothes

15.) Beautiful things (nature)

Note. The numberings, 1.)-18.), relate to the numbers in the final identification of the invariant constituents (ICs) and themes (validation) - validated ICs and themes for the students', parents', and teachers' group chart, (see Appendix H8).

The listed themes are themes that at least three of four students, two of three parents, and two of two teachers/ university liaison considered as important during the interview. 

validation:

\section{Everything related to the arts or the AIC:}

1.) Connection to arts and work

3.) Interest in art

4.) Took art classes

11.) Interdisciplinary learning (AIC)

21.) AIC school positive evaluation

2. Positive effects, characterizations, and experiences of the arts or the AIC:

2.) Experience the arts through daughter

5.) Positive descriptions/ characterizations of art

6.) Positive influence of AIC

7.) Recognition, confirmation, positive feedback from art (intrinsic motivation)

10.) Learning/ recall is supported by AIC

12.) Friends (interactive/ social)

3. Things related to the self-concept:

8.) Positive self-concept through arts

14.) Self-concept

18.) Artwork displayed/ shared

20.) Show/ share artwork

4. Beauty (internal/ external) also in relation to clothes and dressing up:

13.) Beautiful things (for daughter)

5. Various forms of involvement (e.g. family involvement):

9.) Family involvement

15.) Parent involvement/ integration

16.) Parents volunteered

17.) Interest

19.) Involvement (in general)

Note. The numberings, 1.)-21.), relate to the numbers in the final identification of the invariant constituents (ICs) and themes (validation)- validated ICs and themes for the students', parents', and teachers' group chart, (see Appendix H8). 
Appendix H9.3. New common thematic portrayals of the experience for TEACHERS after validation:

\section{Everything related to the arts or the AIC:}

1.) Connection to arts

2.) AIC is not a gender thing (it is not boys versus girls)

11.) Interdisciplinary learning (AIC)

15.) Pro AIC

26.) AIC school positive evaluation

27.) Small AIC schools are different from "normal schools"

28.) Learning through AIC is fun

2. Positive effects, characterizations, and experiences of the arts or the AIC:

6.) Interest in art

8.) Positive descriptions, characterizations of arts, positive influence of AIC

23.) Arts, dance, drama (help surpass shyness and/ or can make you braver)

3. Skills acquired through the AIC:

4.) Problem solving (skills)

5.) No barriers/ tolerance

10.) Learn in different way through AIC

14.) Leadership role (taken over by students)

16.) Parents taught by children

4. Influences of the teachers, the school, and/ or the environment:

3.) Gifted and talented students are usually academically

17.) Role-model/ mentor

18.) Beauty (weight, tall,...)

19.) School has influence on image/ self-concept (boys and girls!)

20.) Teachers have positive influence

24.) Environment

5. Things related to the self-concept:

9.) Positive self-concept through arts

12.) Self-concept

13.) Self-identity (in AIC okay to be different)

21.) Artwork displayed/ shared

6. Beauty (internal/ external), also in relation to clothes and dressing up:

[18.) Beauty (weight, tall,...)] -4 .

7. Various forms of involvement (e.g. family involvement):

22.) Involvement

Note. The numberings, 1.)-28.), relate to the numbers in the final identification of the invariant constituents (ICs) and themes (validation)- validated ICs and themes for the students', parents', and teachers' group chart, see (Appendix H8). 
Appendix H10 - Summary chart for phenomena of all co-research groups and participants

\begin{tabular}{|c|c|c|}
\hline $\begin{array}{l}\text { Students } \\
\text { (BAND, PIANO, DRAWING, } \\
\text { FLUTE) }\end{array}$ & $\frac{\text { Parents }}{\text { (BUSY, CARING, LOVING) }}$ & $\begin{array}{l}\text { Teachers } \\
\text { (INVOLVED, MOTIVATED, } \\
\text { Dr. S.) }\end{array}$ \\
\hline 1.) Adolescent & $\begin{array}{l}\text { 1.) Connection to arts and } \\
\text { work }\end{array}$ & 1.) Connection to arts \\
\hline 2.) Interest in music & $\begin{array}{l}\text { 2.) Experience the arts } \\
\text { through daughter }\end{array}$ & $\begin{array}{l}\text { 2.) No gender thing } \\
\text { (not boys versus girls) }\end{array}$ \\
\hline 3.) Took art classes & 3.) Interest in art & $\begin{array}{l}\text { 3.) Gifted and talented } \\
\text { students are usually } \\
\text { identified academically }\end{array}$ \\
\hline $\begin{array}{l}\text { 4.) Positive descriptions/ } \\
\text { characterizations of art }\end{array}$ & 4.) Took art classes & 4.) Problem-solving (skills) \\
\hline $\begin{array}{l}\text { 5.) Positive self-concept } \\
\text { through arts }\end{array}$ & $\begin{array}{l}\text { 5.) Positive descriptions/ } \\
\text { characterizations of art }\end{array}$ & 5.) No barriers/ tolerance \\
\hline $\begin{array}{l}\text { 6.) Interdisciplinary } \\
\text { Learning }\end{array}$ & 6.) Positive influence of AIC & 6.) Interest in art \\
\hline 7.) Role-model (support) & $\begin{array}{l}\text { 7.) Recognition, } \\
\text { confirmation, positive } \\
\text { feedback from art } \\
\text { (intrinsic motivation) }\end{array}$ & $\begin{array}{l}\text { 7.) Results of arts-based } \\
\text { learning experiences }\end{array}$ \\
\hline $\begin{array}{l}\text { 8.) Teacher's positive } \\
\text { influence }\end{array}$ & $\begin{array}{l}\text { 8.) Positive self-concept } \\
\text { through arts }\end{array}$ & $\begin{array}{l}\text { 8.) Positive descriptions, } \\
\text { characterizations of } \\
\text { arts, positive influence } \\
\text { of AIC }\end{array}$ \\
\hline 9.) Outside (positive) & 9.) Family involvement & $\begin{array}{l}\text { 9.) Positive self-concept } \\
\text { through arts }\end{array}$ \\
\hline $\begin{array}{l}\text { 10.) Learning through AIC } \\
\text { is fun }\end{array}$ & $\begin{array}{l}\text { 10.) Learning/ recall } \\
\text { is supported by AIC }\end{array}$ & $\begin{array}{l}\text { 10.) Learn in different way } \\
\text { through AIC }\end{array}$ \\
\hline 11.) Problem Solving & $\begin{array}{l}\text { 11.) Interdisciplinary } \\
\text { Learning (AIC) }\end{array}$ & $\begin{array}{l}\text { 11.) Interdisciplinary } \\
\text { Learning (AIC) }\end{array}$ \\
\hline $\begin{array}{l}\text { 12.) Think differently, be } \\
\text { creative, imagination }\end{array}$ & $\begin{array}{l}\text { 12.) Friends } \\
\text { (interactive/ social) }\end{array}$ & 12.) Self-concept \\
\hline $\begin{array}{l}\text { 13.) Friends } \\
\text { (interactive/ social) }\end{array}$ & $\begin{array}{l}\text { 13.) Beautiful things } \\
\text { (for daughter) }\end{array}$ & $\begin{array}{l}\text { 13.) Self-identity } \\
\text { (in AIC okay to be } \\
\text { different) }\end{array}$ \\
\hline $\begin{array}{l}\text { 14.) } \frac{\text { Clothes }}{\text { (was mentioned two }} \\
\text { times in themes that did } \\
\text { not make the list) }\end{array}$ & 14.) Self-concept & $\begin{array}{l}\text { 14.) Leadership role } \\
\text { (taken over by } \\
\text { students) }\end{array}$ \\
\hline $\begin{array}{l}\text { 15.) Beautiful things } \\
\text { (nature) }\end{array}$ & $\begin{array}{l}\text { 15.) Parent involvement/ } \\
\text { integration }\end{array}$ & 15.) Pro AIC \\
\hline $\begin{array}{l}\text { 16.) Self-concept } \\
\text { (positive/ negative) }\end{array}$ & 16.) Parents volunteered & $\begin{array}{l}\text { 16.) Parents taught by } \\
\text { children }\end{array}$ \\
\hline $\begin{array}{l}\text { 17.) Endurance (in } \\
\text { practice) } \\
\text { (was mentioned two } \\
\text { times - in themes that did }\end{array}$ & 17.) Interest & 17.) Role-model/ mentor \\
\hline
\end{tabular}




\begin{tabular}{|c|c|c|}
\hline not make the list) & & \\
\hline \multirow[t]{11}{*}{$\begin{array}{l}\text { 18.) No boredom } \\
\text { (arts help out) }\end{array}$} & $\begin{array}{l}\text { 18.) Artwork displayed/ } \\
\text { shared }\end{array}$ & 18.) Beauty (weight, tall,...) \\
\hline & 19.) Involvement & $\begin{array}{l}\text { 19.) School has influence on } \\
\text { Image and self-concept } \\
\text { (boys and girls!) }\end{array}$ \\
\hline & 20.) Show/ share artwork & $\begin{array}{l}\text { 20.) Teachers have positive } \\
\text { influence }\end{array}$ \\
\hline & $\begin{array}{l}\text { 21.) AIC school positive } \\
\text { evaluation }\end{array}$ & $\begin{array}{l}\text { 21.) Artwork displayed/ } \\
\text { shared }\end{array}$ \\
\hline & & 22.) Involvement \\
\hline & & $\begin{array}{l}\text { 23.) Arts, dance, drama } \\
\text { (help surpass shyness/ } \\
\text { can make you braver) }\end{array}$ \\
\hline & & 24.) Environment \\
\hline & & $\begin{array}{l}\text { 25.) Show/ share AIC with } \\
\text { officials }\end{array}$ \\
\hline & & $\begin{array}{l}\text { 26.) AIC school positive } \\
\text { evaluation }\end{array}$ \\
\hline & & $\begin{array}{l}\text { 27.) Small AIC different } \\
\text { from "normal school" }\end{array}$ \\
\hline & & $\begin{array}{l}\text { 28.) Learning through AIC } \\
\text { is fun }\end{array}$ \\
\hline
\end{tabular}

Notes: The same colors mark the same phenomena. 
Appendix H11 - Summary list of chart (Appendix H10) for phenomena of all co-research groups and participants

Appendix H11.1. Phenomena that are representing the groups as a WHOLE (all three groups considered these themes as important):
1.) Interest in music/ art
2.) Positive descriptions/ characterizations of art
3.) Positive self-concept through arts
4.) Interdisciplinary learning
5.) Beautiful things (nature, weight, size)
6.) Self-concept (positive/ negative) 
Appendix H11.2. Phenomena that are representing only two of the groups, instead of all three:
1.) Took art classes
2.) Role-model (support)
3.) Teacher's positive influence
4.) Learning through AIC is fun
5.) Problem solving
6.) Friends (interactive/ social)
7.) Learning/ recall is supported by AIC
8.) (Family) involvement
9.) (Parent) involvement, integration, volunteered
10.) Artwork displayed/shared
11.) AIC school positive evaluation
12.) Connection to arts and work 


\section{Appendix H11.3. Phenomena that are representing only one group (will not be considered for this dissertation).}

1.) Adolescent

2.) Outside (positive)

3.) Think differently, be creative, imagination

4.) Clothes (mentioned two times in themes that did not make the list)

5.) Endurance (in practice); (mentioned two times in themes that did not make the list)

6.) No boredom (arts help out)

7.) Experience the arts through daughter

8.) Recognition, confirmation, positive feedback from art (intrinsic motivation)

9.) Interest

10.) No gender thing (not boys versus girls)

11.) Gifted and talented students are usually identified academically

12.) No barriers/ tolerance

13.) Self-identity (in AIC okay to be different)

14.) Leadership role (taken over by students)

15.) Pro AIC

16.) Parents taught by children

17.) School has influence on image/ self-concept (boys/ girls)

18.) Arts, dance, drama (help surpass shyness/ make you braver

19.) Environment

20.) Show/ share AIC with officials

21.) Small AIC different from "normal school" 


\section{Appendix I -}

Devono's (2009) and Douglas' (2011) accord with Findings for Teachers in Comparison:

\begin{tabular}{|c|c|c|}
\hline Authors: & (Devono, 2009) & (Douglas, 2011) \\
\hline Title of works: & $\begin{array}{l}\text { Teacher decision making and } \\
\text { the implementation of } \\
\text { an integrated arts curriculum }\end{array}$ & $\begin{array}{c}\text { The Arts-Integrated } \\
\text { Curriculum (AIC) and its } \\
\text { possible impact on } \\
\text { the self-concepts of adolescent } \\
\text { girls and their perceptions of } \\
\text { beauty }\end{array}$ \\
\hline \multicolumn{3}{|l|}{ Findings: } \\
\hline $\begin{array}{c}\text { Research narrative } \\
\text { perspective }\end{array}$ & $\begin{array}{l}\text { Research narrative is presented } \\
\text { from teacher's unique } \\
\text { perspective }\end{array}$ & $\begin{array}{l}\text { Research narrative includes } \\
\text { perspective of all the ones that are } \\
\text { involved } \\
\text { (students, parents, teachers) }\end{array}$ \\
\hline Content standards & 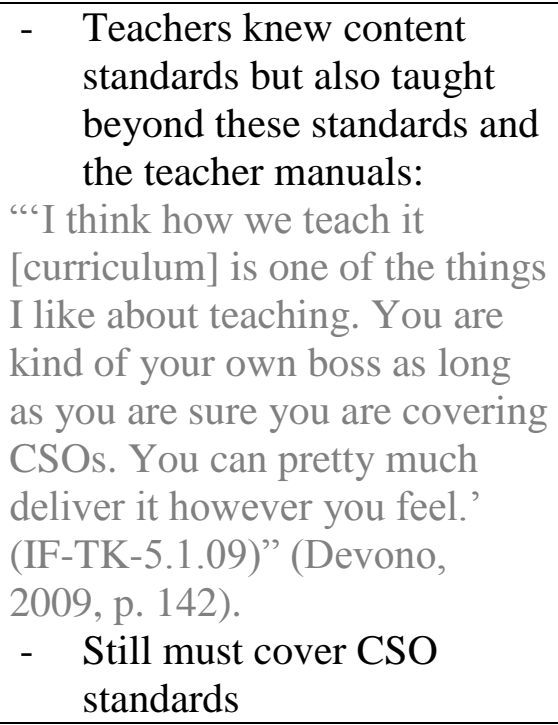 & $\begin{array}{l}\text { INVOLVED: } \\
\text { "So they really didn't (...) } \\
\text { interfered too much or came down } \\
\text { on us and said, 'You are going off } \\
\text { the curriculum here,' because we } \\
\text { really weren't. We were just } \\
\text { doing it in a different way, but our } \\
\text { teaching was always very } \\
\text { purposeful. We knew what our } \\
\text { goals were and it was-one of the } \\
\text { big goals was getting kids to think } \\
\text { about things." }\end{array}$ \\
\hline Accountability & $\begin{array}{l}\text { The arts integration approach } \\
\text { had to have an accountability } \\
\text { element to not be considered as } \\
\text { disconnected and frivolous } \\
\text { activities: } \\
\text { "'We cannot just do it because } \\
\text { we like it. How are we going to } \\
\text { be accountable for that?" (II-TJ- } \\
\text { 4.20.09)" (p. 187). }\end{array}$ & $\begin{array}{l}\text { INVOLVED: } \\
\text { "You read this book and tell me } \\
\text { whether it deserves to be in this } \\
\text { library. It had to meet criteria. } \\
\text { (...)." }\end{array}$ \\
\hline $\begin{array}{c}\text { Students } \\
\text { accomplishments }\end{array}$ & $\begin{array}{l}\text { Teacher(s) show love and } \\
\text { appreciation for students } \\
\text { accomplishments }\end{array}$ & $\begin{array}{l}\text { MOTIVATED: } \\
\text { "And that is what I remember, she } \\
\text { was holding her imaginary bubble. } \\
\text { She was one that I think really did } \\
\text { blossom in that way because she } \\
\text { could offer so much." }\end{array}$ \\
\hline University & - $\quad$ Participation in university & Dr. S.: \\
\hline
\end{tabular}




\begin{tabular}{|c|c|c|}
\hline collaborative & $\begin{array}{l}\text { collaborative was indicative } \\
\text { for desire to immerse class } \\
\text { in arts integration } \\
\text { "I love my job! I am very } \\
\text { satisfied with teaching. I'd like } \\
\text { to see the arts integrated into } \\
\text { all classrooms from pre- } \\
\text { kindergarten to grade twelve } \\
\text { (...).' (II-TA-4.23.09)" (p. } \\
\text { 242). } \\
\text { - Affiliation \& connection }\end{array}$ & $\begin{array}{l}\text { "And I would bring my students } \\
\text { to their classroom to observe so } \\
\text { that the children knew that it was } \\
\text { important what they were doing } \\
\text { because our university students } \\
\text { who wanted to be teachers would } \\
\text { come and study in that } \\
\text { classroom." }\end{array}$ \\
\hline Student's needs & $\begin{array}{l}\text { Individual knowledge of each } \\
\text { student's needs and motivation: } \\
\text { "'We try to teach to each } \\
\text { child's individual needs. It } \\
\text { depends on the students. Also it } \\
\text { is on their interest.' (II-TK- } \\
4.29 .09 \text { )" (p. 260). }\end{array}$ & $\begin{array}{l}\text { Dr. S.: } \\
\text { "Both of you described these were } \\
\text { girls that were struggling in some } \\
\text { way when they came to you, but } \\
\text { what you saw they blossomed into } \\
\text { a leadership position given the } \\
\text { opportunities that you were } \\
\text { providing." }\end{array}$ \\
\hline $\begin{array}{c}\text { Instructional } \\
\text { offerings }\end{array}$ & $\begin{array}{l}\text { Confidence and believe in } \\
\text { charge to control the } \\
\text { instructional offerings: } \\
\text { "I feel like I have quite a bit of } \\
\text { control over my lesson } \\
\text { presentation... I do follow the } \\
\text { content standards, and I use the } \\
\text { phonemic awareness book. I use } \\
\text { what I've been instructed to } \\
\text { use, but I'm able to extend that, } \\
\text { and I think my lesson } \\
\text { presentation is the whole thing." } \\
\text { (II-TA-4.23.09)" (p. 71). }\end{array}$ & $\begin{array}{l}\text { INVOLVED: } \\
\text { "(...) we were allowed to do what } \\
\text { we did because I think we } \\
\text { consistently had high test scores. } \\
\text { So they really didn't-you know, no } \\
\text { one interfered too much or came } \\
\text { down on us and said, 'You are } \\
\text { going off the curriculum here,' } \\
\text { because we really weren't." }\end{array}$ \\
\hline Modeling & Modeling by thinking-out-loud & $\begin{array}{l}\text { INVOLVED: } \\
\text { "(...) modeling is so important. } \\
(\ldots) \text {. So we would have a mirror } \\
\text { and I would look at the mirror and } \\
\text { I would talk out loud. I said, 'I } \\
\text { am thinking out loud, so bear with } \\
\text { me.' I would talk it through. Then } \\
\text { they would know what to look for. } \\
(\text { (...)." }\end{array}$ \\
\hline $\begin{array}{l}\text { Arts integration } \\
\text { instruction }\end{array}$ & $\begin{array}{l}\text { Arts integration instruction has } \\
\text { various processes that lead to a } \\
\text { final product. Look-paint-write: } \\
\text { "Our book is divided up into } \\
\text { themes and it happened to be }\end{array}$ & $\begin{array}{l}\text { INVOLVED: } \\
\text { "Look in the mirror for } 15 \\
\text { minutes. At first you see yourself } \\
\text { and then you begin to see all of } \\
\text { the different things, uniqueness }\end{array}$ \\
\hline
\end{tabular}




\begin{tabular}{|c|c|c|}
\hline & $\begin{array}{l}\text { environment, so I just try and } \\
\text { say, what can I do with the } \\
\text { environment? It's tough. It's } \\
\text { tough to decide. We did put the } \\
\text { pond in, so we thought, well ... } \\
\text { writing. We gave them the day } \\
\text { to look at the pond and all that } \\
\text { was in it ... The next day we } \\
\text { water colored. Then we kept the } \\
\text { paintings and said, OK let's } \\
\text { write about that.' (II-TA- } \\
\text { 4.23.09)"' (p. 243). }\end{array}$ & $\begin{array}{l}\text { about me that is different than you } \\
\text { and you start looking at your nose } \\
\text { and you eye lashes and eyebrows } \\
\text { and lips and they start really } \\
\text { examining it. So then when you } \\
\text { draw, "I didn't know my nose was } \\
\text { that big."' }\end{array}$ \\
\hline Struggling learners & $\begin{array}{l}\text { Teachers draw upon their own } \\
\text { bag of tricks to meet struggling } \\
\text { learners' needs: } \\
\text { "“(...) I like to buddy them up } \\
\text { with someone ... I've asked } \\
\text { them to pantomime to each } \\
\text { other. They take turns being the } \\
\text { leader. Get those kids in here } \\
\text { and let them be introduced to all } \\
\text { this learning.' (IF-TA-5.12.09)" } \\
\text { (p. 244). }\end{array}$ & $\begin{array}{l}\text { MOTIVATED: } \\
\text { "That is the part we tricked them a } \\
\text { little bit, how do you know if you } \\
\text { really want this book unless you } \\
\text { read it?" }\end{array}$ \\
\hline Involvement & $\begin{array}{l}\text { Work with students seen as } \\
\text { conduit to parent and } \\
\text { community involvement }\end{array}$ & $\begin{array}{l}\text { INVOLVED: } \\
\text { "If they wanted to talk to the art } \\
\text { decorator, there were two guys } \\
\text { that came in, two dads." }\end{array}$ \\
\hline Show artwork & $\begin{array}{l}\text { Students are prepared to show } \\
\text { their work: } \\
\text { "(...) take a walk in my room } \\
\text { and let me tell you why we did } \\
\text { this. Actually, let me ask the } \\
\text { kids to tell you why we did this" } \\
\text { (II-TA-4.23.09)" (p. 244). }\end{array}$ & $\begin{array}{l}\text { Dr. S.: } \\
\text { "They had their portfolios and } \\
\text { they would take to the hallway } \\
\text { displays where the art was } \\
\text { celebrated and labeled like an art } \\
\text { gallery with their name and their } \\
\text { title. They would walk them out } \\
\text { there, 'Here is my example of my } \\
\text { self-portrait. Here is my example } \\
\text { of extending the landscaping,' and } \\
\text { here is my example of (....)." }\end{array}$ \\
\hline Confidence & $\begin{array}{l}\text { Confidence \& culture of } \\
\text { teacher confidence: } \\
\text { " (...) confidence is what } \\
\text { Wiles, Bondi (2000) suggest } \\
\text { when they propose that } \\
\text { establishing a culture of teacher } \\
\text { confidence and innovation in } \\
\text { the classroom con improve }\end{array}$ & $\begin{array}{l}\text { Dr. S.: } \\
\text { "She was in charge of the money, } \\
\text { so then you are talking about the } \\
\text { self-concept, confidence and every } \\
\text { contribution is valuable." }\end{array}$ \\
\hline
\end{tabular}




\begin{tabular}{|c|c|c|}
\hline & teaching"" (p. 244). & \\
\hline $\begin{array}{c}21^{\text {st }} \text { Century } \\
\text { Learning/ Skills }\end{array}$ & $\begin{array}{l}21^{\text {st }} \text { Century Learning skills: } \\
-\quad \text { Meet needs } \\
-\quad \text { Problem-solving } \\
-\quad \text { Teamwork } \\
-\quad \text { Change } \\
\text { "'I think the realization of this } \\
21^{\text {st }} \text { Century Learning is the } \\
\text { biggest thing right now. We } \\
\text { have got to be able to meet } \\
\text { what the community needs are. } \\
\text { There is no longer this teach- } \\
\text { regurgitate kind of thing } \\
\text { anymore. Students must be } \\
\text { able to problem solve and work } \\
\text { as a team. That's been the } \\
\text { biggest thing I noticed as a } \\
\text { change.'(IF-TA-5.12.09)" (p. } \\
\text { 244). }\end{array}$ & $\begin{array}{l}\text { INVOLVED: } \\
\text { "I think that is what it was, critical } \\
\text { thinking and problem solving } \\
\text { skills." }\end{array}$ \\
\hline $\begin{array}{c}\text { Teachers } \\
\text { collaboration \& } \\
\text { teamwork }\end{array}$ & $\begin{array}{l}\text { - Collaboration \& working } \\
\text { together for teachers: } \\
\text { "'We all knew what the other } \\
\text { was doing" (II-TI-4.20.09)" (p. } \\
\text { 285). } \\
\text { - Culture of relationships }\end{array}$ & $\begin{array}{l}\text { Dr. S.: } \\
\text { "(...) both classrooms were } \\
\text { working together as a group, they } \\
\text { would be free to taking the parent } \\
\text { over there (...)." }\end{array}$ \\
\hline $\begin{array}{l}\text { Community } \\
\text { involvement }\end{array}$ & $\begin{array}{l}\text { Community (parent) } \\
\text { involvement (go beyond } \\
\text { classroom): } \\
\text { "Every unit has some sort of } \\
\text { introduction to parents." (II-TC- } \\
\text { 5.1.09)" (p. 160). } \\
\text { - Parent newsletters } \\
\text { - Volunteers } \\
\text { - Invitations to special } \\
\text { learning events } \\
\text { - At-home read together } \\
\text { assignments } \\
\text { - Opportunities for parents to } \\
\text { become involved at school } \\
\text { and at home } \\
\text { “Once they start learning more } \\
\text { and get more involved with } \\
\text { their puppet and report, the } \\
\text { excitement grows. Parents get } \\
\text { involved with their children. } \\
\text { (...).' (II-TC-5.1.09)" (p. 306). }\end{array}$ & $\begin{array}{l}\text { INVOLVED: } \\
\text { "(...) so we had kind of guided the } \\
\text { questions for them to talk about, } \\
\text { you know, is he telling the truth? } \\
\text { Is it okay to protect a dog if you } \\
\text { think he is being abused? Is that } \\
\text { right or wrong? That is not my } \\
\text { thinking to say for you, but your } \\
\text { family needs to. So it kind of } \\
\text { brought family together }(. . .) . "\end{array}$ \\
\hline
\end{tabular}




\begin{tabular}{|c|c|c|}
\hline AIC & $\begin{array}{l}\text { Integrated arts approach is used } \\
\text { to deliver e.g. science, social } \\
\text { studies, other subjects focused } \\
\text { lessons }\end{array}$ & $\begin{array}{l}\text { INVOLVED: } \\
\text { "She became a very good reader } \\
\text { (...) her math skills became } \\
\text { stronger because of the money } \\
\text { experience." }\end{array}$ \\
\hline $\begin{array}{l}\text { Positive effects of } \\
\text { arts integration }\end{array}$ & $\begin{array}{l}\text { Arts integration provides } \\
\text { students with opportunities to } \\
\text { build confidence, esteem, and a } \\
\text { pursuit of excellence through } \\
\text { their art products }\end{array}$ & $\begin{array}{l}\text { MOTIVATED: } \\
\text { "(...) a lot of them we saw gain } \\
\text { more self-confidence because } \\
\text { when you are working in the arts, } \\
\text { sometimes there is not a black and } \\
\text { white and right and wrong answer. } \\
\text { So all of their comments were } \\
\text { validated (...)." }\end{array}$ \\
\hline Responsibility & $\begin{array}{ll}\text { - } & \text { Community awareness is } \\
\text { heightened (responsibility) } \\
\text { - } \\
\text { Instructional responsibility } \\
\text { of teachers }\end{array}$ & $\begin{array}{l}\text { MOTIVATED: } \\
\text { "Those kinds of things I am sure } \\
\text { affected girls and boys just } \\
\text { teaching them to be independent } \\
\text { and responsible, (...)." }\end{array}$ \\
\hline $\begin{array}{l}\text { Enrichment of } \\
\text { students learning }\end{array}$ & $\begin{array}{l}\text { Standard-based instruction } \\
\text { combined with } \\
\text { teachers own creativity to } \\
\text { enrich students learning } \\
\text { connections }\end{array}$ & $\begin{array}{l}\text { INOVOLVED: } \\
\text { "(...) they scored higher, but they } \\
\text { were willing to take risk and they } \\
\text { knew to look at all of them } \\
\text { because when you look at a piece } \\
\text { of art, we gave them plenty of } \\
\text { time to look at it and study it and } \\
\text { to examine all angles of it so when } \\
\text { they saw a written question on a } \\
\text { test, they were able, then, to do } \\
\text { that same thing on that test, look } \\
\text { at all of the questions, what are } \\
\text { some of the parts of the question? } \\
\text { And so I think they tested higher." }\end{array}$ \\
\hline Students no. 1 & $\begin{array}{l}\text { Students are number one for } \\
\text { teachers }\end{array}$ & $\begin{array}{l}\text { Dr. S.: } \\
\text { "(...) know the extent of the work } \\
\text { that these wonderful teachers, who } \\
\text { created an amazing classroom } \\
\text { environment and arts-focused } \\
\text { environment that reaped benefits } \\
\text { for all of the children in the room. } \\
(\ldots) . "\end{array}$ \\
\hline $\begin{array}{l}\text { Commitment to arts } \\
\text { integration }\end{array}$ & $\begin{array}{l}\text { Teachers are committed to arts } \\
\text { integration }\end{array}$ & $\begin{array}{l}\text { INVOLVED: } \\
\text { "You have to be committed to } \\
\text { that. And I think it is a big } \\
\text { commitment. It was a lot of } \\
\text { work." }\end{array}$ \\
\hline Instructional & Instructional commitment to & INVOLVED: \\
\hline
\end{tabular}




\begin{tabular}{|c|c|c|}
\hline commitment & $\begin{array}{l}\text { provide: } \\
\text { - Fun: } \\
\text { "'What to teach' translates to } \\
\text { amazing and joy" (p. 247). } \\
\text { "'It is fun for us. It's fun for } \\
\text { the teacher aide and me, and } \\
\text { it's fun for the kids.' (II-TA- } \\
\text { 4.23.09)" (p. 306). } \\
\text { - It is engaging \& provides } \\
\text { real life learning } \\
\text { experiences }\end{array}$ & $\begin{array}{l}\text { "We taught many things and other } \\
\text { people were going to get in the } \\
\text { math book, but I looked at the } \\
\text { book (...) looked at the objectives, } \\
\text { said, "This is what needs to be } \\
\text { taught. I can do it that way." }\end{array}$ \\
\hline Reflection & Reflection is necessary & $\begin{array}{l}\text { INVOLVED: } \\
\text { "(...) we don't spend enough time } \\
\text { even as adults reflecting. I think } \\
\text { we gave them that opportunity to } \\
\text { reflect on what happened, how } \\
\text { they could have done it } \\
\text { differently." }\end{array}$ \\
\hline Role-model & $\begin{array}{l}\text { - Teachers: } \\
\text { "So, I try to orchestrate being } \\
\text { a good role model with what } \\
\text { has to be done. I always tell the } \\
\text { kids, 'You know, this is } \\
\text { something that, as a fourth } \\
\text { grader, you need to know. So } \\
\text { how are we going to go about } \\
\text { getting it?' (IF-TC-6.8.09)" (p. } \\
\text { 101). } \\
\text { - Students: collaboration } \\
\text { with high school student } \\
\text { exchanges }\end{array}$ & $\begin{array}{l}\text { INVOLVED: } \\
\text { "We showed them how. We } \\
\text { would actually model. } \\
\text { MOTIVATED would come in and } \\
\text { she would start talking to } \\
\text { somebody and I would have to go } \\
\text { get her and say, "Excuse me, but I } \\
\text { need to show you this."" }\end{array}$ \\
\hline $\begin{array}{l}\text { AIC } \leftarrow \rightarrow \text { non AIC } \\
\text { (same outcome }\end{array}$ & $\begin{array}{l}\text { Different approaches, same } \\
\text { outcomes }\end{array}$ & $\begin{array}{l}\text { INVOLVED: } \\
\text { "We were just doing it in a } \\
\text { different way, but our teaching } \\
\text { was always very purposeful." }\end{array}$ \\
\hline $\begin{array}{l}\text { Lasting life } \\
\text { experience }\end{array}$ & $\begin{array}{l}\text { Arts integration as lasting life } \\
\text { experience: "'This is what we } \\
\text { all have ... humanity. You can } \\
\text { connect with them any other } \\
\text { way. There is a connection to } \\
\text { the arts that pushes students } \\
\text { forward beyond their own } \\
\text { perceived limitations.' (II-TC- } \\
5.1 .09 \text { )" (p. 251). }\end{array}$ & $\begin{array}{l}\text { INVOLVED: } \\
\text { "(...) she said he is an engineer, } \\
\text { he is going to graduate this year as } \\
\text { an engineering professor. He } \\
\text { wants to teach, anyway, but an } \\
\text { engineering degree and a math } \\
\text { degree. And she said I was his } \\
\text { favorite teacher. I said, 'Why do } \\
\text { you think that?' She said, } \\
\text { 'Because you let him express } \\
\text { himself.", }\end{array}$ \\
\hline
\end{tabular}




\begin{tabular}{|c|c|c|}
\hline Projects & $\begin{array}{l}\text { Project based approach to } \\
\text { learning }\end{array}$ & $\begin{array}{l}\text { MOTIVATED: } \\
\text { "We did lots and lots of brain } \\
\text { storming when we were in these } \\
\text { projects." }\end{array}$ \\
\hline Love for profession & $\begin{array}{l}\text { Teachers love for profession; } \\
\text { they even face stress for } \\
\text { students' sake: } \\
\text { "“(...) I love this job! My kids } \\
\text { now that. It's just who I am. I } \\
\text { can't help who I am, and that's } \\
\text { not a bad thing.' (IF-TC- } \\
6.8 .09) " \text { (p. 252). }\end{array}$ & $\begin{array}{l}\text { Teachers love for teaching was } \\
\text { passed on to students by modeling } \\
\text { teaching for them: } \\
\text { INVOLVED: } \\
\text { "So we really modeled for them } \\
\text { how to go about doing that. (...). } \\
\text { So they loved teaching that. (...)." }\end{array}$ \\
\hline Student motivation & $\begin{array}{l}\text { - Awareness of student } \\
\text { motivation } \\
\text { - S. motivation leads to } \\
\text { community \& parent } \\
\text { involvement } \\
\text { - Success supports } \\
\text { motivation: } \\
\text { “'Success spurs you on to try } \\
\text { or continue with something." } \\
\text { (IF-TK-5.11.09)" (p. 262). } \\
\text { - } \quad \text { Motivation supports student } \\
\text { learning } \\
\text { - Higher interest: } \\
\text { "(...). The girls were so } \\
\text { motivated. This was not only a } \\
\text { classroom lesson ... and then } \\
\text { when you see the Power Point } \\
\text { and the research done, there are } \\
\text { just no questions about high } \\
\text { motivation and high interest } \\
\text { levels. That is just absolutely } \\
\text { awesome.' (IF-TV-5.5.09)" (p. } \\
\text { 307). }\end{array}$ & $\begin{array}{l}\text { INVOLVED: } \\
\text { "All of a sudden, these kids were } \\
\text { reading and then we were writing } \\
\text { to these authors and asking for an } \\
\text { autographed copy for our } \\
\text { classroom. [Intrinsic Motivation]" }\end{array}$ \\
\hline $\begin{array}{l}\text { Student-driven } \\
\text { learning }\end{array}$ & $\begin{array}{l}\text { Focus on student-driven } \\
\text { learning: } \\
\text { "“As far as the plan ... a lot of } \\
\text { times, it is student driven. As } \\
\text { long as we can show that we're } \\
\text { getting through it (the } \\
\text { curriculum), then I'm OK with } \\
\text { it. (...).' (IF-TC-6.8.09)" (p. } \\
\text { 109). } \\
\text { - Instead of teacher-direction } \\
\text { (students have freedom to }\end{array}$ & $\begin{array}{l}\text { INVOLVED: } \\
\text { "They were independent. They } \\
\text { could go get themselves water, } \\
\text { water paints, brushes, whatever } \\
\text { they needed. Then they would } \\
\text { clean them up and go on to the } \\
\text { next thing. They were } \\
\text { independent that way and I guess } \\
\text { we were teaching more of that } \\
\text { independence, you know, 'You } \\
\text { are big now, you can take care of }\end{array}$ \\
\hline
\end{tabular}




\begin{tabular}{|c|c|c|}
\hline & choose) & $\begin{array}{l}\text { yourself,' rather than, 'Okay, you } \\
\text { can't paint until I get everything } \\
\text { out for you.' Of course, we started } \\
\text { like that, but then we showed } \\
\text { them how they could do that } \\
\text { themselves." }\end{array}$ \\
\hline Self-confidence & $\begin{array}{l}\text { Help students build self- } \\
\text { confidence }\end{array}$ & $\begin{array}{l}\text { Dr. S.: } \\
\text { "So when you talk about what is a } \\
\text { leader, this is a child who had } \\
\text { some confidence and I would } \\
\text { think that might be connected to } \\
\text { their self-concept." }\end{array}$ \\
\hline $\begin{array}{l}\text { Children's } \\
\text { performance }\end{array}$ & $\begin{array}{l}\text { Parents want to see their kids } \\
\text { perform: } \\
\text { "'Parents always like to see } \\
\text { their children perform, so they } \\
\text { always turn out for that" (II-TV- } \\
5.1 .09 \text { )" (p. 92). }\end{array}$ & $\begin{array}{l}\text { INVOLVED: } \\
\text { "( }(. .) \text { at the end of our unit, they } \\
\text { taught their unit to their parents so } \\
\text { they became the teacher, }(. . .) . "\end{array}$ \\
\hline Sharing & $\begin{array}{l}\text { - Kids go home and talk } \\
\text { about what they have } \\
\text { learned in school: } \\
\text { "Teaching this way was the } \\
\text { biggest resource, because the } \\
\text { children would go home and } \\
\text { speak about school happenings. } \\
\text { The kids talked to their } \\
\text { parents" (IF-TJ-5.11.09)" (p. } \\
\text { 216). } \\
\text { - Teachers share their } \\
\text { experiences with colleagues }\end{array}$ & $\begin{array}{l}\text { INVOLVED: } \\
\text { "What is lying? I am not going to } \\
\text { tell you if that is lying or not. } \\
\text { You guys talk about that at home } \\
\text { and talked about values. So that } \\
\text { was a good chance for family } \\
\text { discussions at home." }\end{array}$ \\
\hline $\begin{array}{l}\text { Volunteering } \\
\text { (Parents) }\end{array}$ & $\begin{array}{l}\text { Willingness of parents to } \\
\text { volunteer with class projects: } \\
\text { "I I think when parents speak } \\
\text { up, if we know and can ask } \\
\text { them to come in, that it is really } \\
\text { an important time to take } \\
\text { advantage of the people in the } \\
\text { community who have these } \\
\text { special skills.' (IF-TV-5.5.09)" } \\
\text { (p. 257). }\end{array}$ & $\begin{array}{l}\text { INVOLVED: } \\
\text { "If they wanted to talk to the art } \\
\text { decorator, there were two guys } \\
\text { that came in, two dads. I can't } \\
\text { remember, one was an architect } \\
\text { and one was maybe a builder. } \\
\text { They came (...)." }\end{array}$ \\
\hline Display artwork & $\begin{array}{l}\text { Arts integration products that } \\
\text { are evidence of learning are put } \\
\text { out for all to see }\end{array}$ & $\begin{array}{l}\text { Dr. S.: } \\
\text { "They had their portfolios and } \\
\text { they would take to the hallway } \\
\text { displays where the art was } \\
\text { celebrated and labeled like an art }\end{array}$ \\
\hline
\end{tabular}




\begin{tabular}{|c|c|c|}
\hline & & $\begin{array}{l}\text { gallery with their name and their } \\
\text { title. They would walk them out } \\
\text { there, 'Here is my example of my } \\
\text { self-portrait. Here is my example } \\
\text { of extending the landscaping,' and } \\
\text { here is my example of ..., and } \\
\text { things that weren't displayed were } \\
\text { in their portfolio. And they would } \\
\text { take them to their portfolios and } \\
\text { pulled out their art and show them } \\
\text { different examples." }\end{array}$ \\
\hline Different subjects & $\begin{array}{l}\text { All subjects are treated as } \\
\text { equally important }\end{array}$ & $\begin{array}{l}\text { MRD: } \\
\text { "But for you, it didn't matter if it } \\
\text { was math, reading, writing, or } \\
\text { whatever, you integrated the arts } \\
\text { in every subject, right?" } \\
\text { INVOLVED: } \\
\text { "Right (...)." }\end{array}$ \\
\hline $\begin{array}{l}\text { Goal-oriented } \\
\text { instruction }\end{array}$ & $\begin{array}{l}\text { "Despite arts integration, goal- } \\
\text { oriented instruction: } \\
\text { "I I feel like you can still teach } \\
\text { your goals and objectives } \\
\text { through play, through art, } \\
\text { through music. So I try to do } \\
\text { that. I think that is one of the } \\
\text { main things. I would want } \\
\text { people to know how I teach." } \\
\text { (IF-TK-5.11.09)" (p. 260). }\end{array}$ & $\begin{array}{l}\text { INVOLVED: } \\
\text { "(...) our teaching was always } \\
\text { very purposeful. We knew what } \\
\text { our goals were and it was - one of } \\
\text { the big goals was getting kids to } \\
\text { think about things." }\end{array}$ \\
\hline $\begin{array}{l}\text { Structure \& } \\
\text { flexibility }\end{array}$ & $\begin{array}{l}\text { Structure and flexibility coexist } \\
\text { in the arts integration } \\
\text { classroom: } \\
\text { "(...) although sometimes it } \\
\text { looks chaotic, it is organized" } \\
\text { (IF-TK-5.11.09)" (p. 141). }\end{array}$ & $\begin{array}{l}\text { INVOLVED: } \\
\text { "You don't have any discipline } \\
\text { problems. Kids were everywhere. } \\
\text { They were never in their seats - } \\
\text { they were in their seats, but they } \\
\text { were not structured, okay, this is } \\
\text { an English class, sit down and pay } \\
\text { attention, here is the page. They } \\
\text { were all getting up, doing what } \\
\text { they needed, going back to their } \\
\text { seats, proceeding to do what they } \\
\text { needed to do." }\end{array}$ \\
\hline $\begin{array}{l}\text { Multiple arts } \\
\text { exposures }\end{array}$ & $\begin{array}{l}\text { Every lesson had multiple } \\
\text { exposures to the arts (music, } \\
\text { visual arts, movement, } \\
\text { literature) }\end{array}$ & $\begin{array}{l}\text { INVOLVED: } \\
\text { "Then they were to just for } 20 \\
\text { minutes or so as they were } \\
\text { listening to really beautiful } \\
\text { classical music, they were going } \\
\text { to be observing this art." }\end{array}$ \\
\hline
\end{tabular}




\begin{tabular}{|c|c|c|}
\hline Leadership roles & $\begin{array}{l}\text { Leadership roles that served as } \\
\text { a model were taken over by } \\
\text { teachers and student teachers }\end{array}$ & $\begin{array}{l}\text { Dr. S.: } \\
\text { "(...). Both of you described these } \\
\text { were girls that were struggling in } \\
\text { some way when they came to you, } \\
\text { but what you saw they blossomed } \\
\text { into a leadership position given } \\
\text { the opportunities that you were } \\
\text { providing. (...). So when you talk } \\
\text { about what is a leader, this is a } \\
\text { child who had some confidence } \\
\text { and I would think that might be } \\
\text { connected to their self-concept." }\end{array}$ \\
\hline contributions & $\begin{array}{l}\text { Parents were valued for their } \\
\text { contributions of items sent in } \\
\text { and for assistance }\end{array}$ & $\begin{array}{l}\text { INVOLVED: } \\
\text { "(...) one parent I remember }(. . .) \\
\text { they came and wrote you a check } \\
\text { because they said, "This had to } \\
\text { cost you a lot of money." }\end{array}$ \\
\hline $\begin{array}{l}\text { Participation } \\
\text { (parents) }\end{array}$ & $\begin{array}{l}\text { Parents could participate in arts } \\
\text { integration unit activities }\end{array}$ & $\begin{array}{l}\text { INVOLVED: } \\
\text { "(...). Some of these parents came } \\
\text { and they decided they wanted to } \\
\text { paint. So }(. . .) \text { the kids and they } \\
\text { would sit down on the floor and } \\
\text { they would paint." }\end{array}$ \\
\hline Critical thinking & $\begin{array}{l}\text { Critical thinking skill were } \\
\text { acquired by the students } \\
\text { through the arts }\end{array}$ & $\begin{array}{l}\text { INVOLVED: } \\
\text { "I think if anything else, we gave } \\
\text { them critical thinking skills. That } \\
\text { is why they did so well on tests." }\end{array}$ \\
\hline Creativity & Students were creative & $\begin{array}{l}\text { INVOLVED: } \\
\text { "Oftentimes they had to look } \\
\text { beyond the painting. That - I } \\
\text { think if anything else, we gave } \\
\text { them critical thinking skills. That } \\
\text { is why they did so well on tests. } \\
\text { (...). Their creative writing was } \\
\text { better, too." }\end{array}$ \\
\hline $\begin{array}{l}\text { Interdisciplinary } \\
\text { learning }\end{array}$ & $\begin{array}{l}\text { Interdisciplinary learning took } \\
\text { place integrating various } \\
\text { subjects with each other }\end{array}$ & $\begin{array}{l}\text { INVOLVED: } \\
\text { "And that is everything we study. } \\
\text { You know, in addition to } \\
\text { integrating the arts we had a lot of } \\
\text { other things going on at that same } \\
\text { time." }\end{array}$ \\
\hline $\begin{array}{l}\text { Engaging in } \\
\text { activities }\end{array}$ & $\begin{array}{l}\text { Students were involved in } \\
\text { engaging activities }\end{array}$ & $\begin{array}{l}\text { Dr. S.: } \\
\text { "And they would wear costumes } \\
\text { almost every day and they would } \\
\text { use voices and they would use } \\
\text { mannerisms and they would }\end{array}$ \\
\hline
\end{tabular}




\begin{tabular}{|c|c|c|}
\hline & & $\begin{array}{l}\text { become a character in a book and } \\
\text { bring it to life, so constantly } \\
\text { providing a model of engaging in } \\
\text { risk taking as a character, as } \\
\text { another person, as an elaboration } \\
\text { of self and both teachers did that } \\
\text { regularly in the classroom." }\end{array}$ \\
\hline $\begin{array}{l}\text { Cross-cultural } \\
\text { content }\end{array}$ & $\begin{array}{l}\text { Cross-cultural content was } \\
\text { taught through the arts }\end{array}$ & $\begin{array}{l}\text { MOTIVATED: } \\
\text { "(...) is probably rare, that we } \\
\text { truly were multi-cultural because } \\
\text { of where we are in the } \\
\text { community, we had children from } \\
\text { many countries, and not all West } \\
\text { Virginia schools can say that. } \\
(\ldots . .) .\end{array}$ \\
\hline Blossom & $\begin{array}{l}\text { Struggling students blossomed } \\
\text { through the arts }\end{array}$ & $\begin{array}{l}\text { Dr. S.: } \\
\text { "(...). Both of you described these } \\
\text { were girls that were struggling in } \\
\text { some way when they came to you, } \\
\text { but what you saw they blossomed } \\
\text { into a leadership position given } \\
\text { the opportunities that you were } \\
\text { providing. (...)." }\end{array}$ \\
\hline $\begin{array}{l}\text { Fears about arts } \\
\text { integration }\end{array}$ & $\begin{array}{l}\text { Teachers who had no } \\
\text { experiences with the arts before } \\
\text { were scared }\end{array}$ & $\begin{array}{l}\text { INVOLVED: } \\
\text { "And I think not all teachers are } \\
\text { willing to do that. I think the } \\
\text { teacher I mentioned, she wasn't } \\
\text { comfortable." }\end{array}$ \\
\hline Real-life/ real-world & $\begin{array}{l}\text { Real-life/ real-world learning } \\
\text { experiences were experienced } \\
\text { by students (gallery walks, } \\
\text { student art exhibits, dramatic } \\
\text { plays, and musical } \\
\text { performances): } \\
\text { "We made it as real as } \\
\text { possible. (...).' (II-TI-4.20.09)" } \\
\text { (p. 285). }\end{array}$ & $\begin{array}{l}\text { INVOLVED: } \\
\text { "I think we did the art gallery, } \\
\text { what we did for that is, we had } \\
\text { maybe } 500 \text { paintings all over the } \\
\text { room. They would walk in and } \\
\text { they were entering the art gallery. } \\
\text { And we talked a little bit about we } \\
\text { accepted normal recess times, } \\
\text { when they came in, this was a new } \\
\text { experience. We kept them in the } \\
\text { hall and we talked about, 'You are } \\
\text { going to the art gallery and these } \\
\text { are some of the rules,' so they } \\
\text { knew how to behave in a formal } \\
\text { situation." }\end{array}$ \\
\hline $\begin{array}{l}\text { No Singling out of } \\
\text { struggling learners }\end{array}$ & $\begin{array}{l}\text { One is unable to single out } \\
\text { struggling learners: "“We just } \\
\text { learn ... we accepted the child. }\end{array}$ & $\begin{array}{l}\text { INVOLVED: } \\
\text { "You could not have picked her } \\
\text { out of the class to say that is the }\end{array}$ \\
\hline
\end{tabular}




\begin{tabular}{|c|c|c|}
\hline & $\begin{array}{l}\text { That's the one who came to us, } \\
\text { and that's the child we } \\
\text { accepted.' (IF-TJ-5.11.09)" (p. } \\
\text { 213). }\end{array}$ & $\begin{array}{l}\text { one. (...). The other thing, too, is } \\
\text { you would not have walked in a } \\
\text { classroom and said, 'That kid is a } \\
\text { discipline problem.' You would } \\
\text { not have found one kid. Or you } \\
\text { would not have found the kids that } \\
\text { are ADHD. You might have found } \\
\text { autistic kids (...) but most you } \\
\text { would not find (...) you could not } \\
\text { pick out the kids who were } \\
\text { ADHD, LD, BD. You would not } \\
\text { pick out-all of the labels we give } \\
\text { kids now (...) or you could not } \\
\text { pick out the talented and gifted } \\
\text { either." }\end{array}$ \\
\hline Learning styles & $\begin{array}{l}\text { Different learning styles were } \\
\text { acquired during the arts } \\
\text { integration units }\end{array}$ & $\begin{array}{l}\text { INVOLVED: } \\
\text { "We were studying learning } \\
\text { styles. We were studying student- } \\
\text { based learning, so all of these } \\
\text { things came together for us as } \\
\text { professionals. It was very exciting } \\
\text { because we got to do all of that } \\
\text { together." }\end{array}$ \\
\hline Dressing up & $\begin{array}{l}\text { Teachers taught in dresses/ } \\
\text { costumes to engage and } \\
\text { motivate their students }\end{array}$ & $\begin{array}{l}\text { MOTIVATED: } \\
\text { "They also imitated us (...), } \\
\text { because we would dress up. They } \\
\text { could tell when they walked in in } \\
\text { the morning, we often are dressed } \\
\text { - gave them clues about what was } \\
\text { going to happen that day." }\end{array}$ \\
\hline Risk taking & $\begin{array}{l}\text { Teachers supported the students } \\
\text { in becoming comfortable in risk } \\
\text { taking: } \\
\text { "We were taking risks, and we } \\
\text { had to make it comfortable for } \\
\text { them to take risks, and we knew } \\
\text { that." (II-TK-4.20.09)" (p. 280). }\end{array}$ & $\begin{array}{l}\text { Dr. S.: } \\
\text { "(...) so constantly providing a } \\
\text { model of engaging in risk taking } \\
\text { as a character, as another person, } \\
\text { as an elaboration of self and both } \\
\text { teachers did that regularly in the } \\
\text { classroom. (...) It was full of that } \\
\text { risk-taking behavior that was } \\
\text { being modeled by the leader in the } \\
\text { classroom. So then the children } \\
\text { had permission to take risks, too." }\end{array}$ \\
\hline $\begin{array}{l}\text { Dramatic } \\
\text { productions }\end{array}$ & $\begin{array}{l}\text { Students got the chance to } \\
\text { experience and participate in } \\
\text { dramatic productions }\end{array}$ & $\begin{array}{l}\text { MOTIVATED: } \\
\text { "And she then loved the dressing } \\
\text { up and the acting and she would } \\
\text { take on like a leadership-she } \\
\text { would want to do more." }\end{array}$ \\
\hline
\end{tabular}




\begin{tabular}{|c|c|c|}
\hline $\begin{array}{l}\text { Observation } \\
\text { practices }\end{array}$ & $\begin{array}{l}\text { Students underwent observation } \\
\text { practices (e.g. in art galleries) }\end{array}$ & $\begin{array}{l}\text { MOTIVATED: } \\
\text { "That was one thing that we } \\
\text { noticed, is that observation is } \\
\text { really a key thing." }\end{array}$ \\
\hline $\begin{array}{l}\text { Diversity \& respect/ } \\
\text { tolerance }\end{array}$ & $\begin{array}{l}\text { Activities were provided that } \\
\text { helped students to value one } \\
\text { another's diversity and respect } \\
\text { each other = culture of respect } \\
\text { and tolerance }\end{array}$ & $\begin{array}{l}\text { MOTIVATED: } \\
\text { "One of the things is that just } \\
\text { tolerance meant to respect each } \\
\text { other's differences, }(. . .) \text { we had } \\
\text { posters of kids demonstrating } \\
\text { tolerance. (...)." }\end{array}$ \\
\hline $\begin{array}{l}\text { Special needs \& } \\
\text { gifted }\end{array}$ & $\begin{array}{l}\text { "Teaching with art integration } \\
\text { opened up a 'whole range of } \\
\text { abilities in the classroom" (...)" } \\
\text { (p. 286). } \\
\text { All students worked on similar } \\
\text { items: } \\
\text { "So, if a gifted child was doing } \\
\text { a writing peace, we encouraged } \\
\text { him to go beyond what third } \\
\text { graders would do. If a child had } \\
\text { deeper challenges, we would } \\
\text { kneel at the desk and help them } \\
\text { physically move a pencil.' (IF- } \\
\text { TI-5.11.09)" (p. 30). }\end{array}$ & $\begin{array}{l}\text { Dr. S.: } \\
\text { "But they all had to be briefed } \\
\text { with a significant adult. They } \\
\text { made sure they had somebody, } \\
\text { everybody had somebody." }\end{array}$ \\
\hline $\begin{array}{l}\text { Personal time \& } \\
\text { resources }\end{array}$ & $\begin{array}{l}\text { Teachers spent personal time } \\
\text { researching content and } \\
\text { designing instructional } \\
\text { activities: } \\
\text { "'I took personal time to find } \\
\text { ways to connect the community } \\
\text { and instructional resources into } \\
\text { the lesson plans"” (p. 286). }\end{array}$ & $\begin{array}{l}\text { INVOLVED: } \\
\text { "It did. [cost a lot of money]." } \\
\text { Dr. S.: } \\
\text { "Yes, it was [a lot of work]. We } \\
\text { would meet at 7:00 in the morning } \\
\text { to plan and after school and } \\
\text { weekends, late to the evening, we } \\
\text { would be meeting and planning } \\
\text { together." }\end{array}$ \\
\hline Detail & $\begin{array}{l}\text { Students learned to pay } \\
\text { attention to detail: } \\
\text { "Look closely at the entire vase } \\
\text { of flowers. Select one flower } \\
\text { from the arrangement and water } \\
\text { color just that one flower"" (p. } \\
289) \text {. }\end{array}$ & $\begin{array}{l}\text { INVOLVED: } \\
\text { "Look in the mirror for } 15 \\
\text { minutes. At first you see yourself } \\
\text { and then you begin to see all of } \\
\text { the different things, uniqueness } \\
\text { about me that is different than you } \\
\text { and you start looking at your nose } \\
\text { and you eye lashes and eyebrows } \\
\text { and lips and they start really } \\
\text { examining it. So then when you } \\
\text { draw, "I didn't know my nose was } \\
\text { that big." }\end{array}$ \\
\hline Adjustment of & Schedules of students were & Dr. S.: \\
\hline
\end{tabular}




\begin{tabular}{|c|c|c|}
\hline schedules & $\begin{array}{l}\text { adjusted to create more time for } \\
\text { certain activities }\end{array}$ & $\begin{array}{l}\text { "You rearranged the schedule for } \\
\text { the whole day so the parents could } \\
\text { come at any point in the day to } \\
\text { have that full hour with their own } \\
\text { child, boy or girl to get debriefed." }\end{array}$ \\
\hline Process \& product & $\begin{array}{l}\text { Process and product are one } \\
\text { unit. One has to go through the } \\
\text { process to receive a product }\end{array}$ & $\begin{array}{l}\text { Dr. S.: } \\
\text { "Well, you shared your critical } \\
\text { examination of yourself out loud, } \\
\text { so that whole thinking process } \\
\text { was exposed to them before they } \\
\text { did it. They saw that as what you } \\
\text { were thinking through." }\end{array}$ \\
\hline $\begin{array}{l}\text { "Teacher-Learner } \\
\text { exchange principle } \\
\text { (TLE)", (DEVONO, } \\
\text { 2009) }\end{array}$ & $\begin{array}{l}\text { "A reciprocal dynamic, which I } \\
\text { call the 'teacher-learner } \\
\text { exchange principle,' is so } \\
\text { powerful that it is almost } \\
\text { tangible" (p. 305). }\end{array}$ & \\
\hline
\end{tabular}

Note: All of Douglas' (2011) quotes for findings are taken from the transcript for interview number two (T-T\&L-INVOLVED, MOTIVATED, \& Dr. S.-03.05.2011). 